Volume 3 • Number $1 \cdot$ March 2020

\title{
CORONAVIRUS EDITION
}

\section{JOURNAL OF BIOENGINEERING}

\section{AND TECHNOLOGY APPLIED TO HEALTH}

SENAI Institute of Innovation in Advanced Health Systems - ISI/SENAI CIMATEC

\author{
Appendix 1 \\ EUA Case Study Database \\ WHO Case Study Database \\ EDITOR-IN-CHIEF \\ Leone Peter Andrade \\ PUBLISHED BY SENAI CIMATEC \\ Sistema FIEB \\ SENAI \\ CIMATEC \\ PELO FUTURO DA INOVAÇÃo \\ DOI 10.34178/jbth.v3iA1.127
}

March 2020

Printed in Brazil 


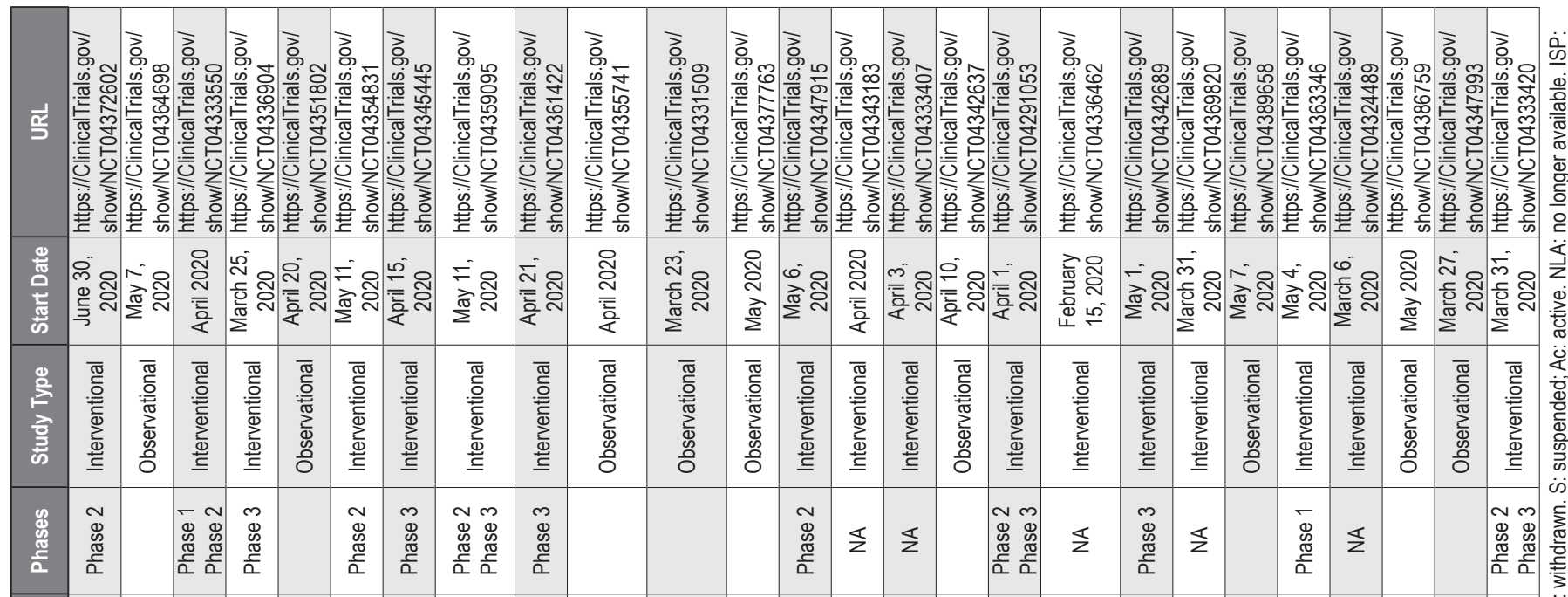

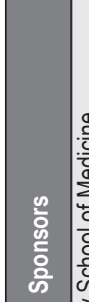

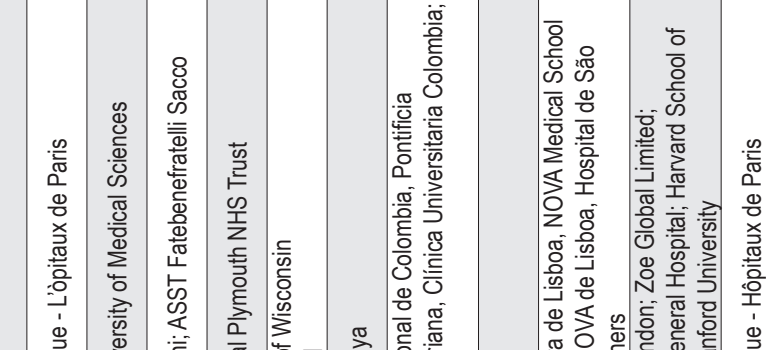

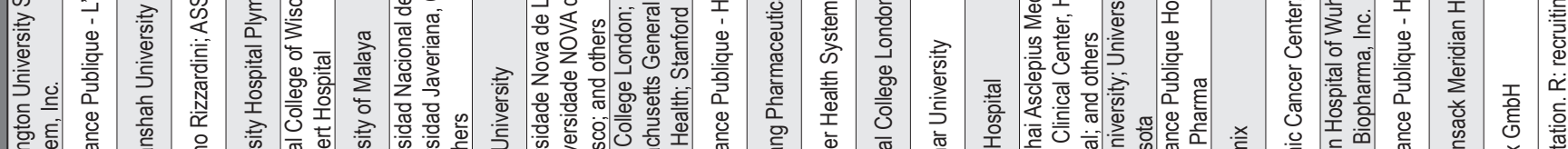

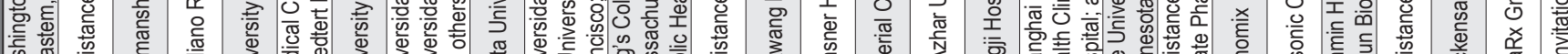

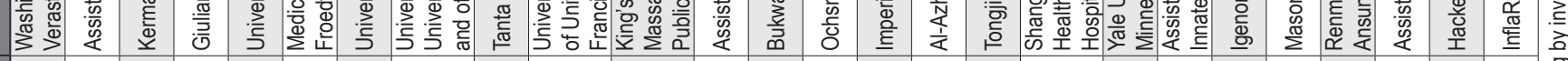

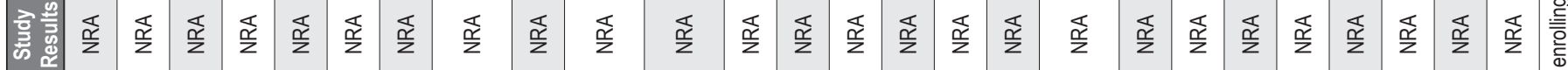

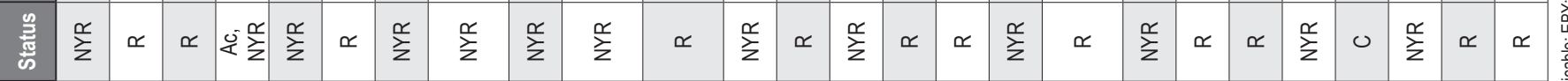

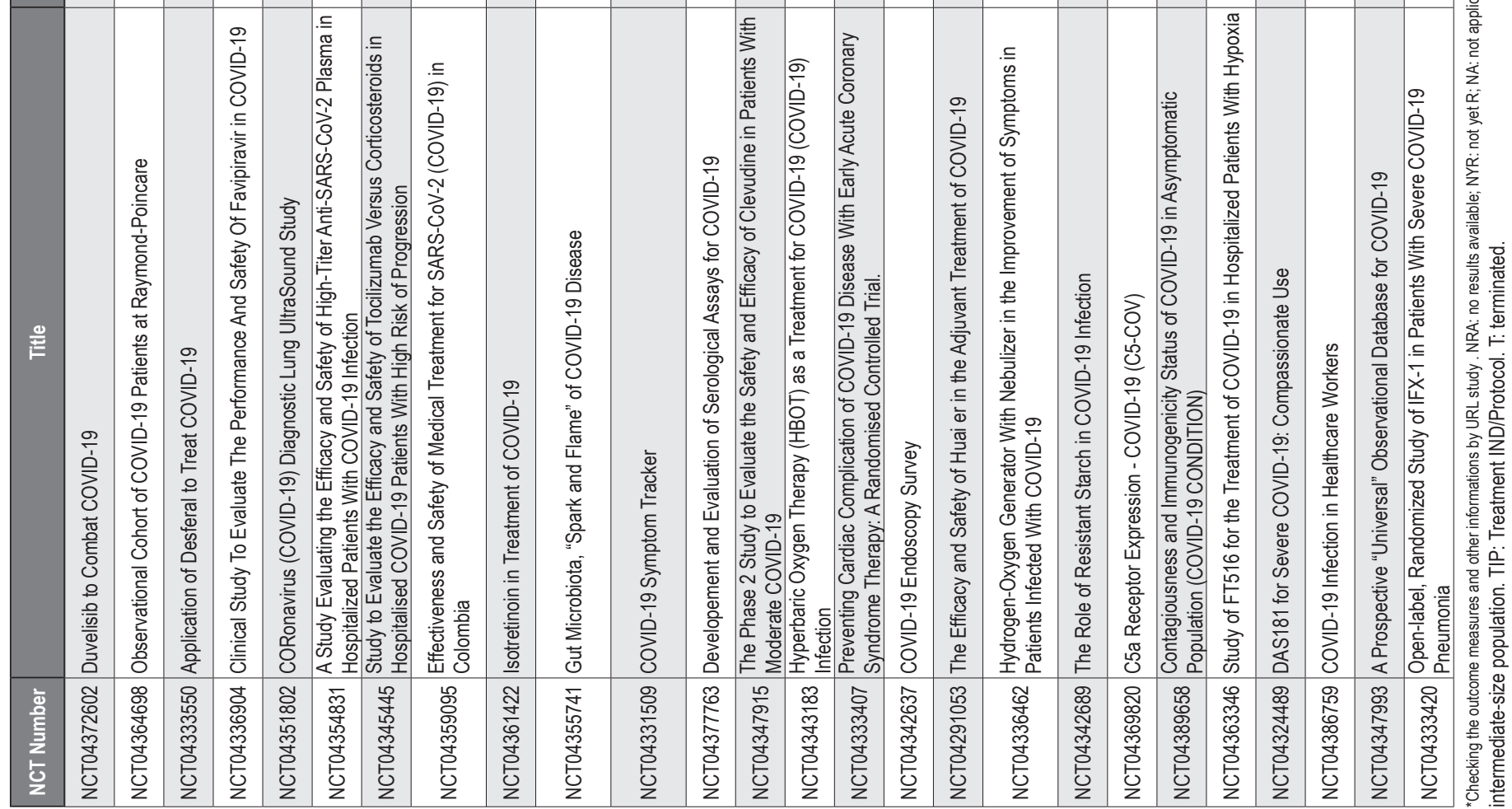




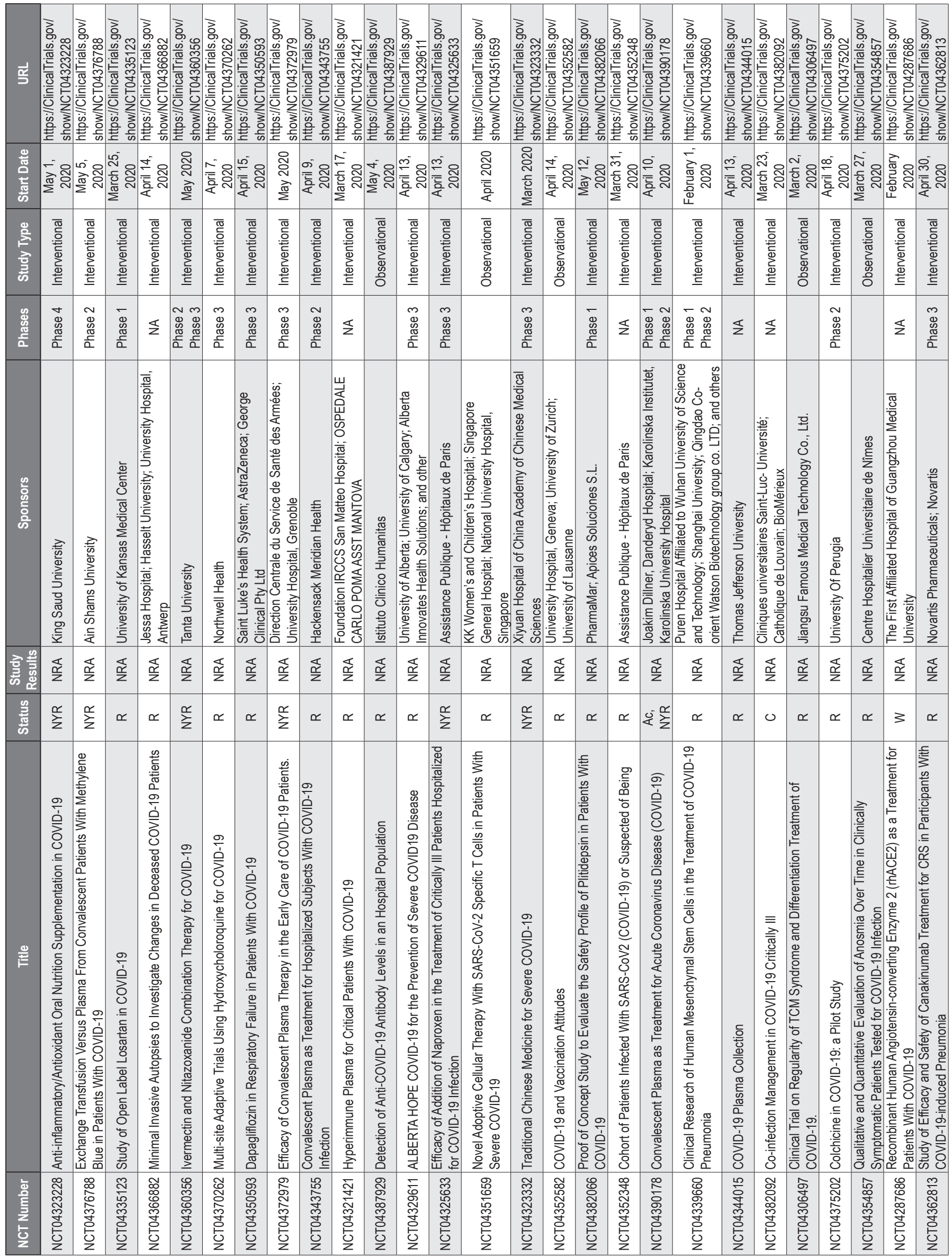




\begin{tabular}{|c|c|c|c|c|c|c|c|c|c|c|c|c|c|c|c|c|c|c|c|c|c|c|c|c|c|}
\hline$\frac{\vec{b}}{5}$ & 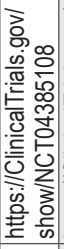 & 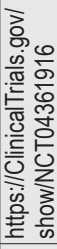 & & 5 & & 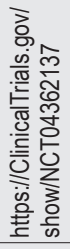 & 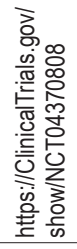 & 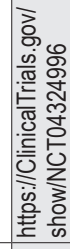 & 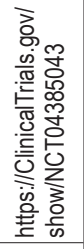 & 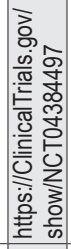 & 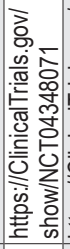 & & & 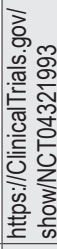 & 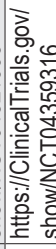 & 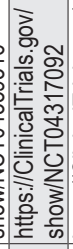 & 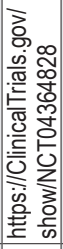 & $\Phi \frac{\delta}{\omega}$ & 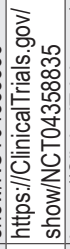 & 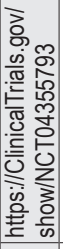 & 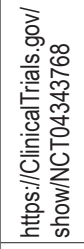 & 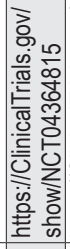 & 豙 & 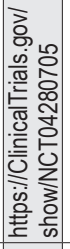 & 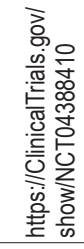 \\
\hline & 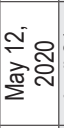 & & & & & 产 & 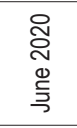 & 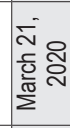 & & 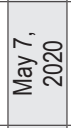 & $\begin{array}{l}\text { 令 } \\
\text { w } \\
\text { ș }\end{array}$ & 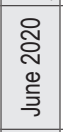 & & & î: & 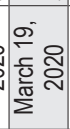 & 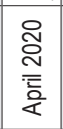 & & ĩ & & कृ & $\begin{array}{l}\text { ते } \\
\stackrel{N}{\mathrm{~J}} \\
\sum\end{array}$ & & 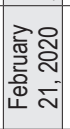 & 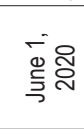 \\
\hline 迳 & 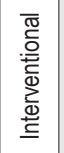 & 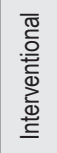 & 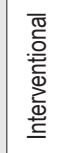 & 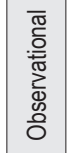 & 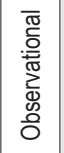 & 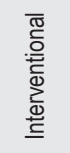 & 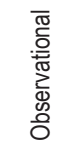 & 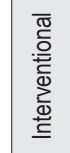 & 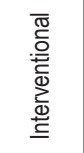 & 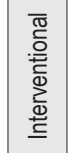 & 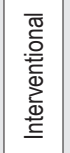 & 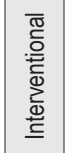 & 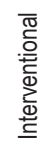 & 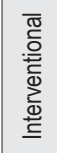 & 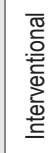 & 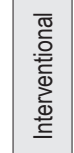 & 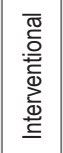 & 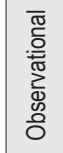 & 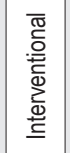 & $\stackrel{n}{F}$ & 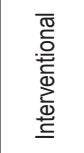 & 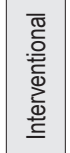 & 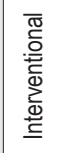 & 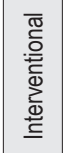 & 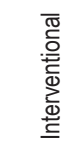 \\
\hline 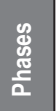 & 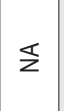 & $\frac{\pi}{z}$ & 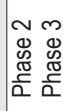 & & & 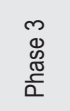 & & 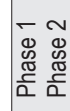 & 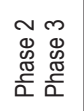 & $\stackrel{8}{\mathscr{I}}$ & $\alpha$ & & $\begin{array}{l}\mathscr{\Phi} \\
\frac{\mathscr{m}}{\frac{0}{\alpha}}\end{array}$ & 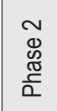 & 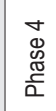 & $\begin{array}{l}\widetilde{N} \\
\mathscr{O} \\
\frac{\pi}{\alpha} \\
\frac{\pi}{\alpha}\end{array}$ & $\Sigma$ & & $\Sigma$ & & 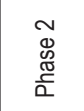 & $\begin{array}{l}m \\
\Phi \\
\Phi \\
\frac{\Phi}{0} \\
\frac{\pi}{\alpha}\end{array}$ & \begin{tabular}{|c}
$\tilde{D}$ \\
$\mathbb{o}$ \\
$\frac{\delta}{\alpha}$ \\
$\frac{\delta}{\alpha}$
\end{tabular} & 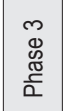 & 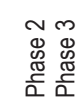 \\
\hline
\end{tabular}
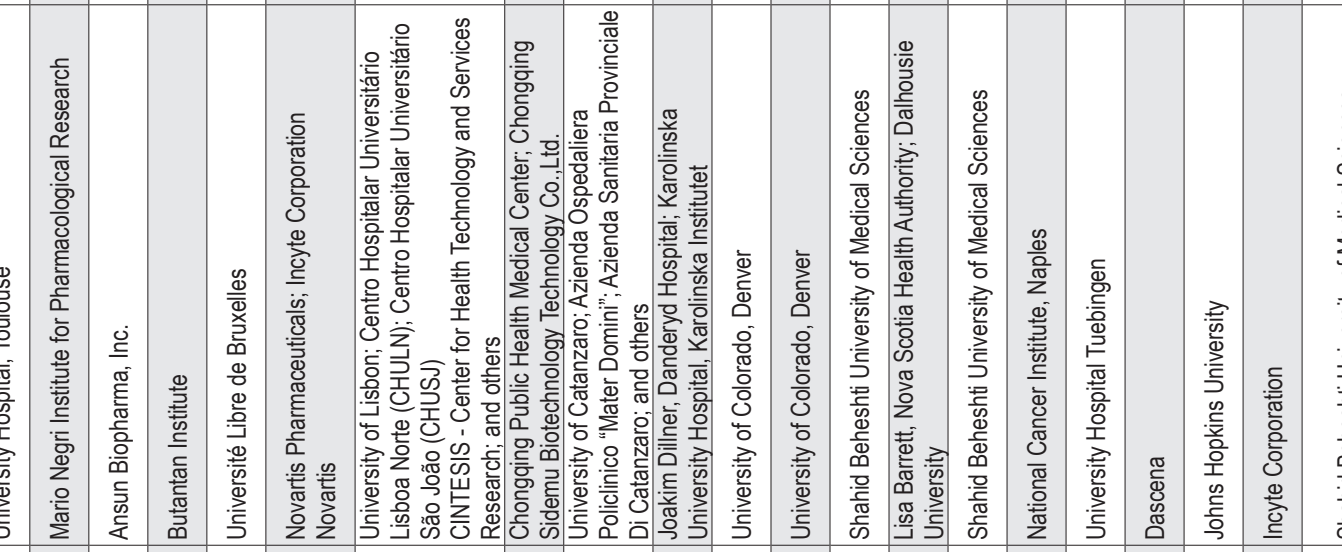

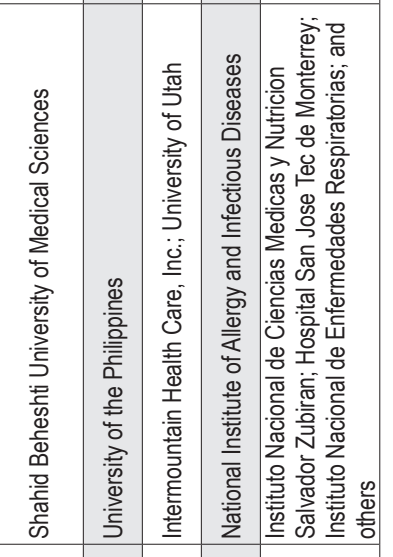

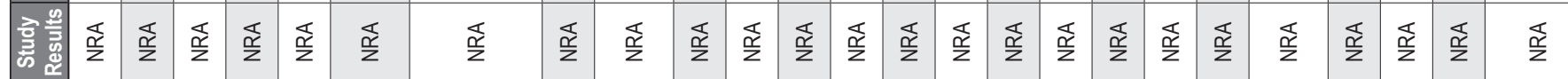

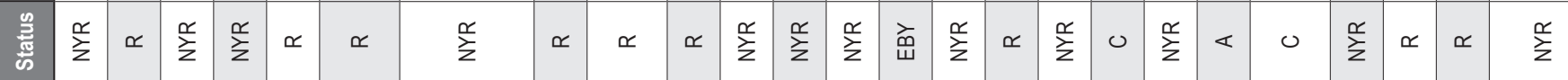

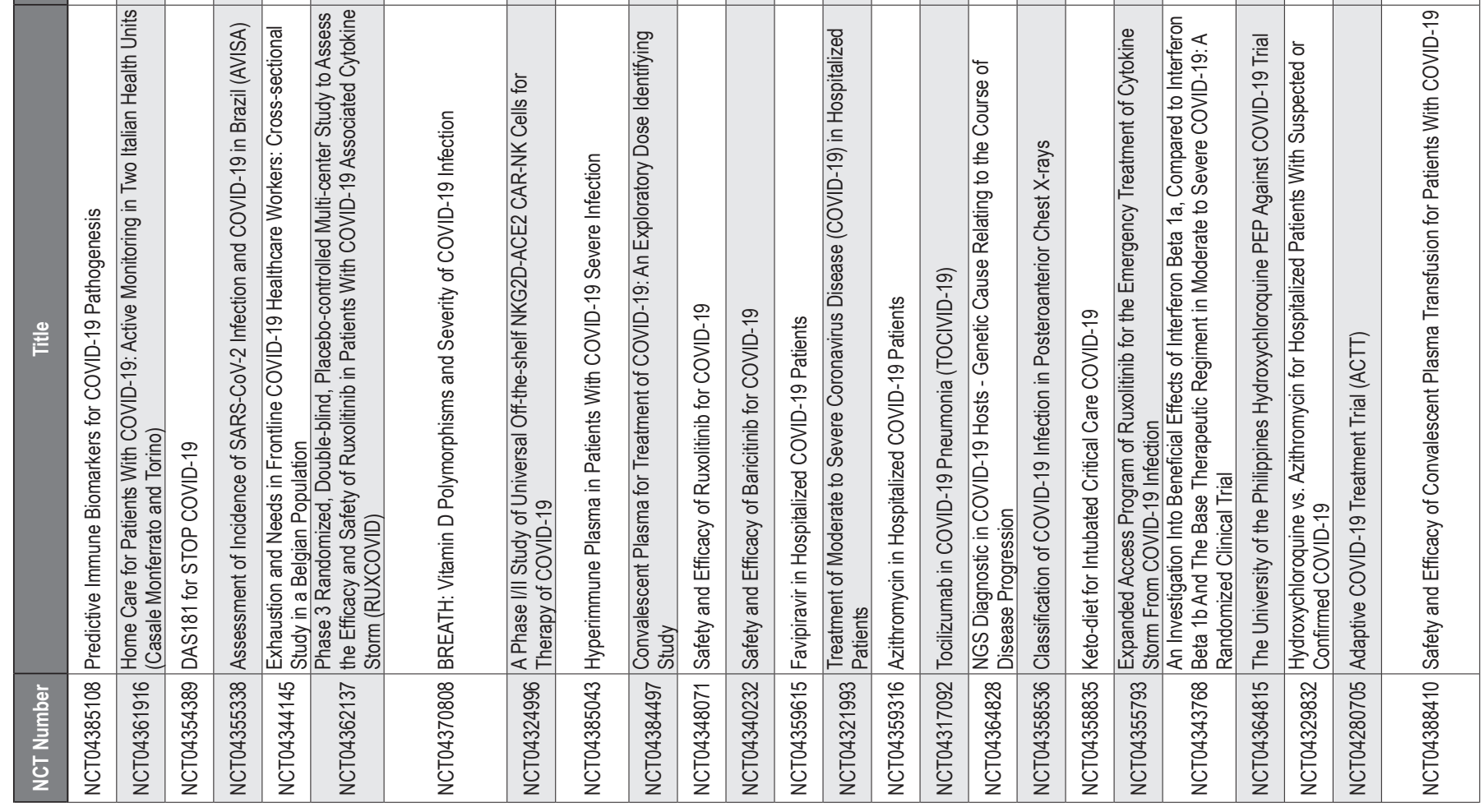




\begin{tabular}{|c|c|c|c|c|c|c|c|c|c|c|c|c|c|c|c|c|c|c|c|c|c|c|c|c|c|}
\hline & 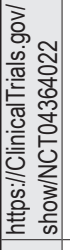 & 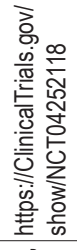 & 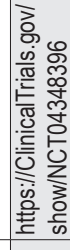 & 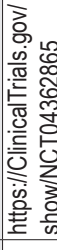 & & $\bar{z}$ & $\frac{\infty}{\pi}$ & 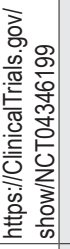 & 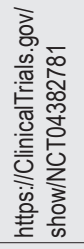 & 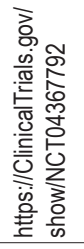 & 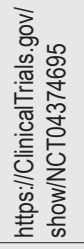 & 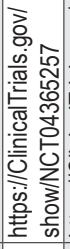 & $\bar{z}$ & & & & & & $\pm \frac{\omega}{\omega}$ & 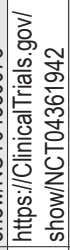 & 定 & 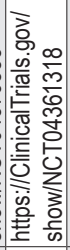 & 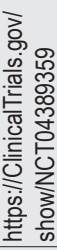 & & \\
\hline & 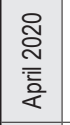 & 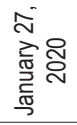 & 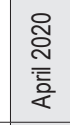 & & & & & & 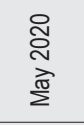 & 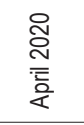 & 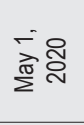 & & & & & & & & & $\begin{array}{c}\text { 令 } \\
\text { 言 } \\
\text { 定 }\end{array}$ & 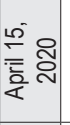 & 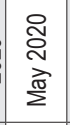 & 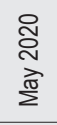 & & \\
\hline$\stackrel{2}{2}$ & 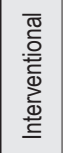 & 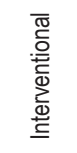 & 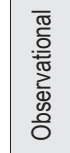 & 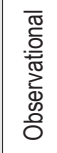 & 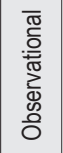 & 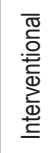 & 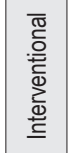 & 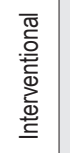 & 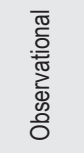 & 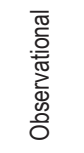 & 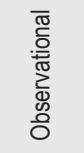 & 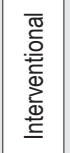 & 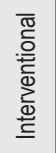 & 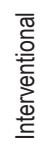 & 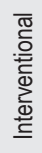 & 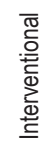 & 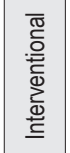 & 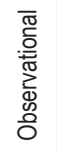 & 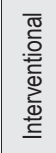 & 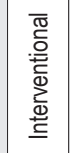 & 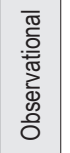 & 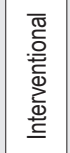 & 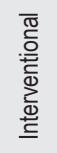 & 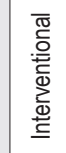 & \\
\hline & 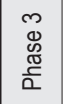 & 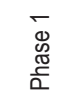 & & & & 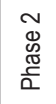 & 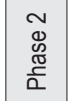 & 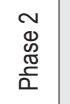 & & & & $\begin{array}{l}\mathbb{N} \\
\mathbb{o} \\
\mathbb{0} \\
\frac{\mathbb{c}}{\alpha}\end{array}$ & 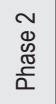 & 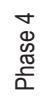 & 遇 & $\begin{array}{l}m \\
\mathbb{\infty} \\
\mathbb{8} \\
\frac{\pi}{0} \\
\frac{0}{\alpha}\end{array}$ & 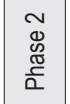 & & $\frac{\pi}{z}$ & 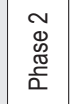 & & & & & \\
\hline
\end{tabular}



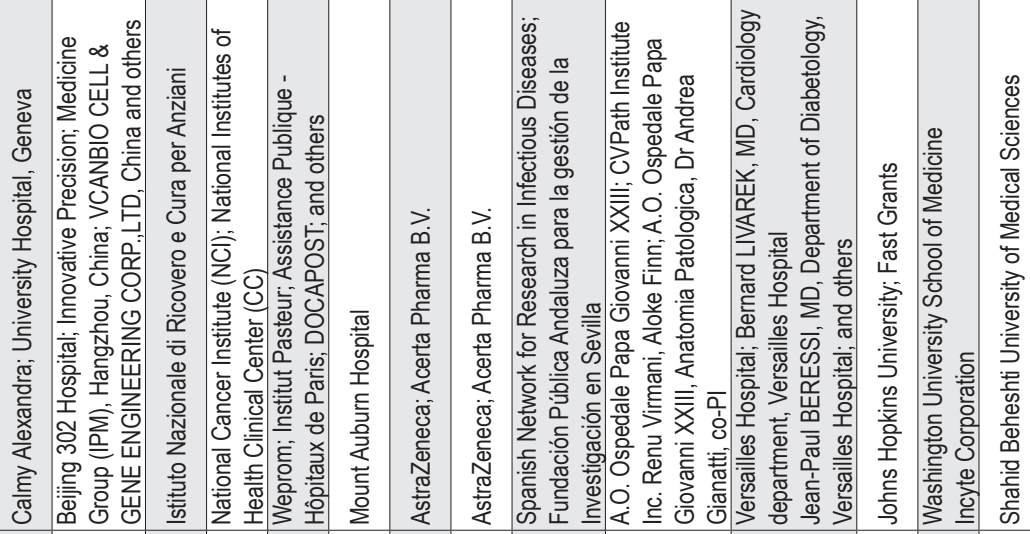

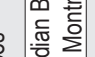
贾

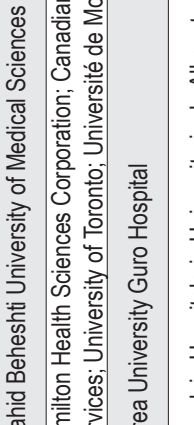

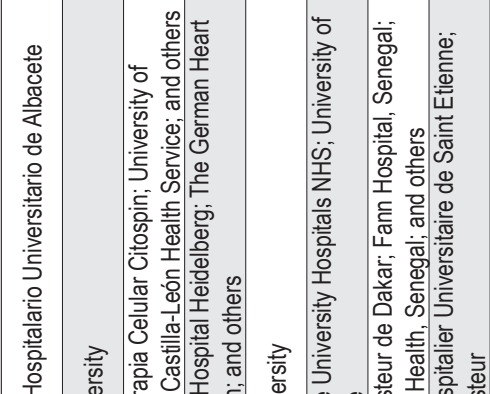

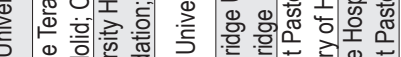

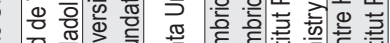

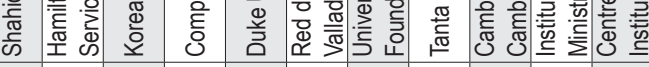

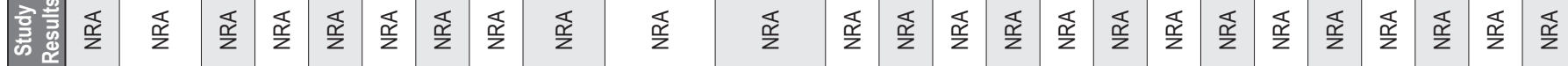

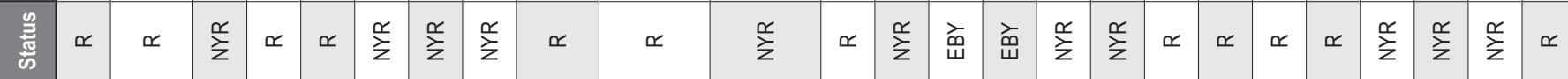

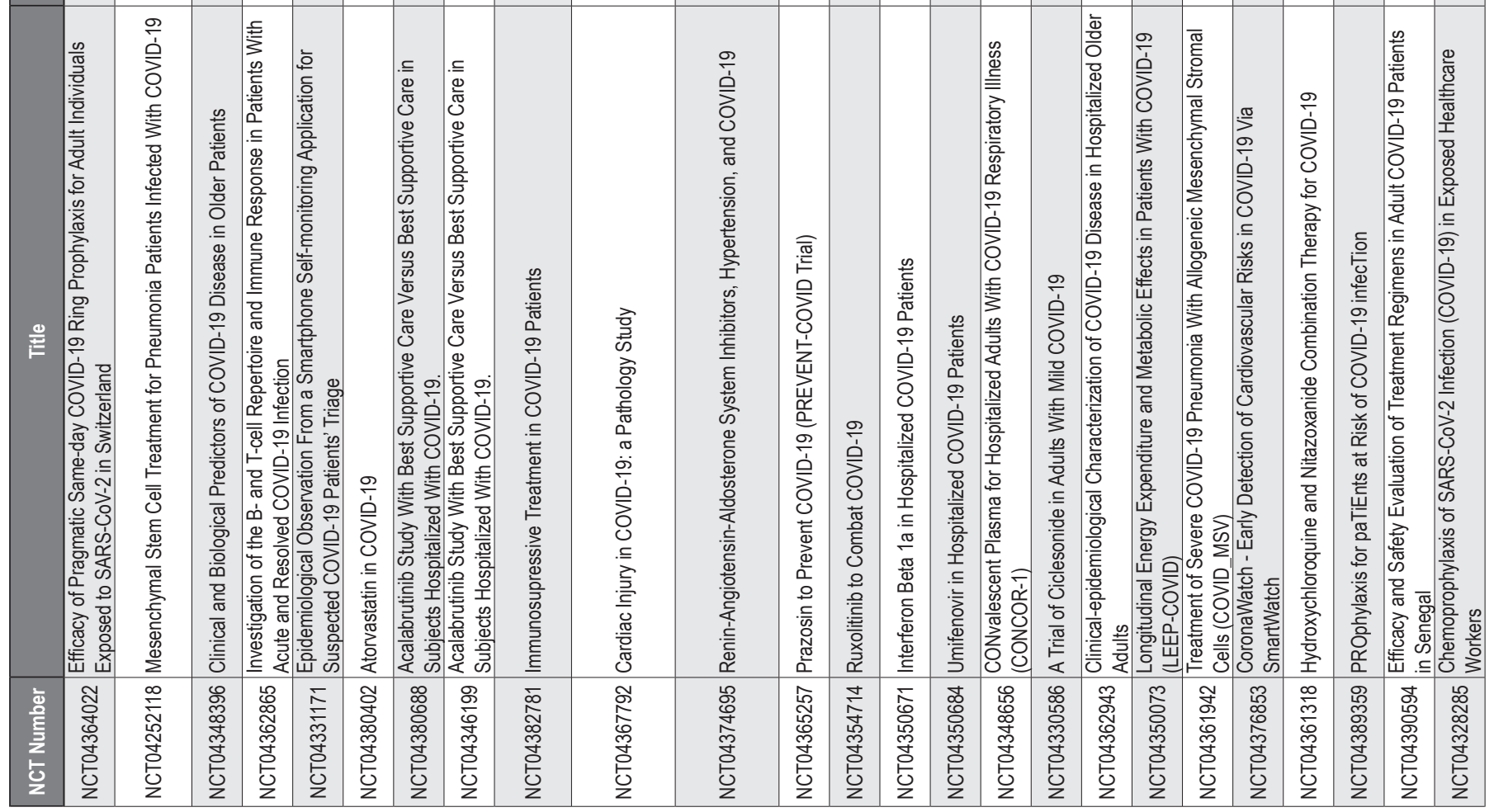




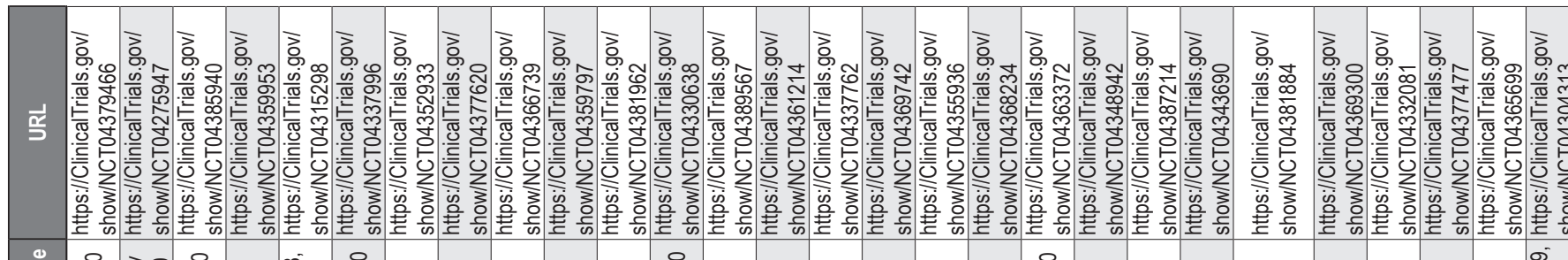

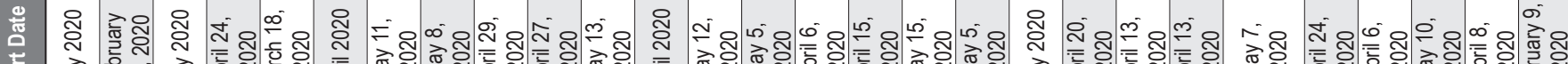

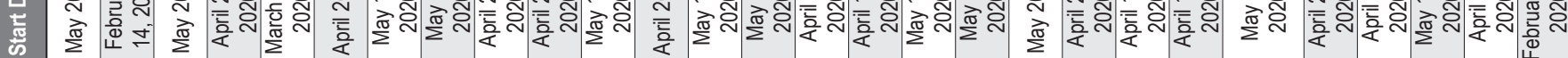

\begin{tabular}{|c|c|c|c|c|c|c|c|c|c|c|c|c|c|c|c|c|c|c|c|c|c|c|c|c|c|c|c|}
\hline$\overline{\mathrm{\sigma}} \overline{\mathrm{\sigma}}$ & 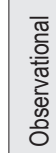 & 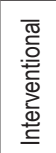 & 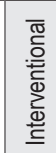 & 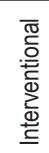 & 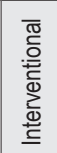 & 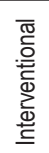 & 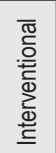 & 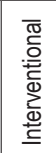 & 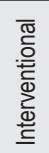 & 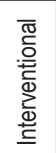 & 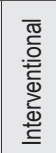 & 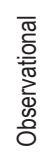 & 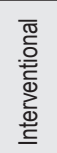 & 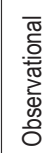 & 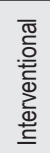 & 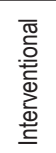 & 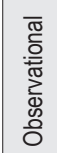 & 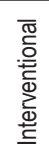 & 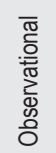 & 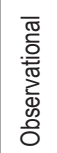 & 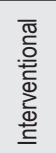 & 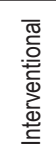 & 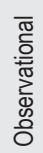 & 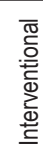 & 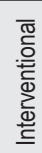 & 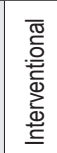 & 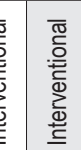 \\
\hline
\end{tabular}

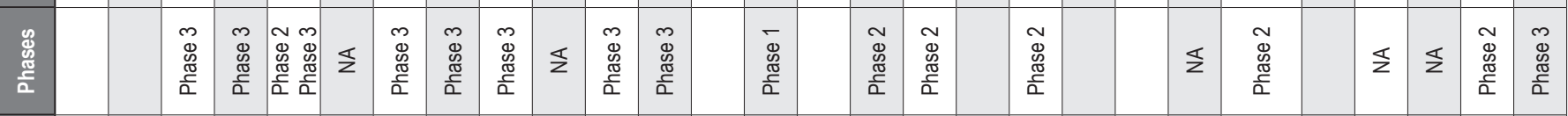

(2)

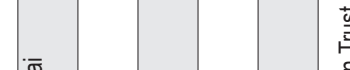

产

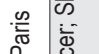

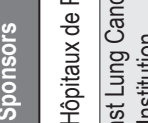

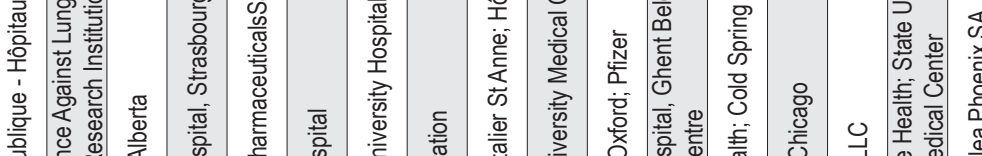

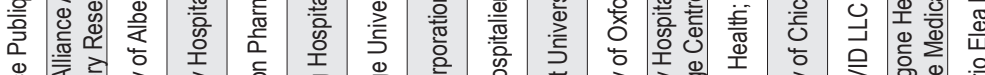

还

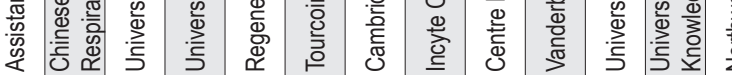

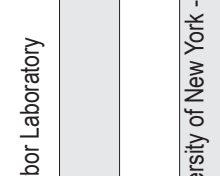

童

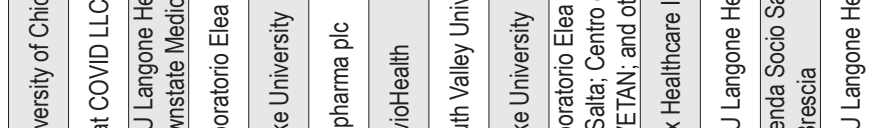

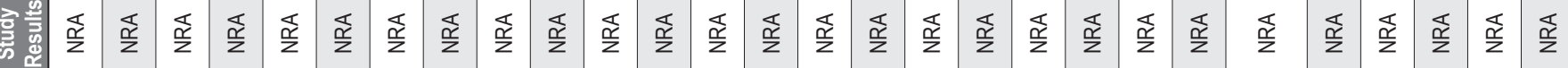
总

\begin{tabular}{|c|c|c|c|c|c|c|c|c|c|c|c|c|c|c|c|c|c|c|c|c|c|c|c|c|c|c|}
\hline 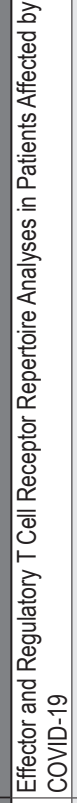 & 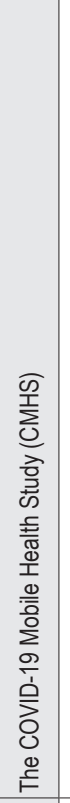 & 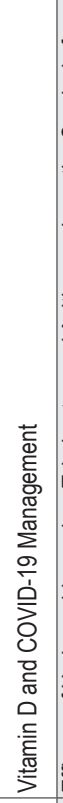 & 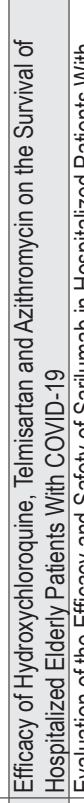 & 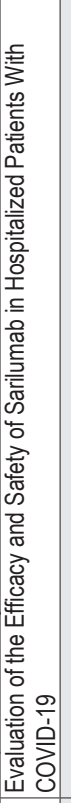 & 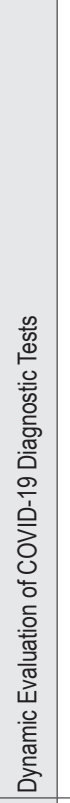 & 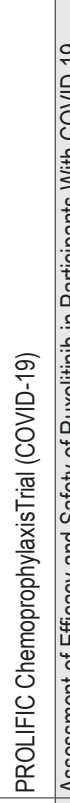 & 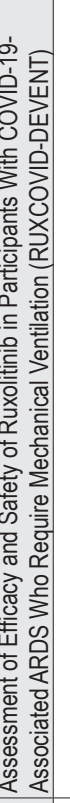 & 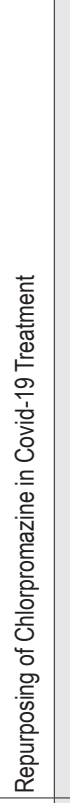 & 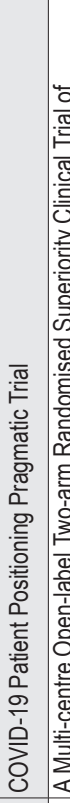 & 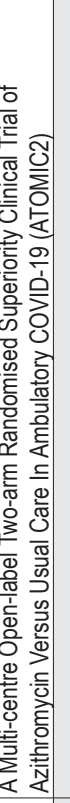 & 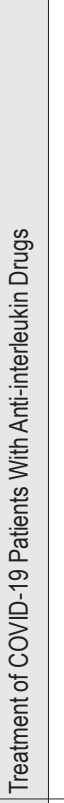 & 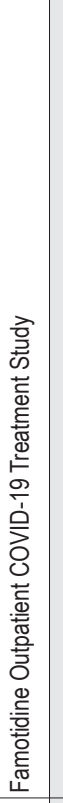 & 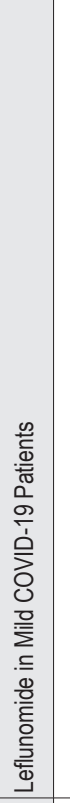 & 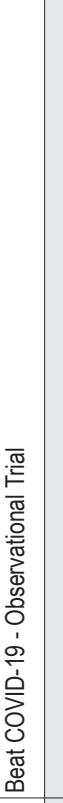 & 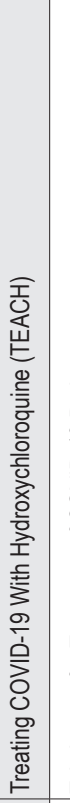 & 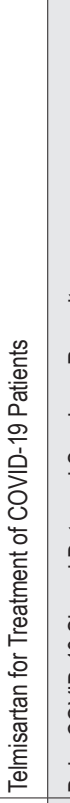 & 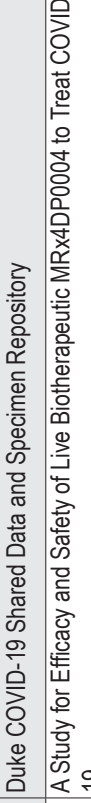 & 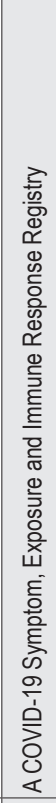 & 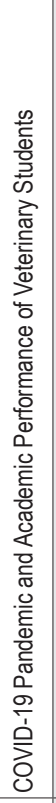 & 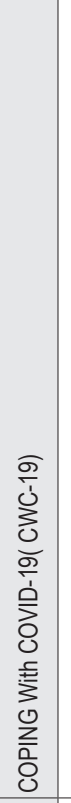 & 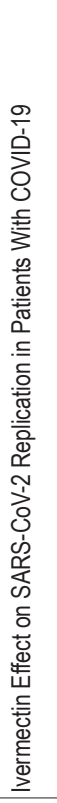 & 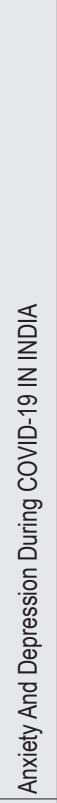 & 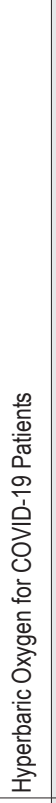 & 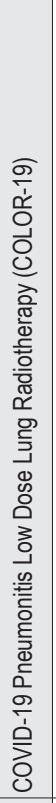 & 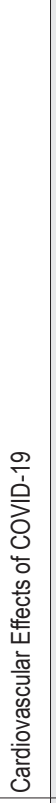 & \\
\hline 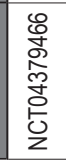 & 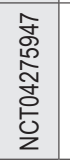 & 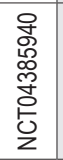 & 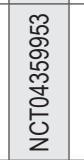 & 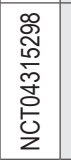 & 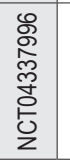 & 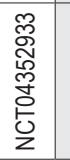 & z & 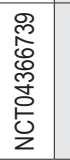 & 勇 & $z$ & 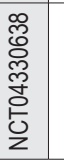 & 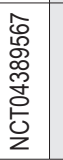 & 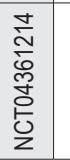 & 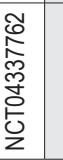 & 志 & 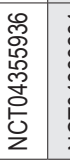 & 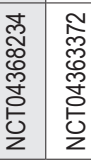 & $\begin{array}{l}\text { 怘 } \\
\text { dog } \\
\text { d }\end{array}$ & 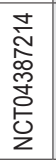 & $\begin{array}{l}\text { : } \\
\text { : }\end{array}$ & $\begin{array}{l}\infty \\
\stackrel{\infty}{0}\end{array}$ & 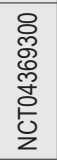 & 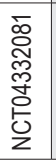 & 喜 & প্. & \\
\hline
\end{tabular}




\begin{tabular}{|c|c|c|c|c|c|c|c|c|c|c|c|c|c|c|c|c|c|c|c|c|c|c|c|c|c|c|}
\hline & 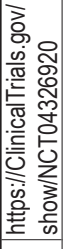 & 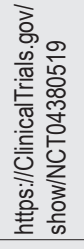 & 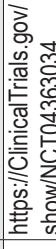 & & & & & 高 & 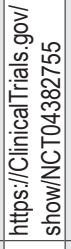 & 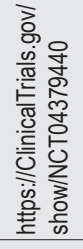 & 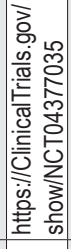 & 吾 & 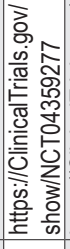 & & 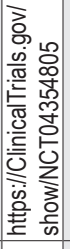 & 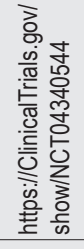 & 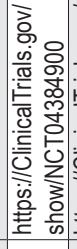 & $\Sigma \bar{\omega}$ & 高 & & & 总 & $f \infty$ & 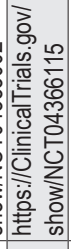 & 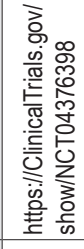 & 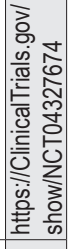 \\
\hline & 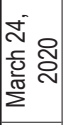 & 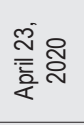 & & & & & & & 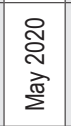 & 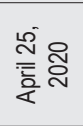 & 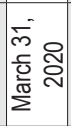 & & & & 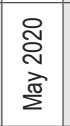 & 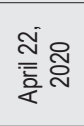 & & & & & & $\frac{j}{2}$ & 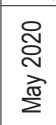 & 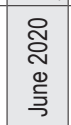 & 家京 & 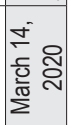 \\
\hline & 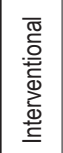 & 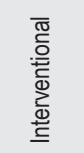 & $\underline{0}$ & 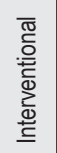 & 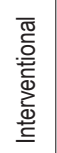 & 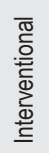 & 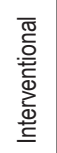 & $\underline{\underline{0}}$ & 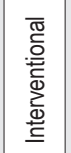 & 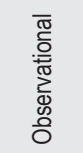 & 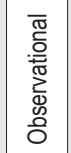 & 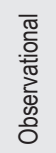 & 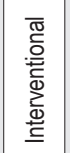 & 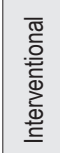 & 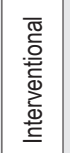 & 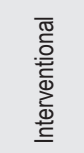 & 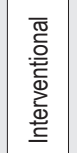 & 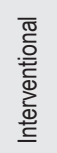 & 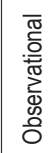 & 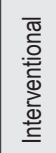 & 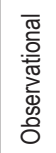 & 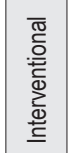 & 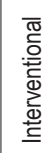 & 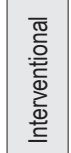 & 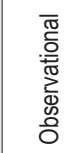 & $\bar{\sigma}$ \\
\hline & \begin{tabular}{|l|} 
\\
$\Phi$ \\
$\Phi$ \\
$\frac{\pi}{\alpha}$ \\
$\frac{0}{\alpha}$ \\
\end{tabular} & 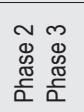 & & 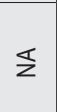 & $\begin{array}{l}\tilde{D} \\
\Phi \\
\frac{\pi}{\alpha} \\
\frac{\pi}{\alpha}\end{array}$ & ఖ & $\Sigma$ & & $\begin{array}{l}\tilde{N} \\
\mathscr{8} \\
\frac{\sigma}{\alpha} \\
\frac{\pi}{\alpha}\end{array}$ & & & & 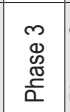 & & & $\begin{array}{l}\text { क } \\
\Phi \\
\frac{\pi}{0} \\
\frac{\pi}{\alpha}\end{array}$ & $\Sigma$ & $\begin{array}{l}\widetilde{N} \\
\stackrel{8}{0} \\
\frac{\pi}{\alpha}\end{array}$ & & $\frac{\$}{z}$ & & $\Sigma$ & \begin{tabular}{|l}
$\overline{8}$ \\
$\stackrel{g}{\alpha}$ \\
$\bar{\alpha}$
\end{tabular} & $\mid \begin{array}{l}\mathscr{\Phi} \\
\frac{\pi}{\alpha} \\
\frac{\pi}{\alpha} \\
0\end{array}$ & & \\
\hline
\end{tabular}
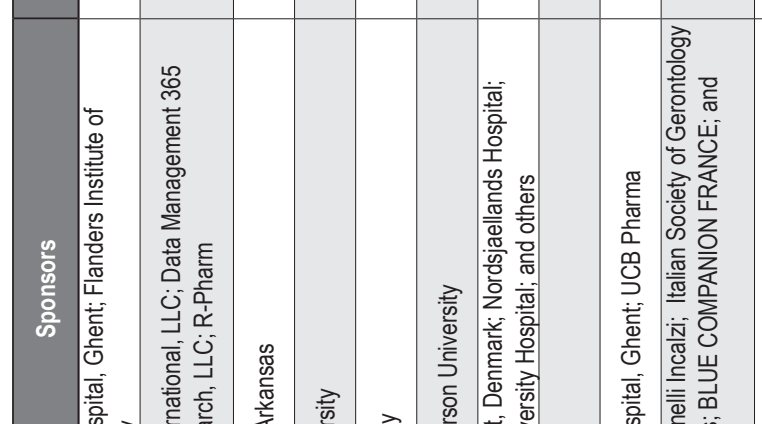

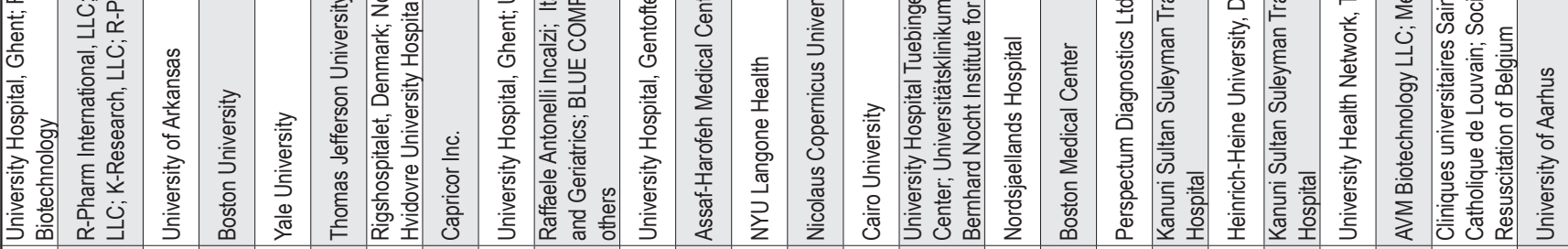

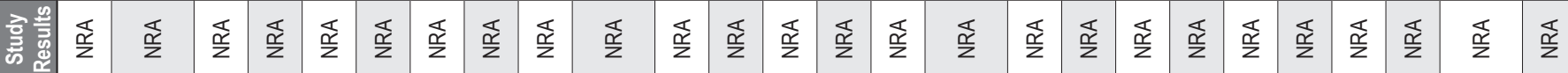

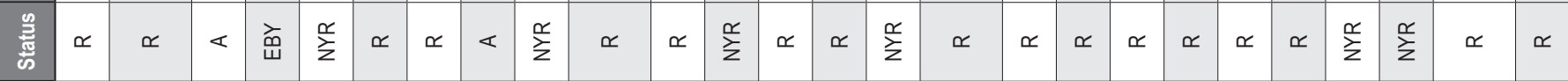

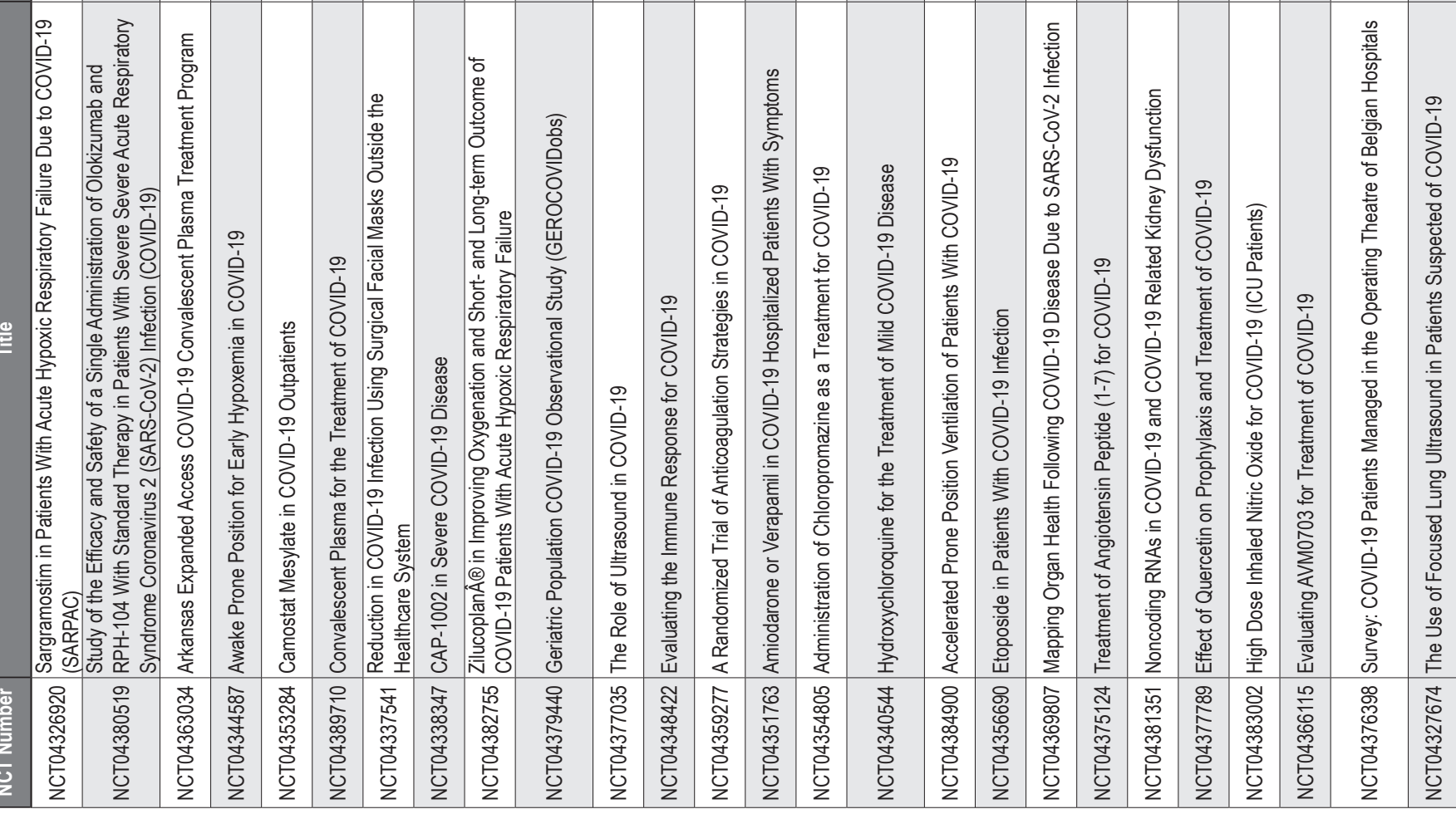




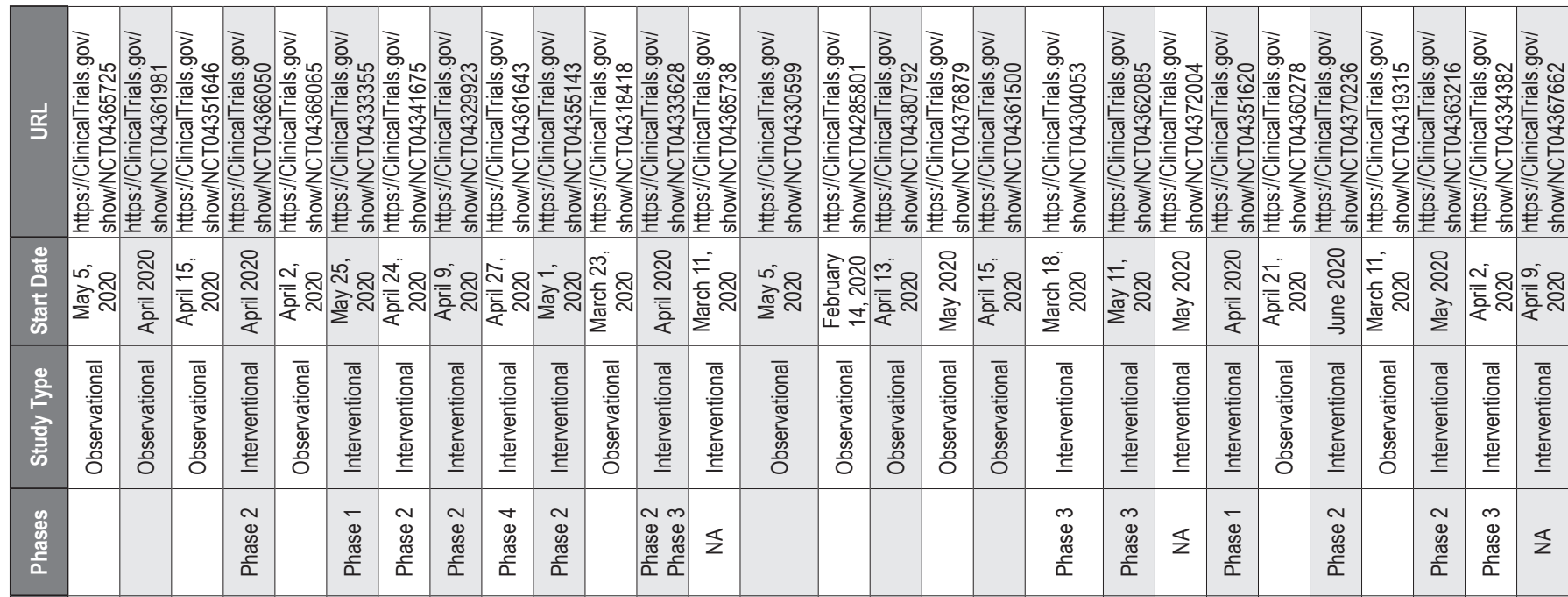
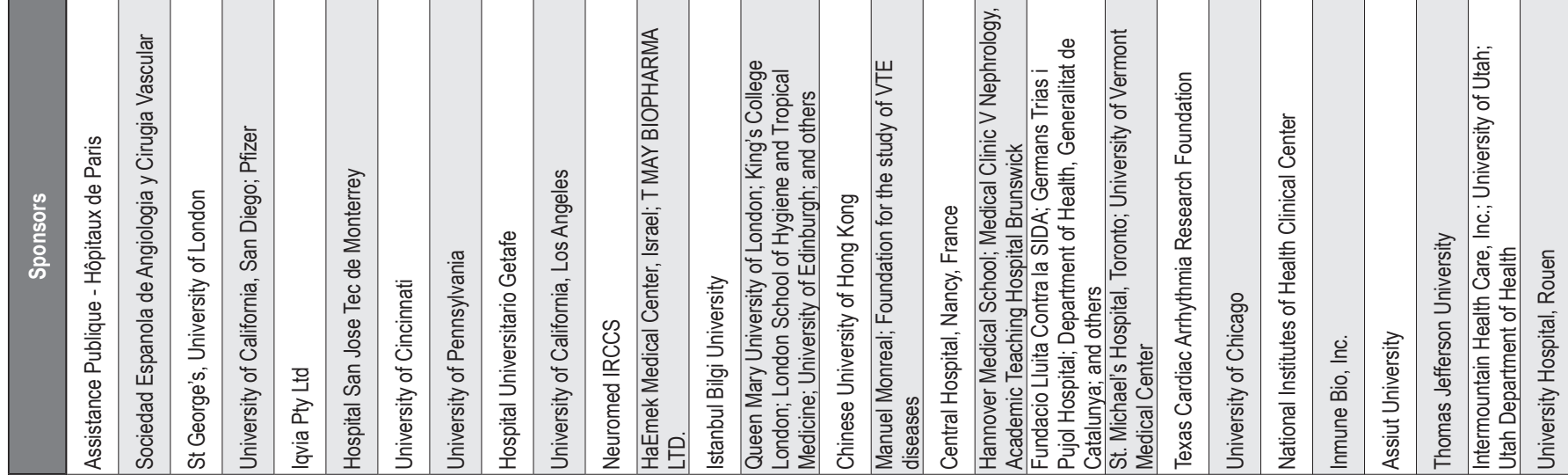

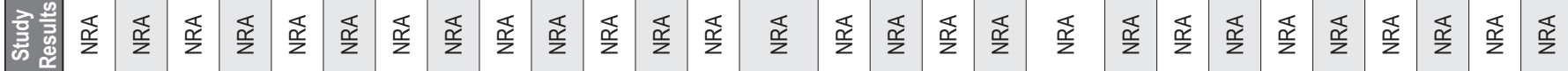

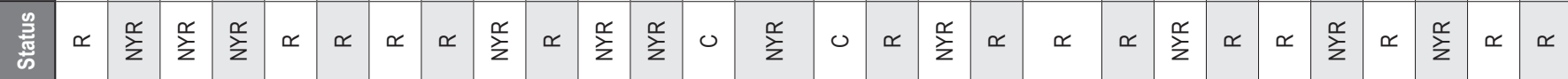

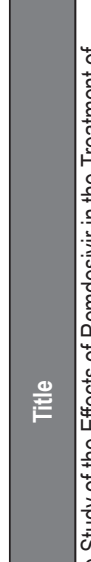

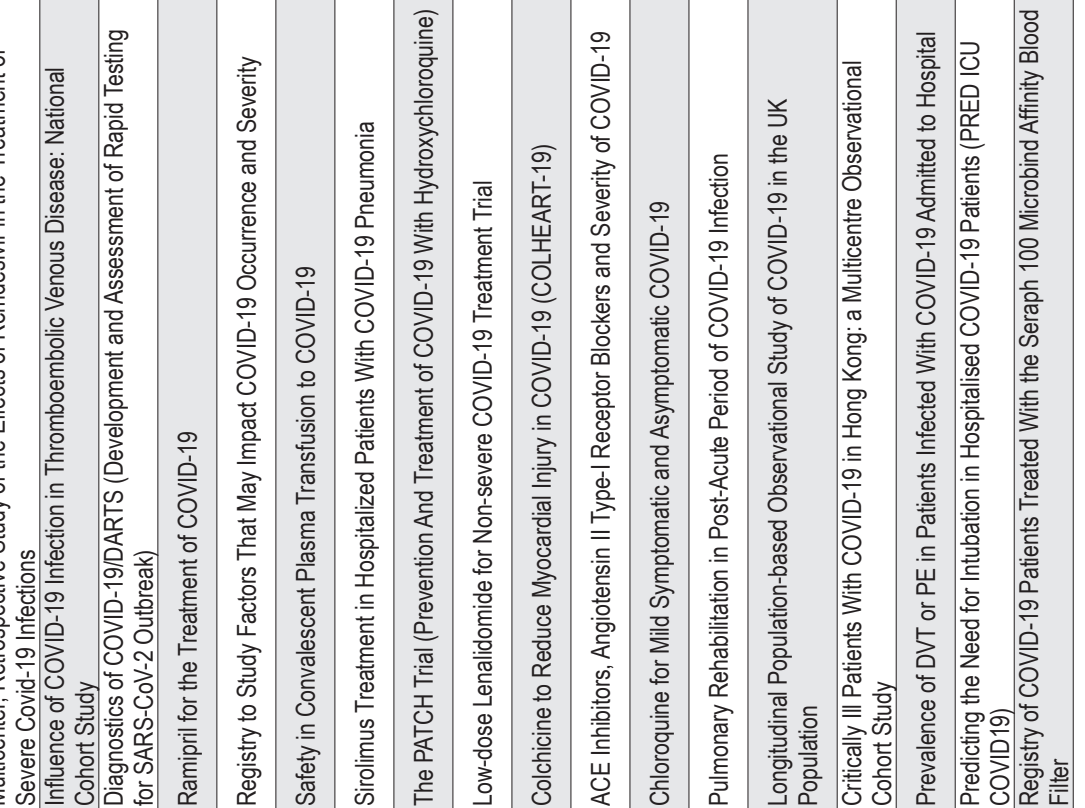

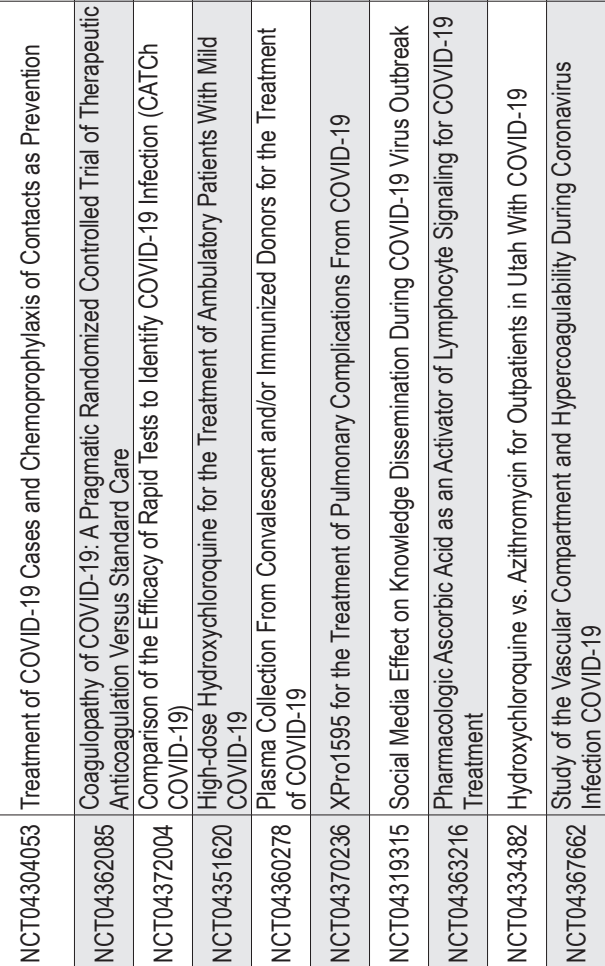




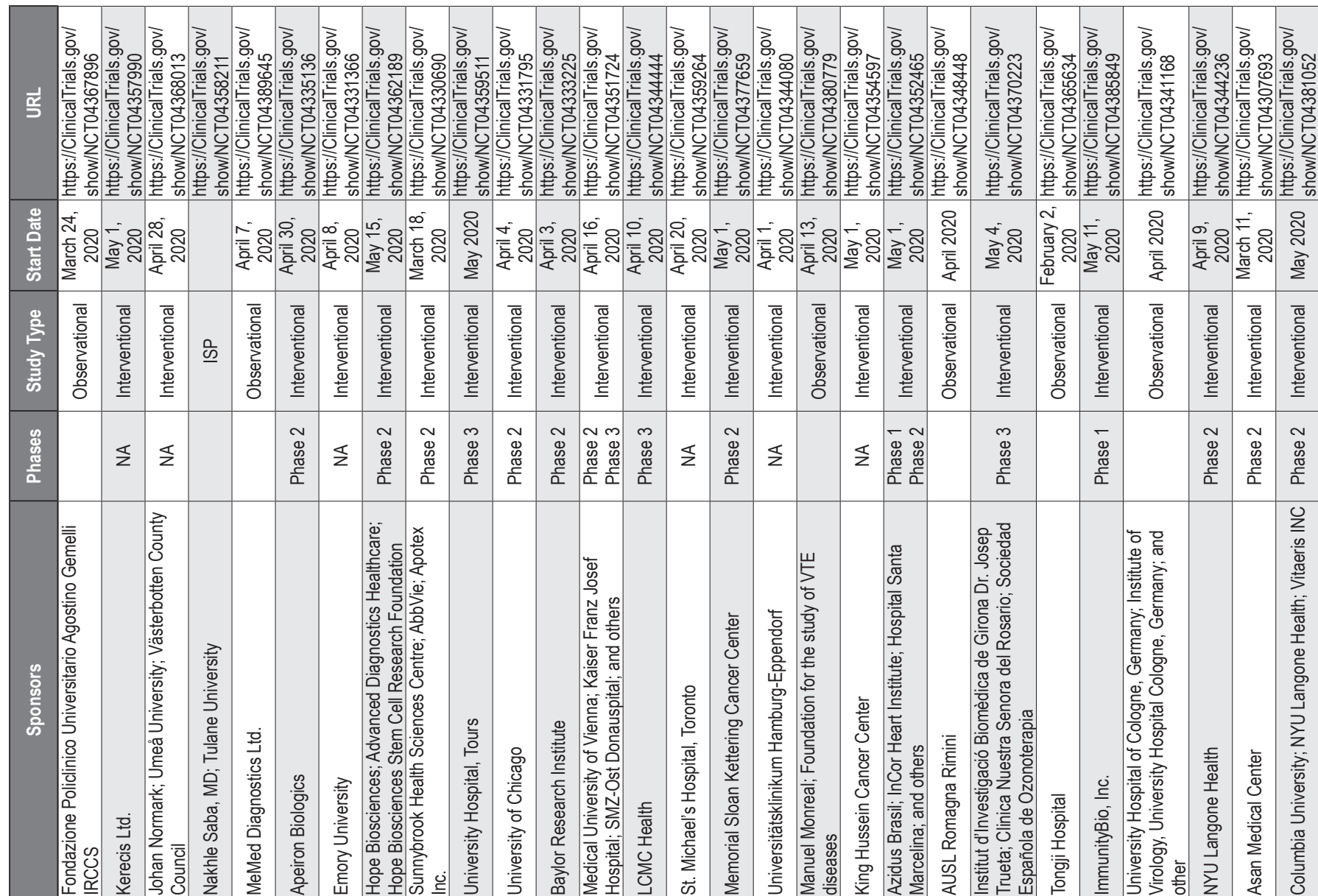

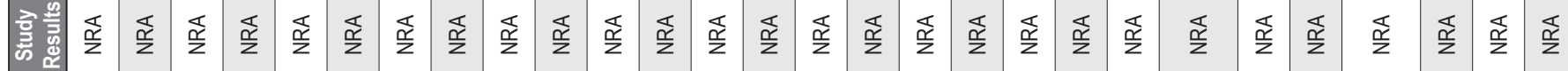

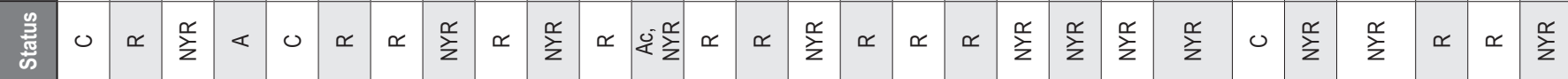

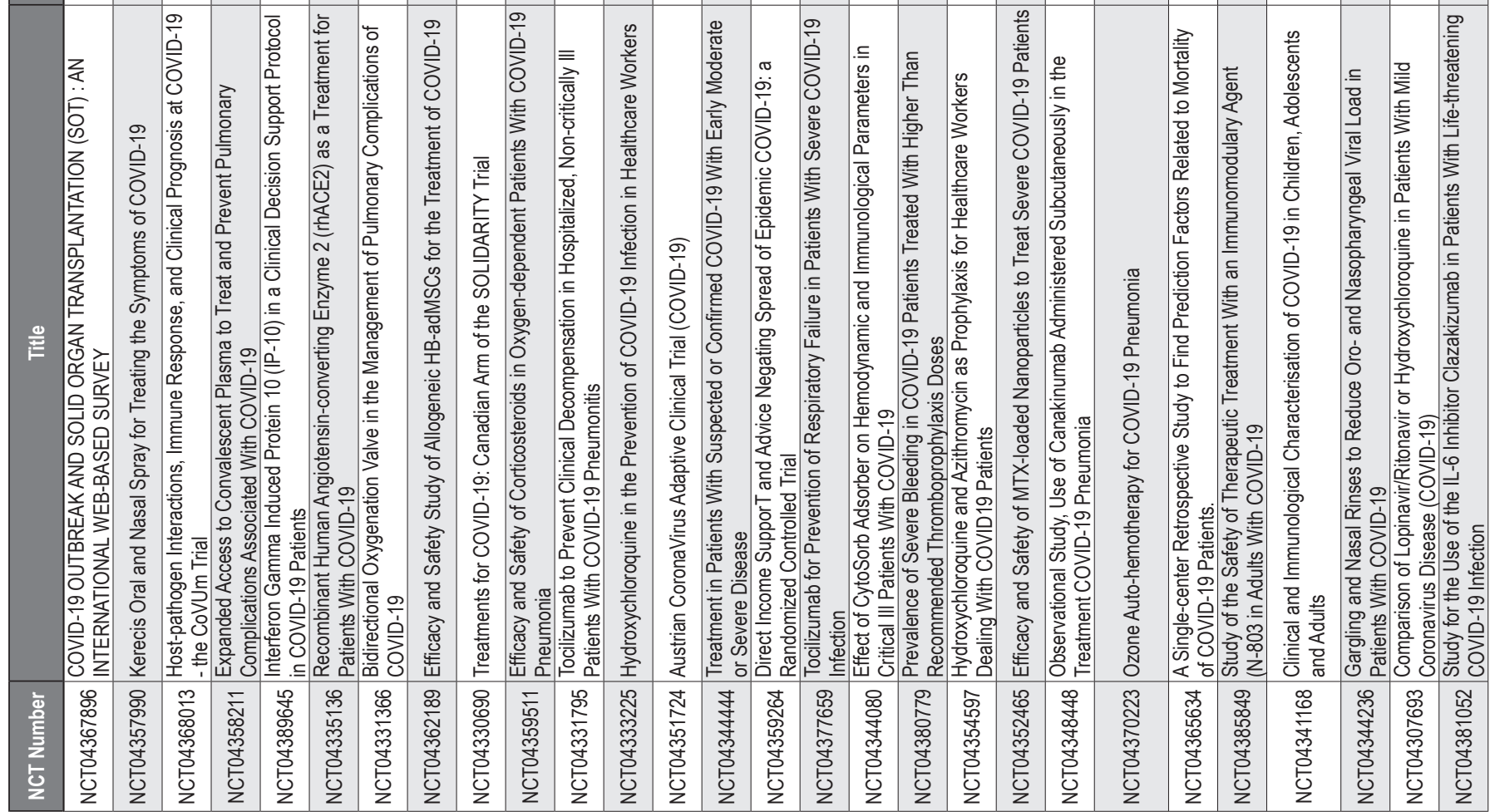




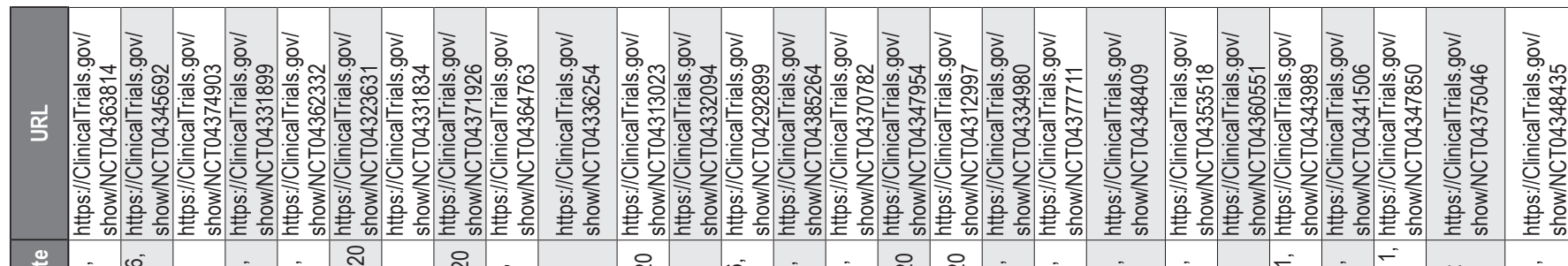

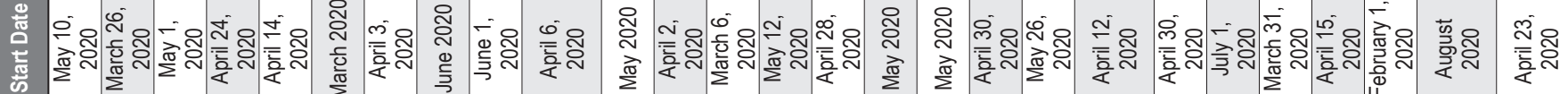

\begin{tabular}{|c|c|c|c|c|c|c|c|c|c|c|c|c|c|c|c|c|c|c|c|c|c|c|c|c|c|c|}
\hline 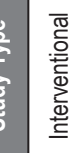 & 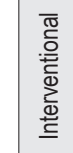 & 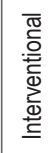 & 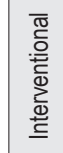 & 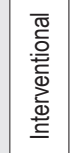 & 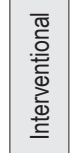 & 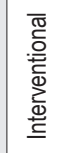 & 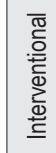 & 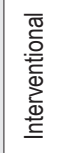 & 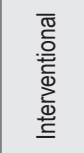 & 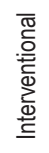 & 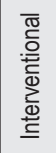 & 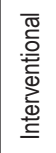 & 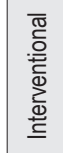 & 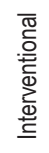 & 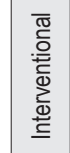 & 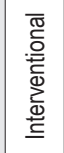 & 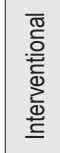 & 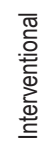 & 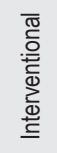 & 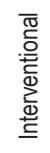 & 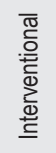 & 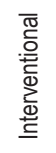 & 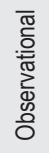 & 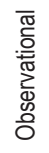 & 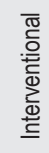 & 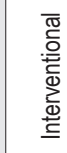 \\
\hline 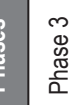 & 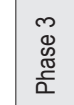 & $\mathbb{z}$ & 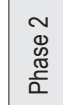 & 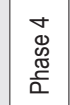 & 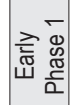 & $\begin{array}{l}0 \\
0 \\
0 \\
\frac{\delta}{\alpha} \\
\frac{\pi}{\alpha}\end{array}$ & $\underline{z}$ & 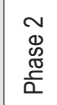 & 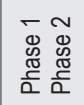 & $\begin{array}{l}\sim \\
\mathbb{8} \\
\widetilde{\pi} \\
\frac{\pi}{\alpha}\end{array}$ & $\begin{array}{l}\sim \\
\mathbb{8} \\
\mathbb{\pi} \\
\frac{\pi}{\alpha} \\
\end{array}$ & $\begin{array}{l}m \\
\mathbb{8} \\
\mathbb{d} \\
\frac{\pi}{\alpha} \\
\end{array}$ & 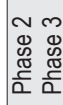 & $\underset{\$}{\stackrel{\$}{\infty}}$ & 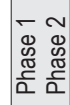 & $\begin{array}{l}\sim \\
\mathcal{D} \\
\frac{\sigma}{\alpha} \\
\frac{\sigma}{\alpha}\end{array}$ & $\begin{array}{l}\bar{\Phi} \\
\stackrel{\pi}{\pi} \\
\frac{\pi}{\alpha}\end{array}$ & 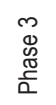 & 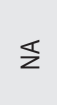 & $\begin{array}{l}\infty \\
\Phi \\
\mathbb{g} \\
\frac{\pi}{\alpha}\end{array}$ & $\begin{array}{l}\mathbb{N} \\
\mathbb{8} \\
\frac{\pi}{\alpha} \\
\frac{\pi}{\alpha}\end{array}$ & 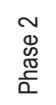 & & & & \\
\hline
\end{tabular}




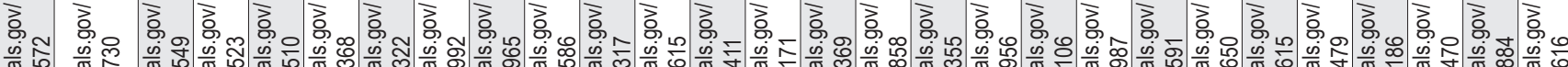

岁

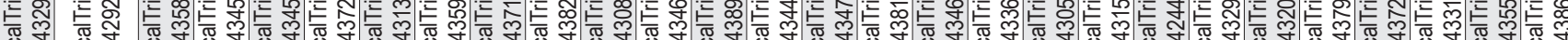
ॠ.

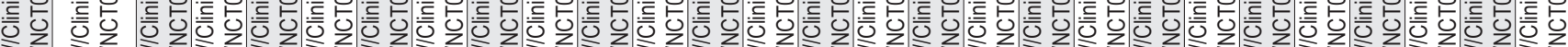

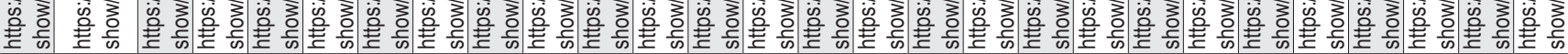

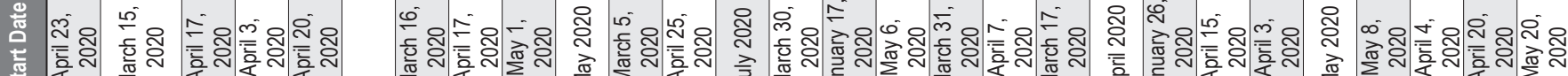

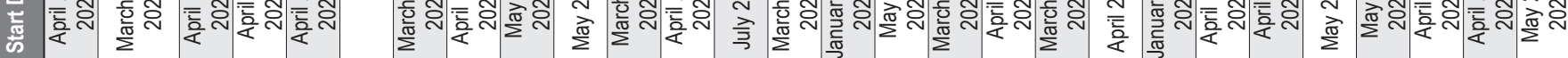

\begin{tabular}{|c|c|c|c|c|c|c|c|c|c|c|c|c|c|c|c|c|c|c|c|c|c|c|c|c|c|c|}
\hline 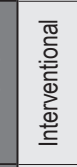 & 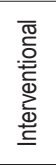 & 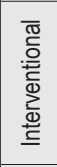 & 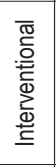 & 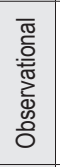 & $\stackrel{\varrho}{F}$ & 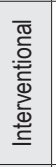 & 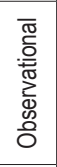 & 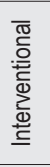 & 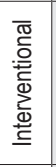 & 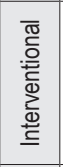 & 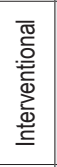 & 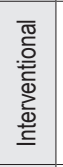 & 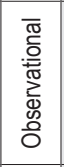 & 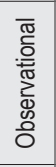 & 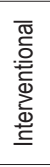 & 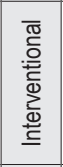 & 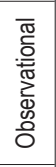 & 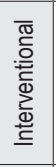 & 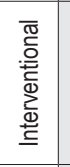 & 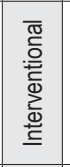 & 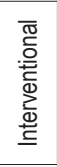 & 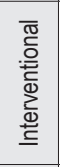 & 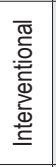 & 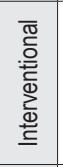 & 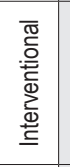 & 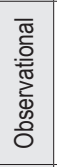 \\
\hline & 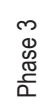 & $\mid \begin{array}{c}\widetilde{D} \\
\mathbb{o} \\
\frac{\pi}{\alpha} \\
\frac{1}{\alpha}\end{array}$ & $\begin{array}{l}\text { o } \\
\Phi \\
\frac{\Phi}{\alpha} \\
\frac{\pi}{\alpha}\end{array}$ & & & 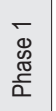 & & $\begin{array}{l}\widetilde{N} \\
\mathscr{o} \\
\frac{\pi}{\alpha} \\
\frac{\pi}{2}\end{array}$ & 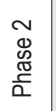 & 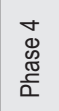 & & & & & 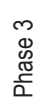 & 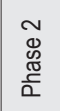 & & 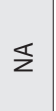 & $\begin{array}{l}\bar{\Phi} \\
\overline{\mathbb{d}} \\
\frac{\pi}{\alpha}\end{array}$ & $\left|\begin{array}{ll}\frac{w}{0} \\
\frac{\pi}{\alpha} \\
\frac{\pi}{\alpha} \\
\end{array}\right|$ & 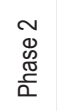 & \begin{tabular}{|l}
0 \\
0 \\
0 \\
$\frac{\pi}{2}$ \\
$\frac{\pi}{\alpha}$
\end{tabular} & $\begin{array}{l}\tilde{N} \\
\mathscr{D} \\
\frac{\tilde{\sigma}}{\alpha} \\
\frac{\pi}{\alpha}\end{array}$ & 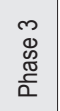 & 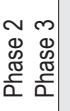 & \\
\hline
\end{tabular}
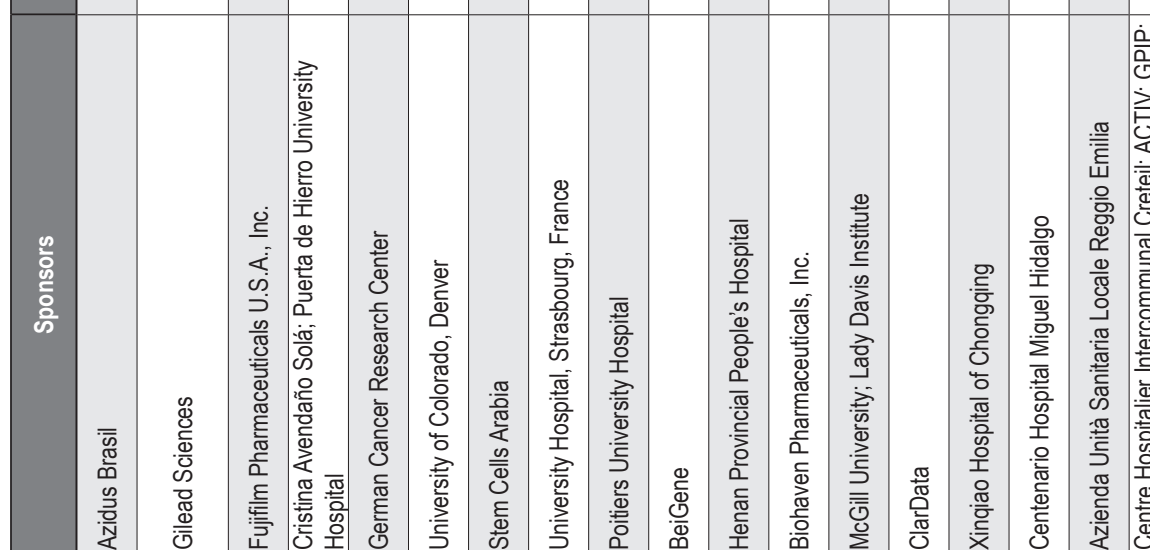

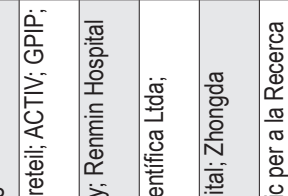

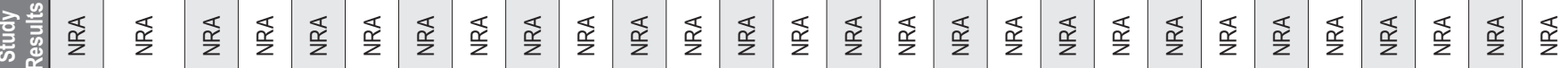

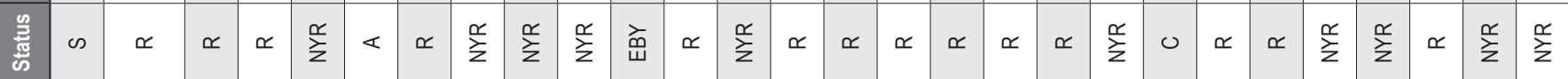

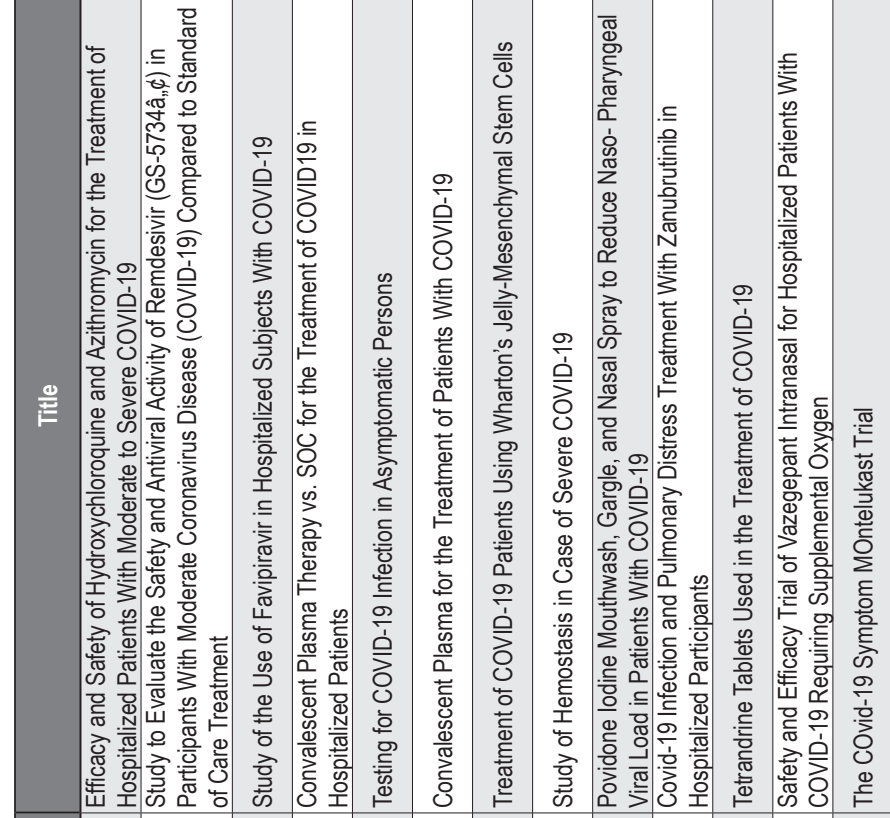
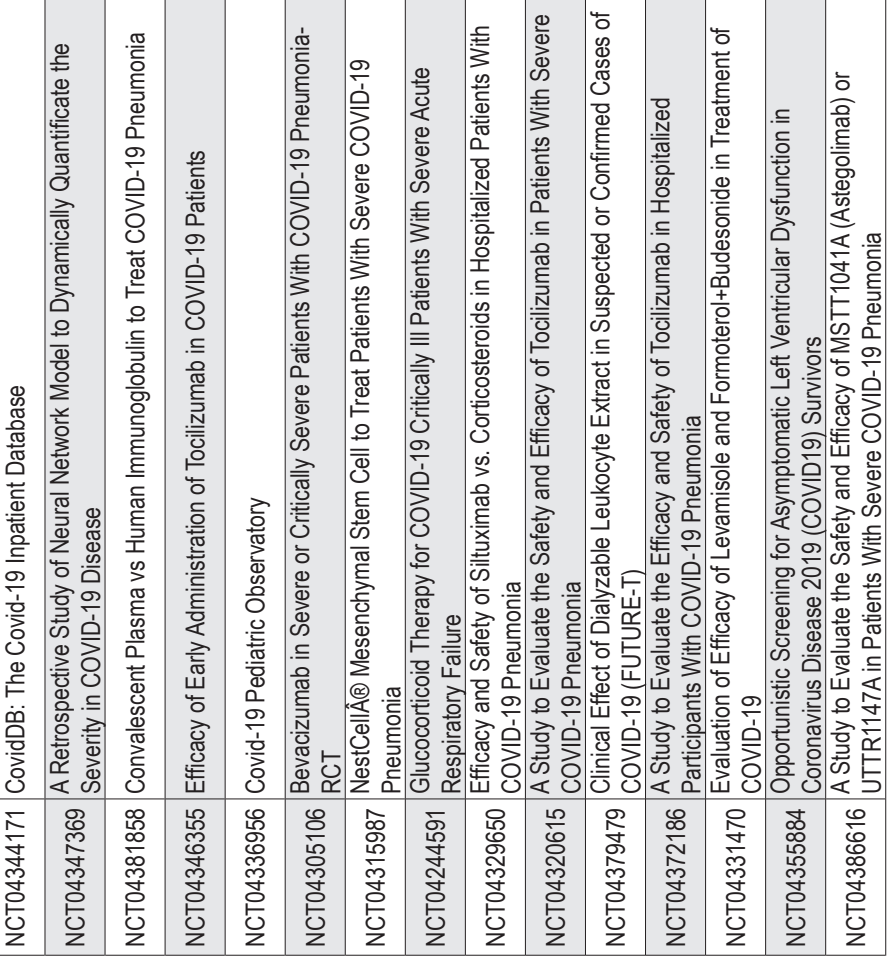

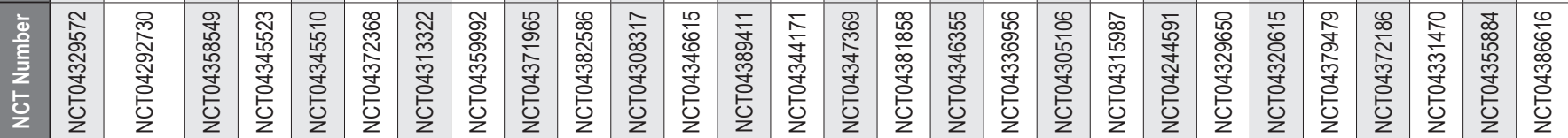




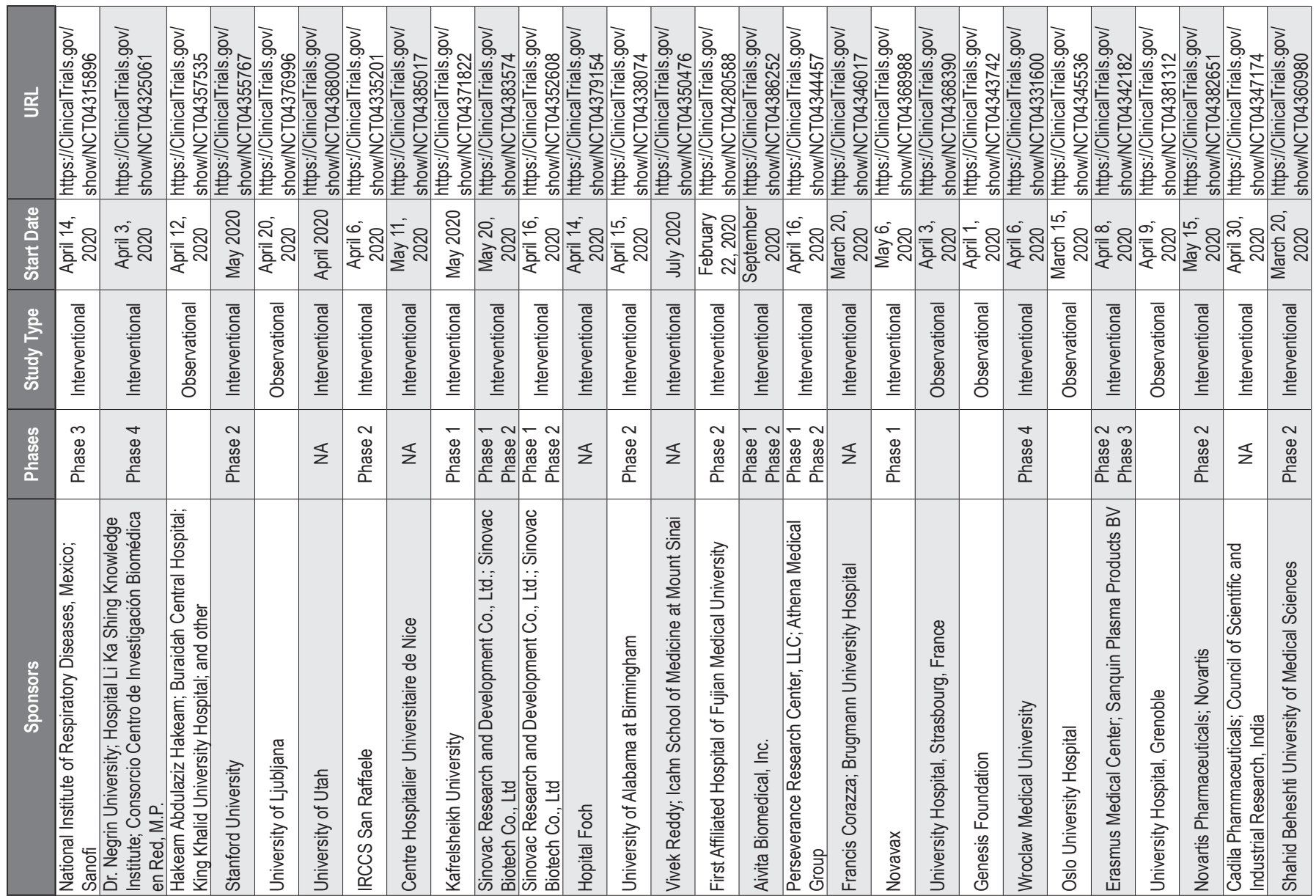

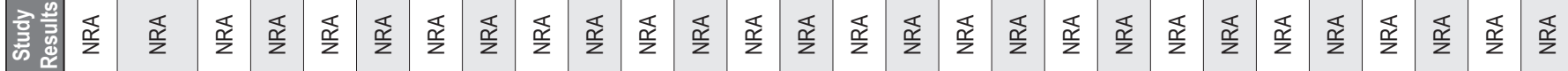

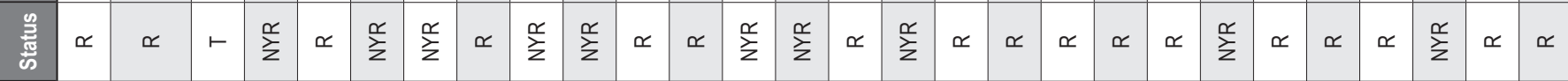

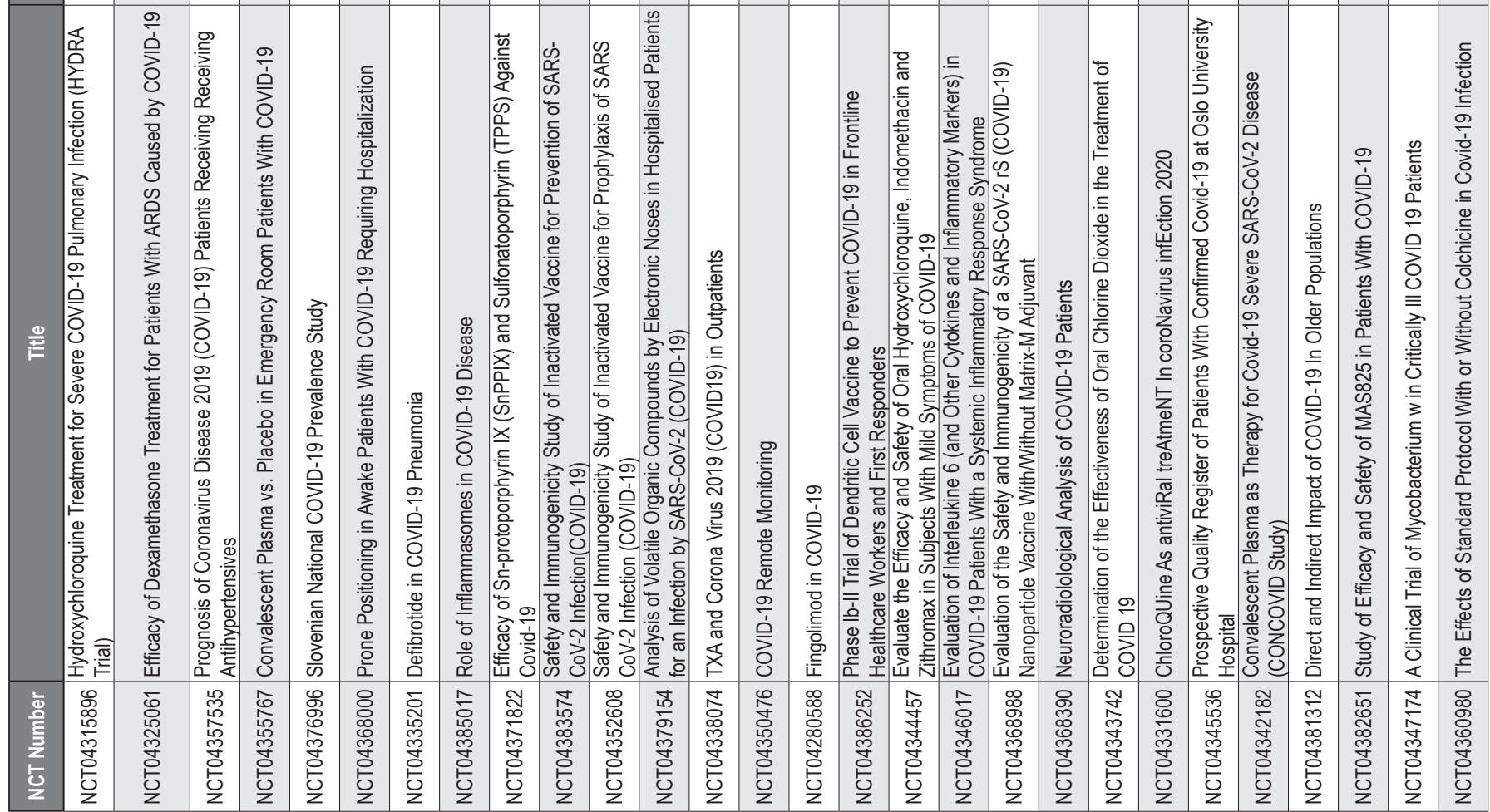




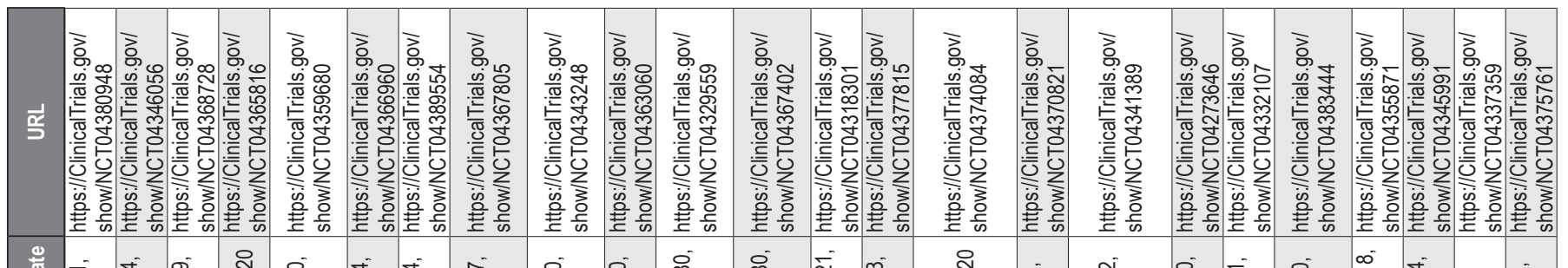

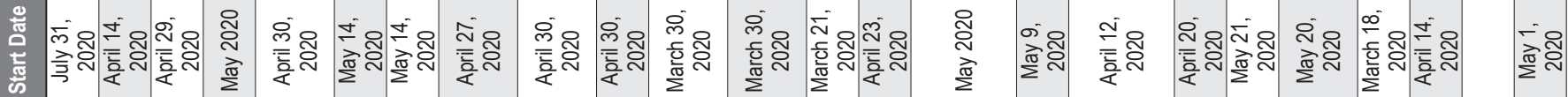

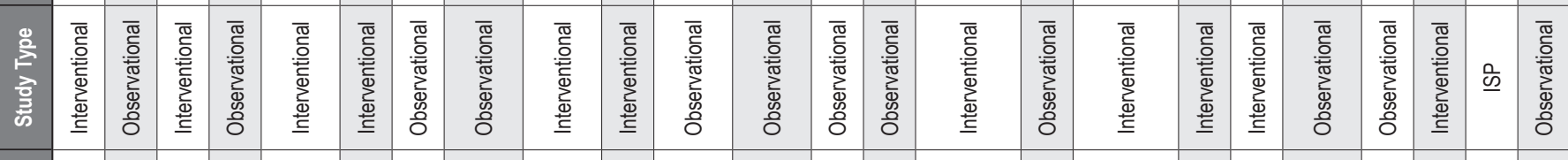

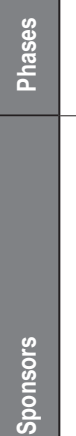

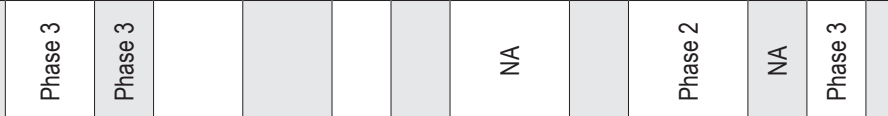

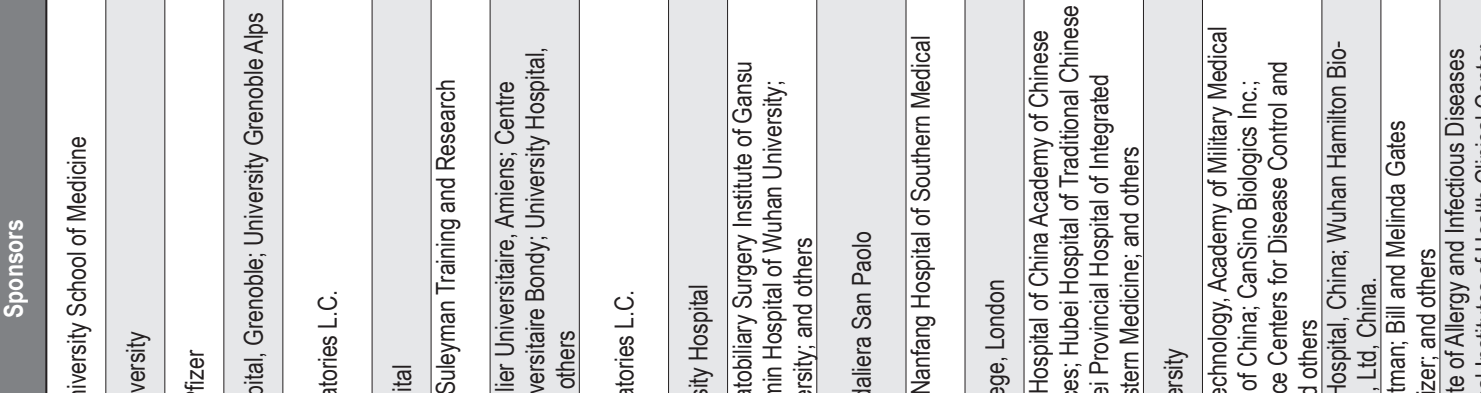

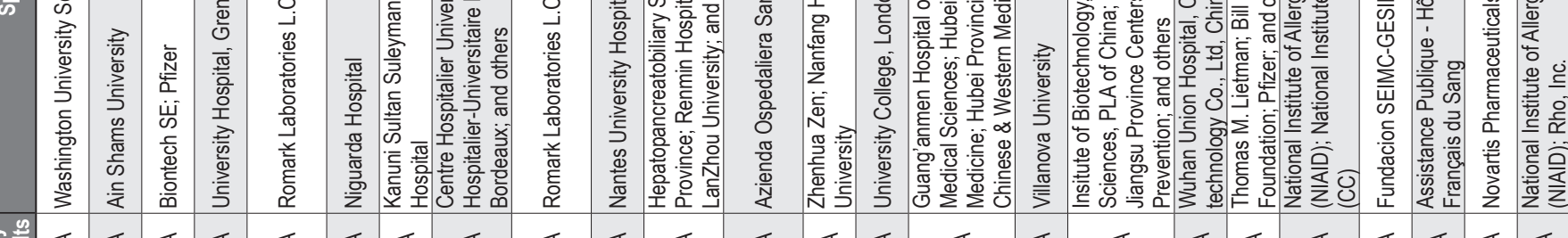

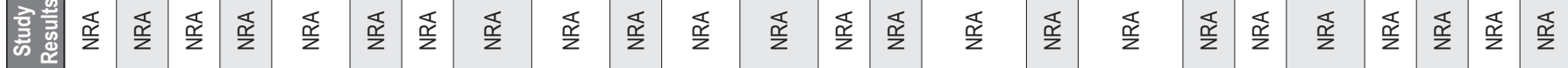

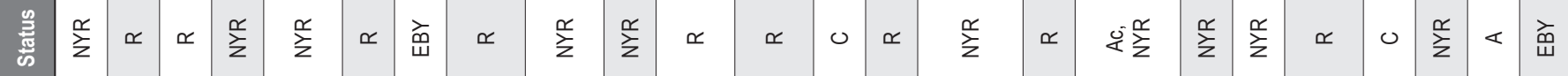

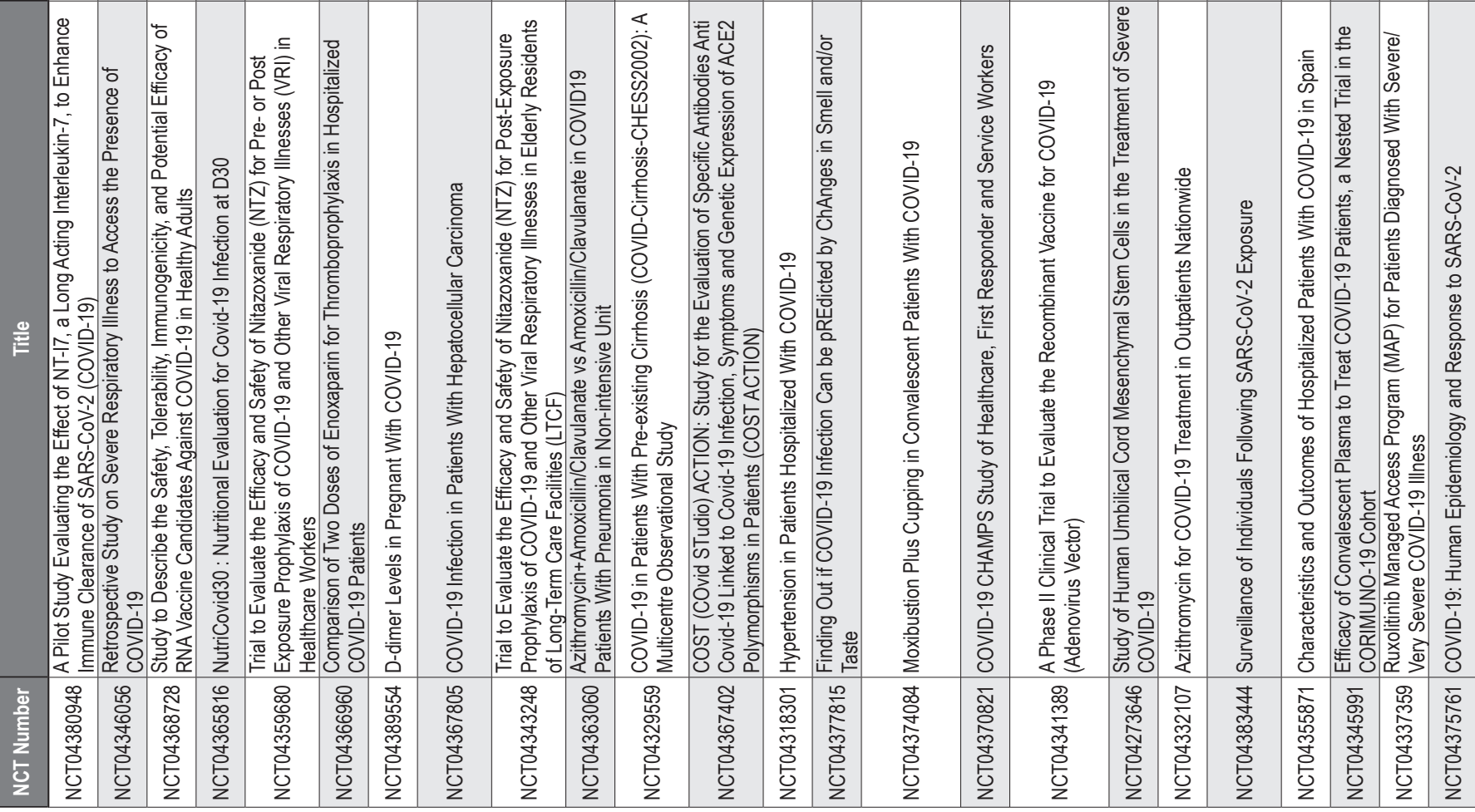




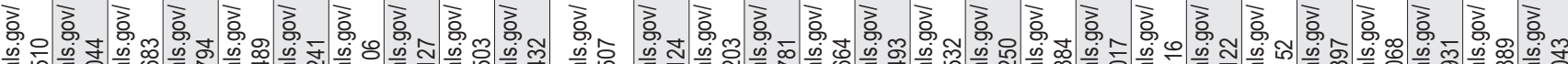

菏

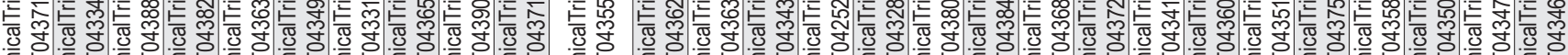
匍

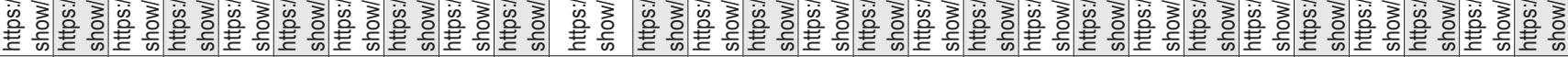

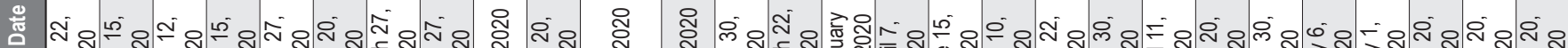

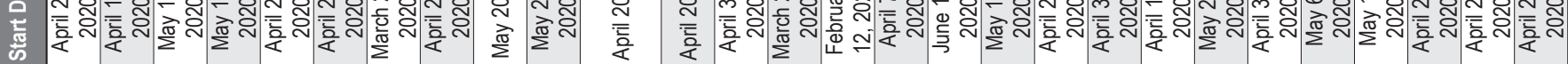

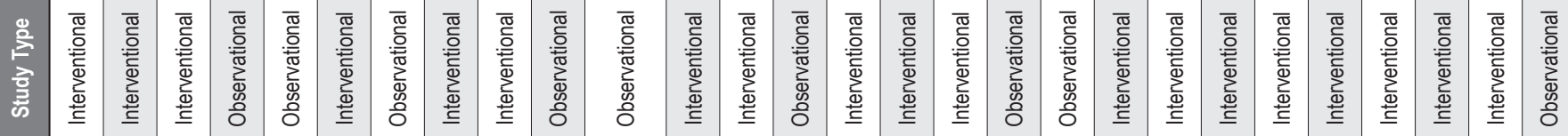

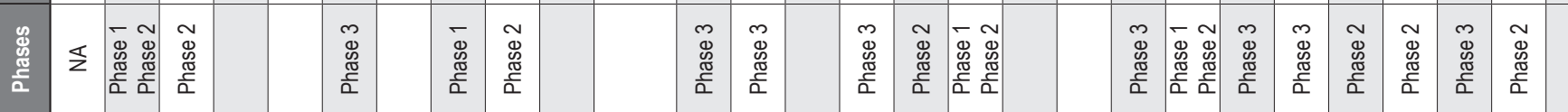

\begin{tabular}{|c|} 
\\
\\
$\frac{2}{0}$ \\
0 \\
0 \\
के \\
के \\
\hline
\end{tabular}

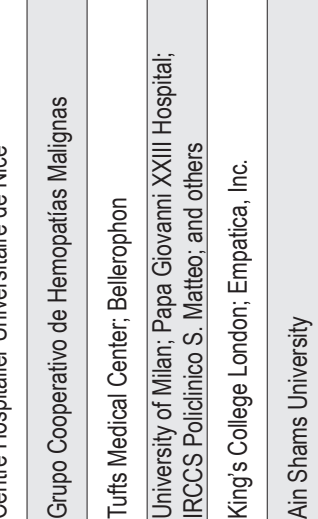

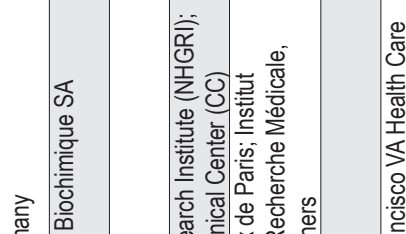

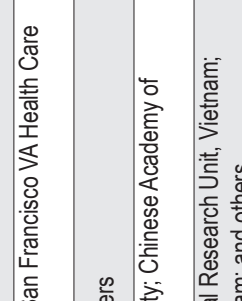

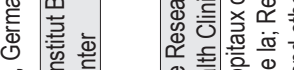

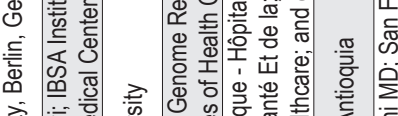

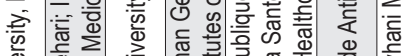

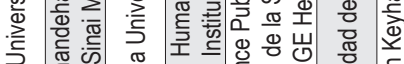

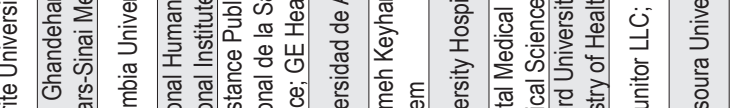

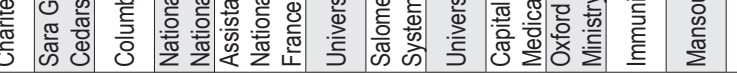

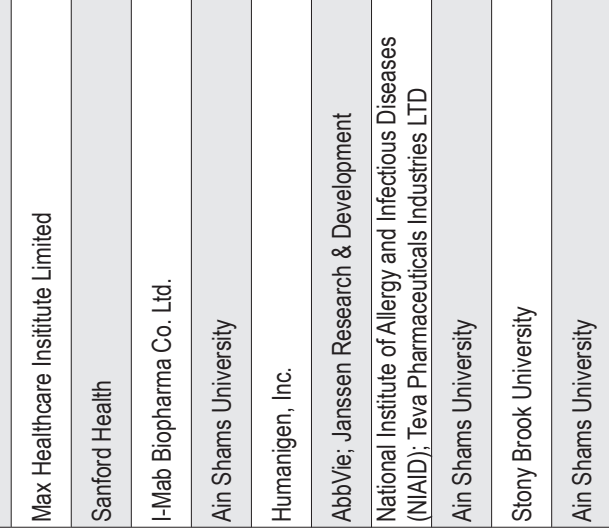

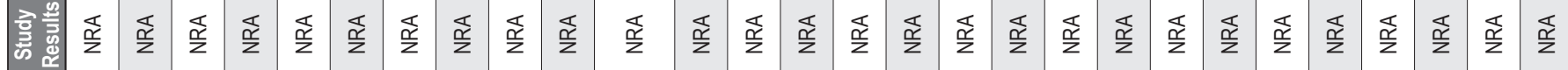

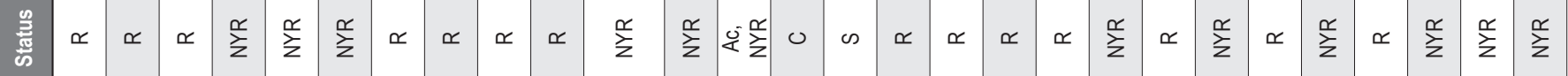
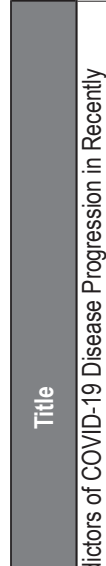

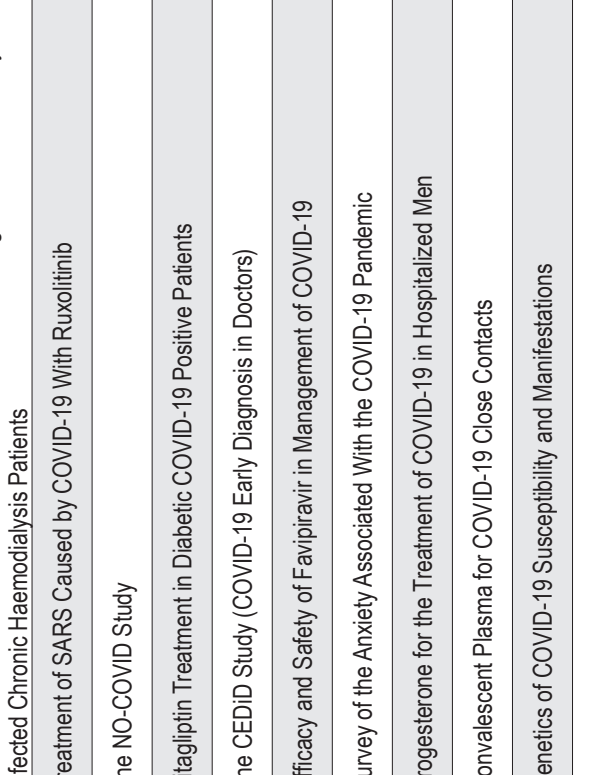

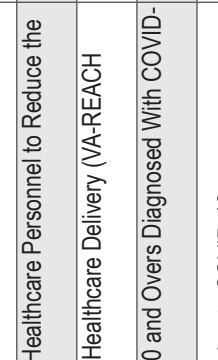

을

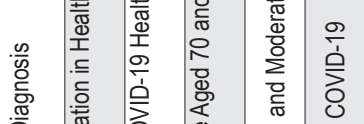

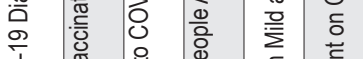

字

ర人

言

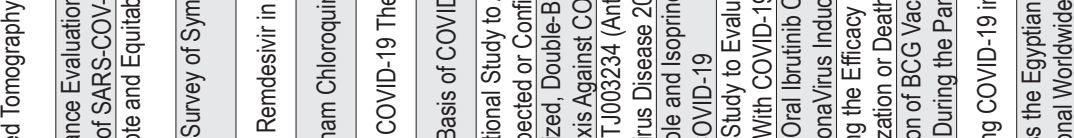

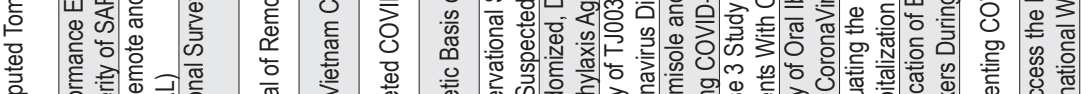

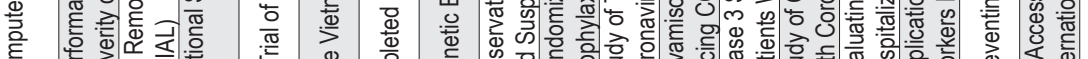

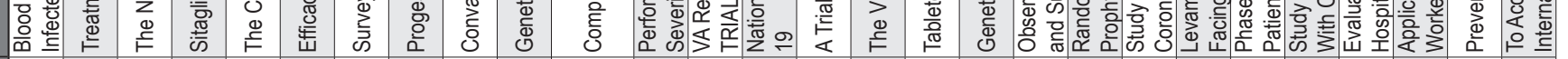

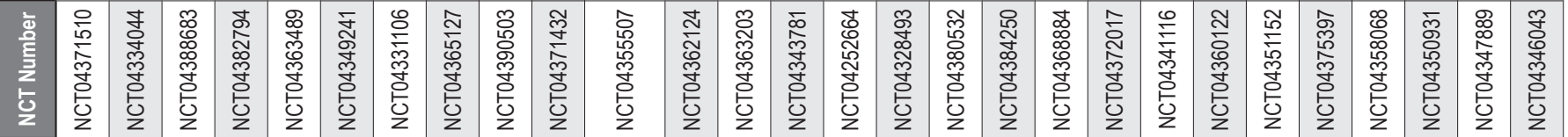




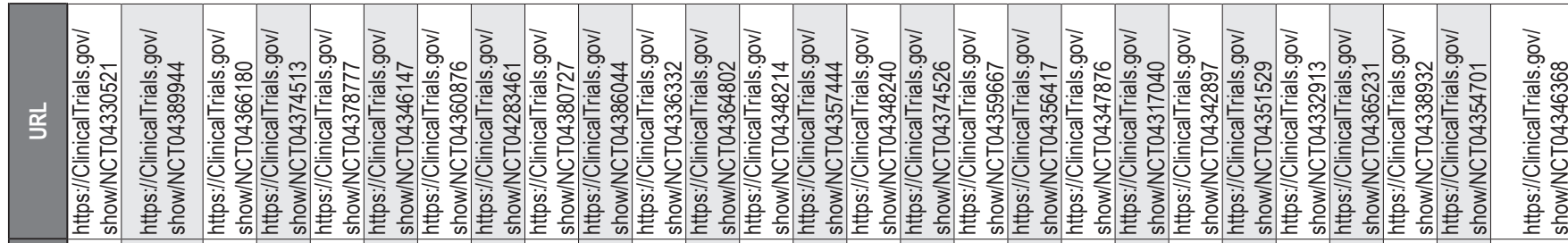

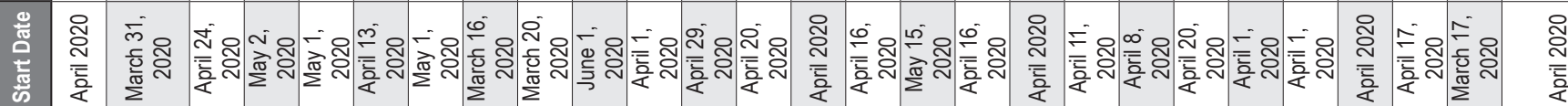

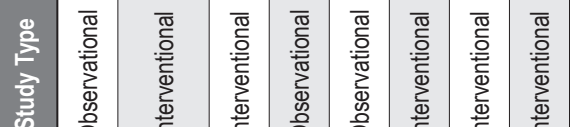

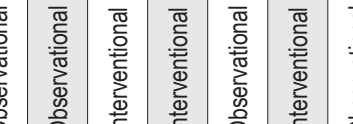

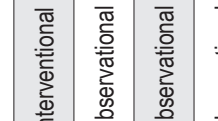

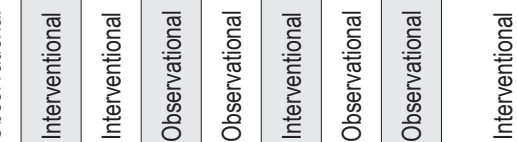

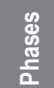

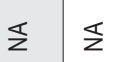

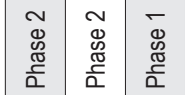

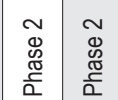

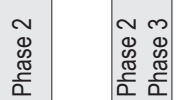

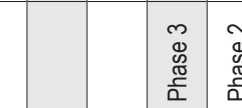

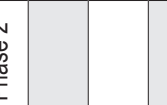

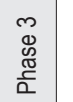

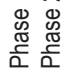

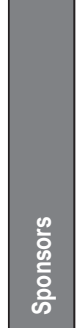

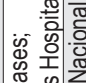

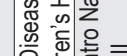

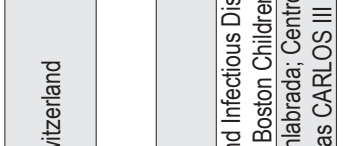

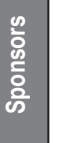

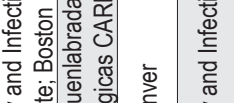

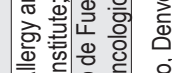

旋

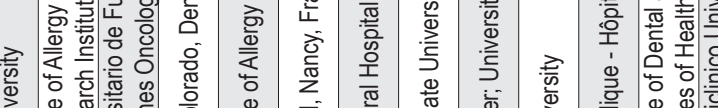

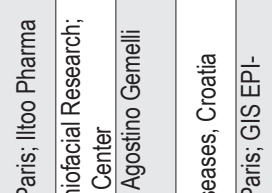

曽

离

证

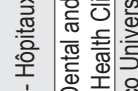

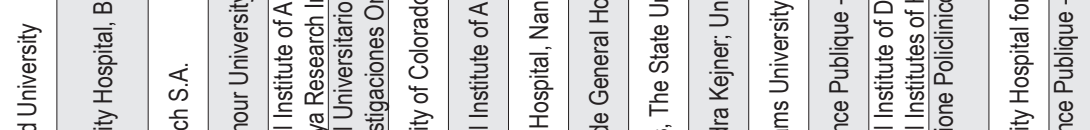

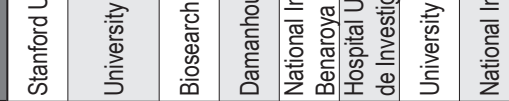

焉

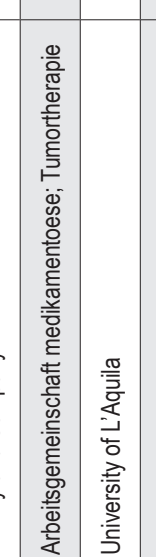

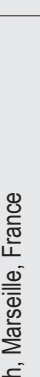

政

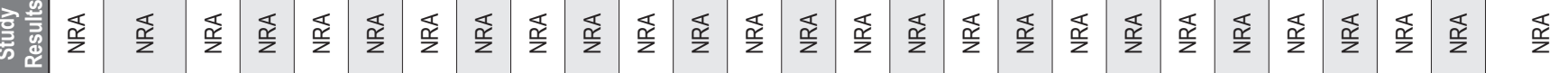

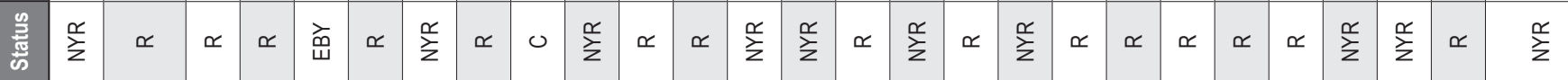
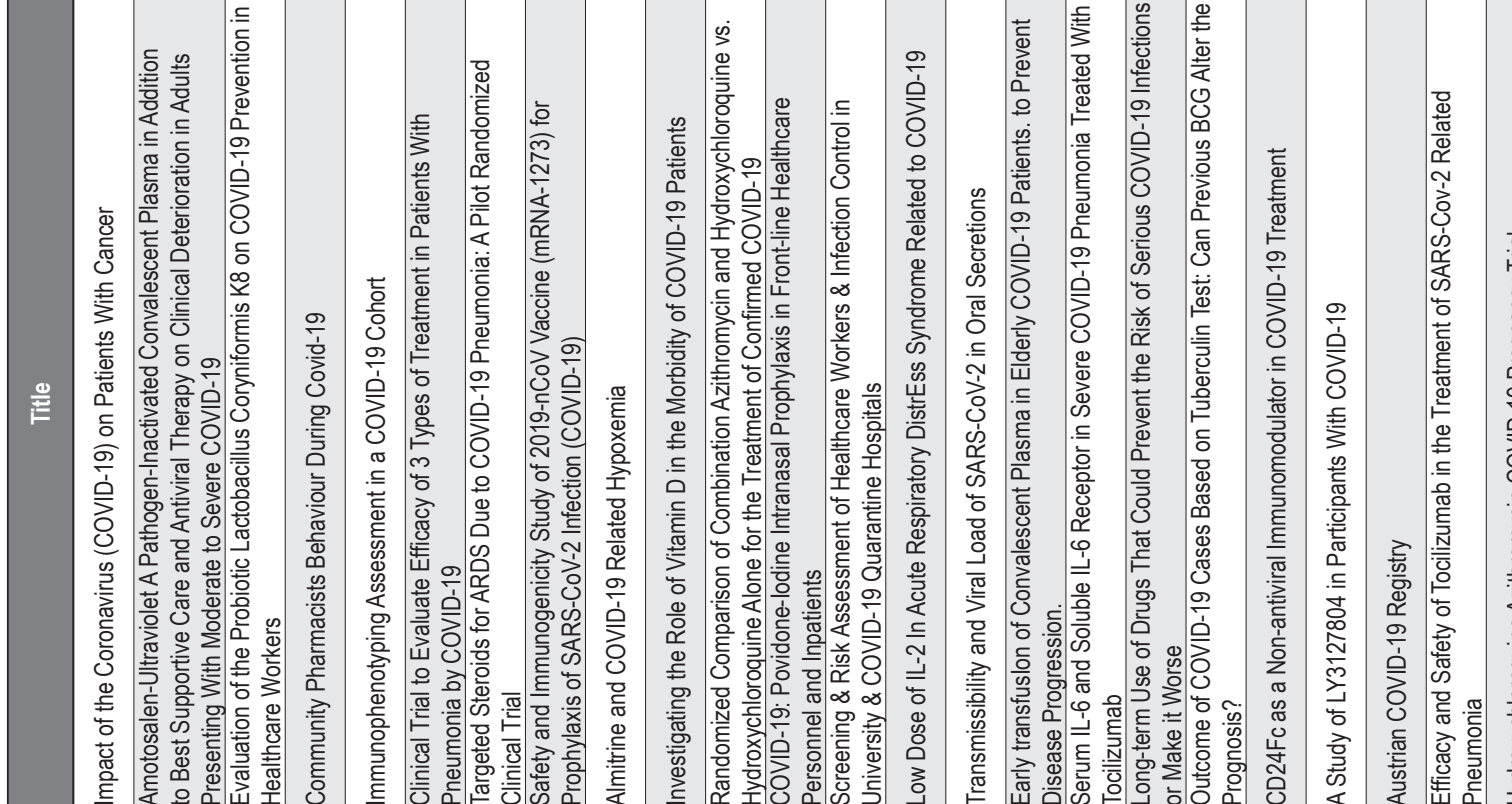

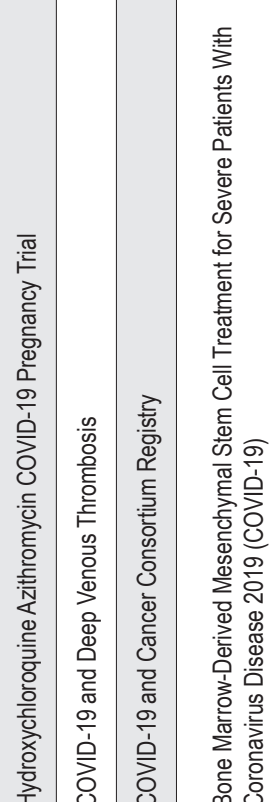

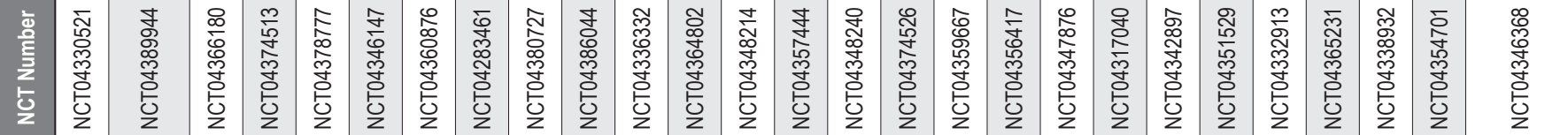




\begin{tabular}{|c|c|c|c|c|c|c|c|c|c|c|c|c|c|c|c|c|c|c|c|c|c|c|c|c|c|}
\hline \multicolumn{8}{|c|}{ 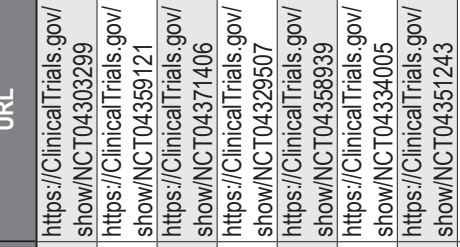 } & 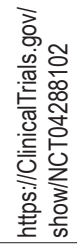 & \multicolumn{2}{|c|}{ 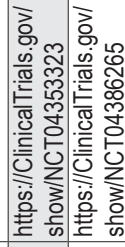 } & 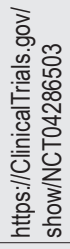 & \multicolumn{3}{|c|}{ 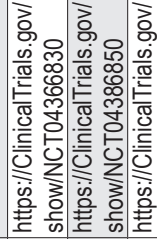 } & \multicolumn{2}{|c|}{ 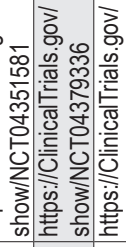 } & 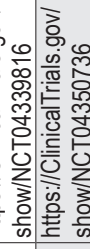 & 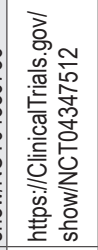 & 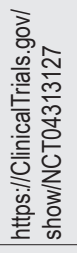 & \multicolumn{3}{|c|}{ 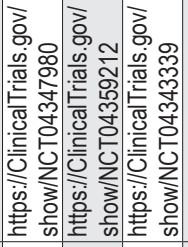 } & 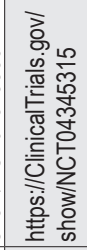 & \multicolumn{2}{|c|}{ 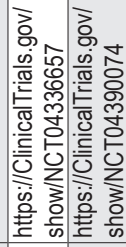 } \\
\hline & & & & & & & & 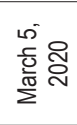 & & & 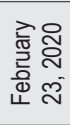 & & & & & & & & 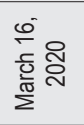 & 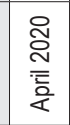 & 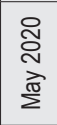 & ฮิ & $\begin{array}{l}\tilde{N} \\
\bar{N} \\
\bar{J} \\
\text { N } \\
\Sigma\end{array}$ & 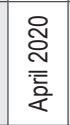 & 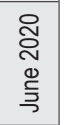 \\
\hline 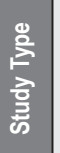 & 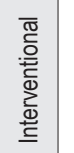 & 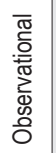 & 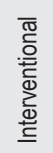 & 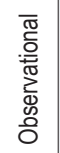 & 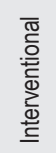 & 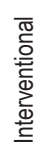 & 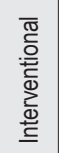 & 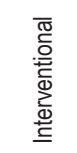 & 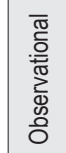 & 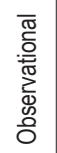 & 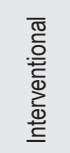 & $\underline{\underline{0}}$ & 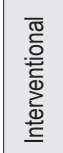 & 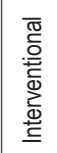 & 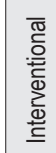 & 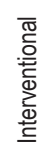 & 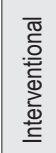 & 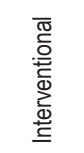 & 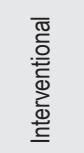 & 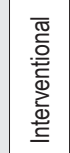 & 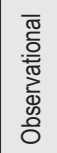 & 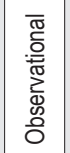 & 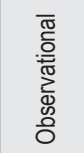 & 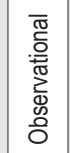 & 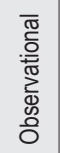 \\
\hline & 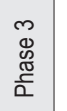 & & & & $\Sigma$ & $\underline{z}$ & $\begin{array}{l}\frac{\mathscr{\Phi}}{2} \\
\frac{\pi}{\alpha}\end{array}$ & $\begin{array}{l}0 \\
0 \\
\frac{\pi}{0}\end{array}$ & & & $\begin{array}{l}\frac{\Phi}{.} \\
\frac{\pi}{\Delta}\end{array}$ & & & $\$$ & m & $\begin{array}{l}\mathbb{m} \\
\mathbb{8} \\
\widetilde{\Xi} \\
\frac{\pi}{\alpha}\end{array}$ & $\begin{array}{l}\frac{\mathscr{m}}{2} \\
\frac{\pi}{\alpha}\end{array}$ & $\frac{c}{a}$ & $\frac{c}{\alpha}$ & & & & & & \\
\hline
\end{tabular}

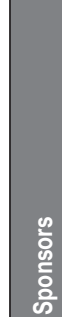

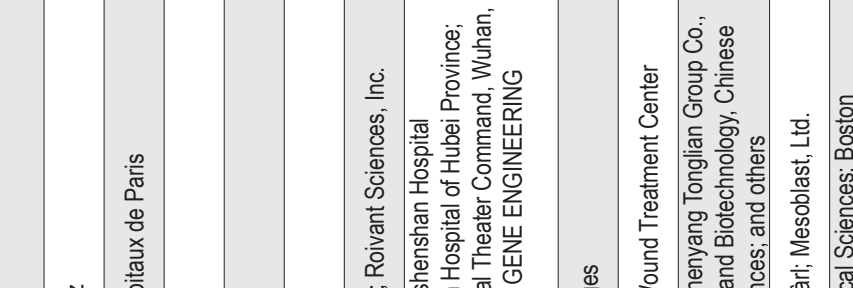

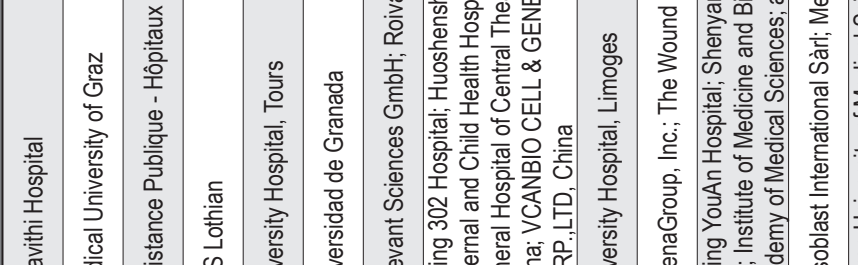

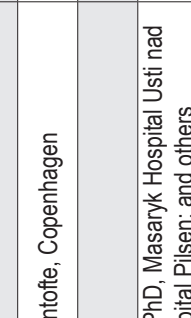

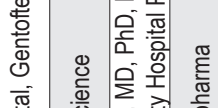

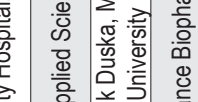

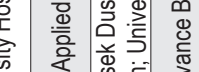

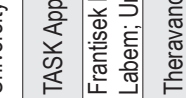

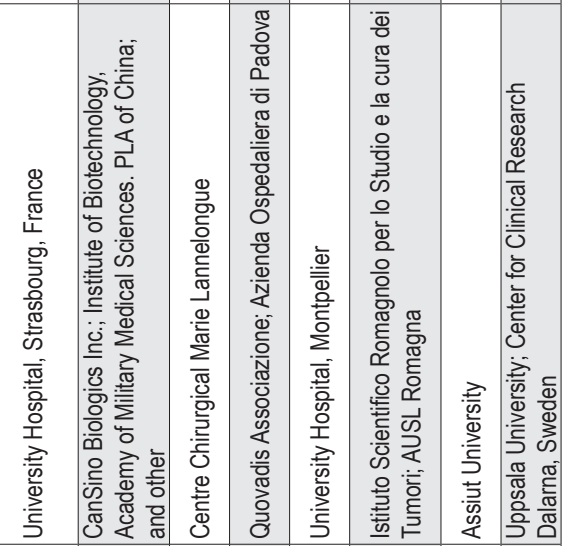
票

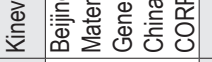

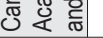
䍃

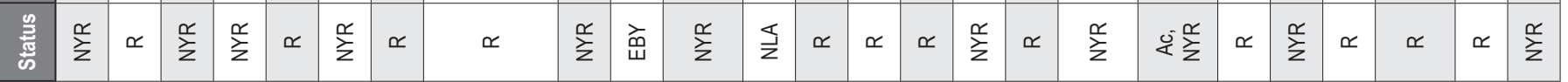

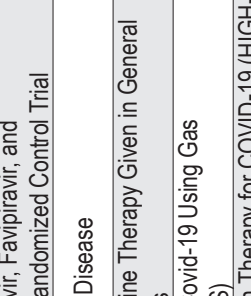

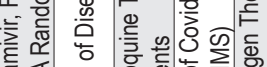

焉㐘

竞

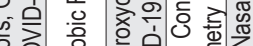

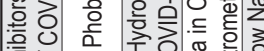

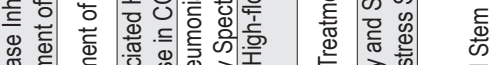

兽

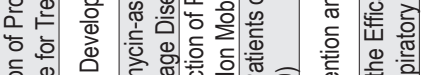

亭 旁

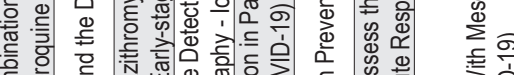

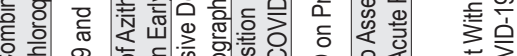

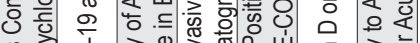

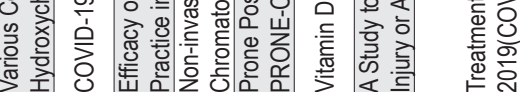

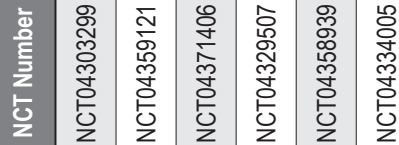

竡

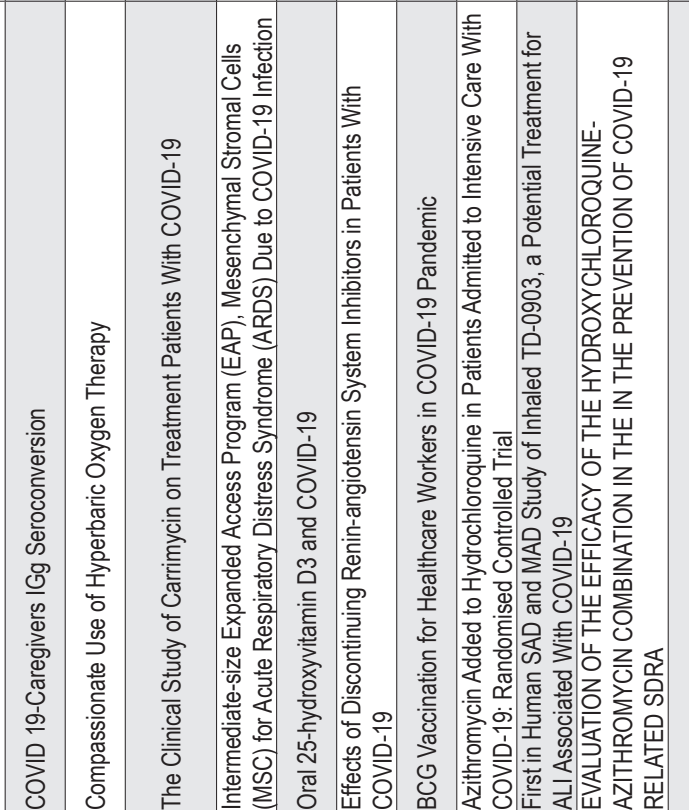

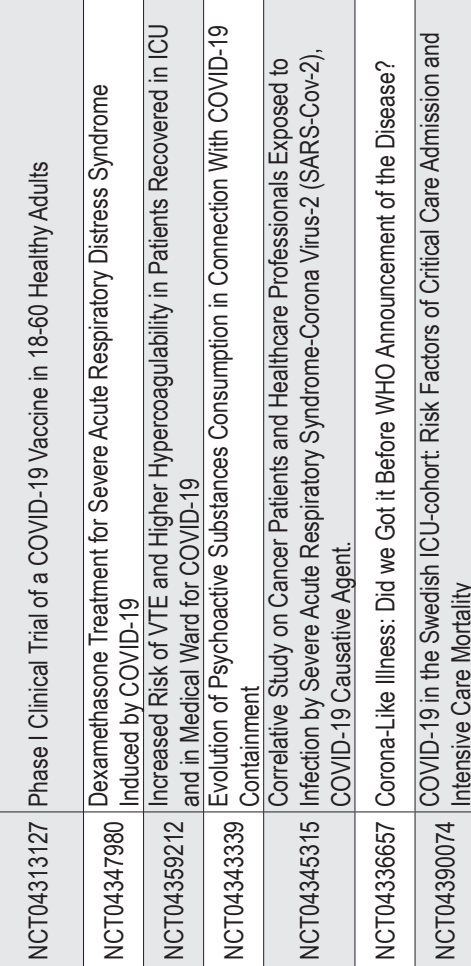




\begin{tabular}{|c|c|c|c|c|c|c|c|c|c|c|c|c|c|c|c|c|c|c|c|c|c|c|c|c|c|c|c|}
\hline$\frac{1}{5}$ & 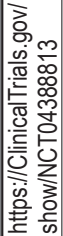 & 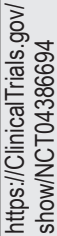 & & & & 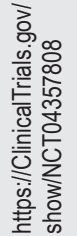 & & & & & & & & & 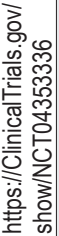 & 陟 & 丞 & ב. & 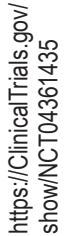 & 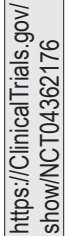 & 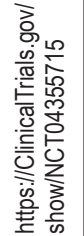 & & 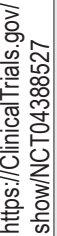 & 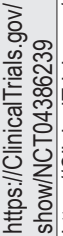 & 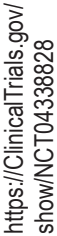 & 总 & : \\
\hline & 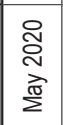 & & & & & 를 & & & & & & & & & & 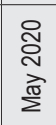 & 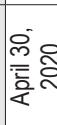 & & i் & 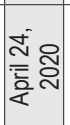 & 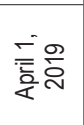 & & oj & 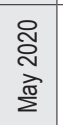 & 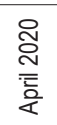 & 胥 & $\frac{-}{\frac{\pi}{\pi}}$ \\
\hline & 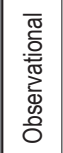 & 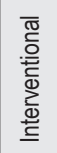 & 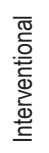 & 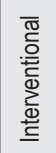 & 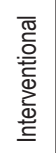 & 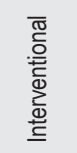 & 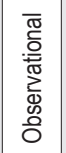 & 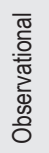 & 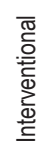 & 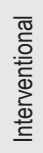 & 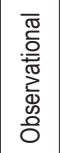 & 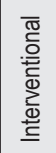 & 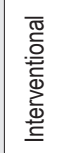 & 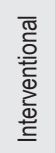 & 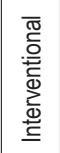 & 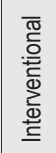 & 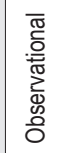 & 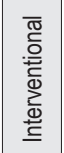 & 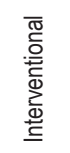 & 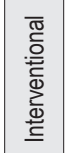 & 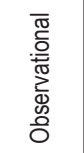 & 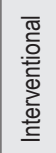 & 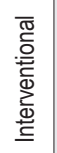 & 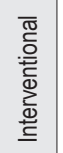 & 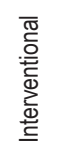 & 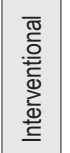 & \\
\hline & & $\underline{z}$ & $\begin{array}{l}\frac{\Phi}{\sigma} \\
\frac{\pi}{\alpha}\end{array}$ & $\frac{\pi}{z}$ & $\frac{\mathbb{s}}{z}$ & 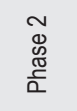 & & & $\begin{array}{l}\frac{d}{d} \\
\frac{d}{\alpha} \\
\frac{d}{\alpha}\end{array}$ & & & & & 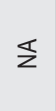 & $\begin{array}{l}D_{0} \\
\end{array}$ & $\underline{z}$ & & $\begin{array}{l}\mathbb{\infty} \\
\mathbb{0} \\
\frac{\pi}{\alpha}\end{array}$ & 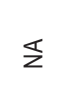 & $\begin{array}{l}\mathscr{0} \\
\frac{\pi}{\alpha} \\
\frac{\pi}{2}\end{array}$ & & $\underline{z}$ & 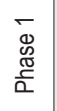 & 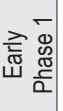 & 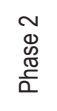 & 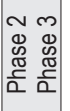 & \\
\hline
\end{tabular}

\begin{tabular}{|} 
\\
$\frac{2}{0}$ \\
$\frac{0}{0}$ \\
$\frac{0}{5}$
\end{tabular}
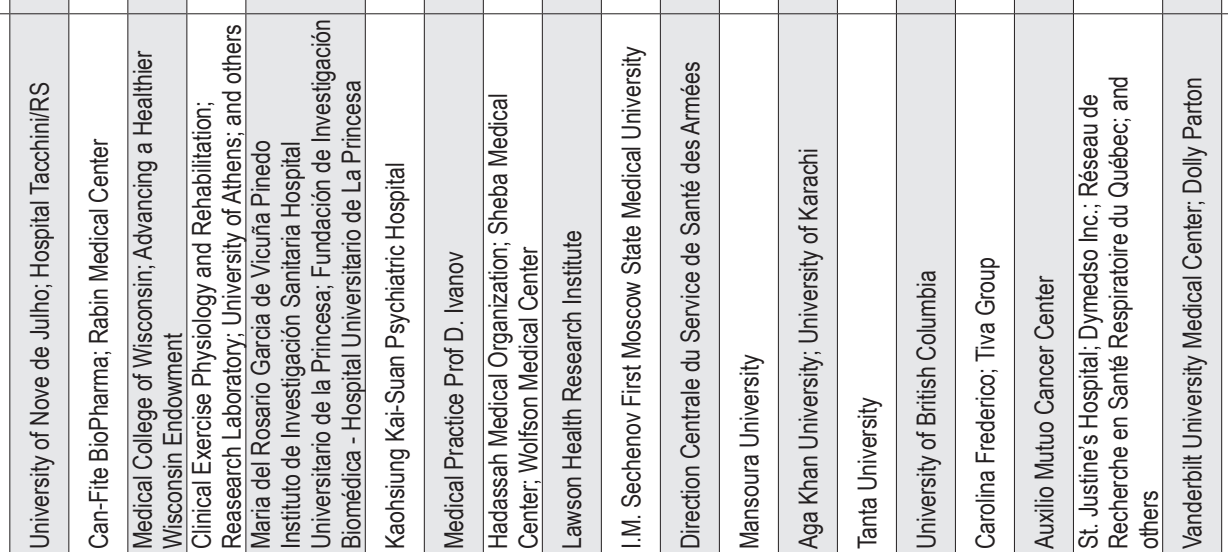

咅产

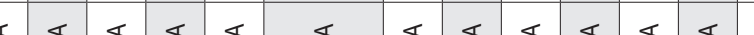

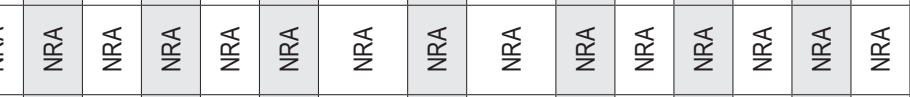

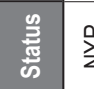

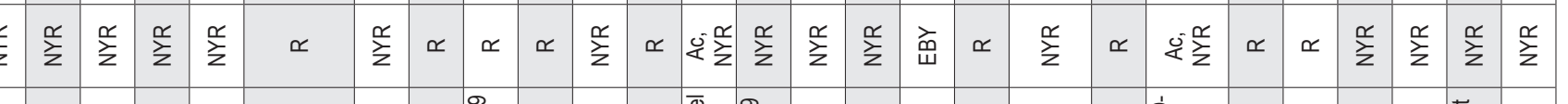

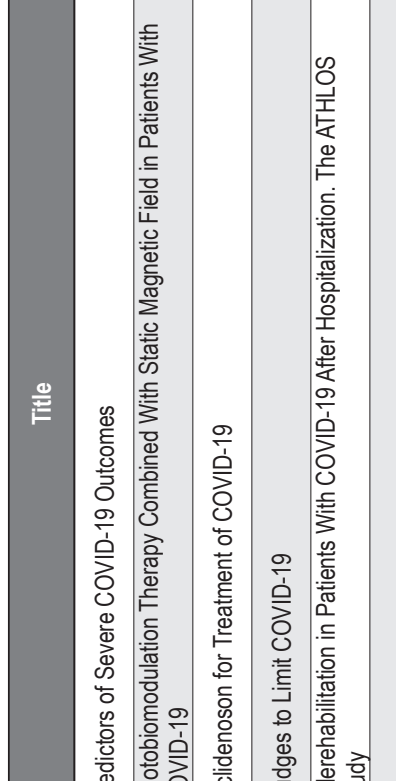

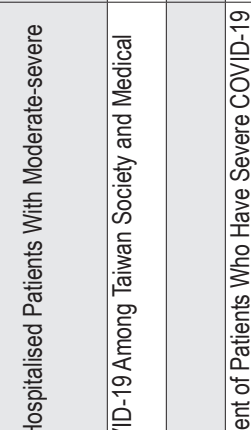

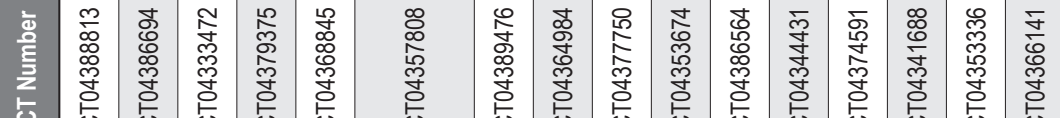

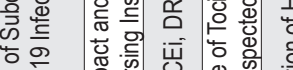

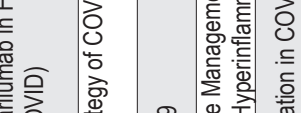

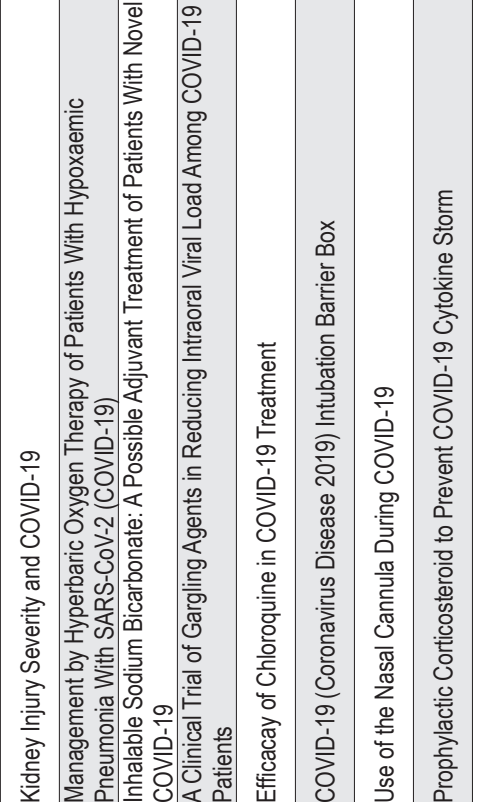

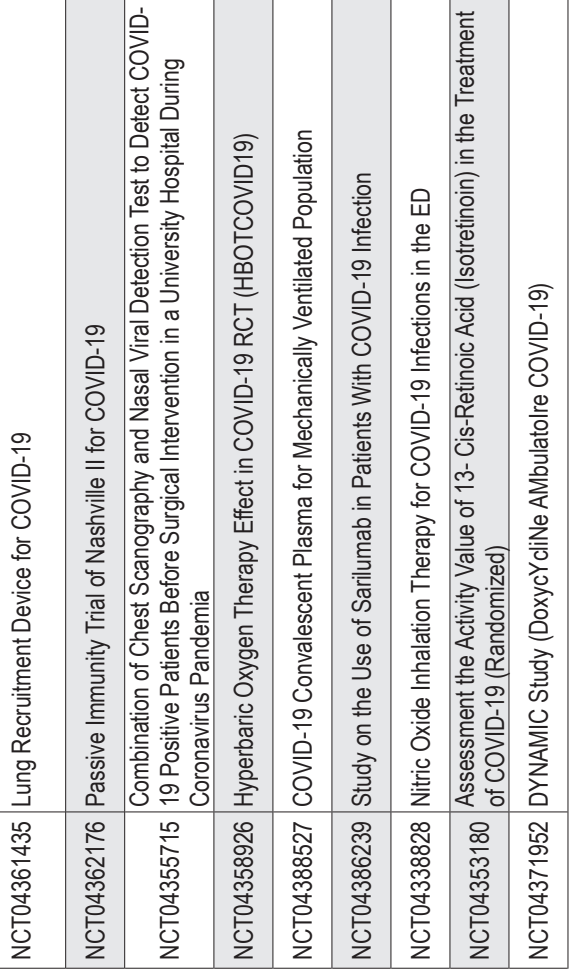




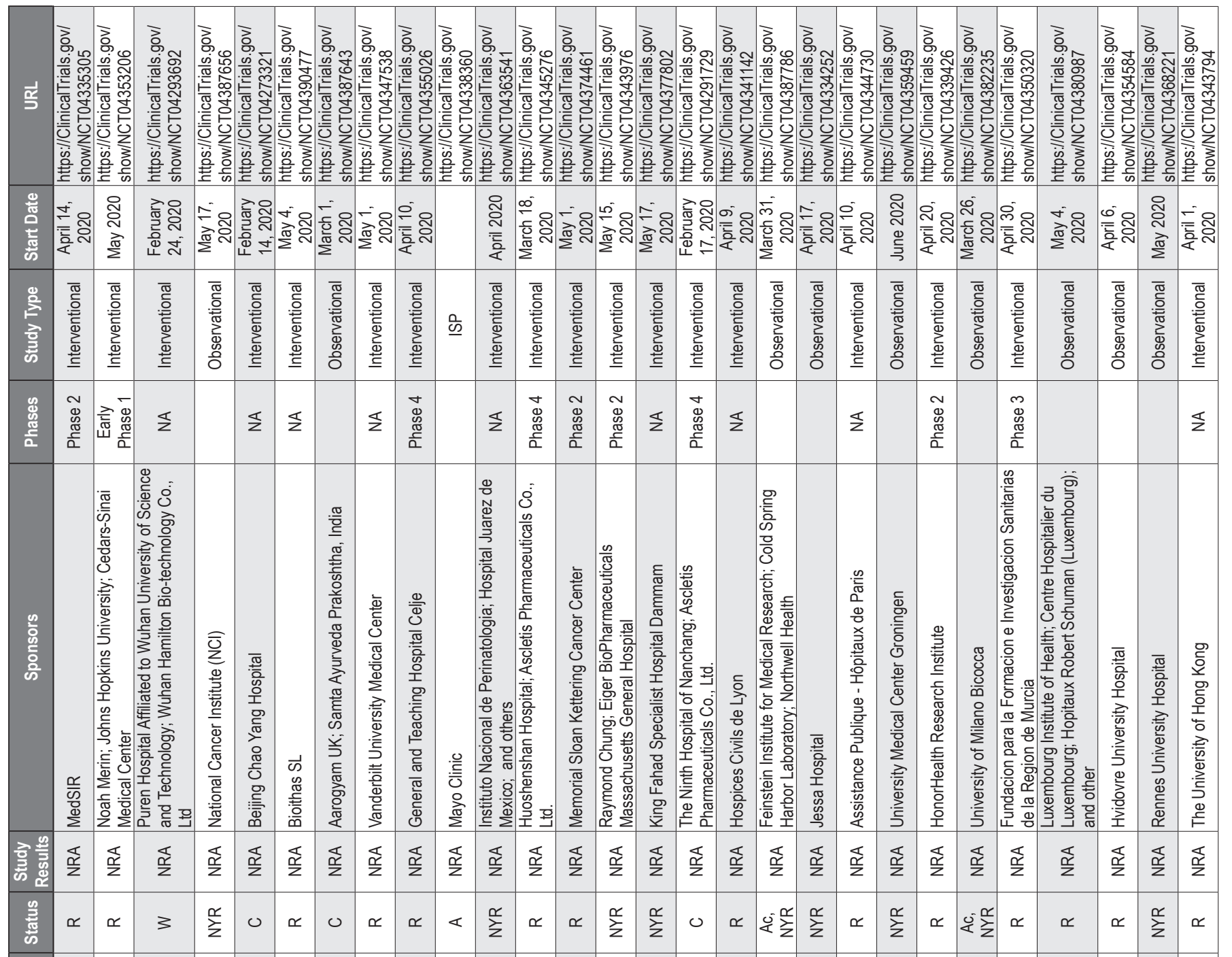

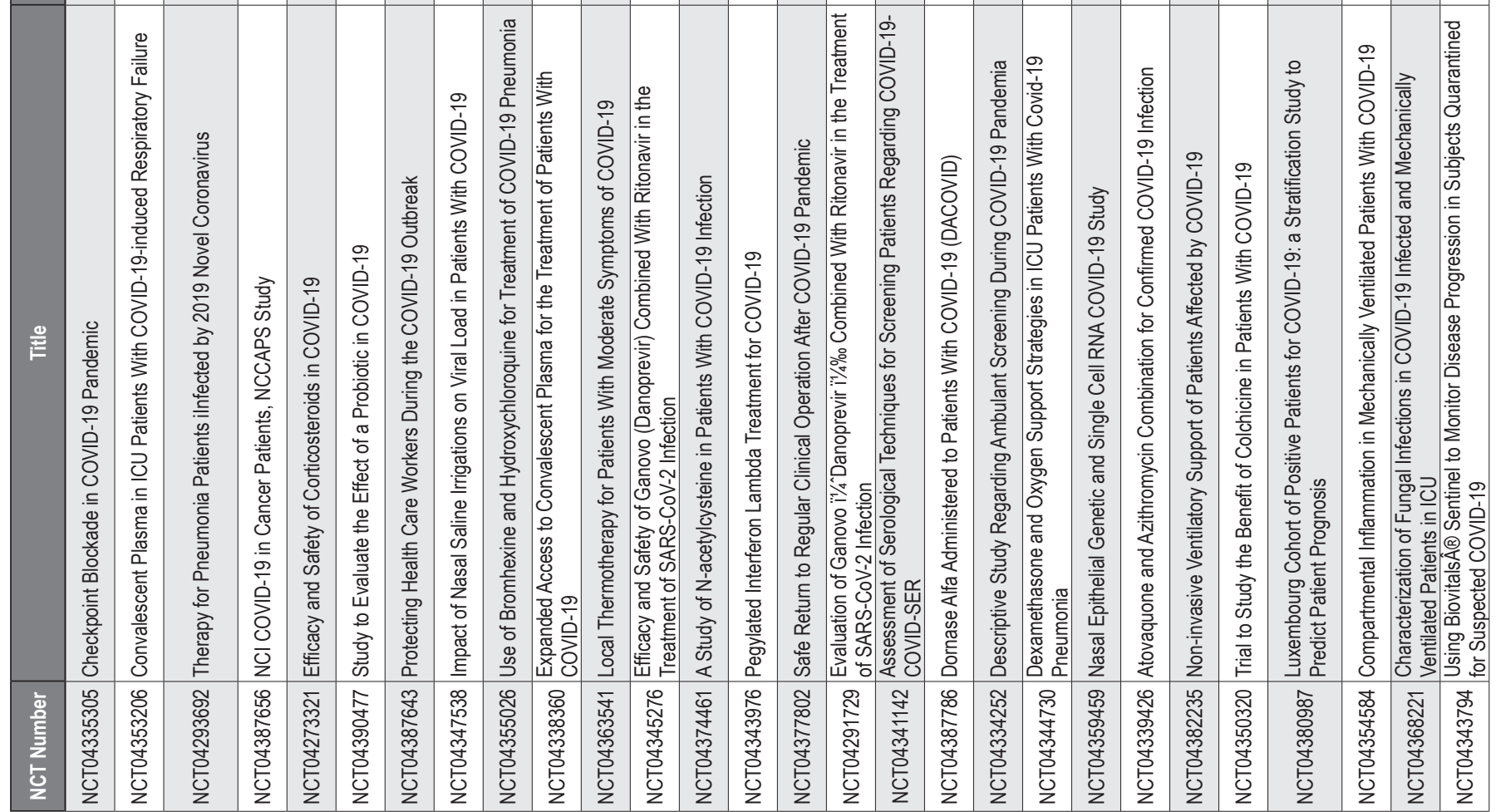




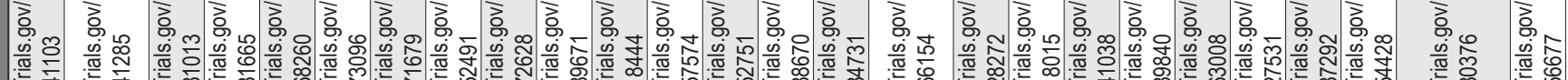

水 吾

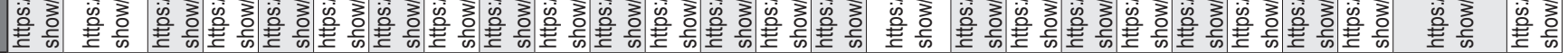

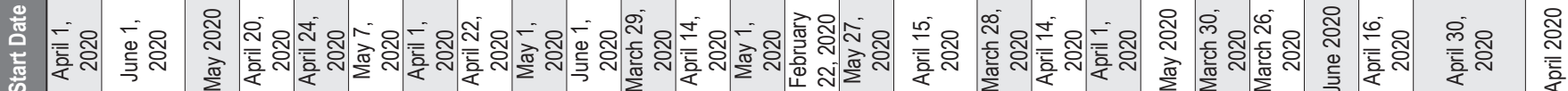

\begin{tabular}{|c|c|c|c|c|c|c|c|c|c|c|c|c|c|c|c|c|c|c|c|c|c|c|c|c|c|}
\hline 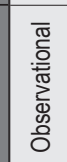 & 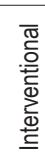 & 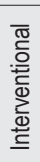 & 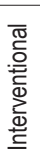 & 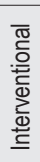 & 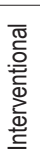 & 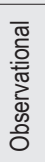 & 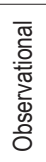 & 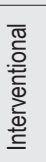 & 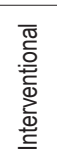 & 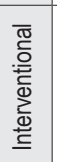 & 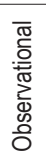 & 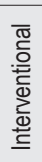 & 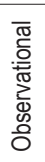 & 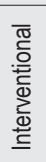 & 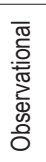 & 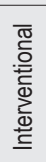 & 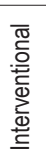 & 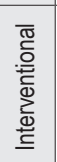 & 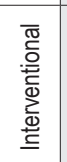 & 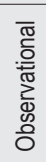 & 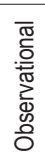 & 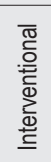 & 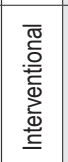 & 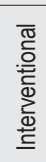 & 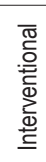 \\
\hline & $\Sigma$ & 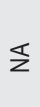 & $\Sigma$ & $\S$ & $\Sigma$ & & & $\begin{array}{l}\sim \\
\mathbb{N} \\
\mathbb{N} \\
\frac{\pi}{\alpha} \\
\end{array}$ & 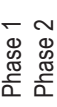 & & & $z$ & & $\begin{array}{l}\text { N } \\
\mathbb{N} \\
\frac{\pi}{\alpha} \\
\frac{\pi}{\alpha}\end{array}$ & & 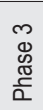 & 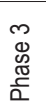 & 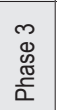 & 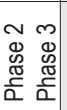 & & & $\frac{\Sigma}{z}$ & $\begin{array}{ll}\approx & m \\
\mathbb{w} & \mathbb{2} \\
\mathbb{\pi} & \mathbb{2} \\
\frac{\pi}{\alpha} & \frac{\pi}{\alpha}\end{array}$ & $\begin{array}{l}\sim \\
\mathbb{8} \\
\tilde{\pi} \\
\frac{\pi}{\alpha}\end{array}$ & 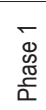 \\
\hline
\end{tabular}

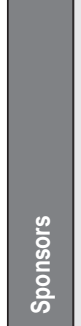

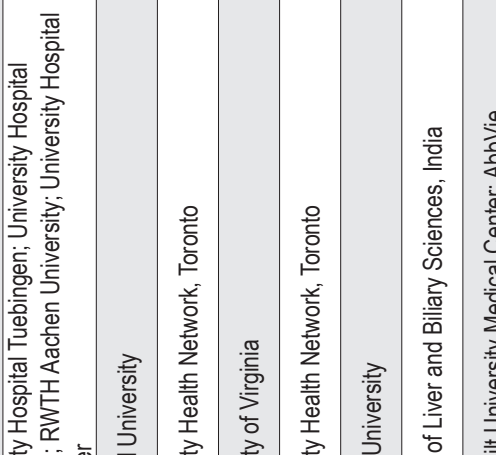

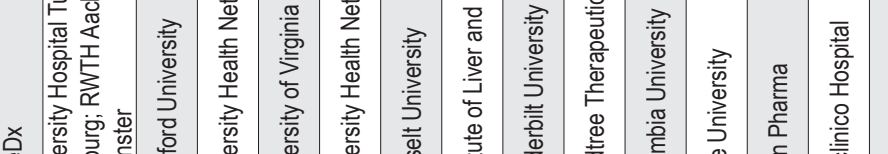

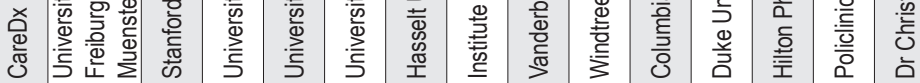
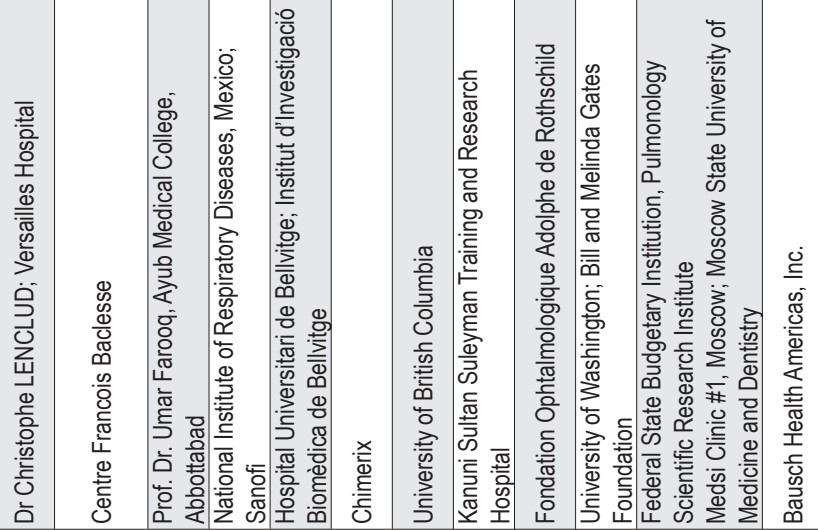

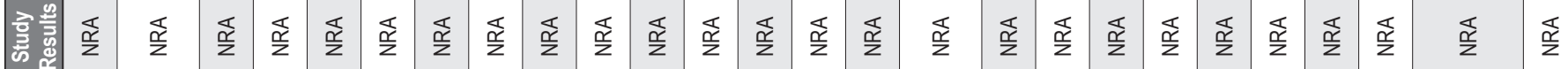

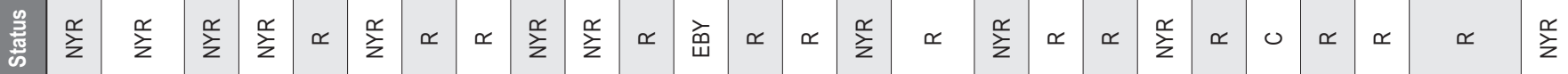

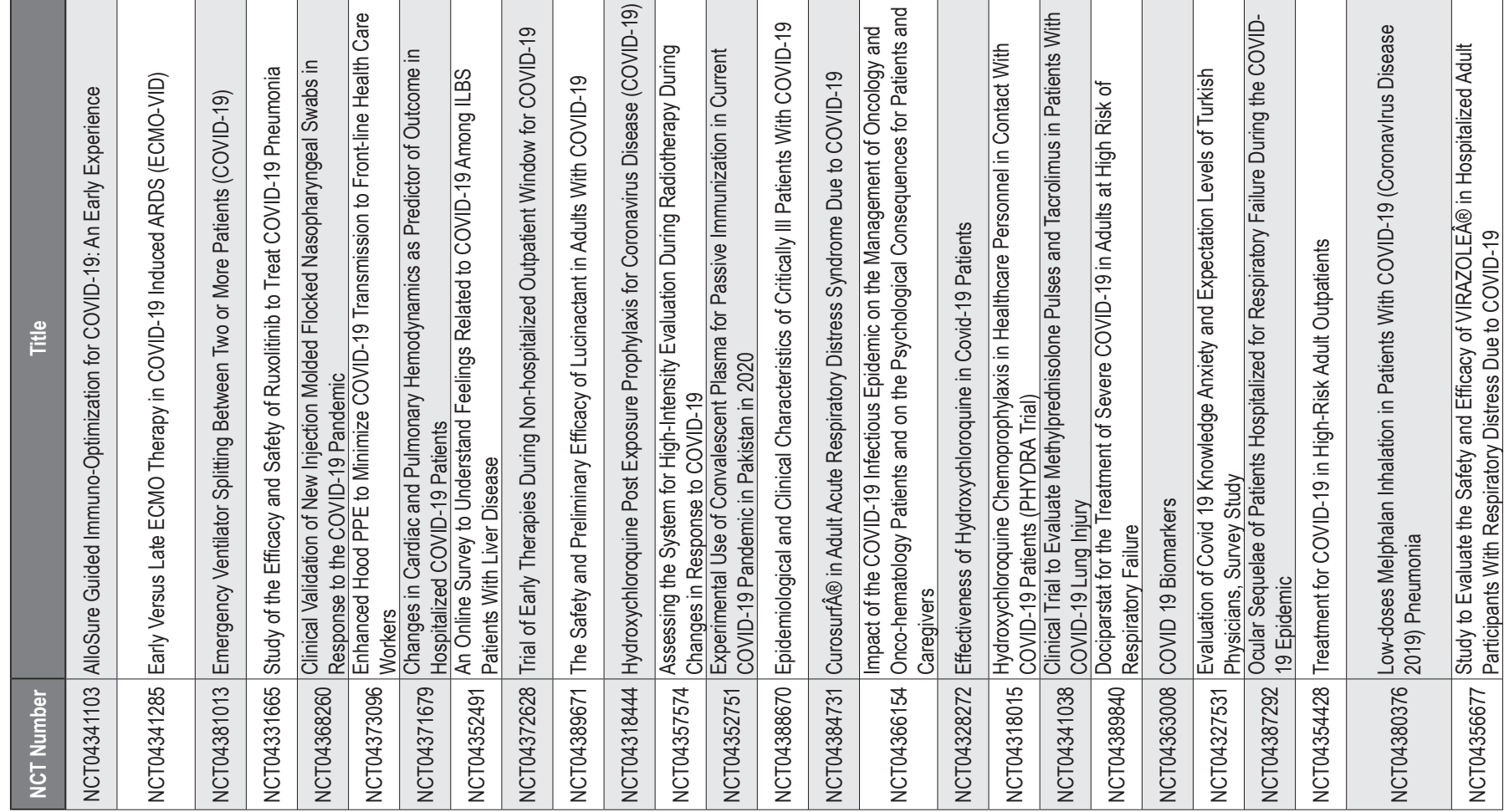




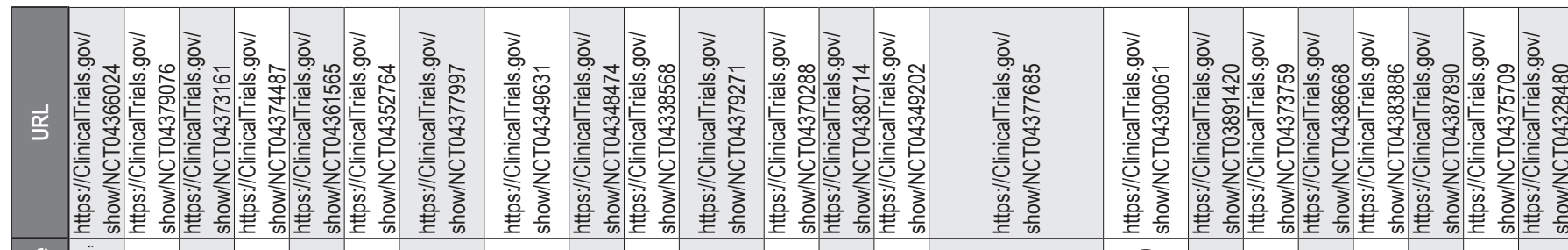

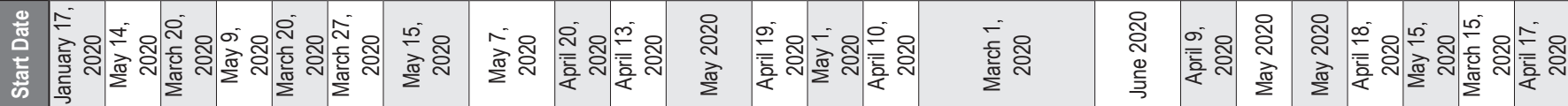

\begin{tabular}{|c|c|c|c|c|c|c|c|c|c|c|c|c|c|c|c|c|c|c|c|c|c|c|}
\hline 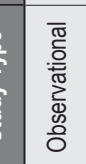 & 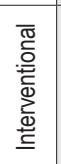 & 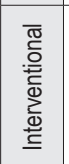 & 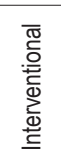 & 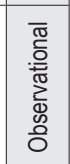 & 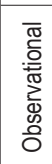 & 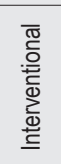 & 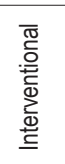 & 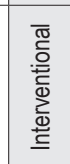 & 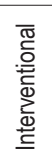 & 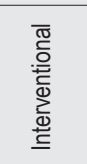 & 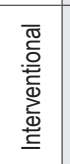 & 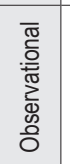 & 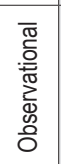 & 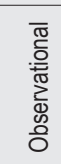 & 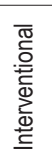 & 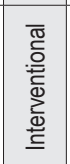 & 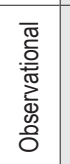 & 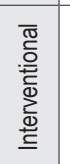 & 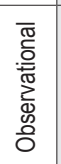 & ळ & & 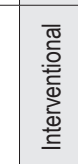 \\
\hline & 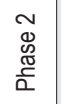 & $\underline{z}$ & 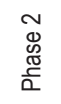 & & & $\begin{array}{l}\text { o. } \\
\text { 鱼 }\end{array}$ & $\begin{array}{l}\text { o. } \\
\text { 兽 }\end{array}$ & 㢇 & $\underline{z}$ & 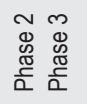 & 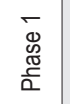 & & & & $\begin{array}{l}\text { 兽 } \\
\frac{a}{\alpha}\end{array}$ & 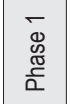 & & $\underline{z}$ & & & & $\frac{\mathrm{g}}{\mathrm{a}}$ \\
\hline
\end{tabular}

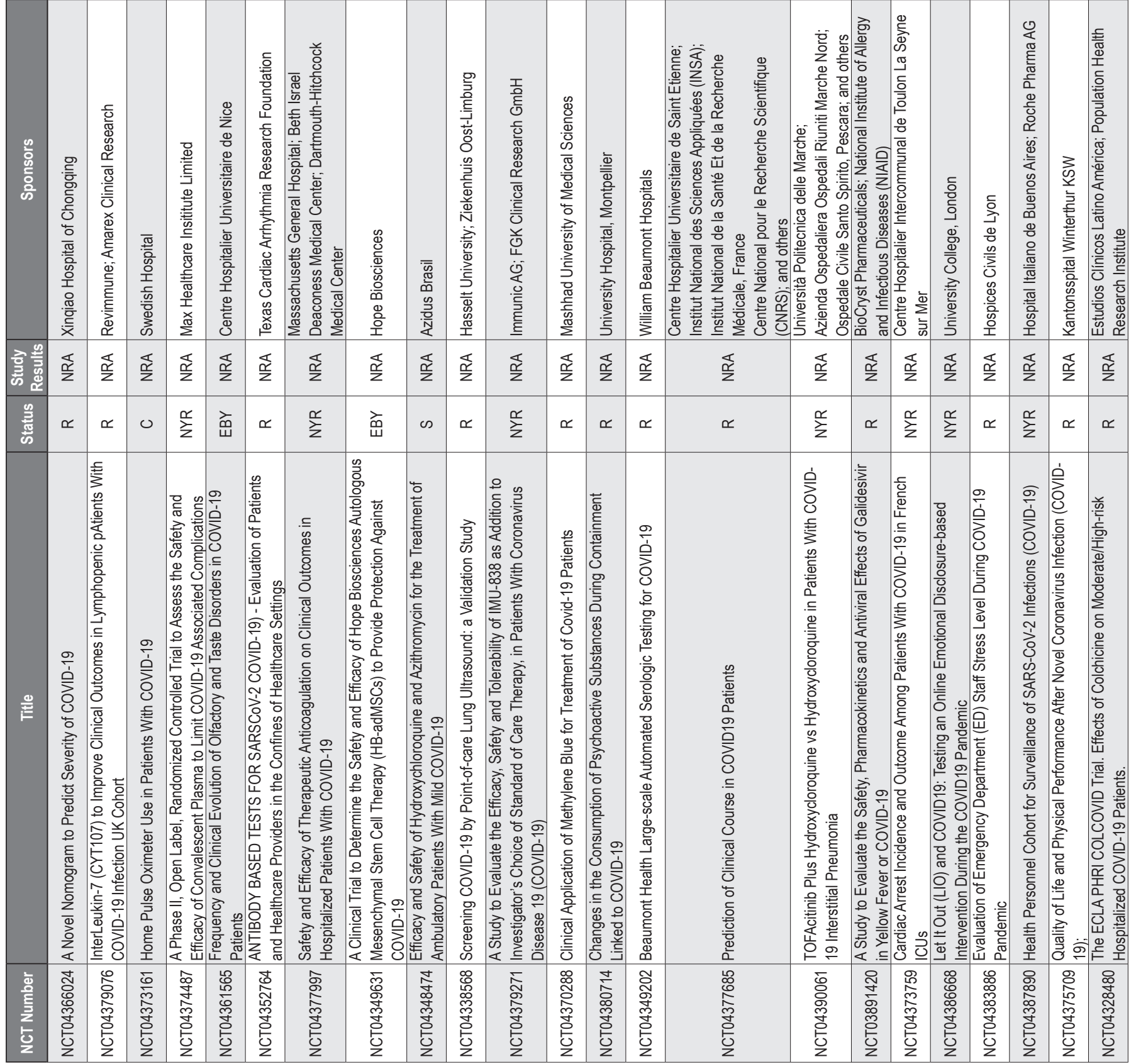




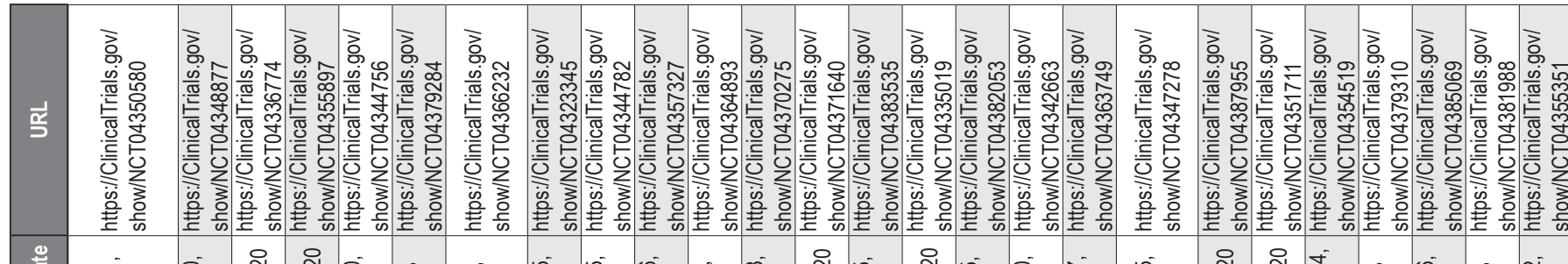

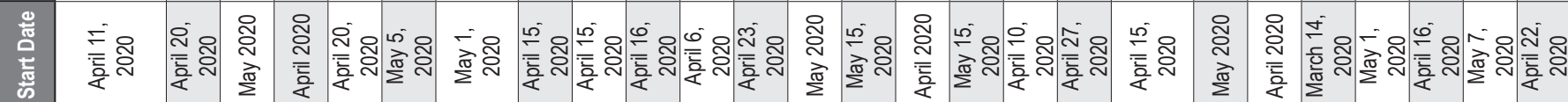

\begin{tabular}{|c|c|c|c|c|c|c|c|c|c|c|c|c|c|c|c|c|c|c|c|c|c|c|c|c|c|}
\hline 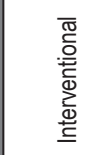 & 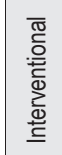 & 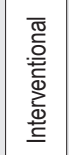 & 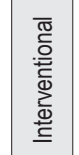 & 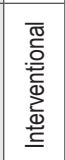 & 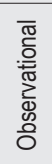 & 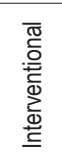 & 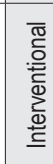 & 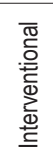 & 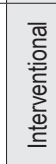 & 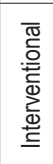 & 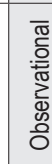 & 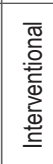 & 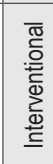 & 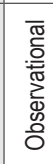 & 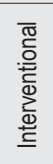 & 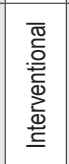 & 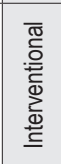 & 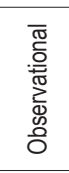 & 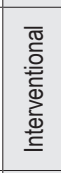 & 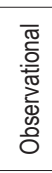 & 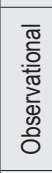 & 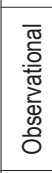 & ฮ & & \\
\hline 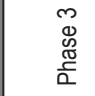 & $\underline{z}$ & & 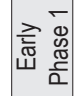 & 惫 & & 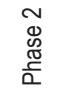 & \begin{tabular}{|l}
$\infty$ \\
$\mathscr{\mathscr { m }}$ \\
$\frac{\pi}{\alpha}$
\end{tabular} & 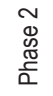 & $\underline{z}$ & $\underline{z}$ & & 惫 $\frac{8}{0}$ & $\underline{\pi}$ & & $\begin{array}{l}\text { 总 } \\
\text { 产 }\end{array}$ & 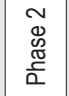 & $\underline{z}$ & & $\frac{\pi}{z}$ & & & & & 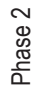 & \\
\hline
\end{tabular}

竞:

오 은

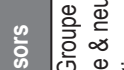

总

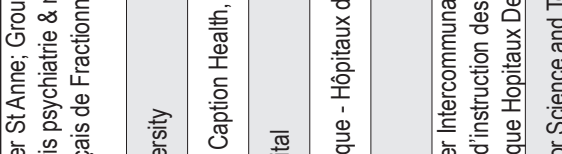

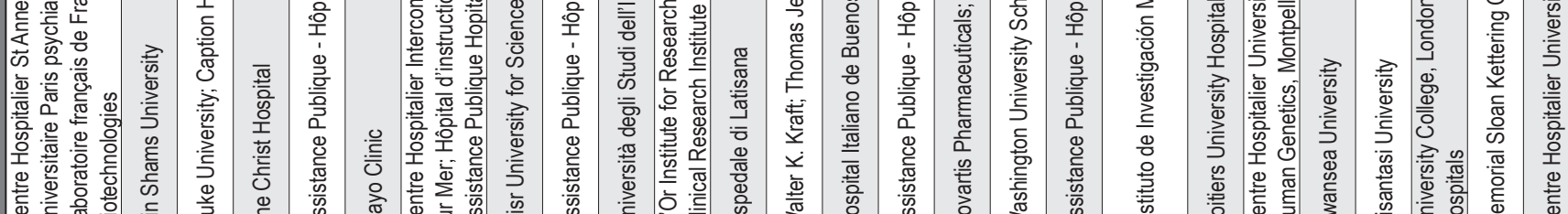

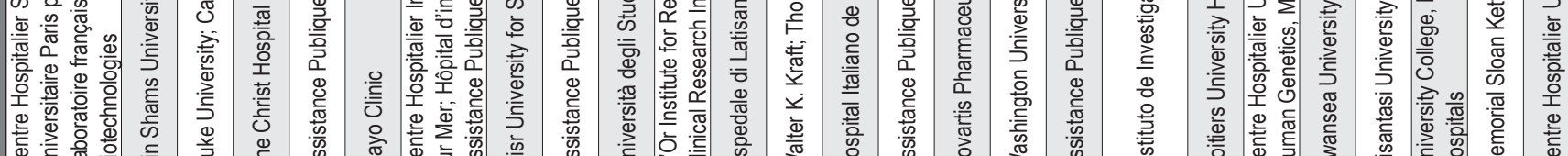

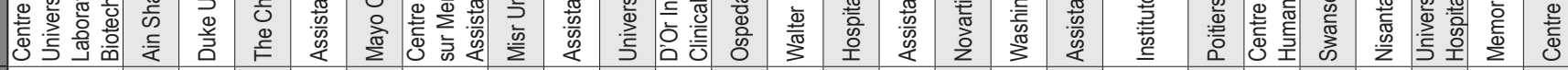

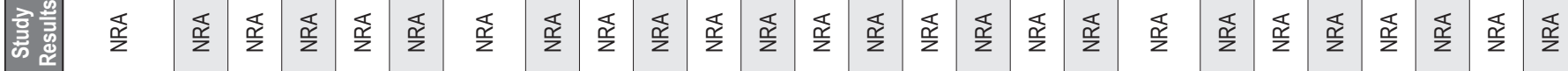
咅

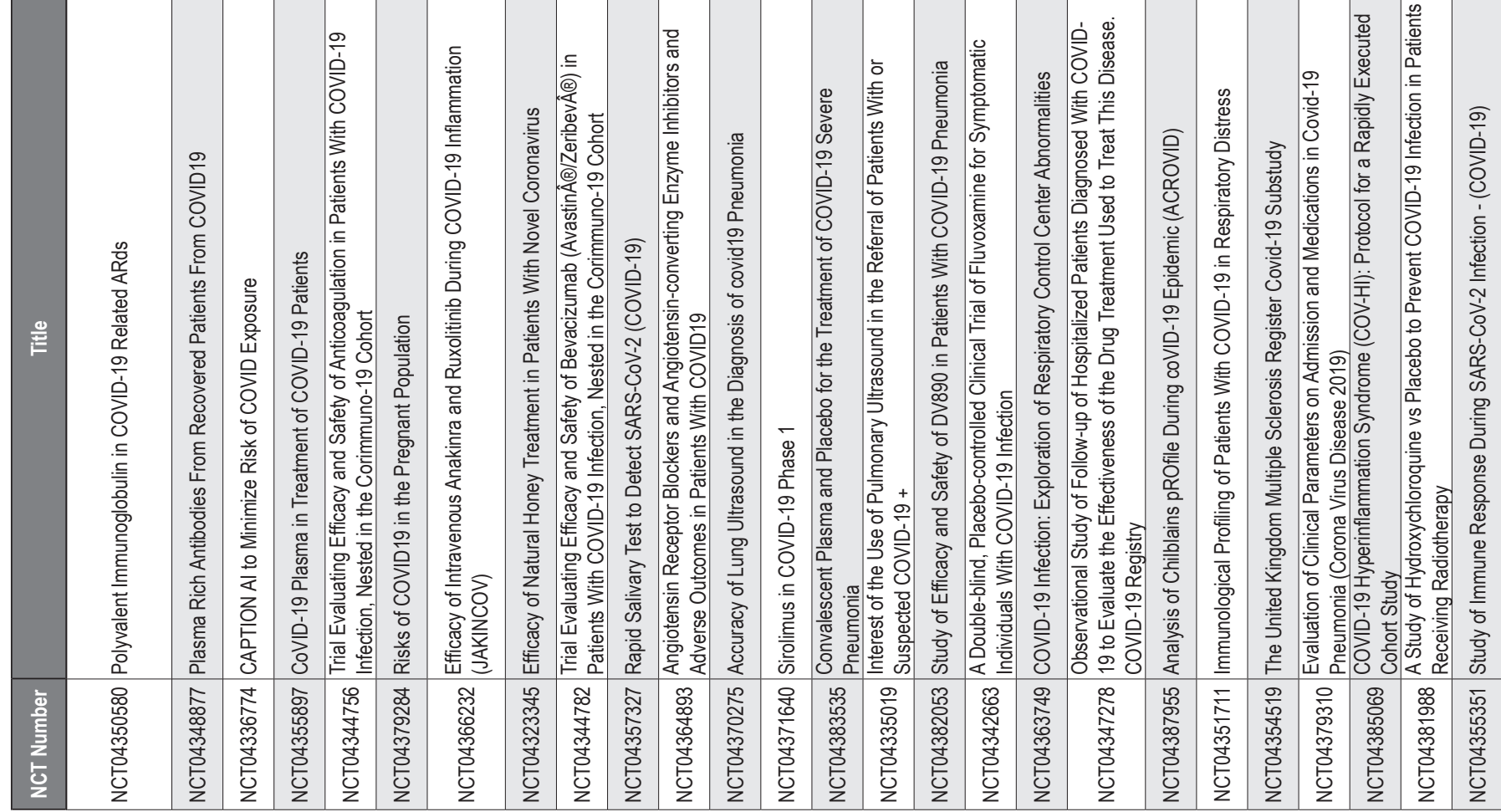




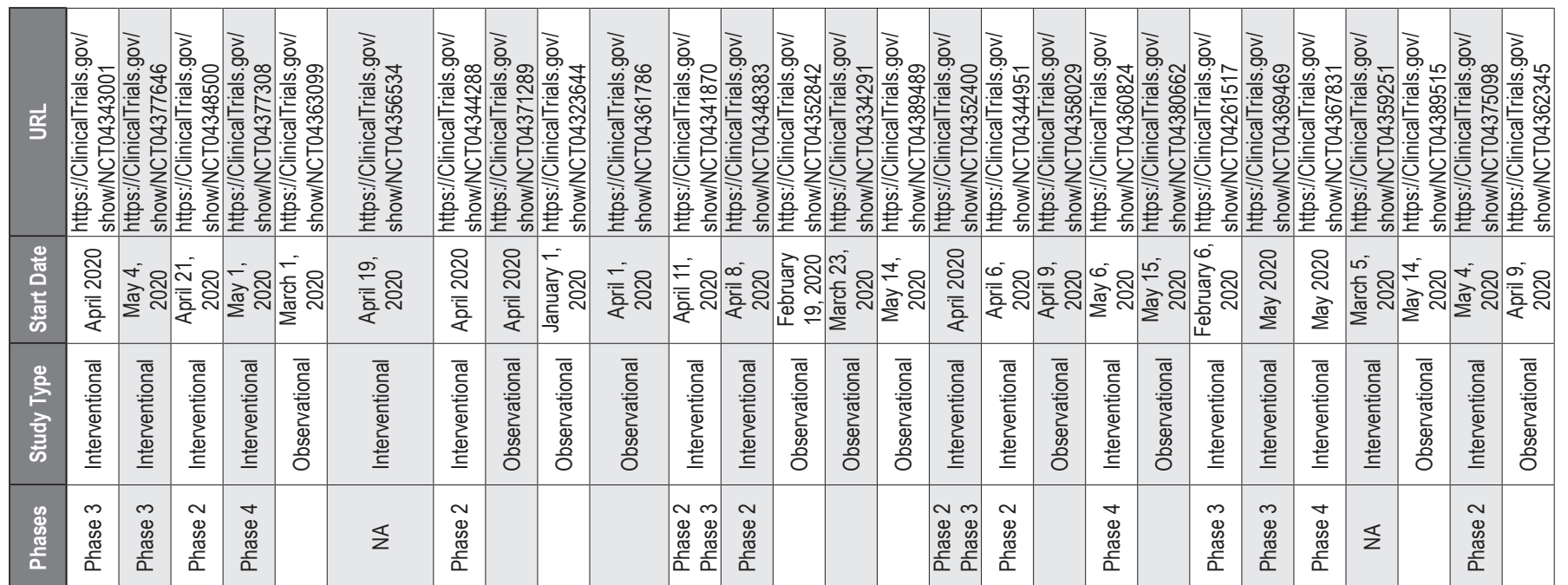

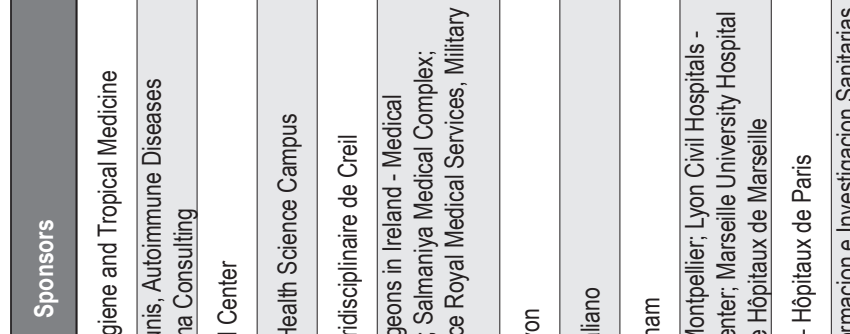

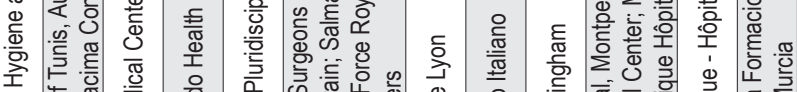

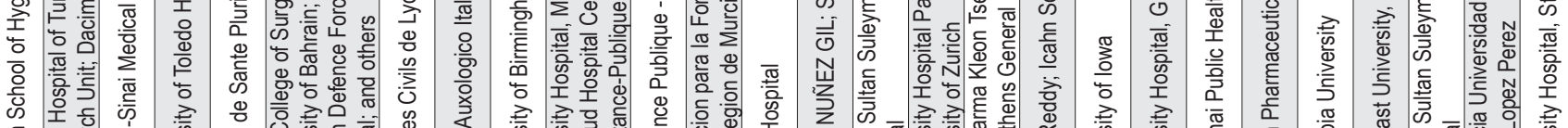

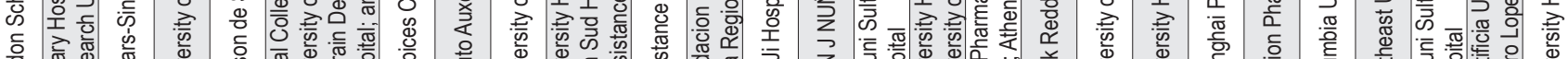

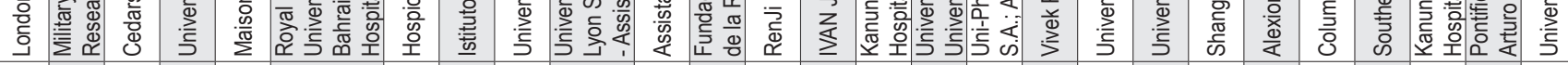

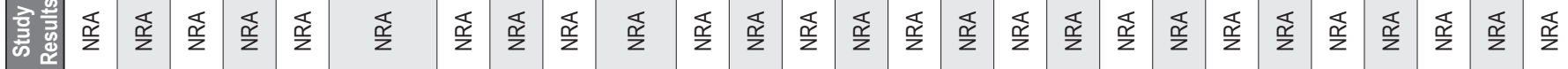

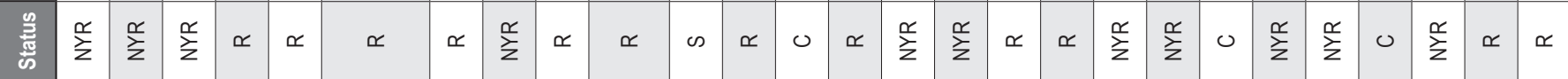

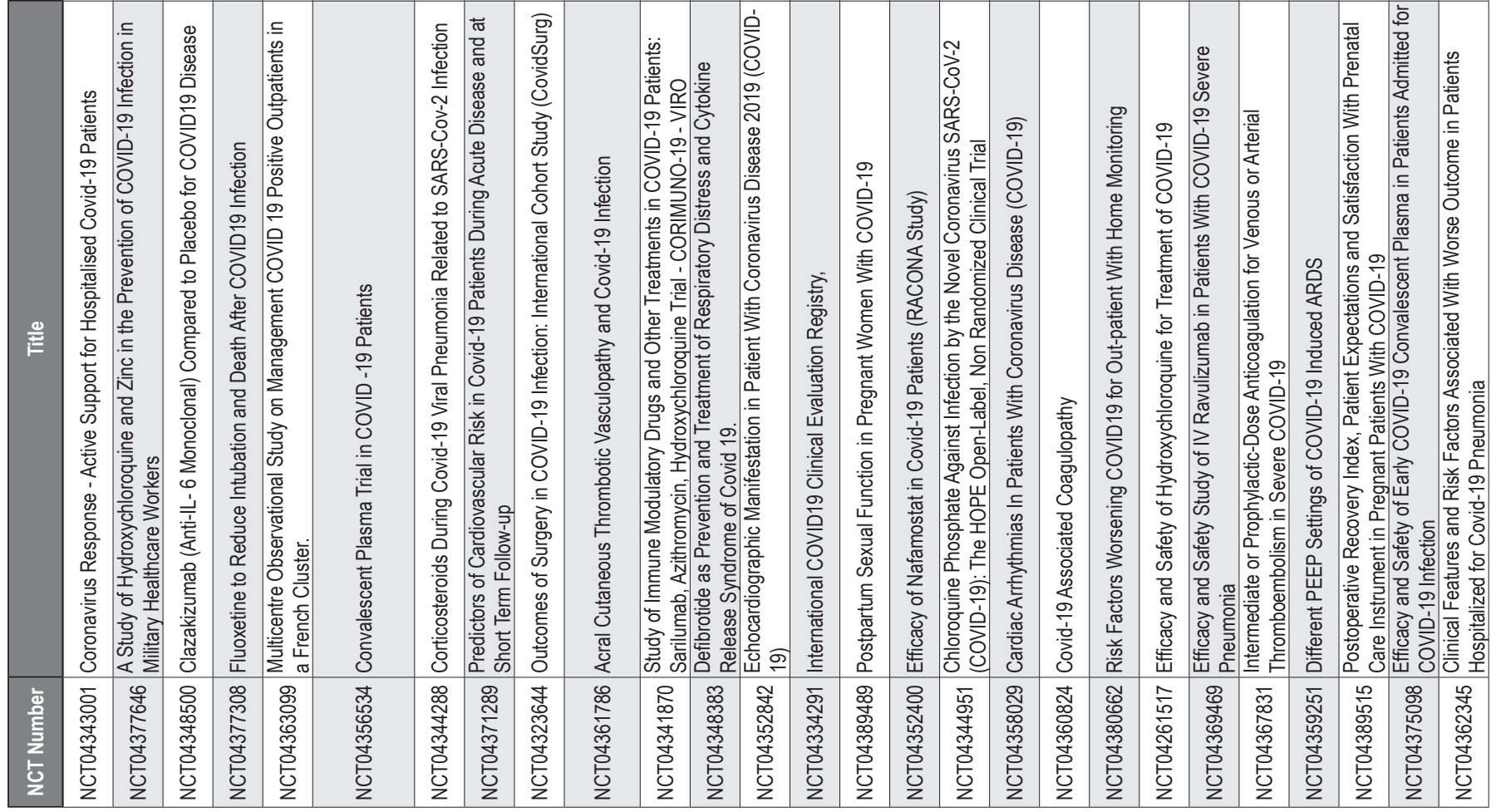




\begin{tabular}{|c|c|c|c|c|c|c|c|c|c|c|c|c|c|c|c|c|c|c|c|c|c|c|c|c|c|}
\hline 桨 & 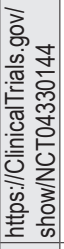 & 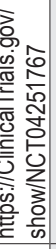 & 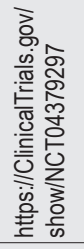 & 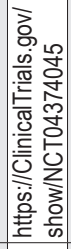 & 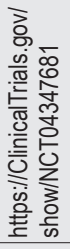 & 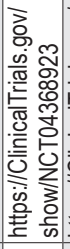 & 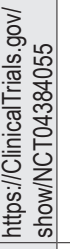 & 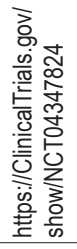 & 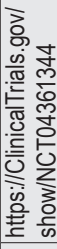 & 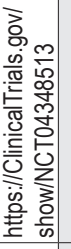 & 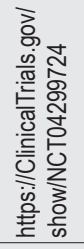 & 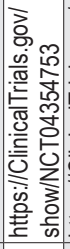 & 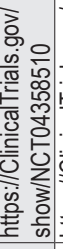 & & $z$ & 竘 & $\$$ & $\overline{0}$ & 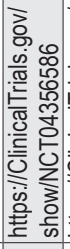 & 总 & 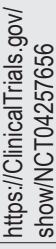 & 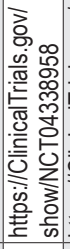 & $\mid$ & 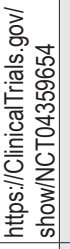 & 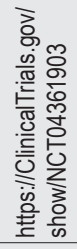 \\
\hline & 틍 & & 웅요 & 商 & 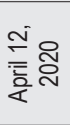 & & & స్. & & & $\begin{array}{l}\text { 워․ } \\
\text { 롱 } \\
\text { 인 } \\
\text { L }\end{array}$ & & & & & & & & & $\stackrel{\text { న్ }}{=}$ & & & & & 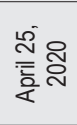 \\
\hline 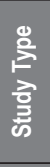 & 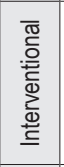 & 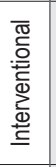 & 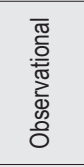 & 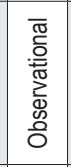 & 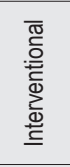 & 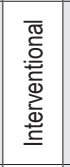 & 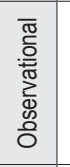 & 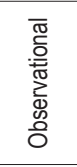 & 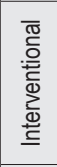 & 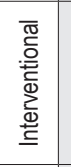 & 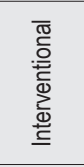 & 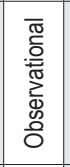 & 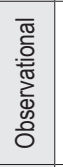 & 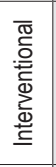 & 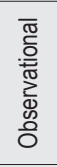 & 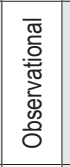 & 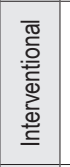 & 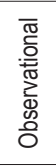 & 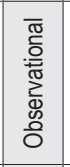 & 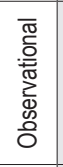 & 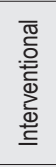 & 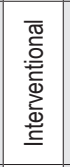 & 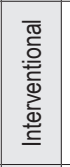 & 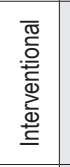 & 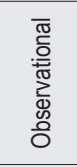 \\
\hline & $\begin{array}{l}\infty \\
\mathbb{D} \\
\frac{\pi}{\alpha} \\
\frac{\pi}{\alpha}\end{array}$ & $\Sigma$ & & & 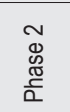 & $\frac{\pi}{z}$ & & & $\mathbb{z}$ & $\begin{array}{l}\sim \\
\mathbb{8} \\
\frac{\pi}{\alpha} \\
\frac{\pi}{\alpha}\end{array}$ & $\begin{array}{l}\overline{8} \\
\overline{8} \\
\frac{\pi}{\alpha}\end{array}$ & & & $\begin{array}{l}\text { ष } \\
\mathbb{8} \\
\frac{\pi}{\alpha}\end{array}$ & & & $\begin{array}{l}\sim \\
\mathscr{g} \\
\frac{\pi}{\alpha} \\
\frac{\pi}{\alpha}\end{array}$ & & & & 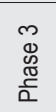 & 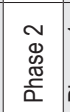 & 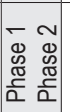 & $\begin{array}{l}\tilde{N} \\
\mathbb{\Phi} \\
\frac{\pi}{\alpha}\end{array}$ & \\
\hline
\end{tabular}

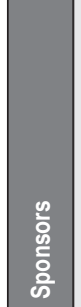

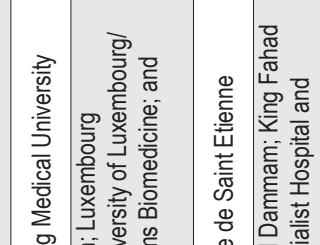

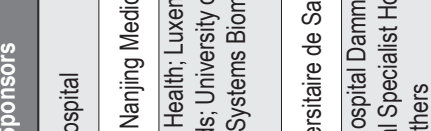

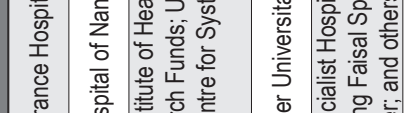

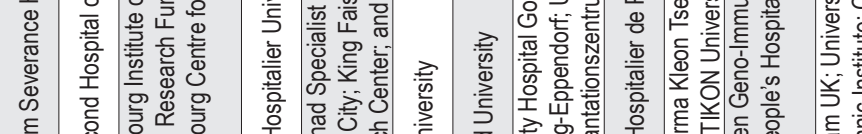

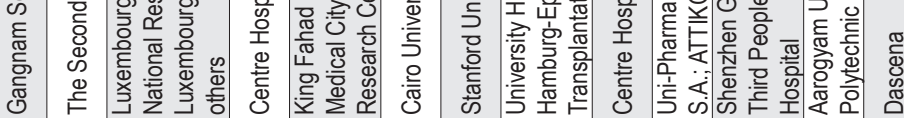

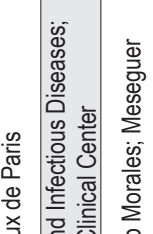

×

产

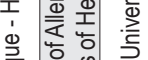

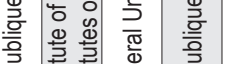

责
产

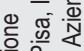

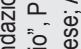

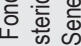

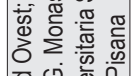

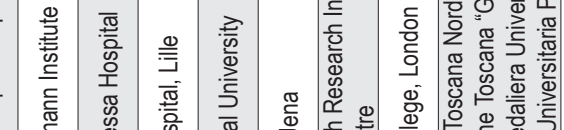
要 要

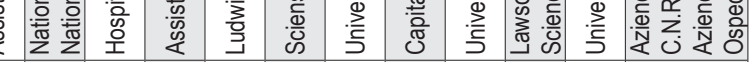

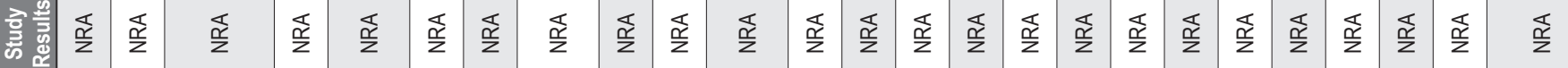

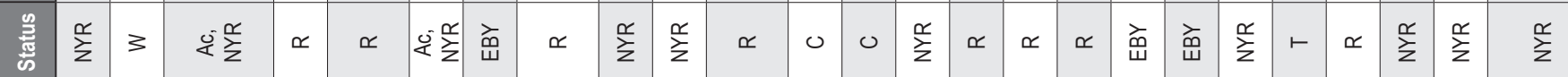

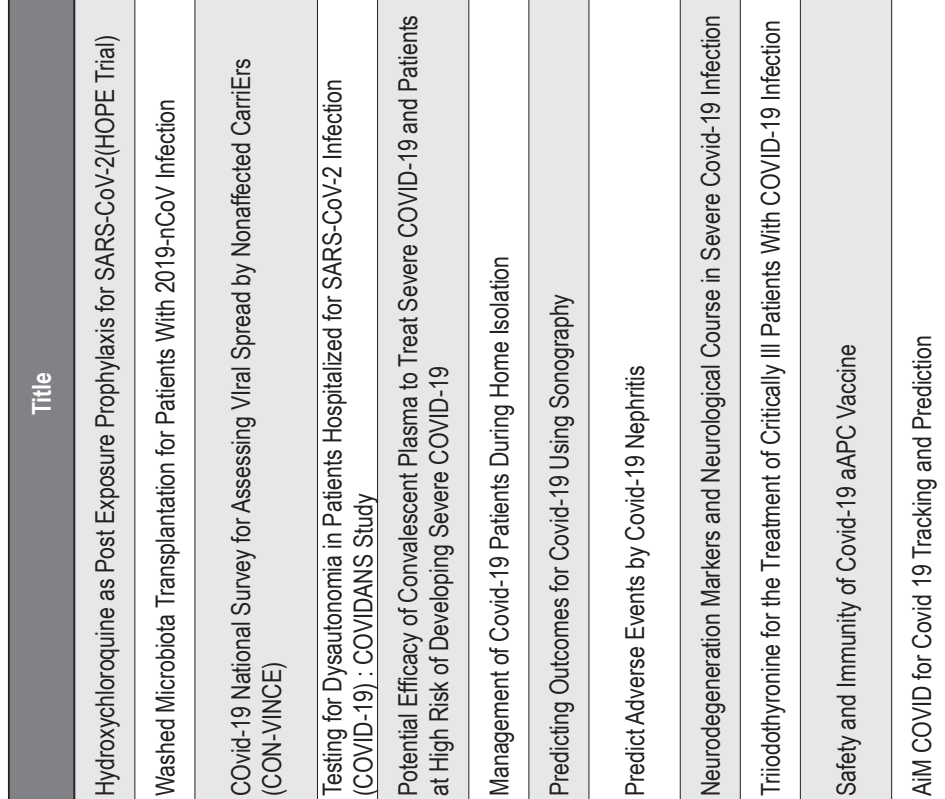

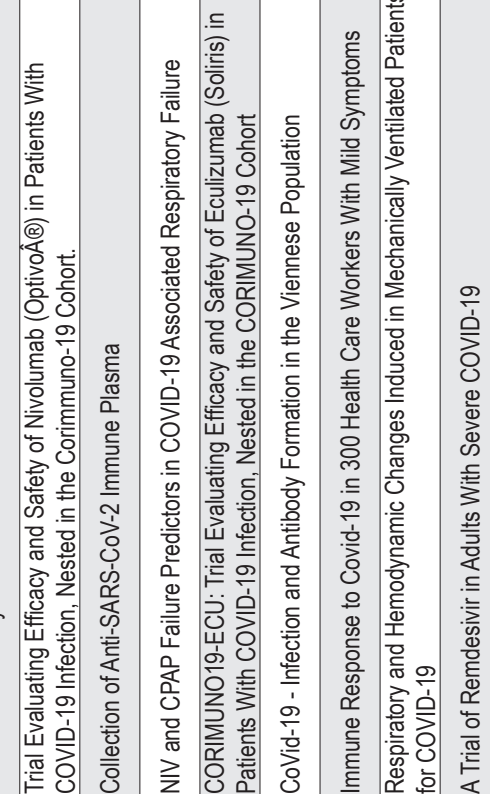

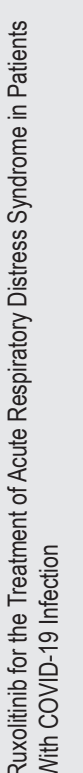

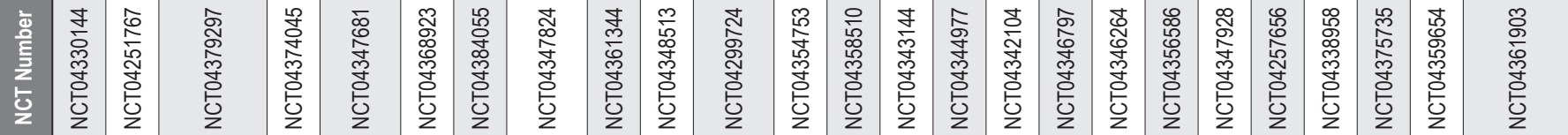




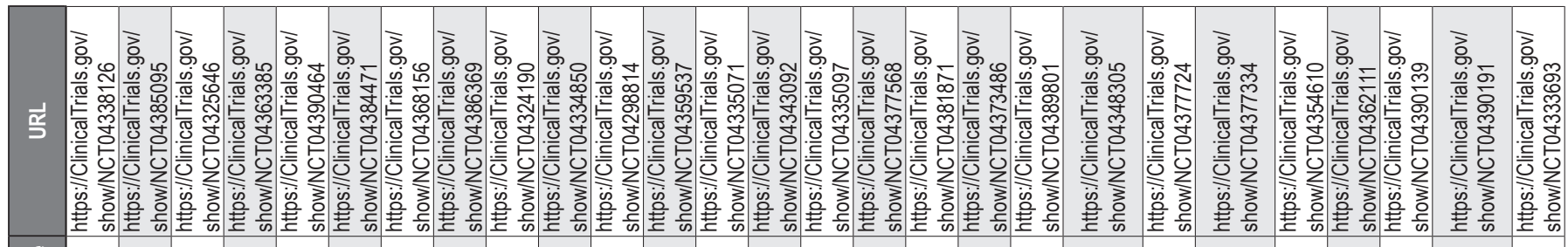

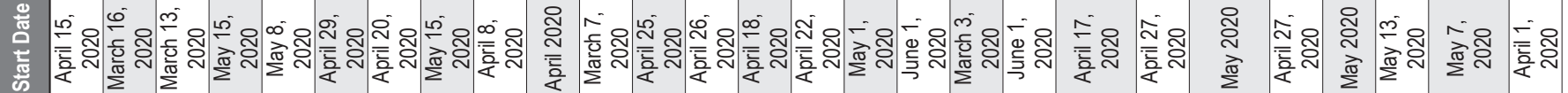

\begin{tabular}{|c|c|c|c|c|c|c|c|c|c|c|c|c|c|c|c|c|c|c|c|c|c|c|c|c|c|}
\hline 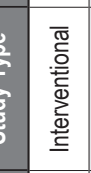 & 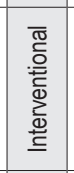 & 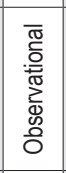 & 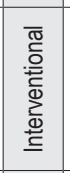 & 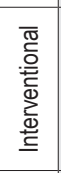 & 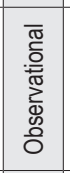 & 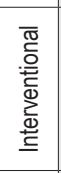 & 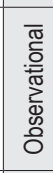 & 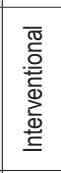 & 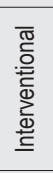 & 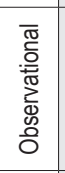 & 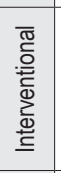 & 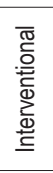 & 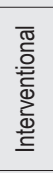 & 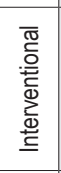 & 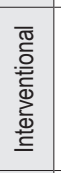 & 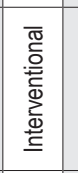 & 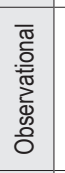 & 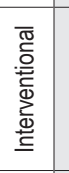 & 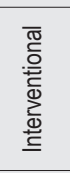 & 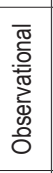 & 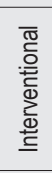 & & & 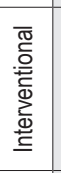 & 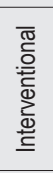 \\
\hline 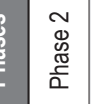 & \begin{tabular}{|l}
$\mathbb{\Xi}$ \\
$\frac{\mathbb{g}}{\alpha}$ \\
$\frac{\pi}{\alpha}$
\end{tabular} & & $\frac{\xi}{z}$ & $\begin{array}{l}\frac{\mathscr{g}}{2} \\
\frac{2}{2}\end{array}$ & & $\frac{\underline{z}}{z}$ & & $\frac{\mathbb{E}}{z}$ & $\frac{\mathbb{s}}{z}$ & & 恖 & $\mathbb{\mathscr { g }}_{\mathbb{8}}$ & $\mathscr{\mathscr { g }}$ & $\frac{\pi}{z}$ & & & & 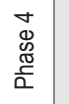 & 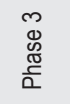 & & $\frac{\pi}{\frac{\pi}{\alpha}}$ & & & & \\
\hline
\end{tabular}

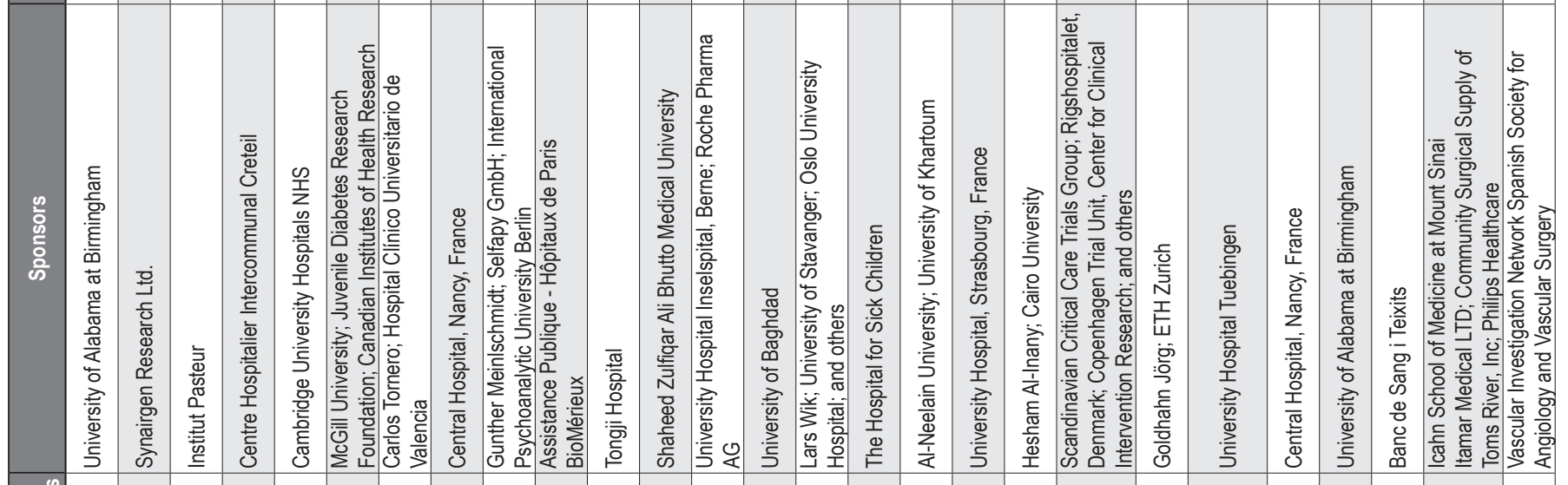

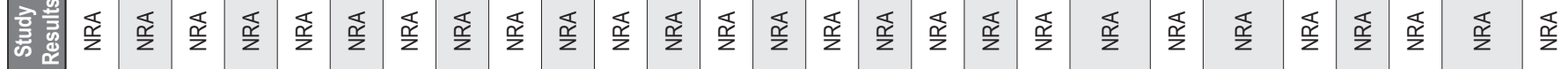

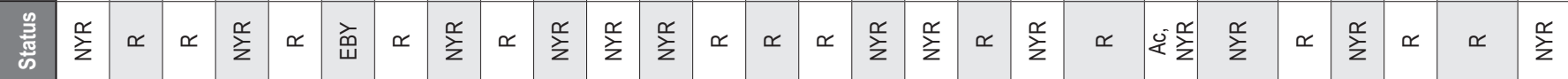

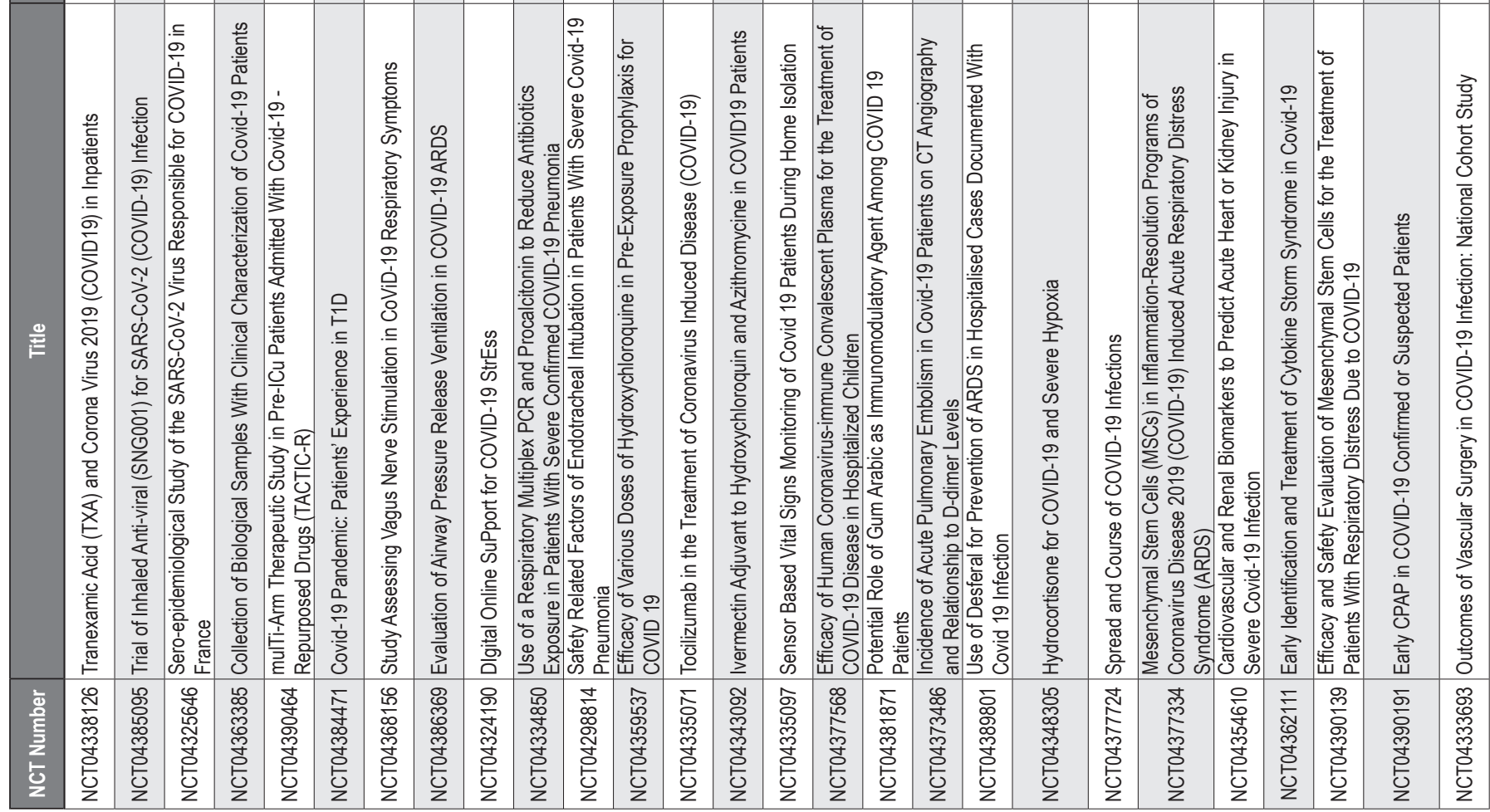




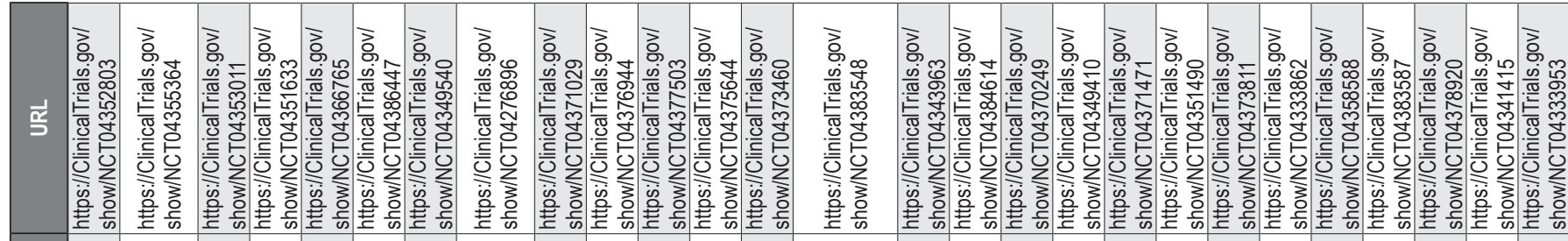

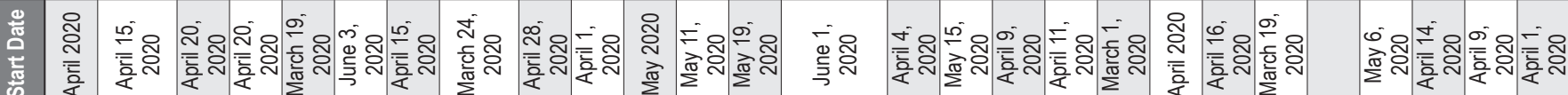

\begin{tabular}{|c|c|c|c|c|c|c|c|c|c|c|c|c|c|c|c|c|c|c|c|c|c|c|c|}
\hline 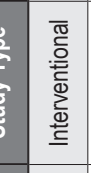 & 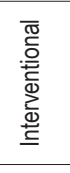 & 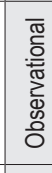 & 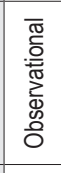 & 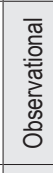 & 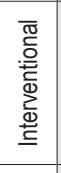 & 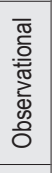 & 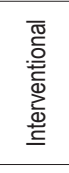 & 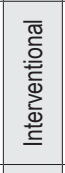 & 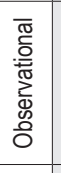 & 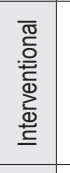 & 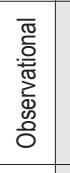 & 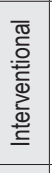 & 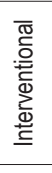 & 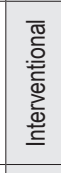 & 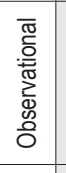 & 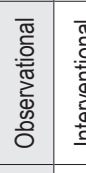 & 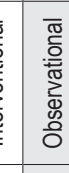 & 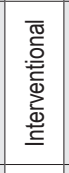 & 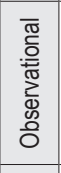 & 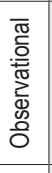 & $\underline{\underline{0}}$ & 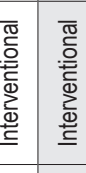 & 区్ \\
\hline 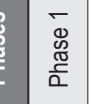 & $\begin{array}{l}\infty \\
\frac{0}{0} \\
\frac{\tilde{m}}{\alpha}\end{array}$ & & & & $\begin{array}{l}\frac{w}{\mathscr{g}} \\
\frac{\mathrm{g}}{\mathrm{a}}\end{array}$ & & 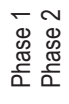 & $\frac{\Sigma}{z}$ & & 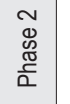 & & 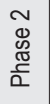 & $z$ & & & & & $\underline{z}$ & & & & 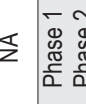 & \\
\hline
\end{tabular}

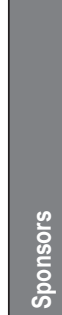

ì

을

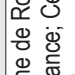

응 뉸 일

훈 옹을

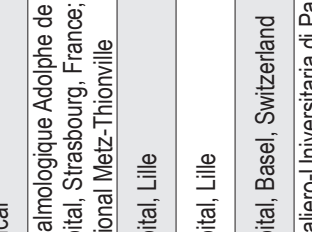

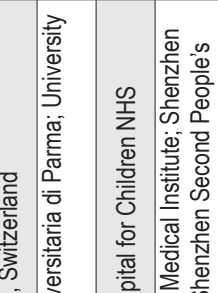

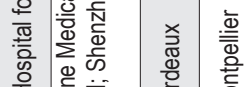

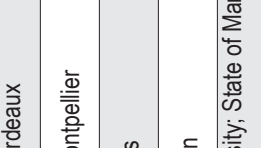

屯 产

之

类

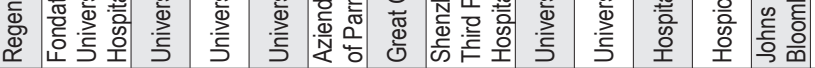

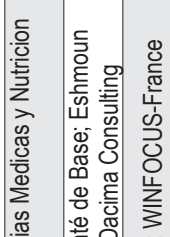

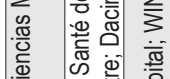

宁 कि

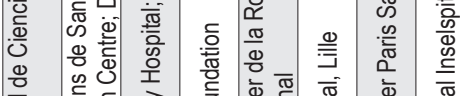

ᄃ

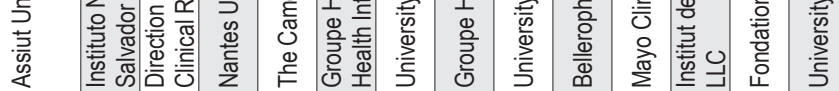

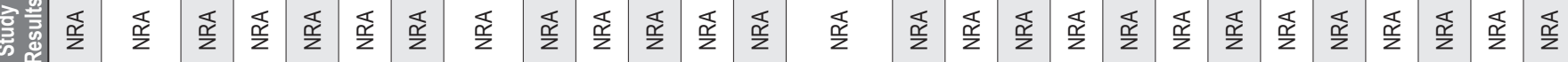

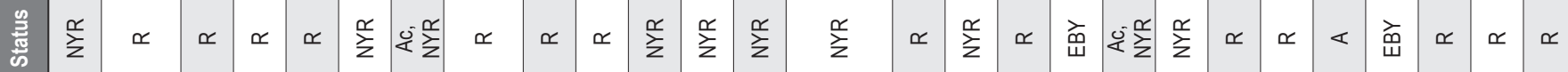

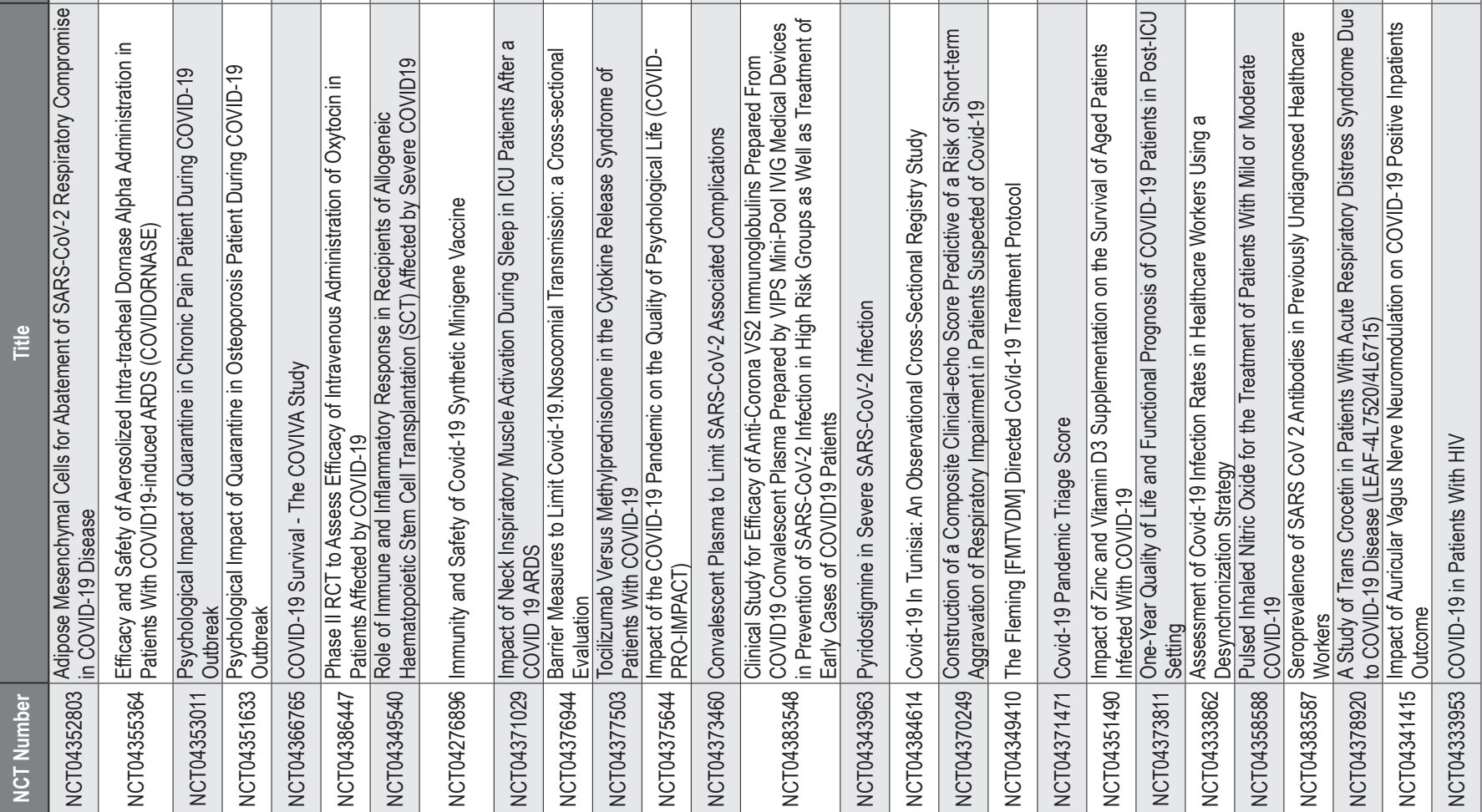




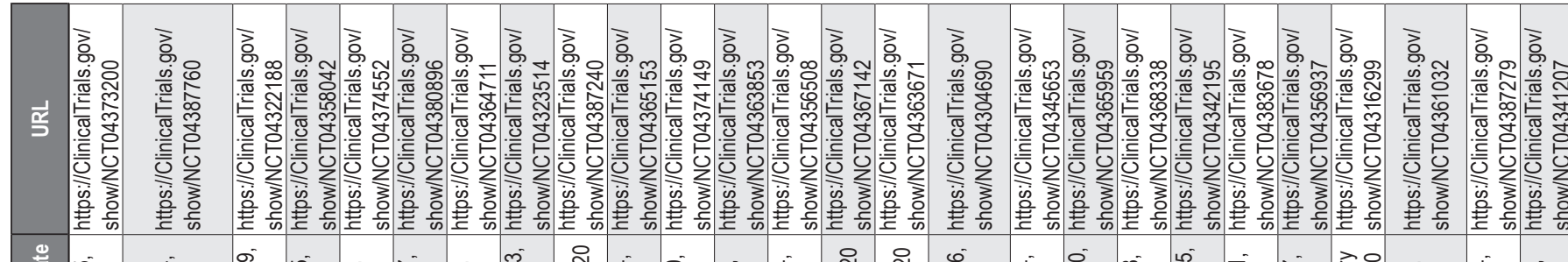

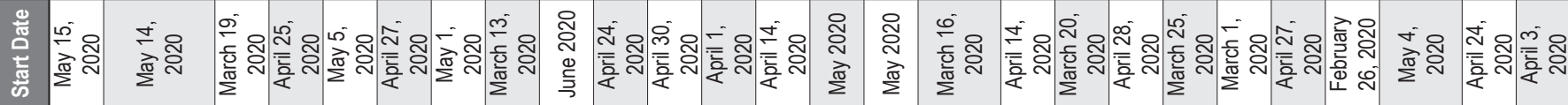

\begin{tabular}{|c|c|c|c|c|c|c|c|c|c|c|c|c|c|c|c|c|c|c|c|c|c|c|c|}
\hline 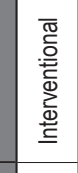 & 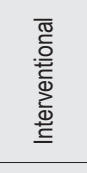 & 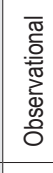 & 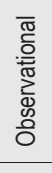 & 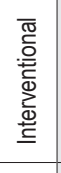 & 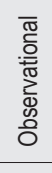 & 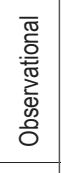 & 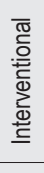 & 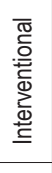 & 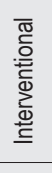 & 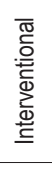 & 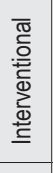 & 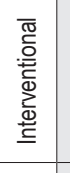 & 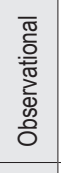 & 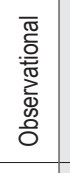 & 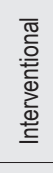 & 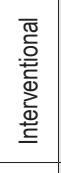 & 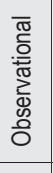 & 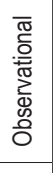 & 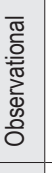 & 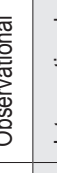 & 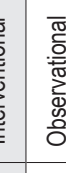 & 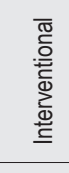 & 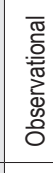 \\
\hline 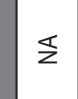 & 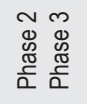 & & & 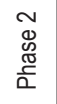 & & & $\sum$ & 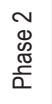 & $\begin{array}{l}\mathscr{\%} \\
\mathbb{\sigma}\end{array}$ & $\begin{array}{l}\mathscr{\mathscr { m }} \\
\frac{0}{0} \\
\end{array}$ & 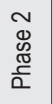 & 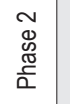 & & & $\frac{\pi}{z}$ & 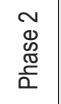 & & & & & & 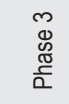 & \\
\hline
\end{tabular}

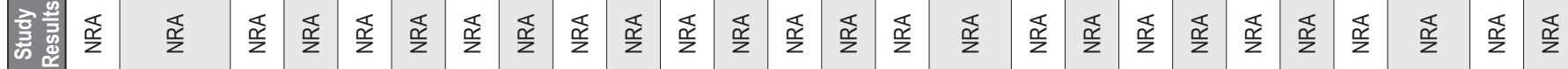

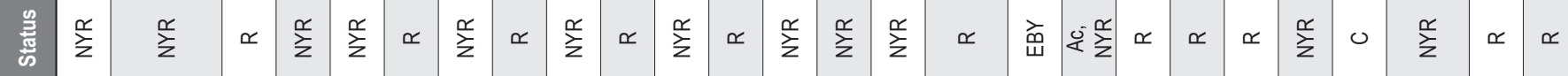

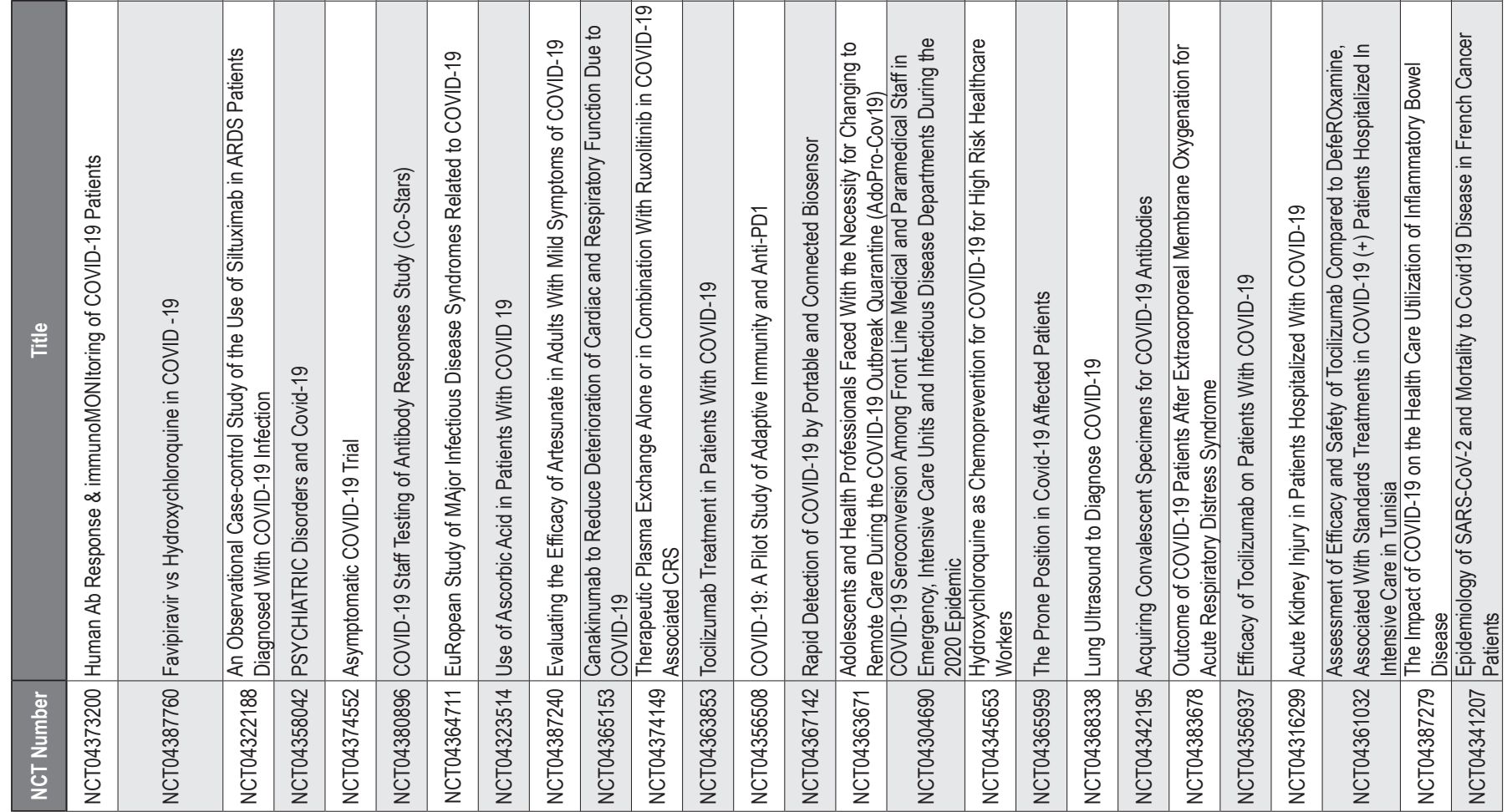




\begin{tabular}{|c|c|c|c|c|c|c|c|c|c|c|c|c|c|c|c|c|c|c|c|c|c|c|c|c|c|c|c|}
\hline$\frac{\vec{z}}{5}$ & 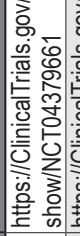 & E & & & 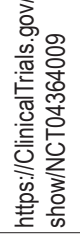 & & 汽 & $\bar{z}_{-}$ & 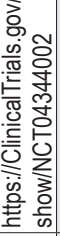 & & & ठ্ল & 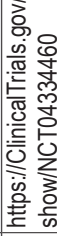 & 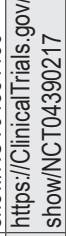 & & $z_{\sharp}$ & 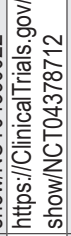 & & $\frac{}{0}$ & 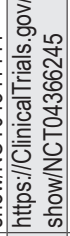 & 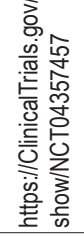 & 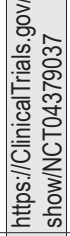 & 豙 & 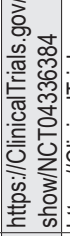 & 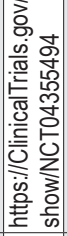 & 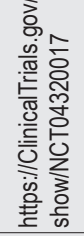 & 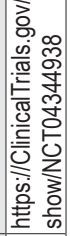 \\
\hline & & & & & స̃. & & & & & & & & & & & & 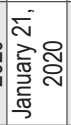 & & & 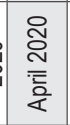 & 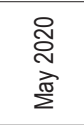 & 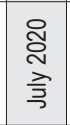 & & 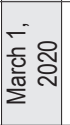 & & 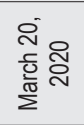 & 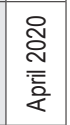 \\
\hline$\frac{\varrho}{2}$ & 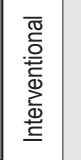 & 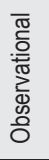 & 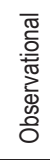 & 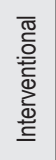 & 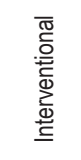 & 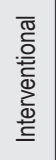 & 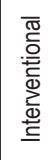 & 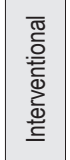 & 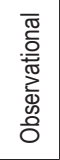 & 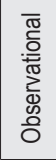 & 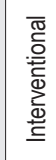 & 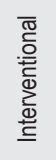 & 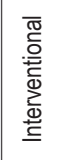 & 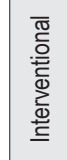 & 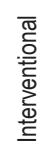 & 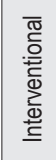 & 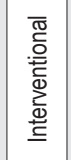 & 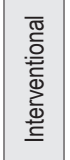 & 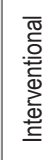 & 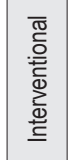 & 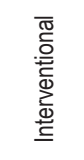 & 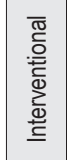 & 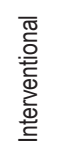 & 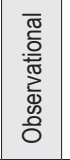 & 哥 & 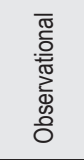 & 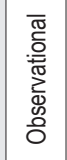 \\
\hline 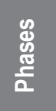 & 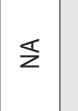 & & & $\mathbb{\Sigma}$ & $\begin{array}{l}\mathscr{8} \\
\mathbb{0} \\
\frac{\pi}{0} \\
\end{array}$ & 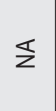 & 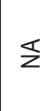 & 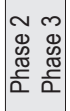 & & & 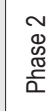 & 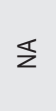 & 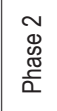 & 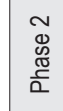 & 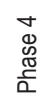 & 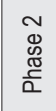 & 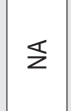 & & $\underset{\infty}{\infty}$ & $\mathbb{\infty}$ & $\begin{array}{l}\mathscr{8} \\
\stackrel{0}{0} \\
\frac{\pi}{\alpha}\end{array}$ & $\underline{z}$ & $\begin{array}{l}\mathscr{\mathscr { D }} \\
\text { 运 }\end{array}$ & & & & \\
\hline
\end{tabular}
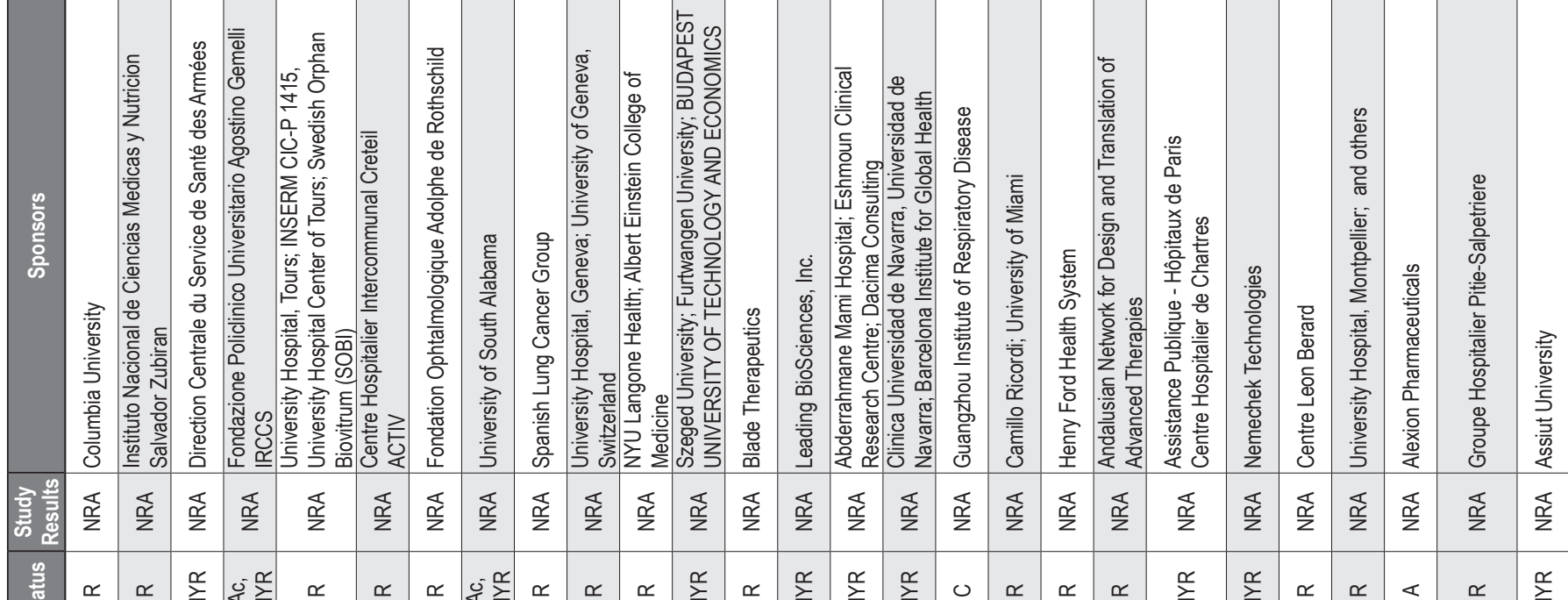

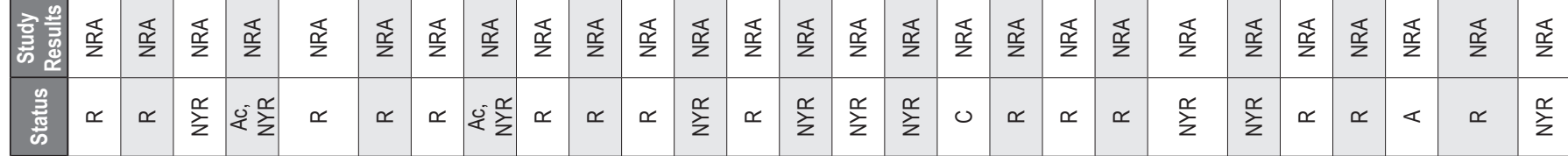
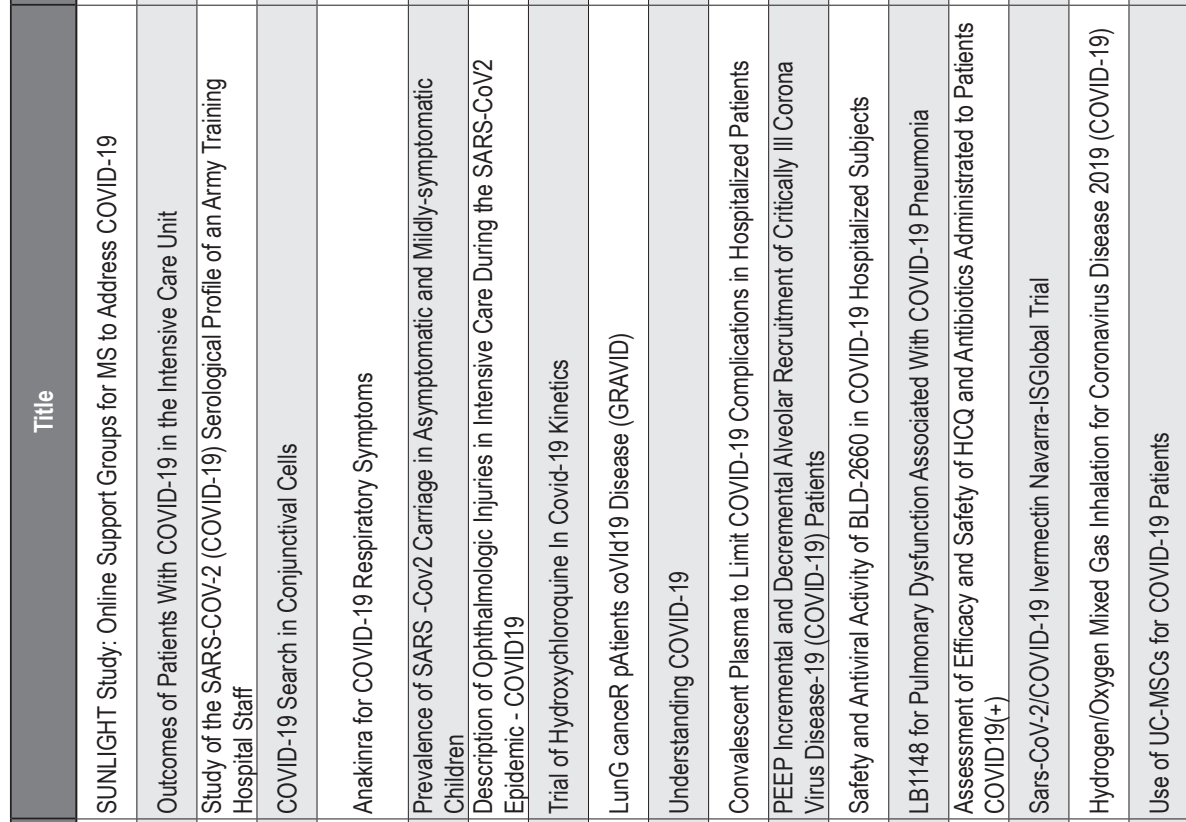

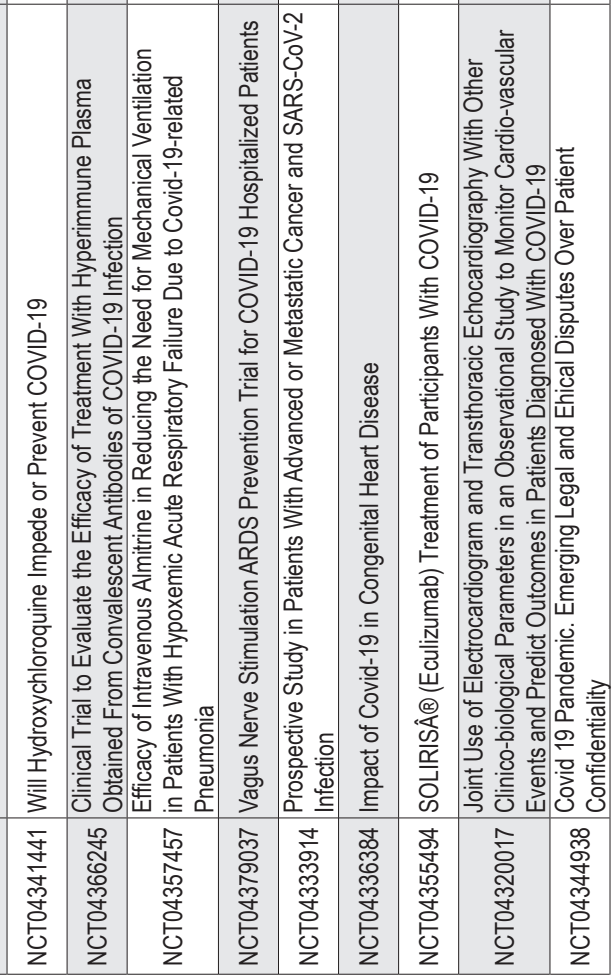




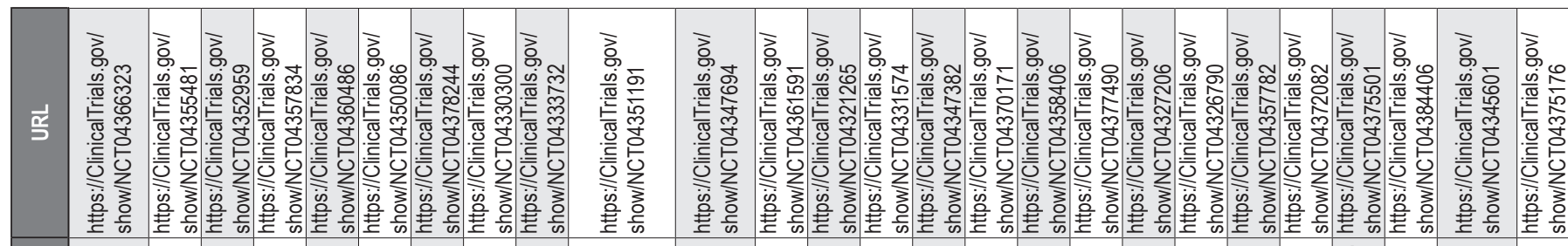

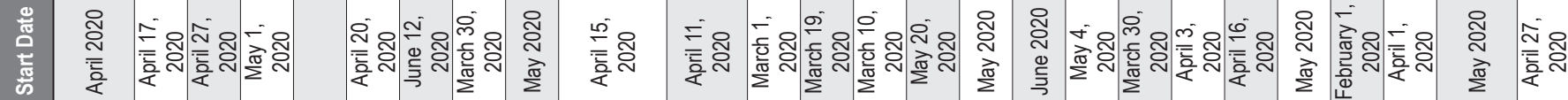

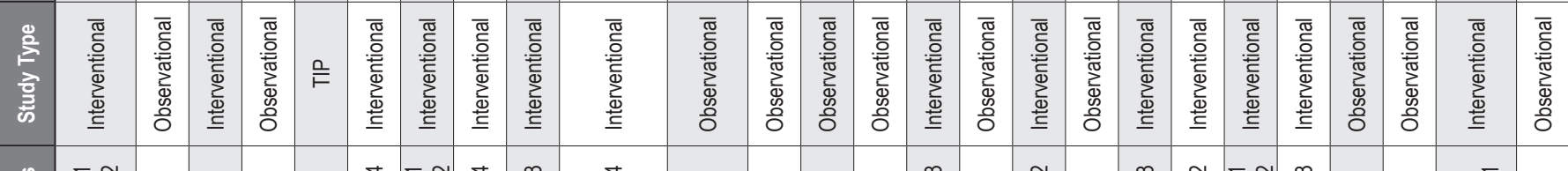

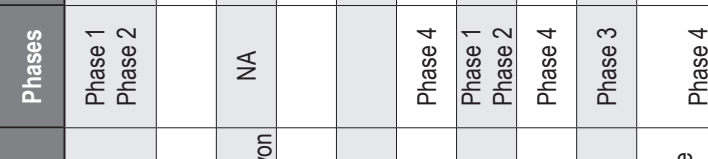

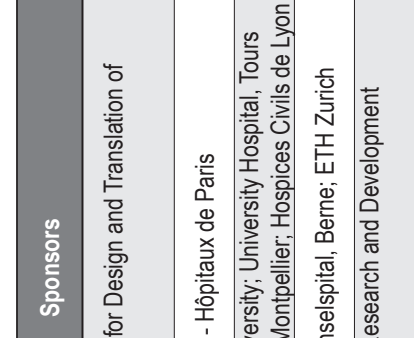

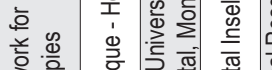

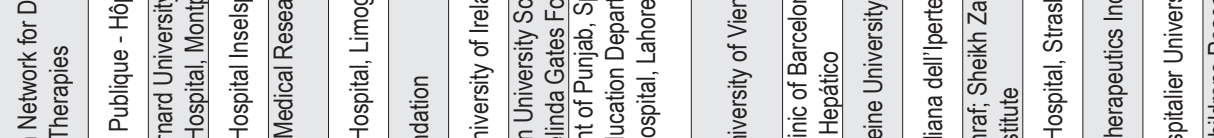

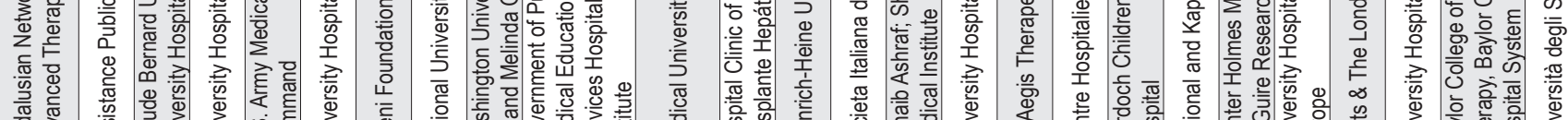

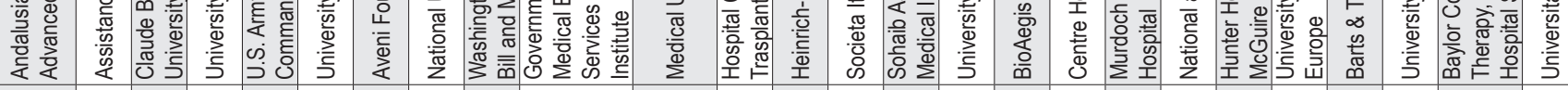

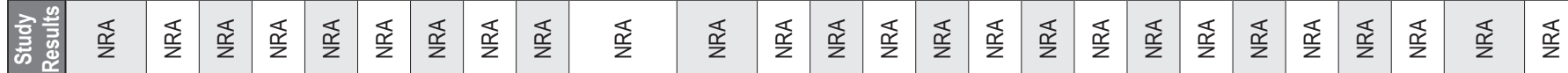

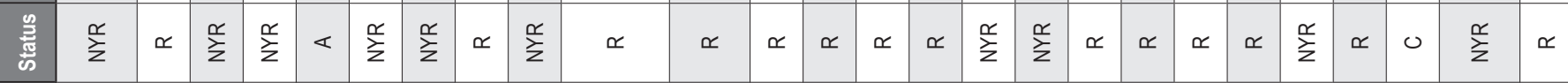

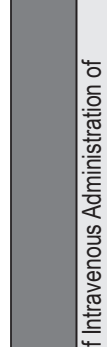

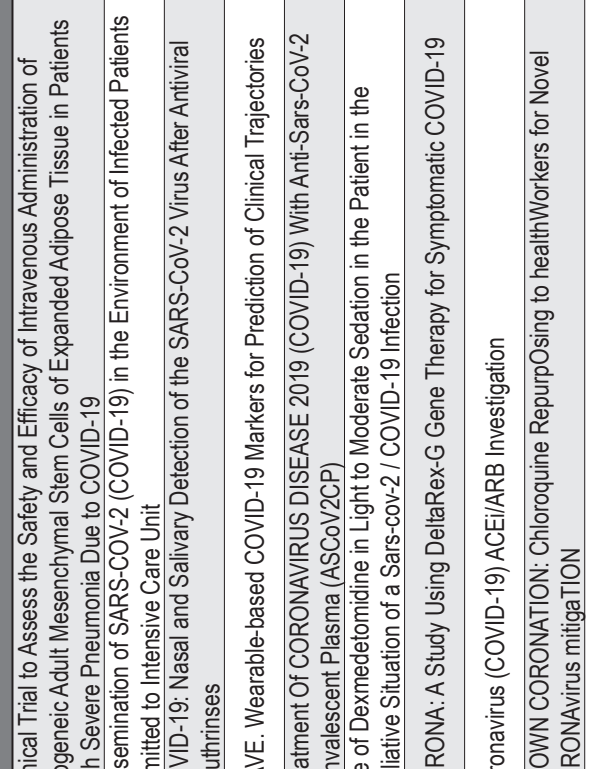

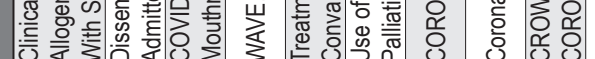

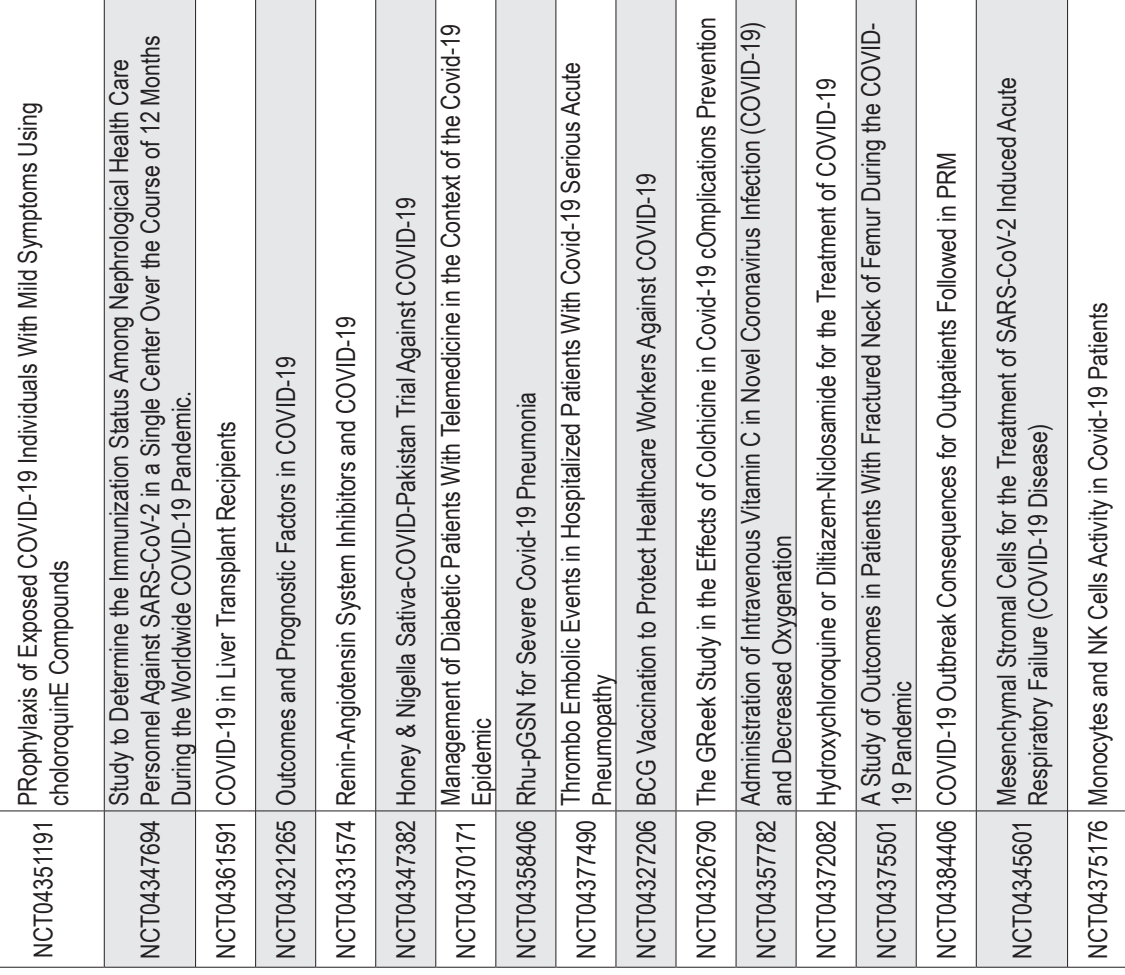




\begin{tabular}{|c|c|c|c|c|c|c|c|c|c|c|c|c|c|c|c|c|c|c|c|c|c|c|c|}
\hline & 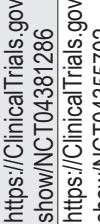 & & & & & & & 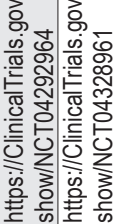 & & & & 隹 & & 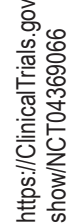 & 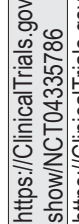 & & 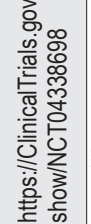 & 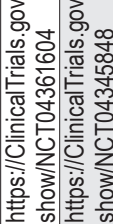 & 产 & 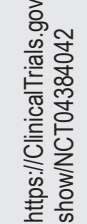 & 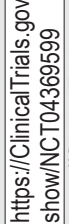 & 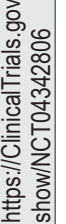 & 㐘 \\
\hline & & & & & & & & & & & & 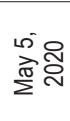 & & 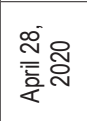 & 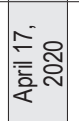 & 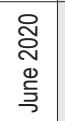 & 言商 & & & 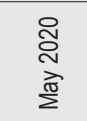 & & & \\
\hline & 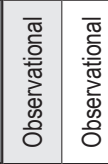 & 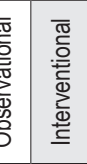 & 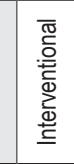 & 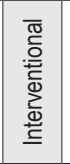 & 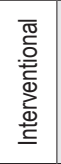 & 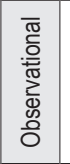 & 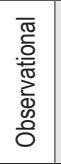 & 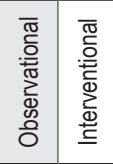 & 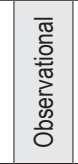 & 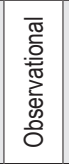 & 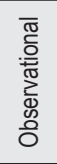 & 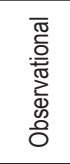 & 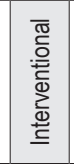 & 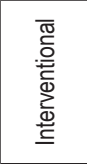 & 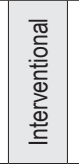 & 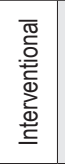 & 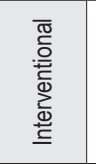 & 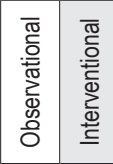 & 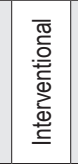 & 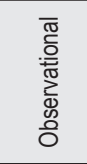 & 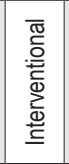 & 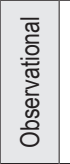 & \\
\hline & & 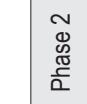 & 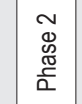 & 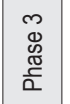 & $\alpha$ & & & & & & & & 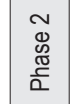 & $\mathbb{z}$ & 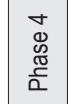 & 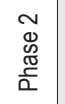 & 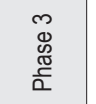 & & & & $\frac{\pi}{2}$ & & \\
\hline
\end{tabular}

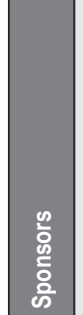

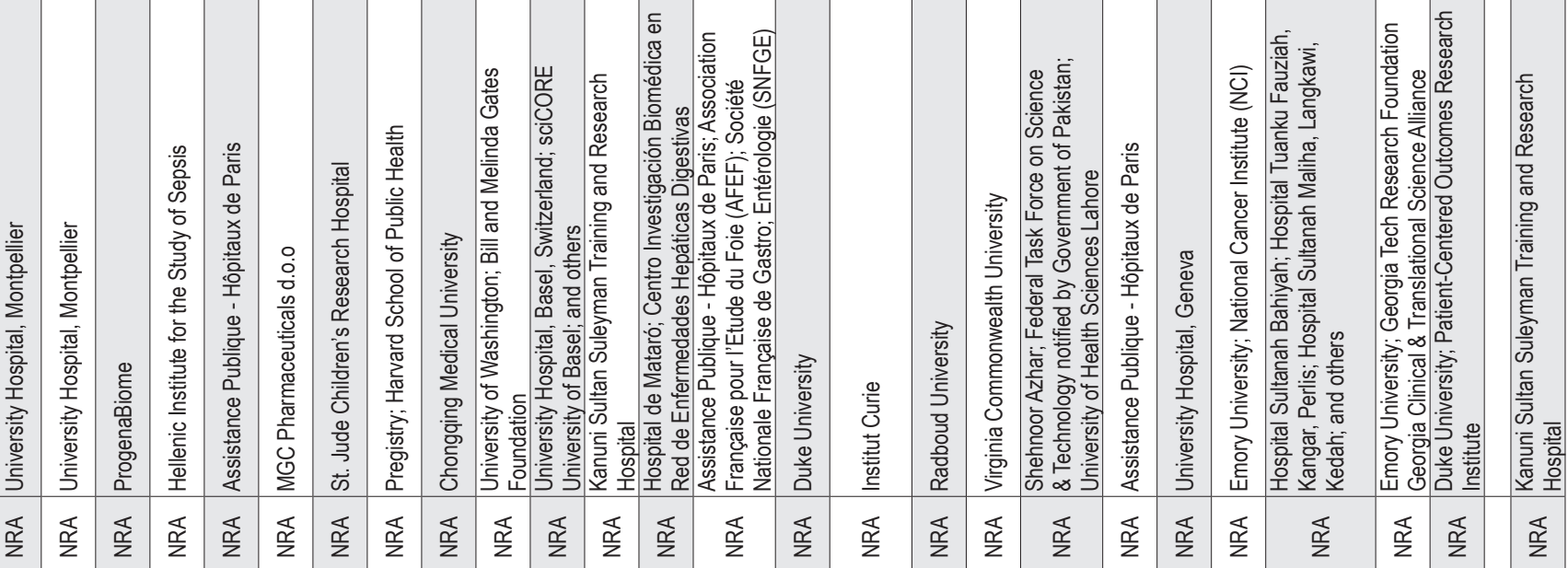

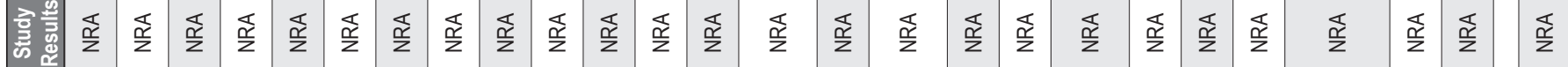

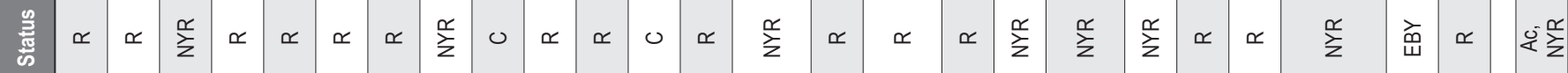

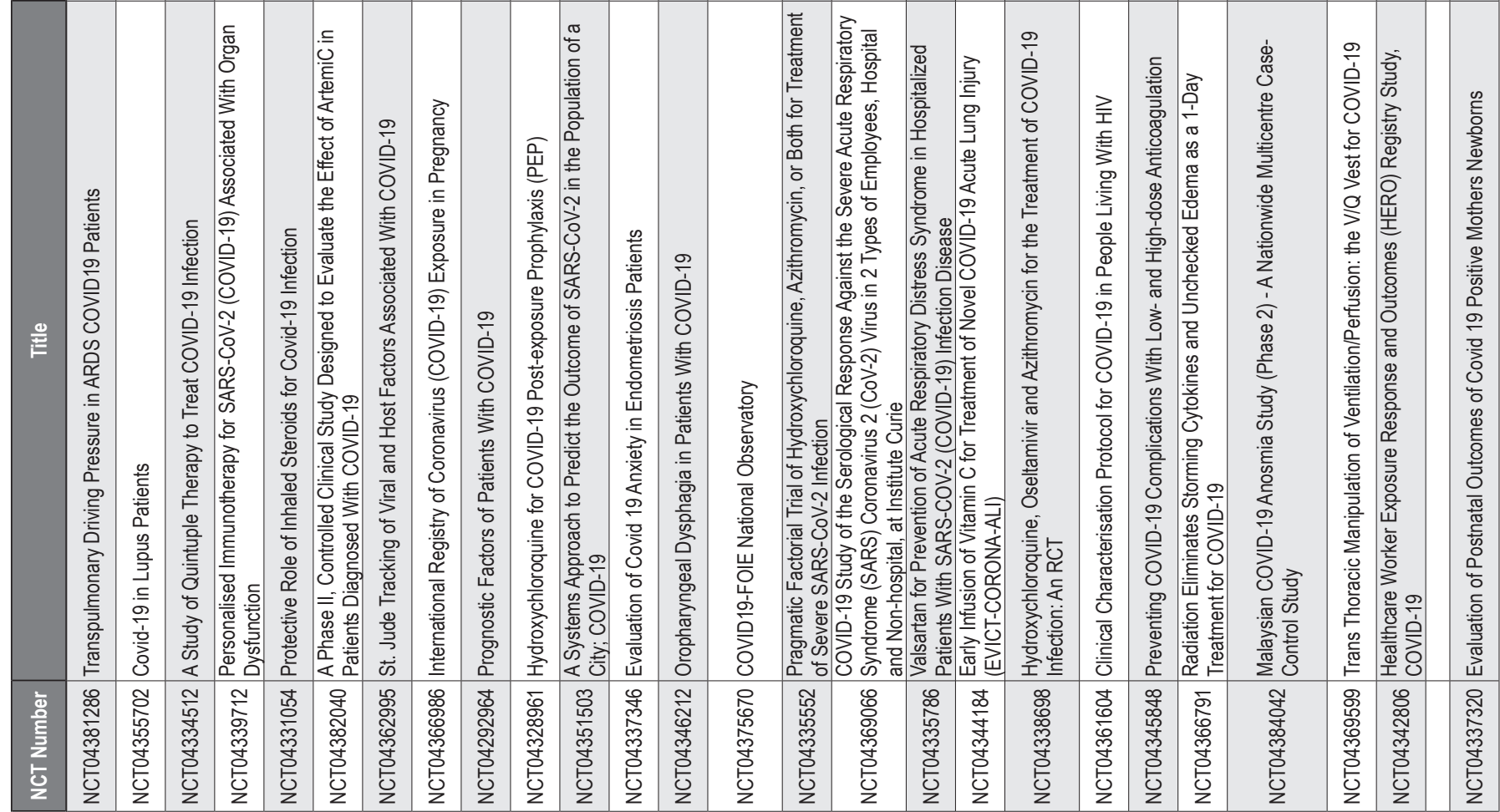




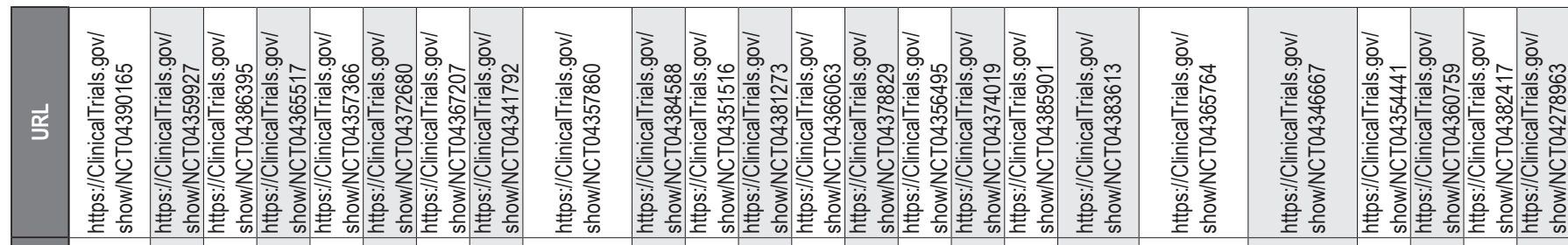

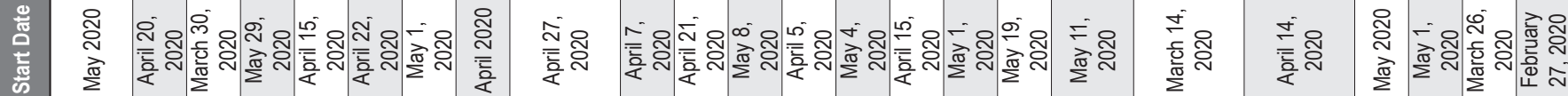

\begin{tabular}{|c|c|c|c|c|c|c|c|c|c|c|c|c|c|c|c|c|c|c|c|c|c|c|}
\hline 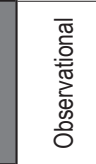 & 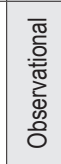 & 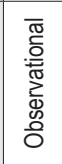 & 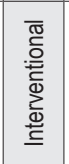 & 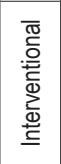 & 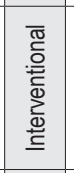 & 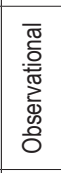 & 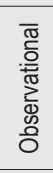 & 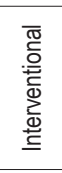 & 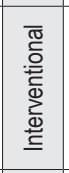 & 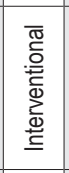 & 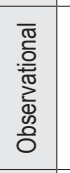 & 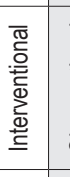 & 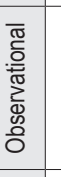 & 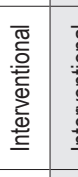 & 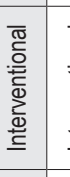 & 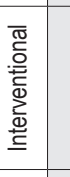 & 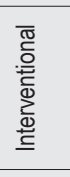 & 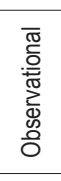 & 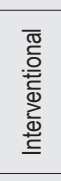 & 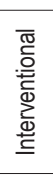 & & \\
\hline & & & 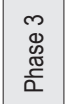 & 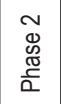 & $\underline{z}$ & & & 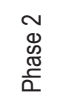 & & & & 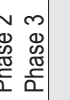 & & & 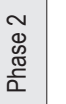 & $\underline{\underline{z}}$ & $\underline{z}$ & & $\begin{array}{l}\text { 响 } \\
\frac{0}{2}\end{array}$ & 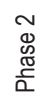 & & \\
\hline
\end{tabular}

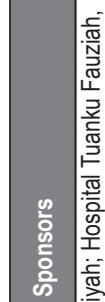

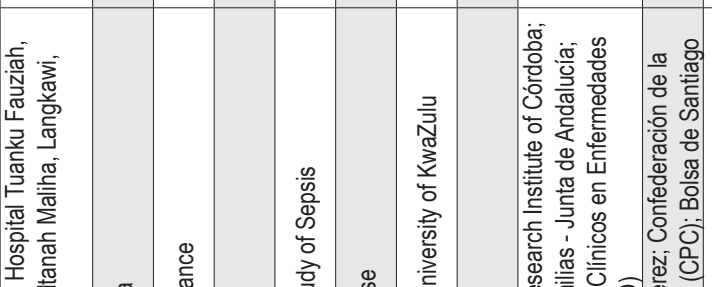

商

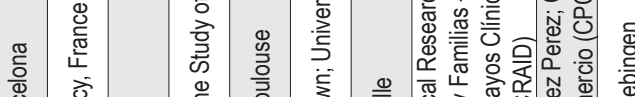

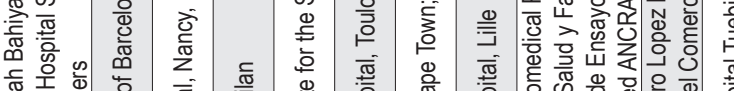

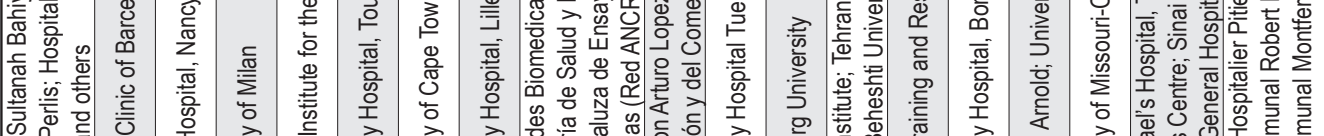

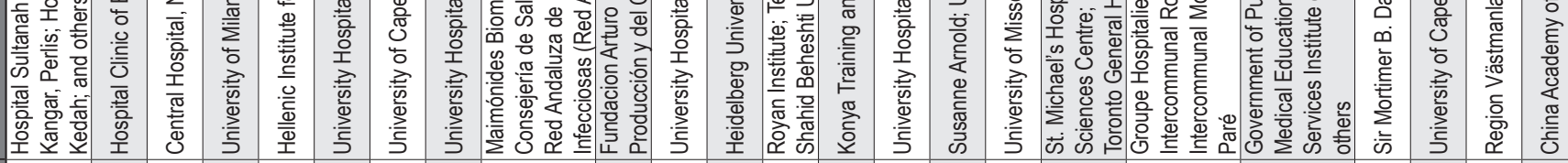

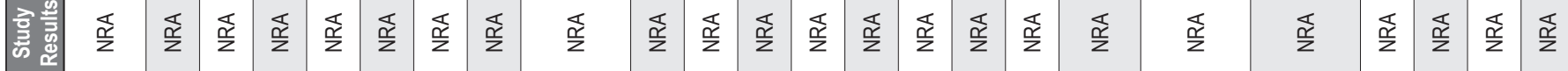

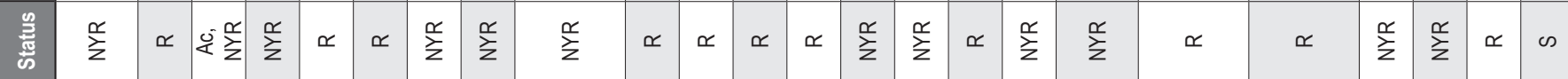

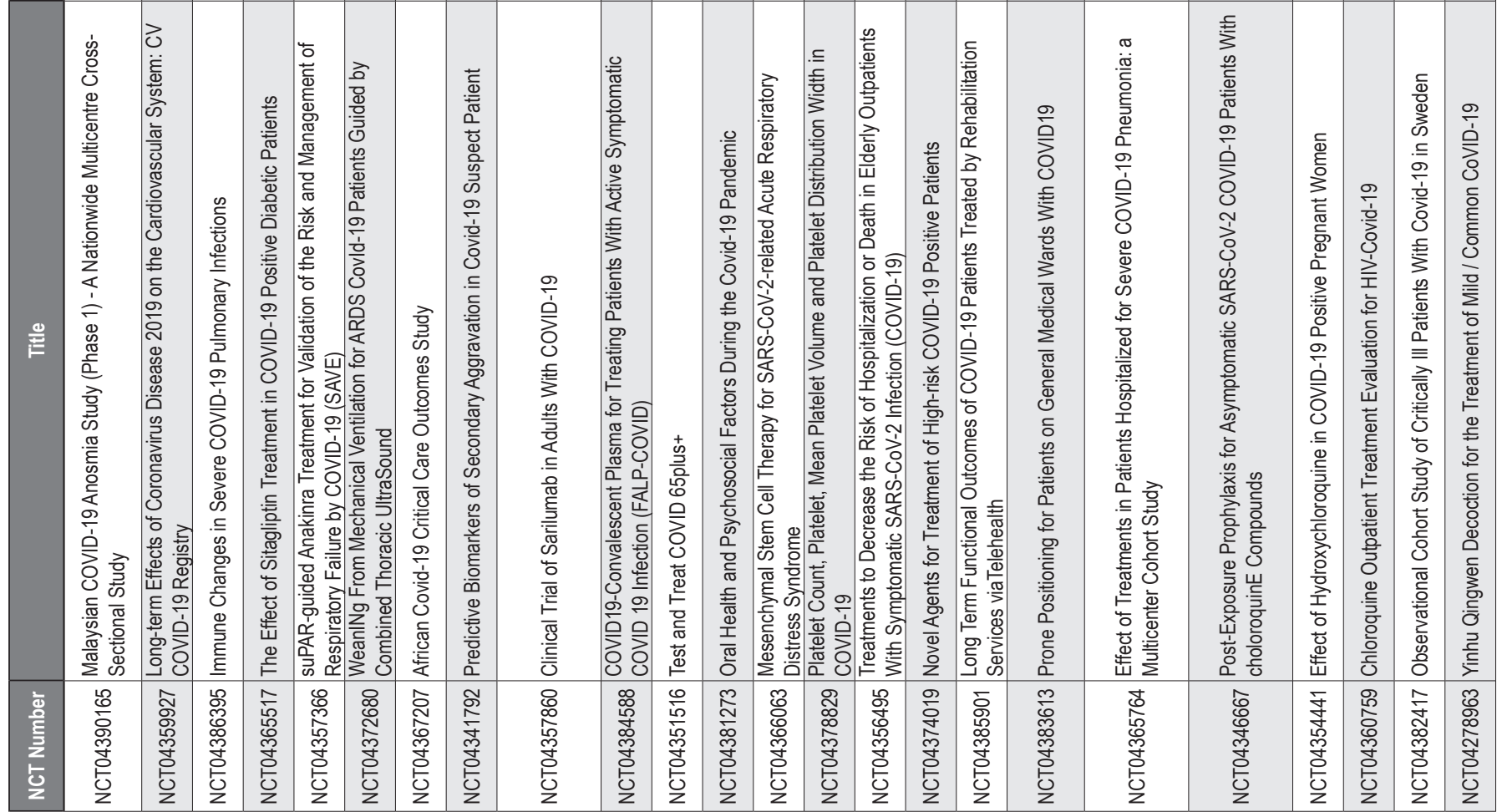




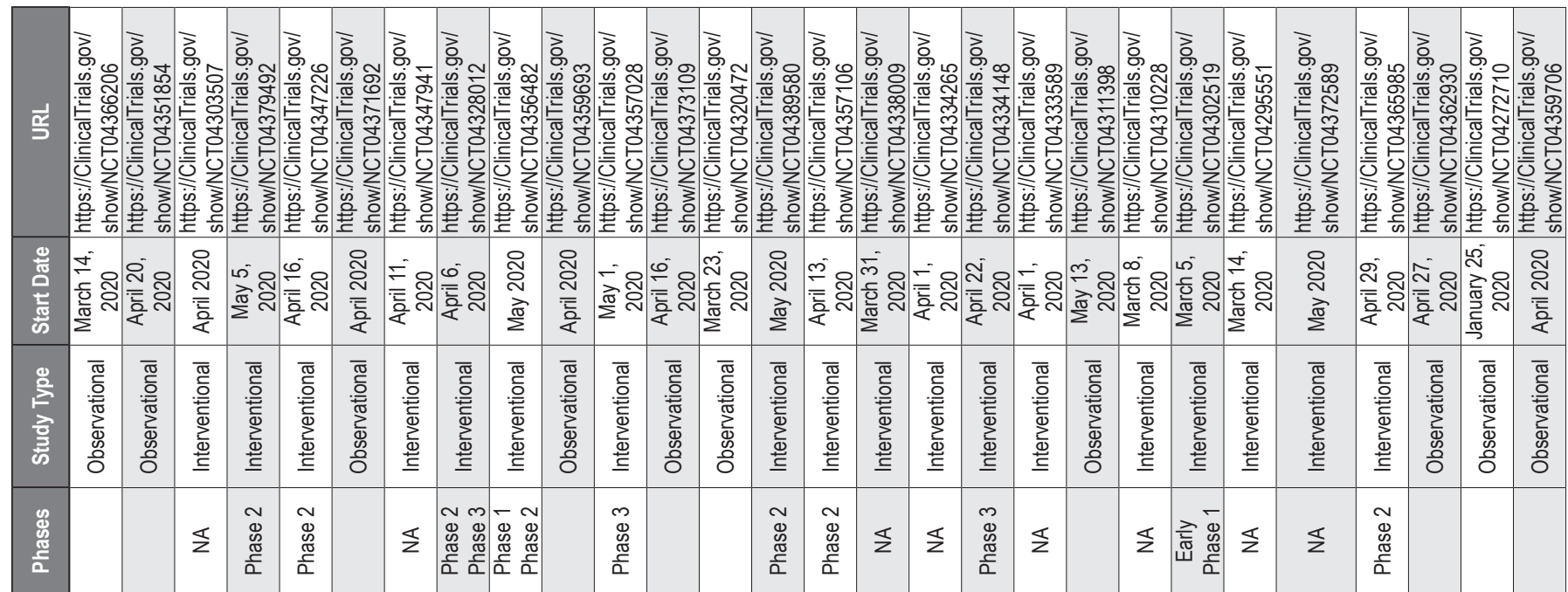

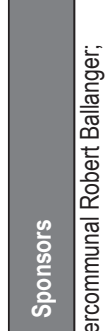

\begin{tabular}{|c|c|c|}
\hline 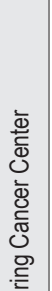 & 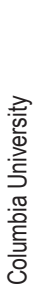 & 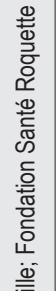 \\
\hline
\end{tabular}

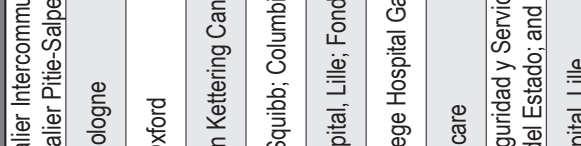

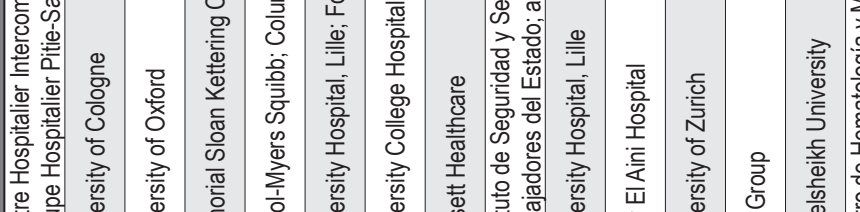

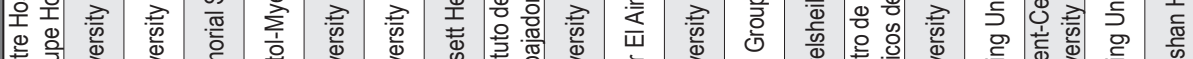

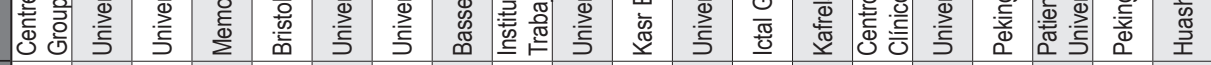
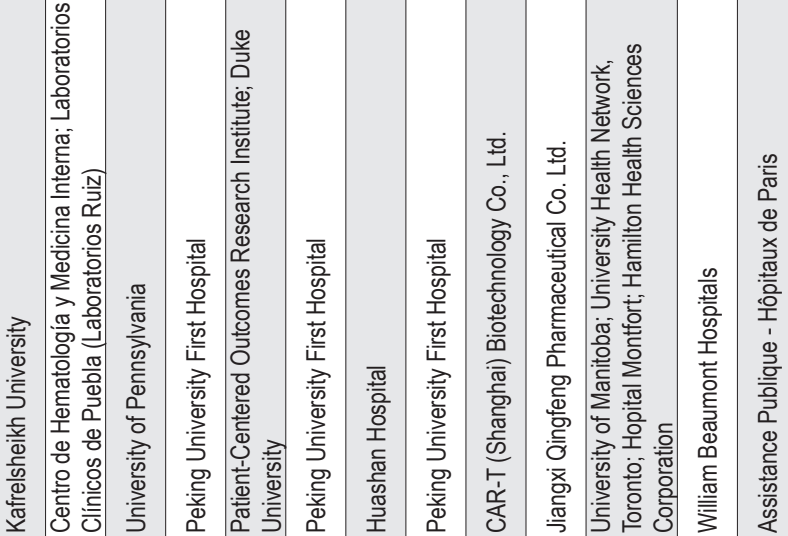

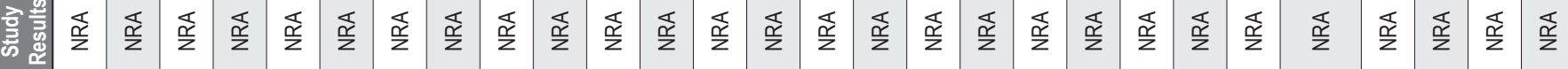

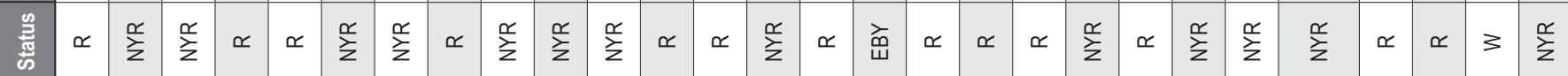

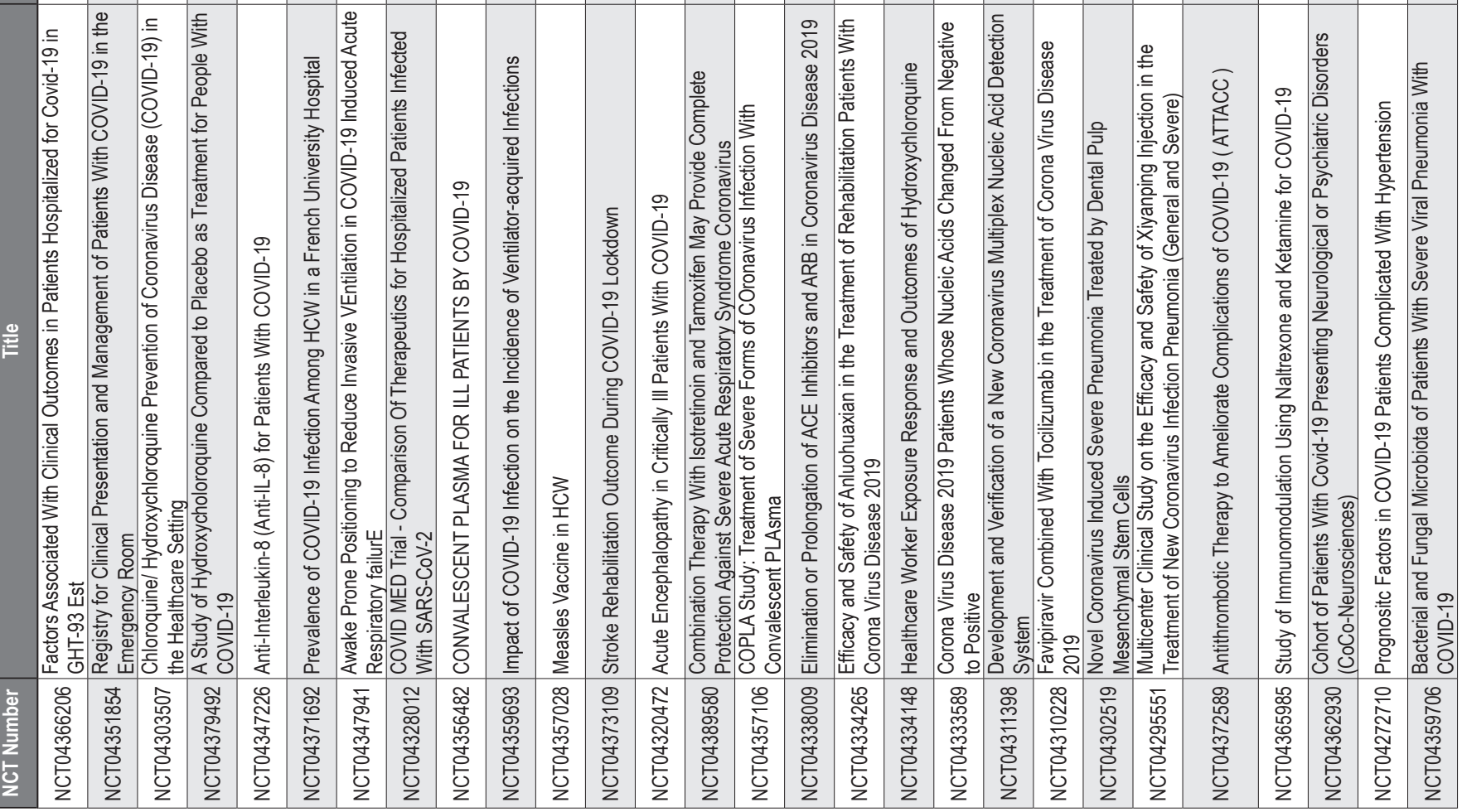




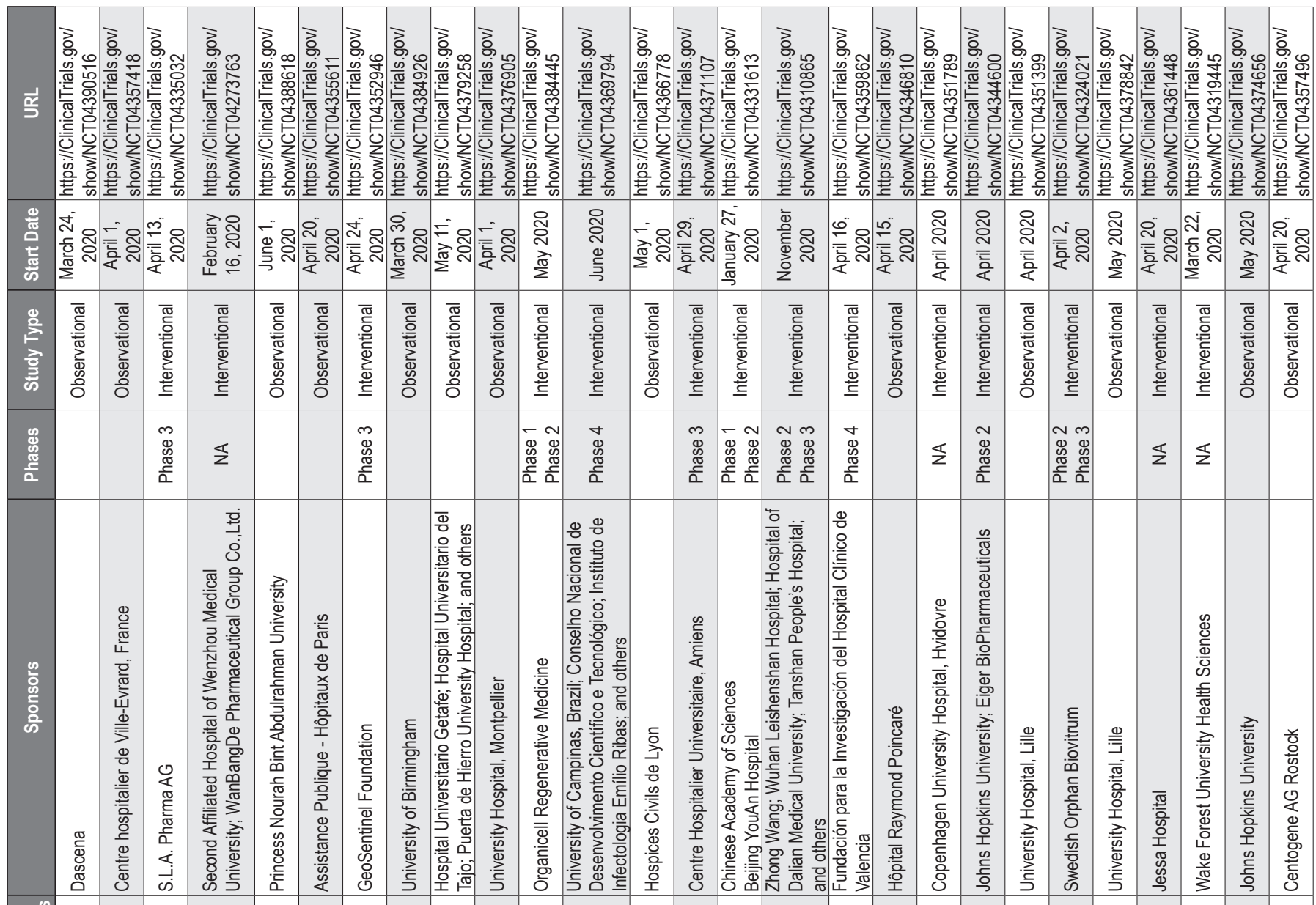

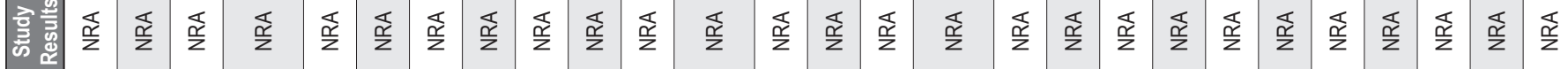

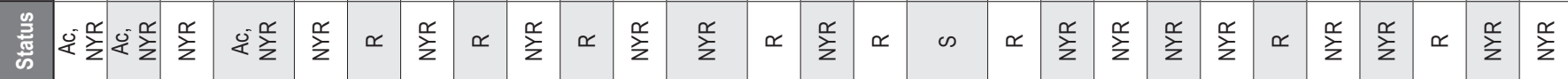

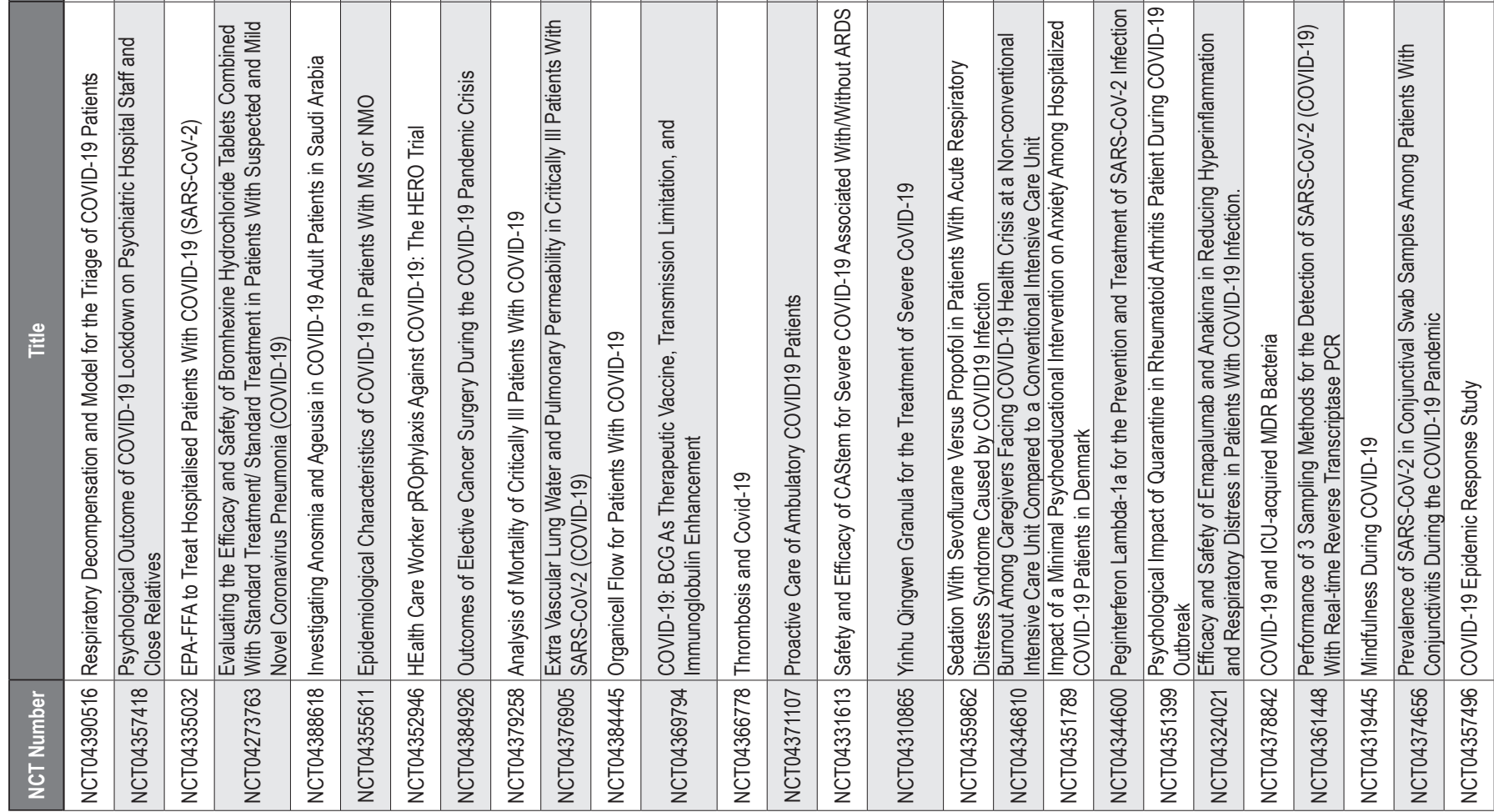




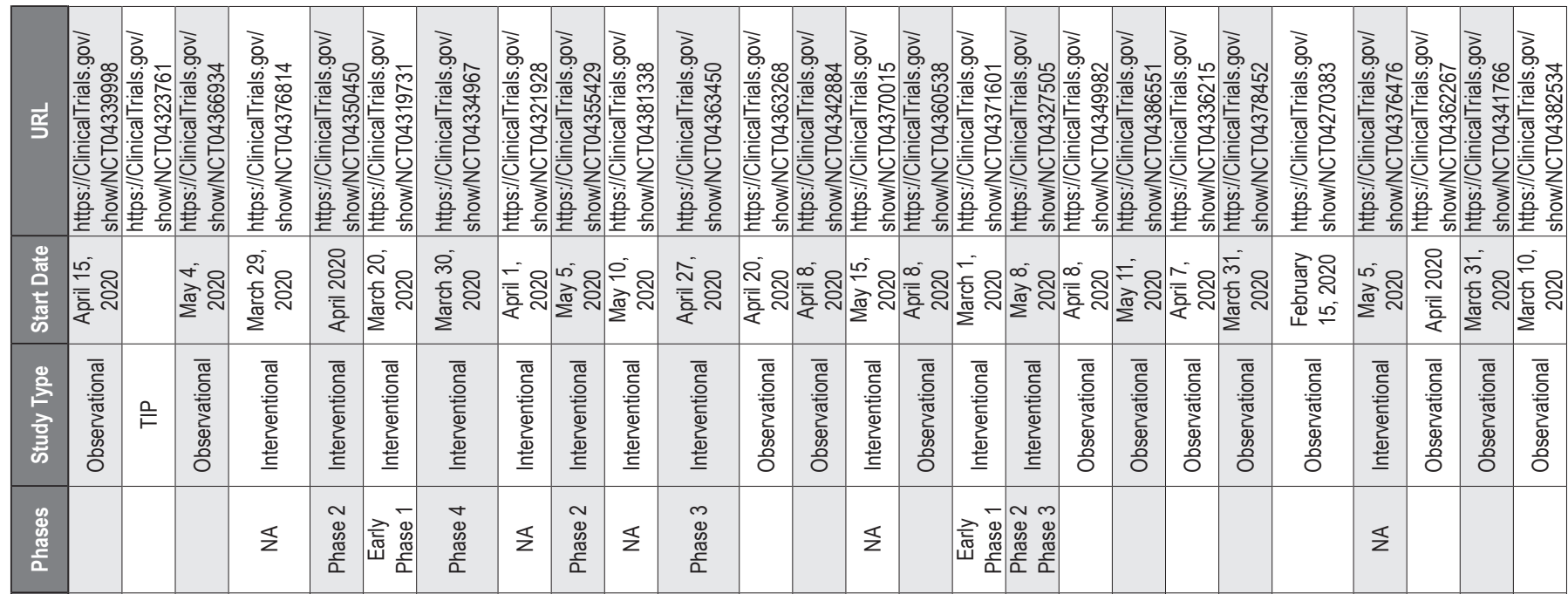

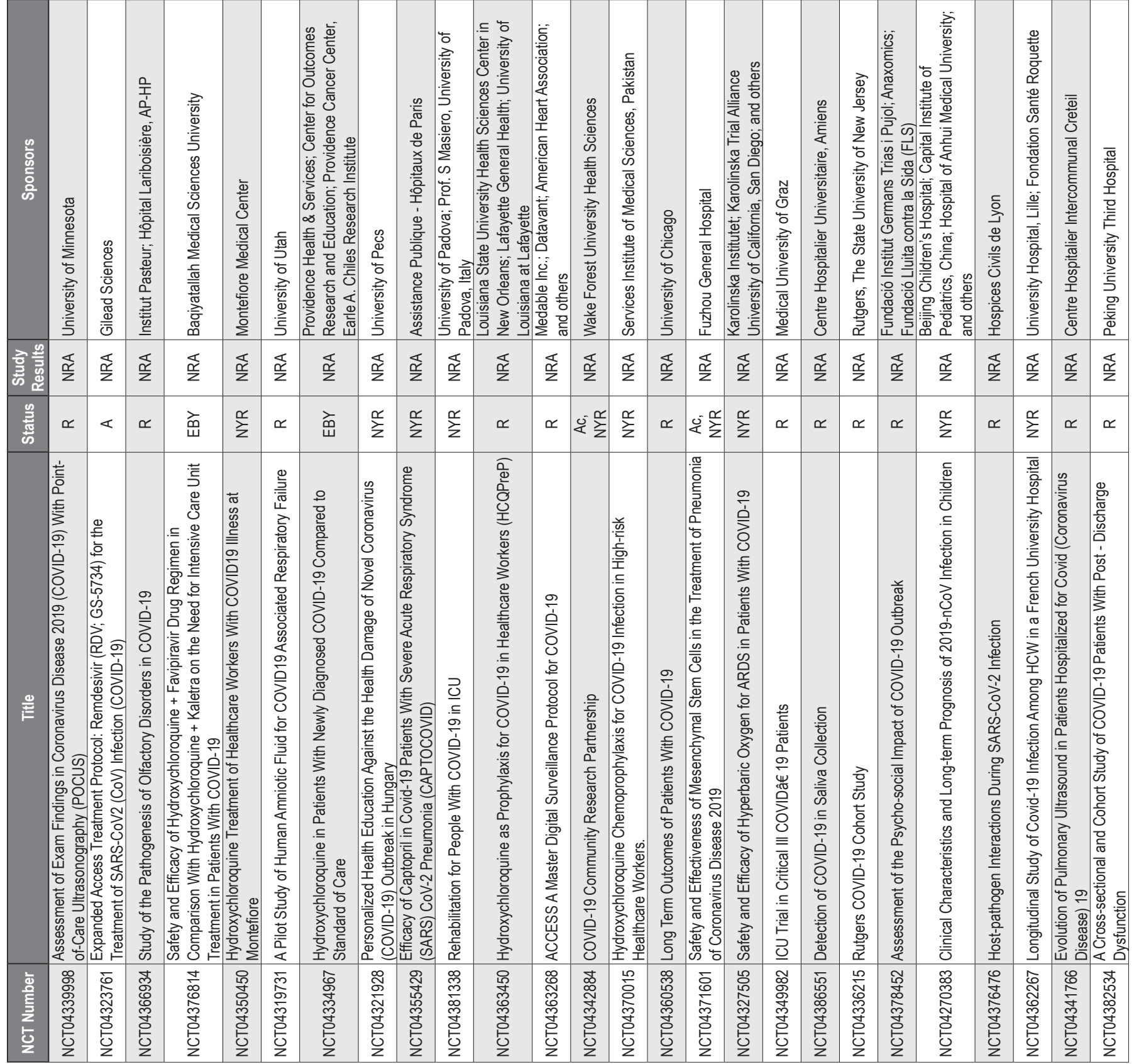




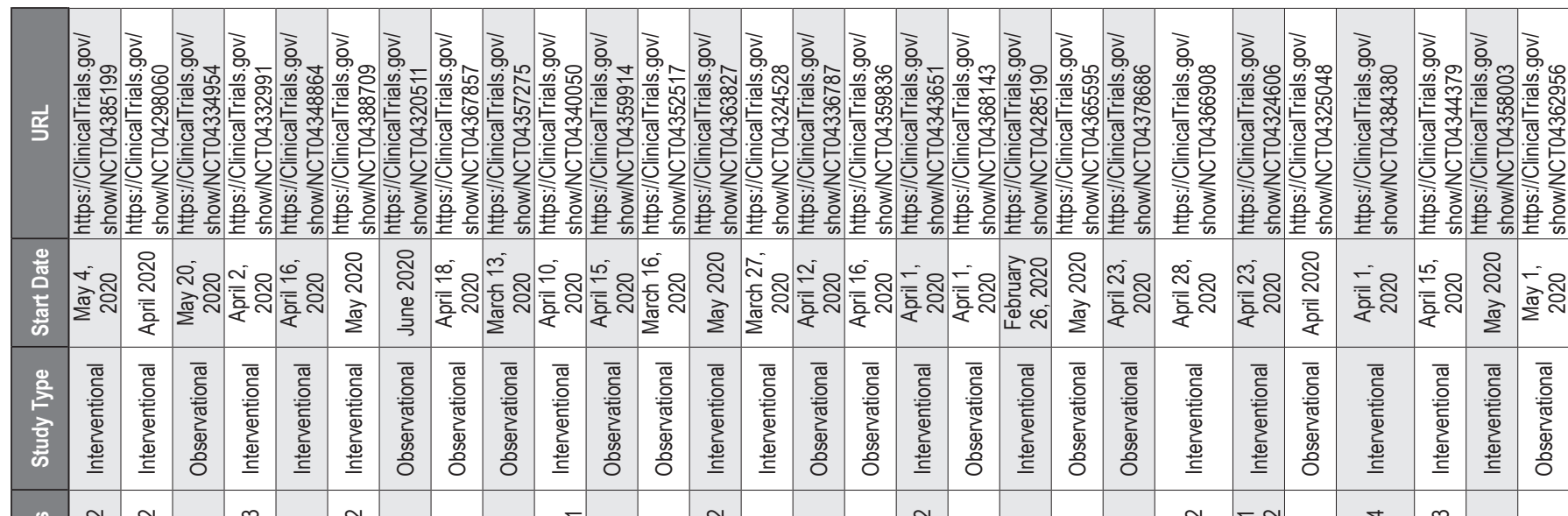

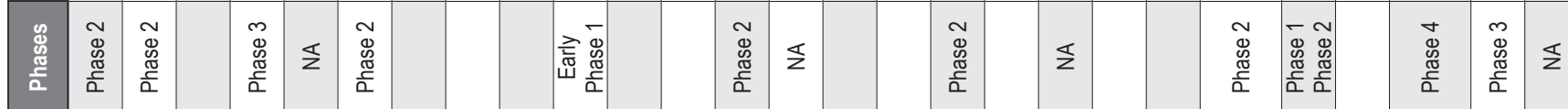

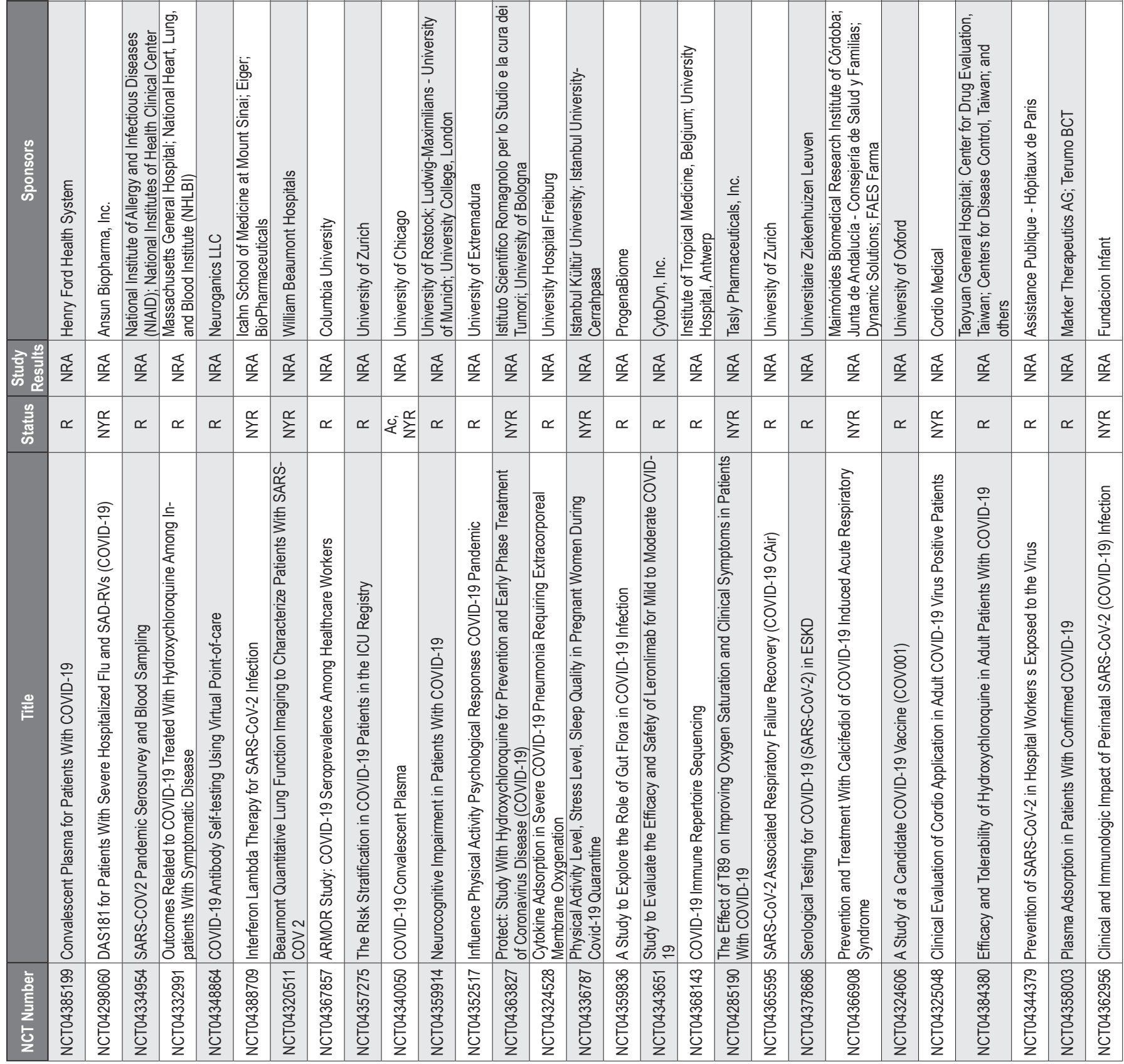




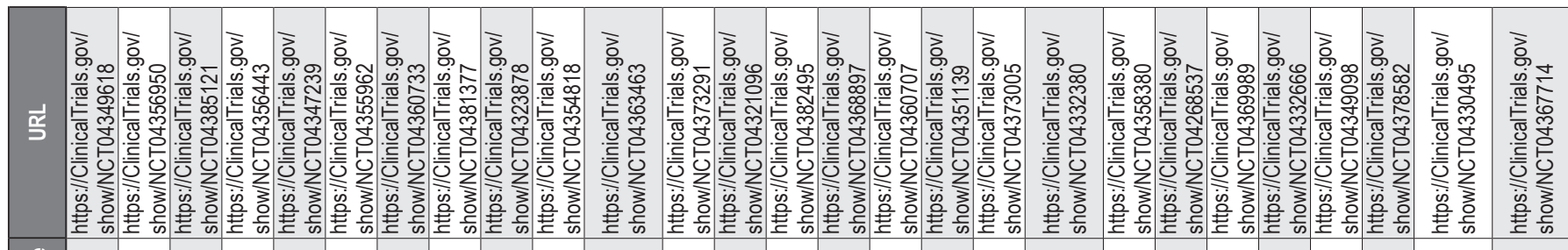

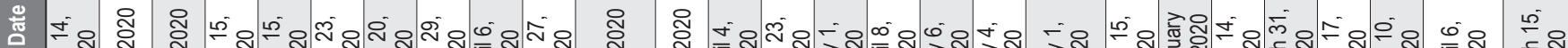

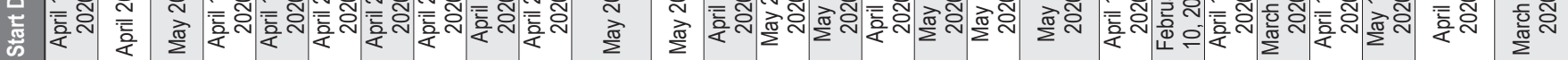

\begin{tabular}{|c|c|c|c|c|c|c|c|c|c|c|c|c|c|c|c|c|c|c|c|c|c|c|c|c|c|c|}
\hline 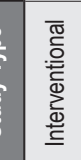 & 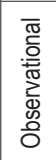 & 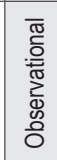 & 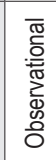 & 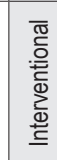 & 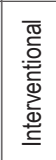 & 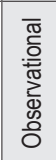 & 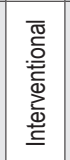 & 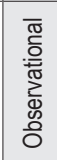 & 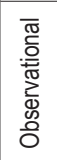 & 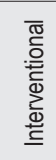 & 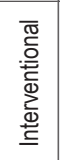 & 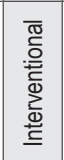 & 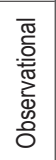 & 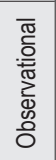 & 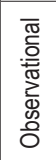 & 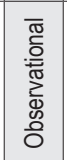 & 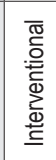 & 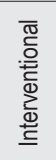 & 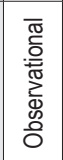 & 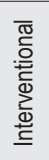 & 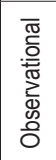 & 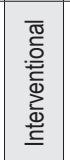 & 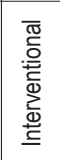 & 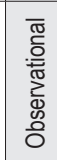 & 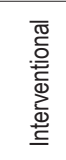 & 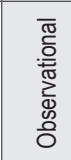 \\
\hline$\frac{\mathbb{k}}{z}$ & & & & $\begin{array}{l}\widetilde{0} \\
\mathscr{0} \\
\frac{\pi}{\alpha}\end{array}$ & $\mid \begin{array}{l}m \\
\Phi \\
\Phi \\
\frac{\pi}{\alpha} \\
\frac{\pi}{\alpha}\end{array}$ & & 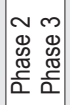 & & & $\Sigma$ & & & & & & & $\Sigma$ & 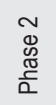 & & 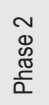 & & & $\begin{array}{l}\sim \\
\text { o } \\
\text { o } \\
\frac{\pi}{\alpha}\end{array}$ & & 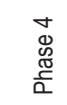 & \\
\hline
\end{tabular}

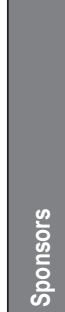

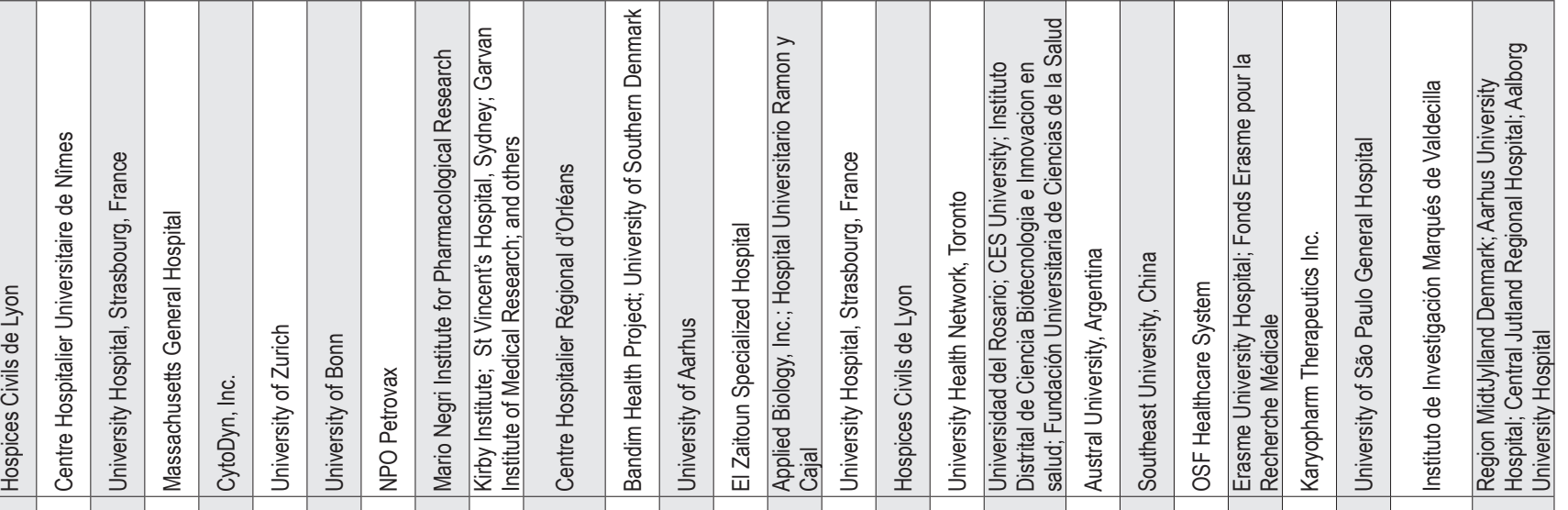

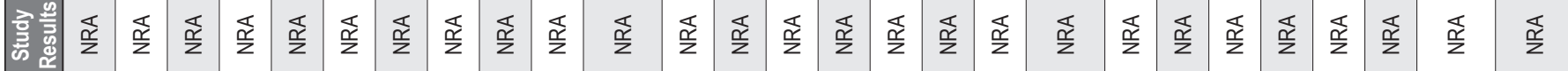

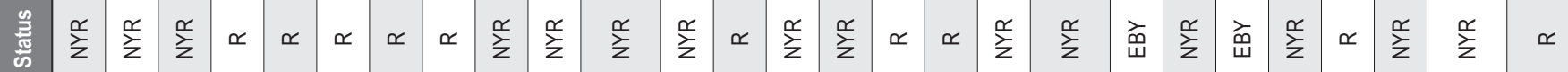

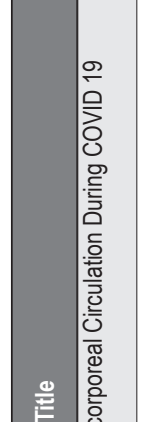

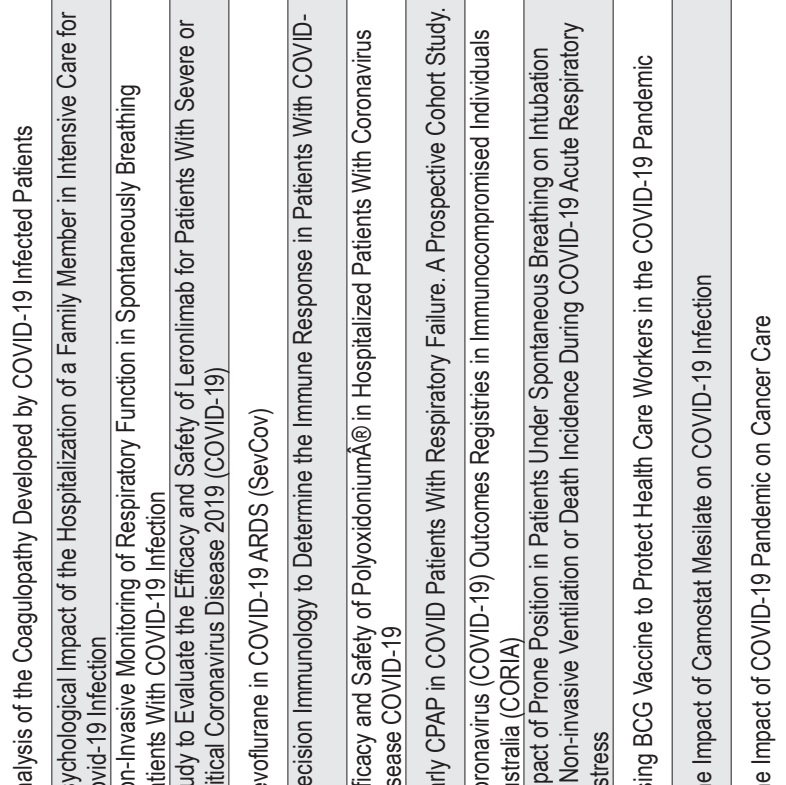

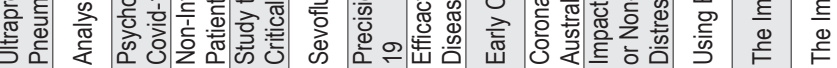

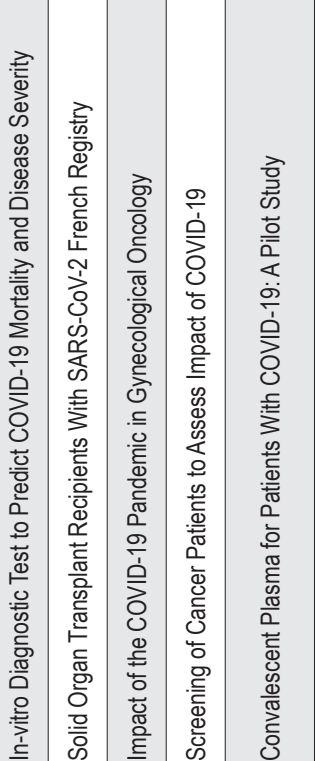

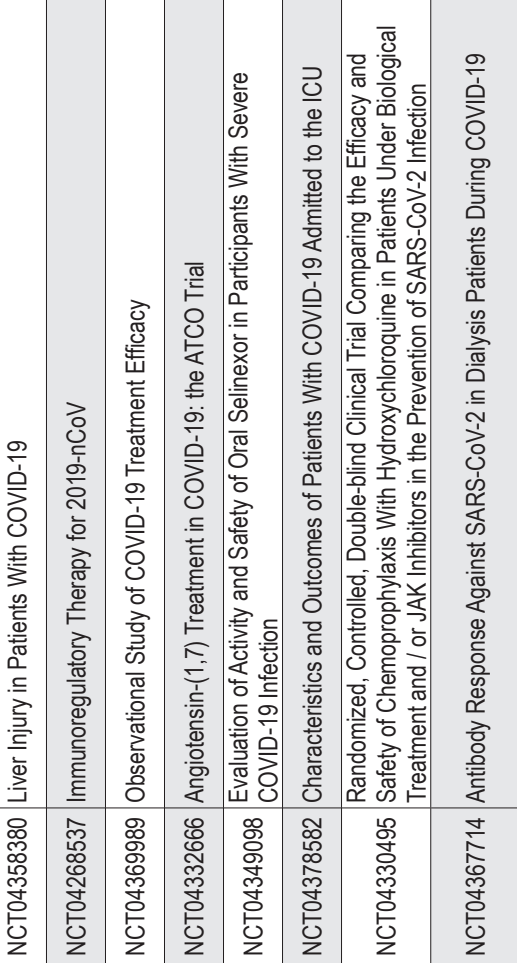




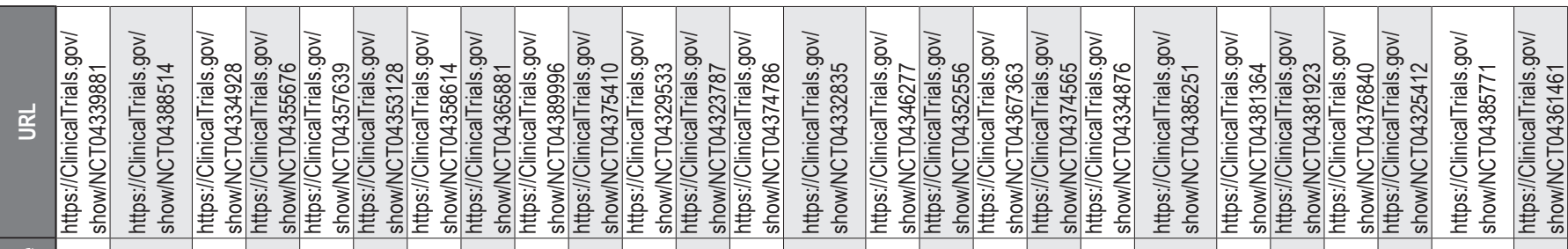

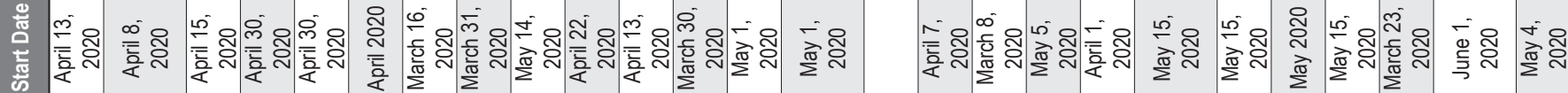

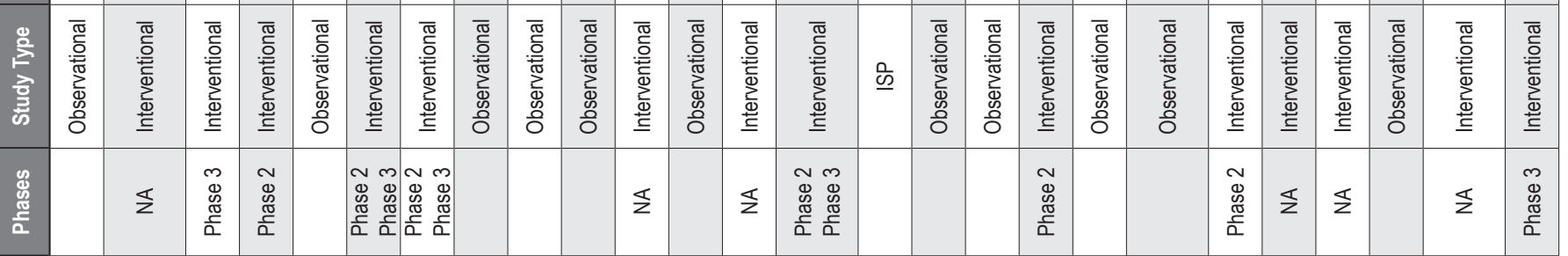

兽

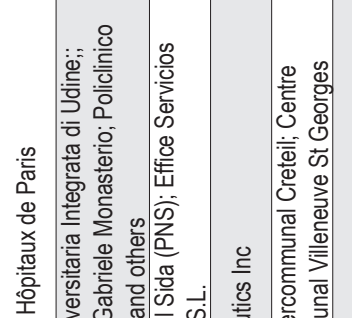

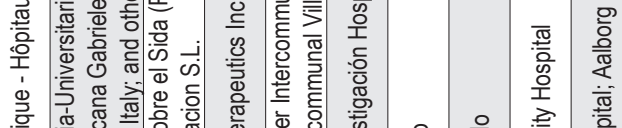

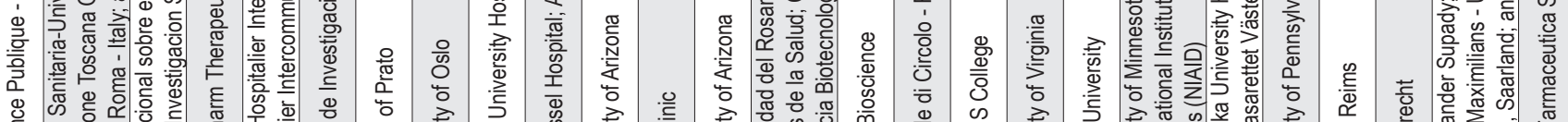

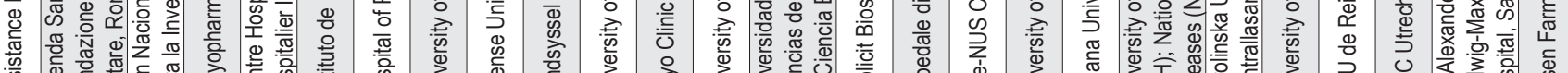

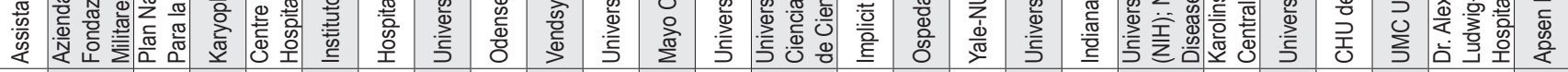

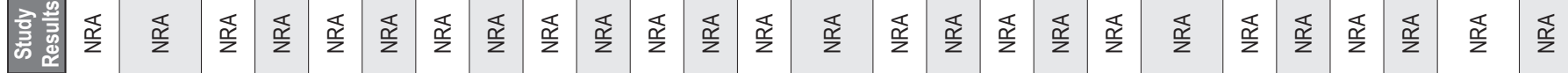

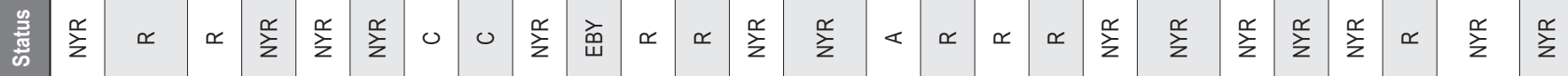

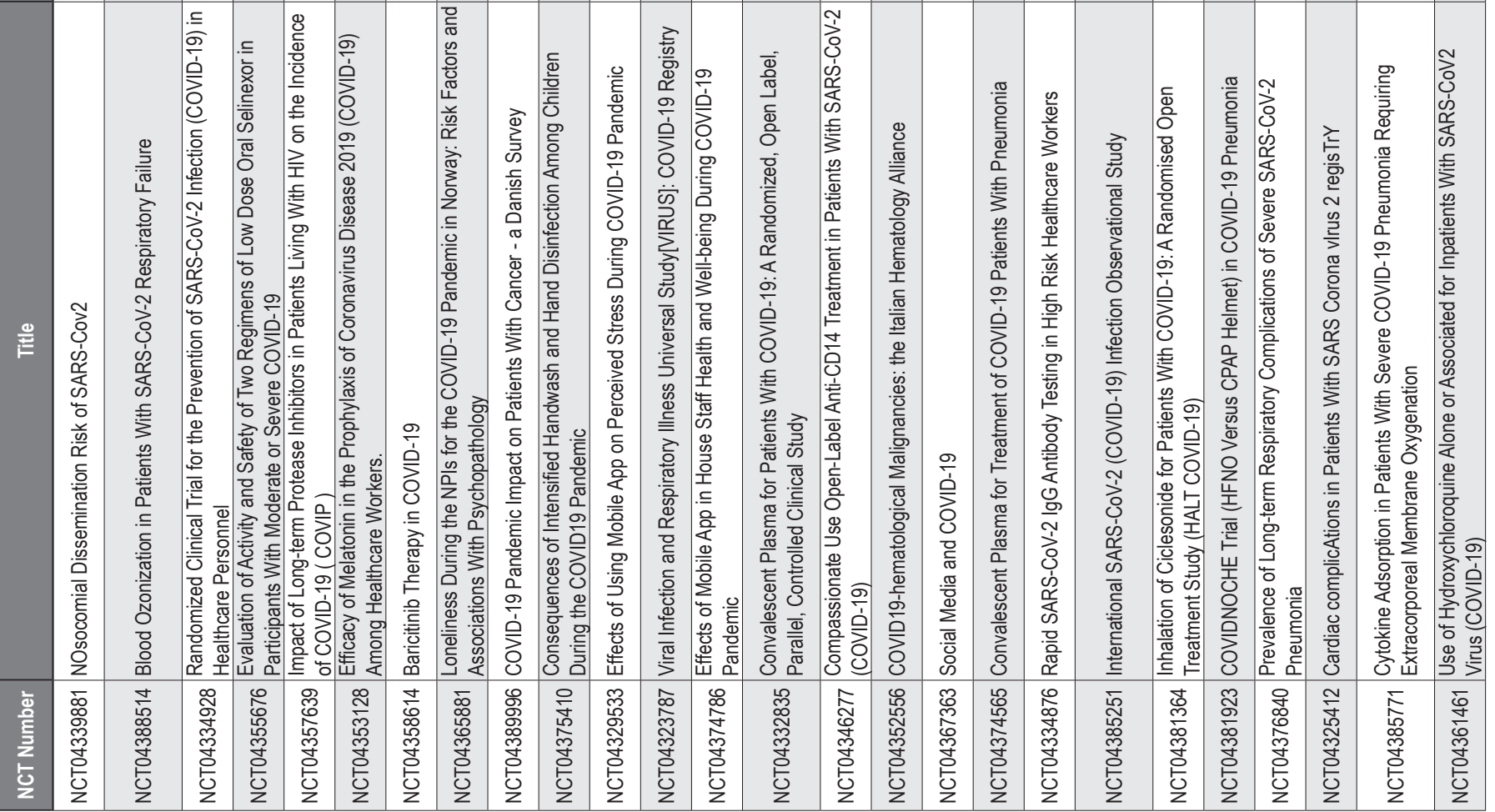




\begin{tabular}{|c|c|c|c|c|c|c|c|c|c|c|c|c|c|c|c|c|c|c|c|c|c|c|c|c|c|c|}
\hline \multicolumn{8}{|c|}{ 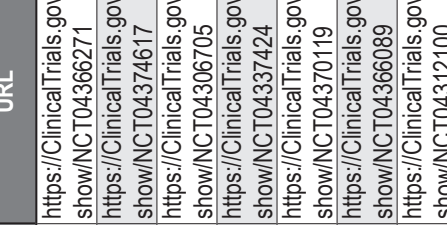 } & 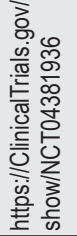 & 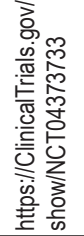 & 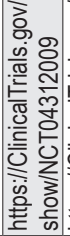 & & 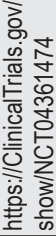 & 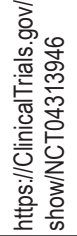 & 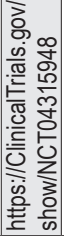 & & & & 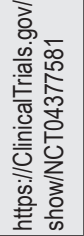 & 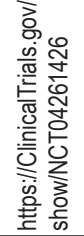 & 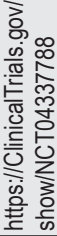 & ల్లె & & & 5 & & \\
\hline & & & & & & & & 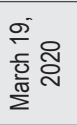 & స్తి & & & & 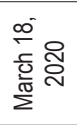 & & & & & 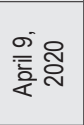 & 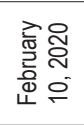 & 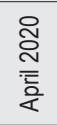 & 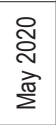 & 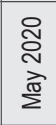 & & & & \\
\hline & 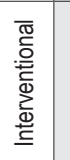 & 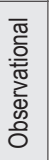 & 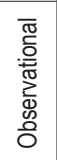 & 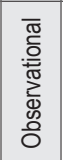 & 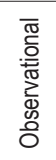 & 离 & 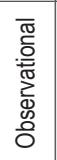 & 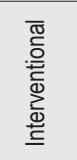 & 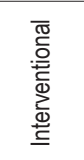 & 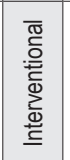 & 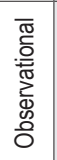 & 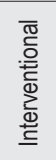 & 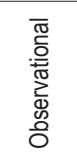 & 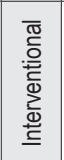 & 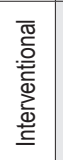 & 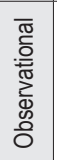 & 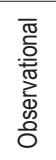 & 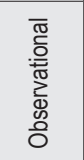 & 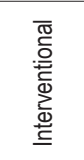 & 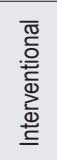 & 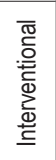 & 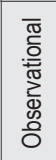 & 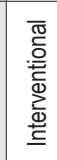 & 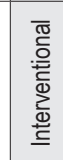 & 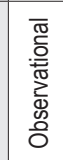 & \\
\hline & $\begin{array}{l}\widetilde{N} \\
\text { 怘 } \\
\frac{\pi}{\alpha}\end{array}$ & & & & & 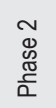 & & 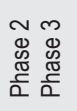 & $\begin{array}{l}\text { क्ष } \\
\text { 兽 } \\
\frac{1}{\alpha}\end{array}$ & $\begin{array}{l}\text { D } \\
\text { 兽 } \\
\frac{\alpha}{\alpha}\end{array}$ & & $\begin{array}{l}\infty \\
\mathbb{W} \\
\mathbb{8} \\
\frac{\pi}{\alpha} \\
\frac{\pi}{\alpha}\end{array}$ & & 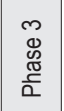 & $\begin{array}{l}\text { o } \\
\text { 蛋 } \\
\frac{a}{\alpha}\end{array}$ & & & & 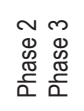 & 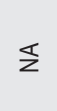 & 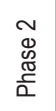 & & & & & \\
\hline
\end{tabular}
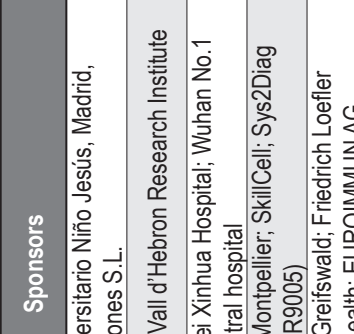

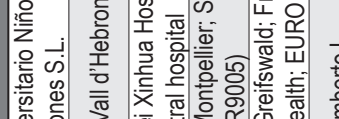
㐘

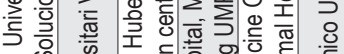

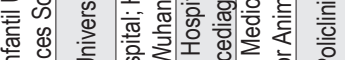

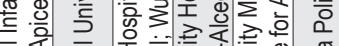

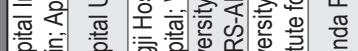

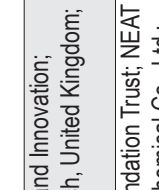

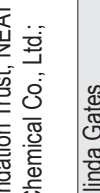

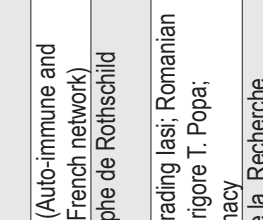

爱

垔

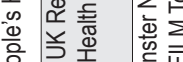

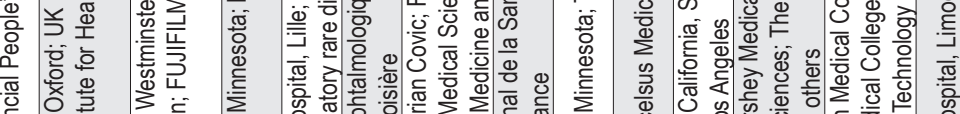

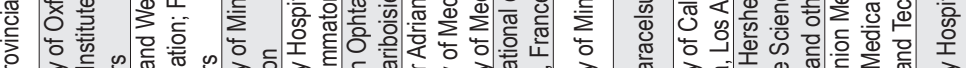
旁

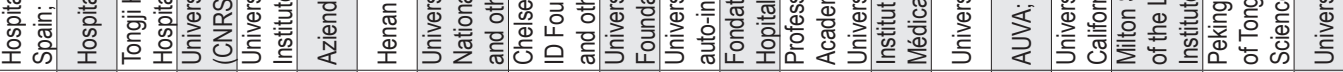

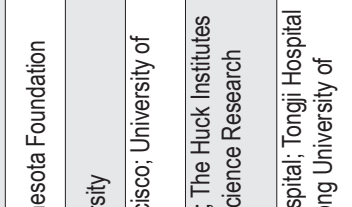

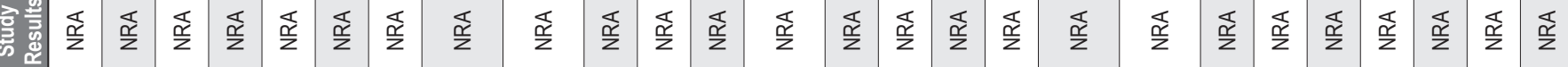

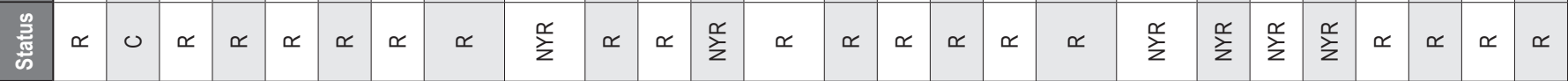

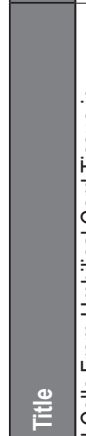

\begin{tabular}{|c|c|c|c|c|c|c|c|c|c|c|}
\hline 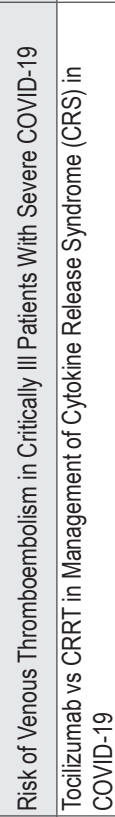 & 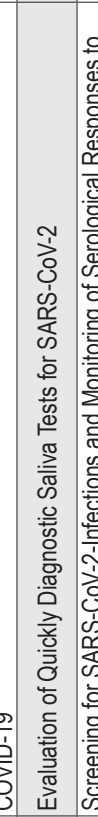 & 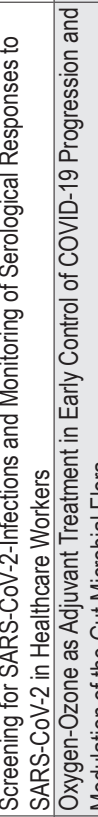 & 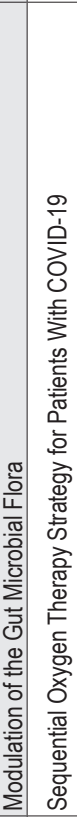 & 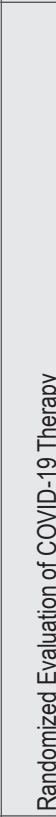 & & 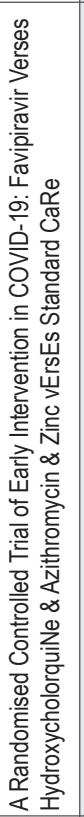 & 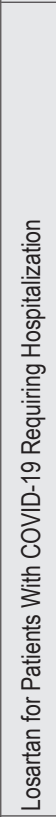 & 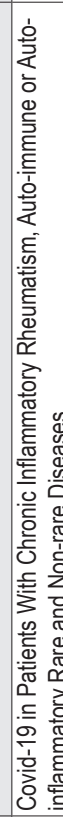 & 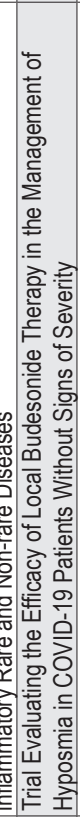 & 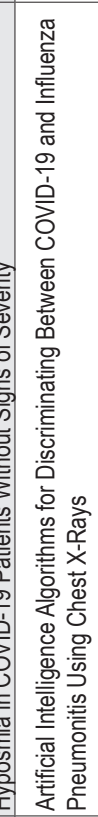 \\
\hline
\end{tabular}

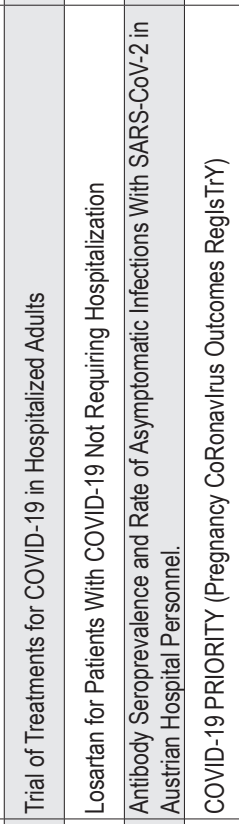

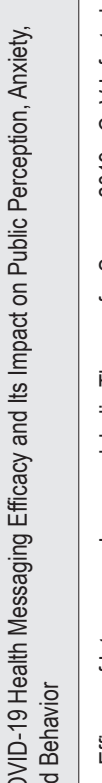

\begin{tabular}{|c|c|c|c|c|c|c|}
\hline 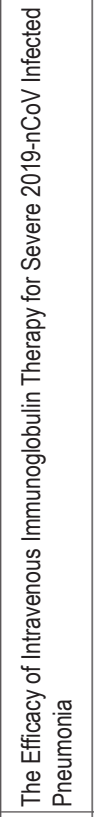 & 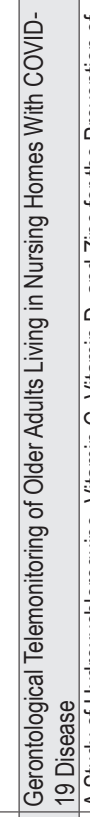 & 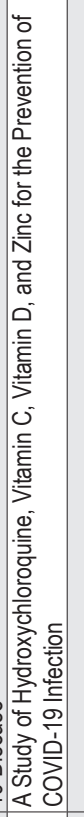 & 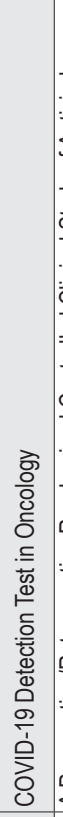 & 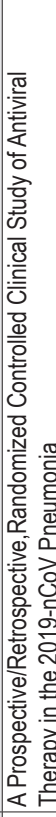 & 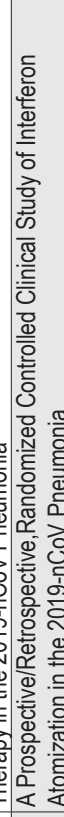 & 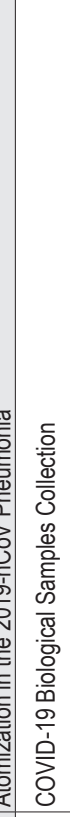 \\
\hline 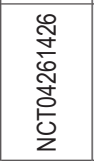 & 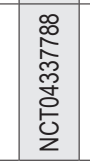 & 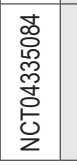 & 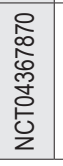 & 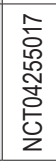 & 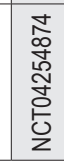 & 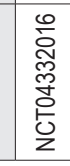 \\
\hline
\end{tabular}




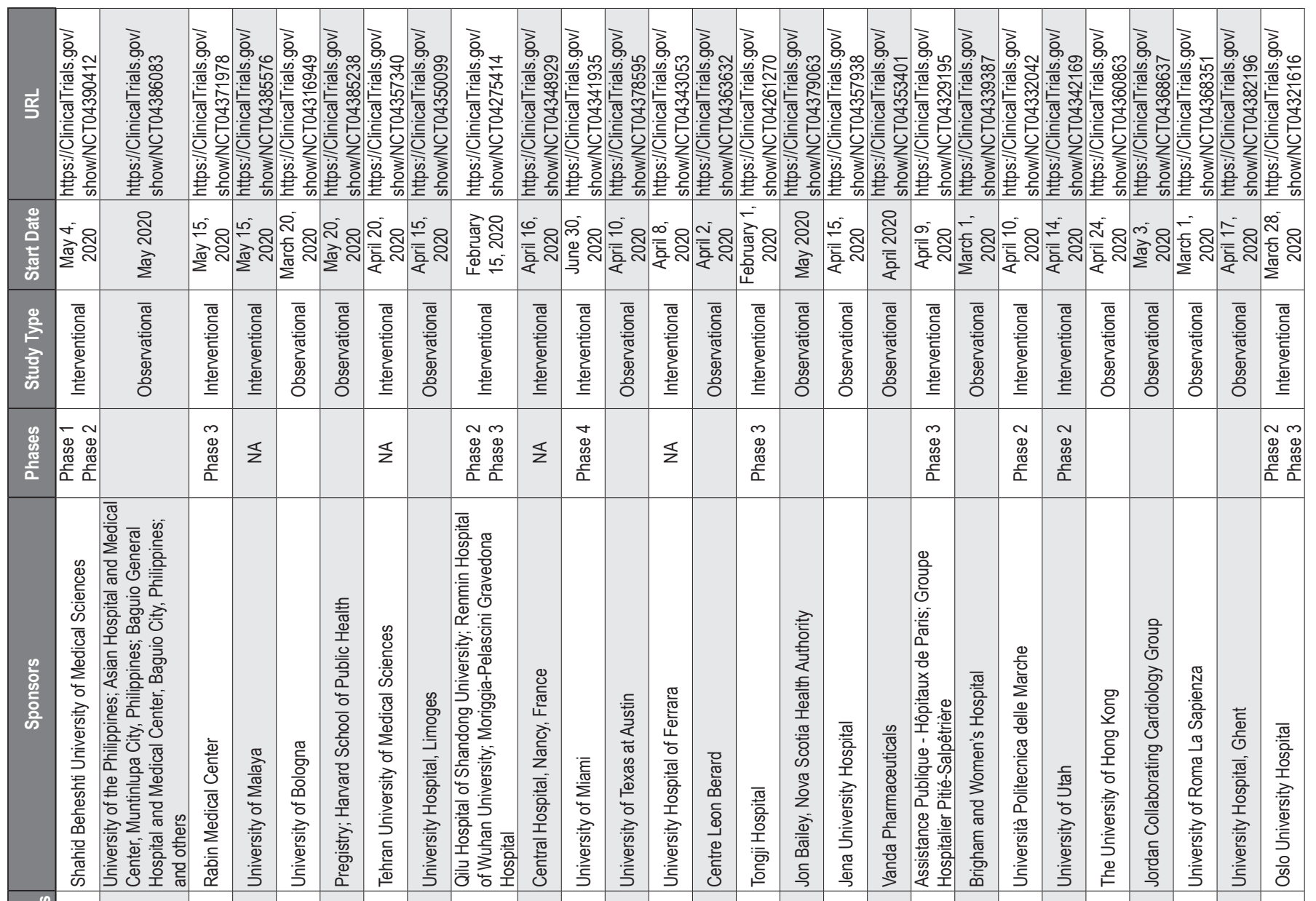

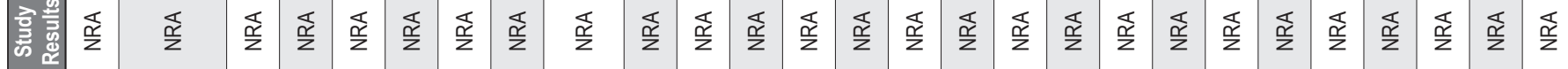

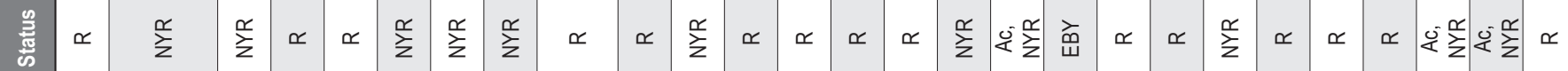

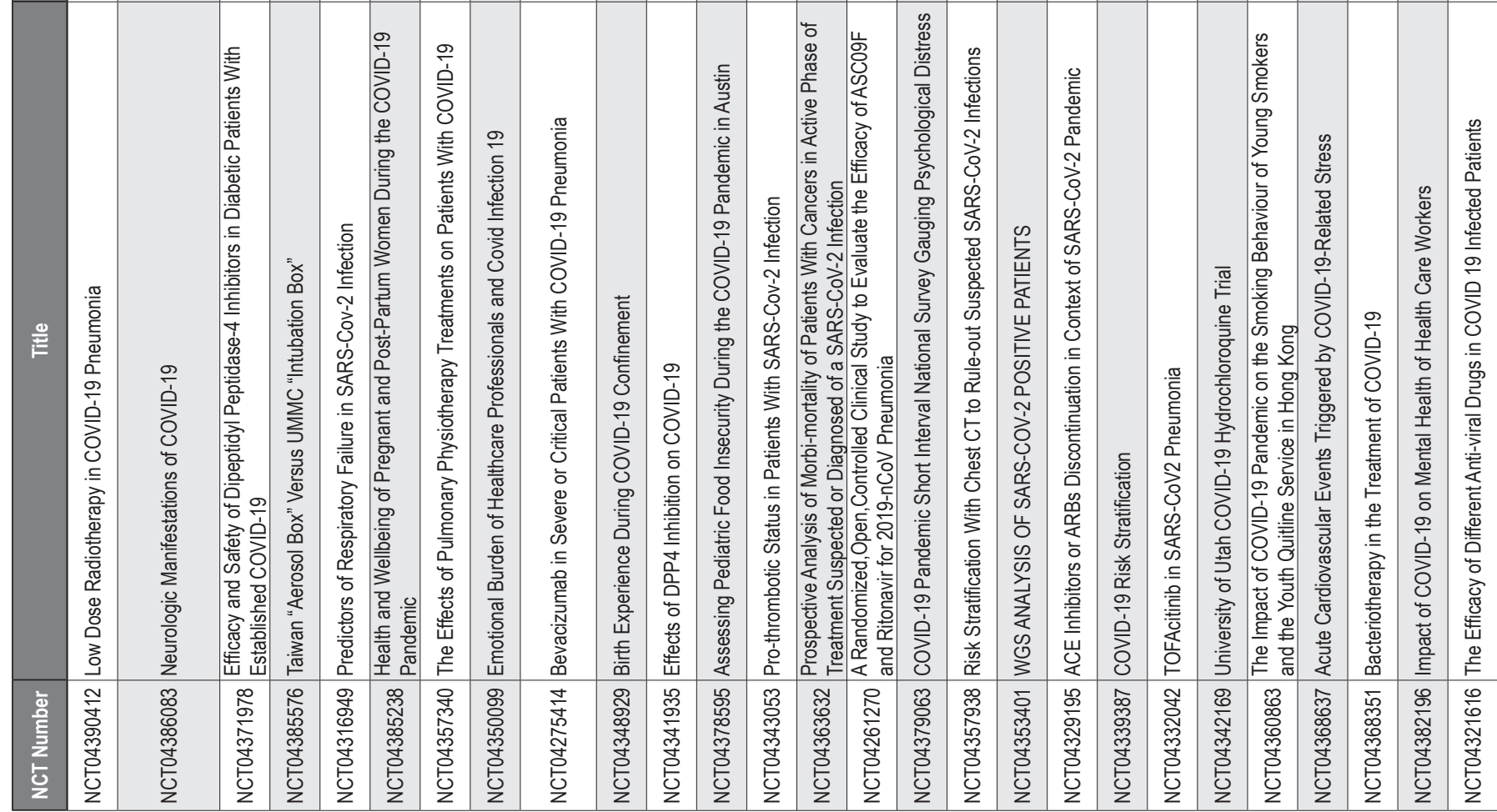




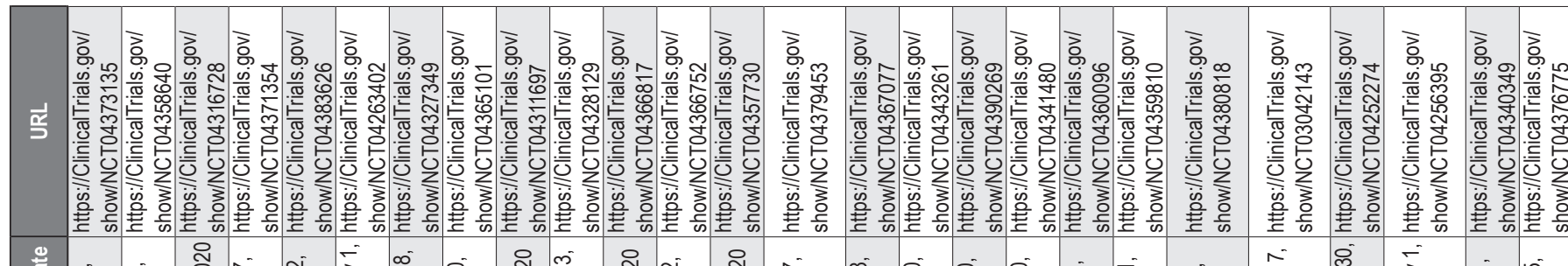

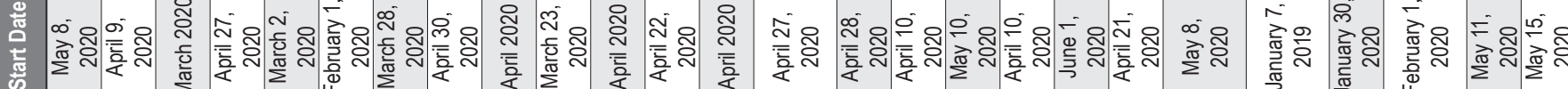

\begin{tabular}{|c|c|c|c|c|c|c|c|c|c|c|c|c|c|c|c|c|c|c|c|c|c|c|c|c|}
\hline 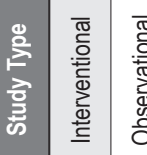 & 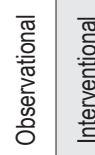 & 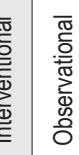 & 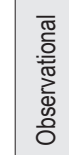 & 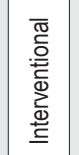 & 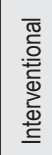 & 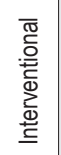 & 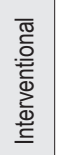 & 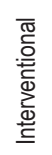 & 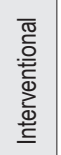 & & 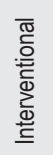 & 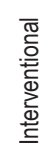 & & 髺 & 产 & 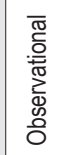 & 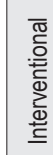 & & & & & & & 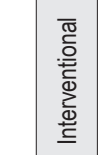 \\
\hline
\end{tabular}

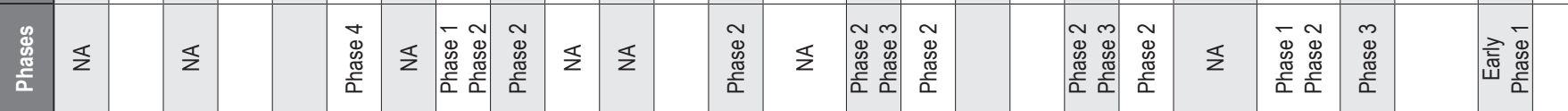

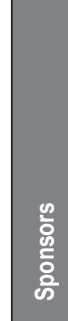

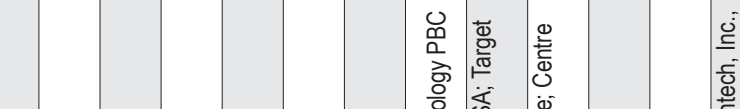

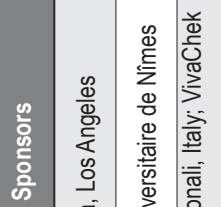

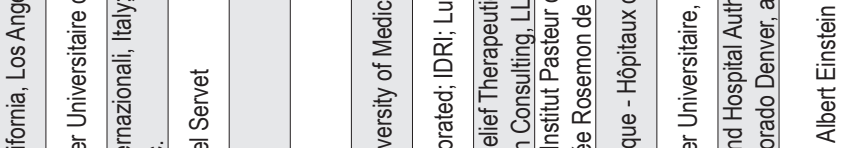

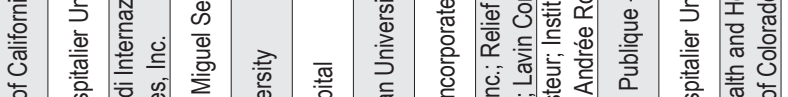

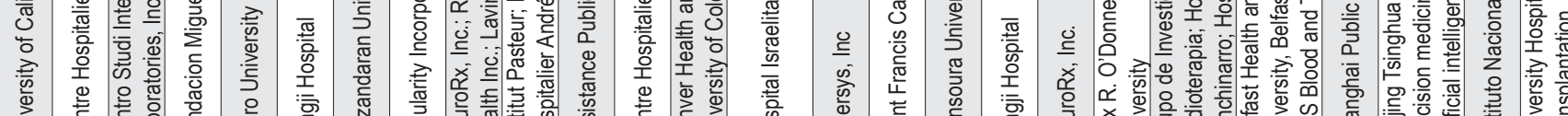

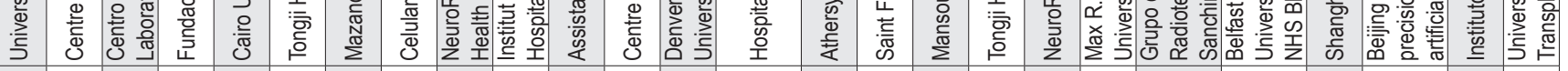

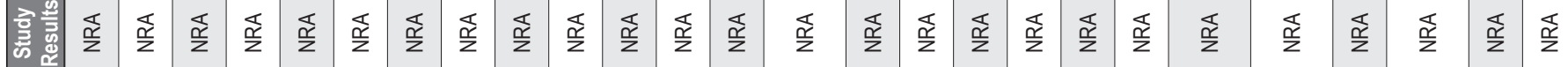

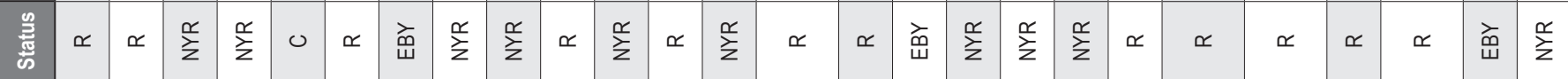

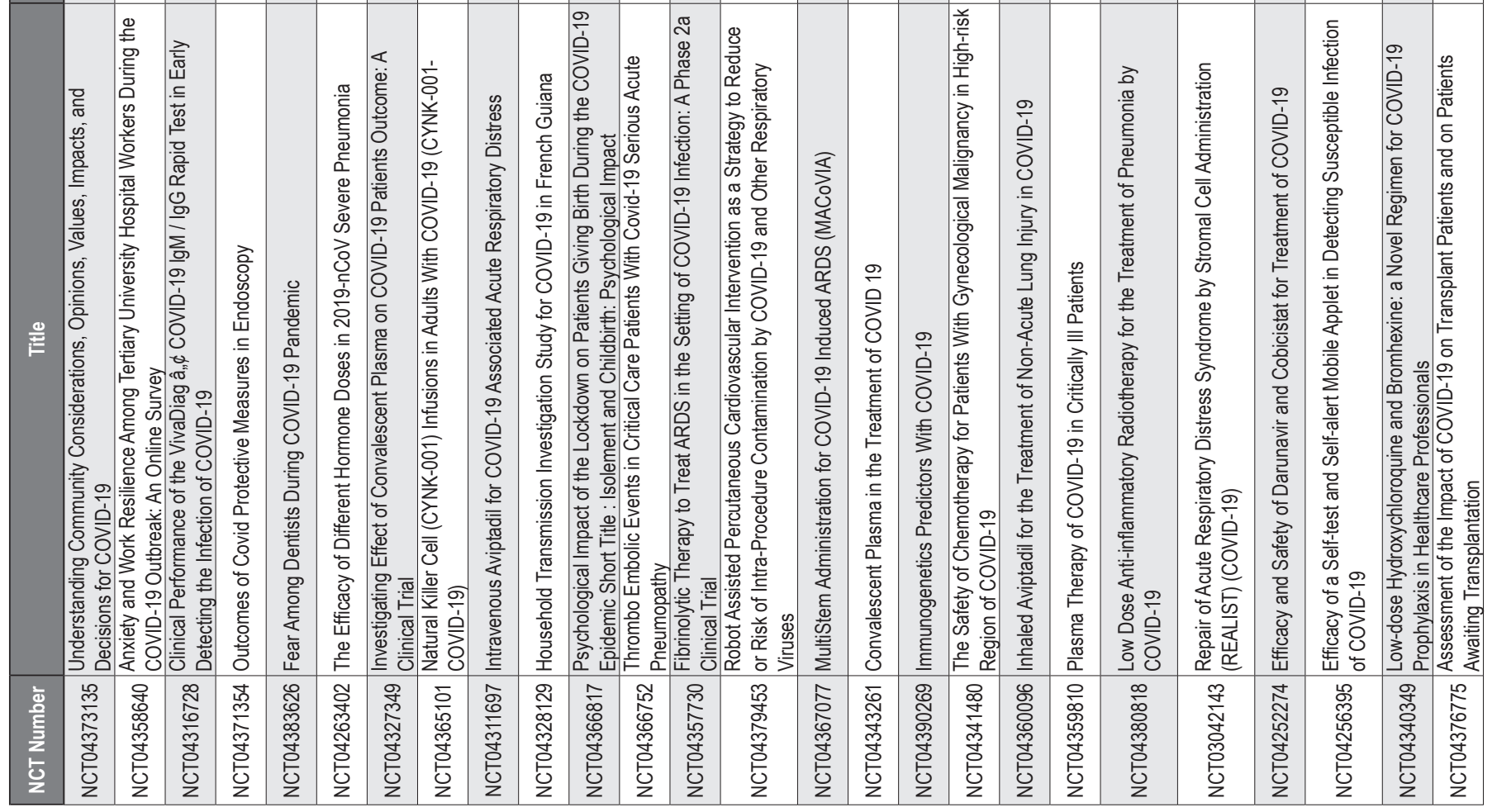




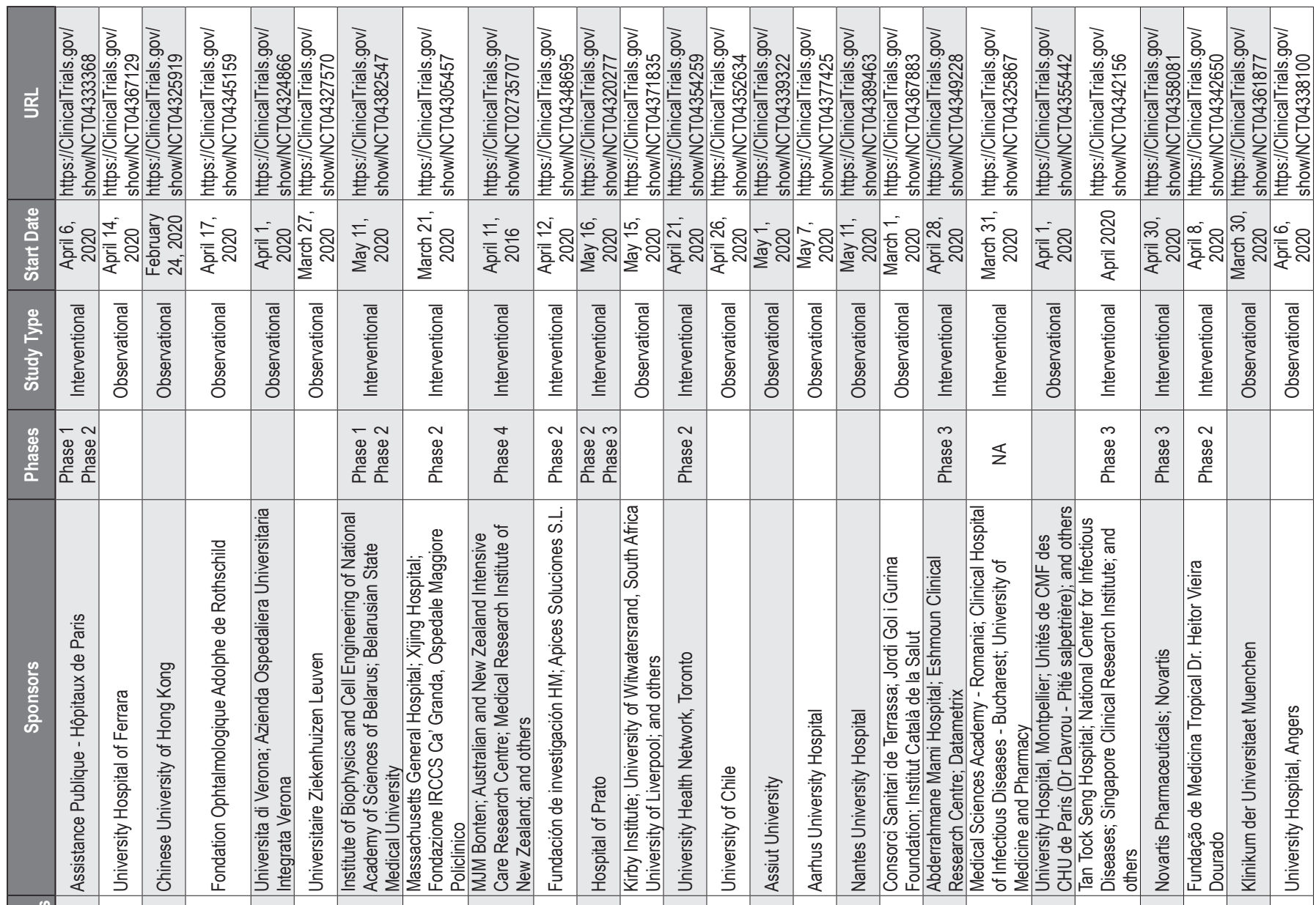

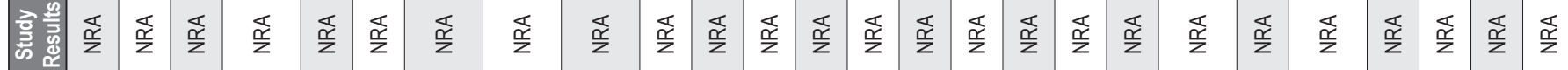

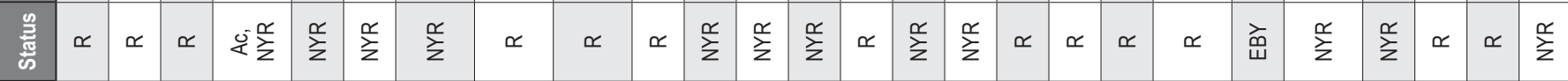

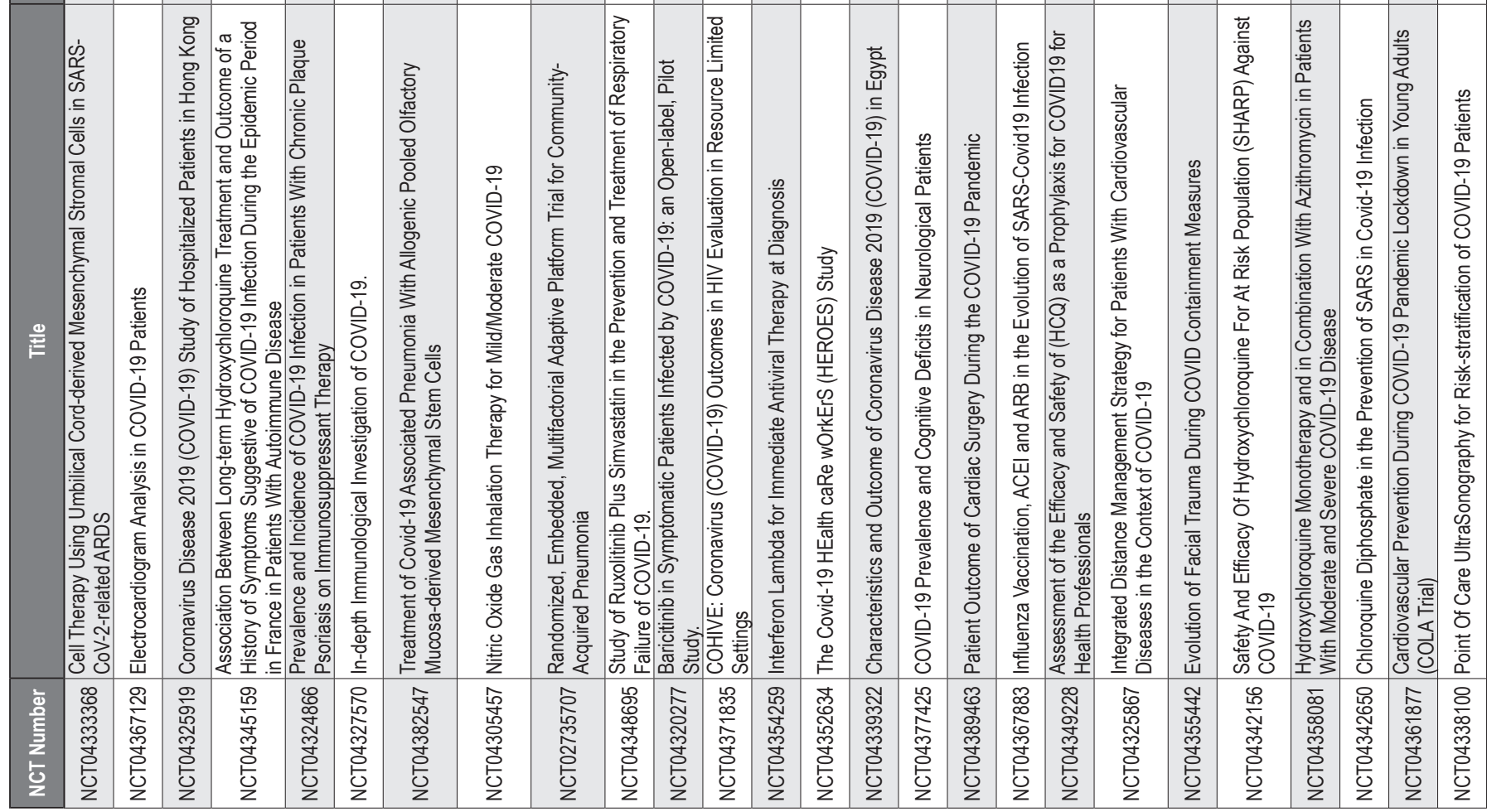




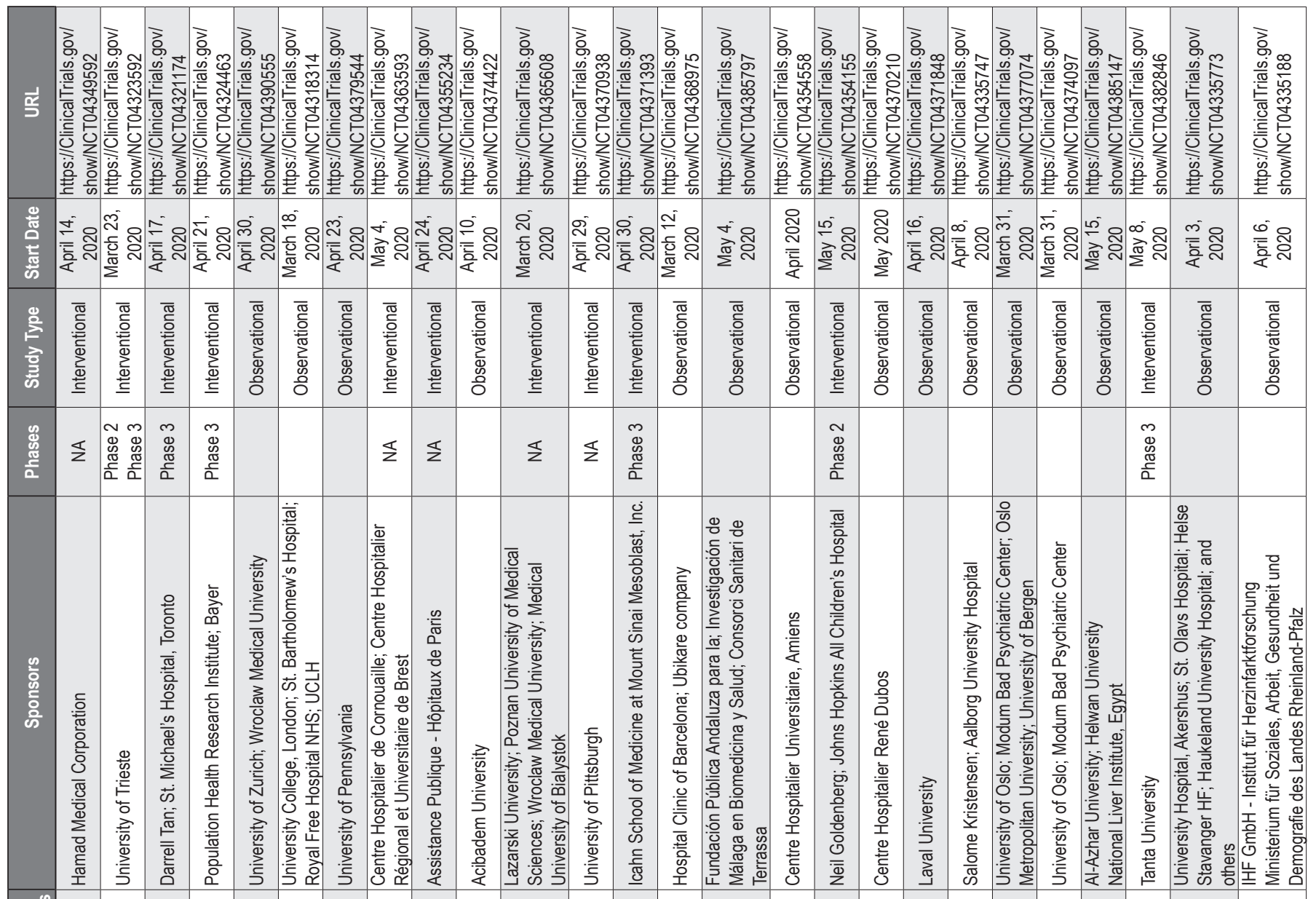

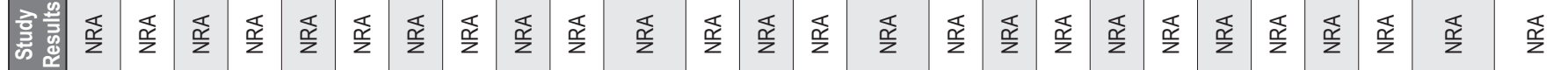

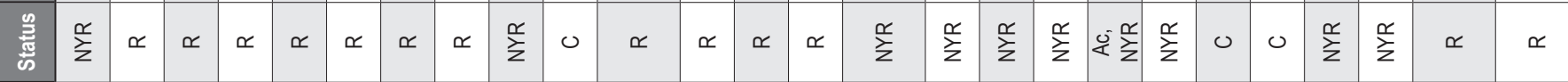

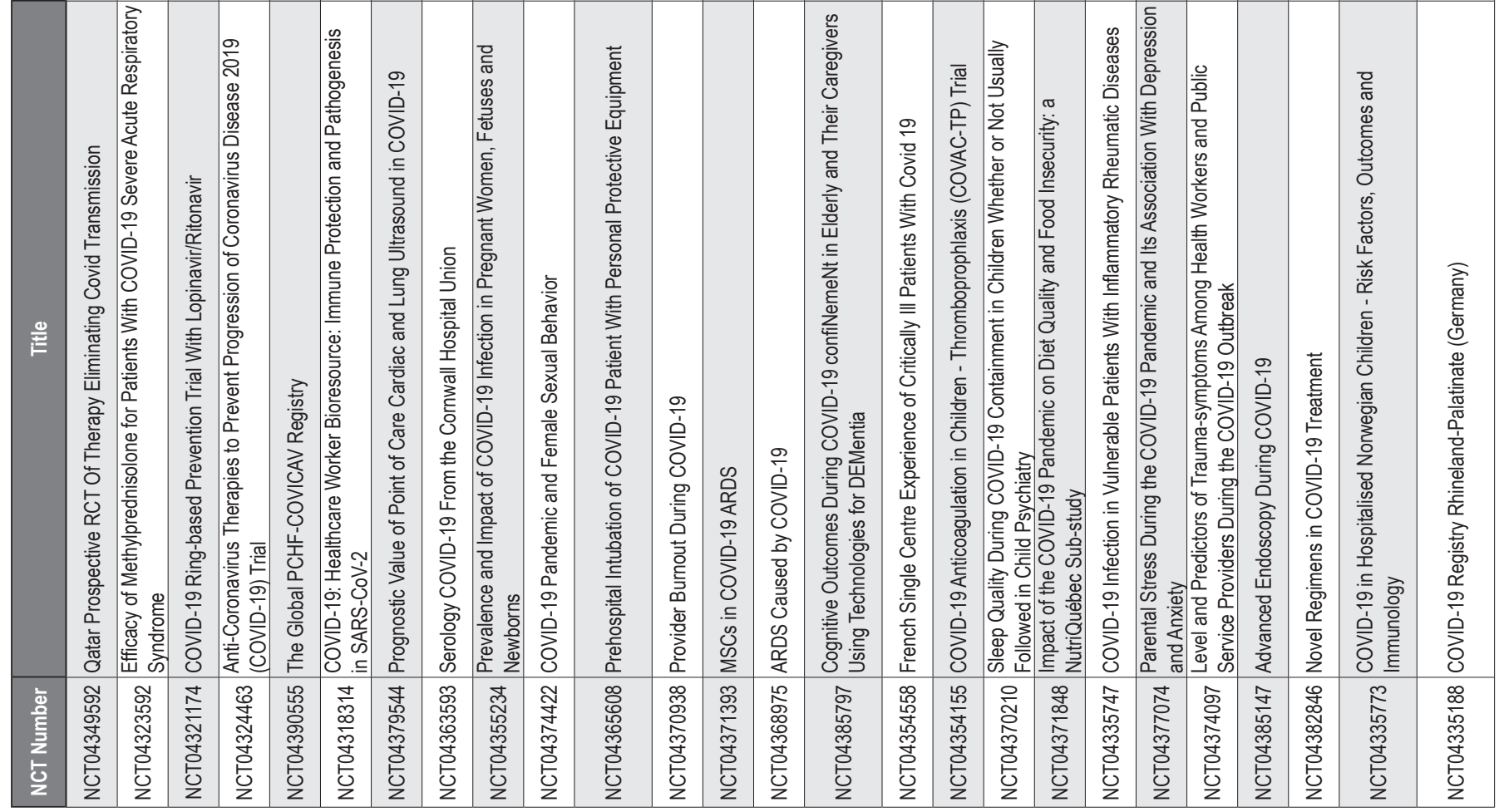




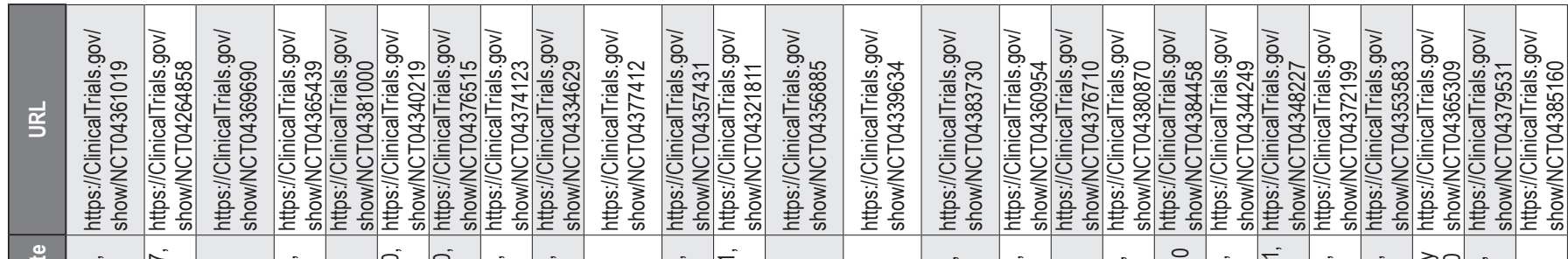

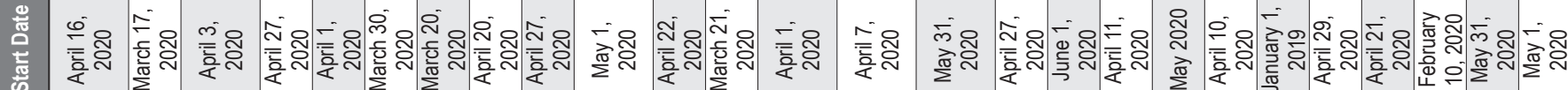

\begin{tabular}{|c|c|c|c|c|c|c|c|c|c|c|c|c|c|c|c|c|c|c|c|c|c|c|c|c|c|}
\hline 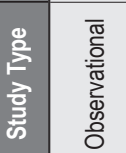 & 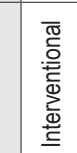 & 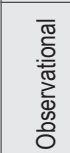 & 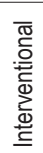 & 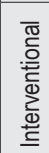 & 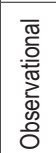 & 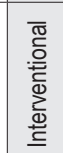 & 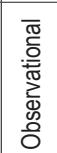 & 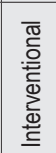 & 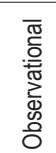 & 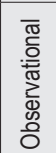 & 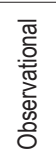 & 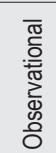 & 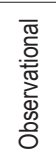 & 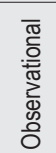 & 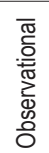 & 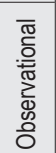 & 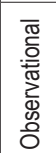 & 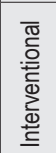 & 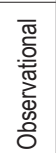 & 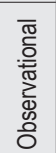 & 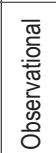 & 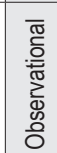 & 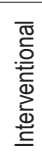 & 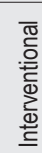 & 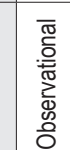 \\
\hline
\end{tabular}

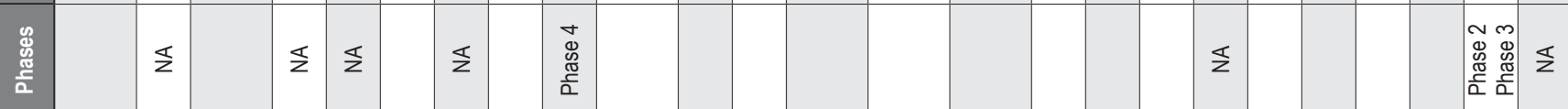

\begin{tabular}{|c|c|c|c|c|c|c|c|c|c|c|c|c|c|c|c|c|c|c|c|c|c|c|c|c|c|}
\hline 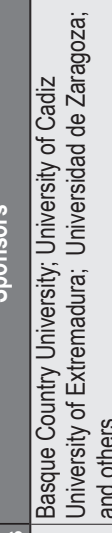 & 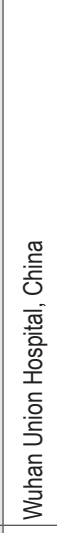 & 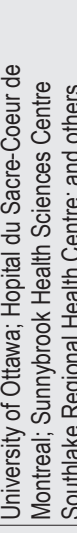 & & 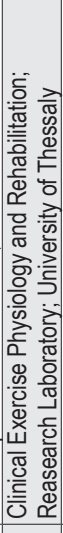 & 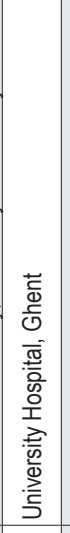 & 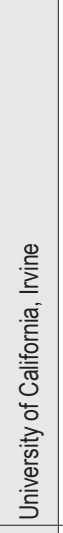 & 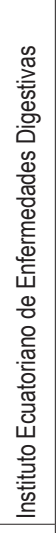 & 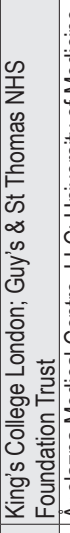 & 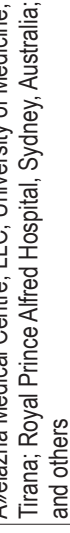 & 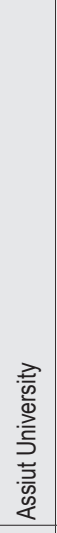 & $\frac{\infty}{\bar{\omega}}$ & 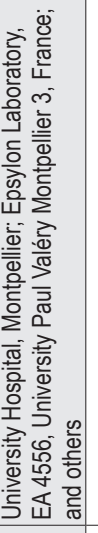 & 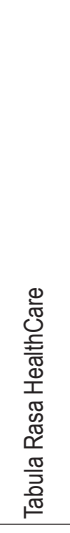 & 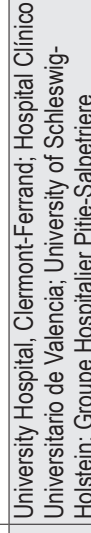 & 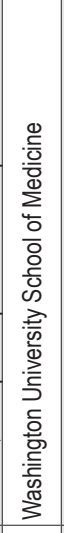 & 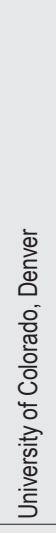 & 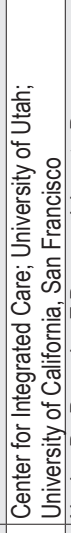 & & 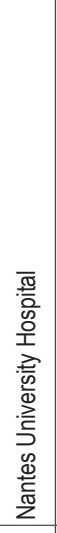 & 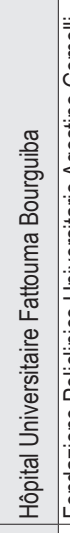 & 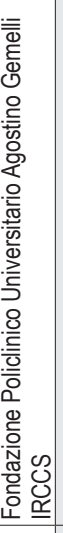 & 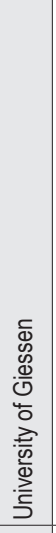 & 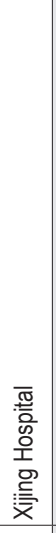 & 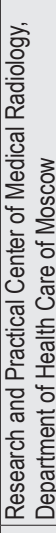 & 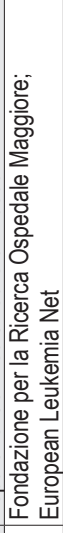 \\
\hline$\frac{\sqrt[5]{2}}{2}$ & $\frac{\underline{p}}{z}$ & $\frac{\sqrt[s]{2}}{\frac{s}{2}}$ & $\frac{\Phi}{z}$ & 旁 & $\frac{\sqrt[s]{z}}{z}$ & 产 & $\frac{\sqrt[s]{z}}{z}$ & $\frac{\mathbb{s}}{z}$ & 絰 & $\frac{\mathbf{s}}{\frac{5}{z}}$ & 鿖 & 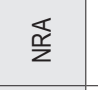 & 蛋 & 产 & 嵒 & $\frac{\widehat{p}}{z}$ & $\frac{\widehat{s}}{z}$ & 丞 & $\frac{\Phi}{z}$ & $\frac{\widehat{s}}{z}$ & $\frac{\widehat{s}}{z}$ & $\frac{\mathbb{s}}{\frac{\hat{s}}{2}}$ & $\stackrel{\mathbb{\underline { \beta }}}{\frac{\underline{p}}{z}}$ & $\frac{\widehat{s}}{z}$ & \\
\hline$\propto$ & $\frac{\alpha}{z}$ & $\simeq$ & $\stackrel{\frac{N}{z}}{\sum}$ & $\simeq$ & $\dot{x} \frac{\alpha}{z}$ & $\dot{x}^{0} \frac{\alpha}{2}$ & $\simeq$ & $\sum_{\Sigma}^{\frac{N}{z}}$ & ए & $\simeq$ & $\propto$ & $\propto$ & 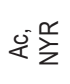 & $\sum_{\Sigma}^{\frac{\alpha}{z}}$ & $\propto$ & $\stackrel{\alpha}{\sum}$ & 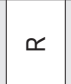 & $\sum_{\Sigma}^{\frac{N}{z}}$ & $\alpha$ & 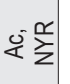 & $\propto$ & $\simeq$ & $\tilde{\Psi}$ & z & \\
\hline
\end{tabular}

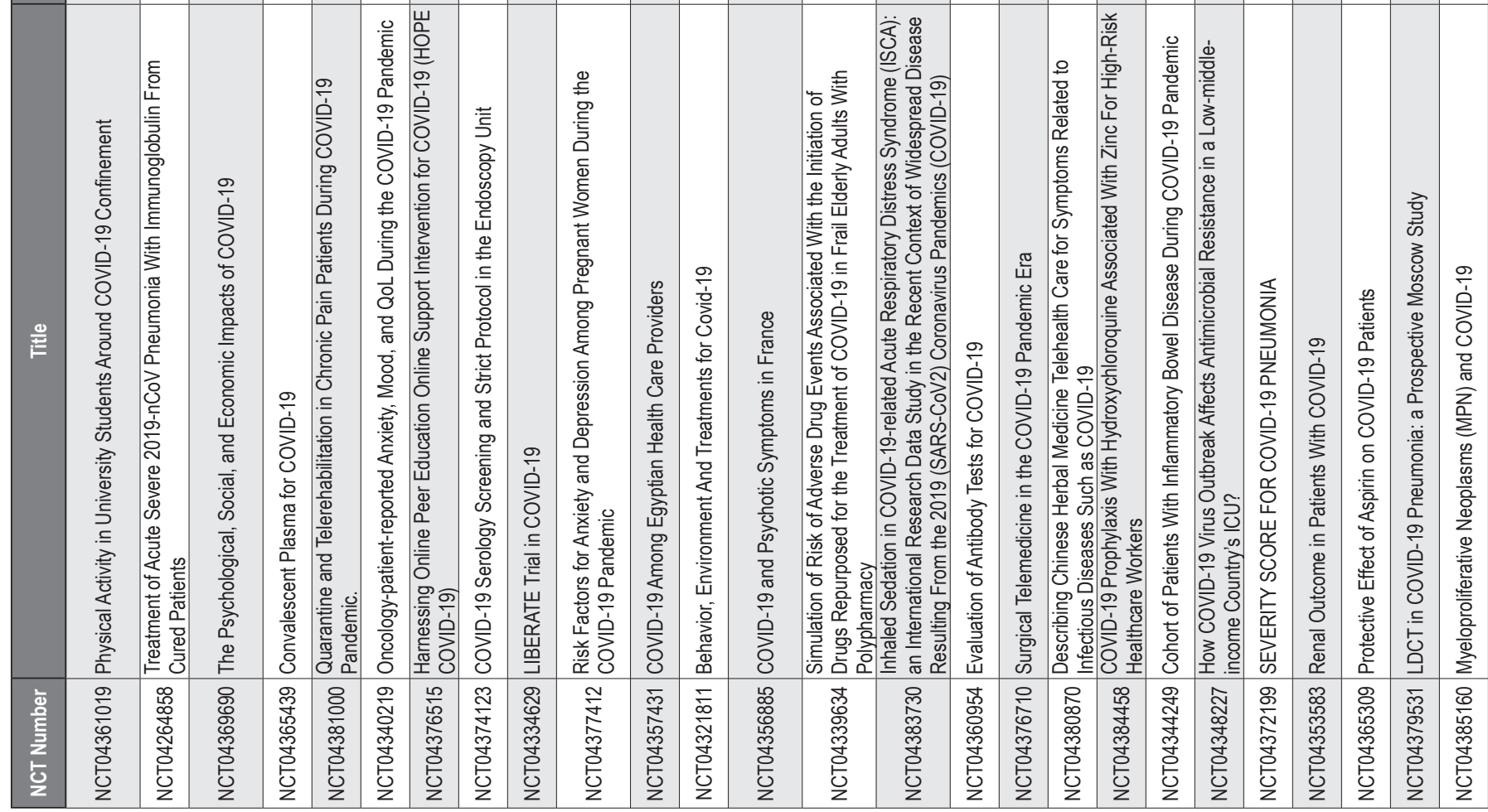




\begin{tabular}{|c|c|c|c|c|c|c|c|c|c|c|c|c|c|c|c|c|c|c|c|c|c|c|c|c|c|c|c|}
\hline$\vec{y}$ & 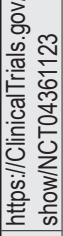 & 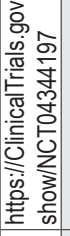 & 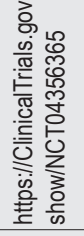 & 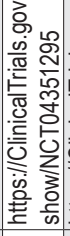 & 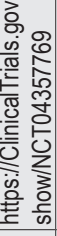 & & & & & 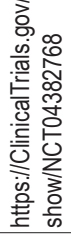 & 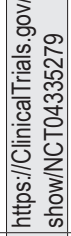 & 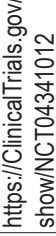 & & 语 讨 & $\frac{y}{2}$ & 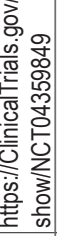 & 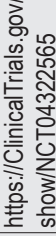 & & 忽 & 㒸 & 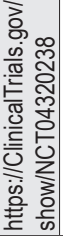 & 曾 & & న్ & 电 & $\pm \bar{\omega}$ & 量 \\
\hline & 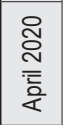 & 交 & 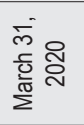 & & & & & & & 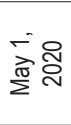 & & & & & & & & & & & & & & & & & \\
\hline & 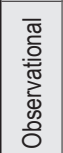 & 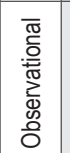 & 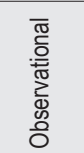 & 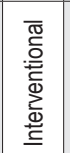 & 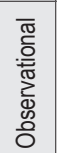 & 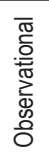 & 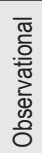 & 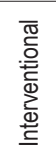 & 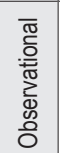 & 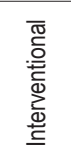 & 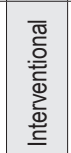 & 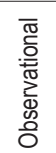 & 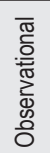 & 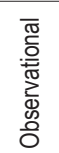 & 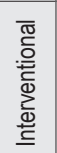 & 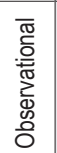 & 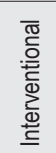 & 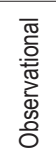 & 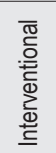 & 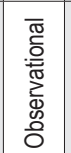 & 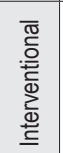 & 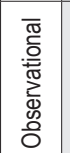 & 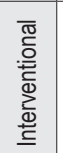 & 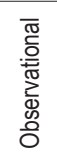 & 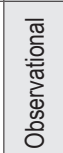 & 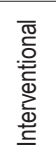 & 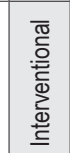 \\
\hline & & & & 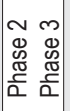 & & & & $\mathbb{z}$ & & $\Sigma$ & $\frac{s}{z}$ & & & & $\begin{array}{l}\frac{\pi}{0} \\
\frac{\pi}{\alpha}\end{array}$ & & \begin{tabular}{|l}
$\mathscr{\Phi}$ \\
$\frac{\pi}{2}$ \\
$\frac{\pi}{\alpha}$
\end{tabular} & & $\mathbb{\Sigma}$ & & 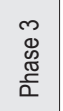 & & 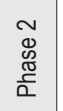 & & & $\begin{array}{l}\widetilde{O} \\
\text { 怘 } \\
\frac{\pi}{\alpha}\end{array}$ & $z$ \\
\hline
\end{tabular}

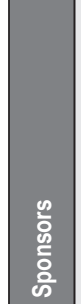

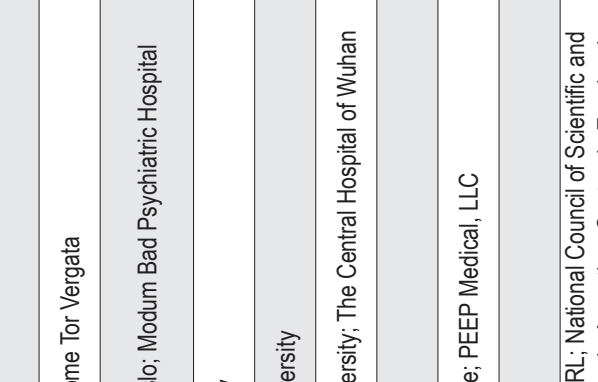

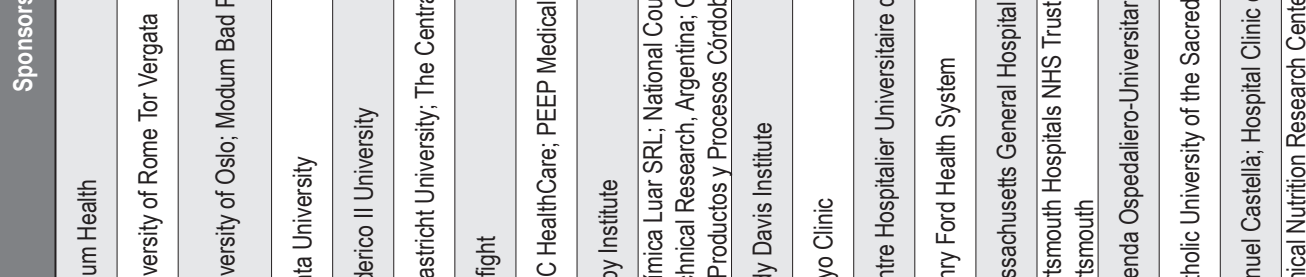

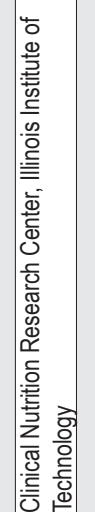

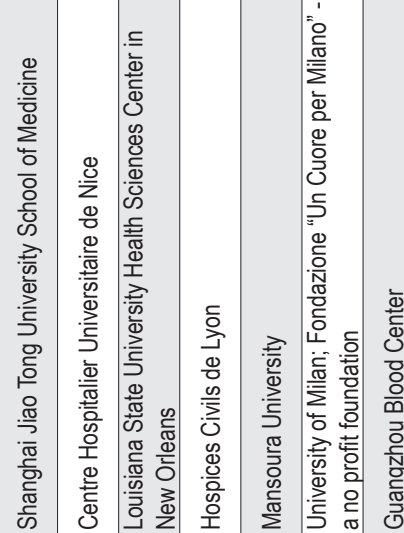

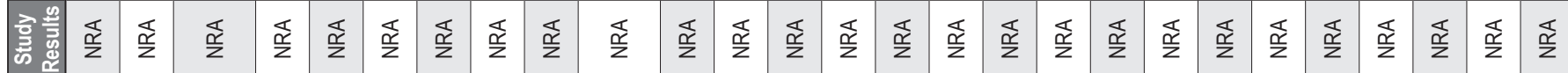

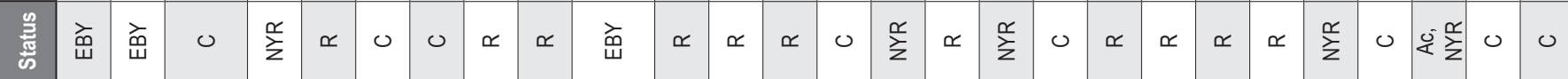

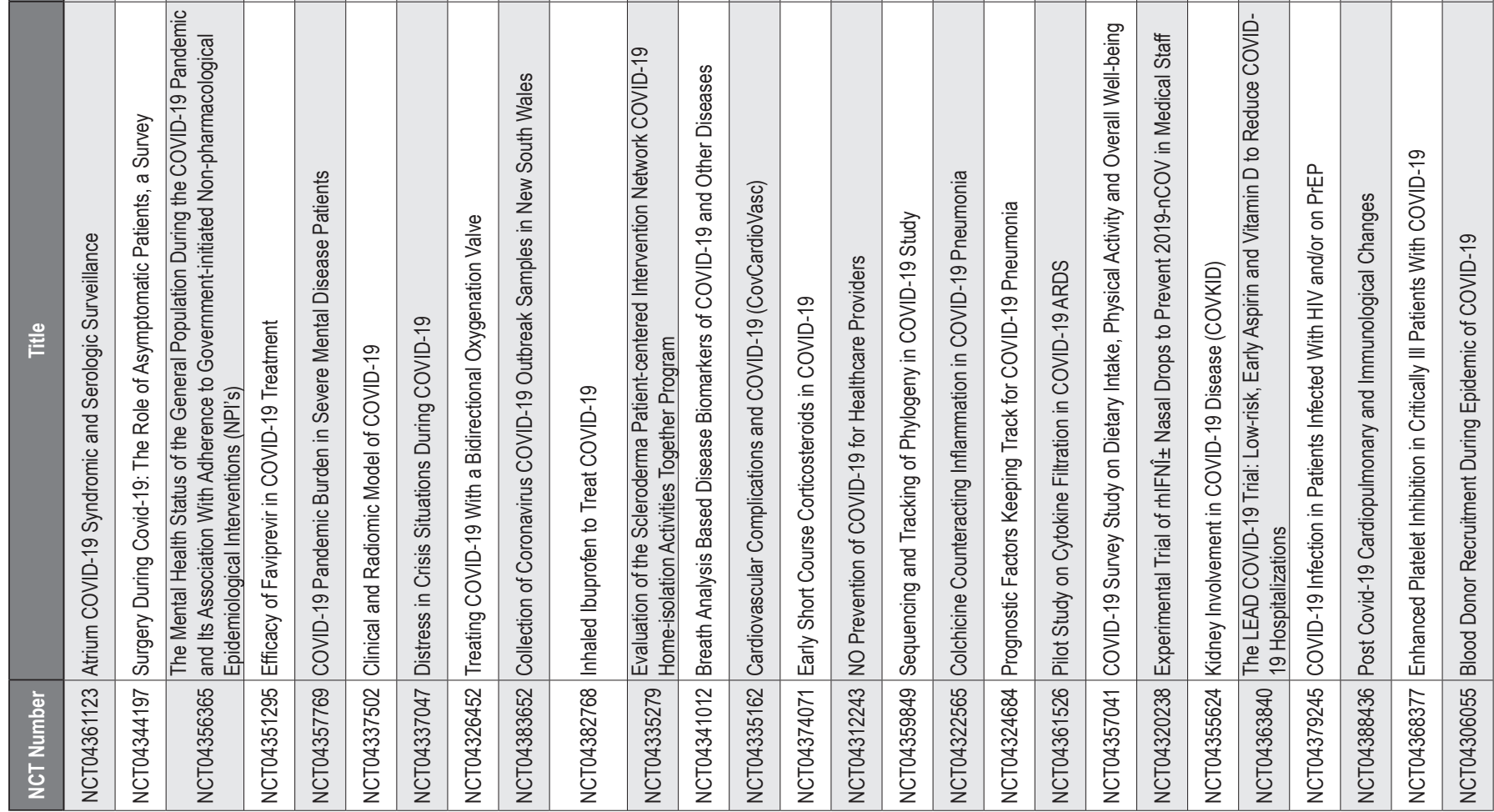




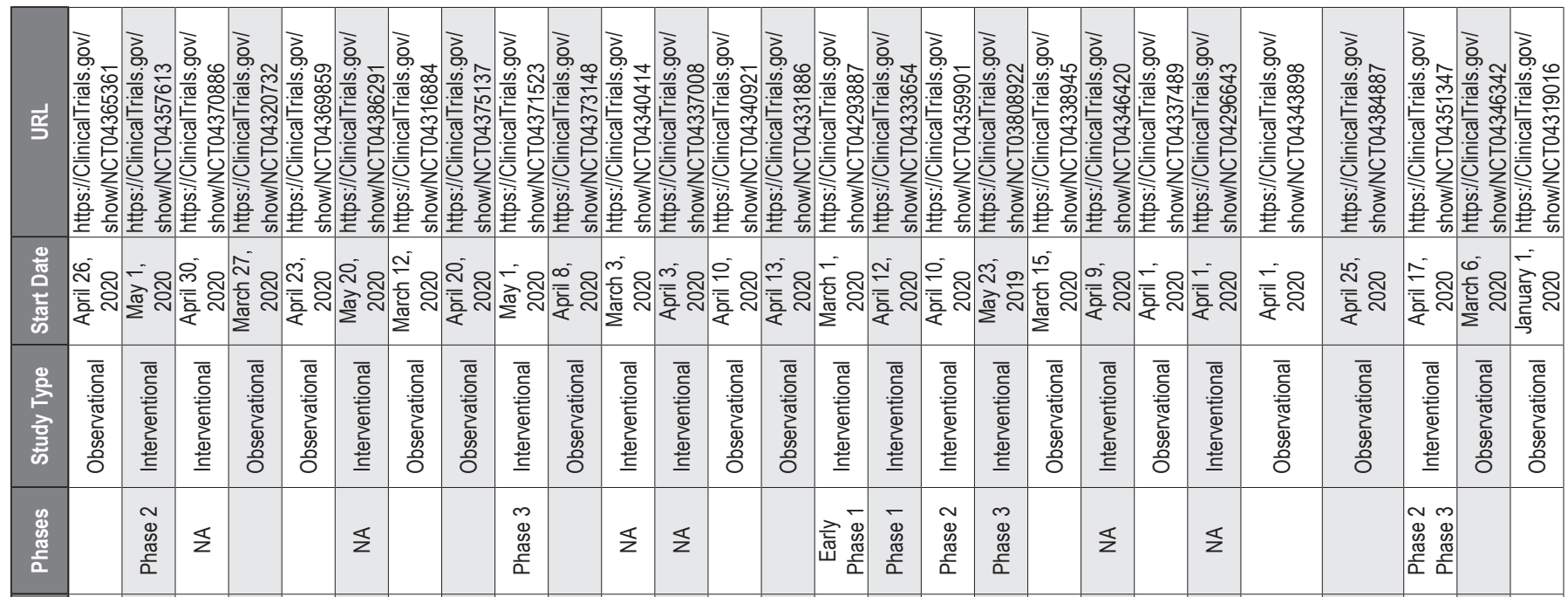
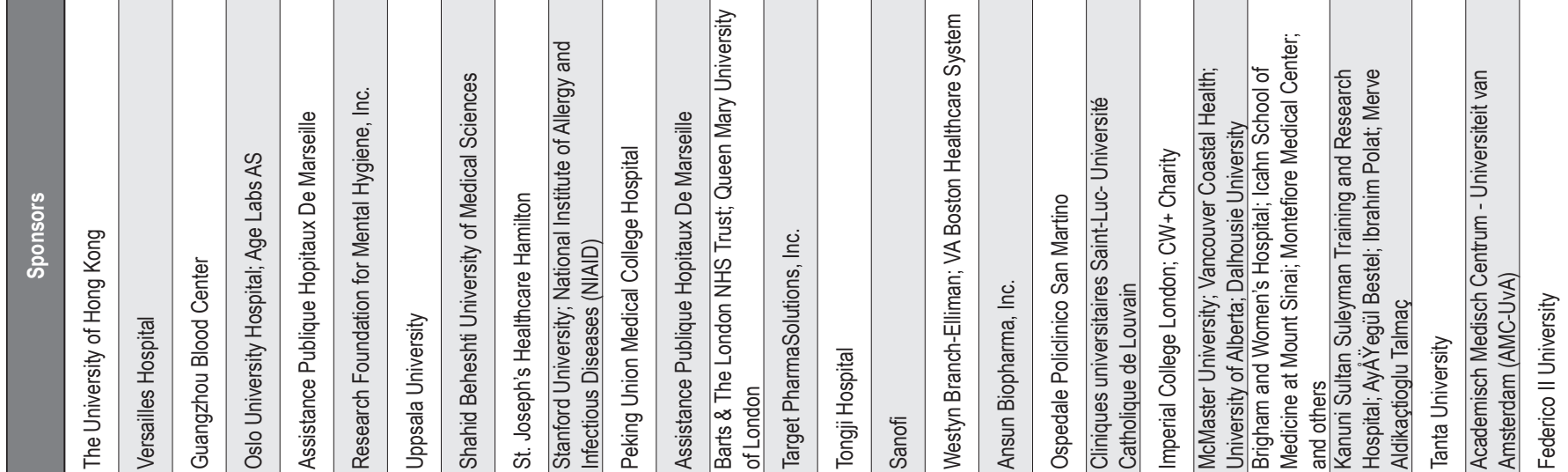

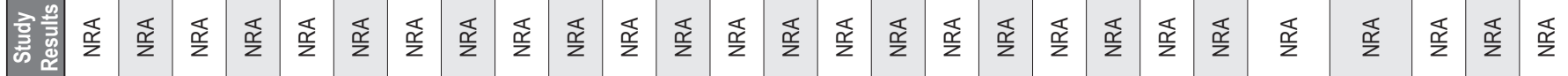

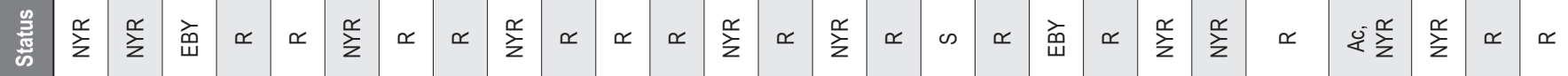

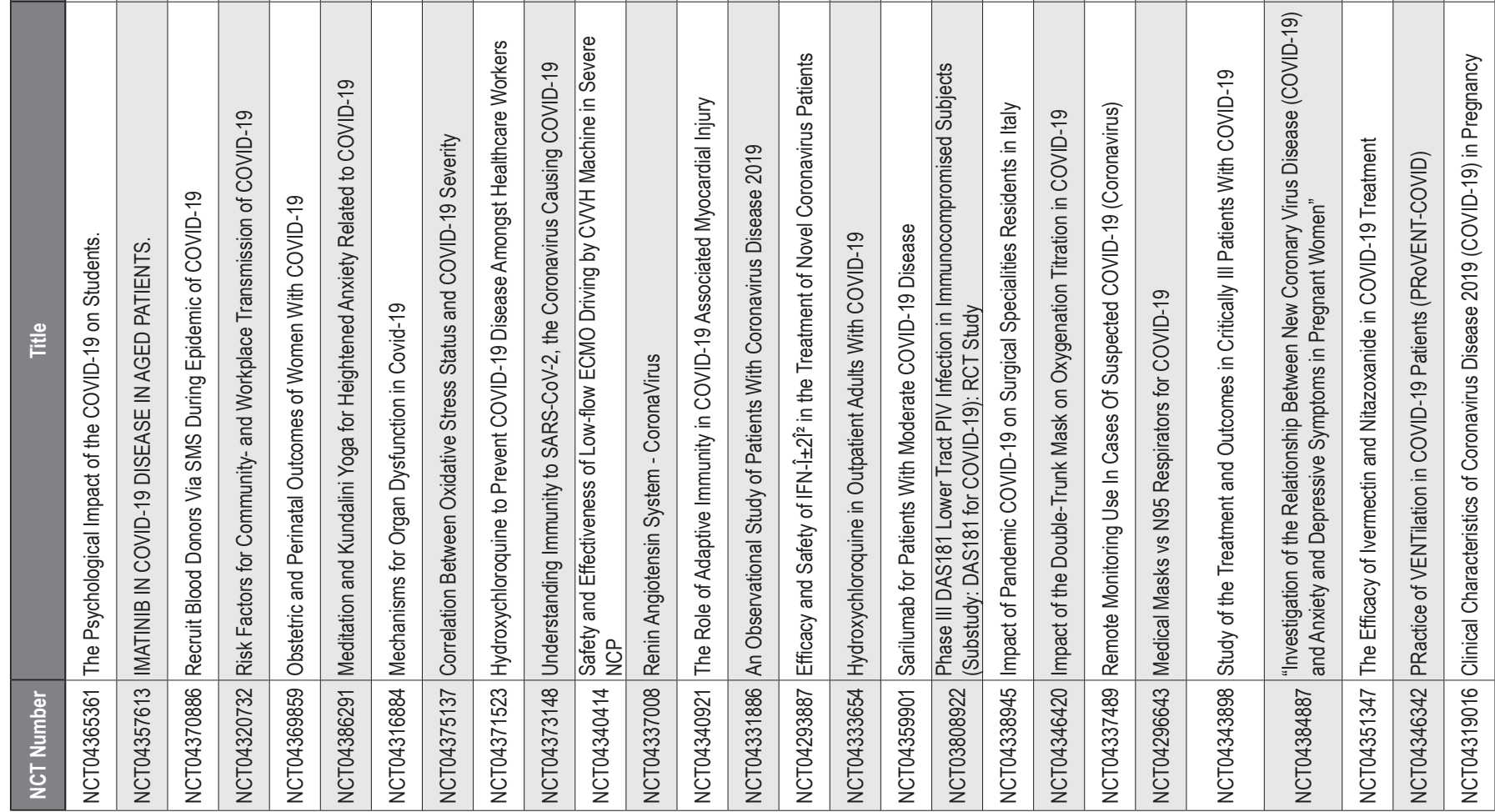




\begin{tabular}{|c|c|c|c|c|c|c|c|c|c|c|c|c|c|c|c|c|c|c|c|c|c|c|c|c|c|c|}
\hline 然 & 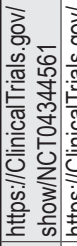 & 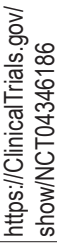 & 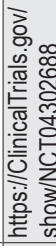 & & & 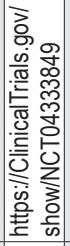 & 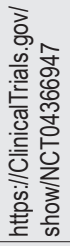 & 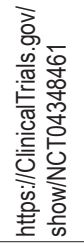 & 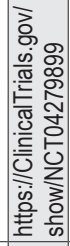 & & 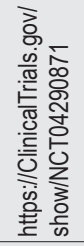 & 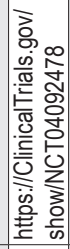 & & & 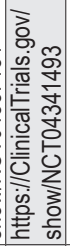 & & \%. & 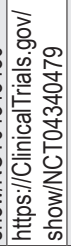 & 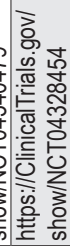 & 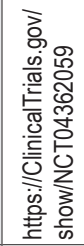 & 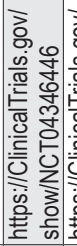 & 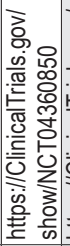 & & & & \\
\hline os & & & هㅇㅇㅇ & & & & 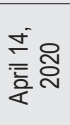 & 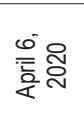 & & & 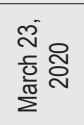 & 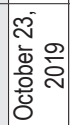 & & 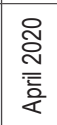 & & & 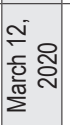 & 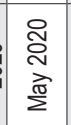 & 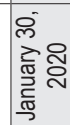 & 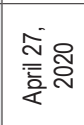 & & & & & & \\
\hline $\begin{array}{l}\text { 을 } \\
\text { 흘 } \\
\text { 흘 }\end{array}$ & 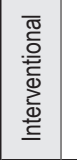 & 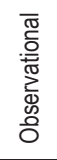 & 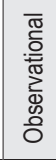 & 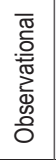 & 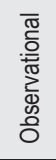 & 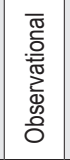 & 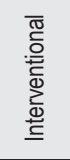 & 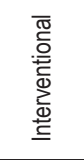 & 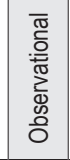 & 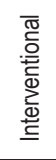 & 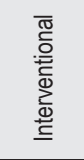 & 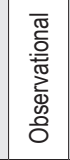 & 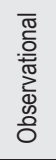 & 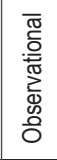 & 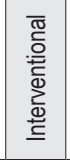 & 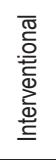 & 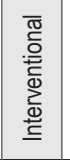 & 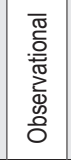 & 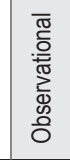 & 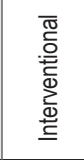 & 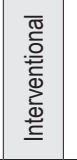 & 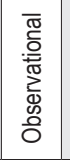 & 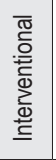 & 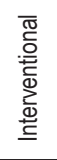 & 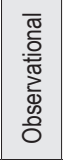 & \\
\hline 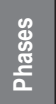 & $\Sigma$ & & & & & & 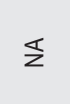 & 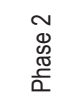 & & $\frac{s}{z}$ & 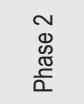 & & & & 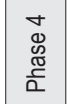 & 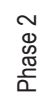 & $\begin{array}{l}\tilde{\Phi} \\
\mathscr{8} \\
\frac{\pi}{\alpha}\end{array}$ & & & 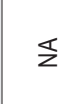 & 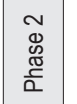 & & 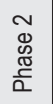 & 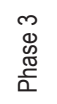 & & \\
\hline
\end{tabular}

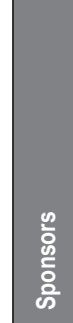

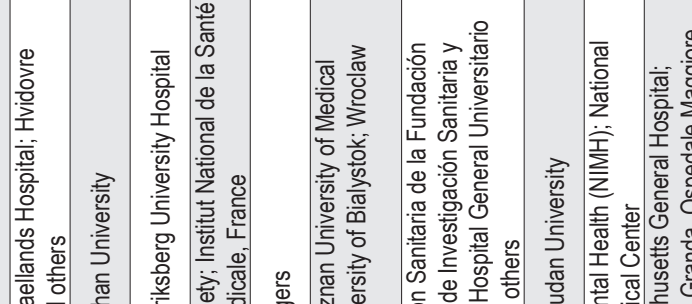

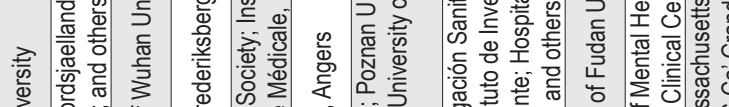

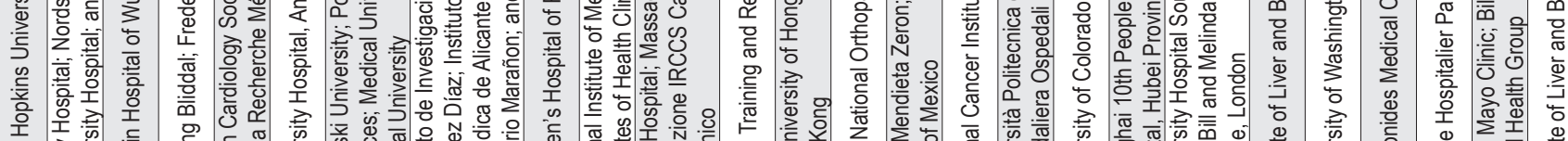

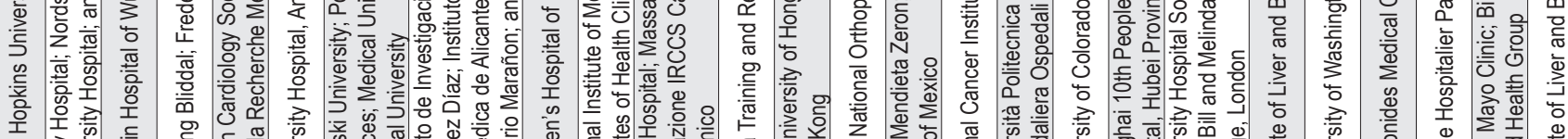

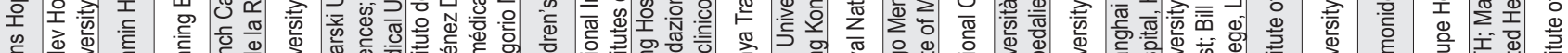
空

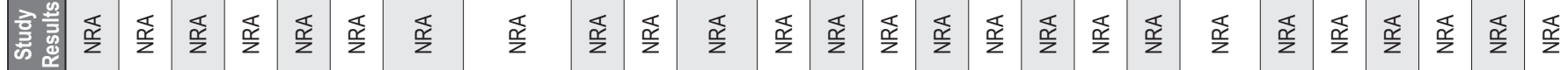

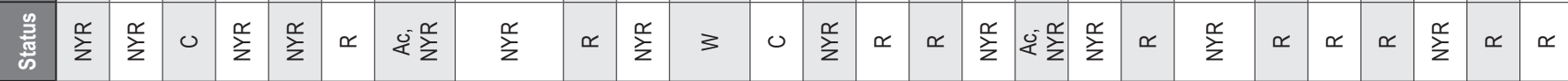

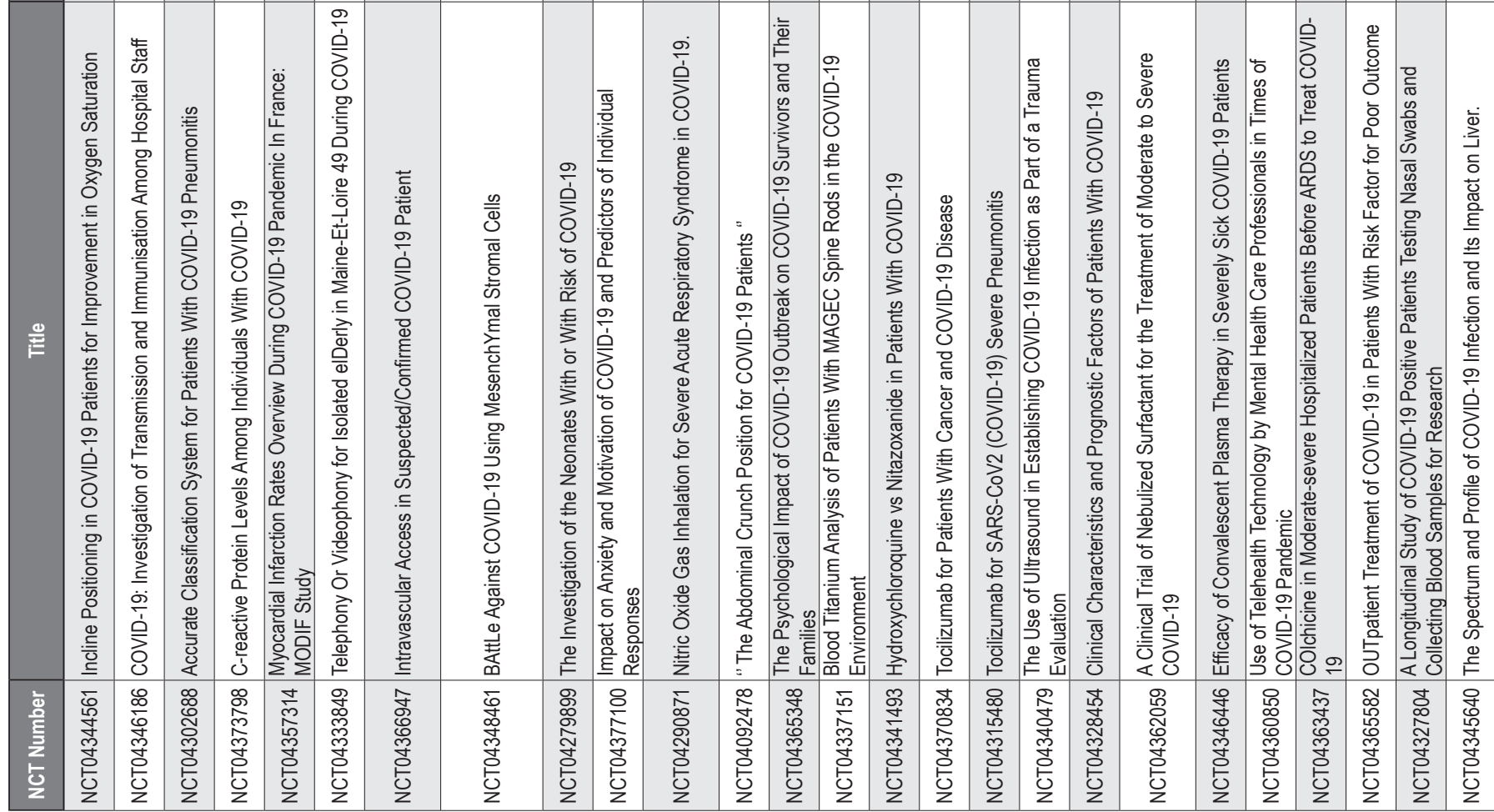




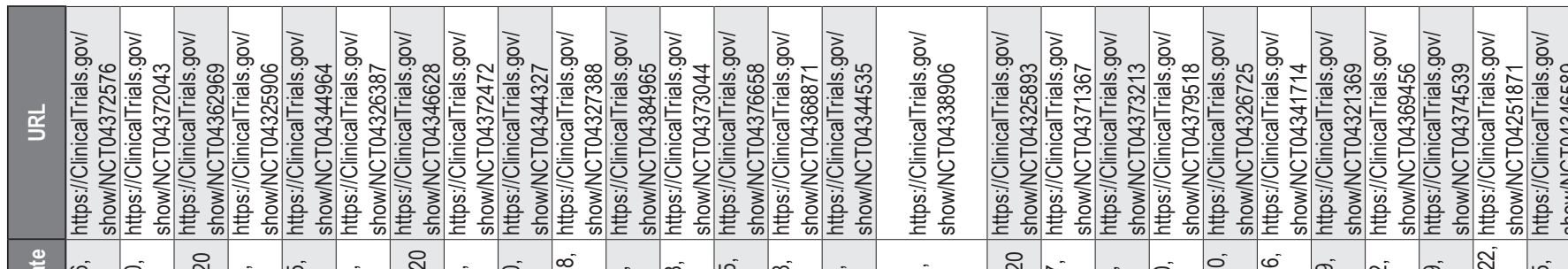

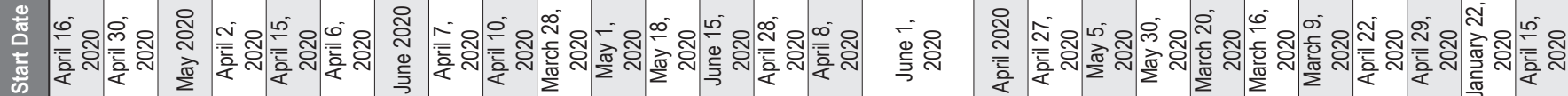

\begin{tabular}{|c|c|c|c|c|c|c|c|c|c|c|c|c|c|c|c|c|c|c|c|c|c|c|c|c|c|c|}
\hline 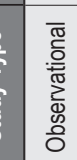 & 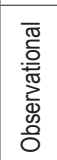 & 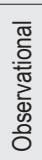 & 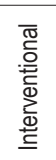 & 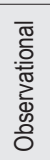 & 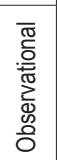 & 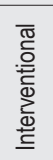 & 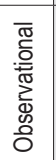 & 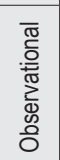 & 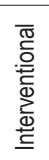 & 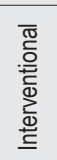 & 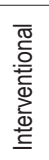 & 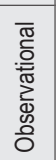 & 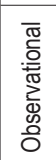 & 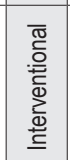 & 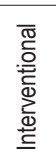 & 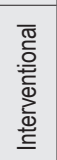 & 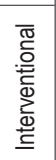 & 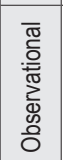 & 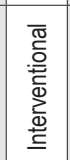 & 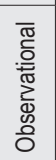 & 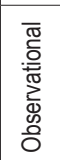 & 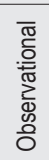 & 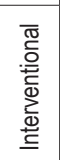 & 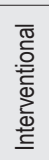 & 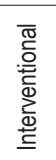 & 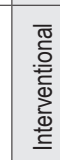 \\
\hline & & & $\underline{z}$ & & & 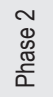 & & & 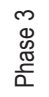 & $\underline{z}$ & 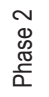 & & & 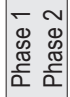 & $\overline{\underline{\alpha}}$ & 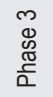 & 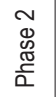 & & 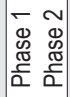 & & & & $\mathbb{z}$ & $\begin{array}{l}\mathbb{\otimes} \\
\frac{\mathbb{\pi}}{\alpha} \\
\frac{1}{2}\end{array}$ & $\underline{z}$ & \\
\hline
\end{tabular}

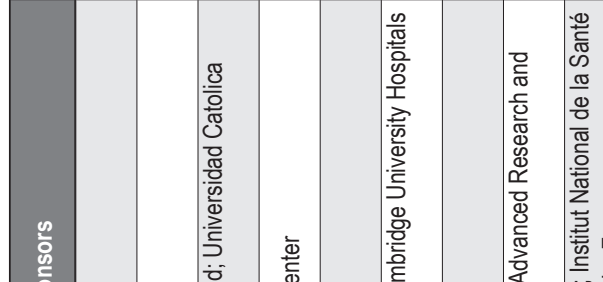

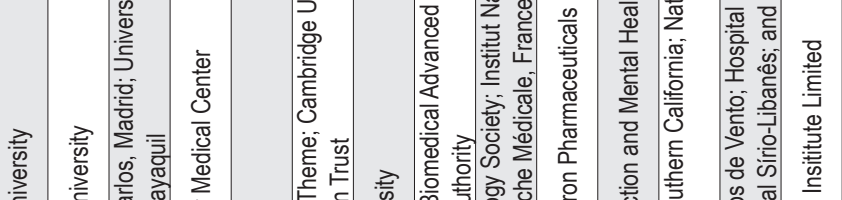

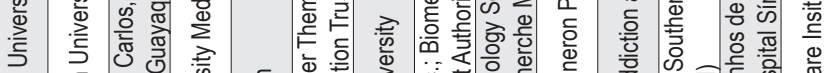

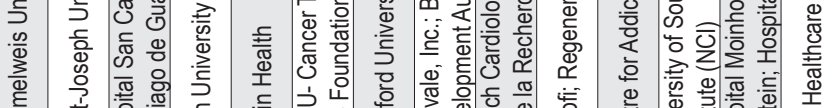

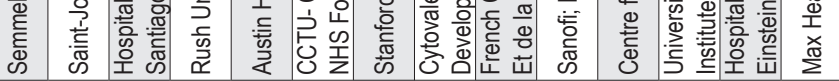

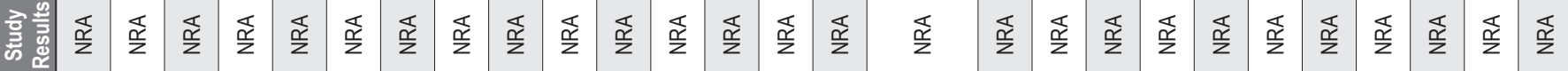

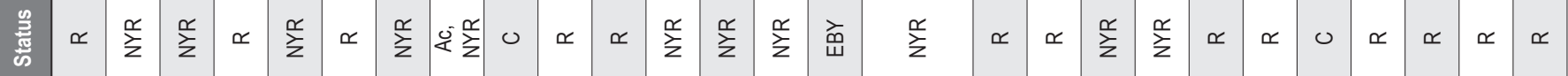

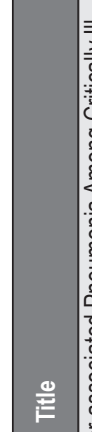

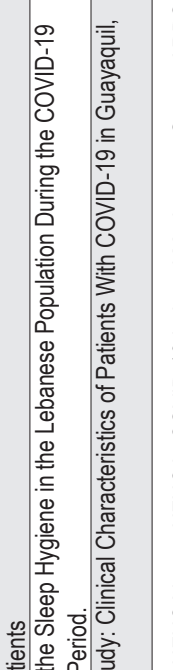

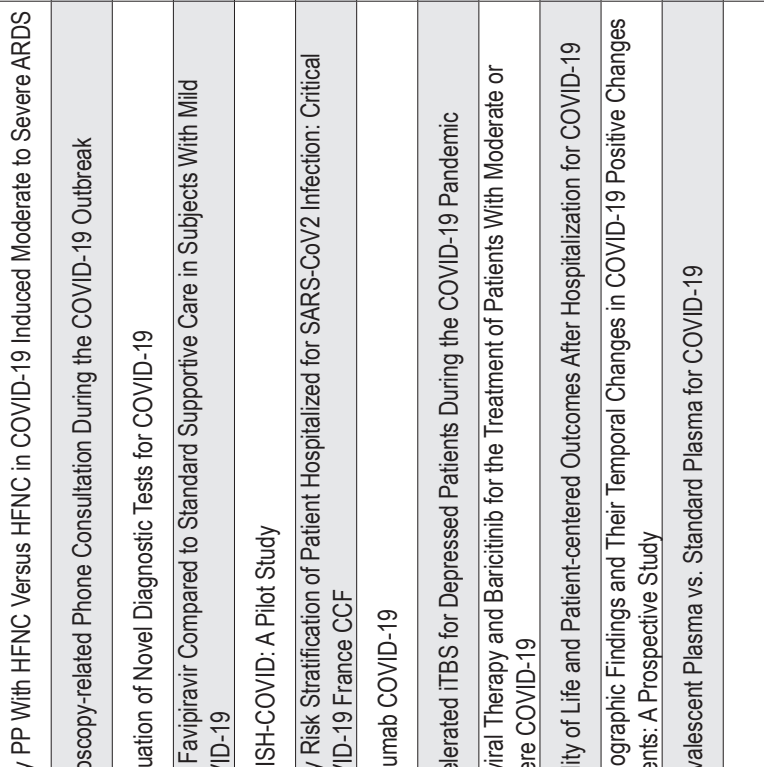

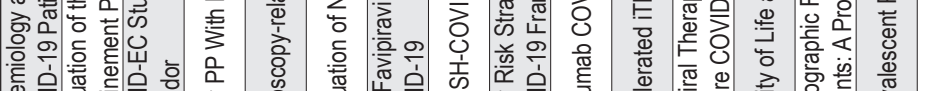

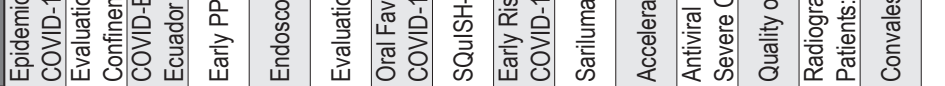

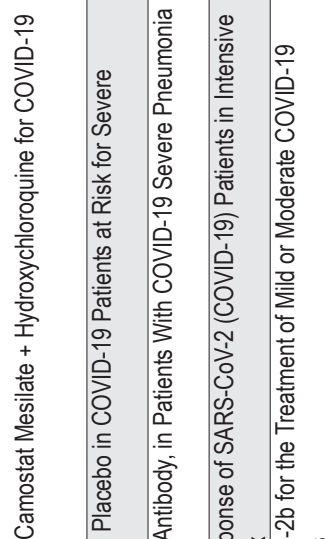

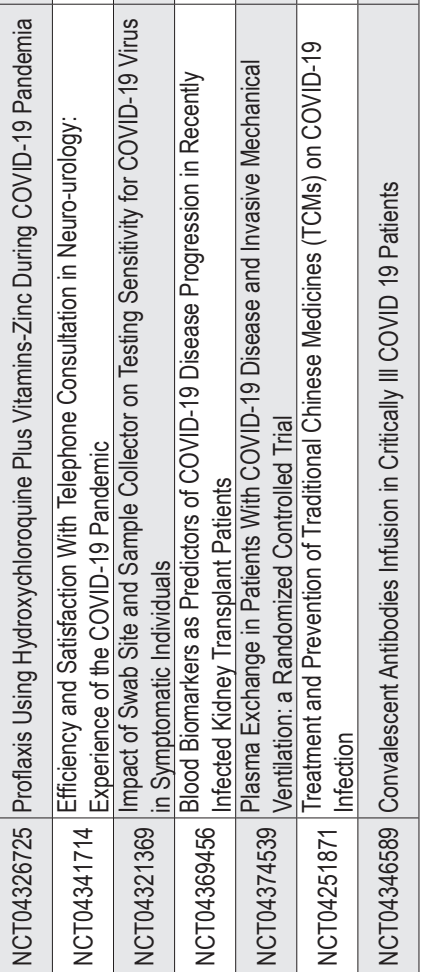




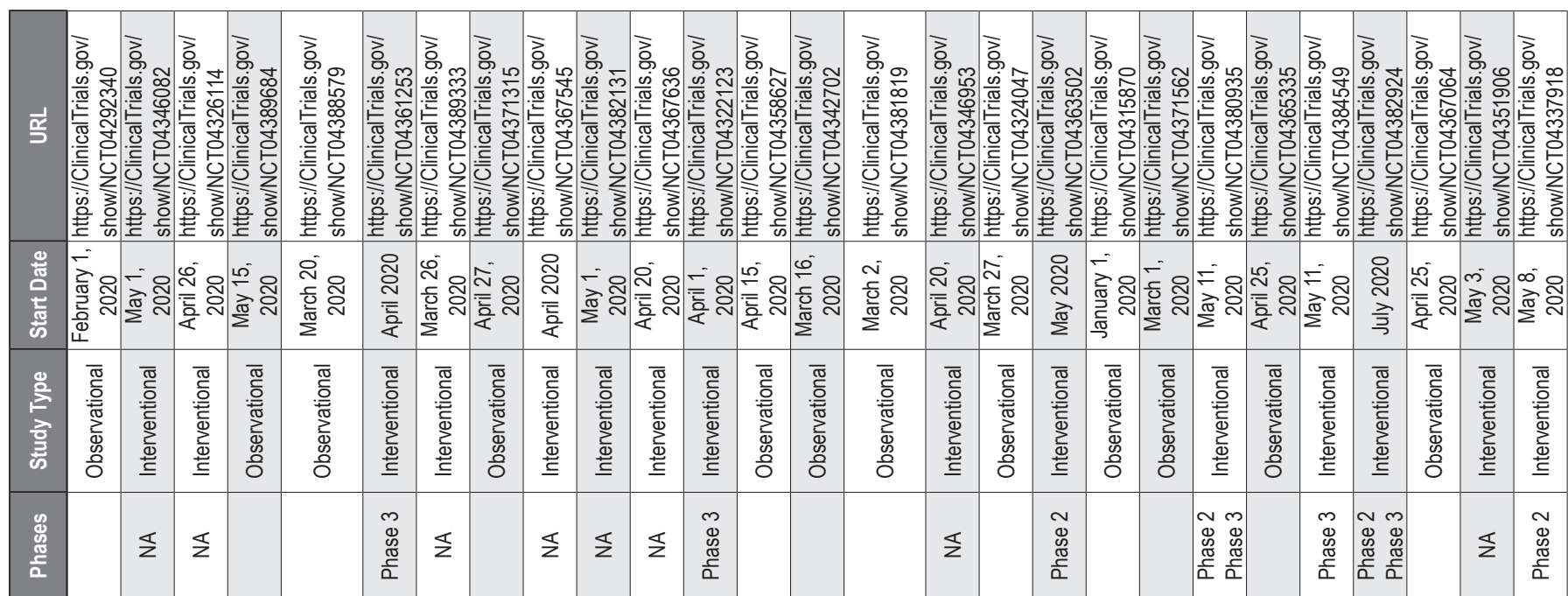

\begin{tabular}{|c|c|c|c|c|c|c|c|c|c|c|c|c|c|c|c|c|c|c|c|c|c|c|c|c|c|}
\hline & 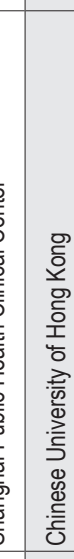 & 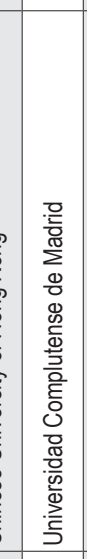 & 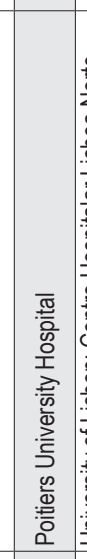 & 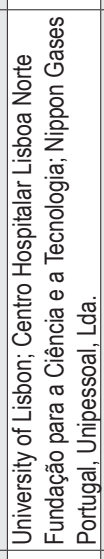 & 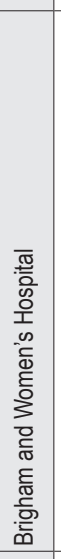 & 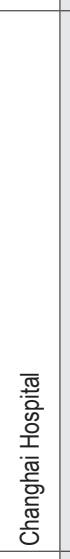 & 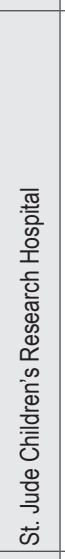 & 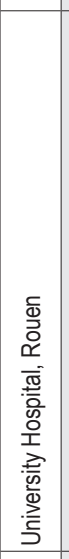 & 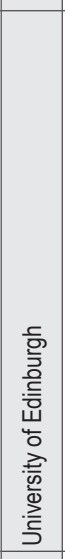 & & & & 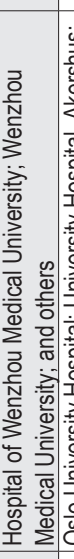 & 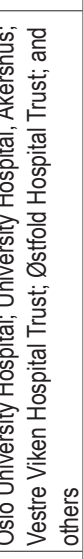 & 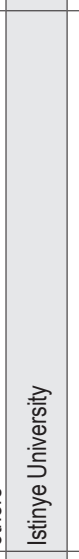 & 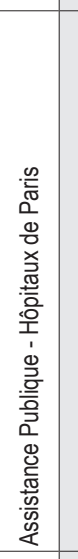 & 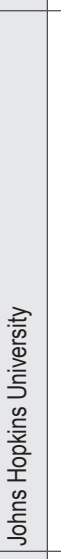 & 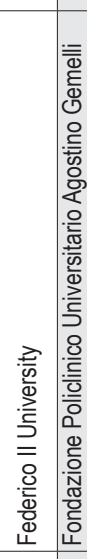 & & & 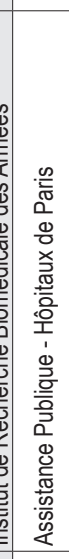 & 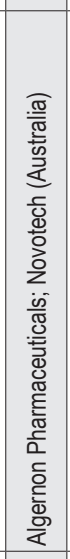 & 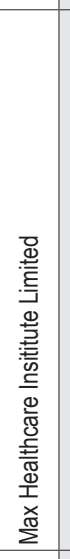 & 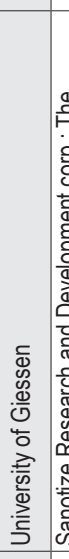 & 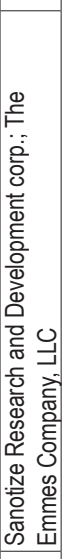 \\
\hline$\frac{\pi}{2}$ & $\frac{5}{2}$ & $\stackrel{\underline{s}}{\underline{z}}$ & $\frac{\widehat{s}}{\frac{\pi}{z}}$ & 妥 & 岸 & $\frac{\bar{r}}{\frac{\pi}{z}}$ & $\frac{\sqrt[3]{z}}{z}$ & 毠 & 妥 & $\frac{\sqrt{s}}{z}$ & $\$$ & $\frac{\vec{s}}{z}$ & $\frac{\widehat{s}}{\frac{\pi}{z}}$ & s & $\frac{\pi}{\frac{\pi}{z}}$ & $\frac{\mathbb{s}}{\frac{\pi}{2}}$ & 妥 & $\frac{\Phi}{\frac{s}{2}}$ & $\$$ & s & 妥 & 妥 & $\frac{\widehat{s}}{\frac{\pi}{z}}$ & $\underset{\frac{s}{z}}{\frac{s}{z}}$ & \\
\hline r & $\frac{\alpha}{\Sigma}$ & $\frac{⿱ 巛 口}{\Sigma}$ & $\stackrel{\mu}{\Sigma}$ & 0 & $\sum_{\Sigma}^{\infty}$ & $\propto$ & $\propto$ & $\propto$ & 些 & $\sum_{\Sigma}^{\mu}$ & $\infty$ & $\frac{\mathbb{N}}{\Sigma}$ & 安䍃 & $\propto$ & $\frac{\alpha}{\Sigma}$ & $\propto$ & $\sum_{\Sigma}^{\alpha}$ & $\propto c$ & u & 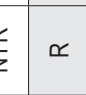 & $\frac{\alpha}{\Sigma}$ & $\frac{\infty}{\Sigma}$ & $\simeq$ & $\propto$ & \\
\hline 妾 & 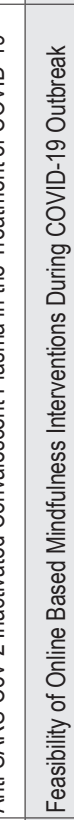 & 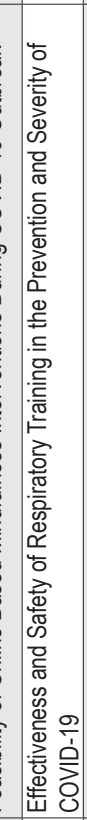 & 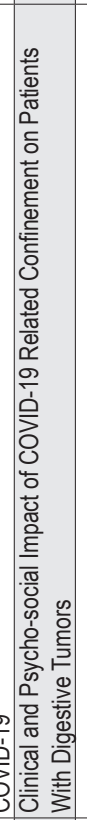 & 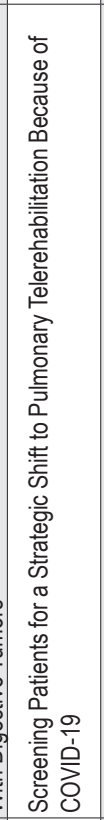 & 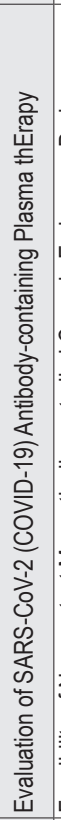 & 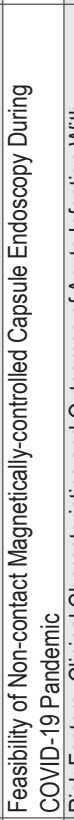 & 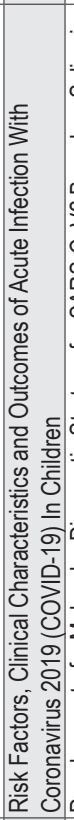 & 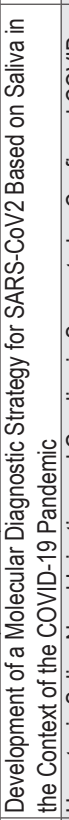 & 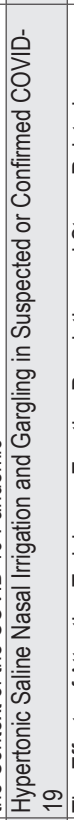 & 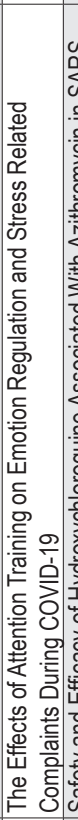 & 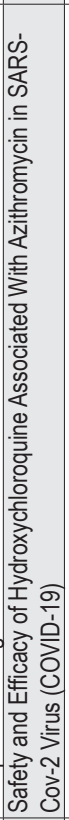 & 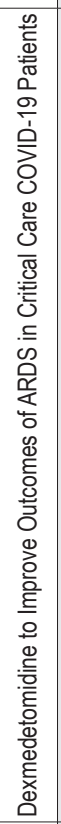 & 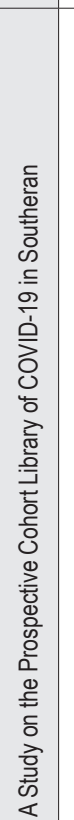 & 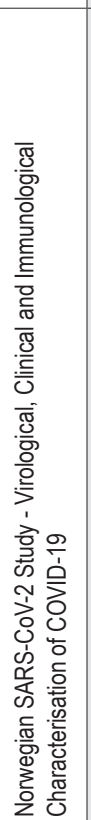 & 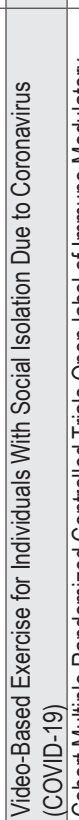 & 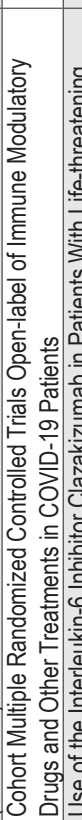 & & 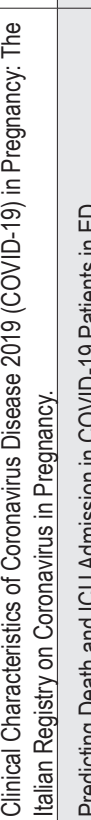 & 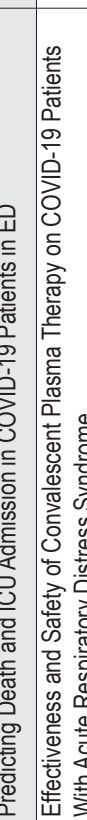 & 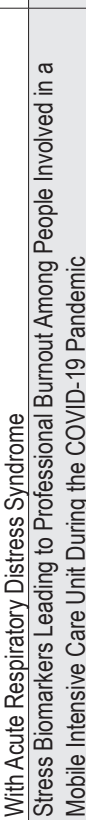 & 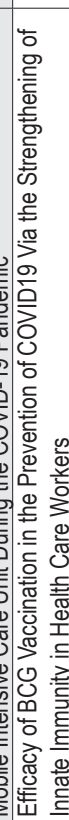 & 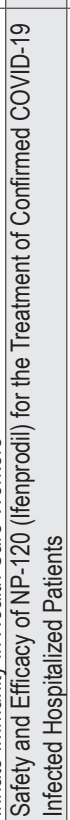 & 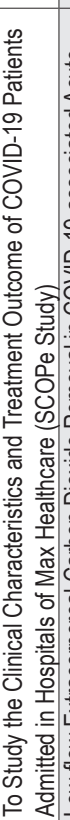 & 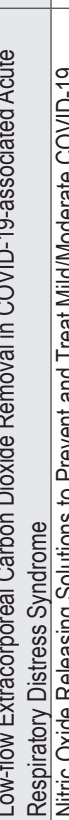 & 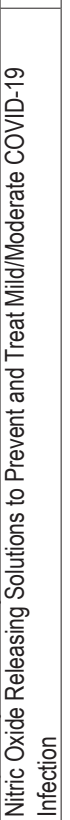 \\
\hline & 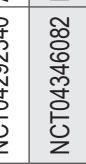 & 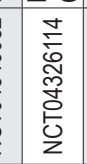 & 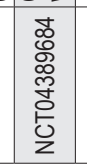 & $\begin{array}{l}\text { 总 } \\
\text { 总 } \\
\text { 总 } \\
z\end{array}$ & 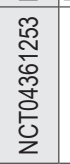 & 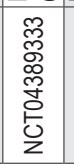 & & 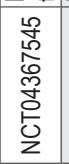 & & 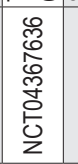 & 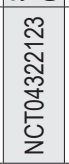 & & ฉั & $\frac{v}{z}$ & 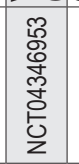 & 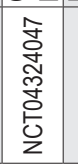 & & 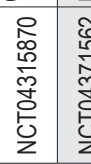 & 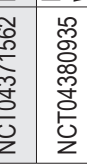 & & 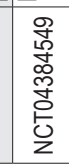 & 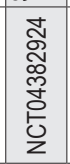 & 畩 & 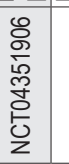 & \\
\hline
\end{tabular}




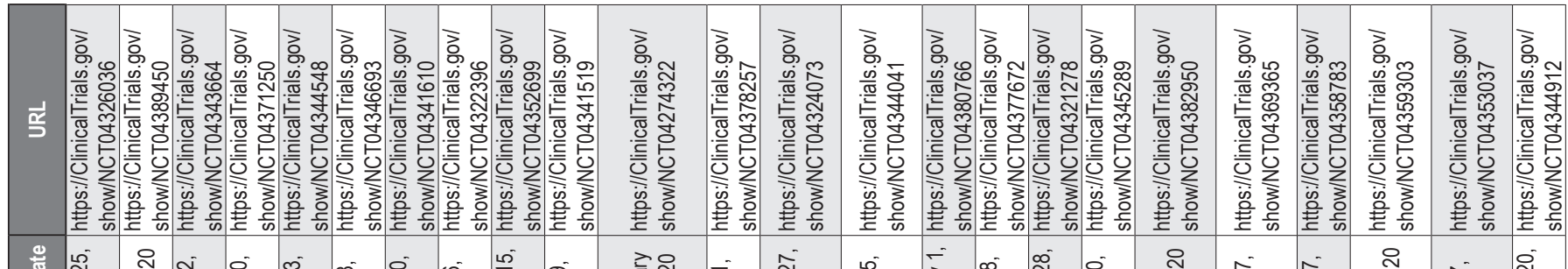

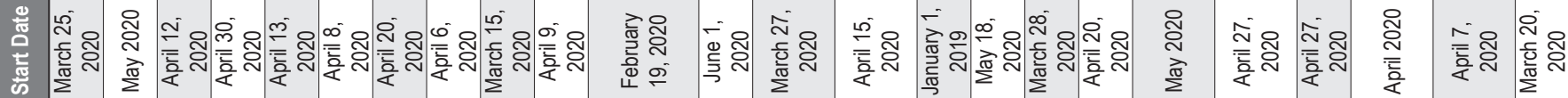

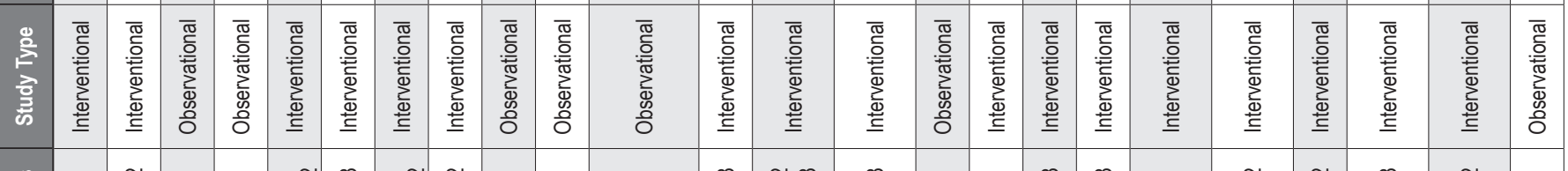

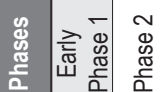

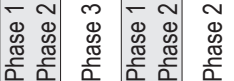

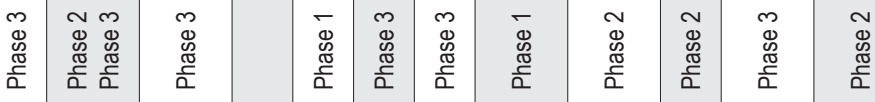

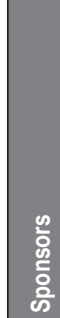

\begin{tabular}{|c|c|c|c|c|c|c|c|c|c|c|c|c|c|c|c|c|c|c|c|c|c|c|}
\hline & 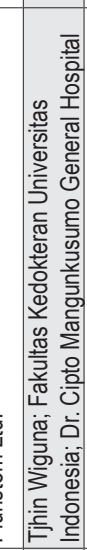 & 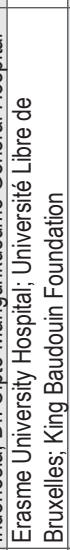 & 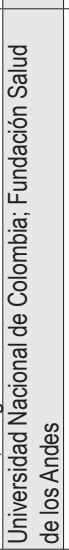 & 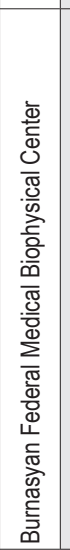 & 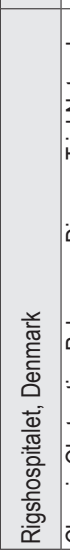 & 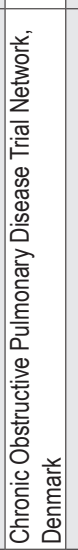 & 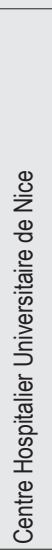 & $\begin{array}{ll} & \\
& \\
& \\
0\end{array}$ & 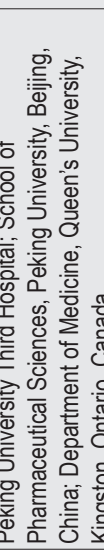 & \begin{tabular}{|l}
$\overline{\bar{z}}$ \\
$\overline{\bar{y}}$ \\
$\bar{\delta}$
\end{tabular} & 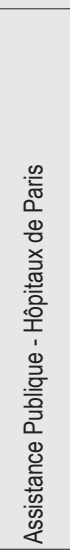 & 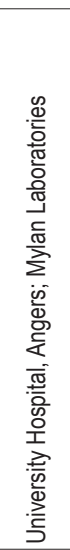 & 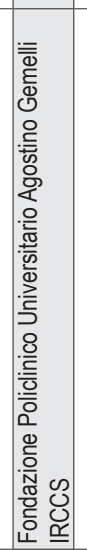 & & 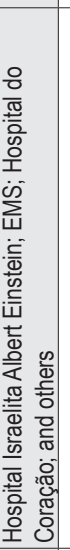 & 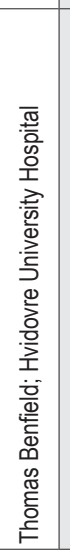 & 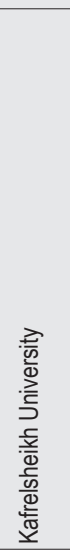 & 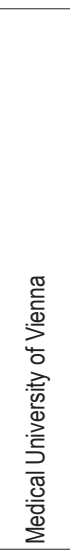 & 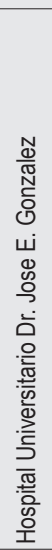 & 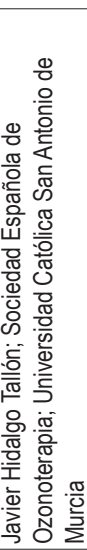 & 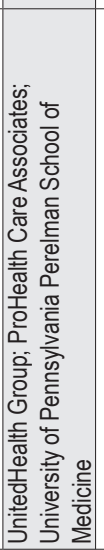 & : \\
\hline & $\$$ & $\frac{\mathbb{s}}{z}$ & $\frac{\mathbb{s}}{\underline{2}}$ & 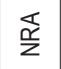 & $\frac{\pi}{\frac{\pi}{z}}$ & (5) & 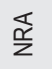 & 蛋 & $\frac{\underline{s}}{z}$ & 2 & $\frac{\sqrt[s]{2}}{\frac{\pi}{2}}$ & $\frac{\widehat{s}}{2}$ & 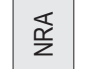 & $\frac{\mathbb{s}}{z}$ & $\frac{\pi}{\frac{5}{2}}$ & $\$$ & $\frac{\pi}{\frac{\pi}{2}}$ & 㞼 & & & \$ & \\
\hline
\end{tabular}

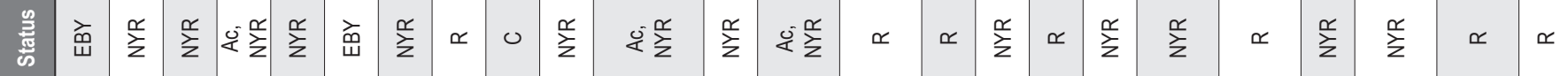

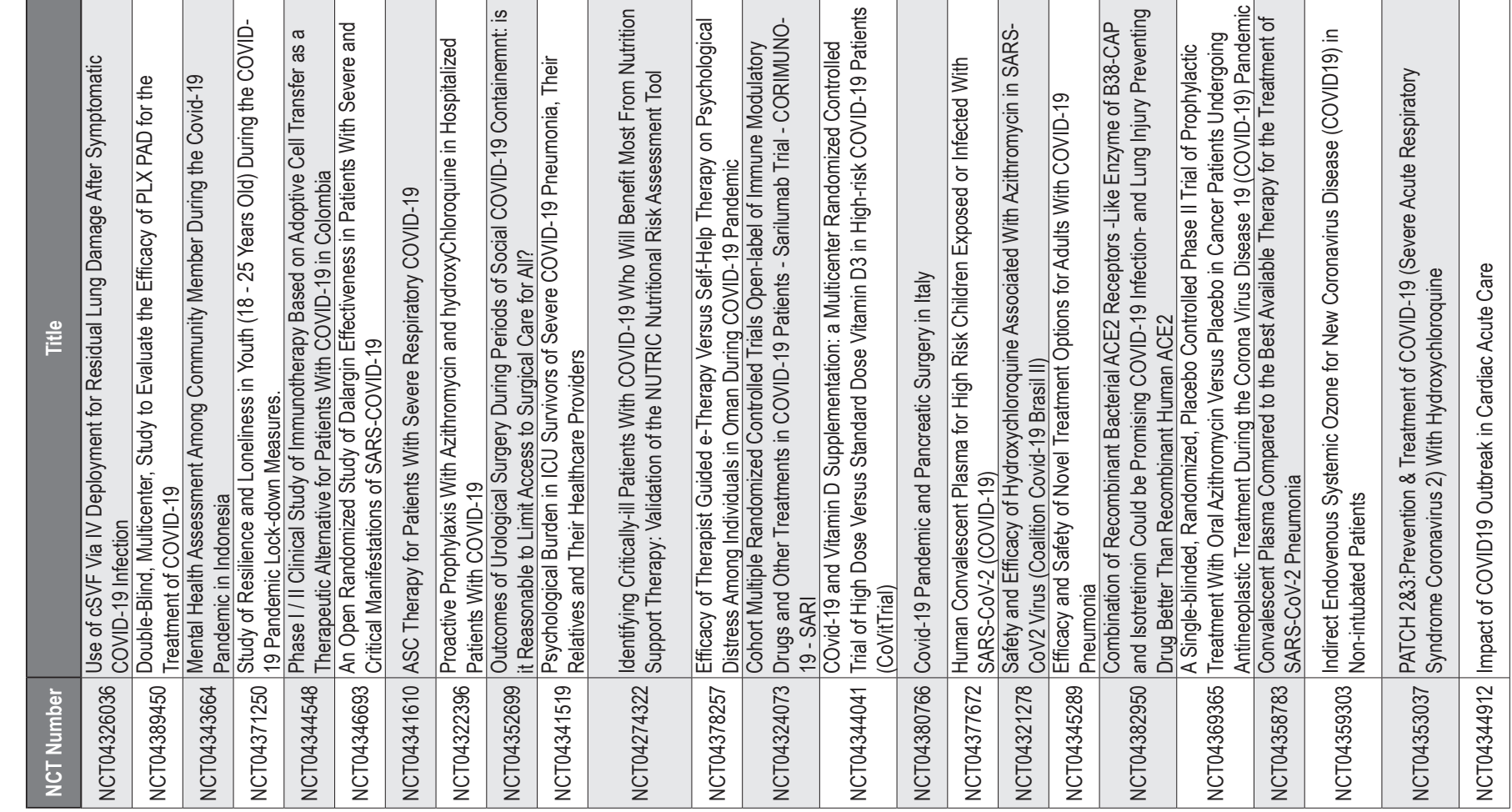




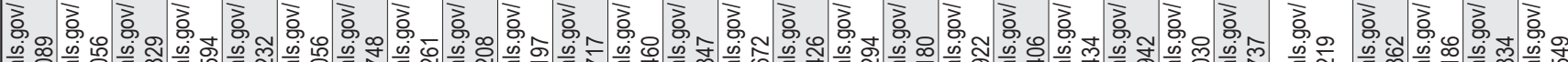

岁

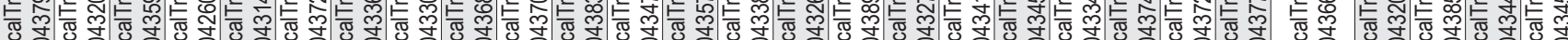

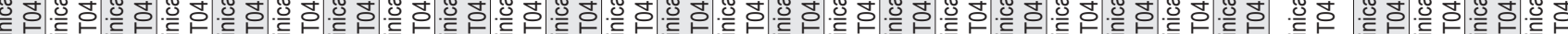

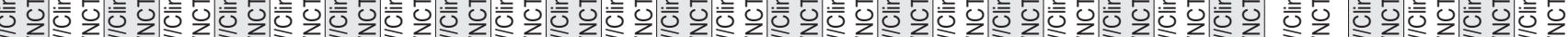

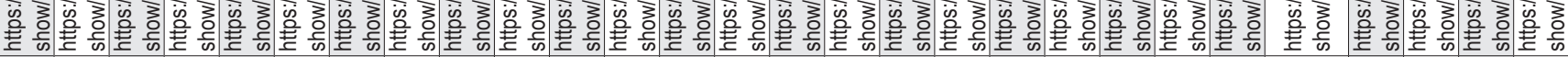

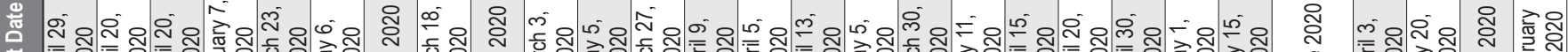

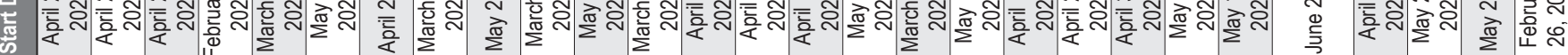

\begin{tabular}{|c|c|c|c|c|c|c|c|c|c|c|c|c|c|c|c|c|c|c|c|c|c|c|c|c|c|c|}
\hline 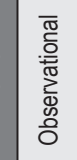 & 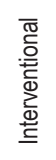 & 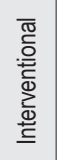 & 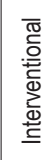 & 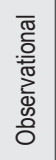 & 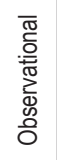 & 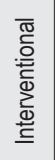 & 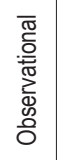 & 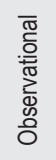 & 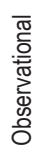 & 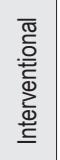 & 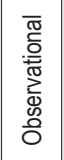 & 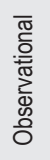 & 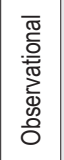 & 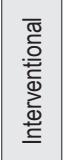 & 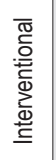 & 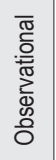 & 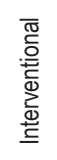 & 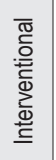 & 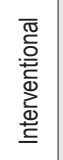 & 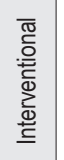 & 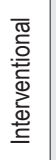 & 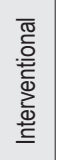 & 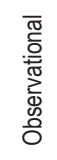 & 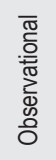 & 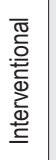 & 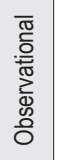 \\
\hline & $\frac{s}{z}$ & $\begin{array}{l}\widetilde{N} \\
\mathbb{\infty} \\
\frac{\pi}{\alpha} \\
\frac{0}{\alpha}\end{array}$ & 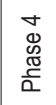 & & & $\begin{array}{l}0 \\
0 \\
心 \\
\frac{0}{\alpha} \\
\frac{\pi}{\alpha}\end{array}$ & & & & $\begin{array}{l}\infty \\
\mathbb{8} \\
\mathbb{\pi} \\
\frac{\pi}{\alpha} \\
\end{array}$ & & & & \begin{tabular}{|l}
$\infty$ \\
$\Phi$ \\
$\Phi$ \\
$\frac{\pi}{0}$ \\
$\alpha$
\end{tabular} & $\Sigma$ & & $\Sigma$ & $\begin{array}{l}m_{0} \\
\mathbb{8} \\
\frac{\pi}{\alpha} \\
\frac{\pi}{2}\end{array}$ & $\Sigma$ & 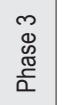 & $\frac{\mathbb{s}}{z}$ & $\Sigma$ & & & 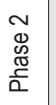 & \\
\hline
\end{tabular}

$\frac{2}{2}$

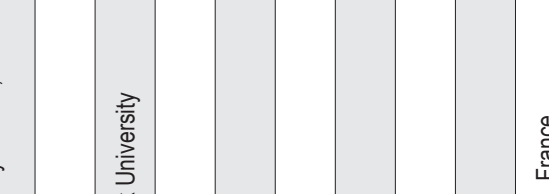

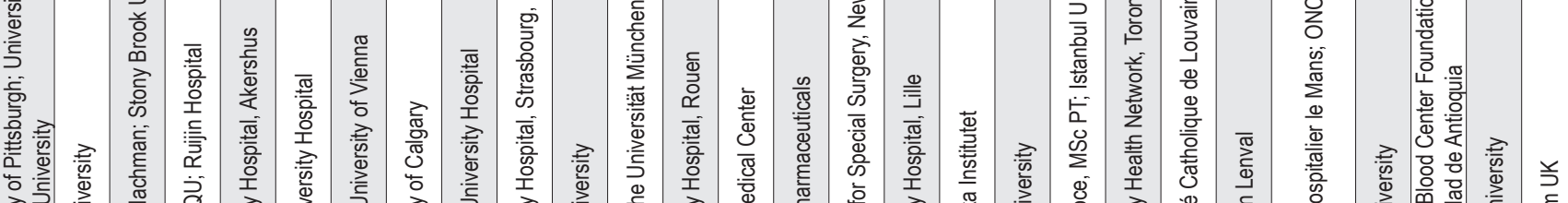

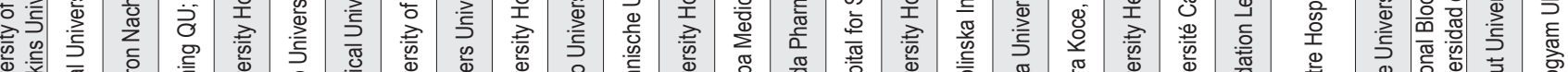

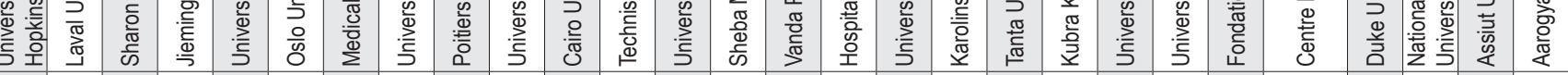

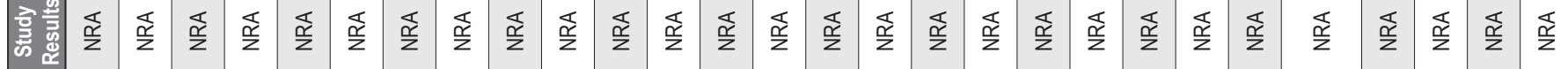

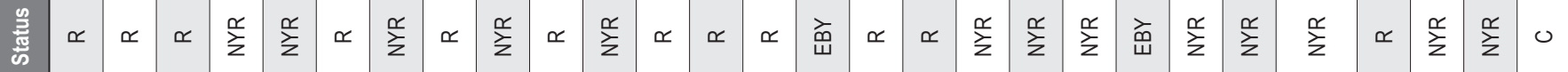

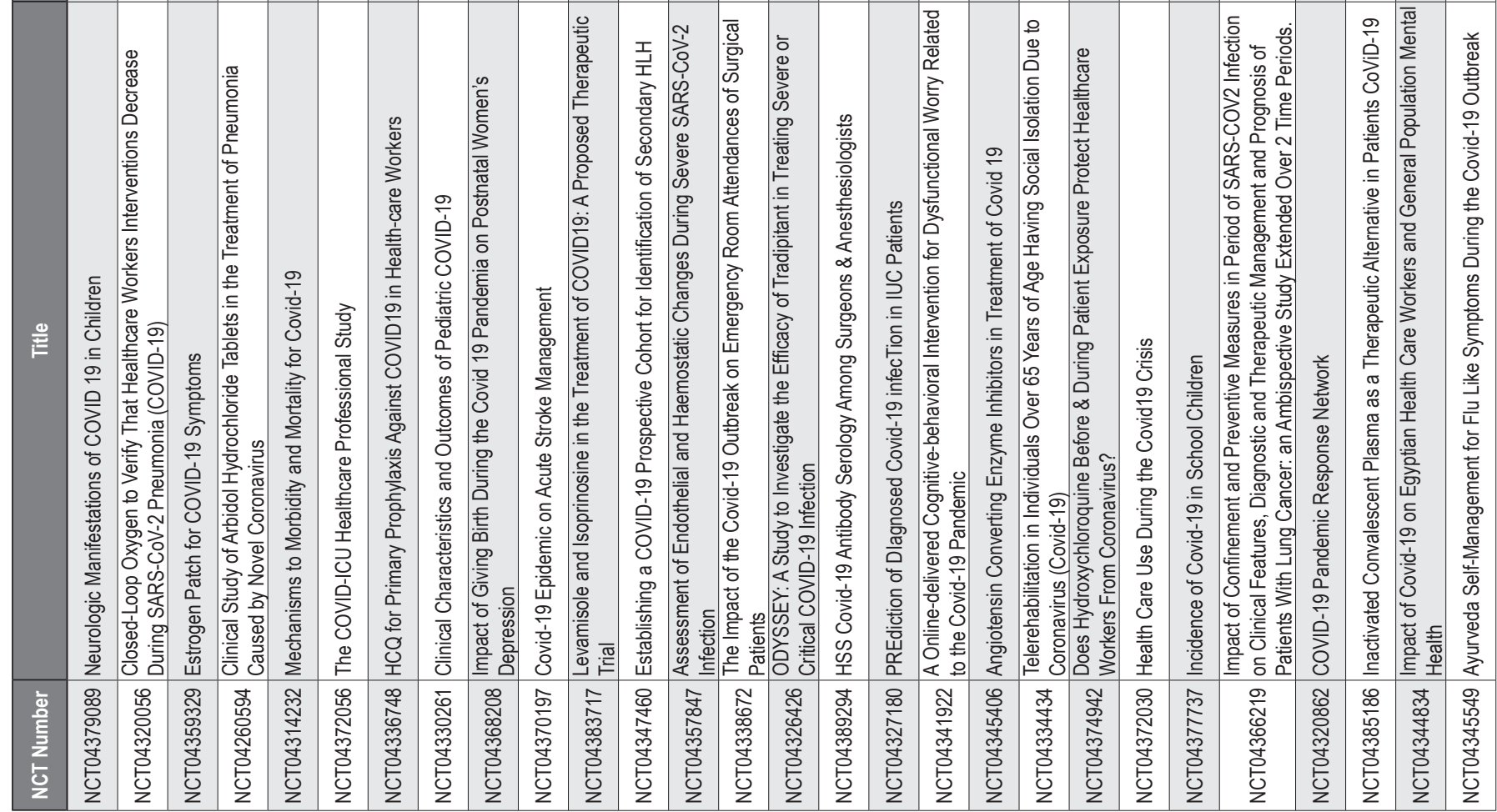




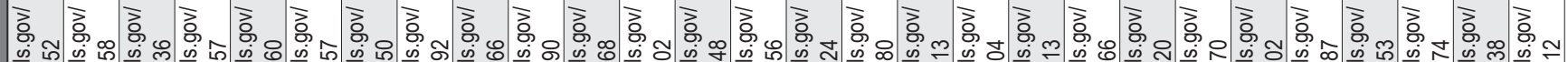

呫

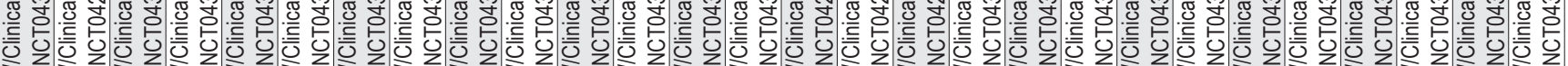

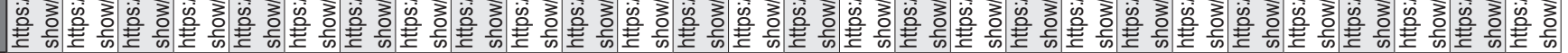

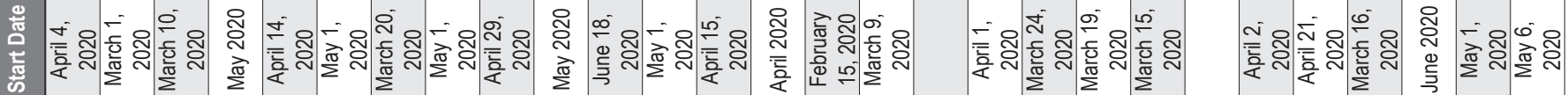

\begin{tabular}{|c|c|c|c|c|c|c|c|c|c|c|c|c|c|c|c|c|c|c|c|c|c|c|c|c|c|c|c|}
\hline 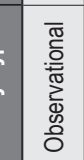 & 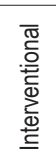 & 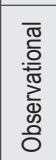 & 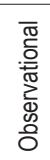 & 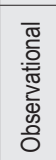 & 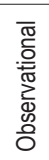 & 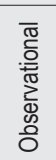 & 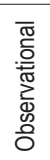 & 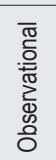 & 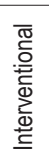 & 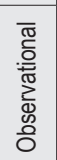 & 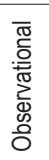 & 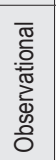 & 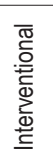 & 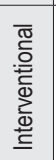 & 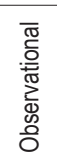 & $\stackrel{\varrho}{F}$ & 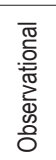 & 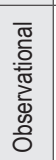 & 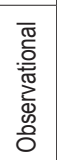 & 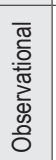 & $\underline{\underline{0}}$ & 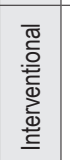 & 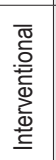 & 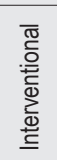 & 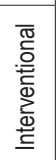 & 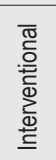 & 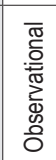 \\
\hline & 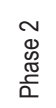 & & & & & & & & $\begin{array}{l}\text { o } \\
\text { o } \\
\frac{\pi}{\alpha}\end{array}$ & & & & $\underline{z}$ & $\begin{array}{l}\mathscr{8} \\
\mathbb{\pi} \\
\frac{\pi}{\alpha}\end{array}$ & & & & & & & & $\begin{array}{l}\sim \\
\mathbb{8} \\
\mathbb{\pi} \\
\frac{\pi}{\alpha}\end{array}$ & 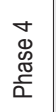 & 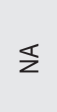 & 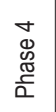 & $\underline{z}$ & \\
\hline
\end{tabular}

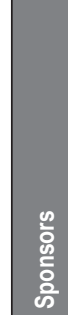

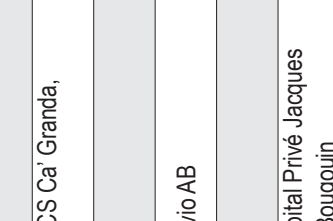

के

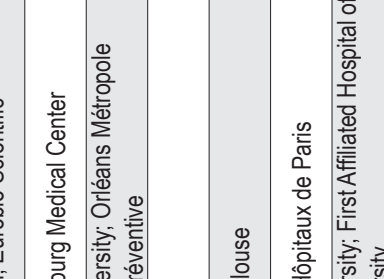

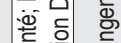

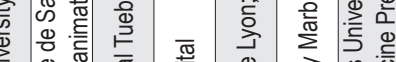

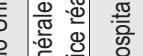

竞

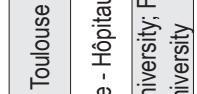

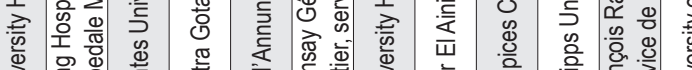

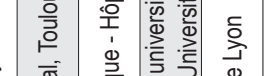

范

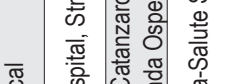

T)

焉

离产

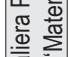

:

荧

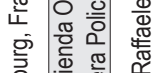

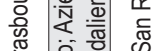

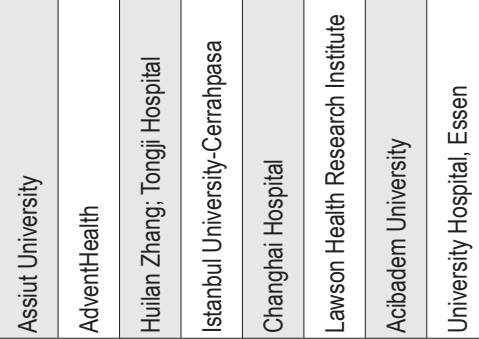

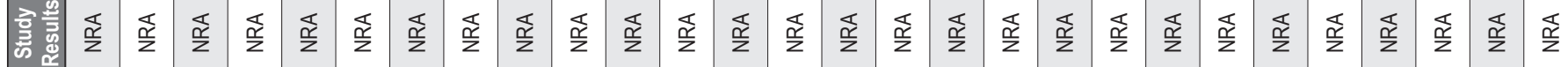

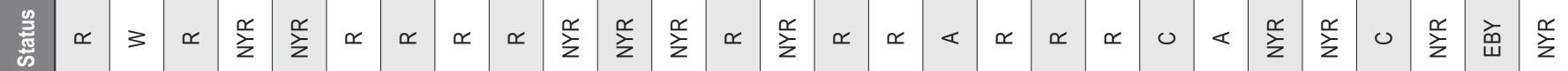

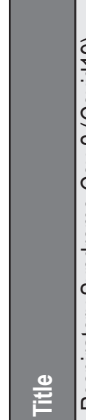

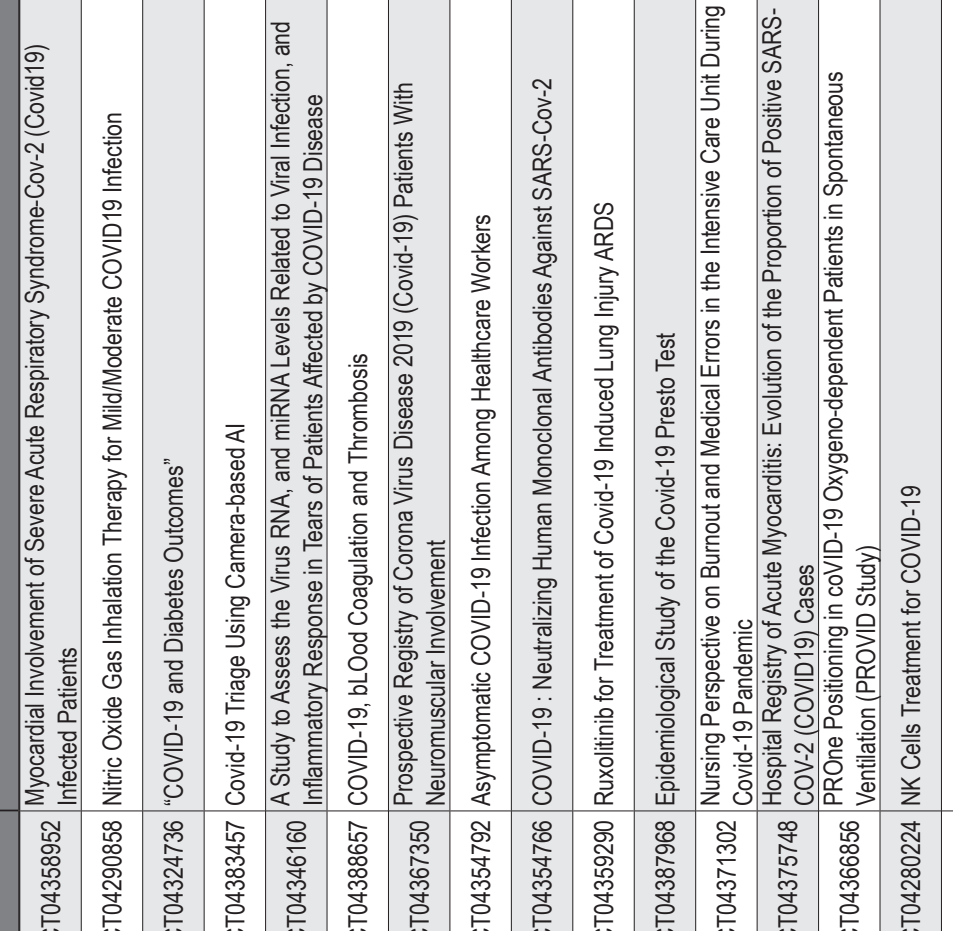

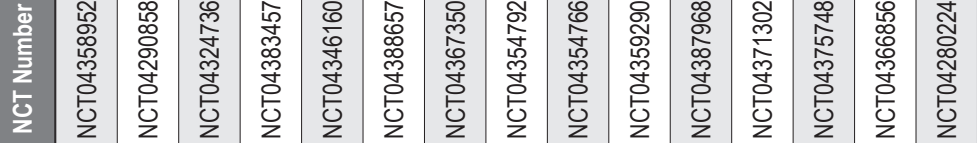

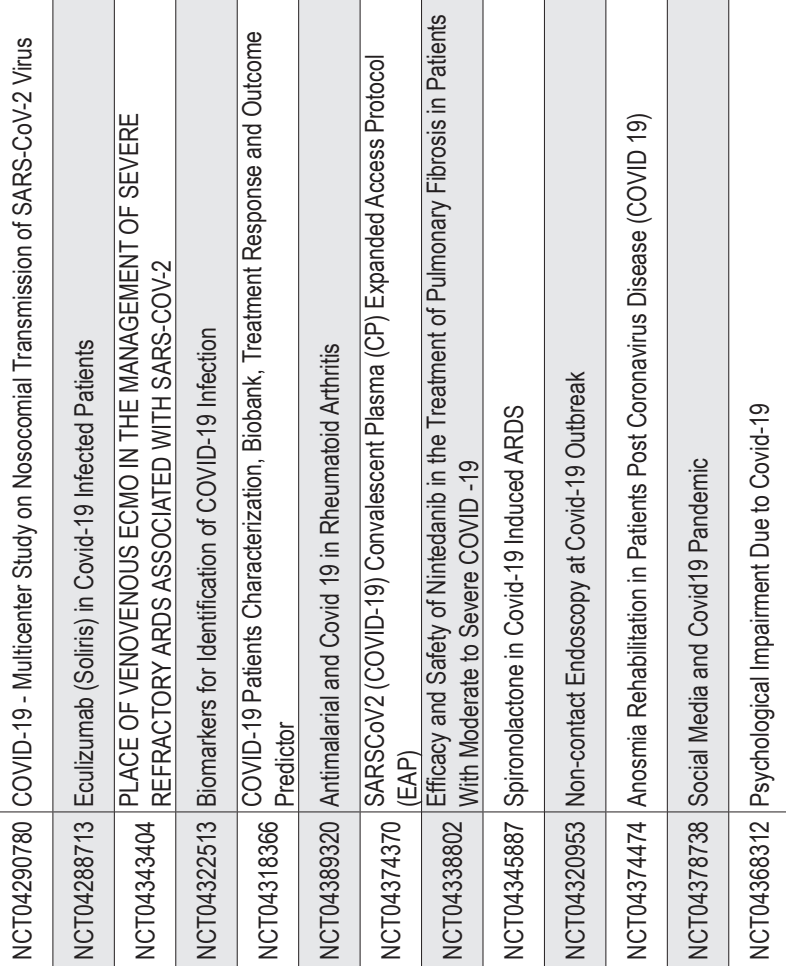




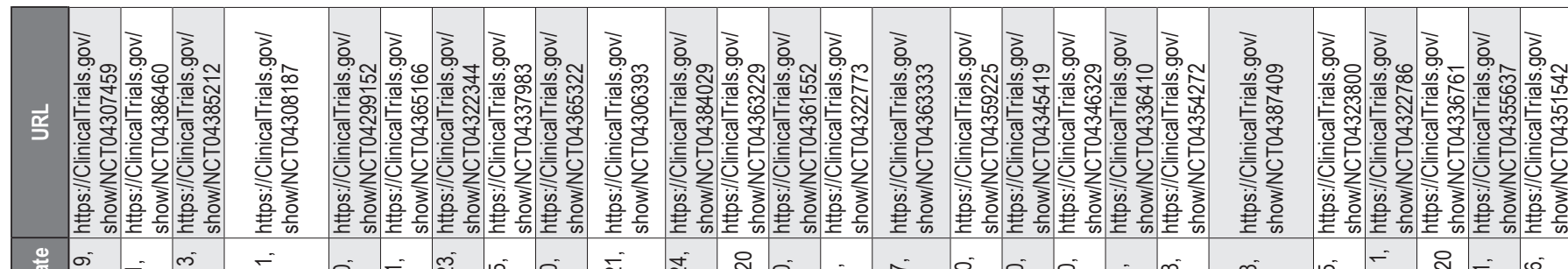

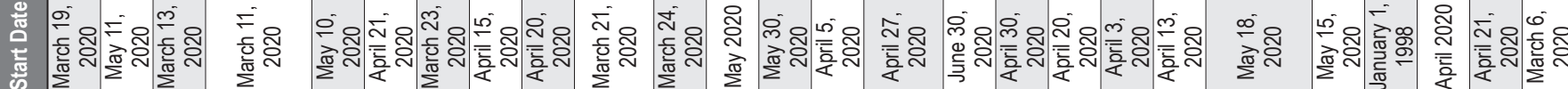

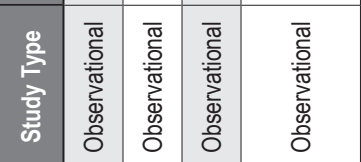

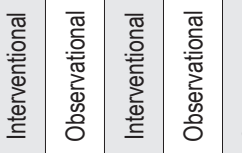

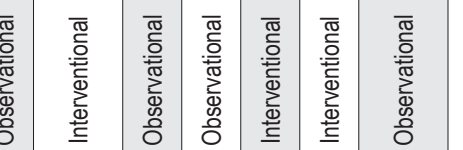

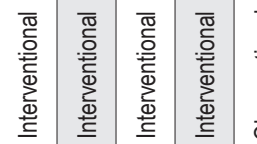

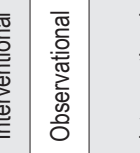

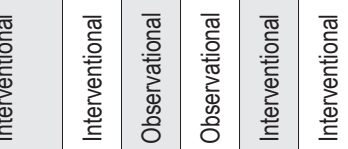

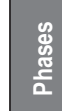

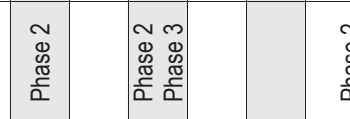

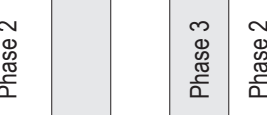

惫
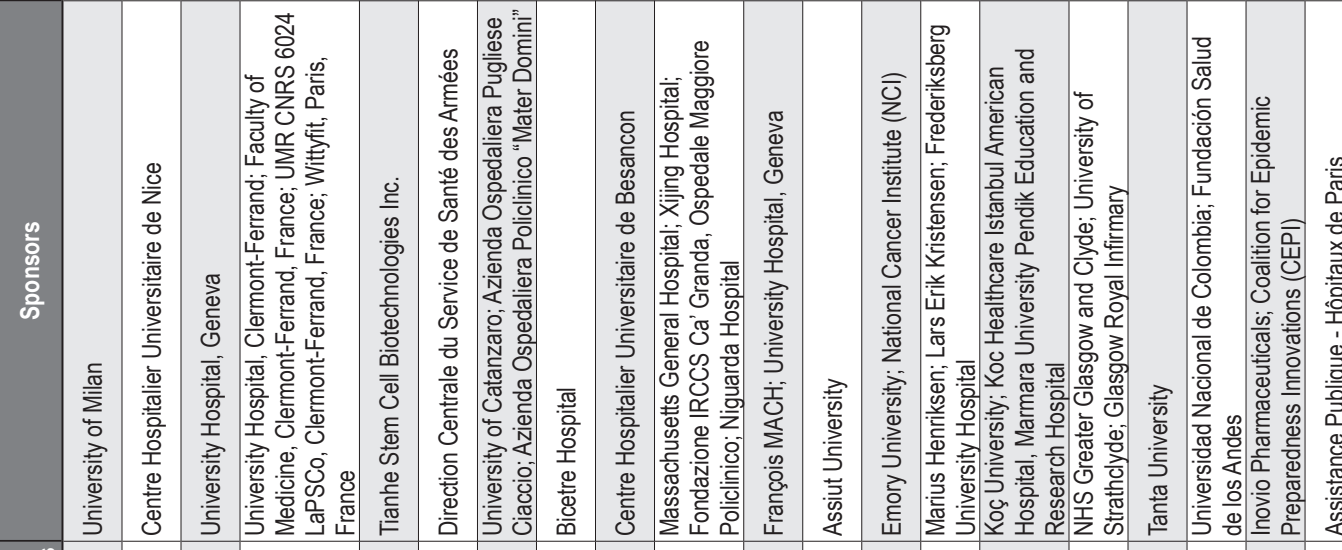

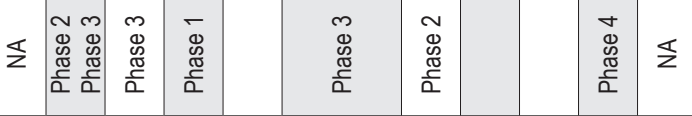

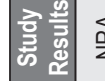

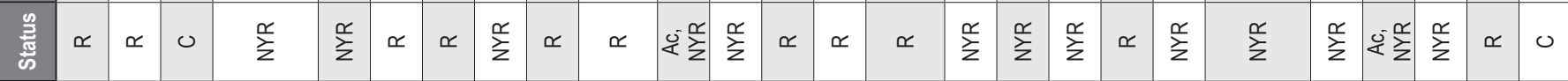

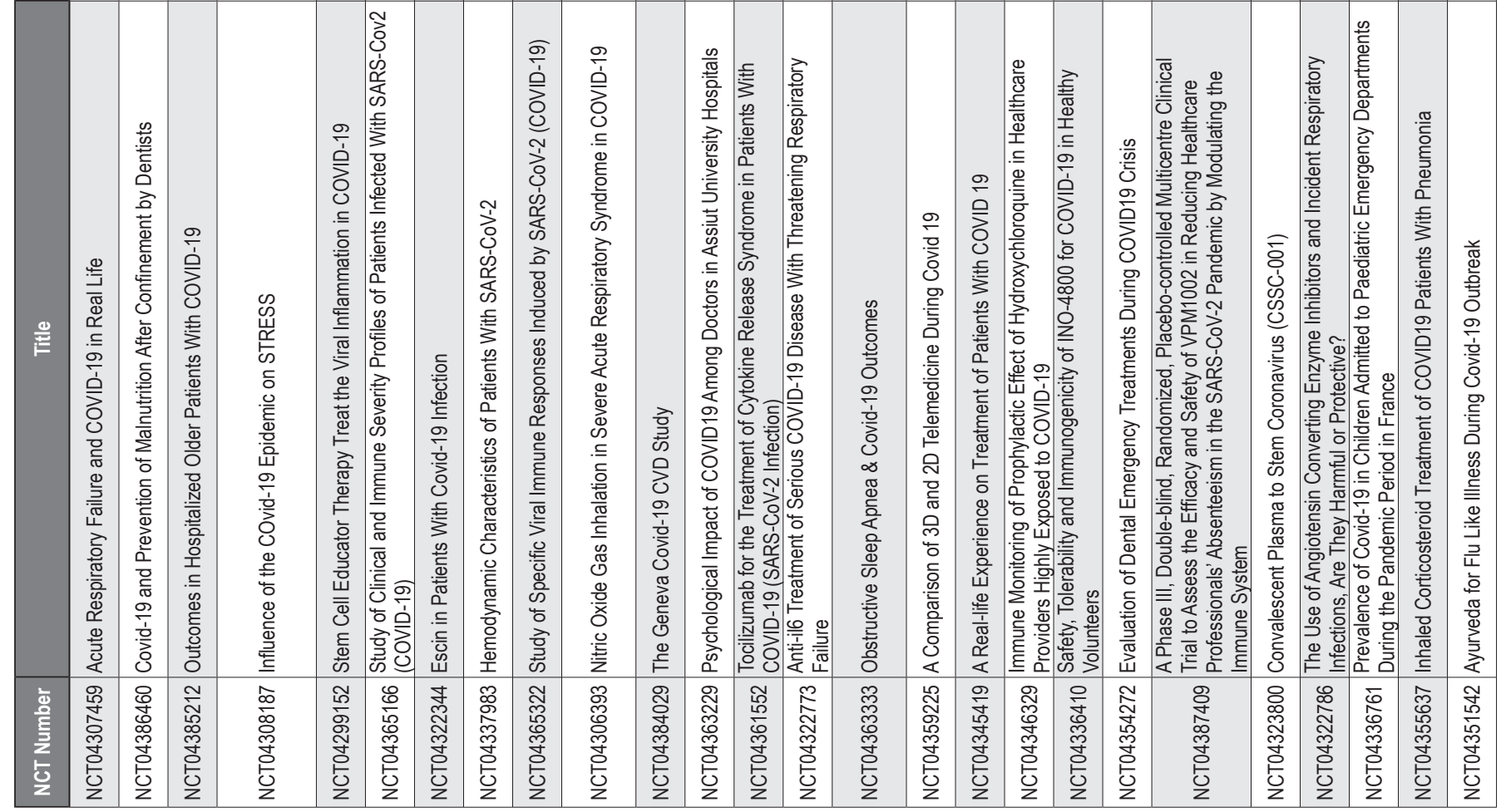




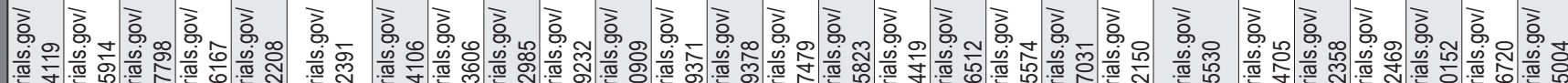

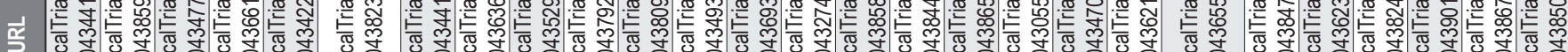

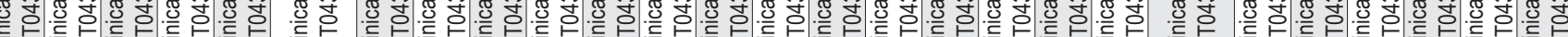

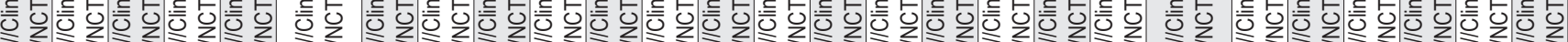

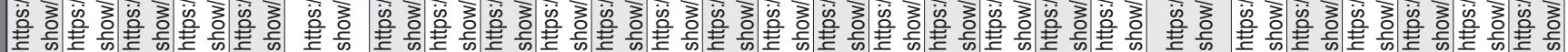

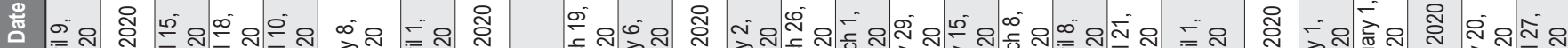

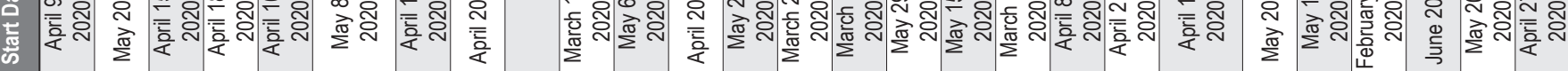

\begin{tabular}{|c|c|c|c|c|c|c|c|c|c|c|c|c|c|c|c|c|c|c|c|c|c|c|c|c|c|c|}
\hline $\begin{array}{l}\overline{\widetilde{\sigma}} \\
\text { 总 }\end{array}$ & 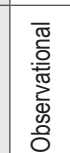 & 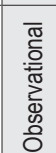 & 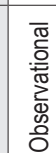 & 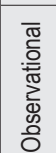 & 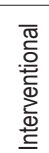 & 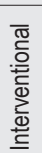 & 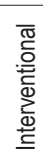 & $\underline{0}$ & 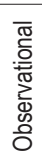 & 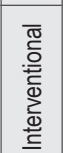 & 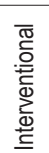 & 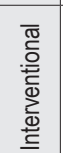 & 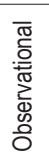 & 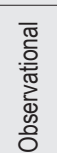 & 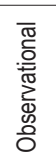 & 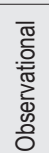 & 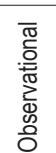 & 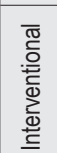 & 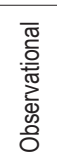 & 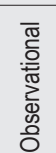 & 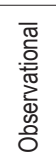 & 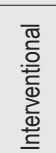 & 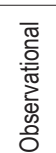 & 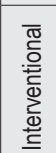 & 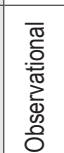 & $\mid$\begin{tabular}{|l}
$\overline{\widetilde{g}}$ \\
흠
\end{tabular} \\
\hline
\end{tabular}

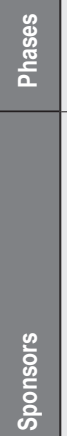

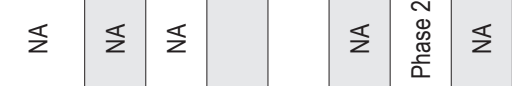

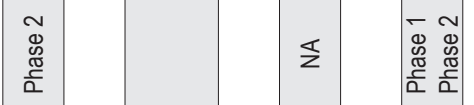

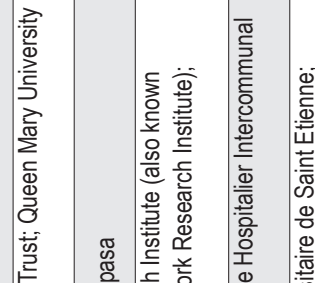

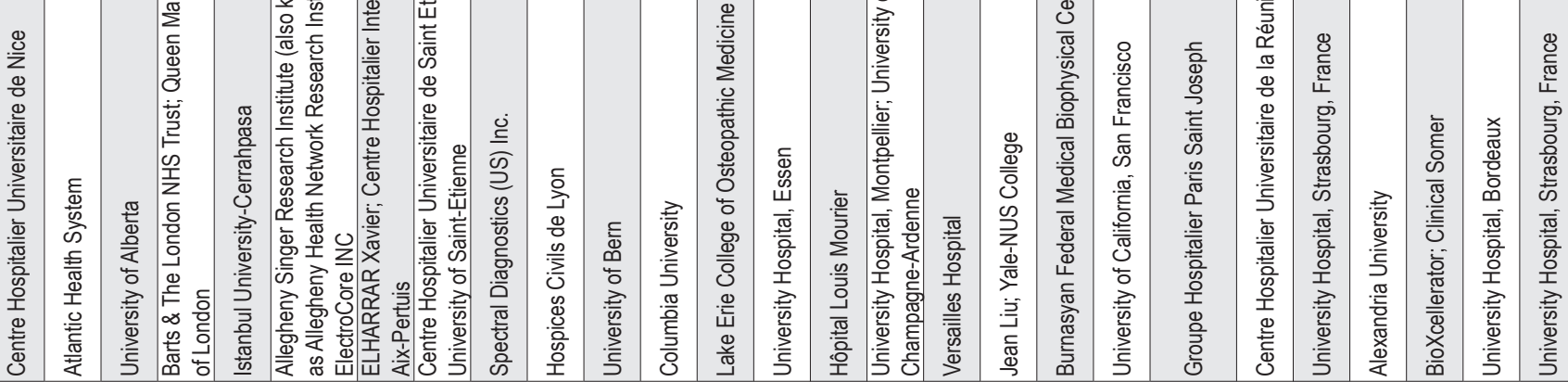

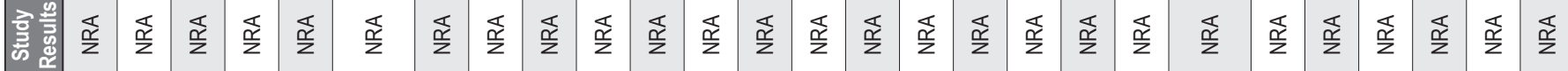

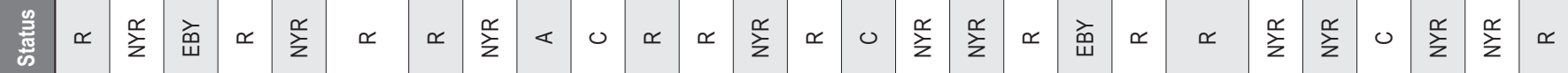

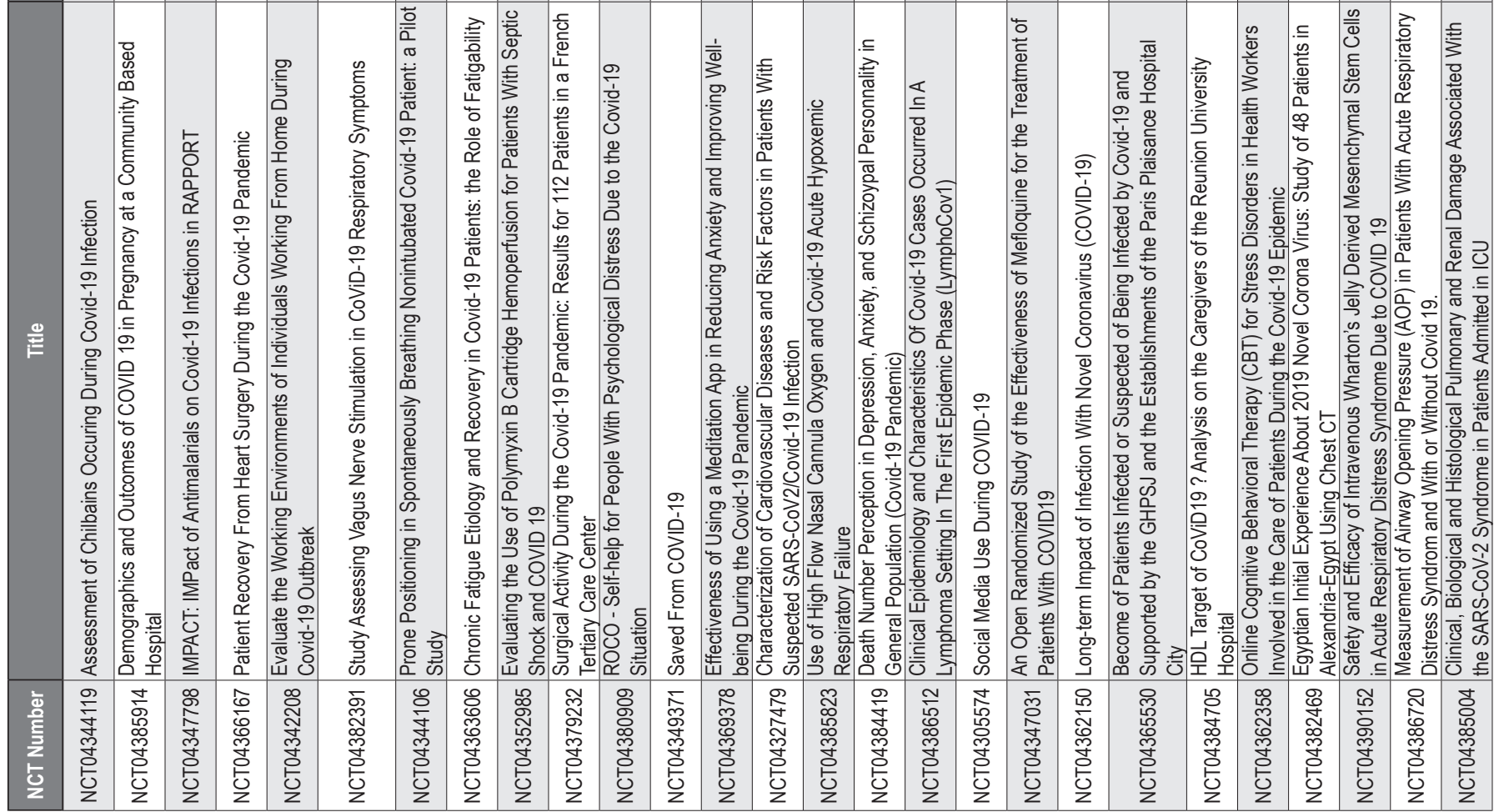




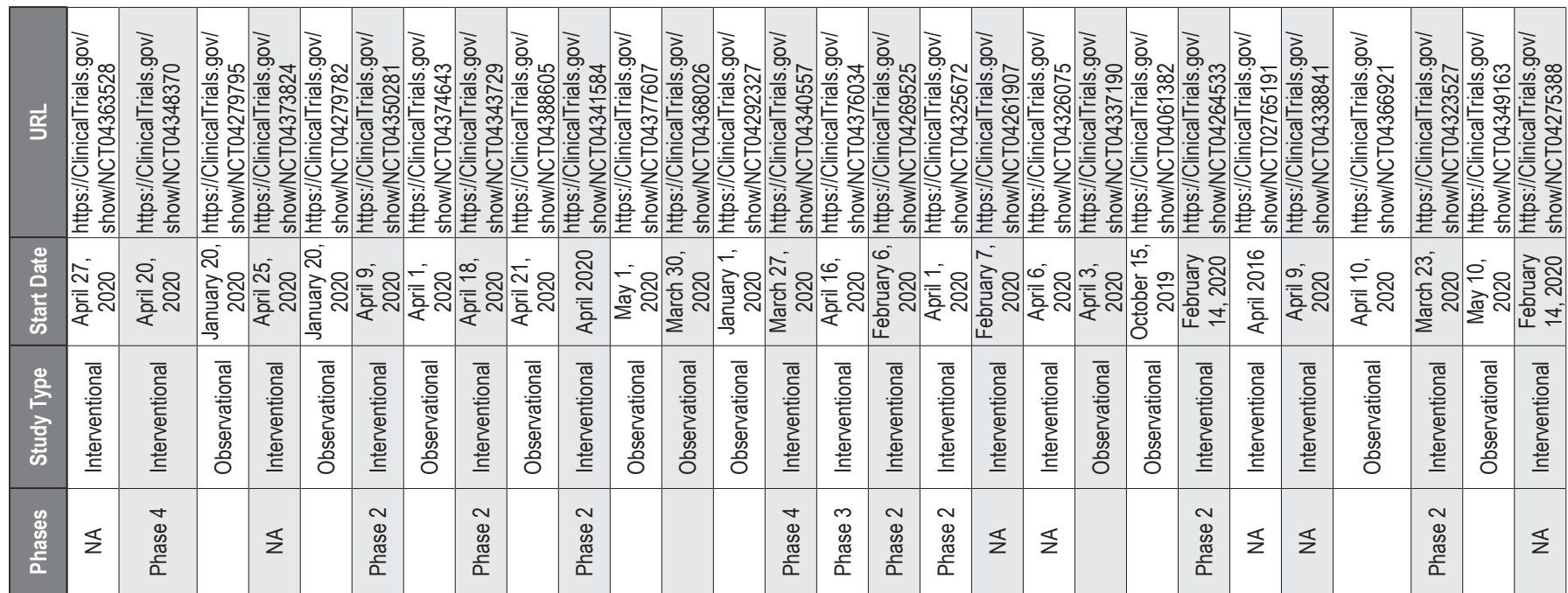

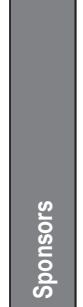

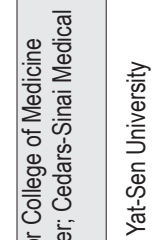

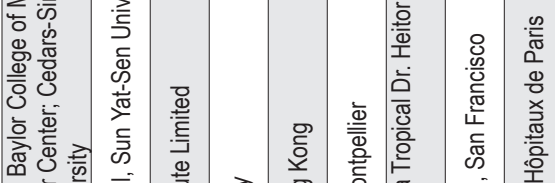

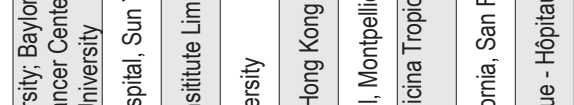

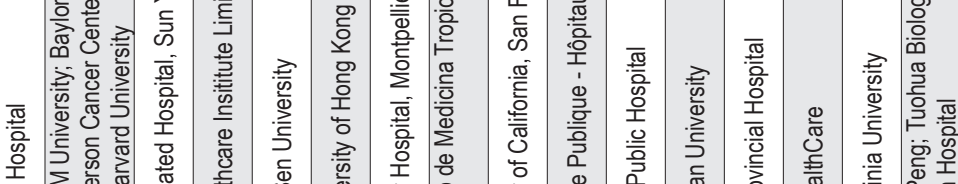

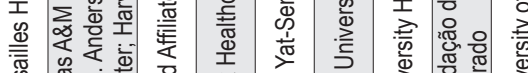

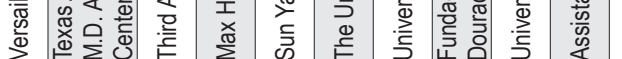

The

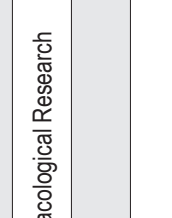

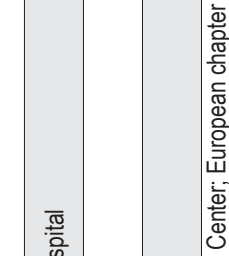

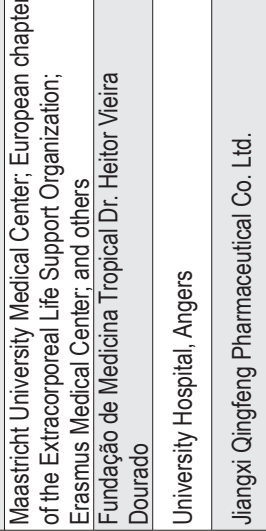

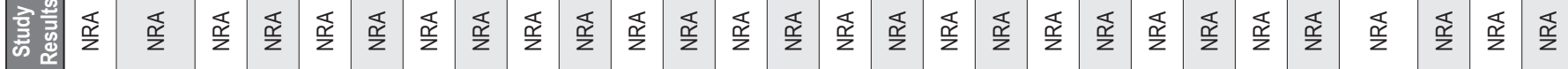

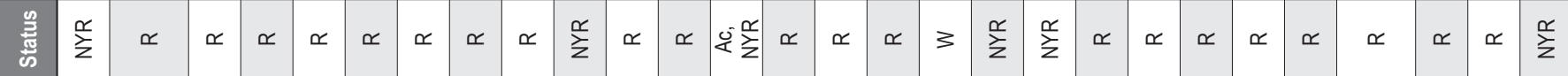

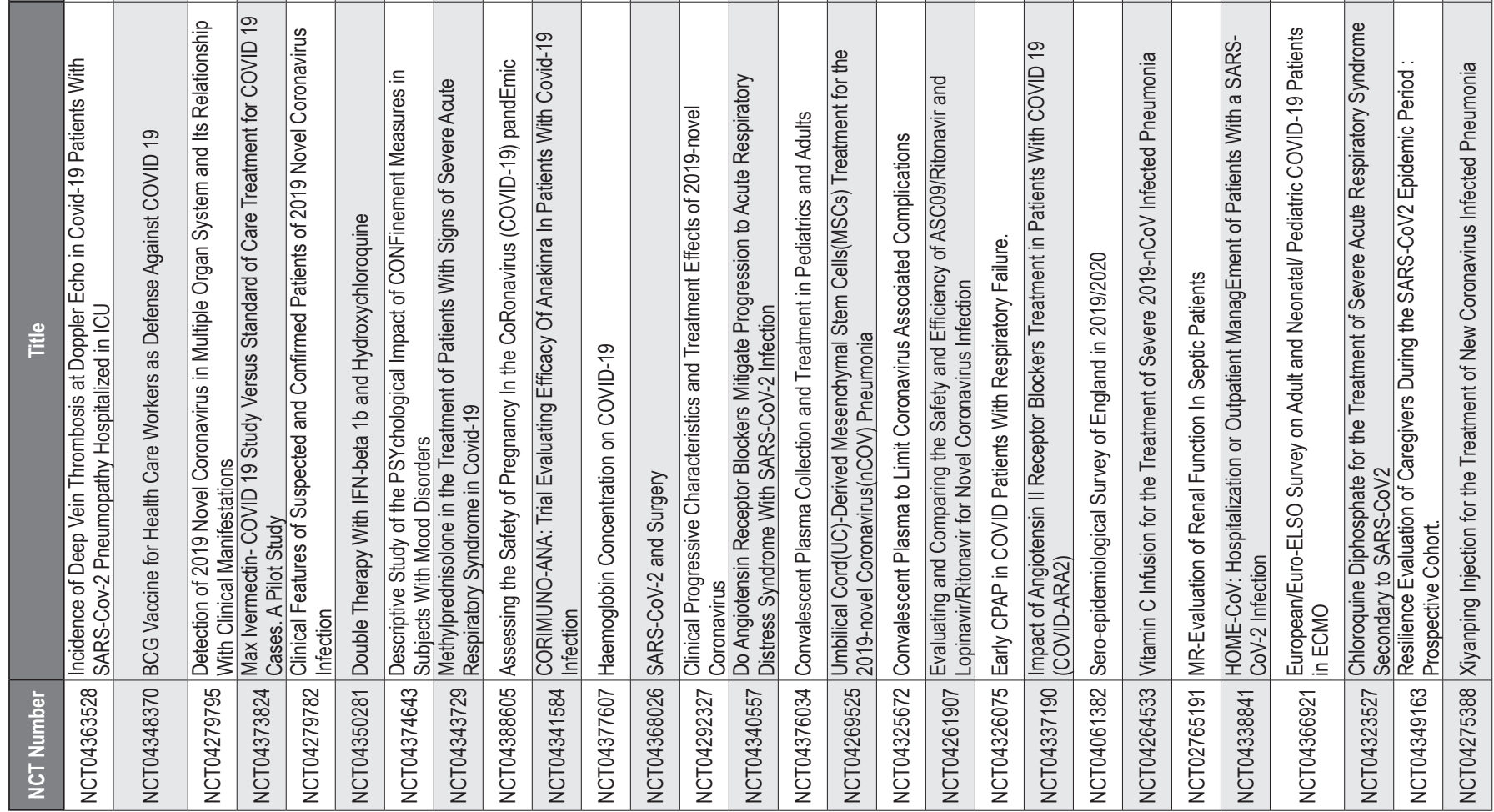




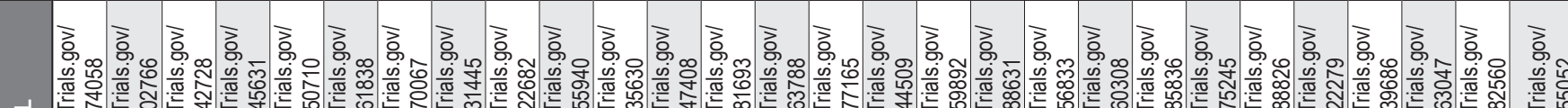 然 吾

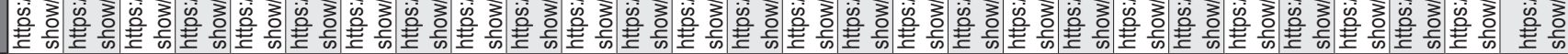

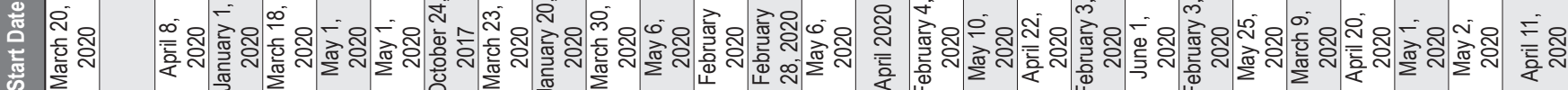

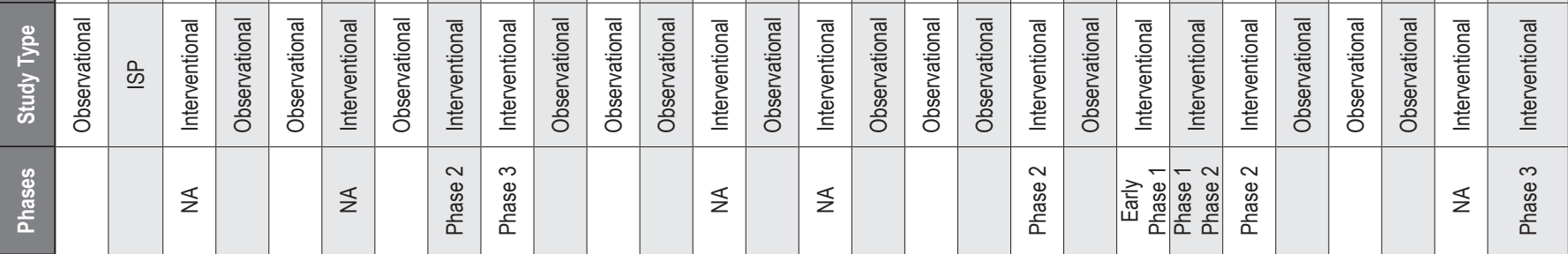

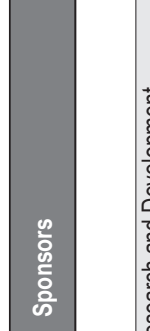

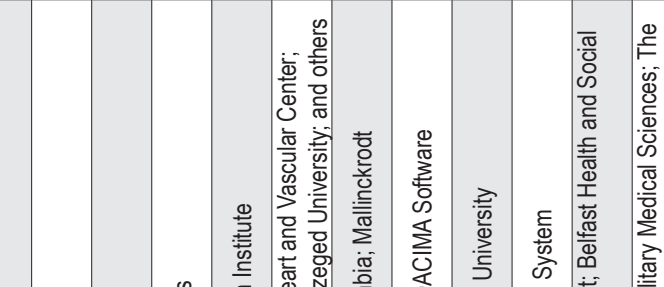
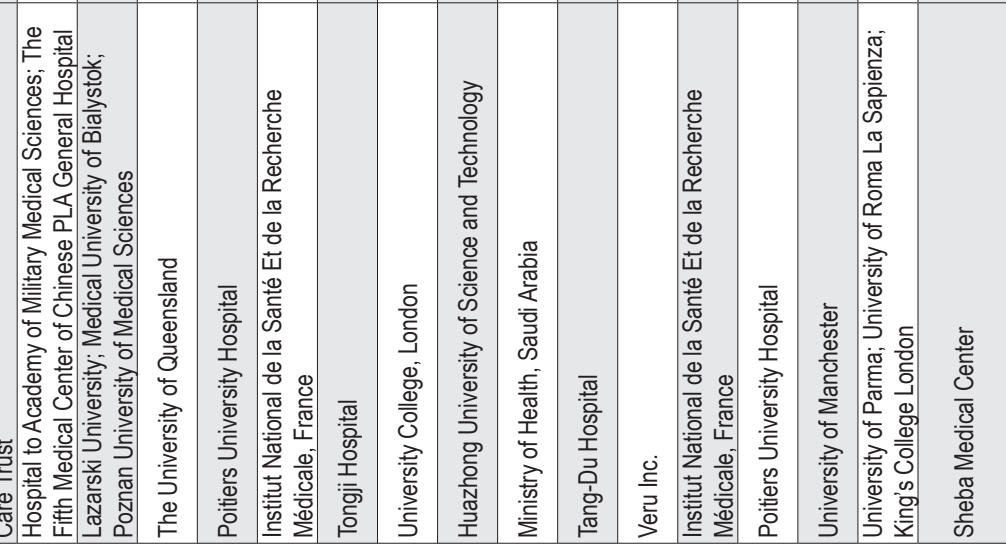

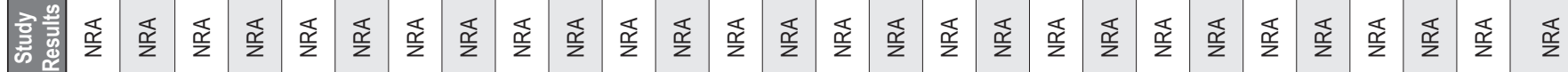

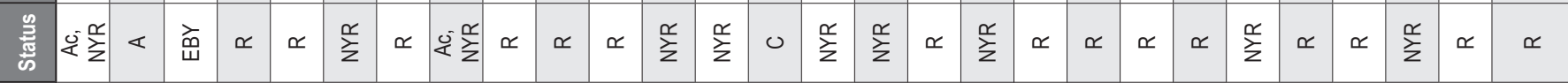

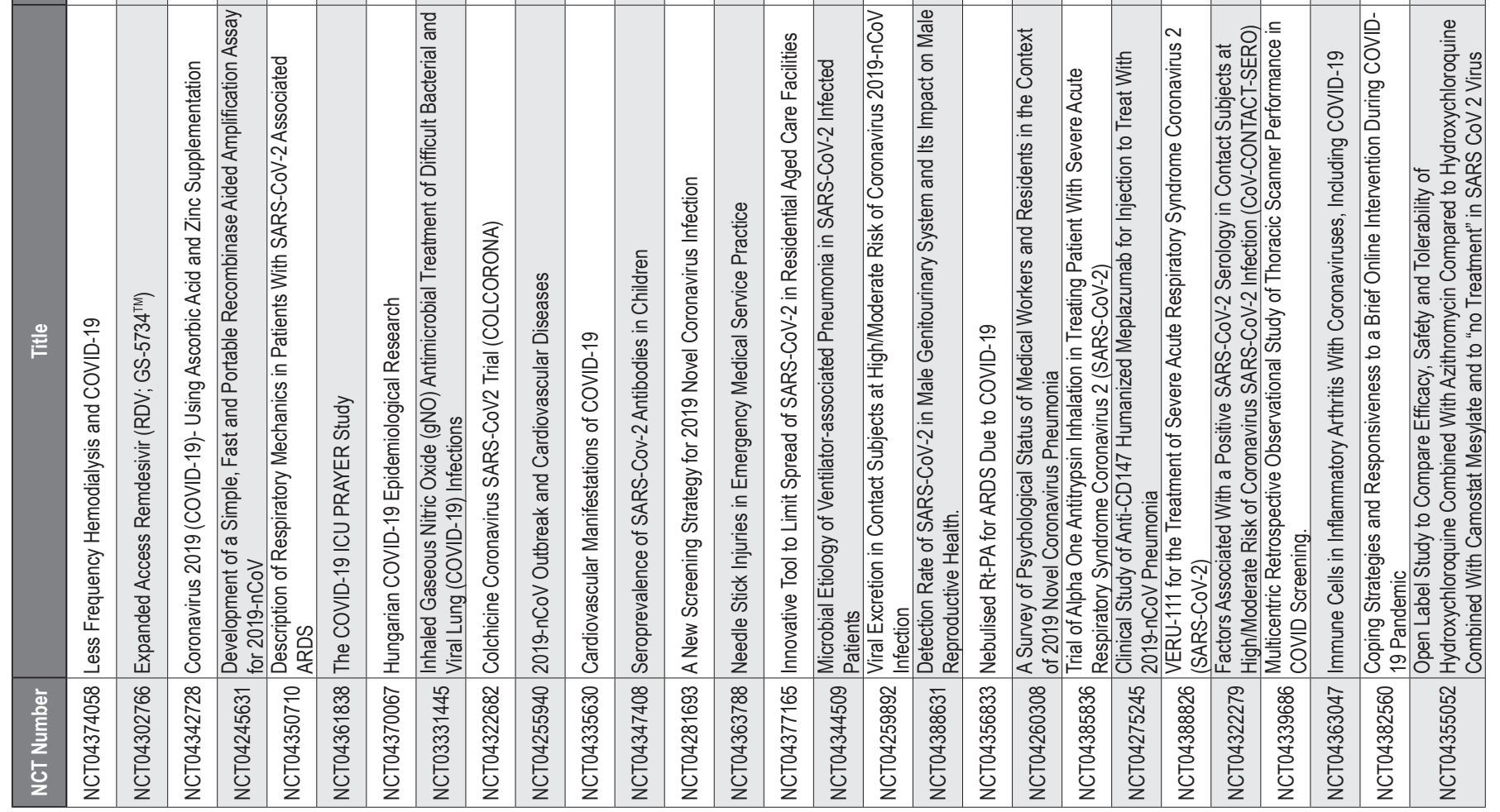




\begin{tabular}{|c|c|c|c|c|c|c|c|c|c|c|c|c|c|c|c|c|c|c|c|c|c|c|c|c|c|c|c|}
\hline & 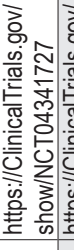 & 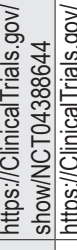 & 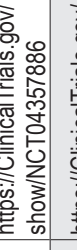 & 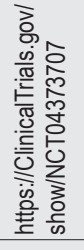 & 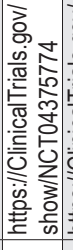 & 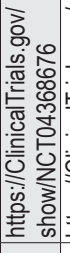 & 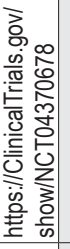 & 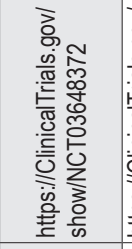 & 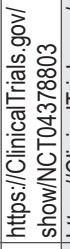 & & 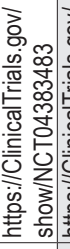 & & & 吕 & 言 & & - & & 象 & 递 & & 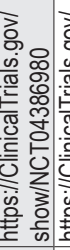 & 递 & & & & \\
\hline 窵 & 言 & 졿유 & 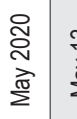 & 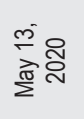 & & & : & $\begin{array}{l}\overline{\bar{\delta}} \\
\overline{\bar{d}} \\
\overline{0}\end{array}$ & & & & & & & & & & & & & & & & & & & \\
\hline 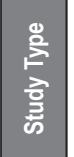 & 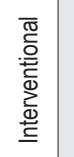 & 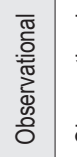 & 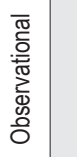 & 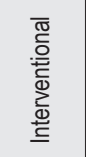 & 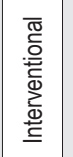 & 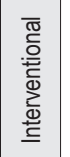 & 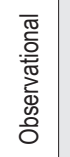 & 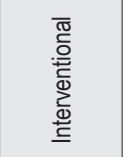 & 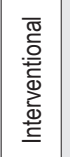 & 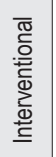 & 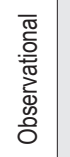 & 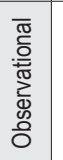 & $\sum_{\substack{\bar{D} \\
\mathbb{Q}}}$ & 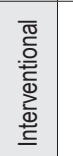 & 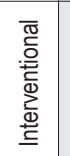 & 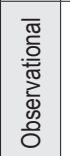 & 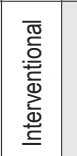 & 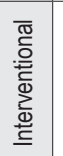 & 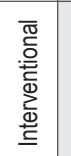 & 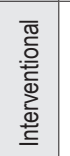 & 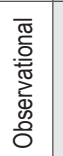 & 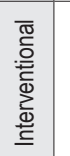 & 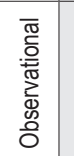 & & & & \\
\hline & 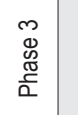 & & & $\underset{\mathscr{J}}{\mathscr{J}}$ & $\Sigma$ & 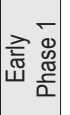 & & 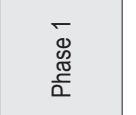 & z & 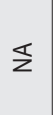 & & & $\frac{5}{z}$ & $\underline{z}$ & $\begin{array}{l}\mathscr{D} \\
\stackrel{\mathbb{D}}{0} \\
\frac{\pi}{0}\end{array}$ & & $\underline{z}$ & 怨 & 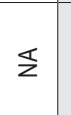 & & & 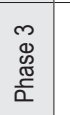 & & & & & \\
\hline & 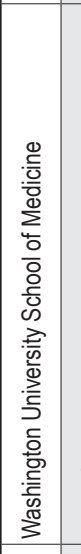 & 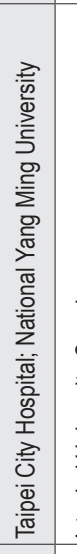 & $\begin{array}{ll} & \\
\end{array}$ & 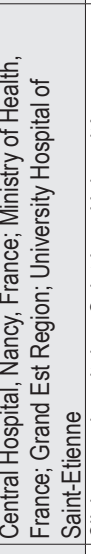 & 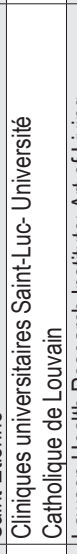 & 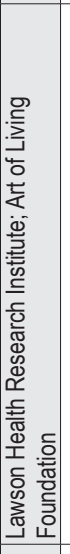 & 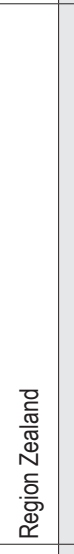 & 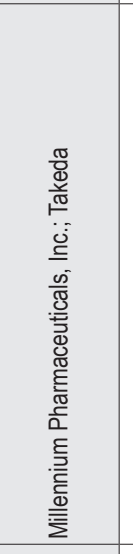 & 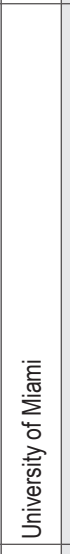 & 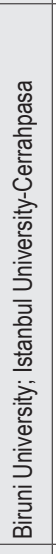 & 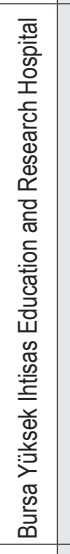 & 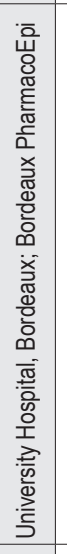 & 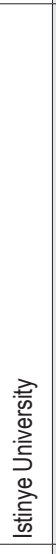 & 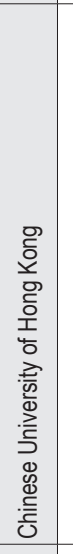 & 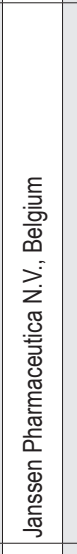 & 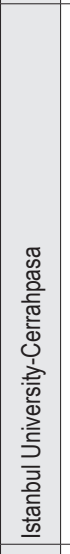 & 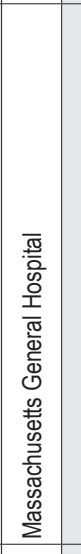 & 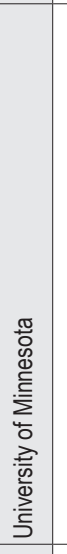 & 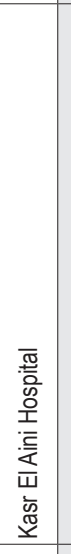 & 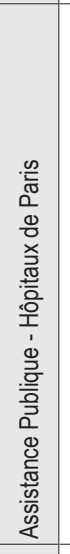 & 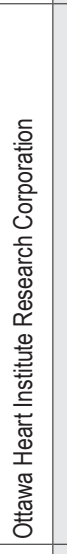 & 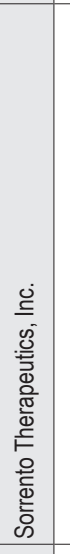 & 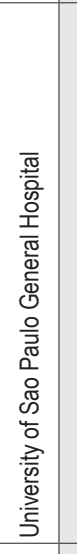 & 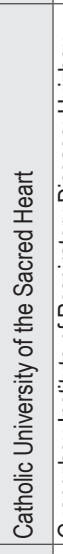 & 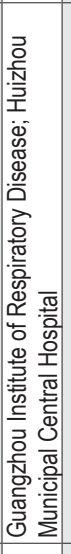 & 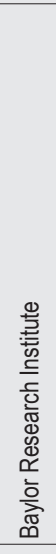 & \\
\hline & $\widehat{d}$ & $\frac{\mathbb{p}}{z}$ & $\frac{\mathbb{\alpha}}{2}$ & 蛋 & $\frac{\mathbb{s}}{z}$ & $\frac{\underline{p}}{z}$ & $\frac{\mathbb{p}}{z}$ & 产 & $\frac{\mathbb{s}}{z}$ & & $\frac{\underline{p}}{z}$ & $\frac{\sqrt[s]{z}}{z}$ & $\frac{\mathbb{r}}{z}$ & $\frac{\text { 多 }}{z}$ & $\frac{\sqrt{s}}{z}$ & $\frac{\widehat{s}}{\frac{\hat{s}}{z}}$ & z & 孚 & $\bar{z}$ & z & $\bar{z}$ & 虹 & $\bar{z}$ & $\frac{\mathbb{p}}{z}$ & & z & \\
\hline & $\propto$ & $\simeq$ & $\sum_{\Sigma}^{\alpha}$ & $\propto$ & $\propto$ & $\frac{1}{2}$ & 0 & & & & $\sum^{\frac{N}{z}}$ & & & $\sum_{\Sigma}^{\frac{N}{z}}$ & & $\sum_{\Sigma}^{\frac{x}{z}}$ & $\sum_{\Sigma}^{\frac{x}{z}}$ & 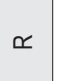 & 产 & 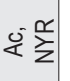 & $\frac{\alpha}{\Sigma}$ & $z$ & & $\propto$ & & & \\
\hline & 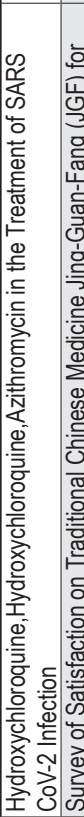 & 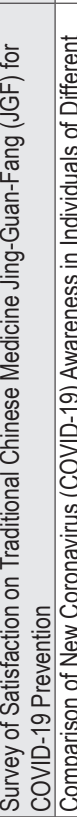 & 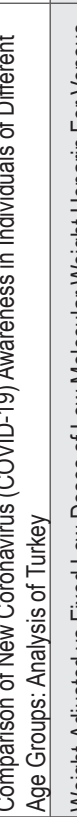 & 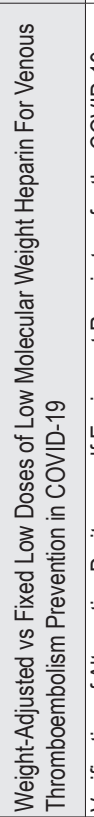 & 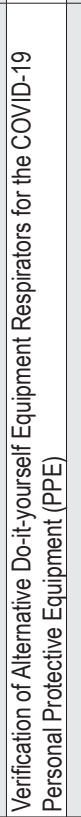 & 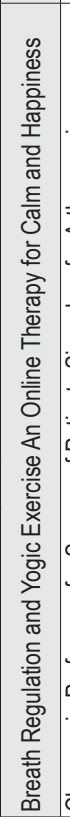 & 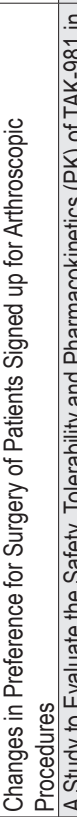 & 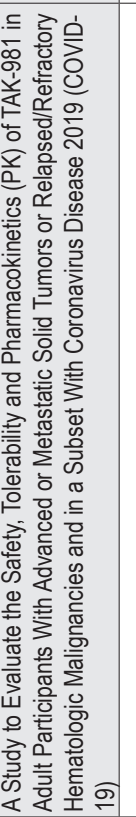 & 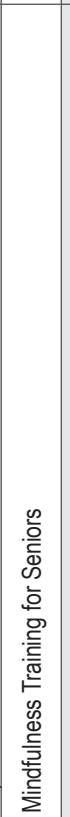 & 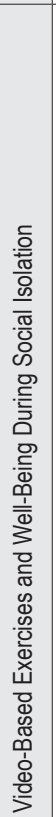 & 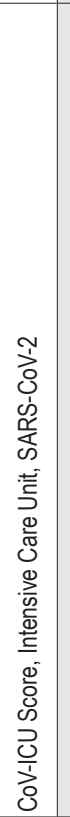 & 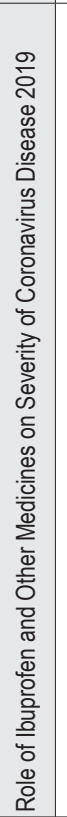 & 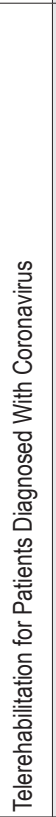 & 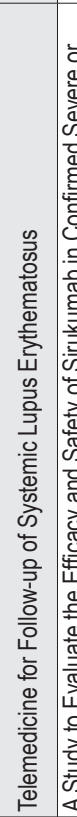 & 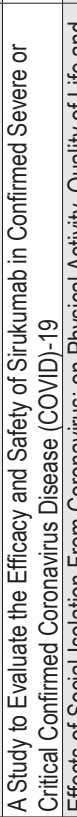 & 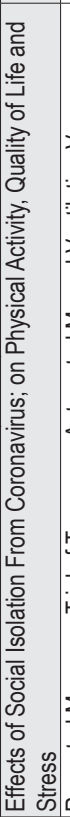 & 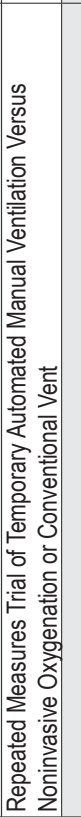 & 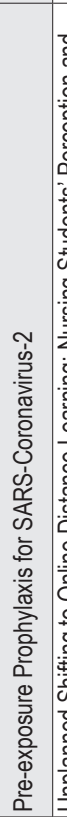 & 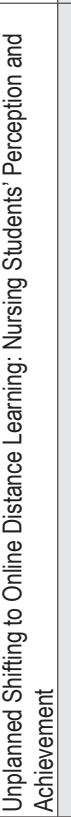 & 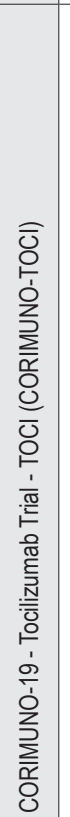 & 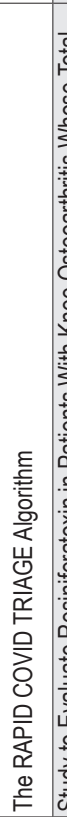 & 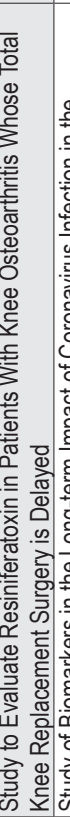 & 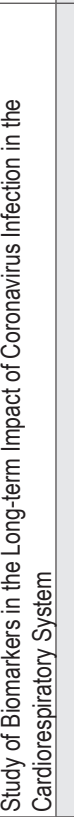 & 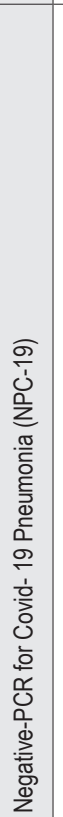 & 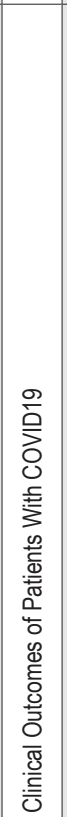 & 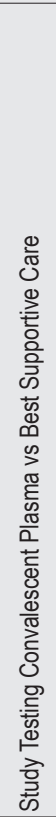 & 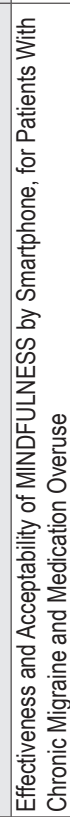 \\
\hline 这 & 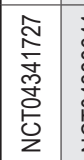 & 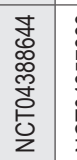 & 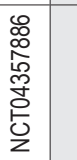 & 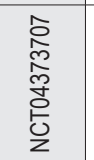 & 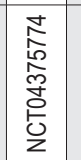 & $\begin{array}{l}0 \\
0.0 \\
00 \\
0 \\
0 \\
0 \\
2 \\
2\end{array}$ & $\begin{array}{l}\infty \\
\stackrel{0}{0} \\
\stackrel{0}{9} \\
\stackrel{0}{0} \\
\frac{0}{z}\end{array}$ & 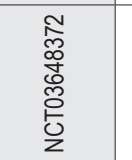 & 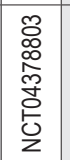 & 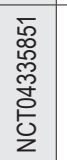 & 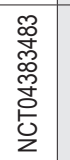 & 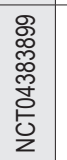 & 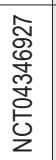 & 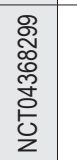 & 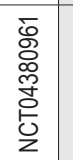 & 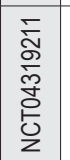 & 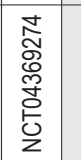 & 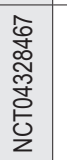 & 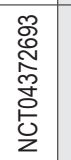 & 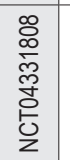 & 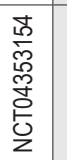 & 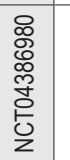 & 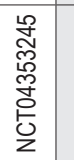 & 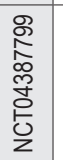 & $\begin{array}{l}\text { 管 } \\
\text { ปै }\end{array}$ & 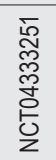 & \\
\hline
\end{tabular}




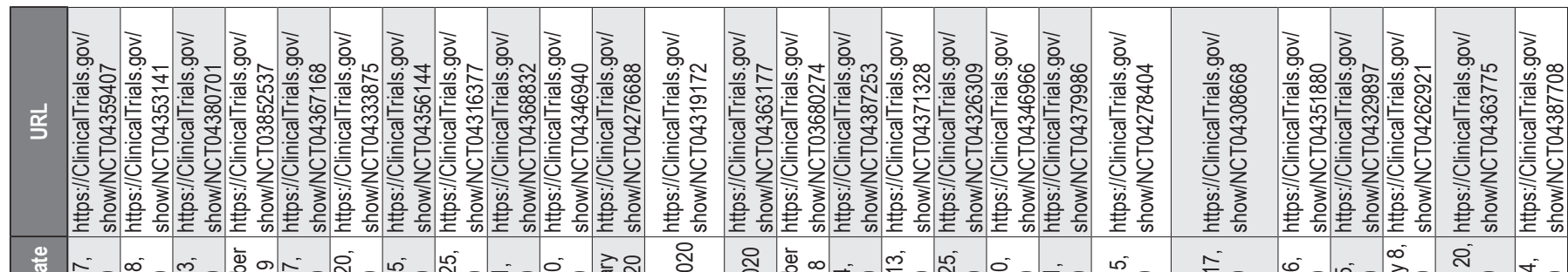

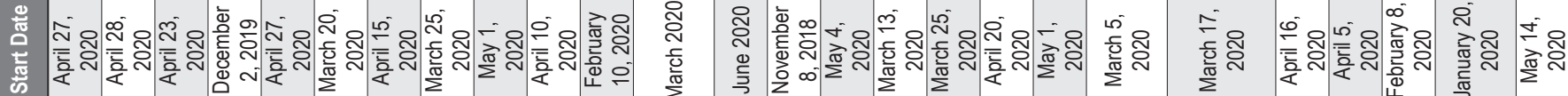

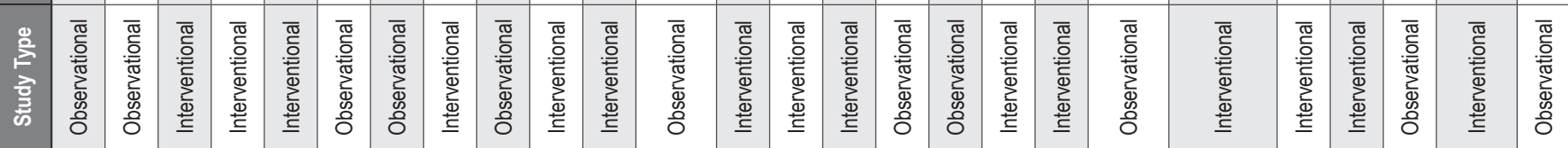

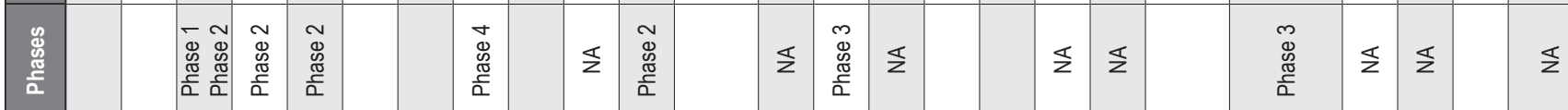

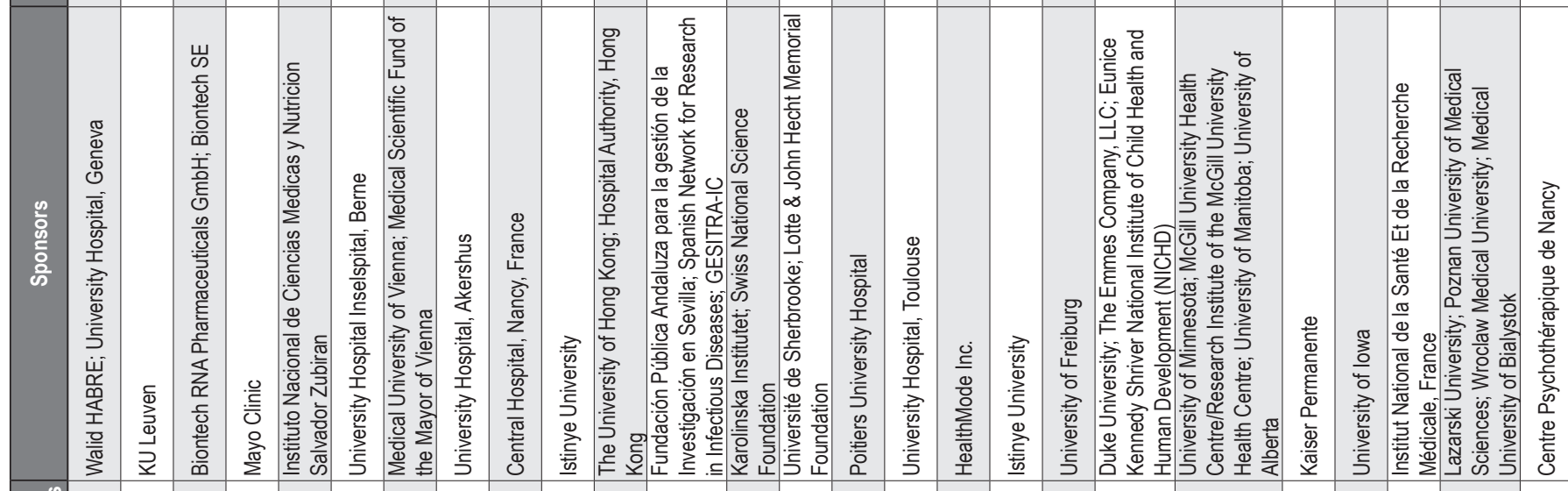

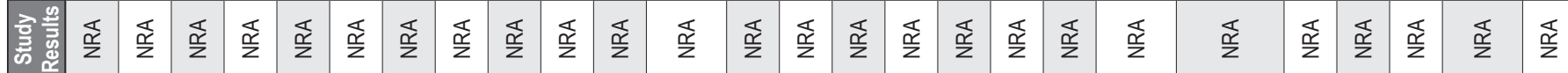

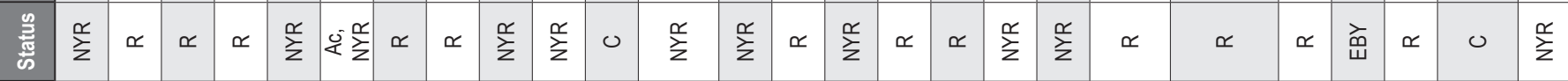

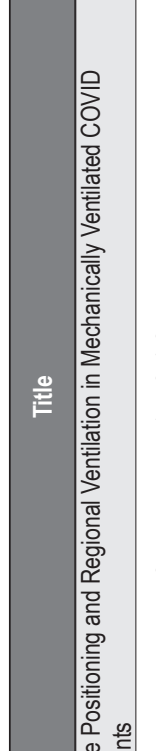

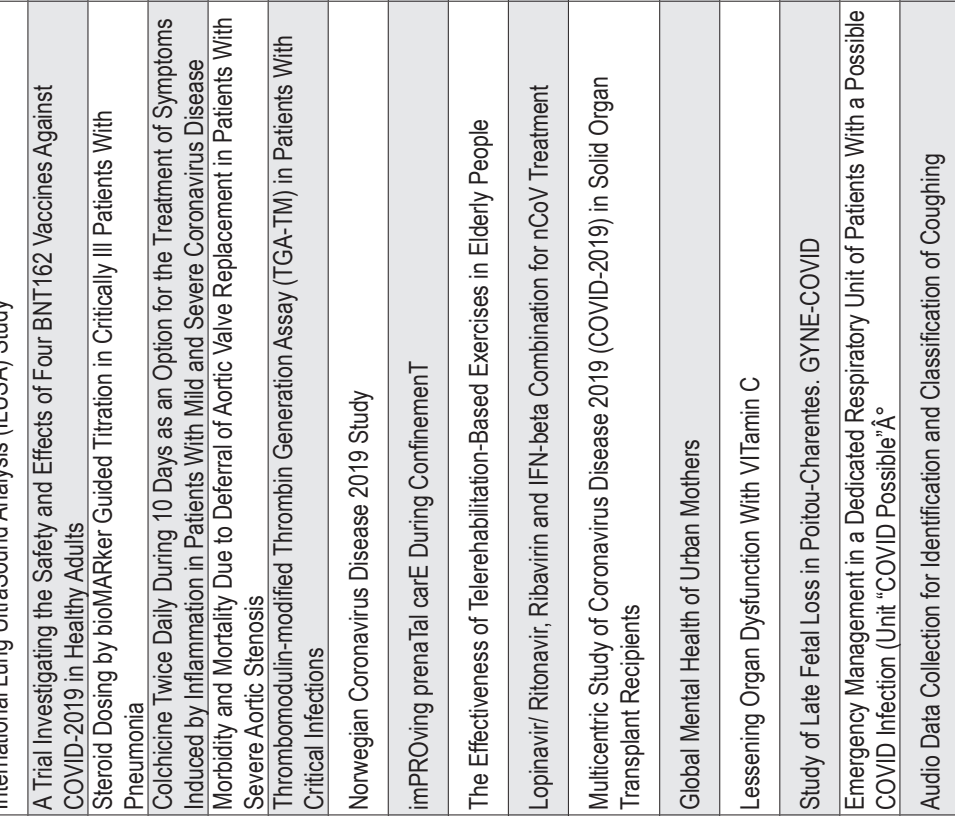

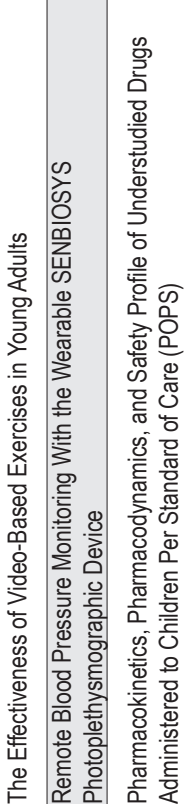

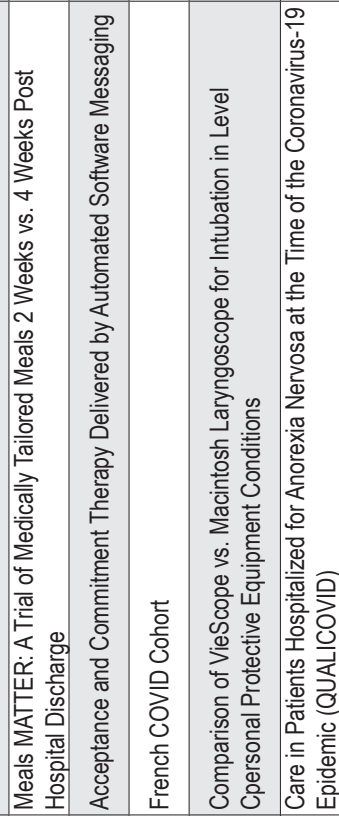




\begin{tabular}{|c|c|c|c|c|c|}
\hline 岁 & 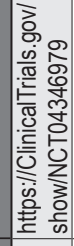 & 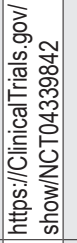 & 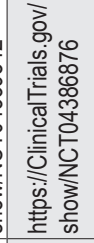 & 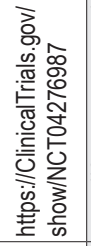 & 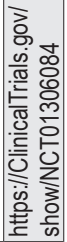 \\
\hline & & & 离 & 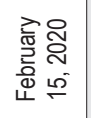 & 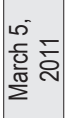 \\
\hline 产 & 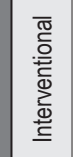 & 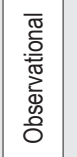 & 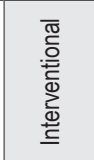 & 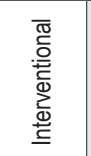 & 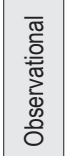 \\
\hline $\begin{array}{l}\frac{8}{8} \\
\frac{8}{0} \\
\frac{3}{0}\end{array}$ & $\sum$ & & $\begin{array}{l}\mathscr{D} \\
\mathbb{\pi} \\
\frac{\pi}{\alpha}\end{array}$ & 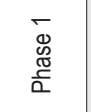 & \\
\hline के & 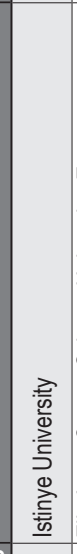 & 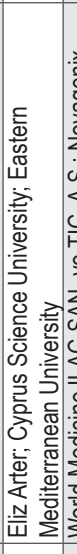 & 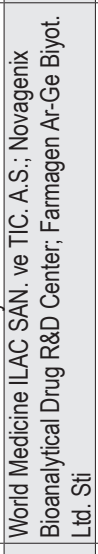 & 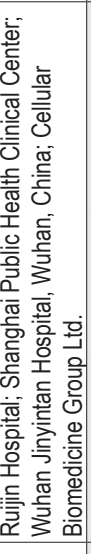 & 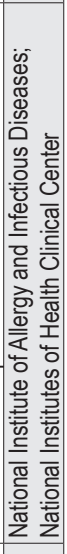 \\
\hline 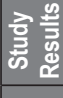 & 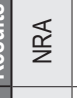 & $\frac{\mathbb{p}}{z}$ & 氶 & 㞼 & 㕸 \\
\hline 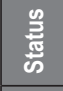 & $\frac{\alpha}{z}$ & $\propto$ & 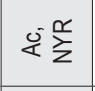 & $\frac{\alpha}{\Sigma}$ & 怠 \\
\hline 童 & 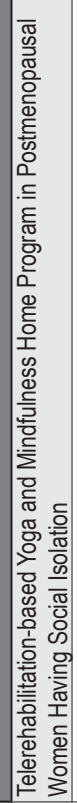 & 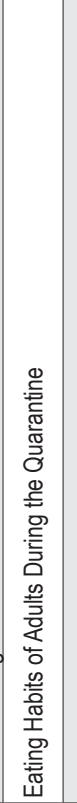 & 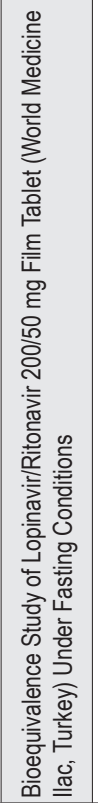 & 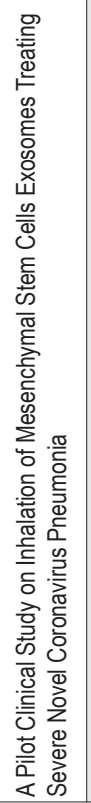 & 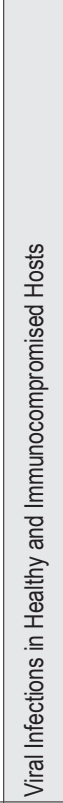 \\
\hline 点 & 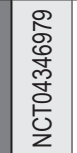 & 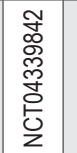 & 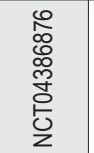 & 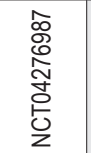 & 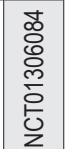 \\
\hline
\end{tabular}




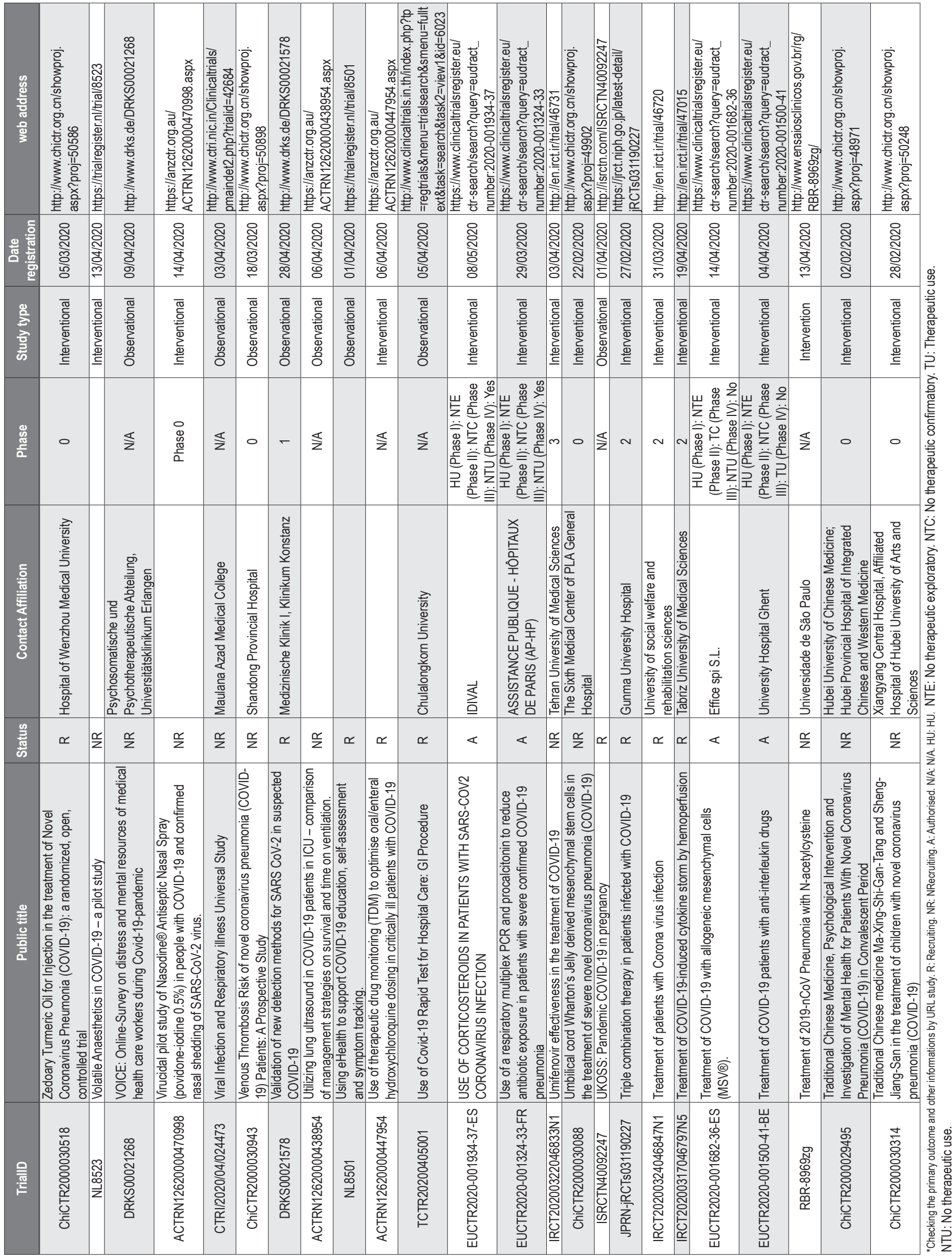




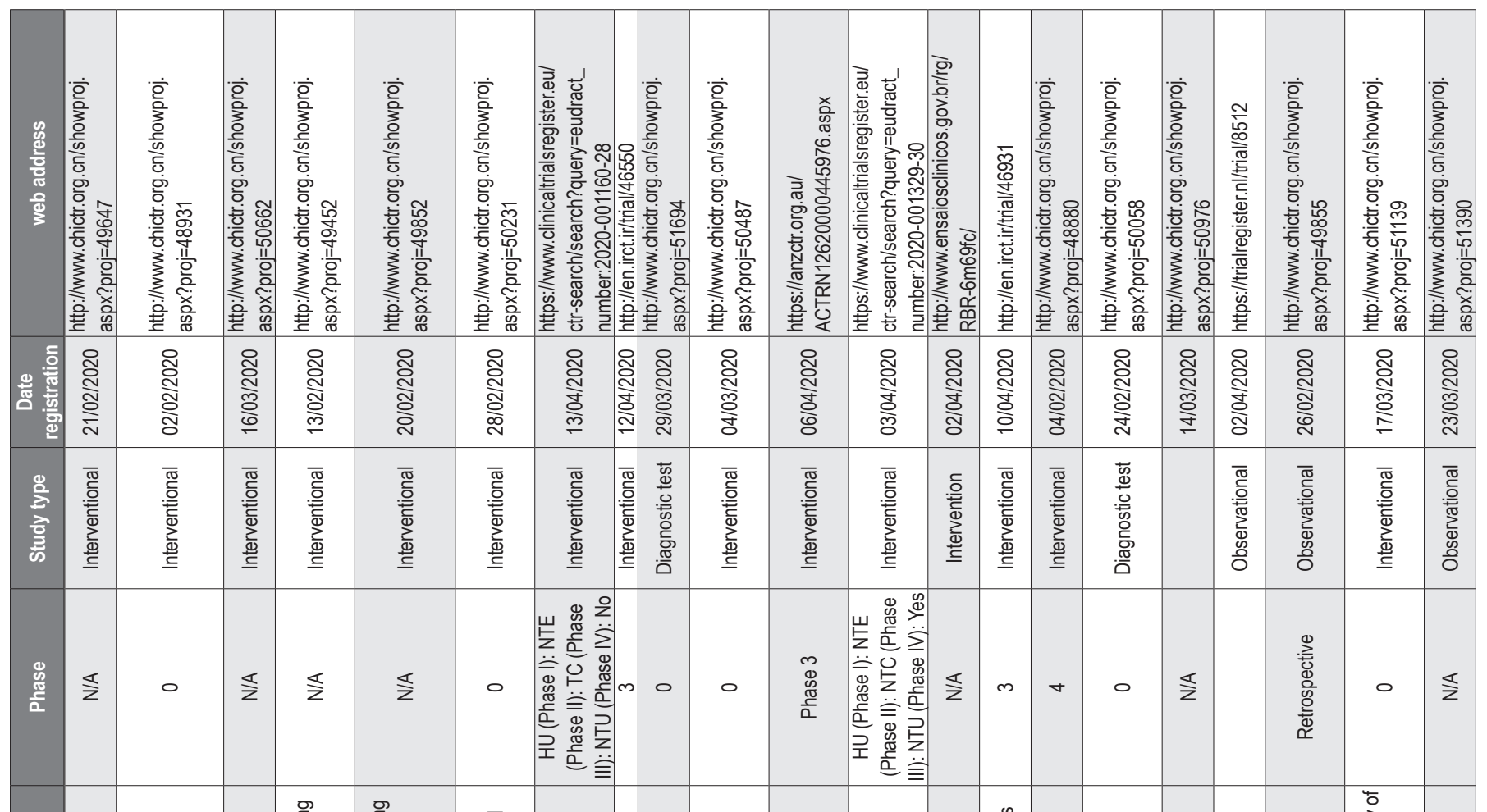

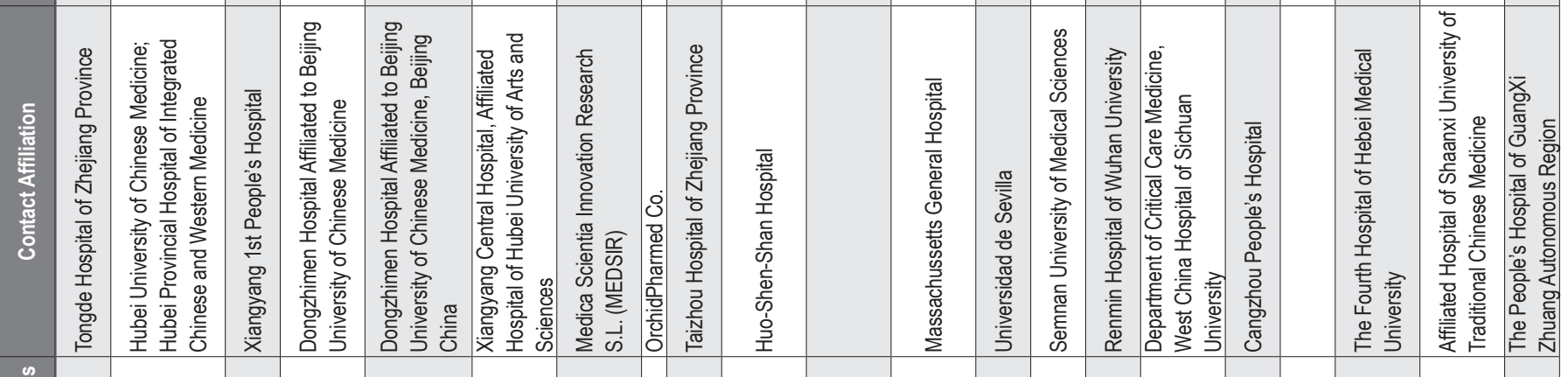

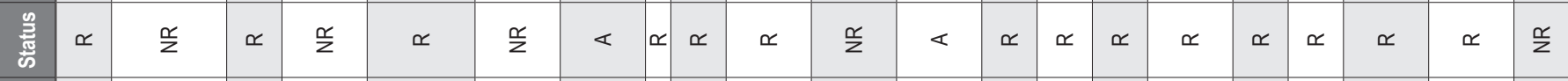

\begin{tabular}{|c|c|c|c|c|c|c|c|c|c|c|c|c|c|c|c|c|c|c|}
\hline & & & & & \multirow{2}{*}{\multicolumn{2}{|c|}{ 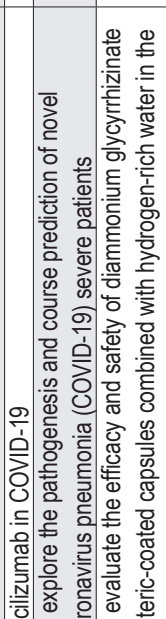 }} & & & & & & & & \multirow{2}{*}{\multicolumn{3}{|c|}{ 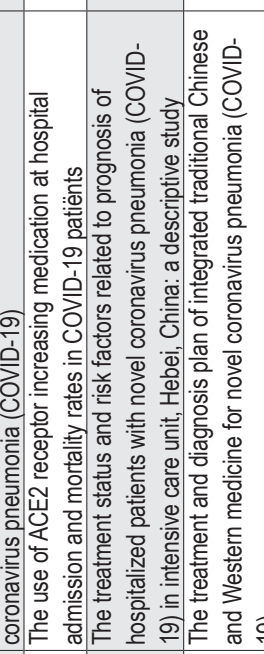 }} & \multirow[b]{2}{*}{ 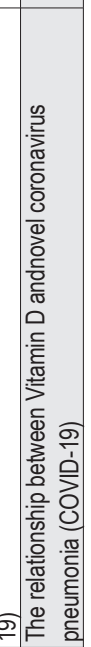 } \\
\hline & & 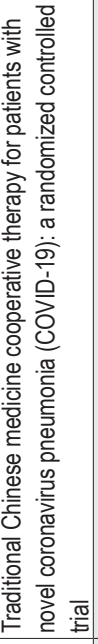 & 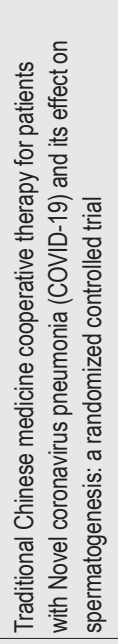 & 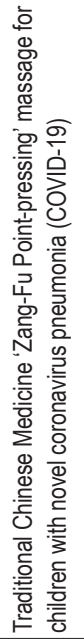 & 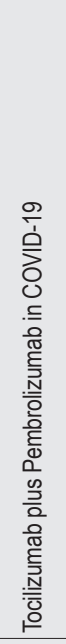 & & & 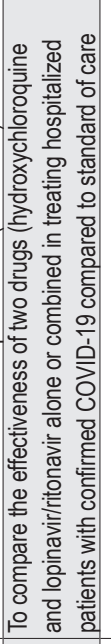 & 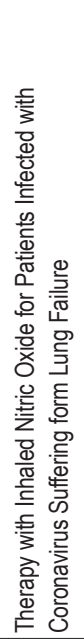 & 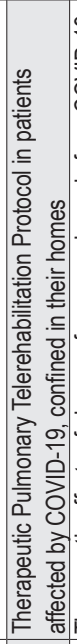 & & & 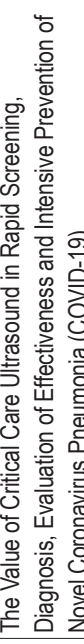 & 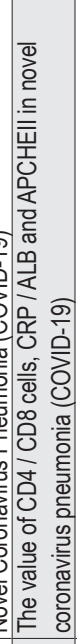 & & & & \\
\hline 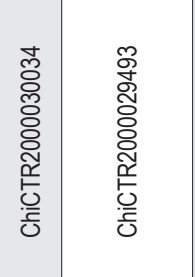 & 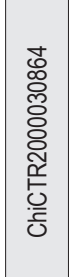 & 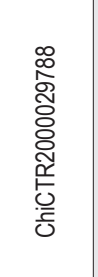 & 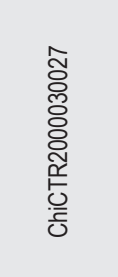 & 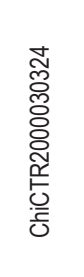 & 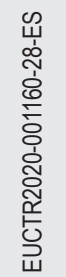 & 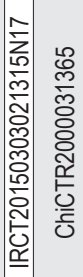 & 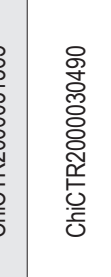 & 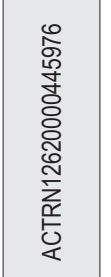 & 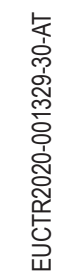 & 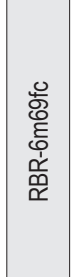 & & 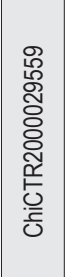 & 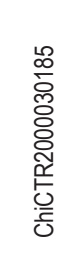 & 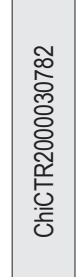 & & & & 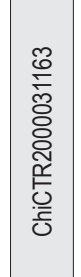 \\
\hline
\end{tabular}




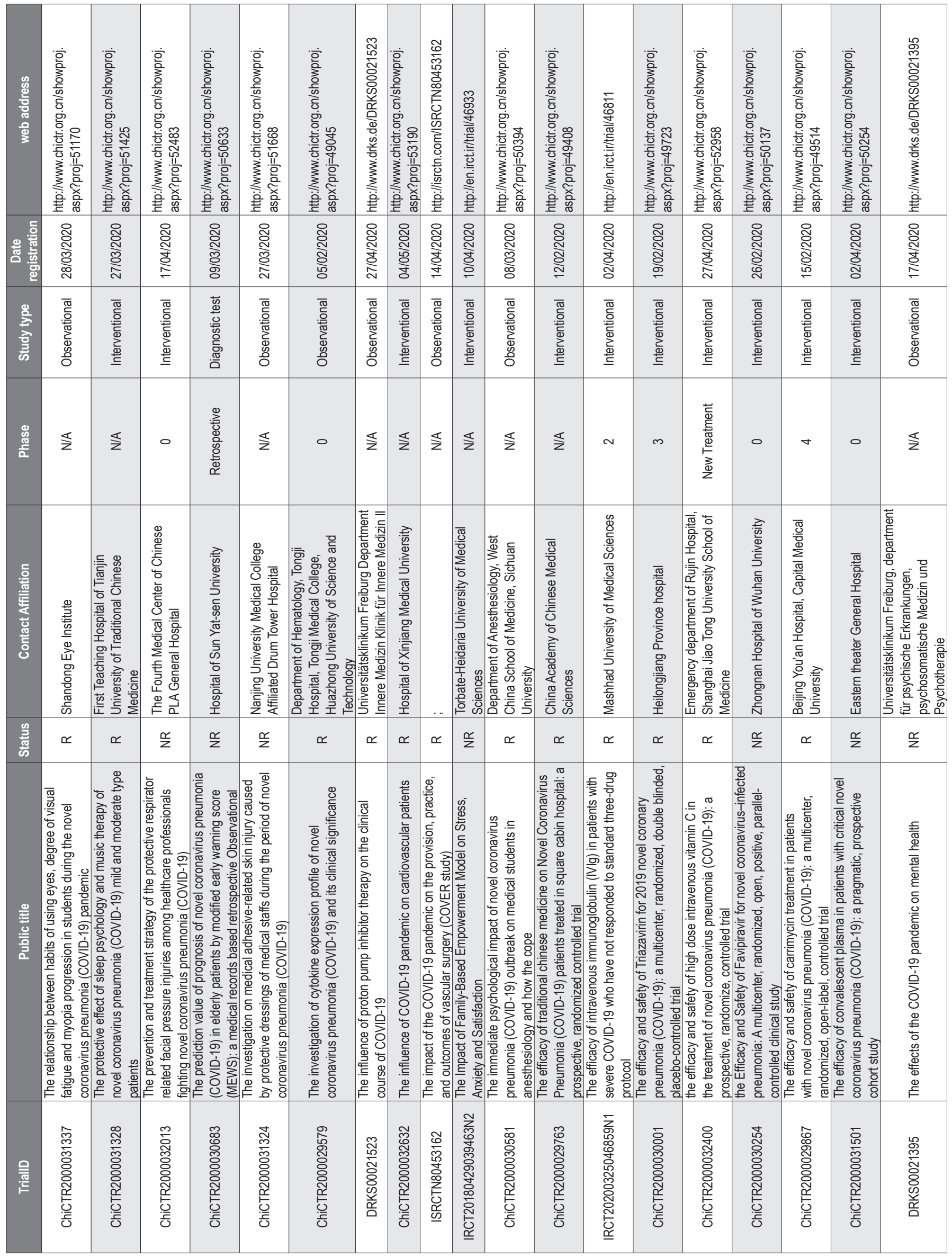




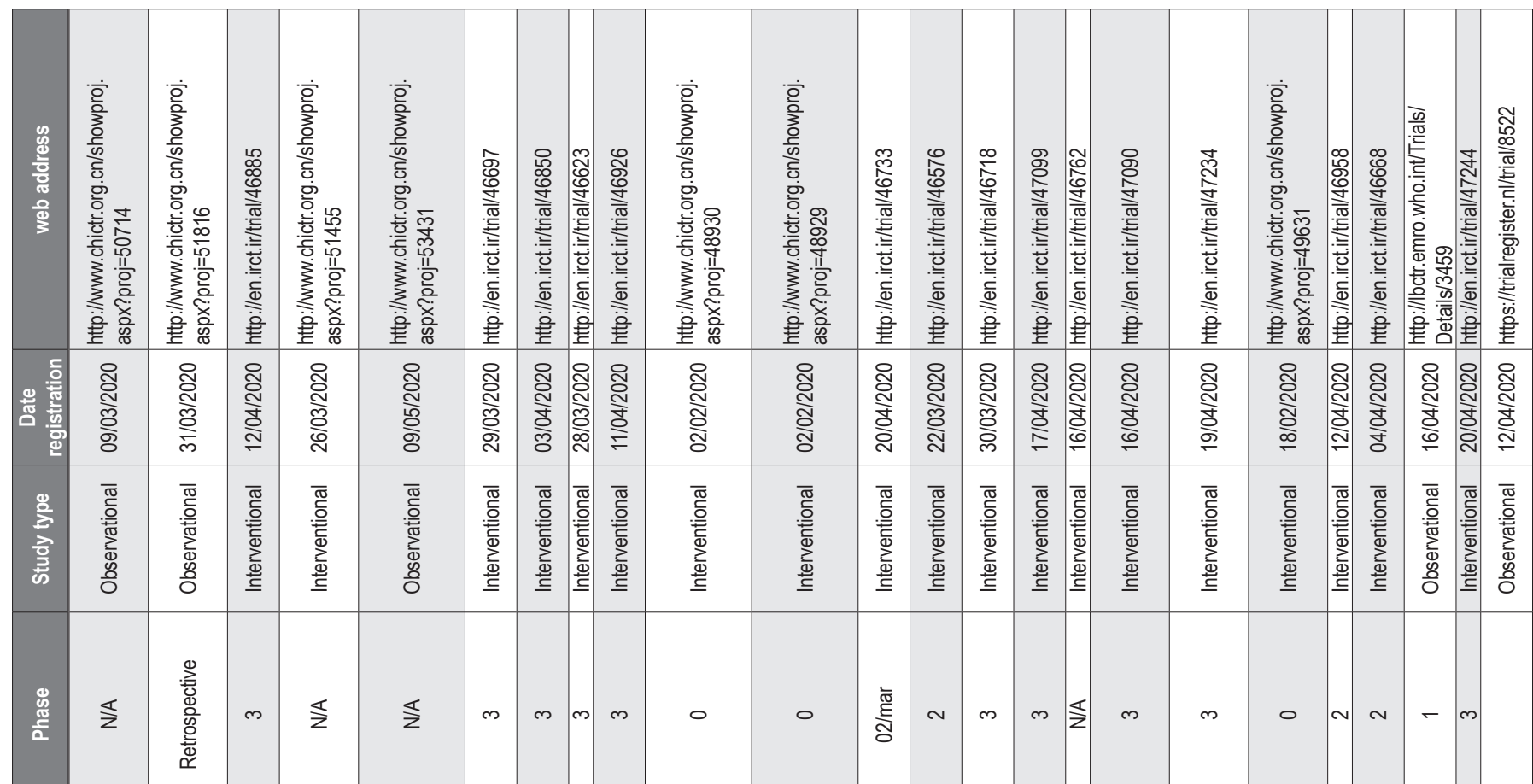

\begin{tabular}{|c|c|c|c|c|c|c|c|c|c|c|c|c|c|c|c|c|c|c|c|}
\hline 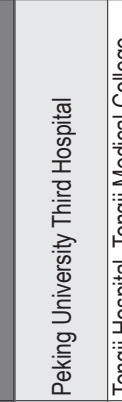 & 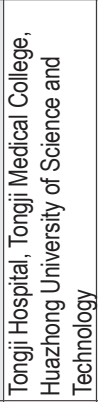 & 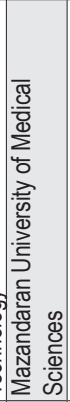 & 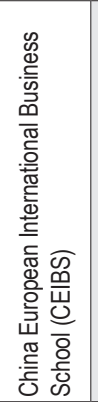 & 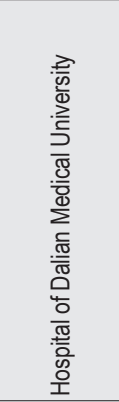 & 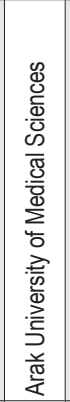 & 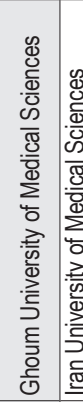 & 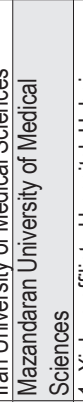 & 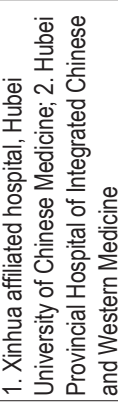 & 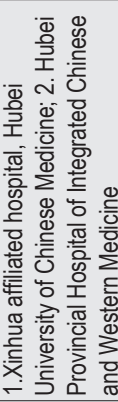 & 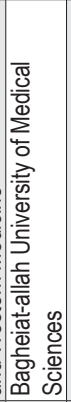 & 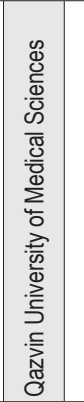 & 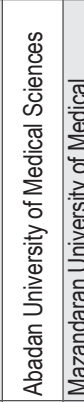 & 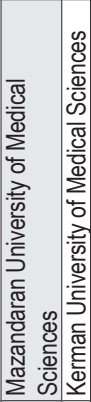 & 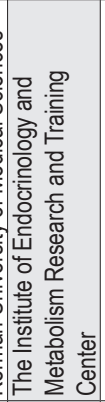 & 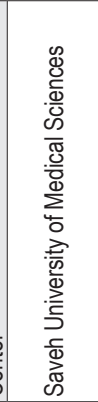 & 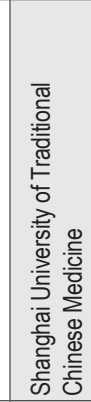 & 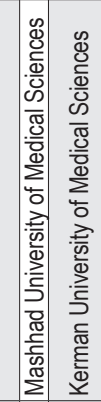 & 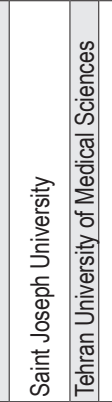 & \\
\hline$\simeq$ & $\frac{\underline{\alpha}}{z}$ & $\frac{x}{z}$ & $\frac{\alpha}{z}$ & $\frac{⿱}{z}$ & $\propto$ & $\alpha$ & $\{\simeq$ & $\frac{\alpha}{z}$ & $\frac{x}{z}$ & $\frac{\alpha}{z}$ & $\frac{\alpha}{z}$ & $\frac{\alpha}{z}$ & $\propto$ & $\propto$ & $\propto$ & $\propto$ & $\propto \propto$ & $\frac{\alpha}{z}\left|\frac{\alpha}{z}\right|$ & \\
\hline 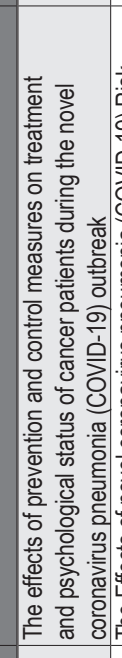 & 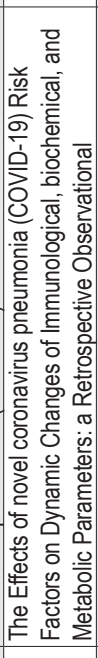 & 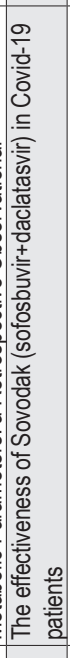 & 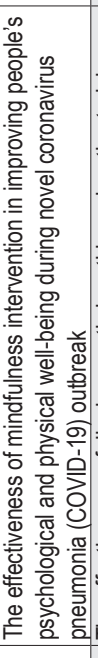 & 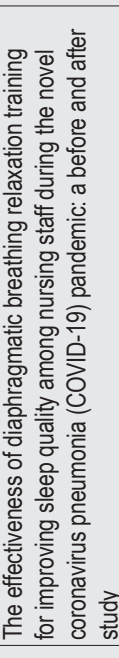 & 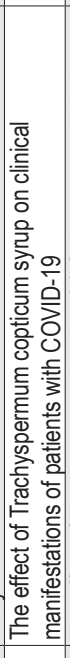 & 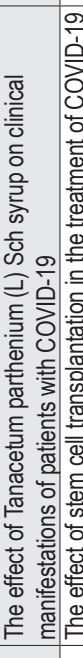 & 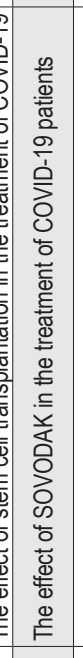 & 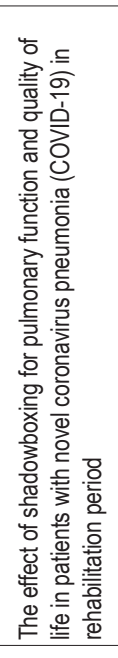 & 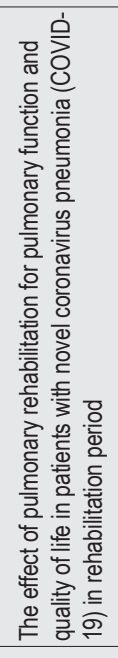 & 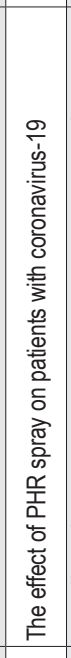 & 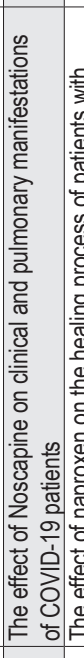 & 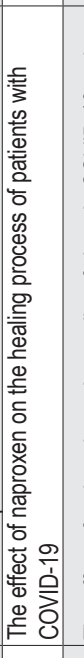 & 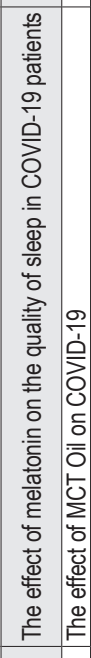 & 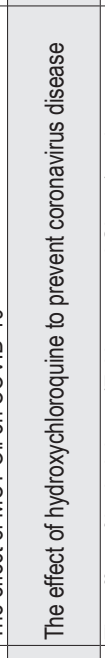 & 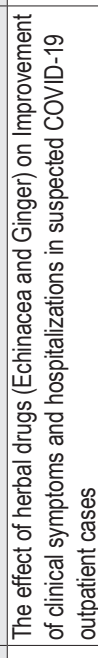 & 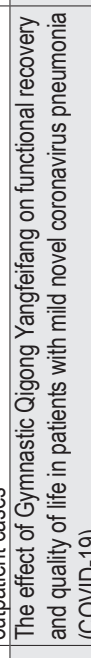 & 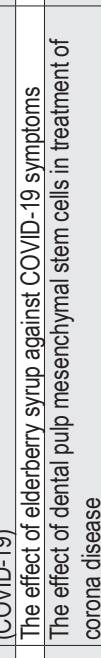 & 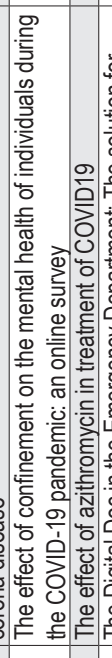 & 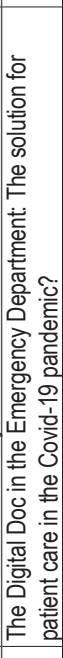 \\
\hline 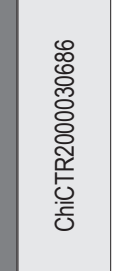 & 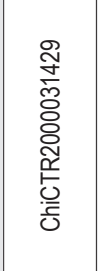 & 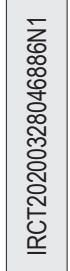 & 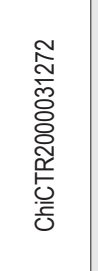 & 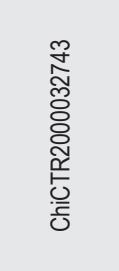 & 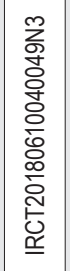 & 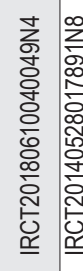 & 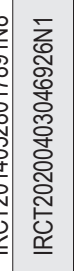 & 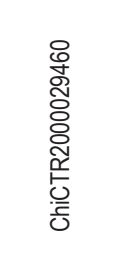 & 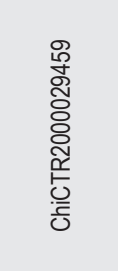 & 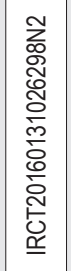 & 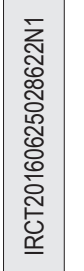 & 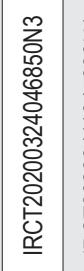 & 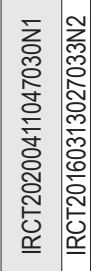 & 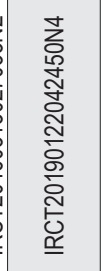 & 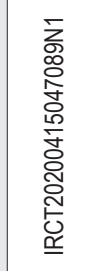 & 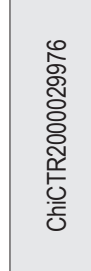 & 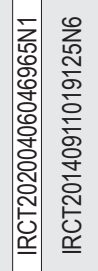 & 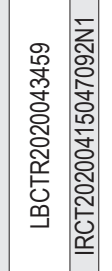 & 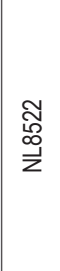 \\
\hline
\end{tabular}




\begin{tabular}{|c|c|c|c|c|c|c|c|c|c|c|c|c|c|c|c|c|c|c|}
\hline 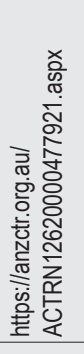 & 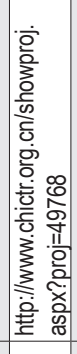 & 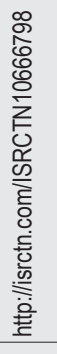 & 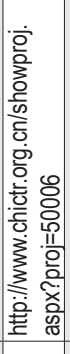 & 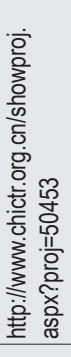 & 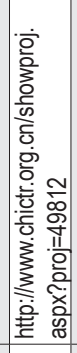 & 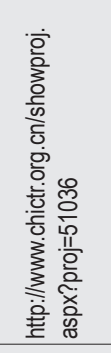 & 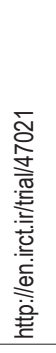 & 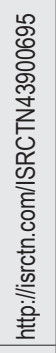 & 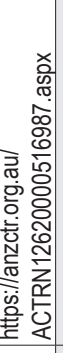 & 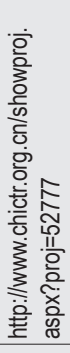 & 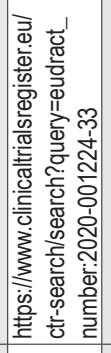 & 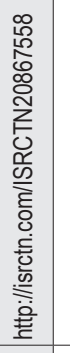 & 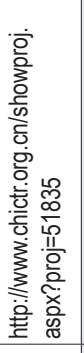 & 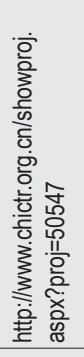 & 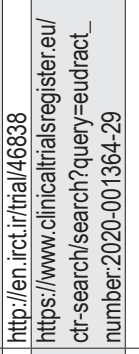 & 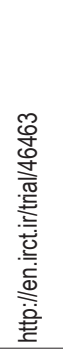 & 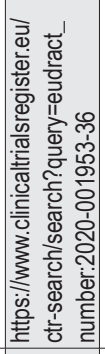 & 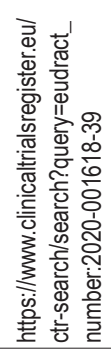 \\
\hline $\begin{array}{l}\text { م્ } \\
\text { Nิ } \\
\stackrel{0}{0} \\
\end{array}$ & 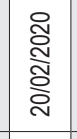 & 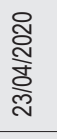 & 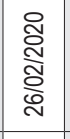 & 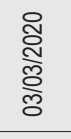 & 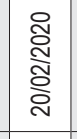 & 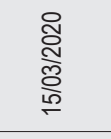 & $\begin{array}{l}\text { ̊ิ̀ } \\
\text { ָे }\end{array}$ & 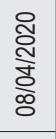 & 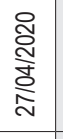 & 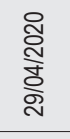 & 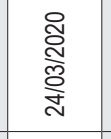 & 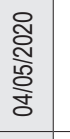 & 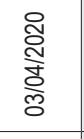 & $\begin{array}{l}\text { ్․ } \\
\text { ָे } \\
\text { ờ }\end{array}$ & 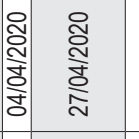 & 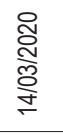 & 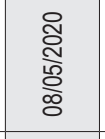 & $\begin{array}{l}\text { तิ } \\
\text { Nิ } \\
\text { స̃ } \\
\text { N }\end{array}$ \\
\hline 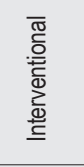 & 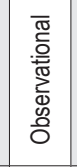 & 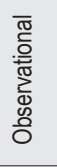 & 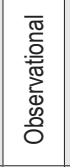 & 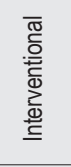 & 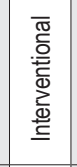 & 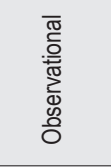 & 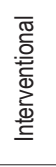 & 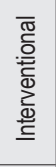 & 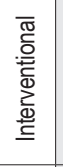 & 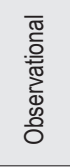 & 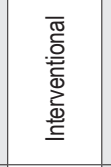 & 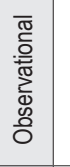 & 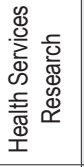 & 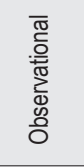 & 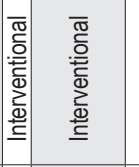 & 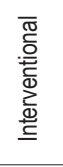 & 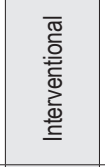 & 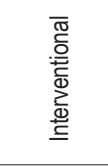 \\
\hline$\stackrel{x}{z}$ & $\stackrel{\mathbb{z}}{\mathbf{z}}$ & $\stackrel{\Sigma}{z}$ & 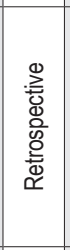 & $\nabla$ & $\stackrel{\Sigma}{z}$ & 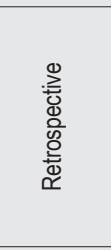 & 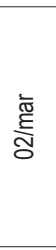 & $\underline{\mathbf{z}}$ & $\stackrel{\$}{z}$ & $\stackrel{\Sigma}{z}$ & 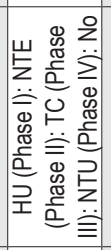 & $\stackrel{\underline{z}}{z}$ & $\stackrel{x}{z}$ & 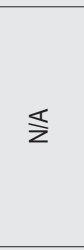 & 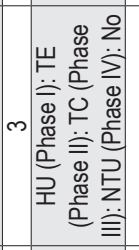 & $m$ & 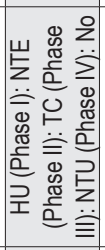 & 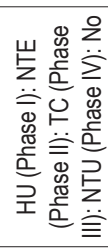 \\
\hline & 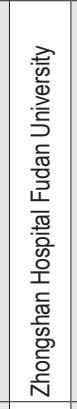 & & 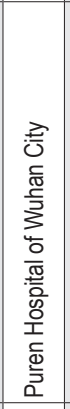 & 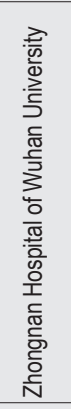 & 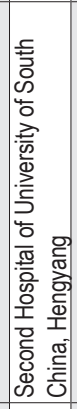 & 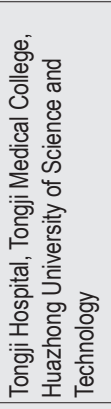 & 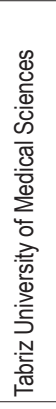 & & & 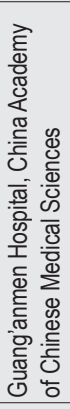 & 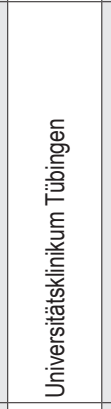 & & 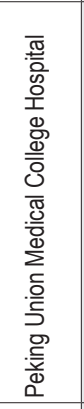 & 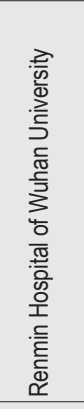 & 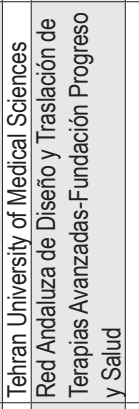 & 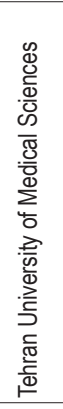 & 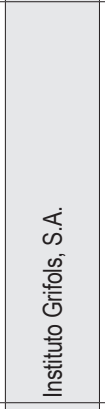 & 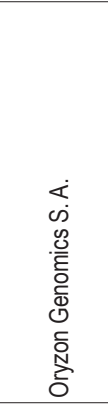 \\
\hline
\end{tabular}

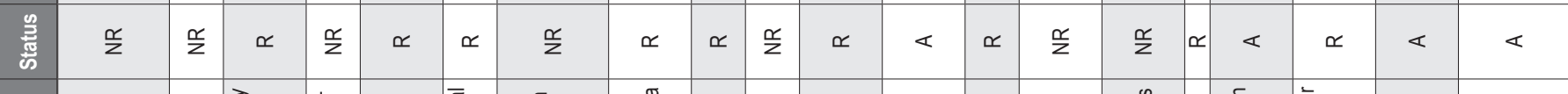

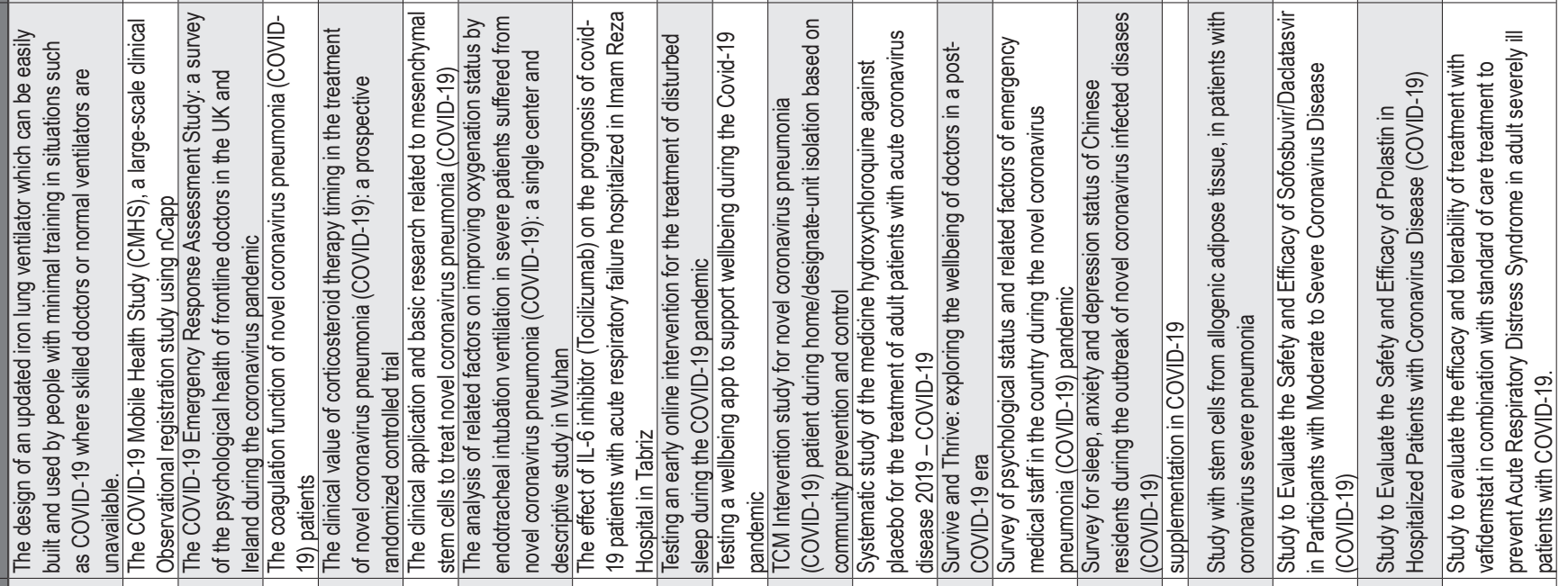

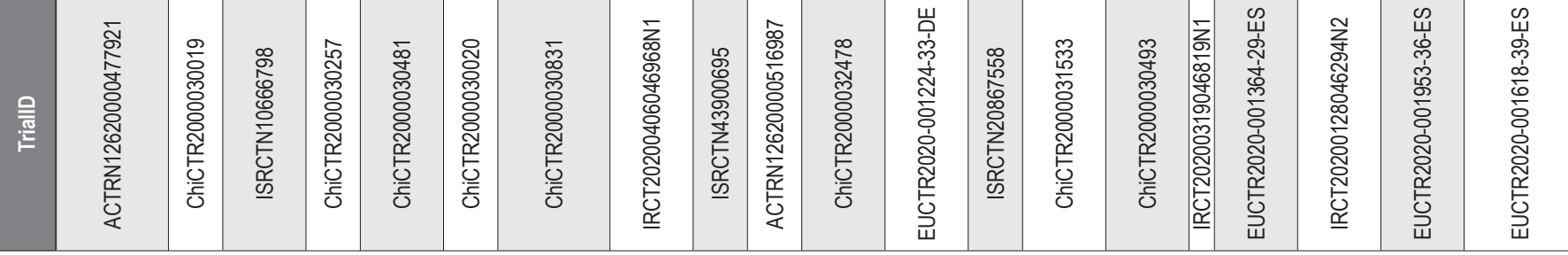




\begin{tabular}{|c|c|c|c|c|c|c|c|c|c|c|c|c|c|c|c|c|c|c|c|}
\hline 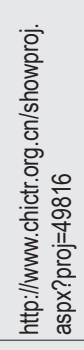 & 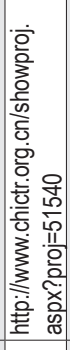 & 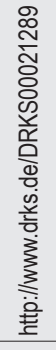 & 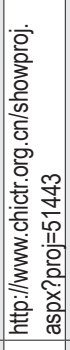 & 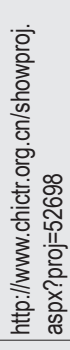 & 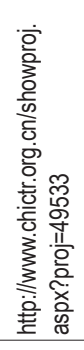 & 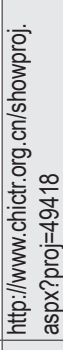 & 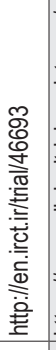 & 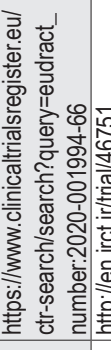 & 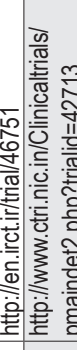 & 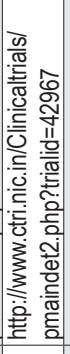 & 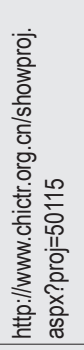 & 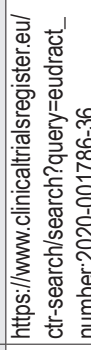 & 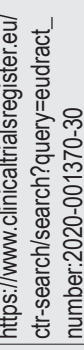 & 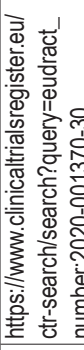 & 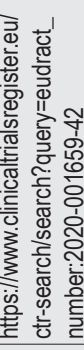 & 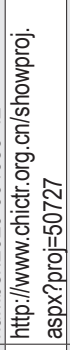 & 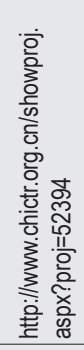 & 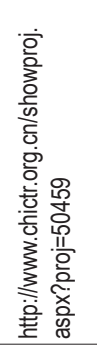 & 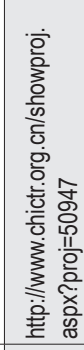 \\
\hline $\begin{array}{l}\text { ָิ } \\
\text { స్ } \\
\text { స. }\end{array}$ & 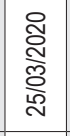 & 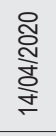 & 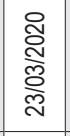 & 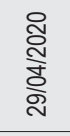 & 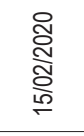 & $\begin{array}{l}\text { त్ } \\
\text { స్. } \\
\text { ิㅗㄷ }\end{array}$ & 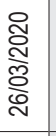 & 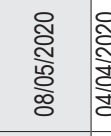 & 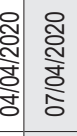 & 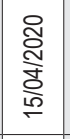 & 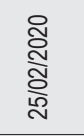 & $\begin{array}{l}\text { ণิ } \\
\text { ָे } \\
\text { 온 }\end{array}$ & 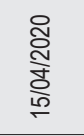 & 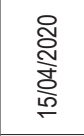 & 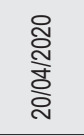 & 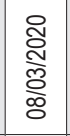 & 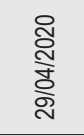 & 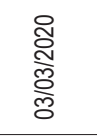 & 8 \\
\hline 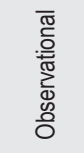 & 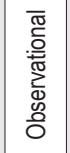 & 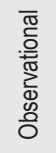 & 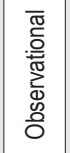 & 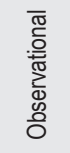 & 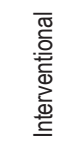 & 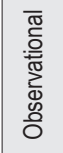 & 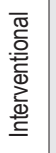 & 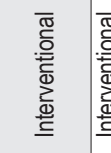 & 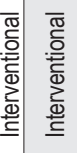 & 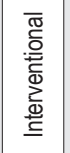 & 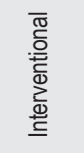 & 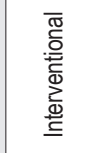 & 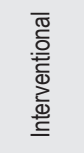 & 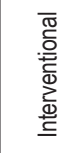 & 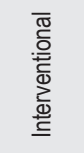 & 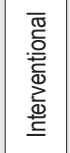 & 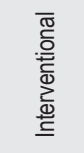 & 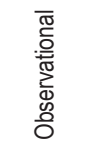 & 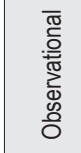 \\
\hline 0 & $\$$ & $\stackrel{\mathbb{z}}{z}$ & $\$$ & $\frac{\$}{z}$ & 0 & $\stackrel{s}{z}$ & $m$ & 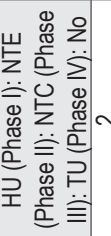 & 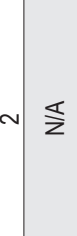 & 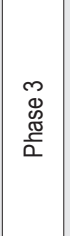 & $\stackrel{\frac{1}{z}}{z}$ & 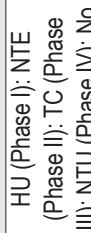 & & $\frac{0}{0}$ & 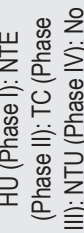 & 0 & $\nabla$ & 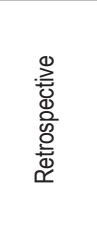 & \\
\hline
\end{tabular}

\begin{tabular}{|c|c|c|c|c|c|c|c|c|c|c|c|c|c|c|c|c|c|c|c|}
\hline 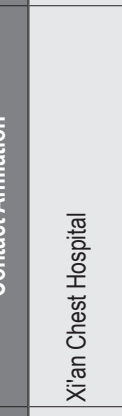 & 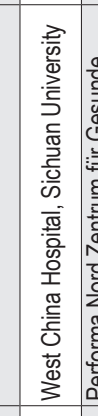 & 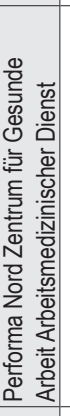 & 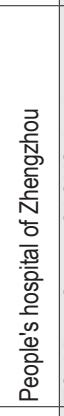 & 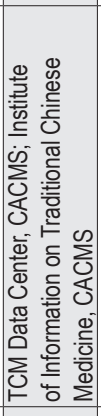 & 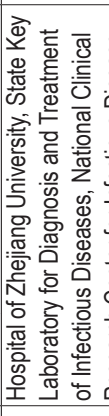 & $\begin{array}{l}\frac{5}{5} \\
\frac{5}{5} \\
\frac{5}{5} \\
\frac{5}{2} \\
\end{array}$ & 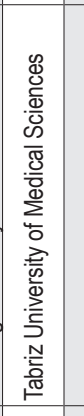 & 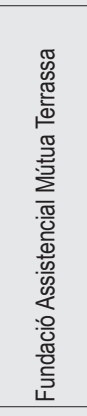 & 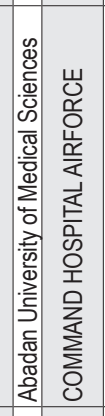 & 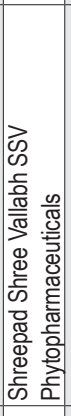 & 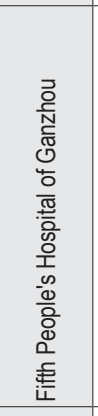 & 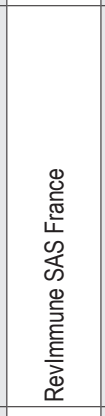 & 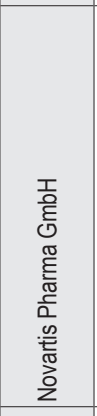 & & 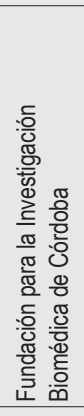 & 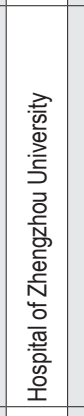 & 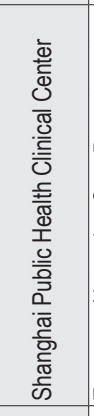 & 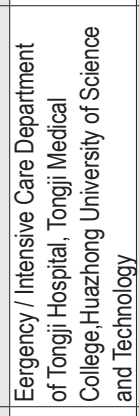 & 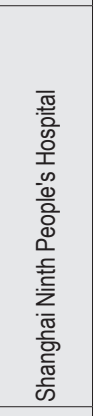 \\
\hline$\simeq$ & $\propto$ & $\simeq$ & $\simeq$ & $\frac{\alpha}{z}$ & $\propto$ & $\simeq$ & $\frac{\alpha}{z}$ & $\varangle$ & $\simeq \quad \frac{\alpha}{z}$ & $\frac{\alpha}{z}$ & $\simeq$ & $\varangle$ & $\ll$ & $\varangle$ & 8 & $\propto$ & $\frac{\alpha}{z}$ & $\frac{\alpha}{z}$ & $\propto$ \\
\hline 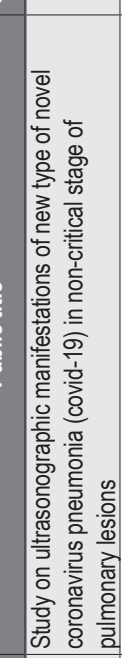 & 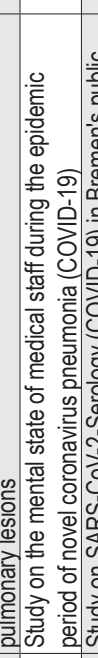 & 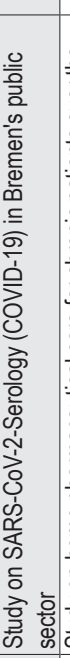 & 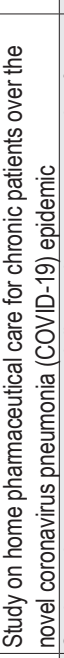 & 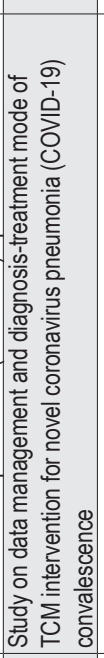 & 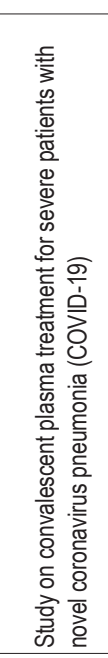 & 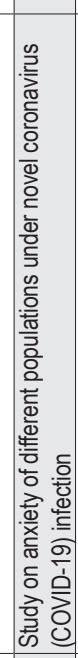 & 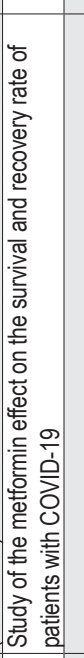 & 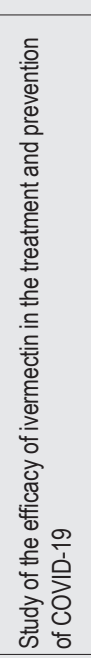 & 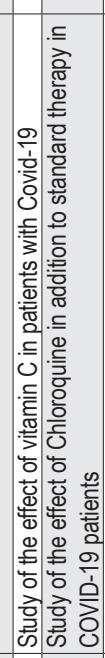 & 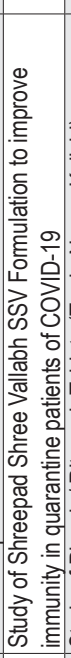 & 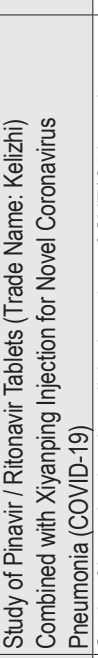 & 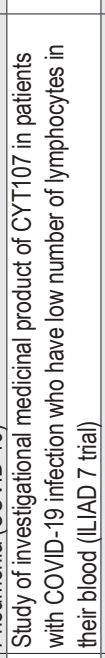 & 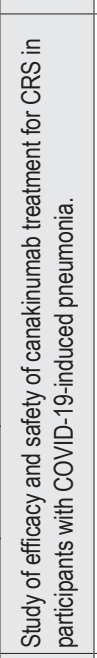 & 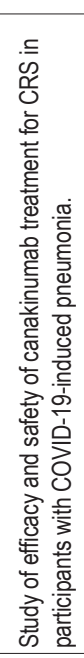 & 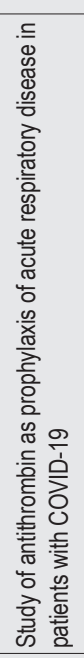 & 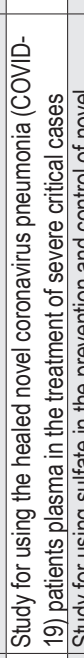 & 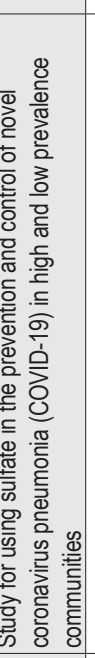 & 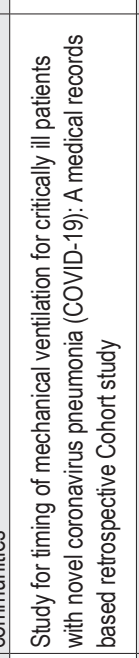 & 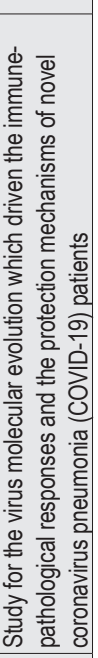 \\
\hline 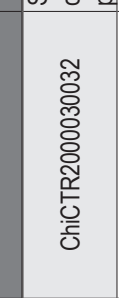 & 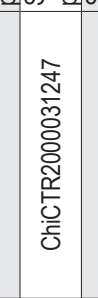 & 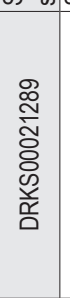 & 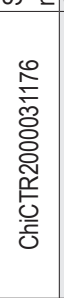 & 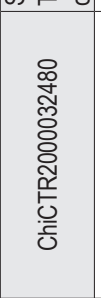 & 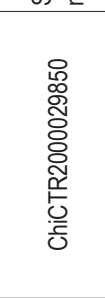 & 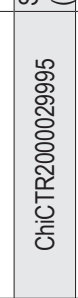 & 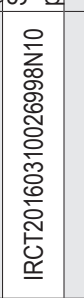 & 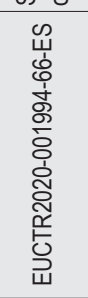 & 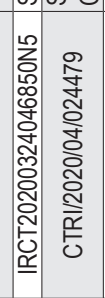 & 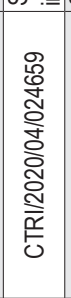 & 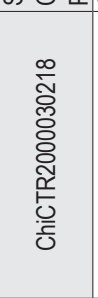 & 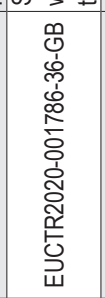 & 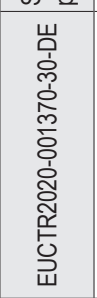 & 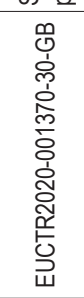 & 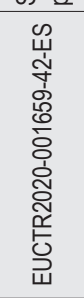 & 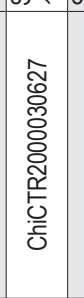 & 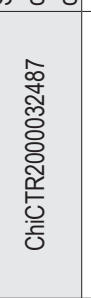 & 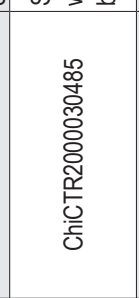 & 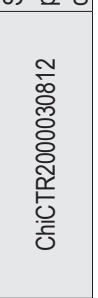 \\
\hline
\end{tabular}




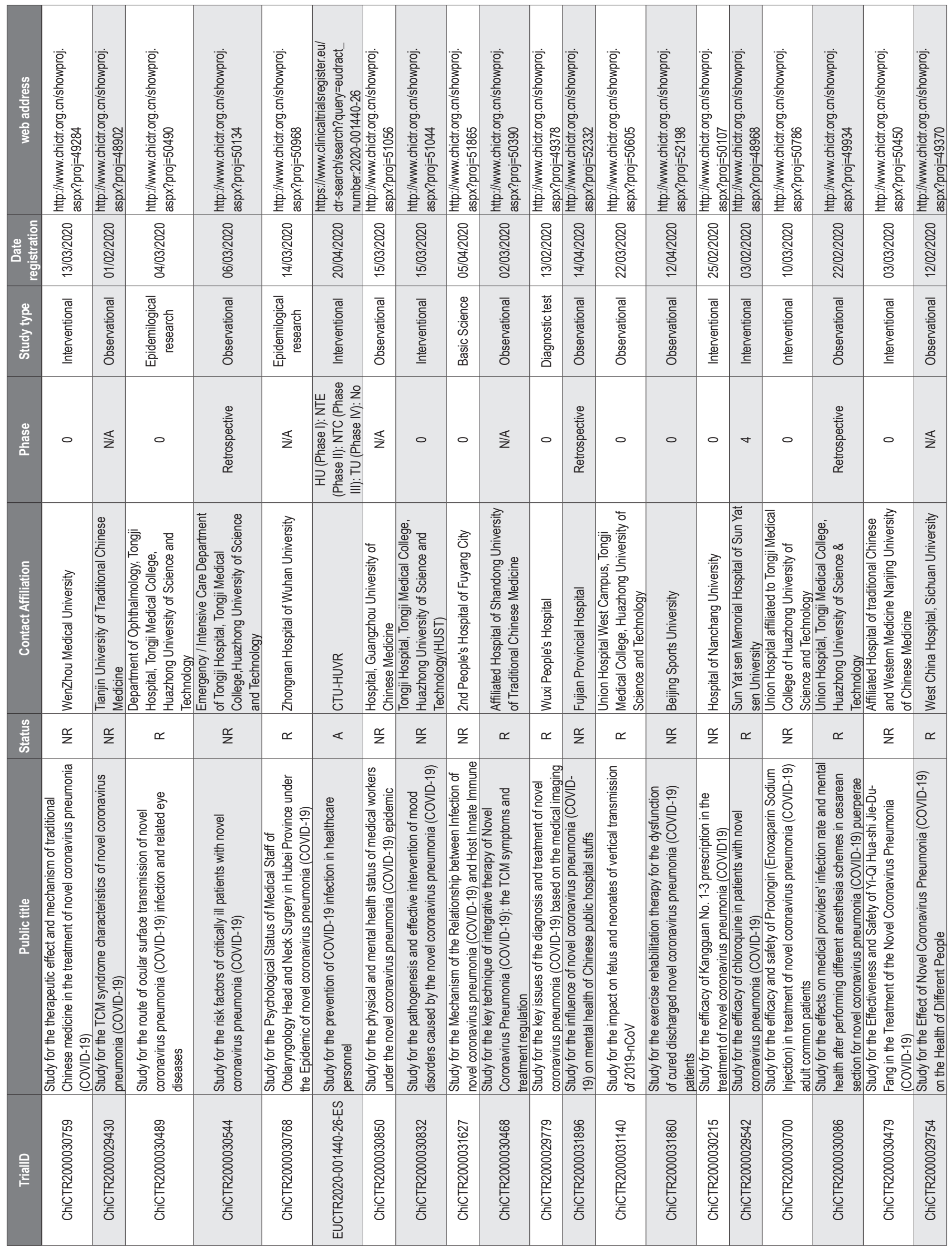




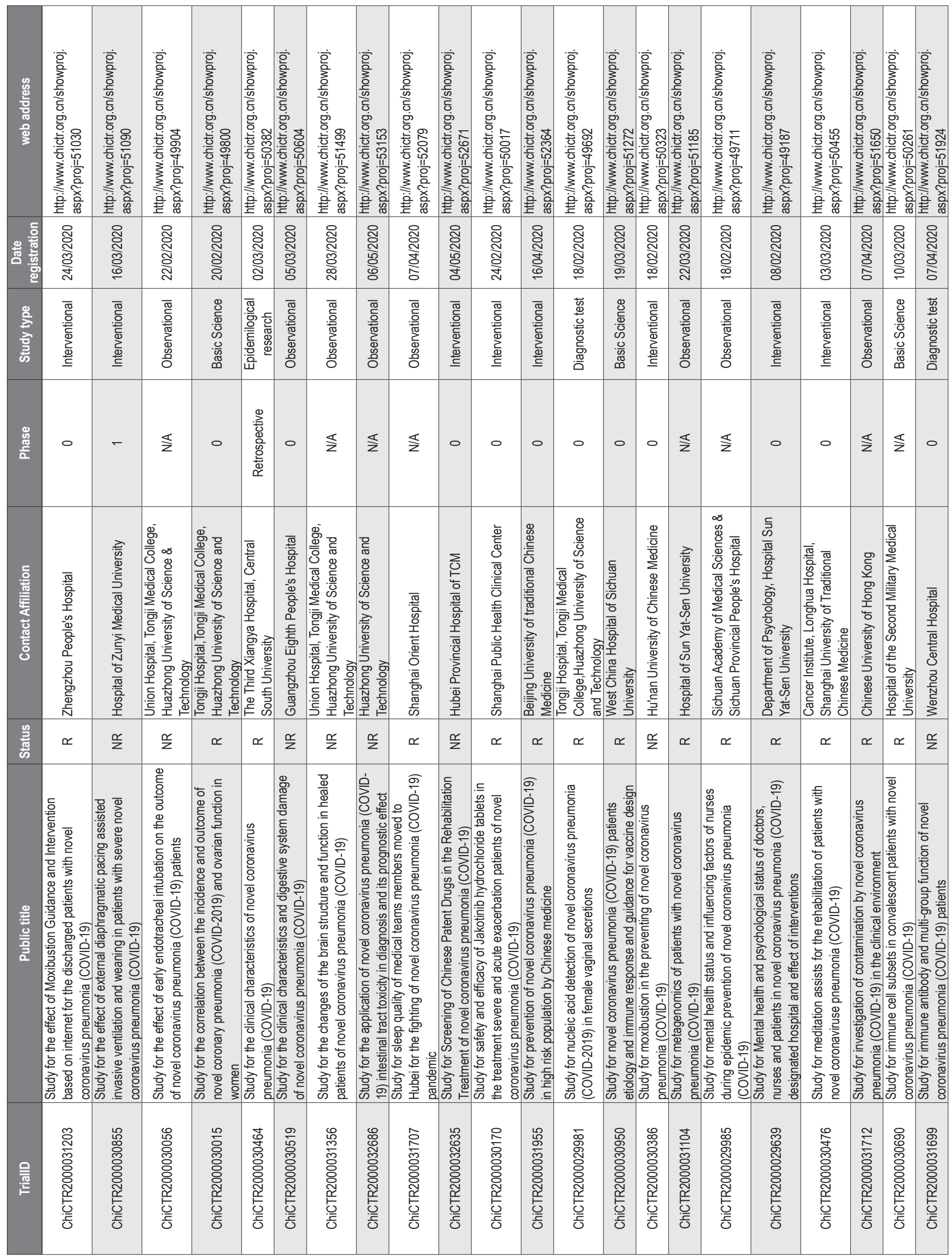




\begin{tabular}{|c|c|c|c|c|c|c|c|c|c|c|c|c|c|c|c|c|c|c|c|}
\hline 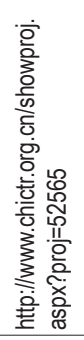 & 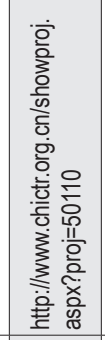 & 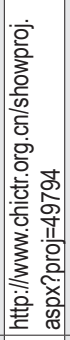 & 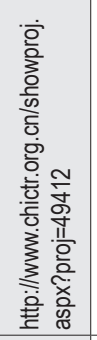 & 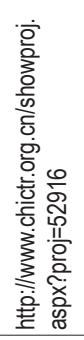 & 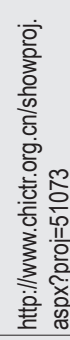 & 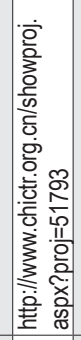 & 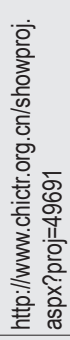 & 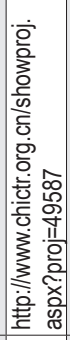 & 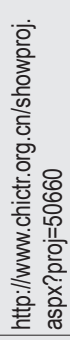 & 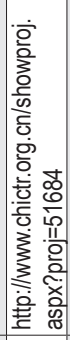 & 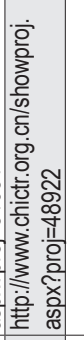 & 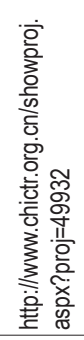 & 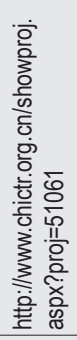 & 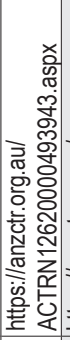 & 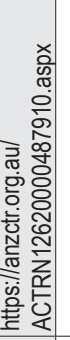 & 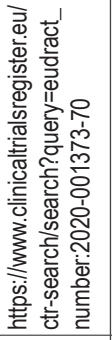 & 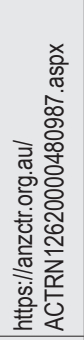 & 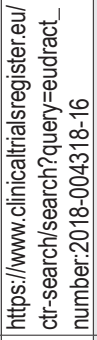 & 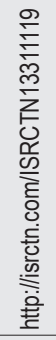 \\
\hline $\begin{array}{l}\text { ్ㅗ } \\
\text { 옹 } \\
\text { 을 }\end{array}$ & 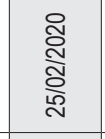 & 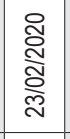 & 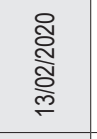 & $\begin{array}{l}\text { ్. } \\
\text { N. } \\
\text { 产 } \\
\text { N }\end{array}$ & $\begin{array}{l}\stackrel{్}{0} \\
\stackrel{\text { }}{0} \\
\stackrel{0}{0}\end{array}$ & 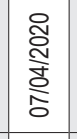 & 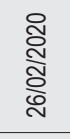 & 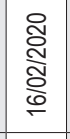 & 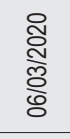 & 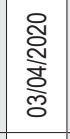 & 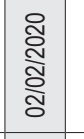 & $\begin{array}{l}\text { ָิ } \\
\text { స్ } \\
\text { స్ } \\
\end{array}$ & $\begin{array}{l}\text { న్ } \\
\stackrel{\text { }}{\infty} \\
\stackrel{\infty}{\circ}\end{array}$ & 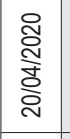 & 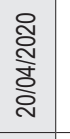 & 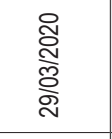 & $\begin{array}{l}\text { त्र } \\
\text { N̦ } \\
\text { 응 }\end{array}$ & 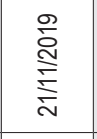 & 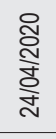 \\
\hline 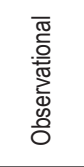 & 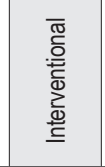 & 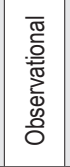 & 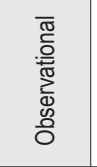 & 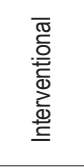 & 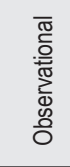 & 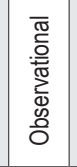 & 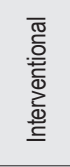 & 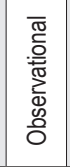 & 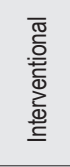 & 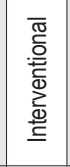 & 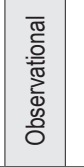 & 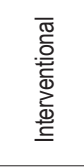 & 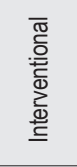 & 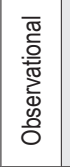 & 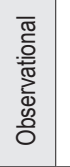 & 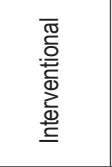 & 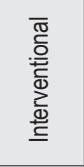 & 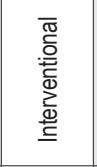 & 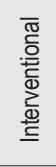 \\
\hline 0 & 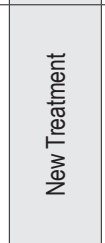 & $\stackrel{\mathbb{3}}{\mathbf{z}}$ & $\stackrel{\Sigma}{z}$ & 0 & $\stackrel{\underline{z}}{z}$ & $\stackrel{\mathbb{s}}{\mathbf{z}}$ & 0 & 0 & 0 & - & $\stackrel{5}{z}$ & $\stackrel{x}{z}$ & $\stackrel{x}{z}$ & $\stackrel{\mathbb{s}}{z}$ & $\stackrel{\$}{z}$ & 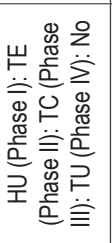 & $\stackrel{\mathbb{s}}{z}$ & 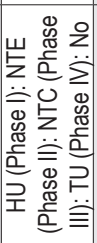 & 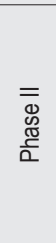 \\
\hline 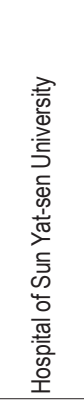 & 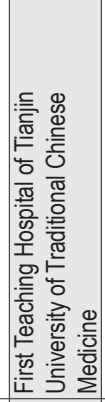 & 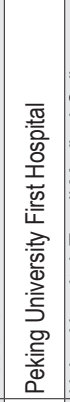 & 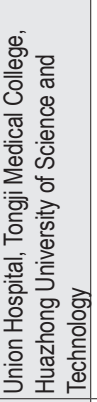 & 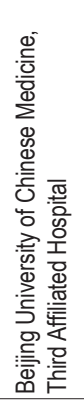 & 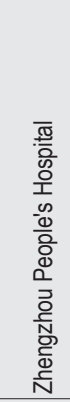 & 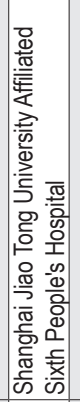 & 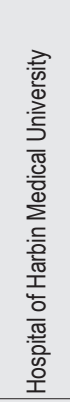 & 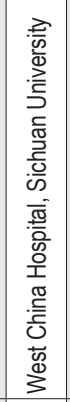 & 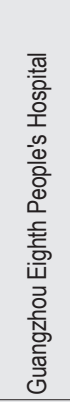 & 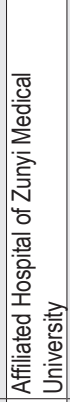 & 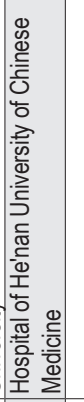 & 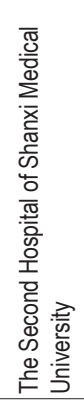 & 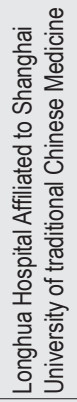 & & & 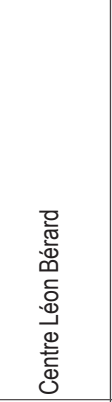 & & 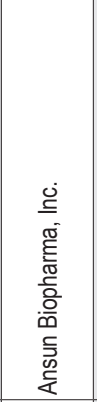 & \\
\hline
\end{tabular}

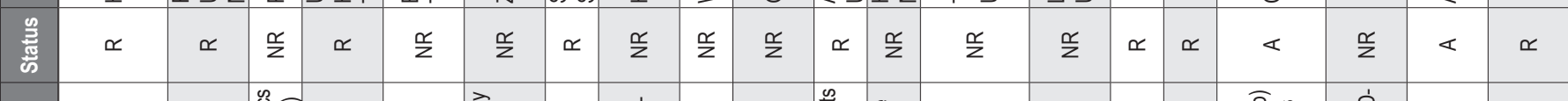

\begin{tabular}{|c|c|c|c|c|c|c|c|c|c|c|c|c|c|c|c|c|c|c|c|c|c|c|}
\hline 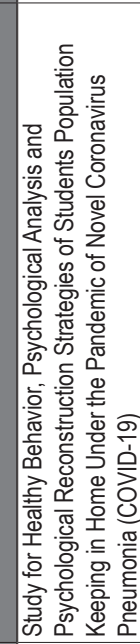 & 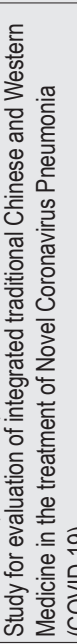 & 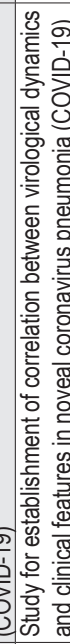 & 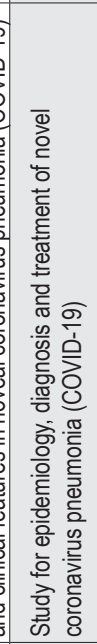 & 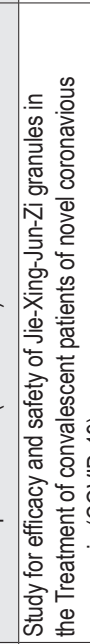 & 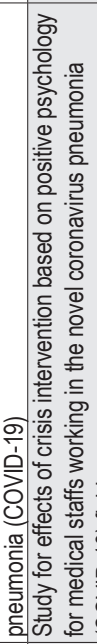 & 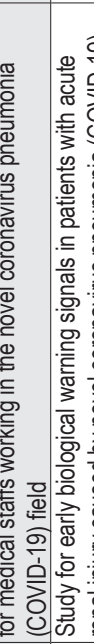 & 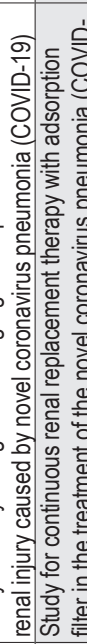 & 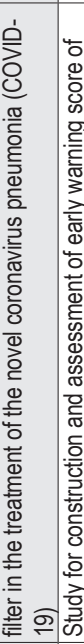 & 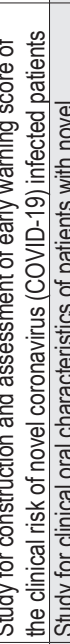 & 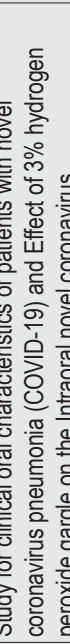 & 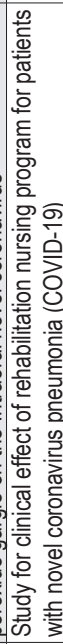 & 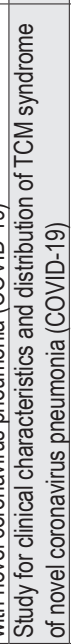 & 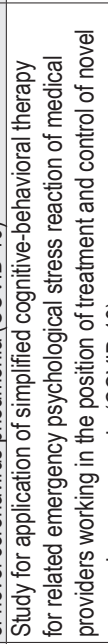 & 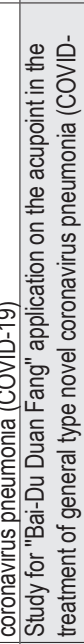 & & & & & & 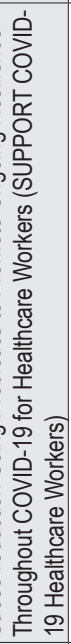 & 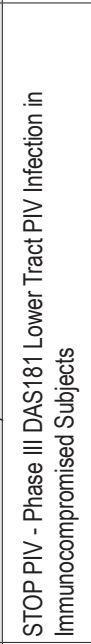 & 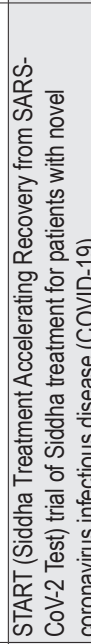 \\
\hline 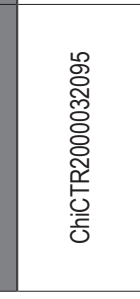 & 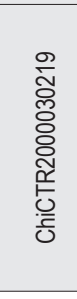 & 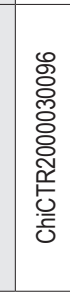 & 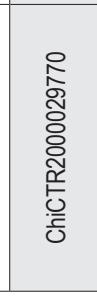 & 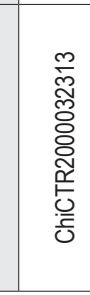 & 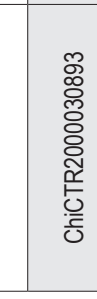 & to. & 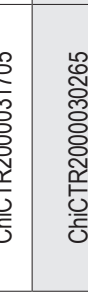 & & 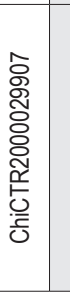 & 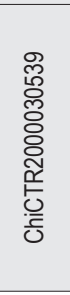 & 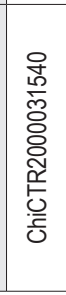 & 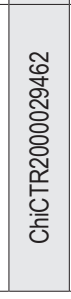 & 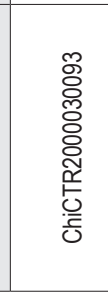 & & & & & 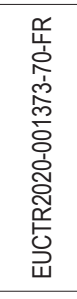 & & 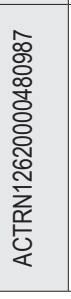 & $\widetilde{\Psi}$ & \\
\hline
\end{tabular}




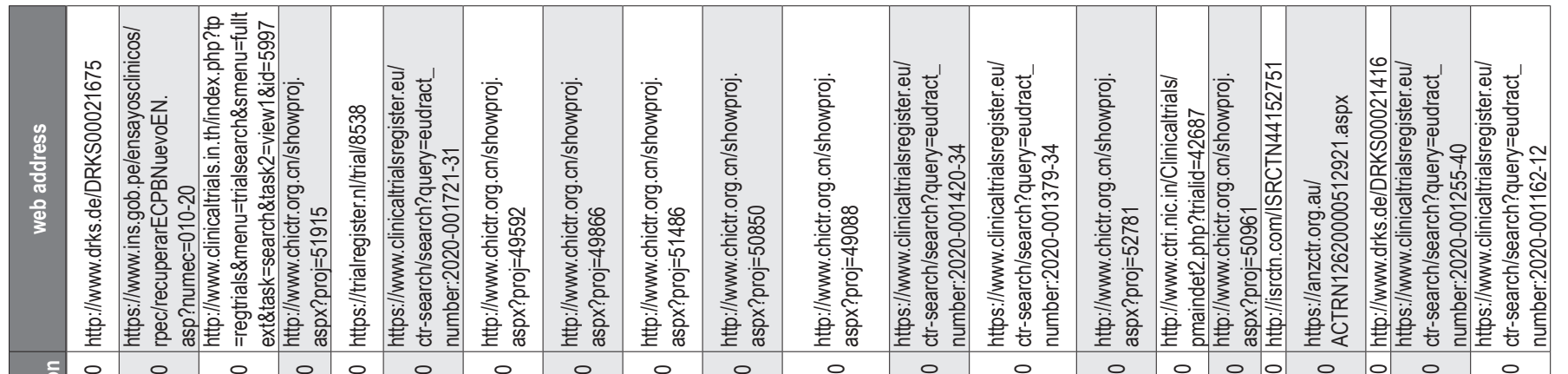

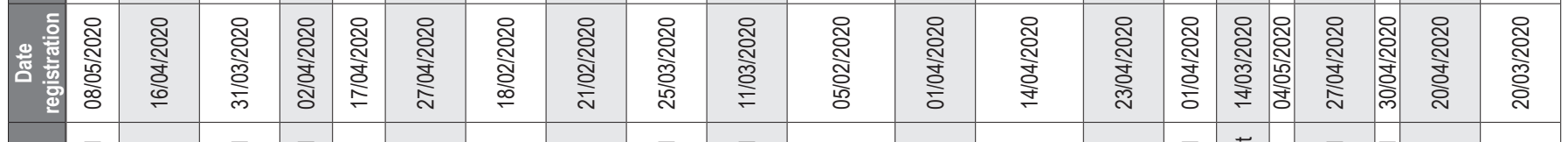

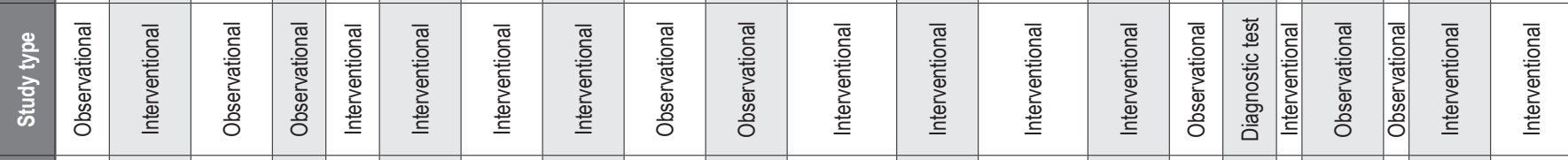

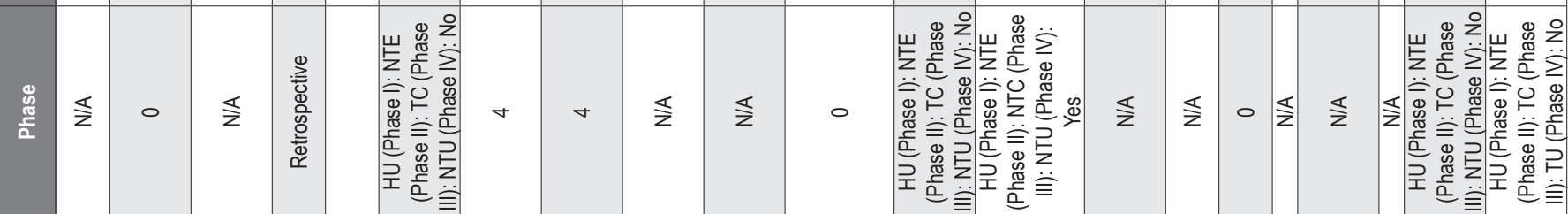

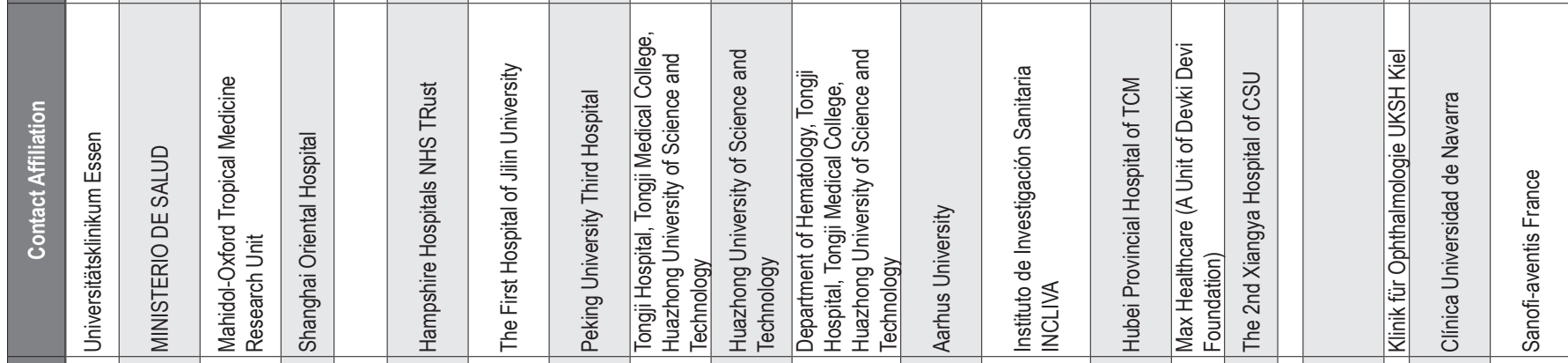

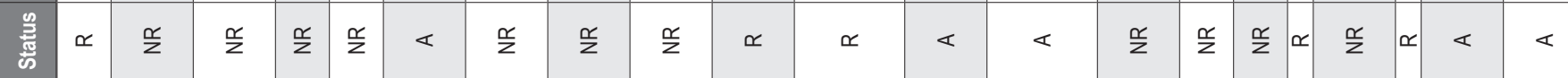

\begin{tabular}{|c|c|c|c|c|c|c|c|c|c|c|c|c|c|c|c|c|c|c|c|}
\hline 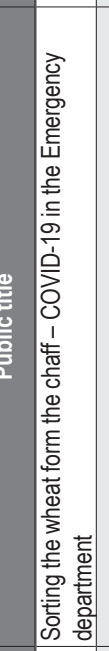 & 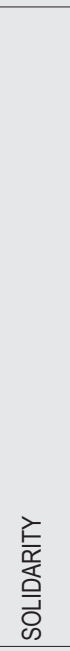 & 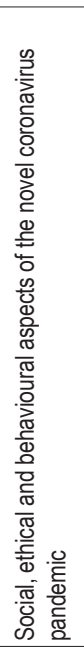 & 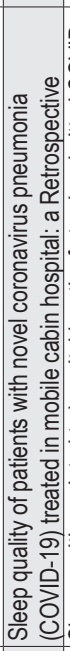 & 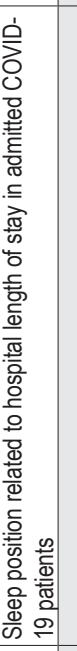 & 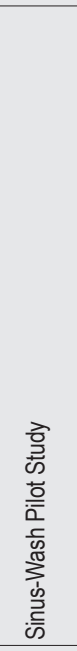 & 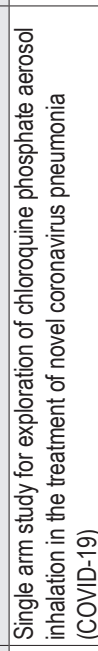 & 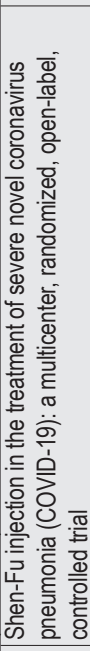 & 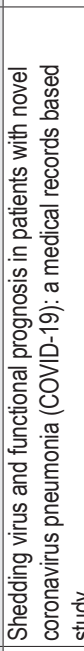 & 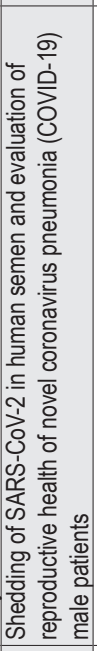 & 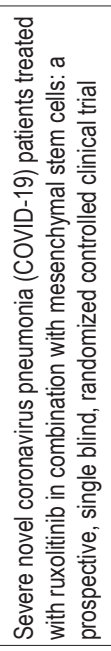 & 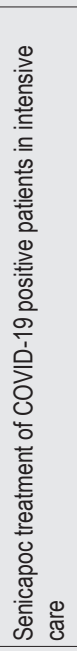 & 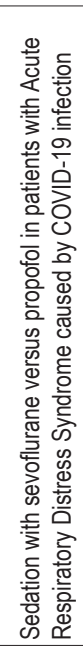 & 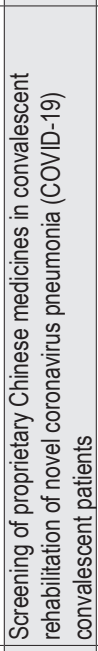 & 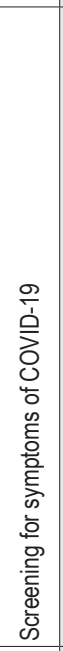 & 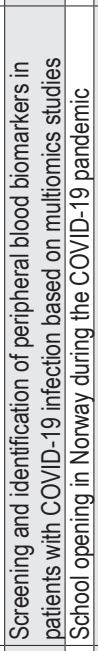 & 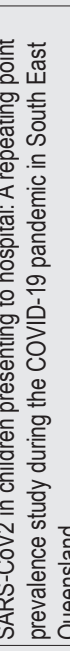 & 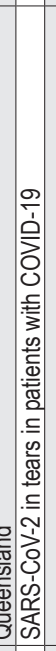 & 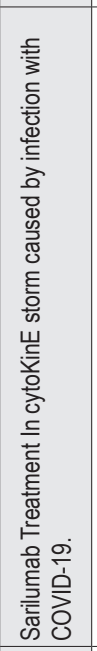 & 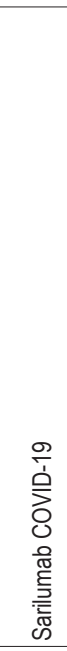 \\
\hline 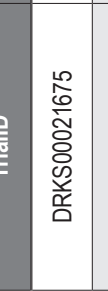 & 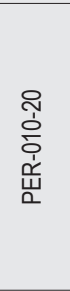 & 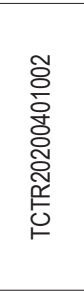 & 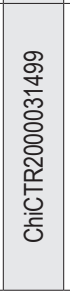 & 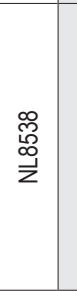 & 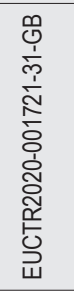 & 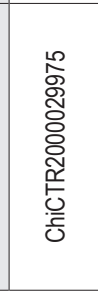 & 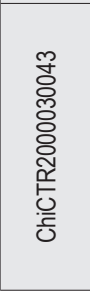 & 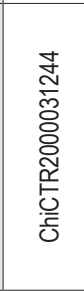 & 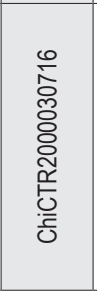 & 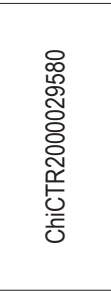 & 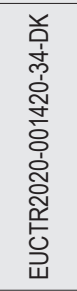 & 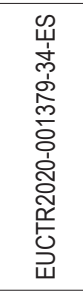 & 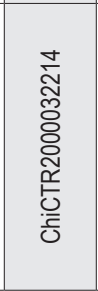 & 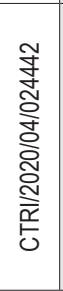 & 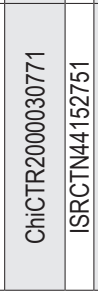 & 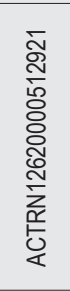 & 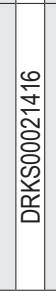 & 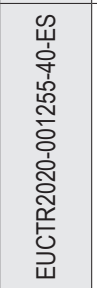 & \\
\hline
\end{tabular}




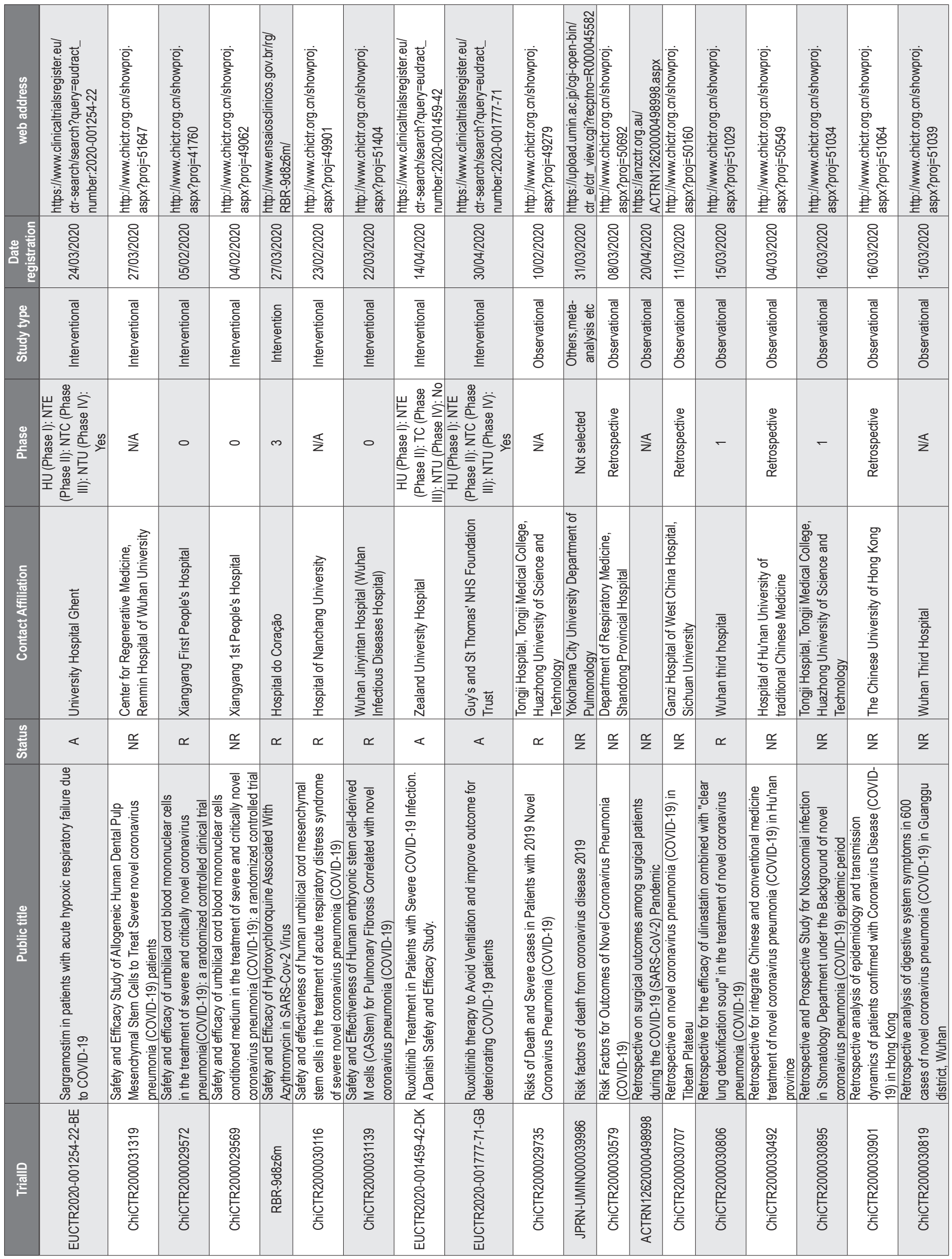




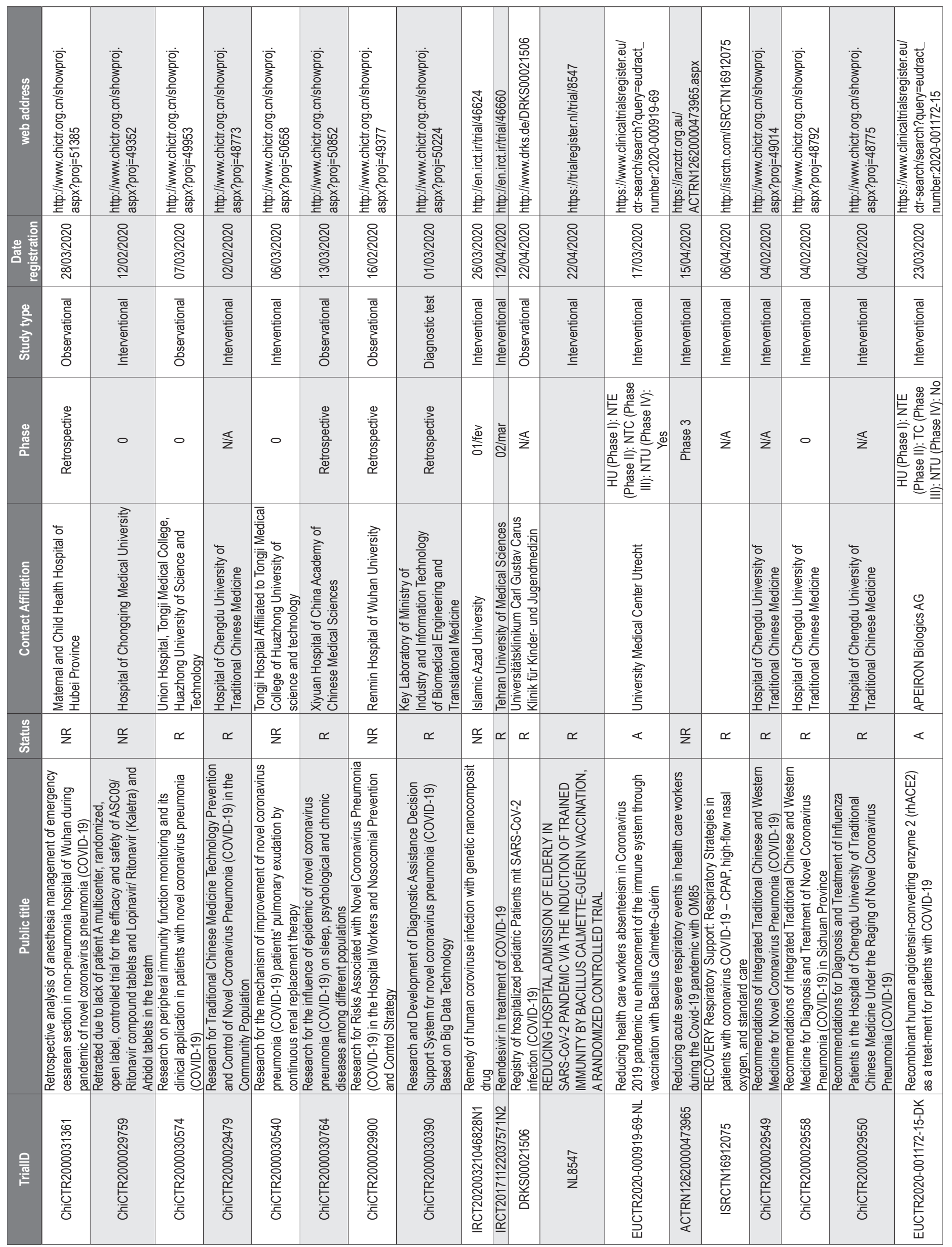




\begin{tabular}{|c|c|c|c|c|c|c|c|c|c|c|c|c|c|c|c|c|c|c|}
\hline 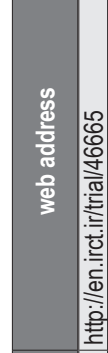 & 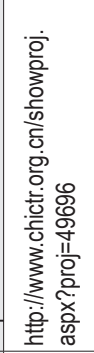 & 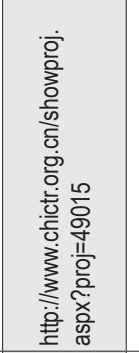 & 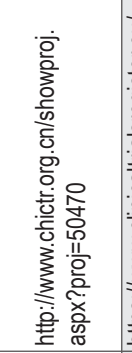 & 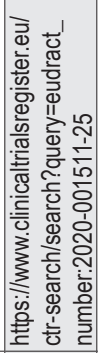 & 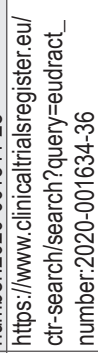 & 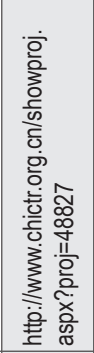 & 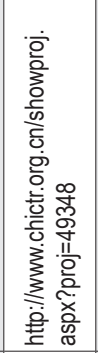 & 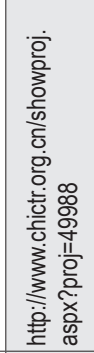 & 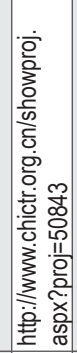 & 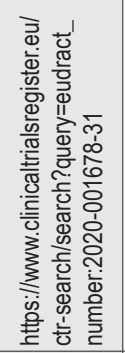 & 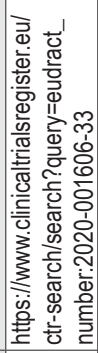 & 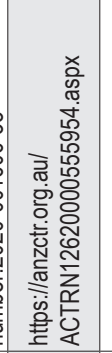 & 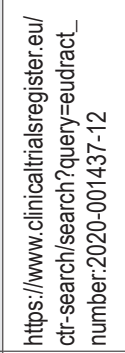 & 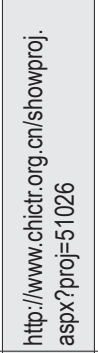 & 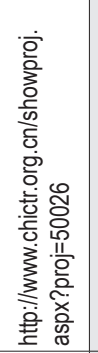 & 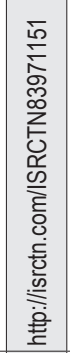 & 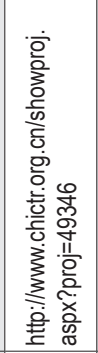 & 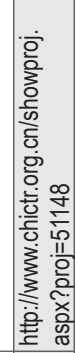 \\
\hline 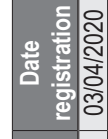 & 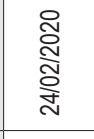 & $\begin{array}{l}\text { స్ } \\
\text { నิ } \\
\text { ঠे }\end{array}$ & 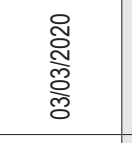 & $\begin{array}{l}\text { कूे } \\
\text { के } \\
\text { के } \\
\text { के }\end{array}$ & 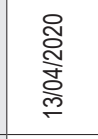 & 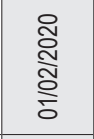 & 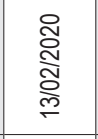 & 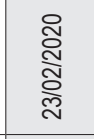 & 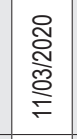 & 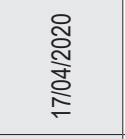 & 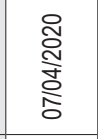 & 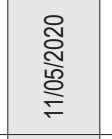 & 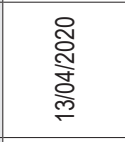 & $\begin{array}{l}\text { ్ָ } \\
\text { ָू }\end{array}$ & 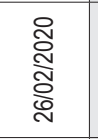 & 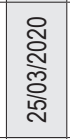 & 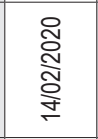 & ָָ \\
\hline 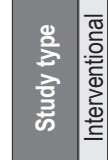 & 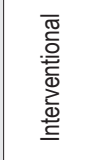 & 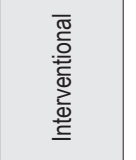 & 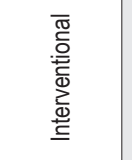 & 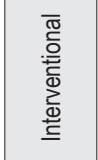 & 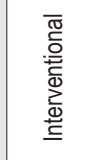 & 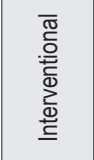 & 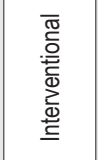 & 壳 & 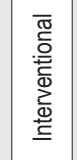 & 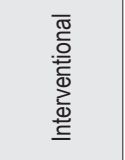 & $\sum_{0,0}^{\bar{d}}$ & 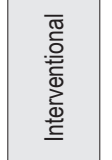 & $\sum_{\substack{\infty \\
0}}^{\infty}$ & 产 & 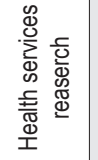 & 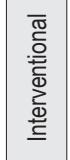 & 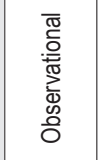 & 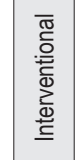 \\
\hline & 0 & 0 & $\checkmark$ & 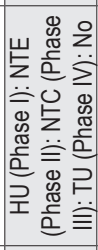 & 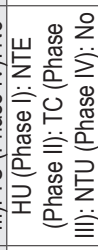 & 0 & 0 & 0 & $\nabla$ & 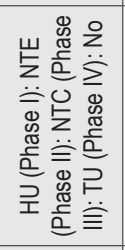 & 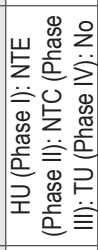 & 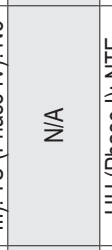 & 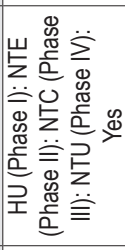 & ب. & $\stackrel{s}{z}$ & $\begin{array}{l}\overline{8} \\
\text { o. } \\
\frac{0}{\alpha}\end{array}$ & $\frac{1}{z}$ & $\frac{s}{z}$ \\
\hline 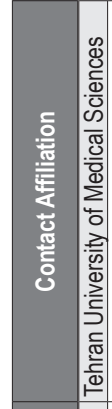 & 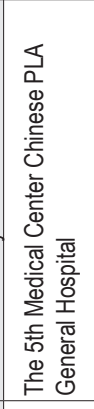 & 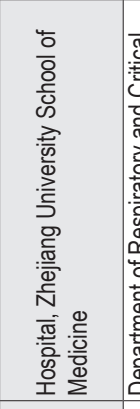 & 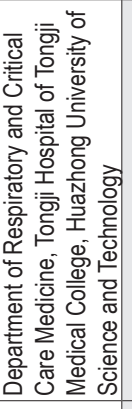 & 要 & 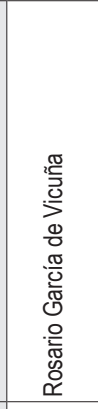 & 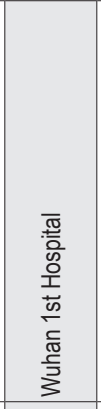 & 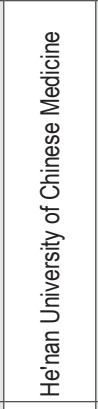 & 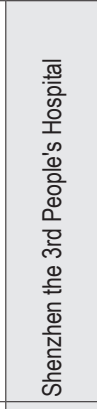 & 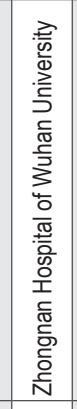 & 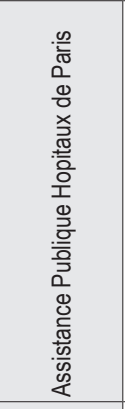 & 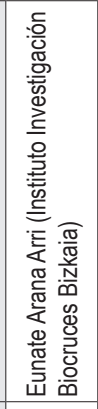 & & 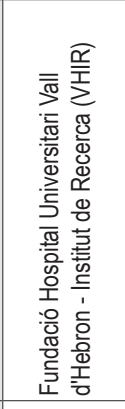 & 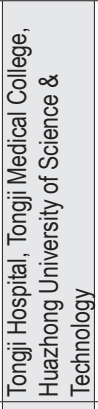 & 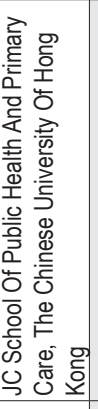 & & 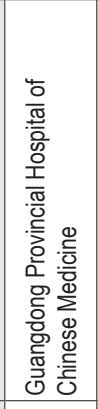 & 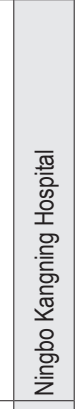 \\
\hline & $\frac{x}{z}$ & $\frac{\alpha}{z}$ & $\simeq$ & $\ll$ & $\ll$ & $\frac{\alpha}{z}$ & $\propto$ & $\propto$ & $\propto$ & & & $\frac{\alpha}{2}$ & & $\frac{\underline{\alpha}}{z}$ & $\propto$ & $\simeq$ & $\alpha$ & $\frac{\underline{\alpha}}{z}$ \\
\hline E. & 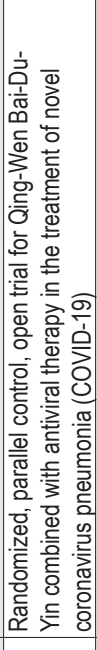 & 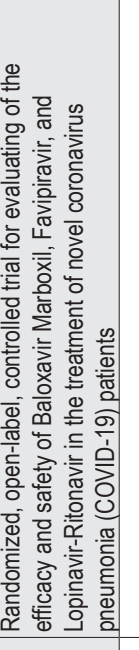 & 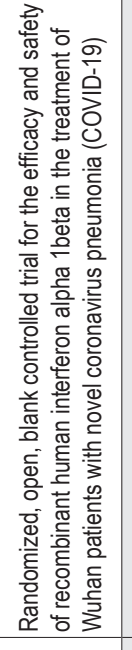 & 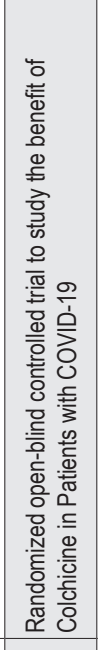 & 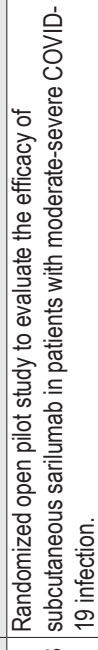 & 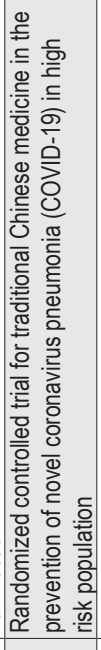 & 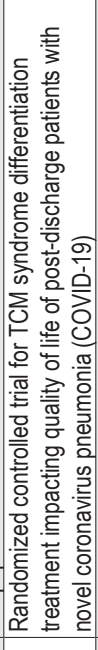 & 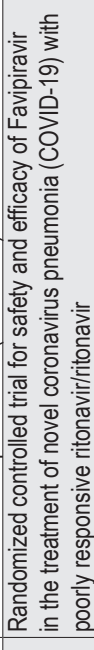 & 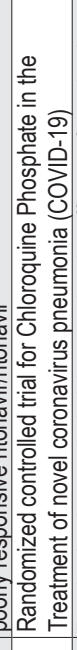 & 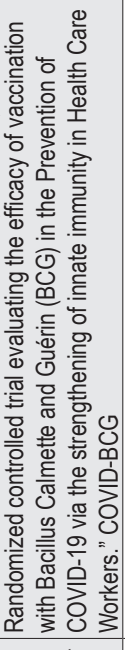 & 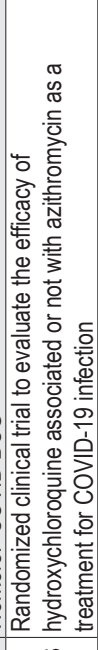 & 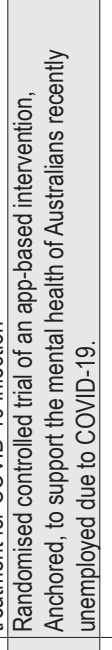 & 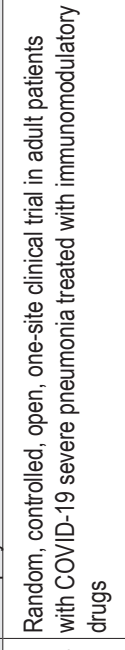 & 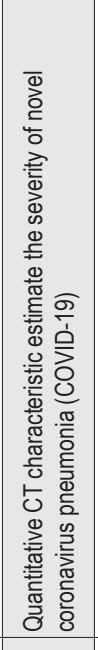 & 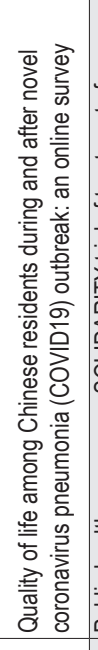 & 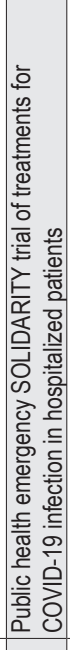 & 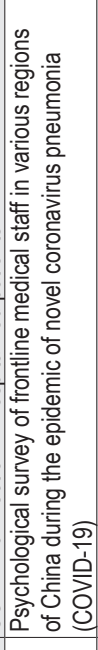 & 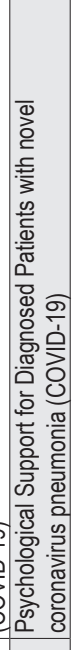 \\
\hline$\frac{f}{5}$ & 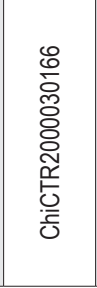 & 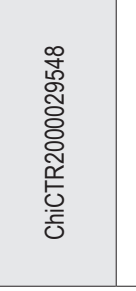 & 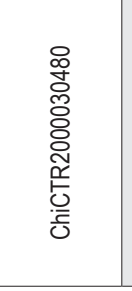 & 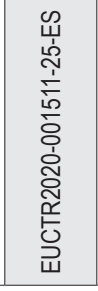 & 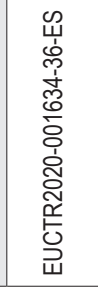 & 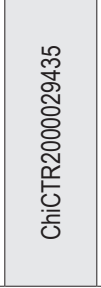 & 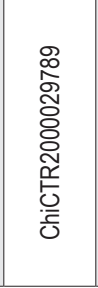 & 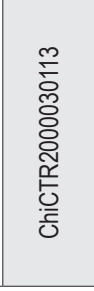 & 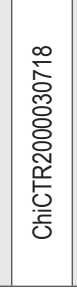 & 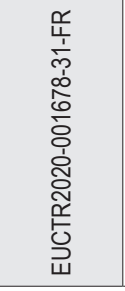 & 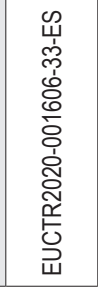 & 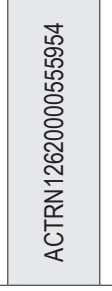 & 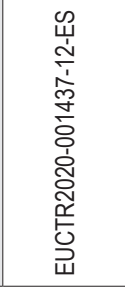 & 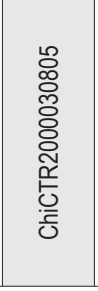 & 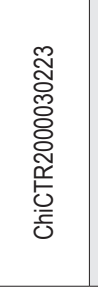 & 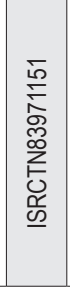 & 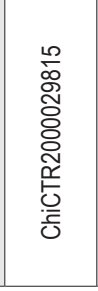 & 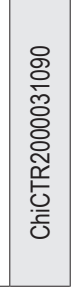 \\
\hline
\end{tabular}




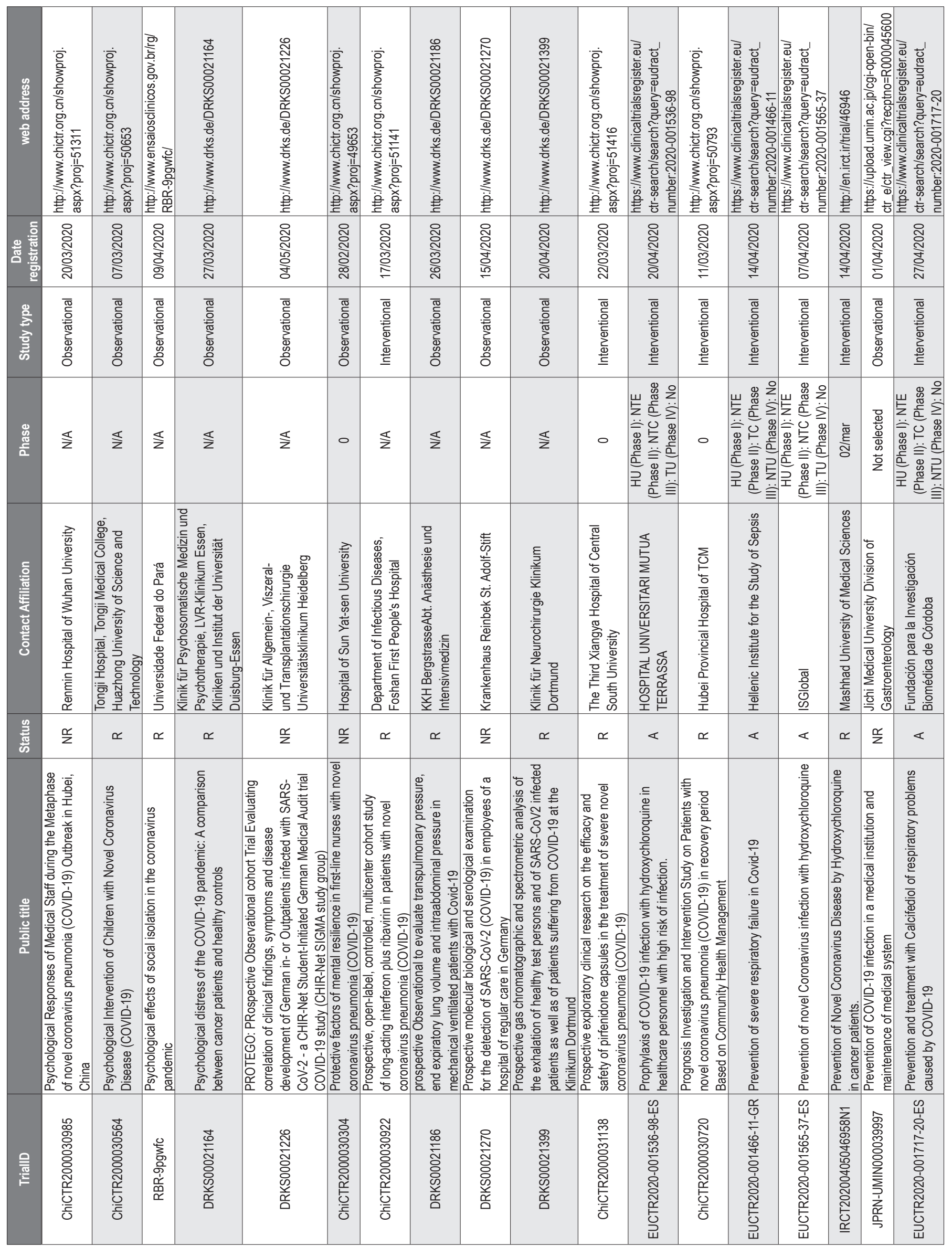




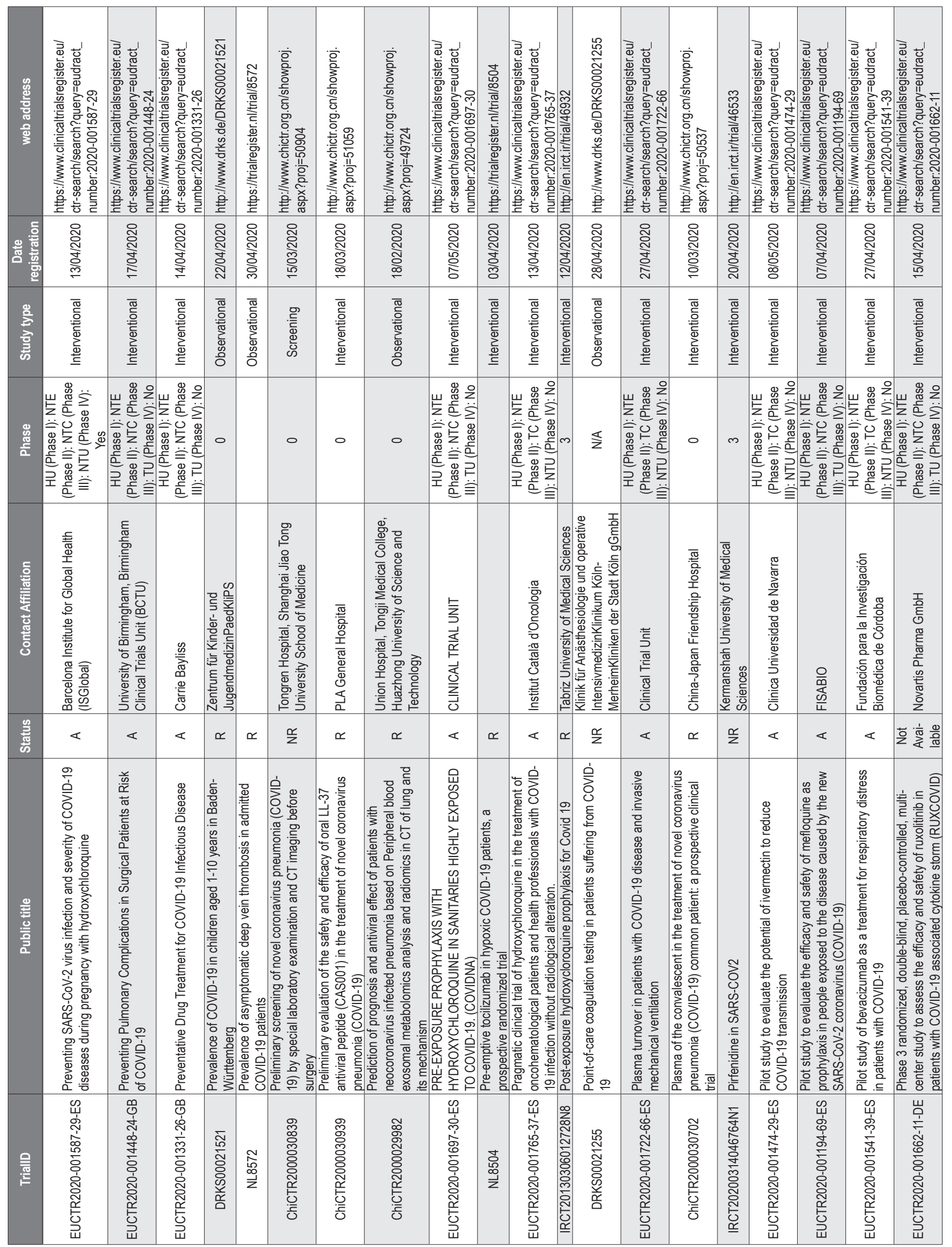




\begin{tabular}{|c|c|c|c|c|c|c|c|c|c|c|c|c|c|c|c|c|c|c|c|}
\hline 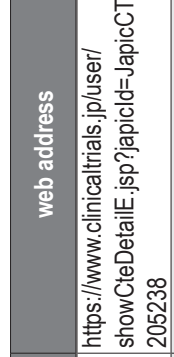 & 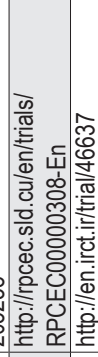 & 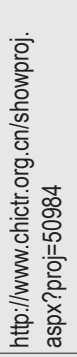 & 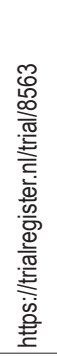 & 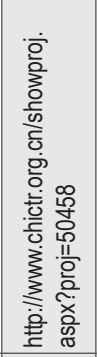 & 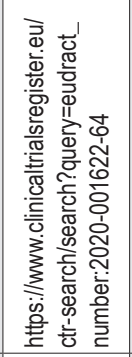 & 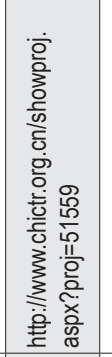 & 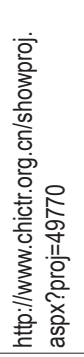 & 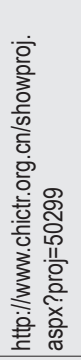 & 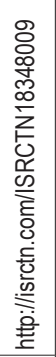 & 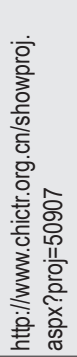 & 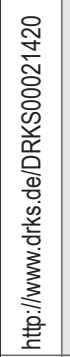 & 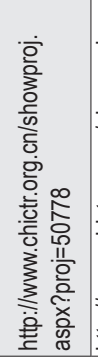 & 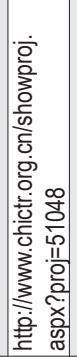 & 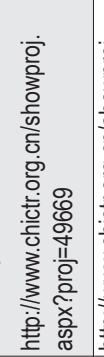 & 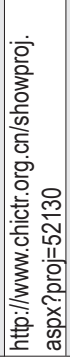 & 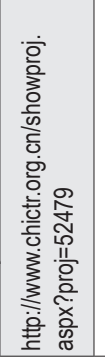 & 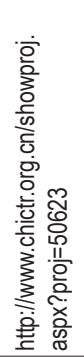 & 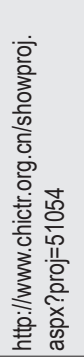 & 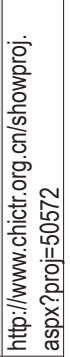 \\
\hline 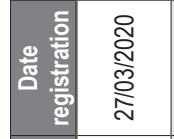 & 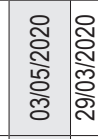 & 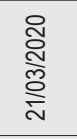 & 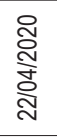 & 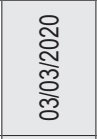 & 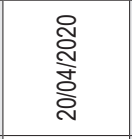 & 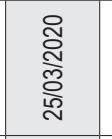 & 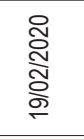 & 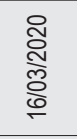 & 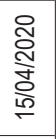 & 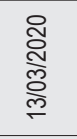 & 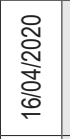 & 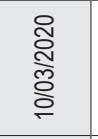 & 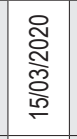 & 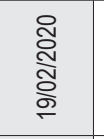 & 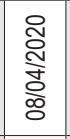 & 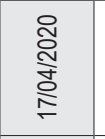 & 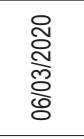 & 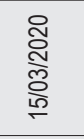 & \begin{tabular}{|l|} 
న్ \\
ô. \\
o. \\
\end{tabular} \\
\hline 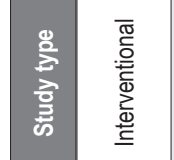 & 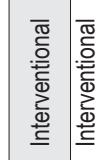 & 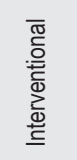 & 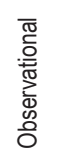 & 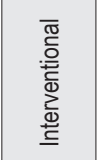 & 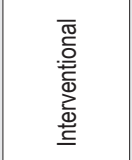 & 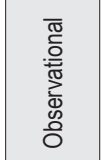 & 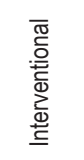 & 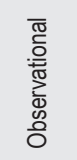 & 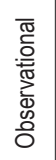 & 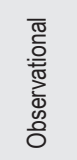 & 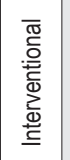 & 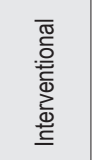 & 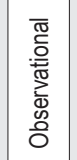 & 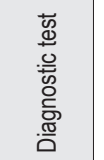 & 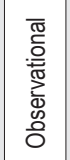 & 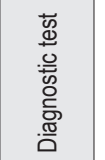 & 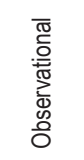 & 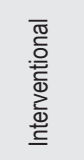 & 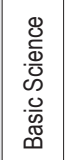 \\
\hline$m$ & - & 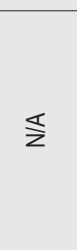 & & 0 & 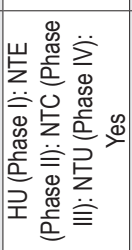 & $z$ & $\$$ & 0 & 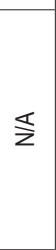 & 0 & - & $\nabla$ & 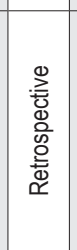 & $\stackrel{\frac{1}{z}}{z}$ & $\underline{z}$ & 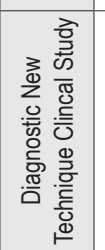 & $\frac{s}{z}$ & $\sigma$ & \\
\hline & 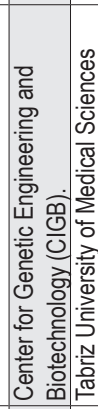 & 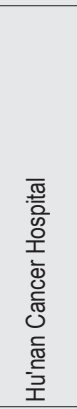 & & 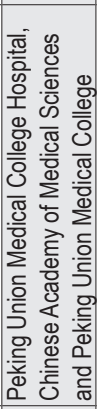 & 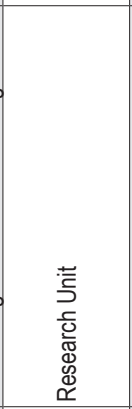 & 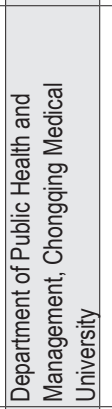 & 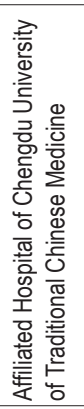 & 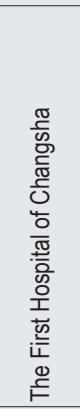 & & 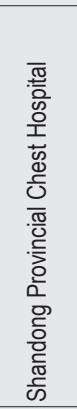 & 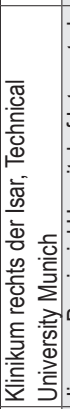 & 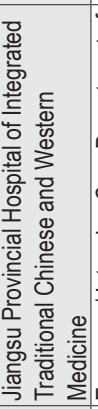 & 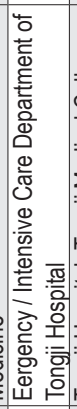 & 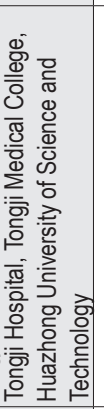 & 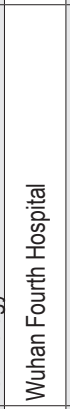 & 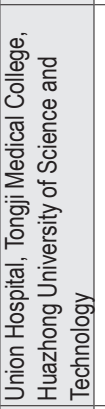 & 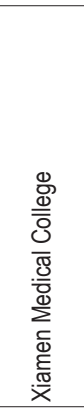 & 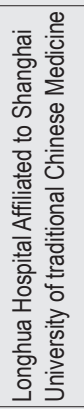 & 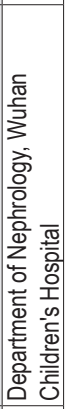 \\
\hline
\end{tabular}

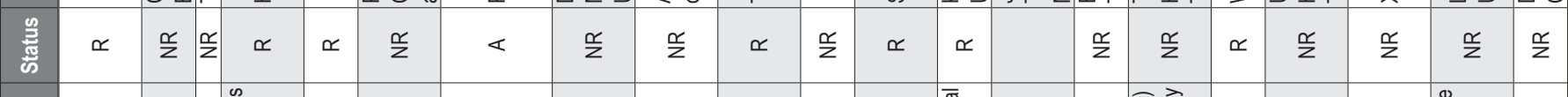

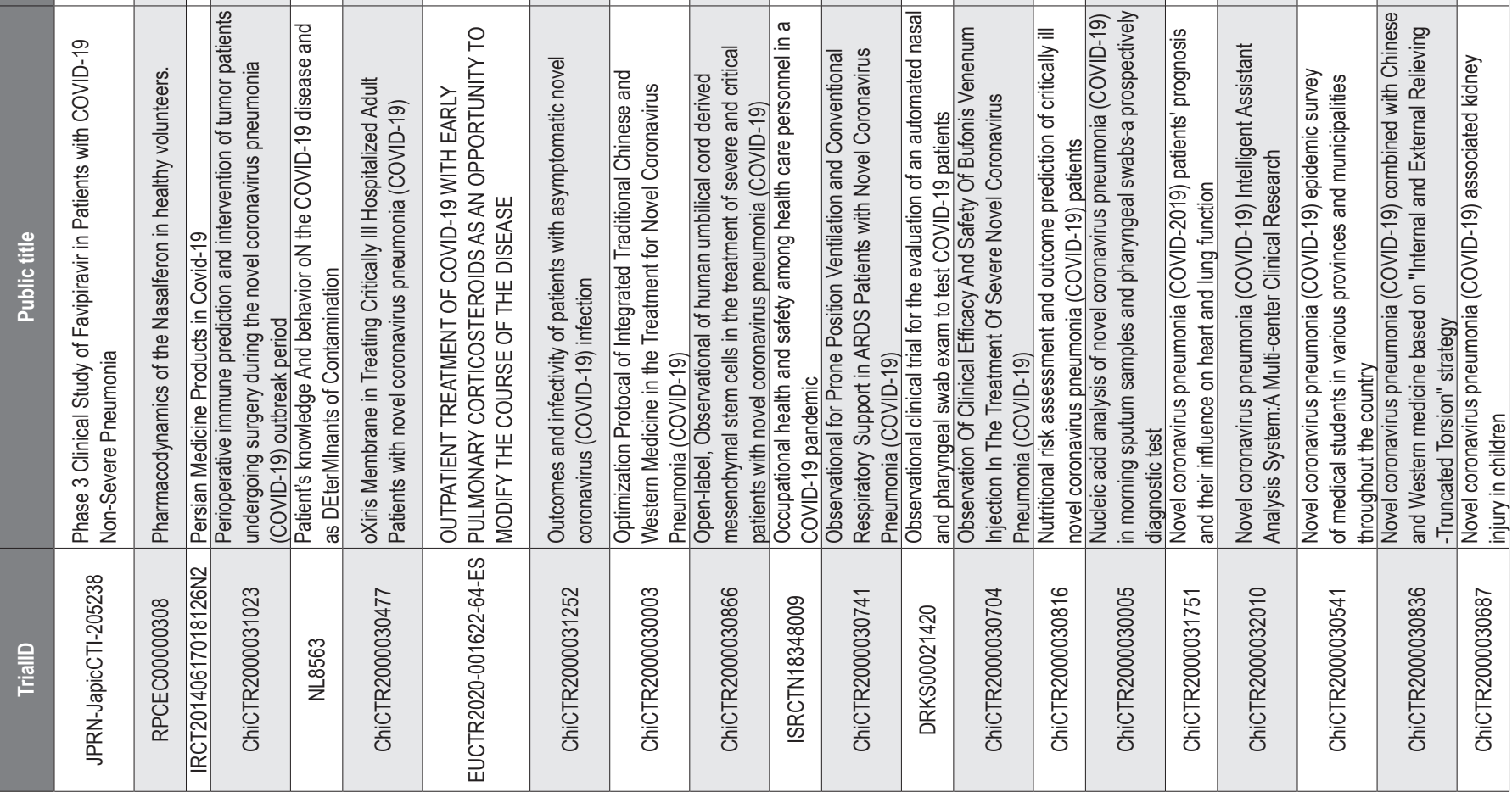




\begin{tabular}{|c|c|c|c|c|c|c|c|c|c|c|c|c|c|c|c|c|c|}
\hline 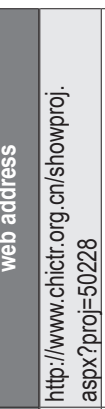 & 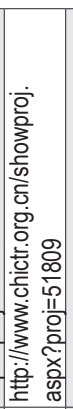 & 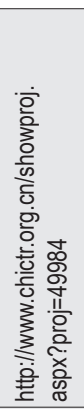 & 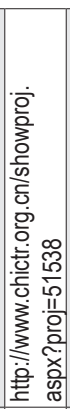 & 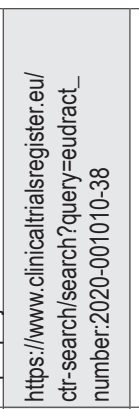 & 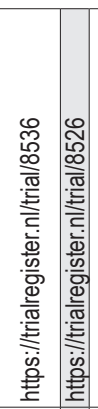 & 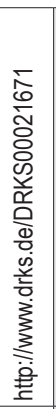 & 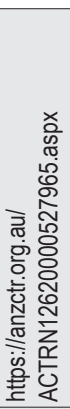 & 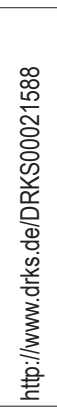 & 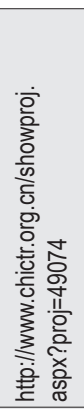 & 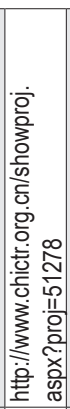 & 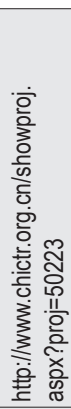 & 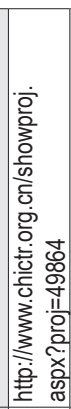 & 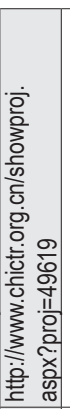 & 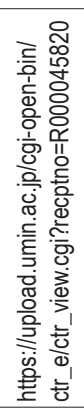 & 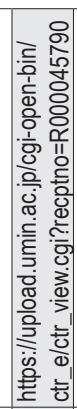 & 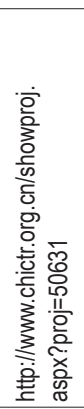 & 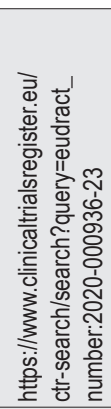 \\
\hline 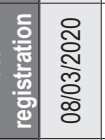 & 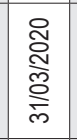 & 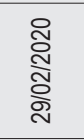 & 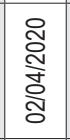 & 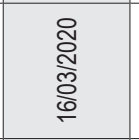 & 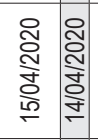 & 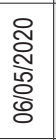 & 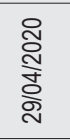 & 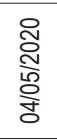 & 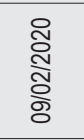 & 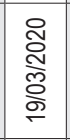 & 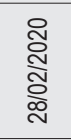 & 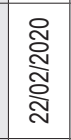 & $\begin{array}{l}\text { ญ్ } \\
\text { ญิ } \\
\text { ळ }\end{array}$ & 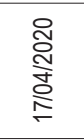 & 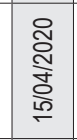 & 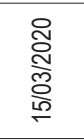 & 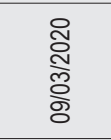 \\
\hline 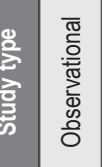 & 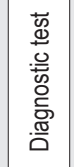 & 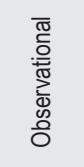 & 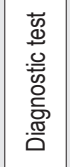 & 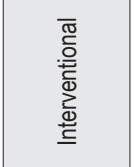 & 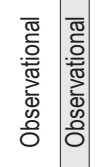 & 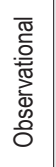 & 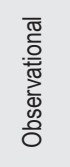 & 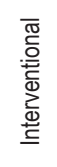 & 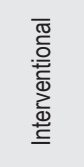 & 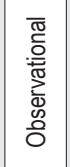 & 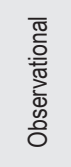 & 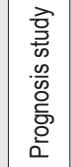 & 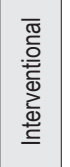 & 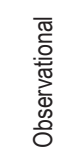 & 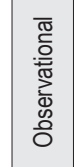 & 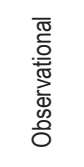 & 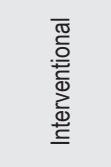 \\
\hline 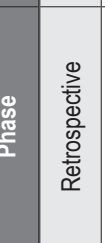 & 0 & $\frac{\pi}{z}$ & 0 & 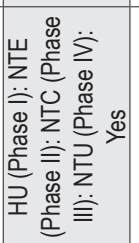 & & & $\stackrel{\varsigma}{z}$ & $\stackrel{s}{z}$ & $\underline{\Sigma}$ & 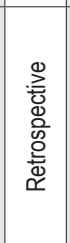 & 0 & 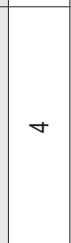 & 0 & $\frac{\pi}{z}$ & 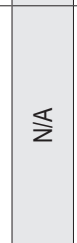 & 0 & 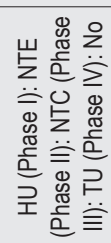 \\
\hline
\end{tabular}

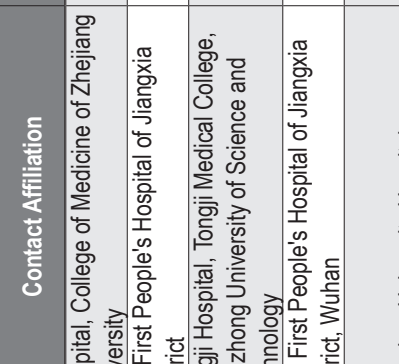

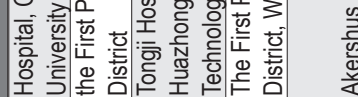

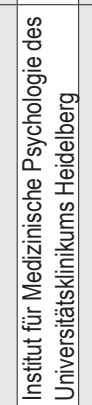

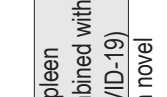

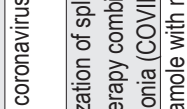

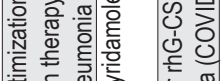

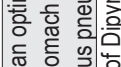

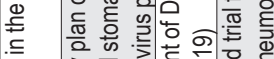
总 卷 엉 突 응 은 흔 高高

$\frac{1}{1}$

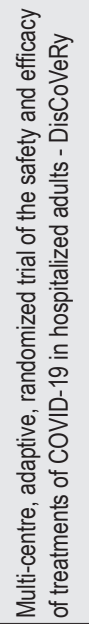

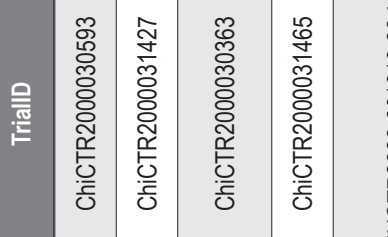
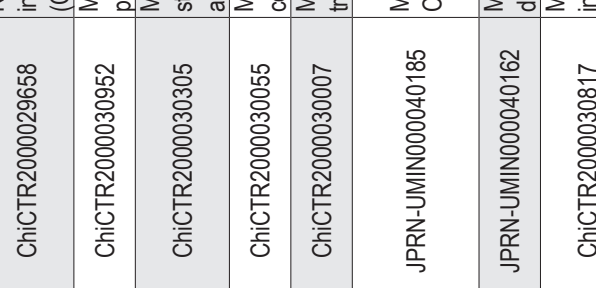

똟 


\begin{tabular}{|c|c|c|c|c|c|c|c|c|c|c|c|c|c|c|c|c|c|c|c|c|c|c|c|}
\hline 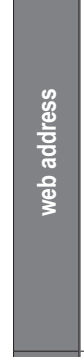 & 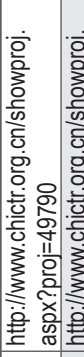 & 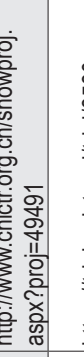 & 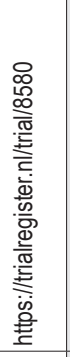 & 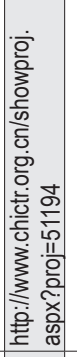 & 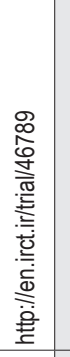 & 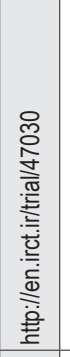 & 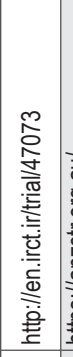 & 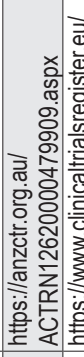 & 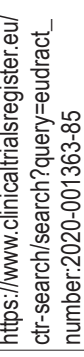 & 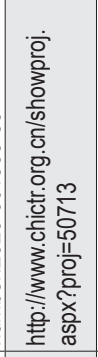 & 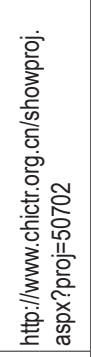 & 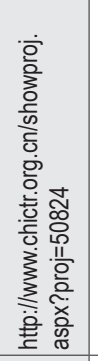 & 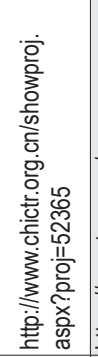 & 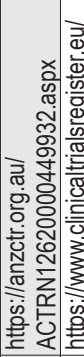 & & 产 & 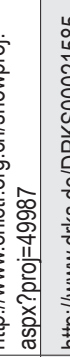 & 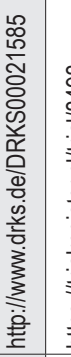 & 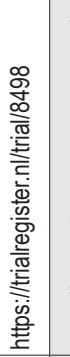 & 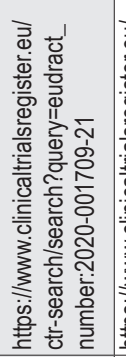 & 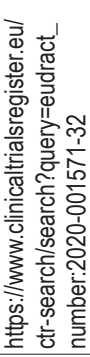 & 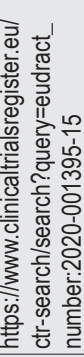 & 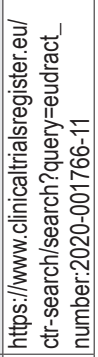 \\
\hline & \begin{tabular}{|l|} 
్. \\
N. \\
응 \\
\end{tabular} & 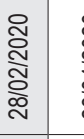 & 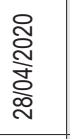 & 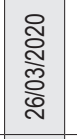 & 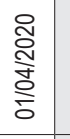 & \begin{tabular}{|l|} 
్. \\
No \\
क्. \\
\end{tabular} & 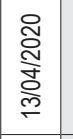 & 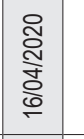 & 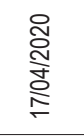 & 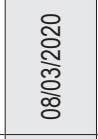 & 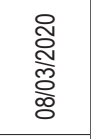 & 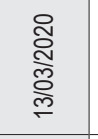 & 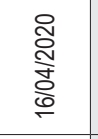 & 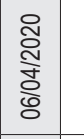 & 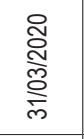 & 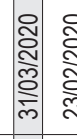 & & 尺ี & 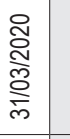 & 0 & 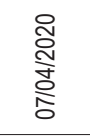 & 管 & 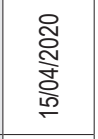 \\
\hline 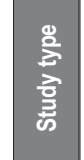 & 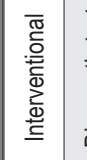 & 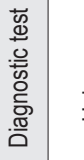 & 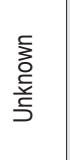 & 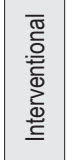 & 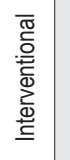 & 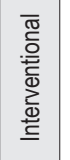 & 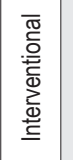 & $\begin{array}{l}\text { 흘 } \\
\text { 总 } \\
\text { o. } \\
\text { o }\end{array}$ & 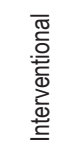 & 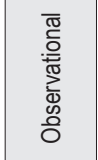 & 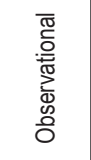 & 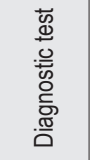 & d) & 胥 & 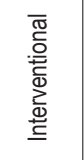 & & & 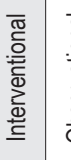 & $\mathbb{\pi}$ & 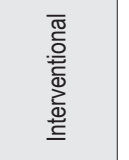 & 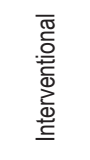 & & 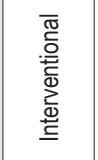 \\
\hline 窇 & $\sigma$ & 0 & & 0 & - & $\frac{\vec{\omega}}{\sigma}$ & 胥 & 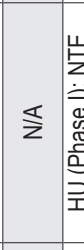 & 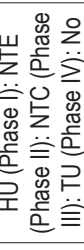 & $\begin{array}{l}\text { o. } \\
\text { oñ } \\
\text { ond }\end{array}$ & 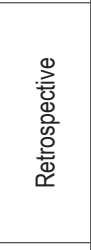 & 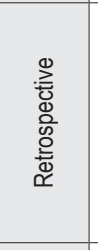 & 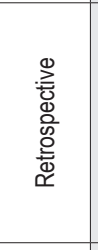 & 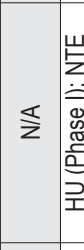 & 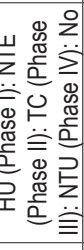 & & $\leqq$ & $\frac{5}{z}$ & & 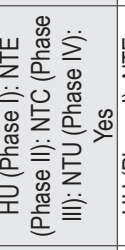 & 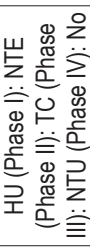 & 는 & 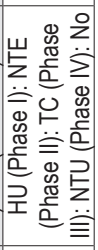 \\
\hline & 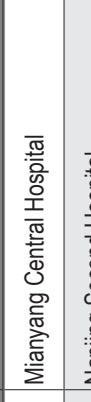 & 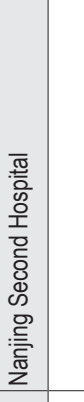 & & 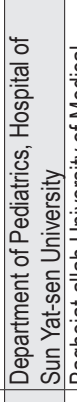 & & 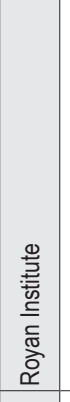 & 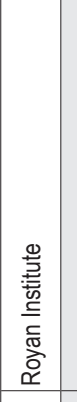 & & 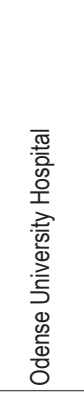 & 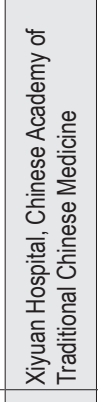 & 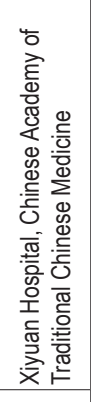 & 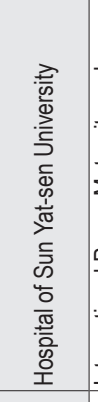 & 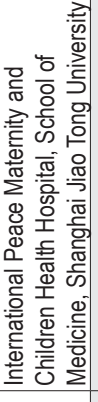 & & 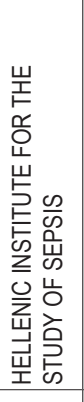 & & 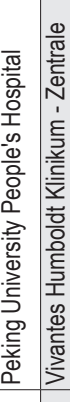 & 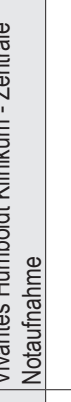 & & $\begin{array}{l}\frac{\underline{\pi}}{2} \\
\mathbb{0} \\
\underline{0} \\
\underline{\underline{\underline{x}}}\end{array}$ & 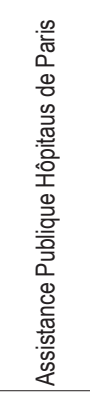 & 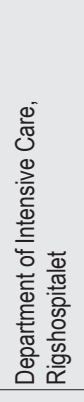 & 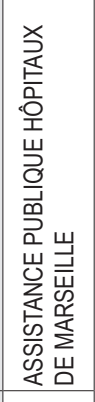 \\
\hline 㺼 & $\alpha$ & $\propto$ & $\simeq$ & $\frac{\alpha}{z}$ & $\frac{\alpha}{z}$ & 号 & $\propto$ & $\frac{x}{z}$ & 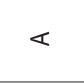 & $\frac{\alpha}{z}$ & $\frac{\alpha}{z}$ & $\frac{\underline{x}}{z}$ & $\propto$ & $\frac{\alpha}{z}$ & $\varangle$ & $\propto \frac{\alpha}{2}$ & $\frac{⿱}{z}$ & $\propto$ & & & & & \\
\hline $\begin{array}{l}\frac{0}{5} \\
\frac{0}{0}\end{array}$ & 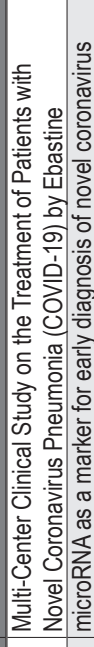 & 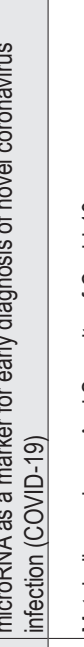 & 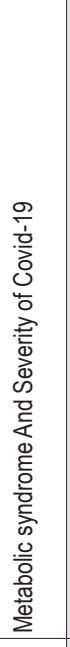 & 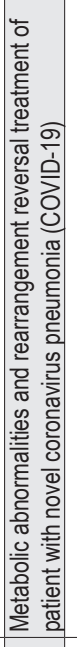 & 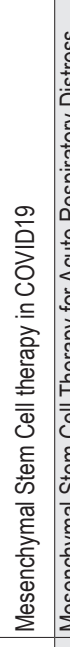 & 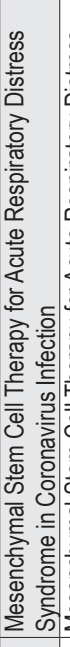 & 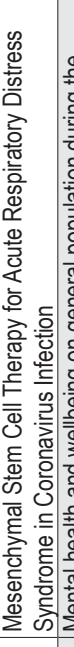 & 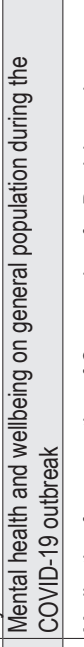 & 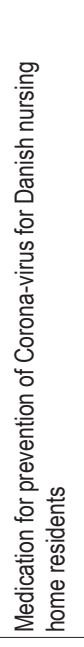 & 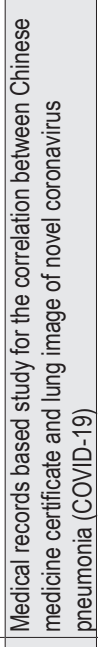 & 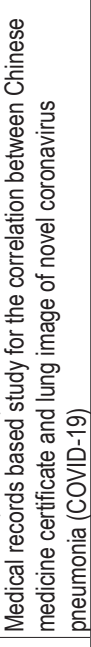 & 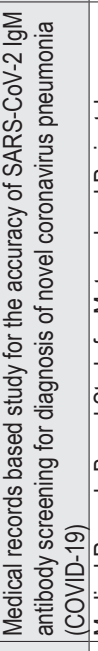 & 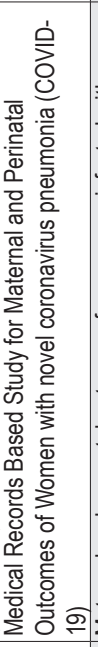 & 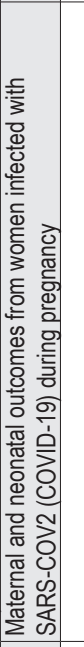 & 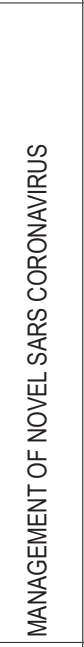 & 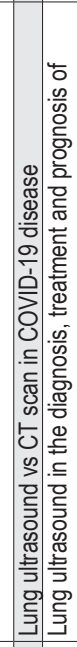 & 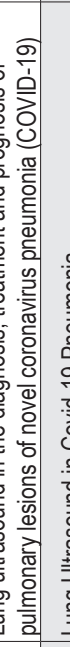 & 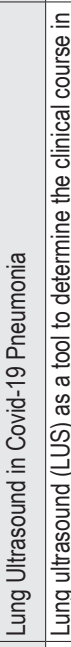 & 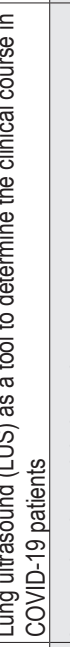 & 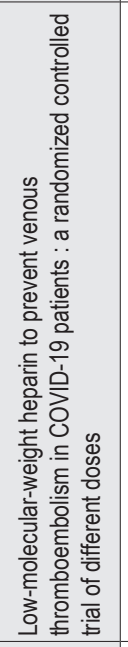 & 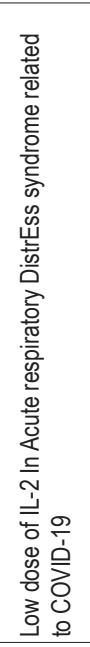 & 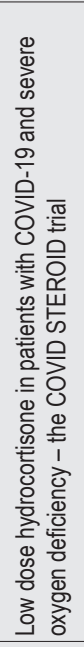 & 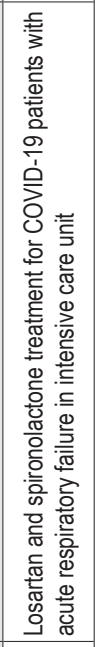 \\
\hline 题 & 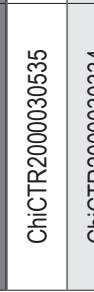 & 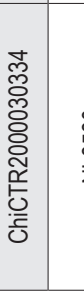 & 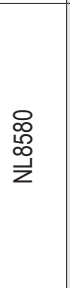 & 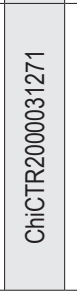 & 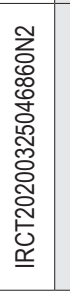 & 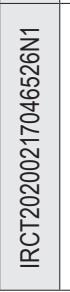 & 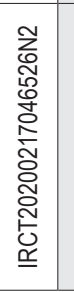 & 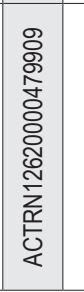 & 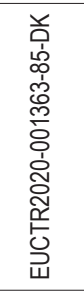 & 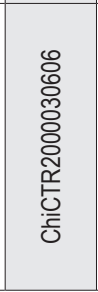 & 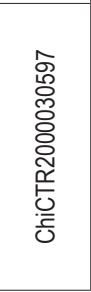 & 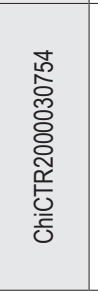 & 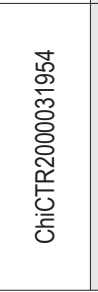 & 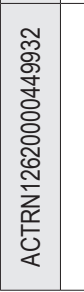 & 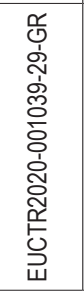 & 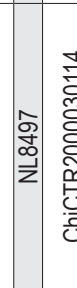 & t: & 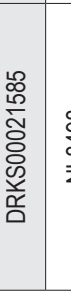 & $z$ & 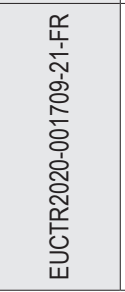 & 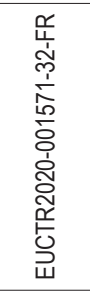 & 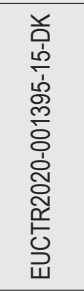 & 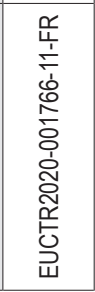 \\
\hline
\end{tabular}




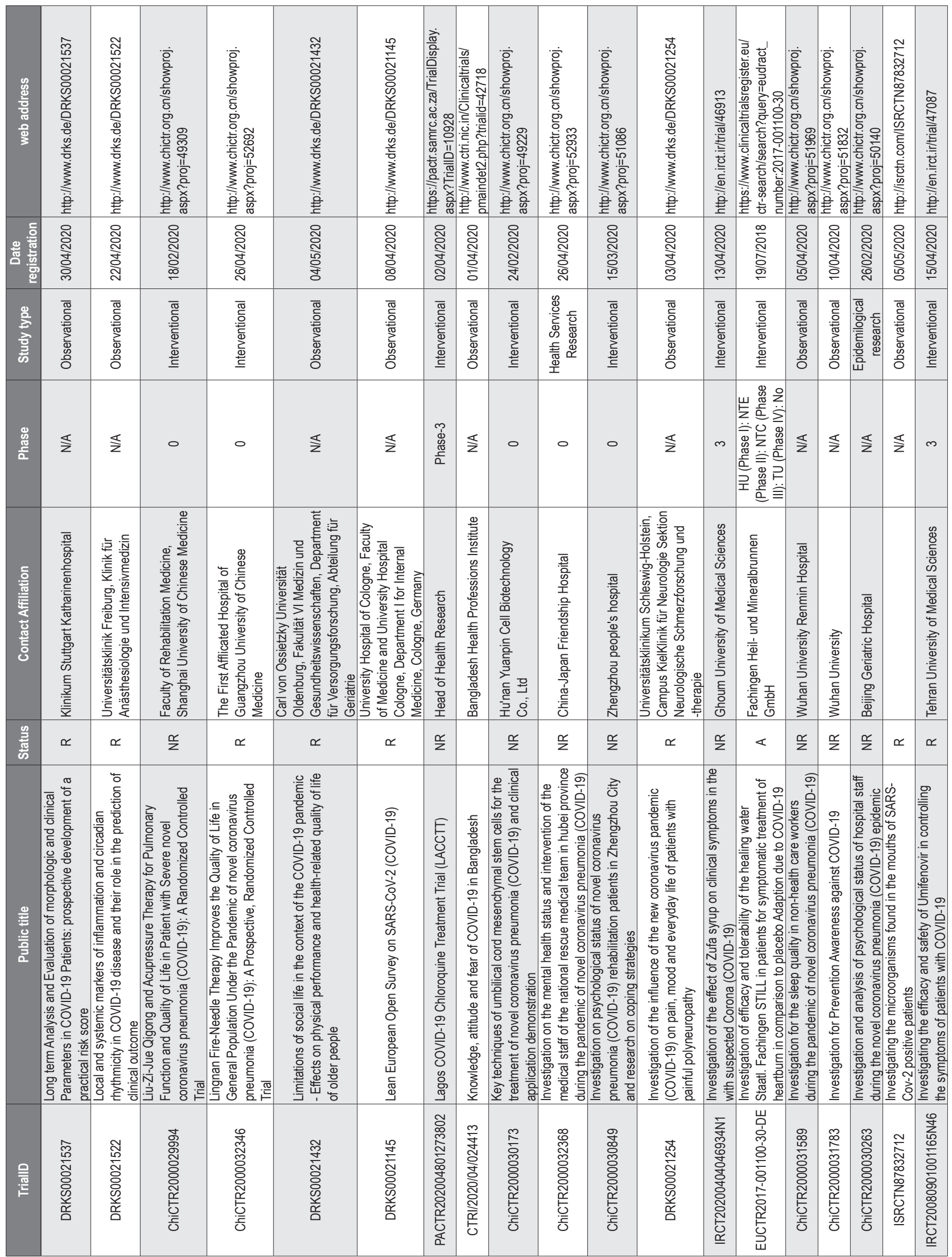




\begin{tabular}{|c|c|c|c|c|c|c|c|c|c|c|c|c|c|c|c|c|c|c|c|c|}
\hline 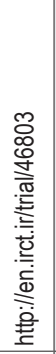 & 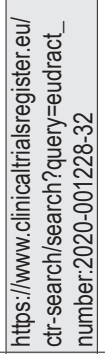 & 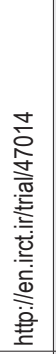 & 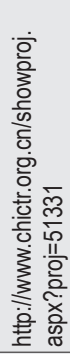 & 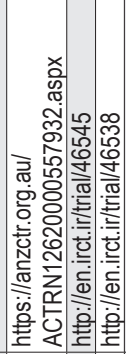 & 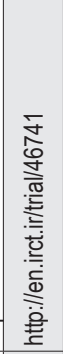 & 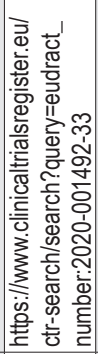 & 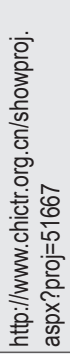 & 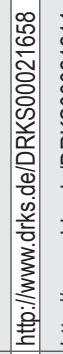 & 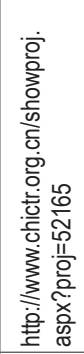 & 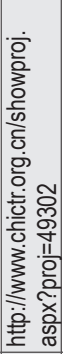 & 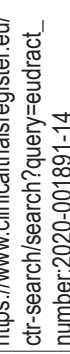 & 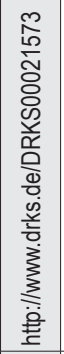 & 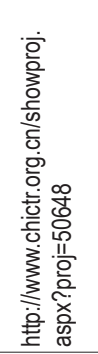 & 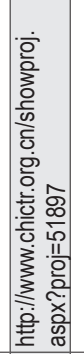 & 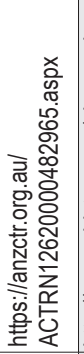 & 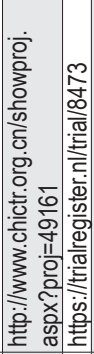 & 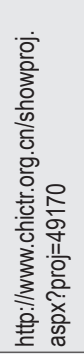 & 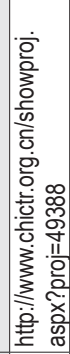 & 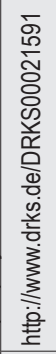 & 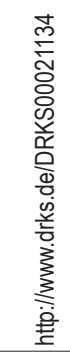 \\
\hline 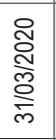 & 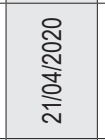 & 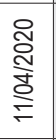 & 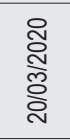 & 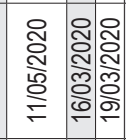 & 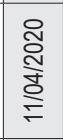 & 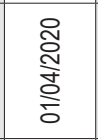 & $\begin{array}{l}\text { D్ } \\
\text { ָ̃ } \\
\stackrel{\text { N }}{\text { N }}\end{array}$ & 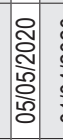 & $\begin{array}{l}\text { 오 } \\
\text { Nิ } \\
\text { 옹 }\end{array}$ & 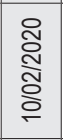 & 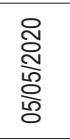 & 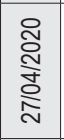 & 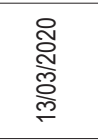 & $\begin{array}{l}\text { त్ } \\
\text { స్ } \\
\text { तิ }\end{array}$ & 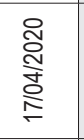 & 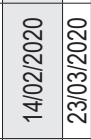 & 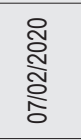 & 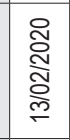 & $\begin{array}{l}\text { Nิ } \\
\text { ปิ } \\
\text { స్ }\end{array}$ & $\stackrel{\rho}{\circ}$ \\
\hline 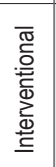 & 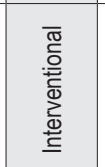 & 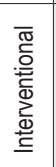 & 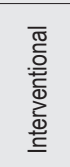 & 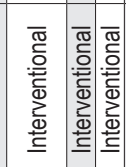 & 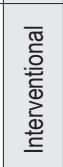 & 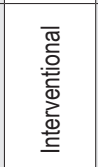 & 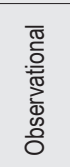 & 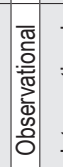 & 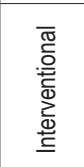 & 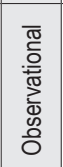 & 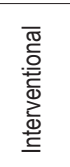 & 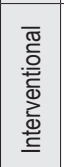 & 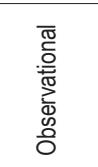 & 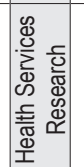 & 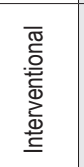 & 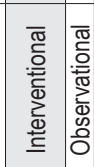 & 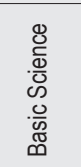 & 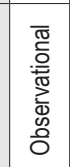 & 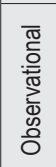 & 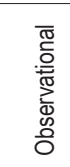 \\
\hline
\end{tabular}

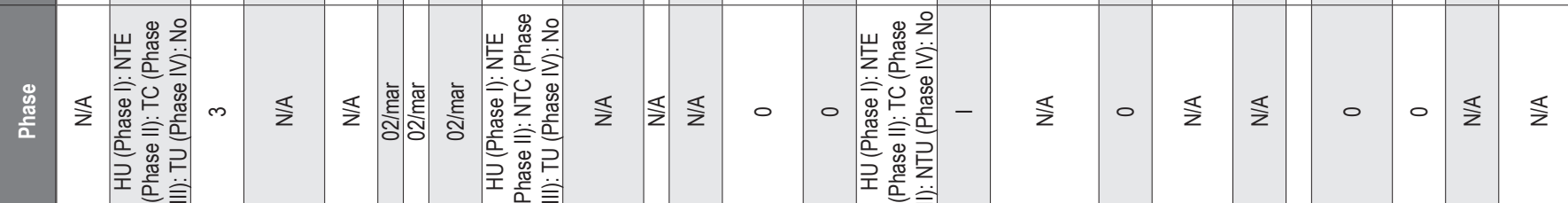

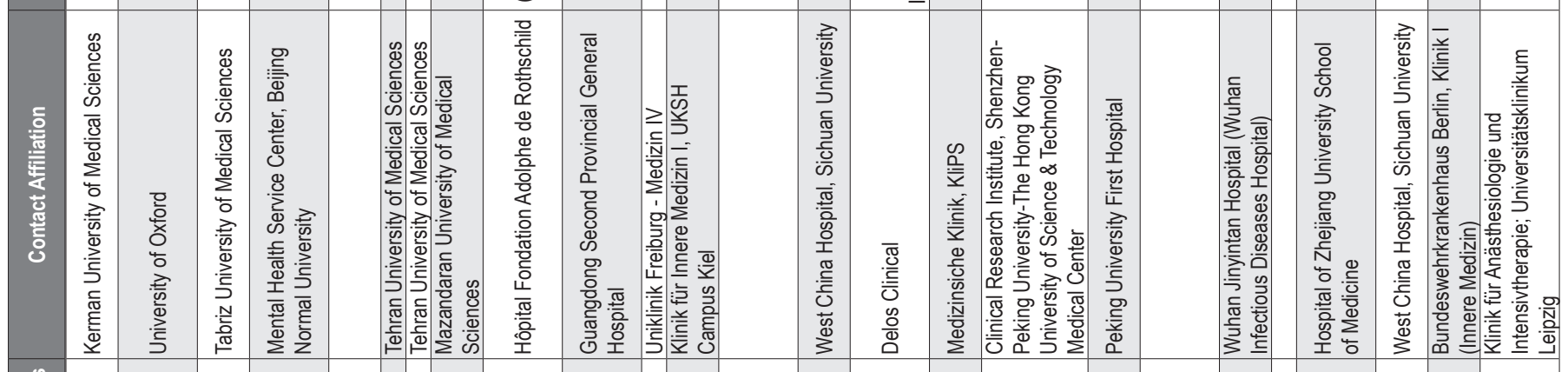

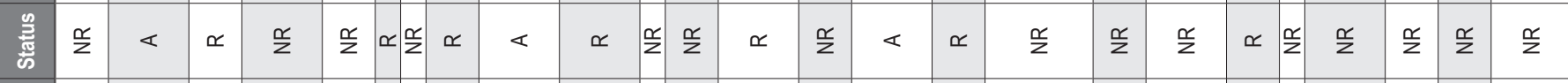

\begin{tabular}{|c|c|c|c|c|c|c|c|c|c|c|c|c|c|c|c|c|c|c|c|c|c|c|}
\hline 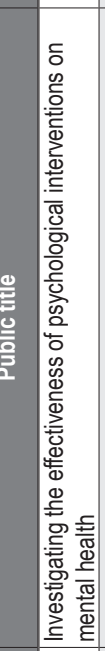 & 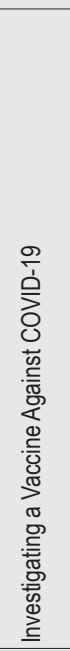 & 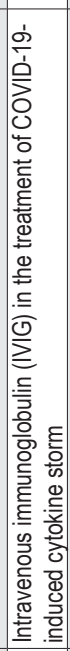 & 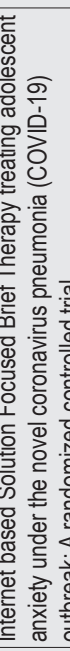 & 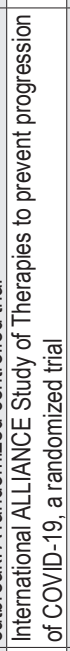 & 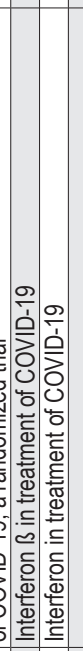 & 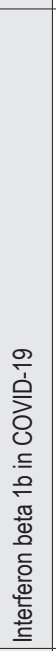 & 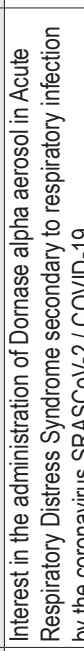 & 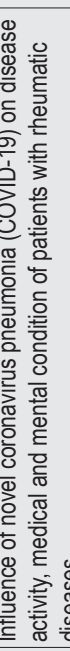 & & 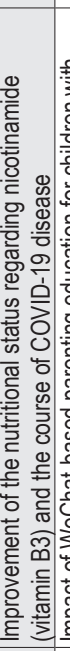 & 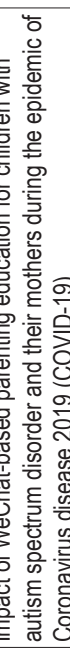 & 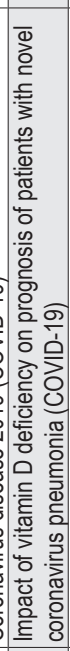 & 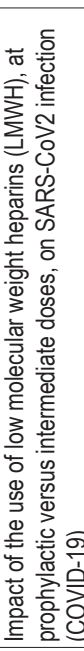 & 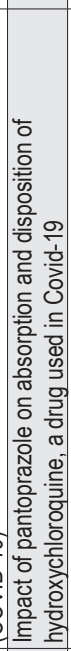 & 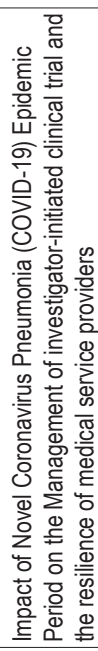 & 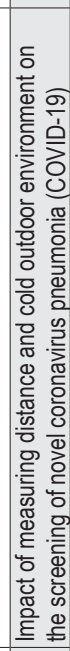 & & 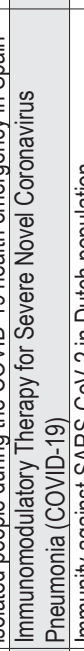 & 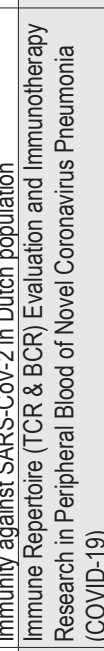 & & 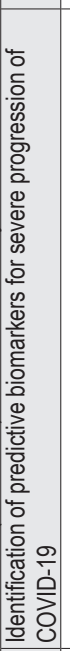 & 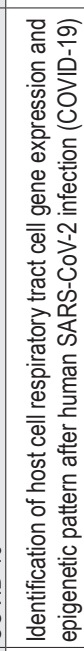 \\
\hline 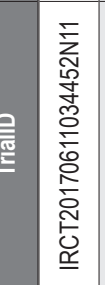 & 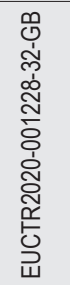 & 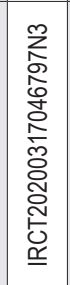 & 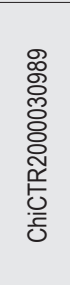 & 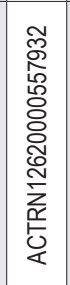 & 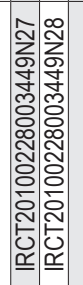 & 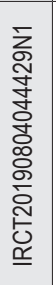 & 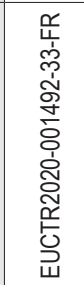 & 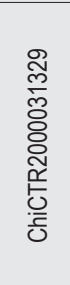 & & 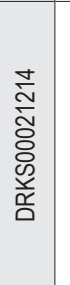 & 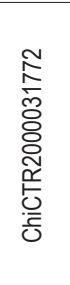 & 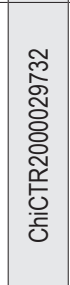 & 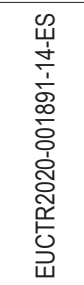 & 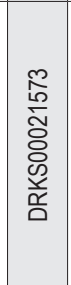 & 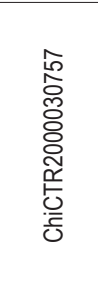 & 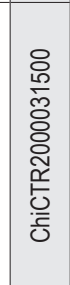 & 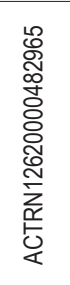 & 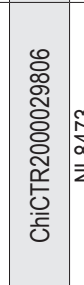 & 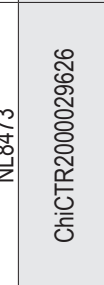 & $\begin{array}{l}\text { ¿ } \\
\text { S్ }\end{array}$ & 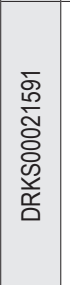 & \\
\hline
\end{tabular}




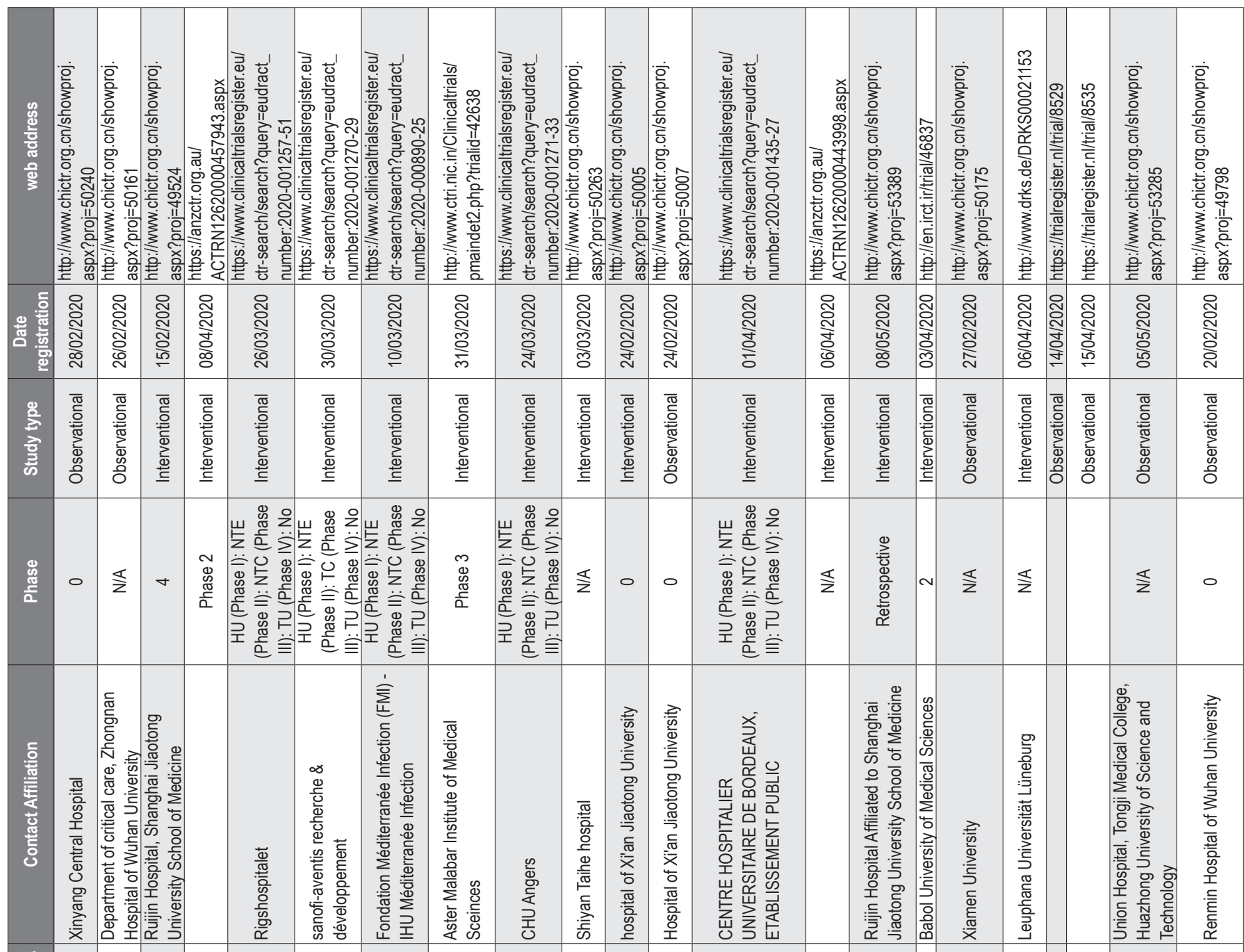

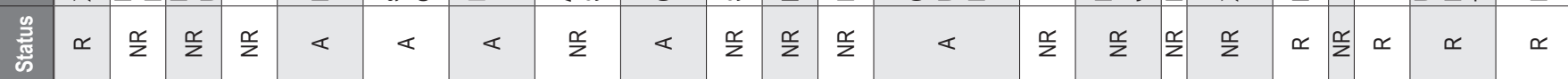

\begin{tabular}{|c|c|c|c|c|c|c|c|c|c|c|c|c|c|c|c|c|c|c|c|c|c|}
\hline 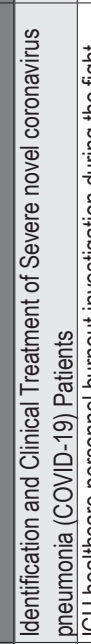 & 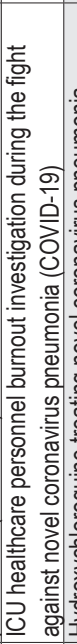 & 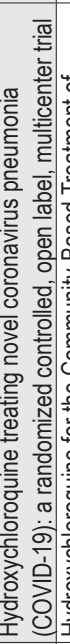 & 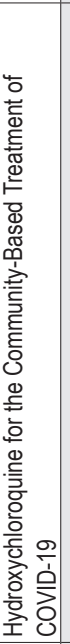 & 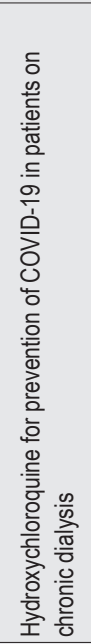 & 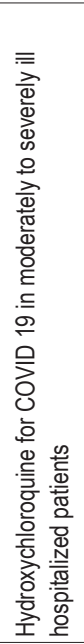 & 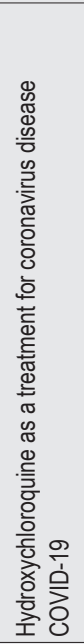 & 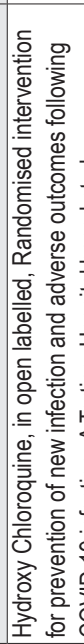 & $\begin{array}{l}\text {. } \\
\text { d. }\end{array}$ & 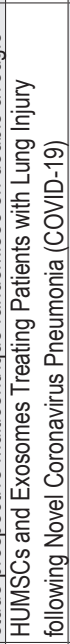 & 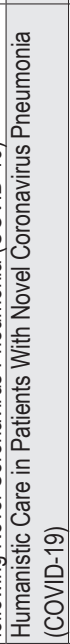 & 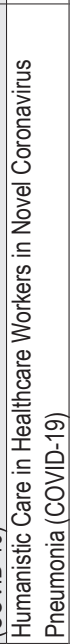 & 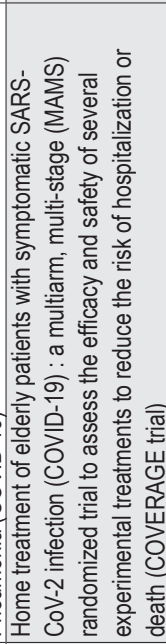 & 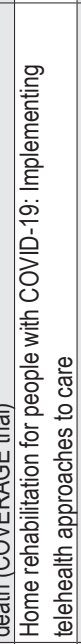 & 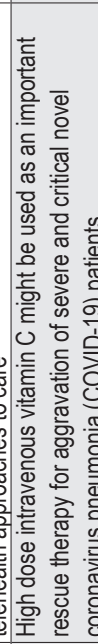 & 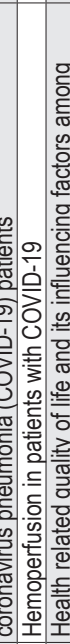 & 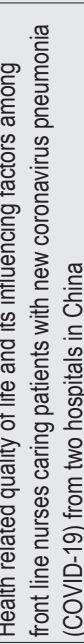 & & & 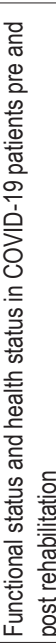 & 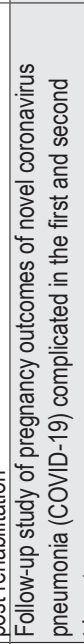 & 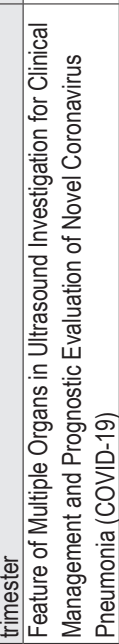 \\
\hline 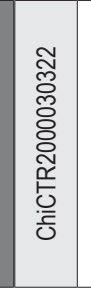 & 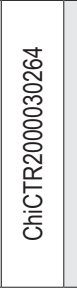 & 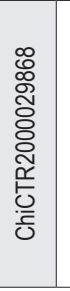 & 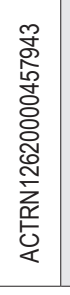 & 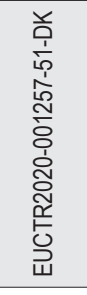 & 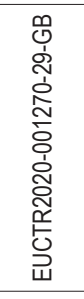 & 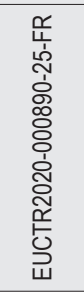 & 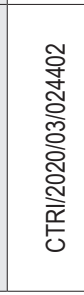 & 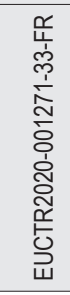 & 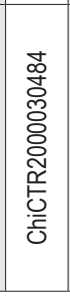 & 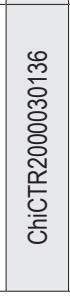 & 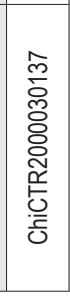 & 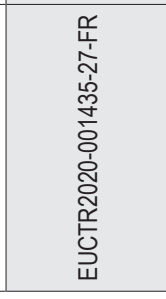 & 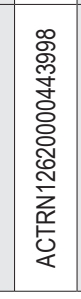 & 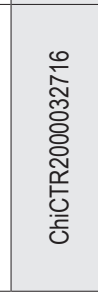 & 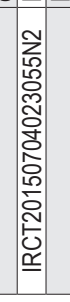 & 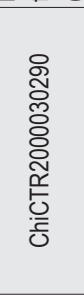 & $\stackrel{5}{\stackrel{5}{\delta}}$ & & & & \\
\hline
\end{tabular}




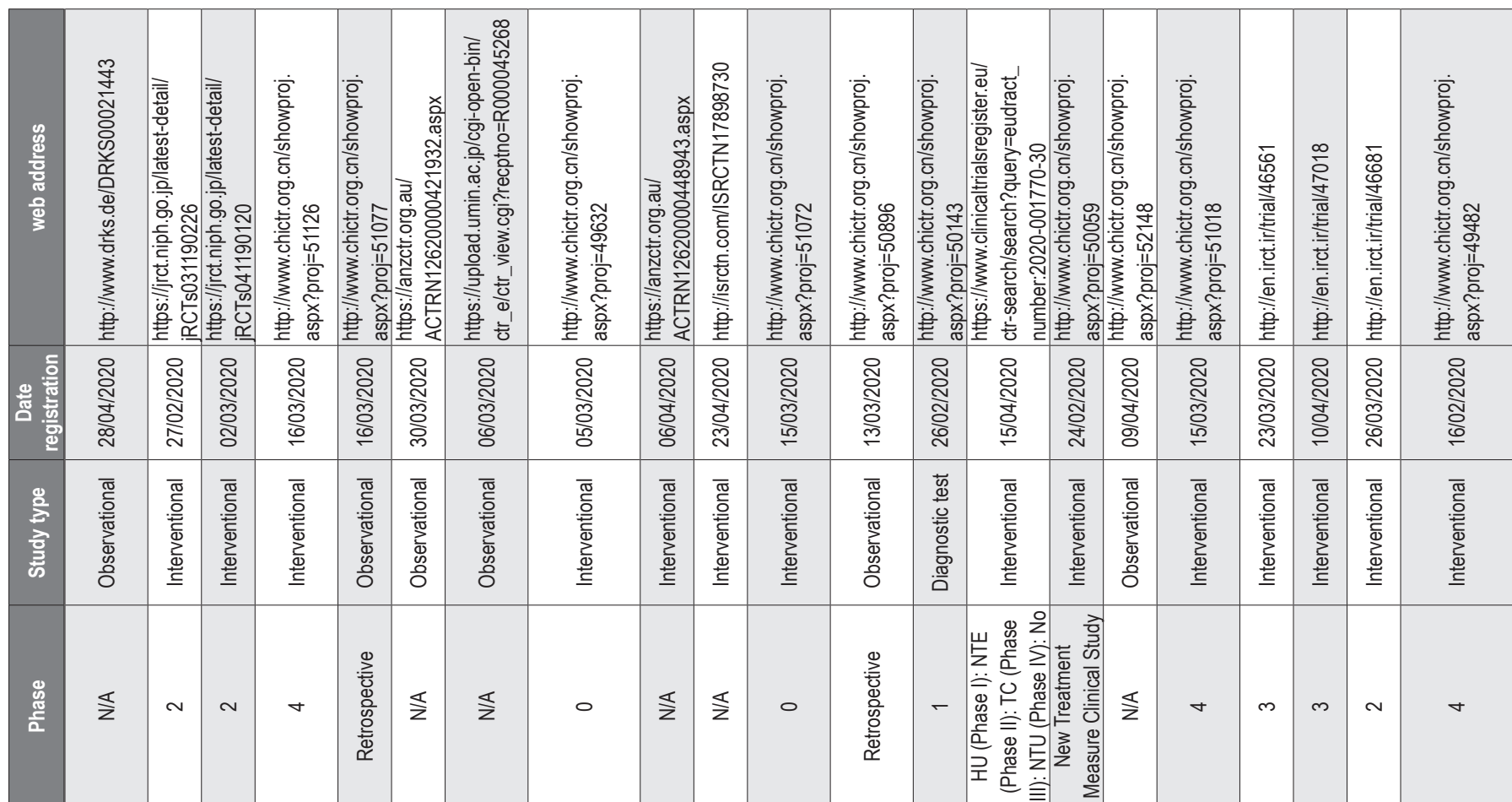

\begin{tabular}{|c|c|c|c|c|c|c|c|c|c|c|c|c|c|c|c|c|c|c|c|c|}
\hline 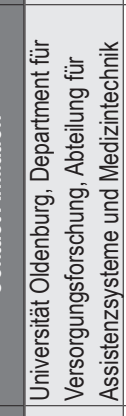 & 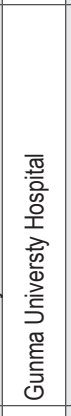 & 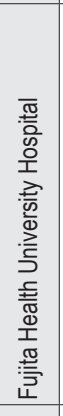 & 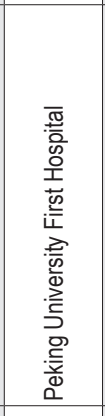 & 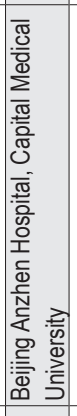 & & 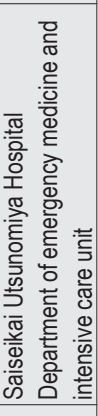 & 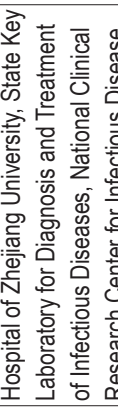 & & & 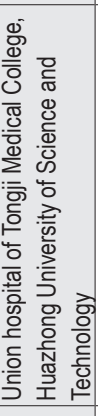 & 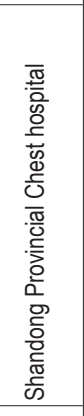 & 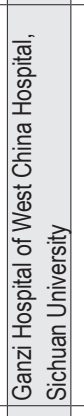 & 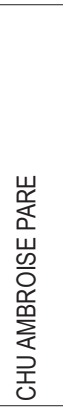 & 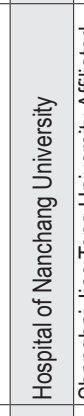 & 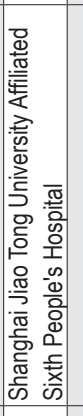 & 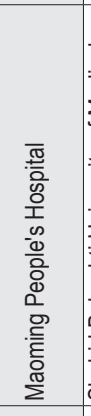 & 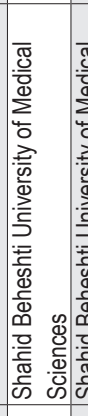 & 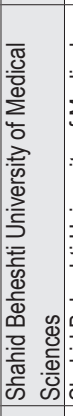 & 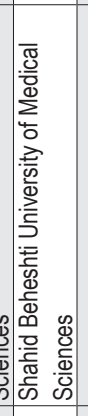 & 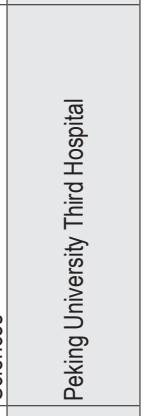 \\
\hline$\propto$ & $\propto$ & $\propto$ & $\simeq$ & $\frac{\alpha}{z}$ & $\simeq$ & $\propto$ & $\simeq$ & $\frac{\underline{x}}{z}$ & $\propto$ & $\simeq$ & $\simeq$ & $\frac{\alpha}{z}$ & $\varangle$ & $\propto$ & $\frac{⿱}{z}$ & $\simeq$ & $\frac{\alpha}{z}$ & $\simeq$ & $\simeq$ & $\simeq$ \\
\hline 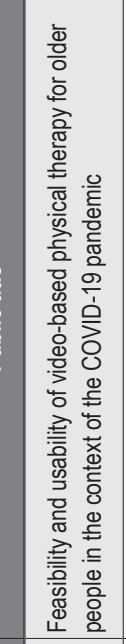 & 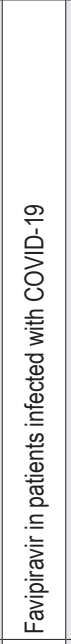 & 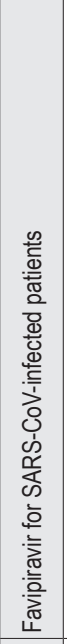 & 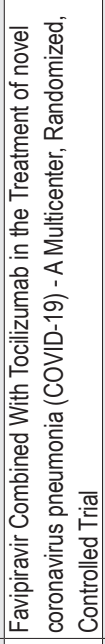 & 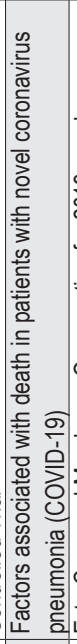 & 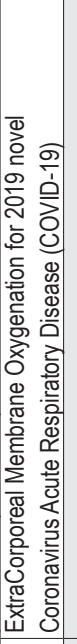 & 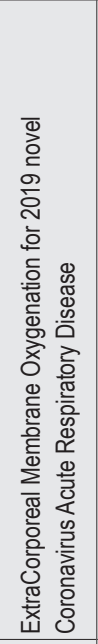 & 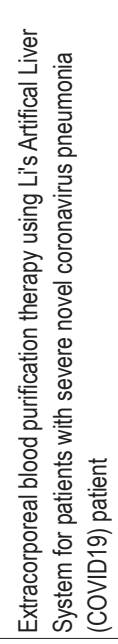 & 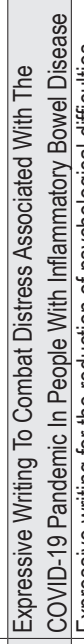 & 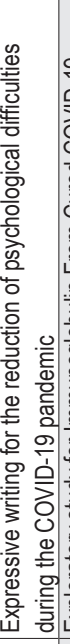 & 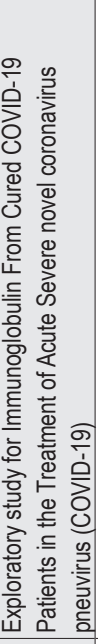 & 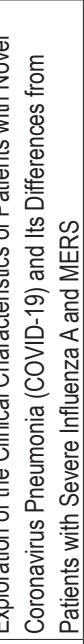 & 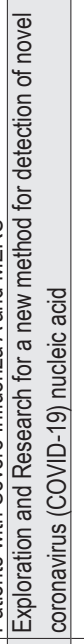 & 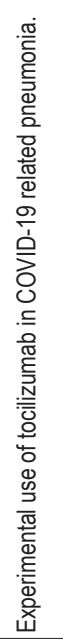 & 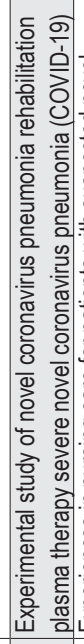 & 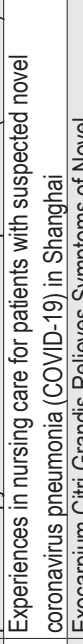 & 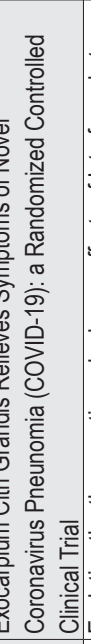 & 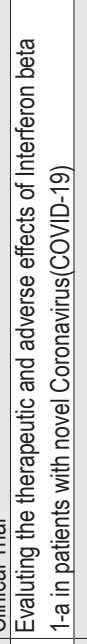 & 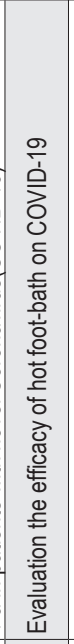 & 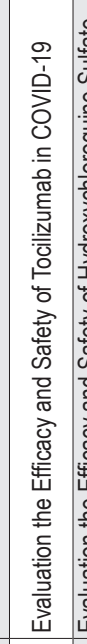 & 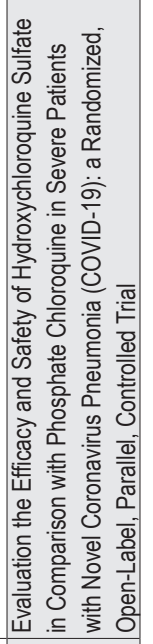 \\
\hline 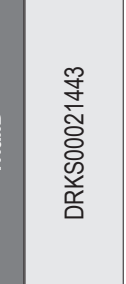 & 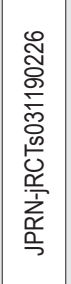 & 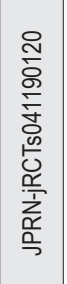 & 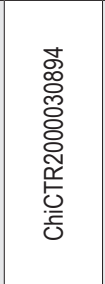 & 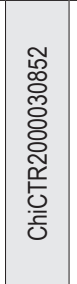 & 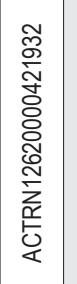 & 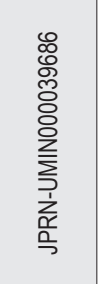 & 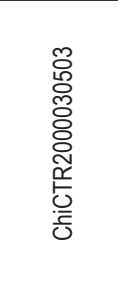 & 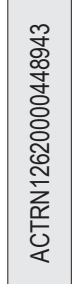 & 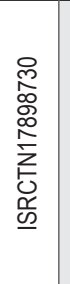 & 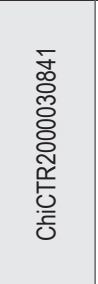 & 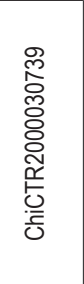 & 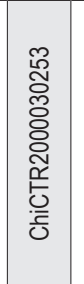 & 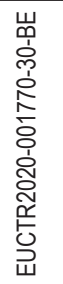 & 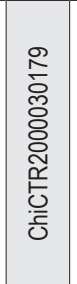 & 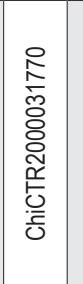 & 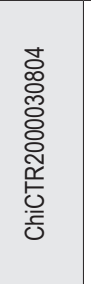 & 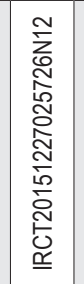 & 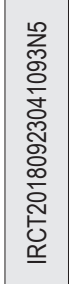 & 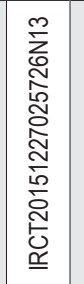 & 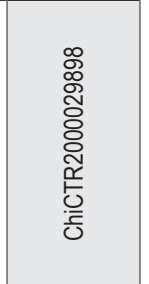 \\
\hline
\end{tabular}




\begin{tabular}{|c|c|c|c|c|c|c|c|c|c|c|c|c|c|c|c|c|c|c|c|c|}
\hline 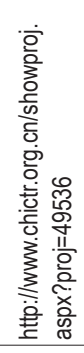 & 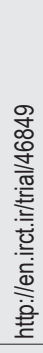 & 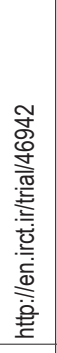 & 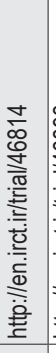 & 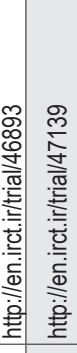 & 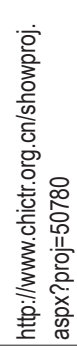 & 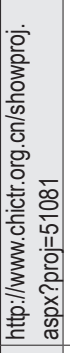 & 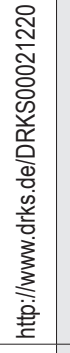 & 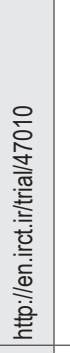 & 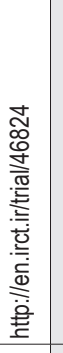 & 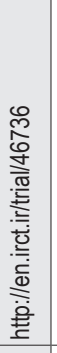 & 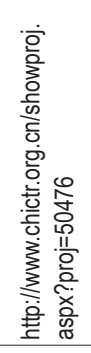 & 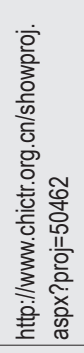 & 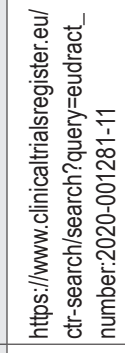 & 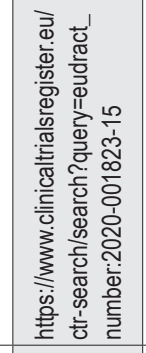 & 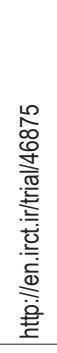 & 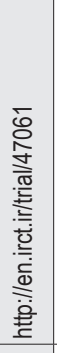 & 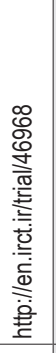 & 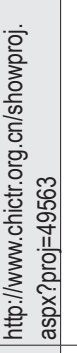 & 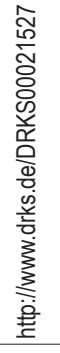 & 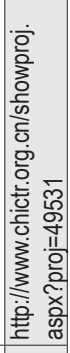 \\
\hline $\begin{array}{l}\text { స్ } \\
\text { స్ } \\
\stackrel{0}{0}\end{array}$ & $\begin{array}{l}\text { ते } \\
\text { సे. } \\
\text { के }\end{array}$ & 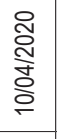 & 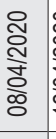 & 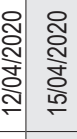 & 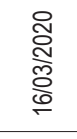 & 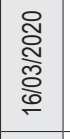 & 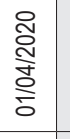 & 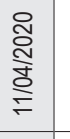 & 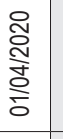 & 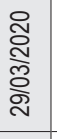 & 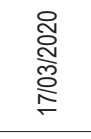 & 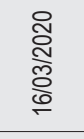 & 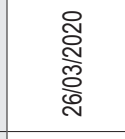 & $\begin{array}{l}\text { Nิ } \\
\text { Nิ } \\
\text { 옹 }\end{array}$ & $\begin{array}{l}\text { ్․ } \\
\text { ิㅗㅇ } \\
\text { 옹 }\end{array}$ & 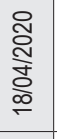 & 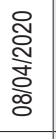 & $\begin{array}{l}\text { त్ } \\
\text { స్ } \\
\text { 은 }\end{array}$ & 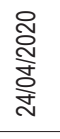 & 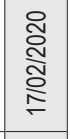 \\
\hline 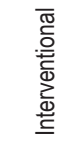 & 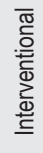 & 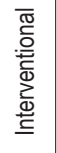 & 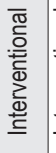 & 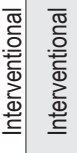 & 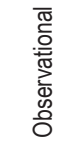 & 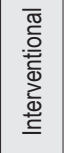 & 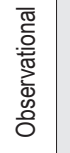 & 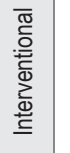 & 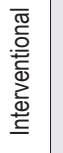 & 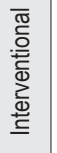 & 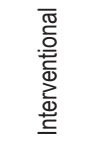 & 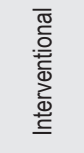 & 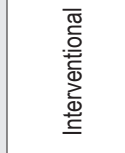 & 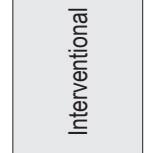 & 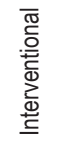 & 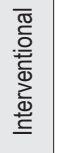 & 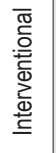 & 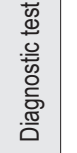 & 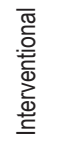 & 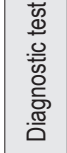 \\
\hline$\nabla$ & $m$ & $m$ & $m$ & $\sim \sim$ & $\stackrel{\$}{z}$ & 0 & & m & $m$ & $m$ & $\frac{\mathbf{s}}{\mathbf{z}}$ & $\frac{\$}{z}$ & 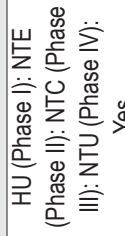 & 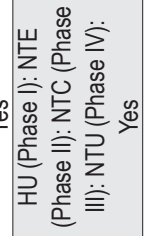 & $m$ & $m$ & $m$ & 0 & $\stackrel{s}{z}$ & 0 \\
\hline
\end{tabular}

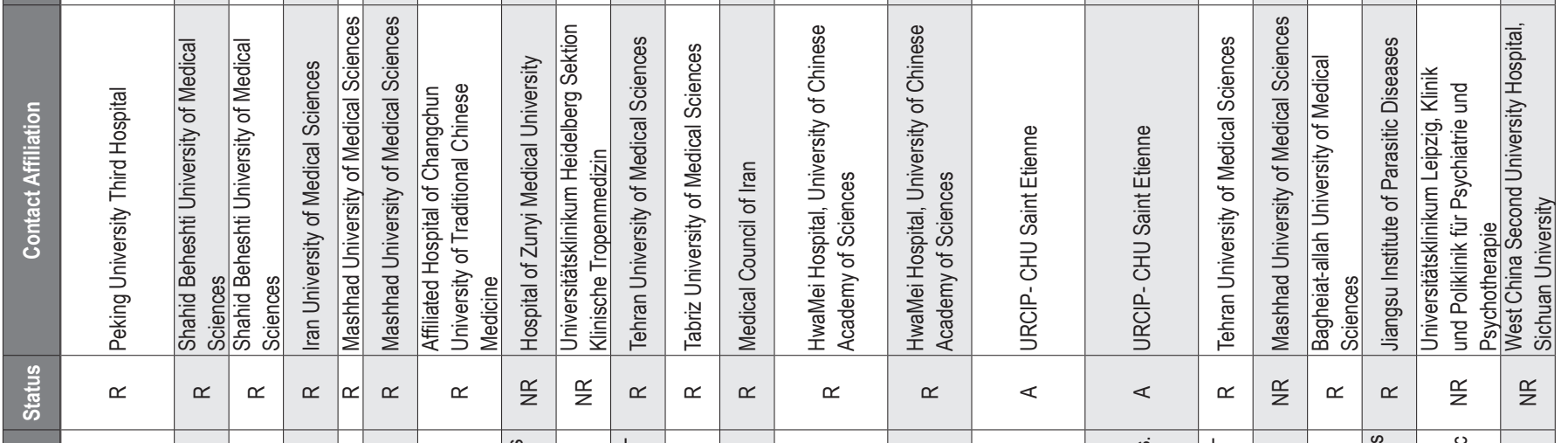

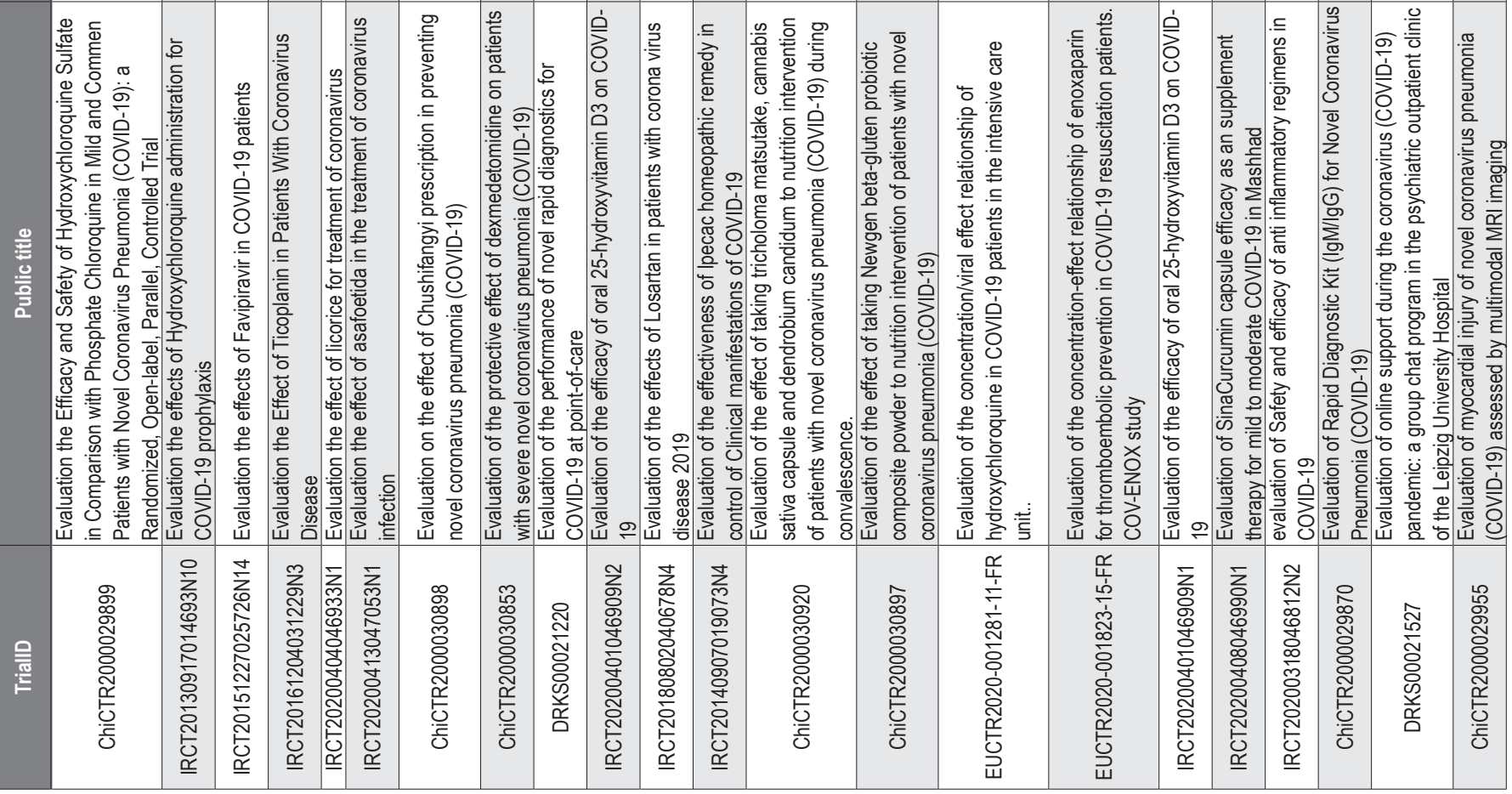




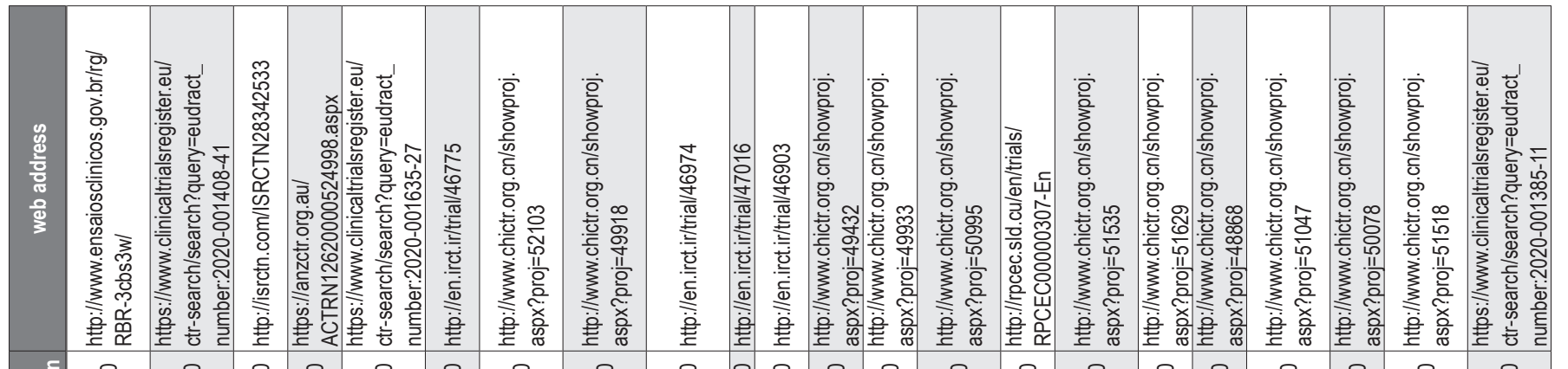

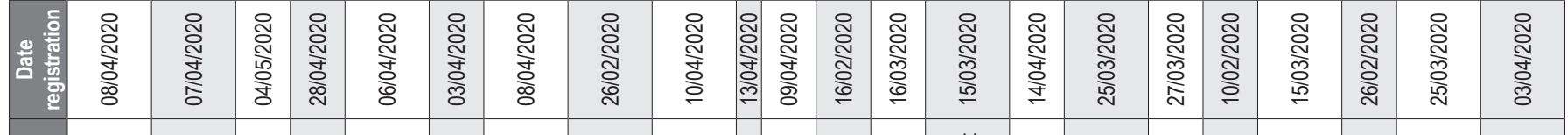

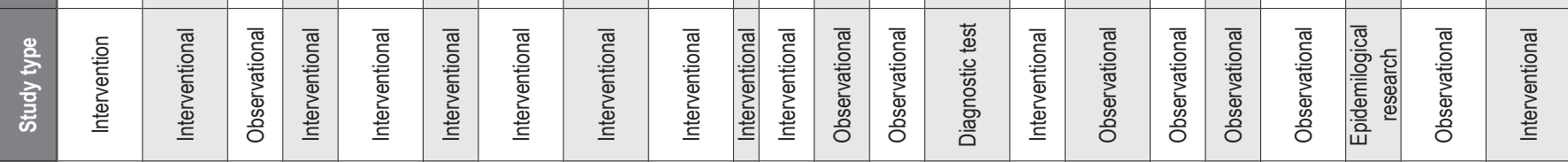

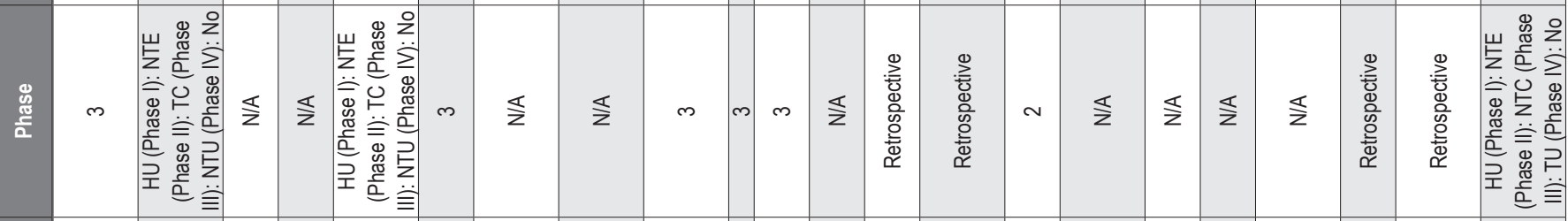

\begin{tabular}{|c|c|c|c|c|c|c|c|c|c|c|c|c|c|c|c|c|c|c|c|c|}
\hline 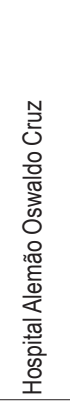 & 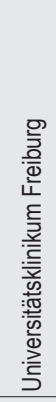 & & & 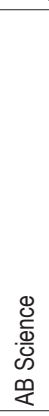 & 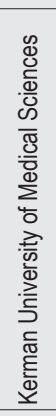 & 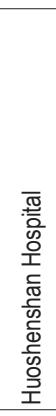 & 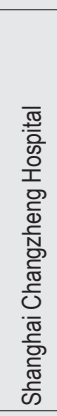 & 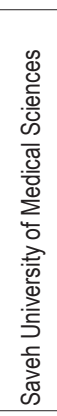 & 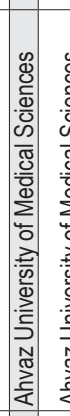 & 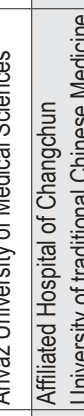 & 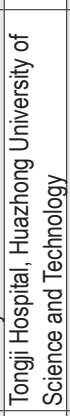 & 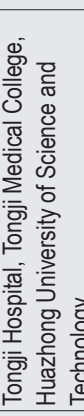 & 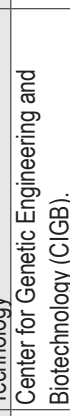 & 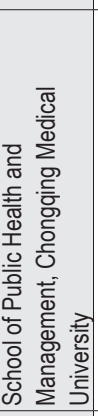 & & 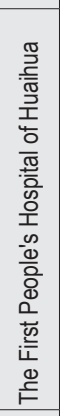 & 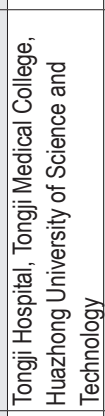 & 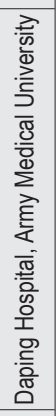 & 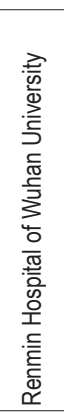 & $\begin{array}{l}\text { 葸 } \\
\end{array}$ \\
\hline$\frac{\alpha}{z}$ & $\ll$ & $\propto$ & $\frac{\underline{x}}{z}$ & $\ll$ & $\simeq$ & $\propto$ & $\simeq$ & $\frac{\mathscr{N}}{z}$ & $x$ & \begin{tabular}{r|r}
$x$ & $\propto$
\end{tabular} & $\propto$ & $\frac{\mathscr{N}}{2}$ & $\propto$ & $\frac{\alpha}{z}$ & $\simeq$ & $\simeq$ & $\frac{\underline{\alpha}}{z}$ & $\frac{\mathscr{\alpha}}{z}$ & $\simeq$ & $\ll$ \\
\hline
\end{tabular}

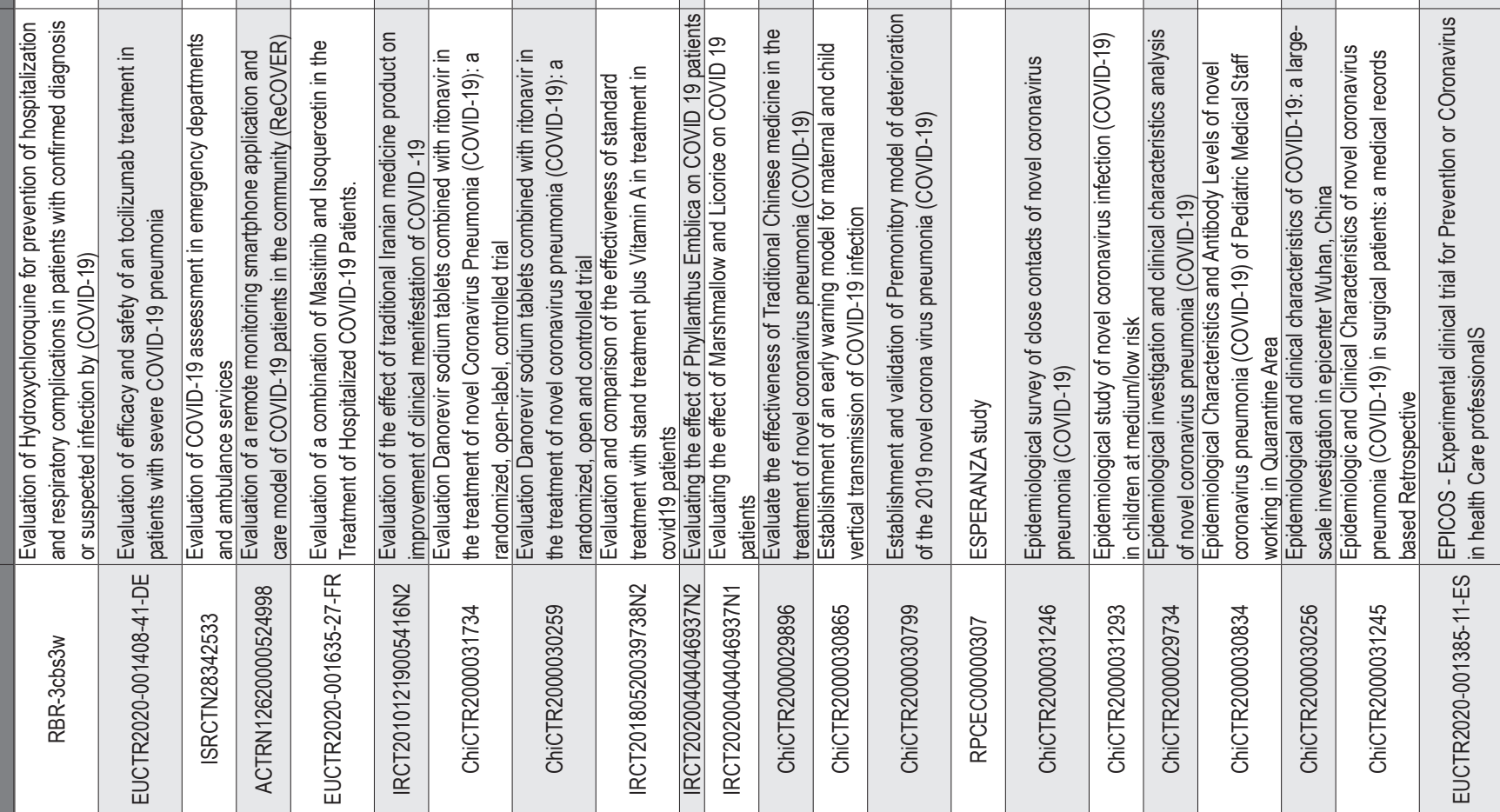




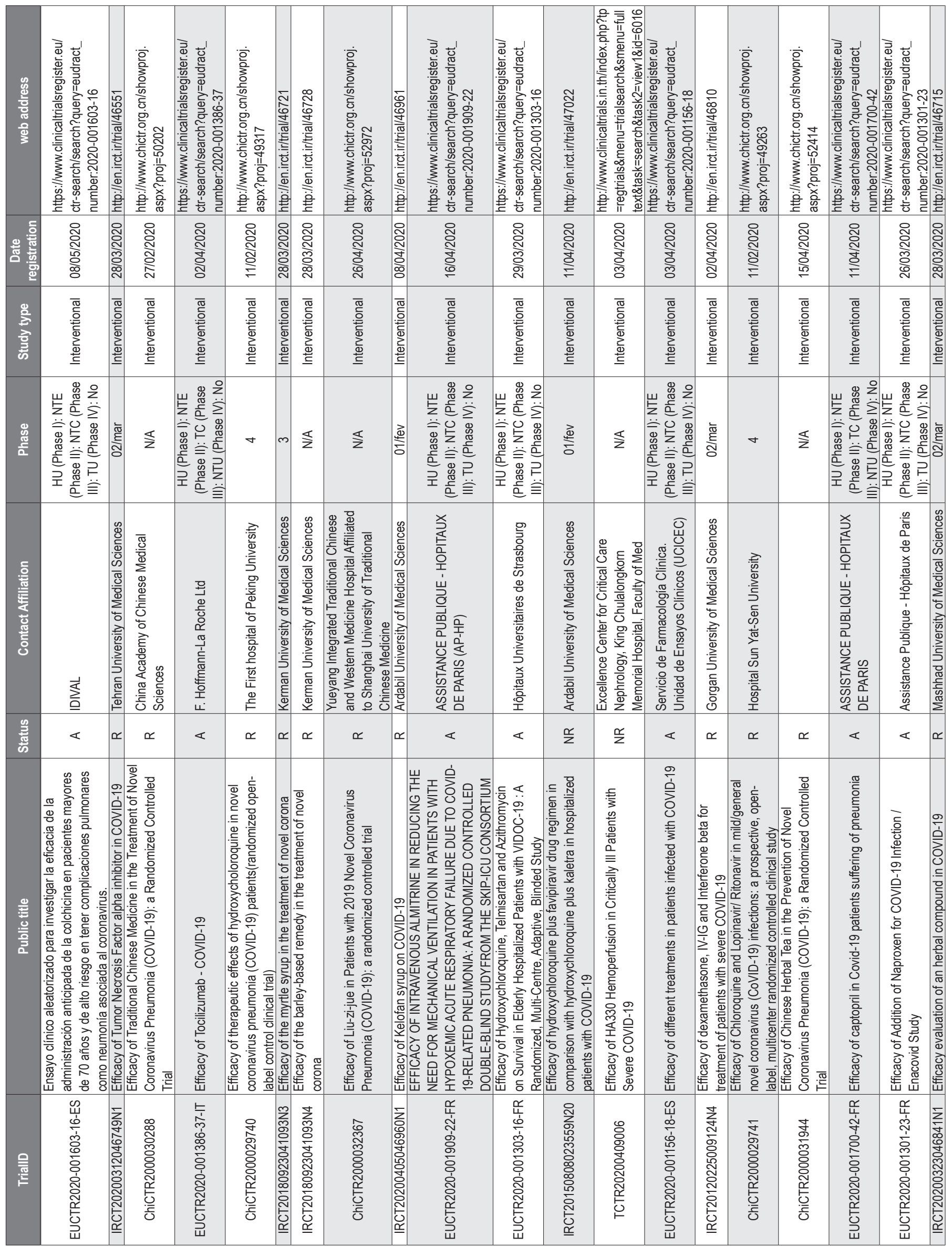




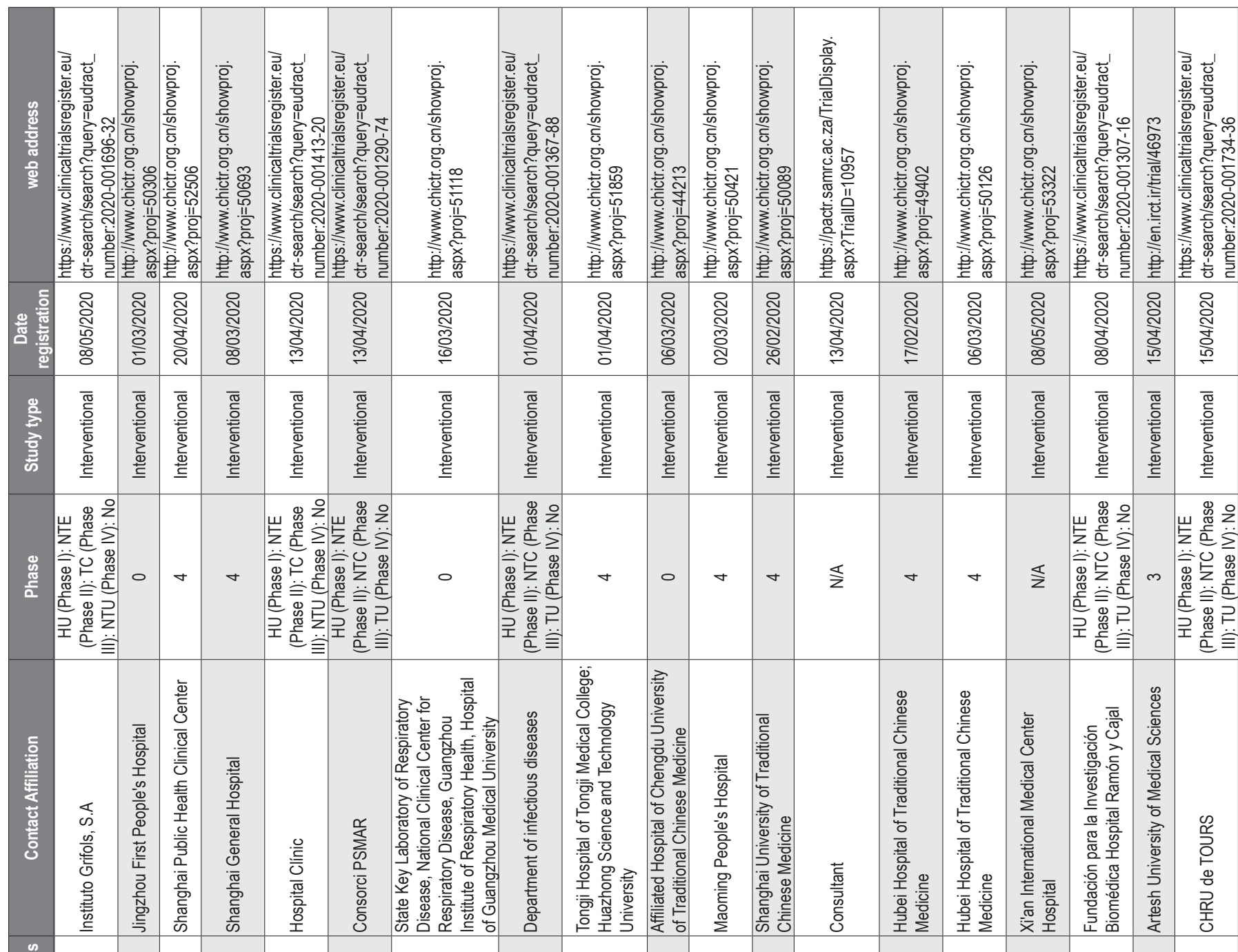

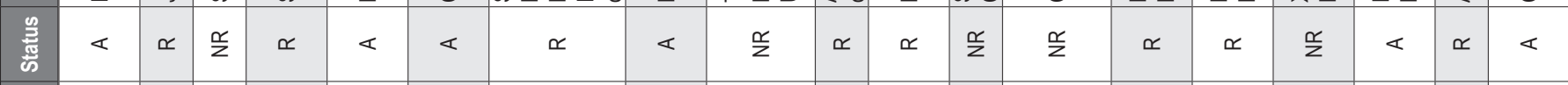

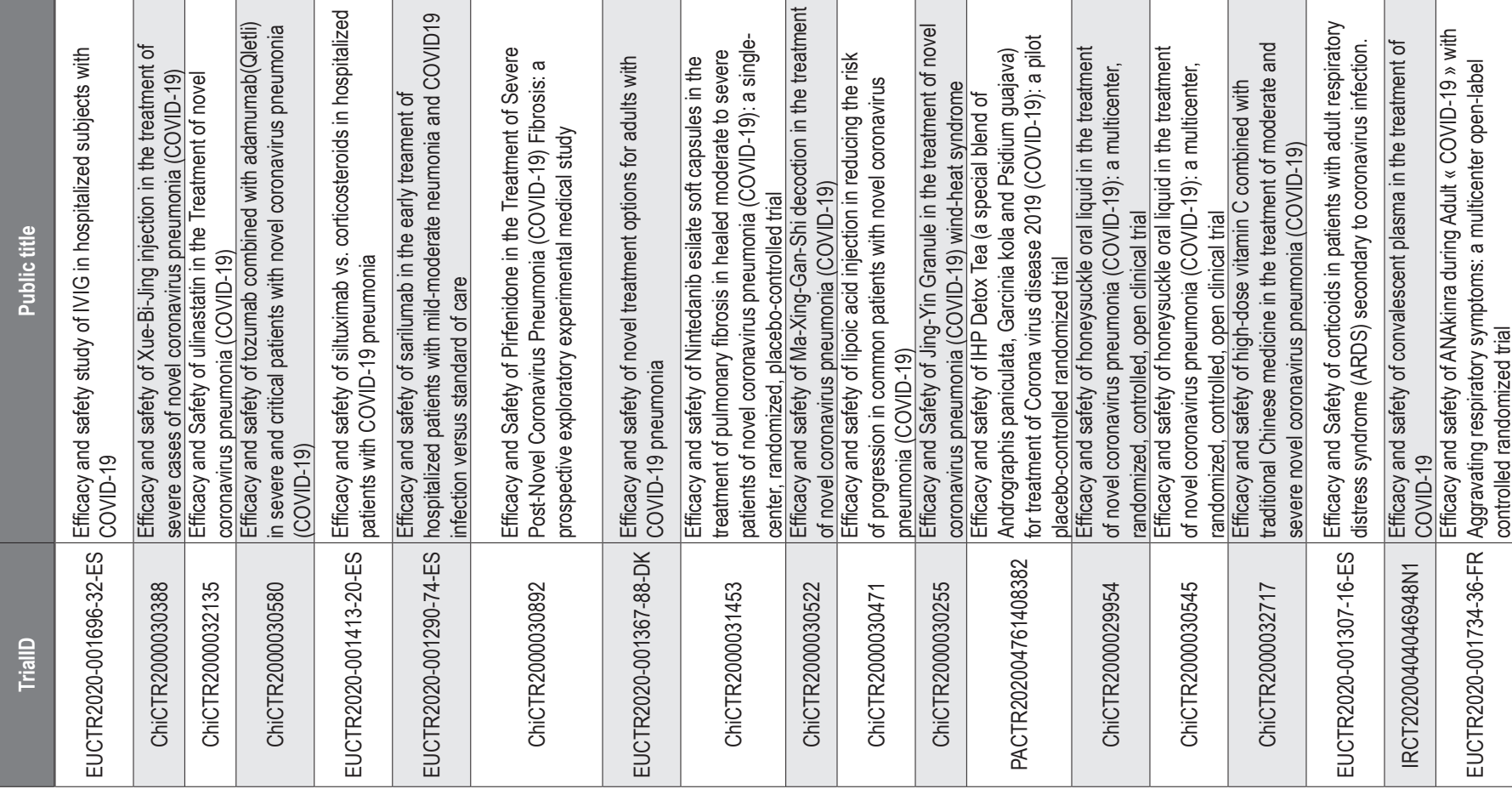




\begin{tabular}{|c|c|c|c|c|c|c|c|c|c|c|c|c|c|c|c|c|c|c|c|}
\hline 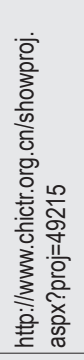 & 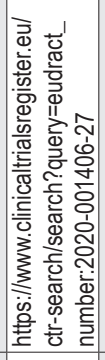 & 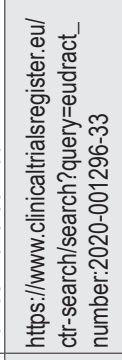 & 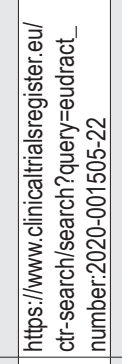 & 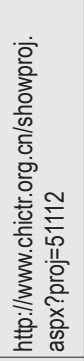 & 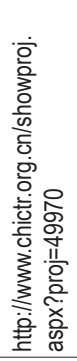 & 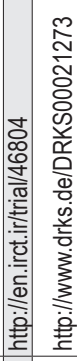 & 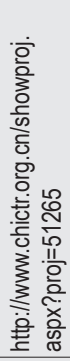 & 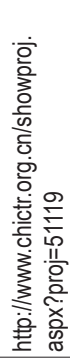 & 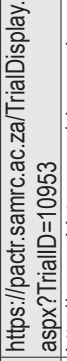 & 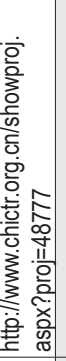 & 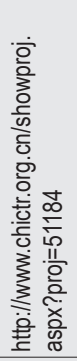 & 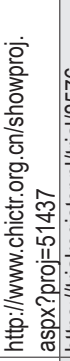 & 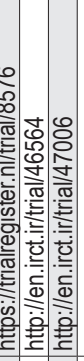 & 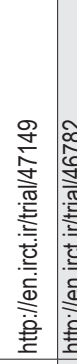 & 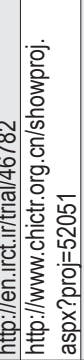 & 㝘 & 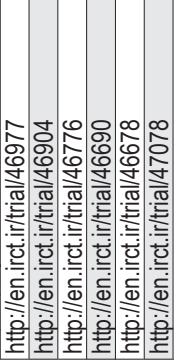 & 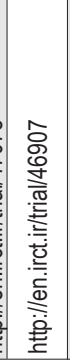 & 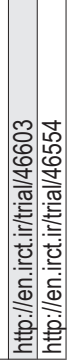 \\
\hline $\begin{array}{l}\text { ָิ } \\
\text { స్̃ } \\
\text { ळ. }\end{array}$ & 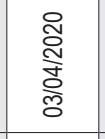 & 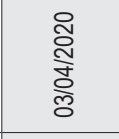 & 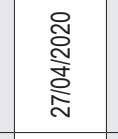 & 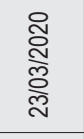 & 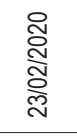 & 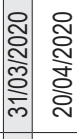 & $\begin{array}{l}\text { तิ } \\
\text { लू } \\
\text { के }\end{array}$ & 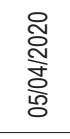 & 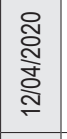 & 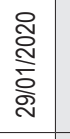 & 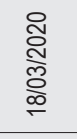 & $\begin{array}{l}\text { ָั } \\
\text { लू } \\
\text { ले }\end{array}$ & 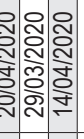 & 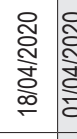 & 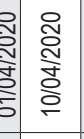 & 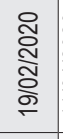 & 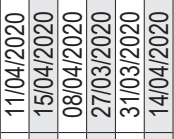 & 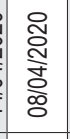 & 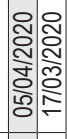 \\
\hline 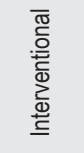 & 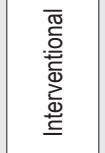 & 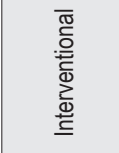 & 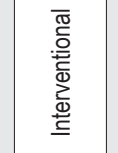 & 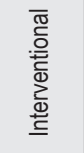 & 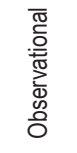 & 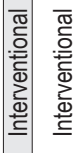 & 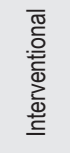 & 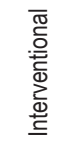 & 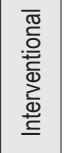 & 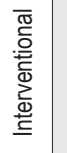 & 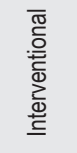 & 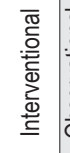 & 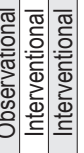 & 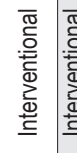 & 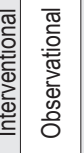 & 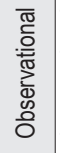 & 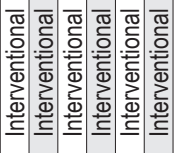 & 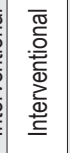 & $\frac{1}{d x}$ \\
\hline 0 & 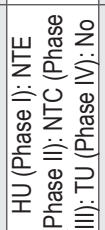 & 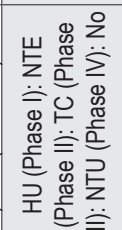 & 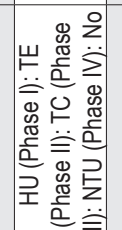 & 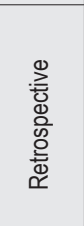 & $\stackrel{\$}{z}$ & $\frac{5}{2}$ & $\nabla$ & $\stackrel{\$}{z}$ & $\stackrel{\mathbb{z}}{z}$ & 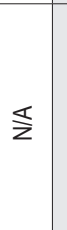 & 0 & $\bar{z}$ & $\bar{\Sigma}$ & 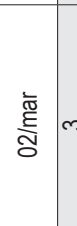 & 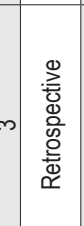 & $\stackrel{s}{z}$ & 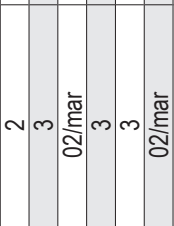 & 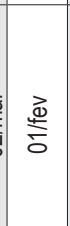 & \\
\hline
\end{tabular}

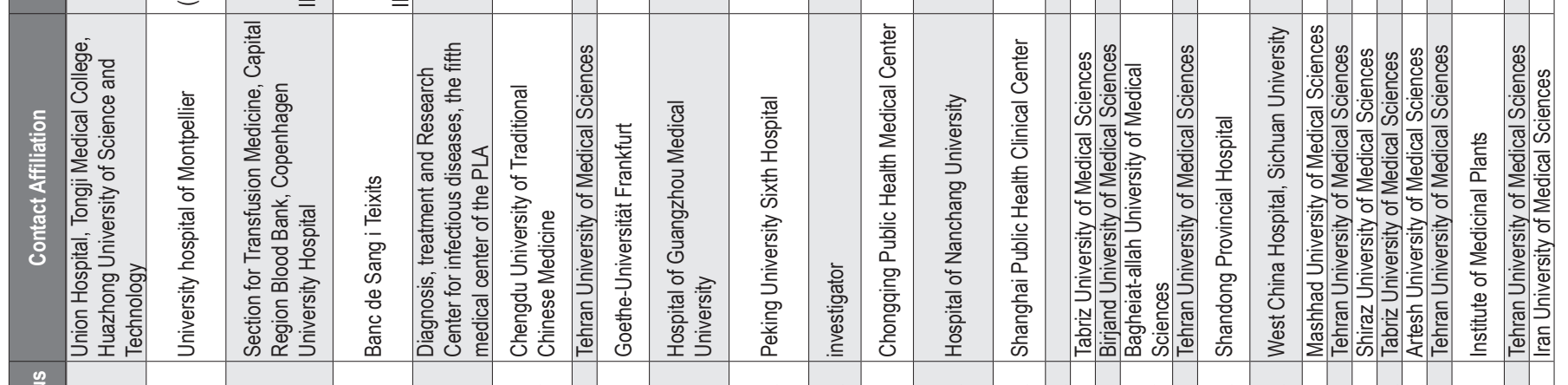

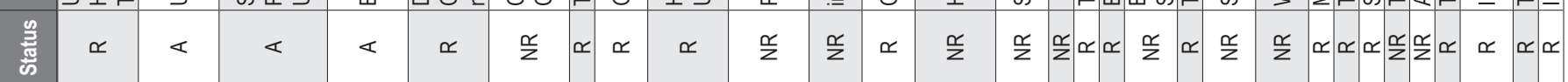

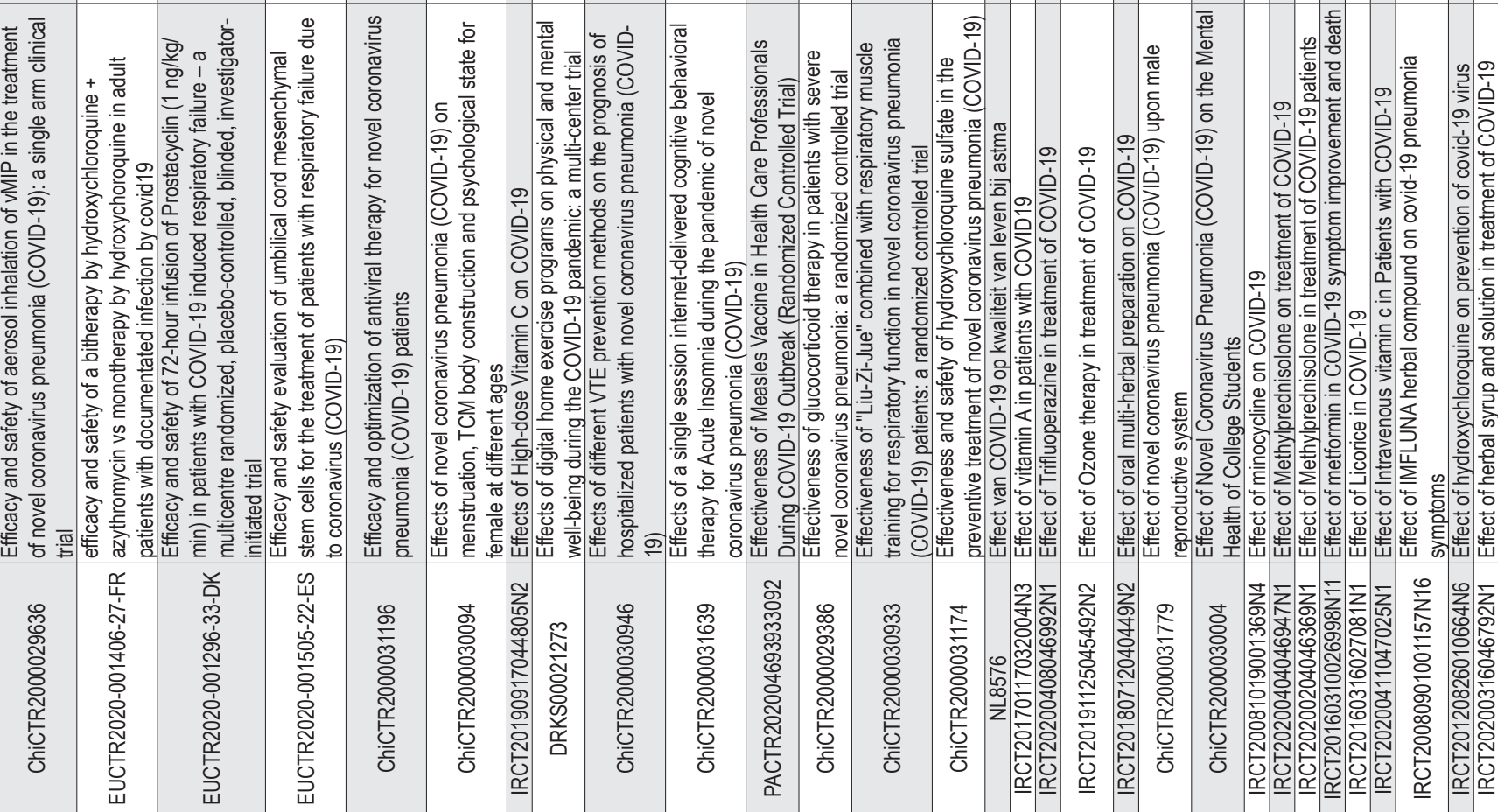




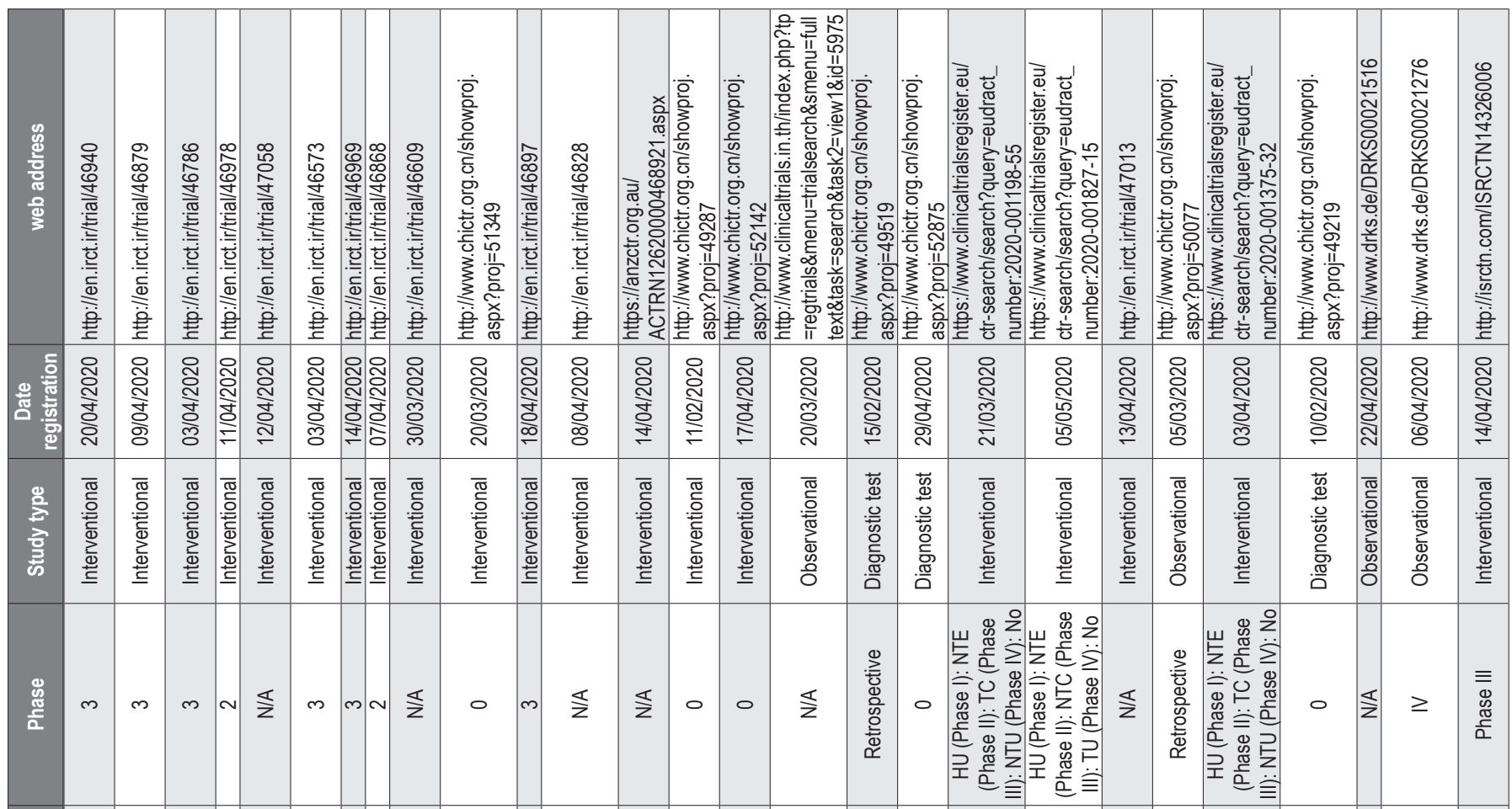

\begin{tabular}{|c|c|c|c|c|c|c|c|c|c|c|c|c|c|c|c|c|c|c|c|c|c|c|c|c|}
\hline 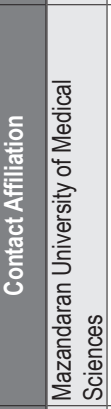 & 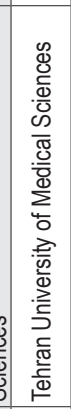 & 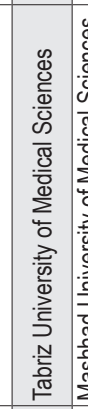 & 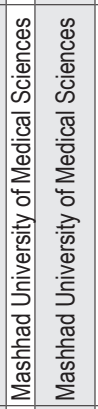 & 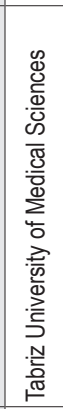 & 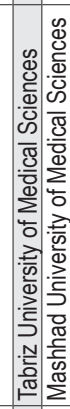 & 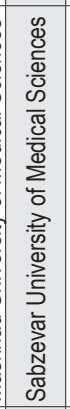 & 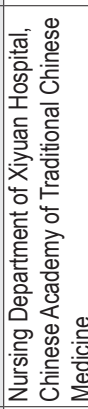 & 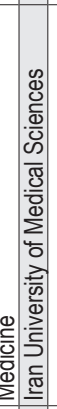 & 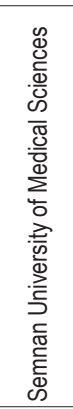 & & 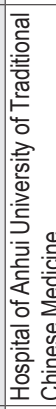 & 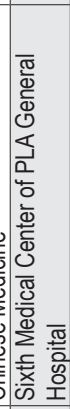 & $\frac{0}{\frac{0}{1}}$ & 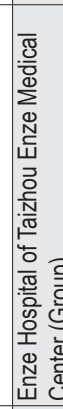 & 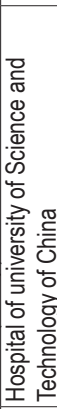 & & 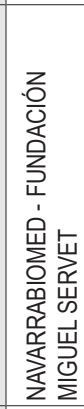 & 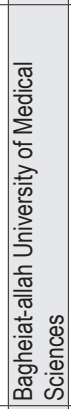 & 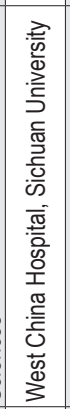 & $\begin{array}{l}0 \\
\sum_{J}\end{array}$ & 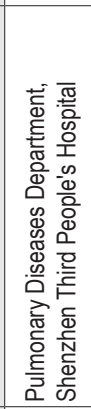 & & 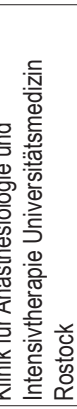 & \\
\hline$\simeq$ & $\simeq$ & $\simeq$ & $\propto \propto$ & $\simeq$ & $\propto \propto$ & $\simeq$ & $\simeq$ & $\frac{\alpha}{z}$ & $\simeq$ & $\frac{\alpha}{z}$ & $\propto$ & $\frac{\alpha}{z}$ & $\frac{\alpha}{z}$ & $\simeq$ & $\frac{⿱}{\frac{N}{z}}$ & $\ll$ & $<$ & $\frac{\alpha}{z}$ & $\frac{\mathscr{r}}{z}$ & $<$ & $\underline{\underline{g}}$ & $\propto$ & & \\
\hline 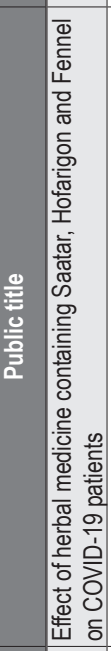 & 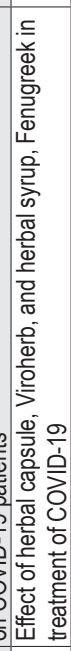 & 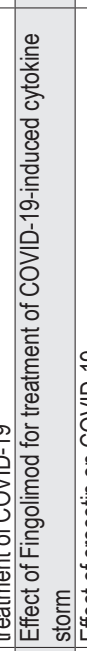 & 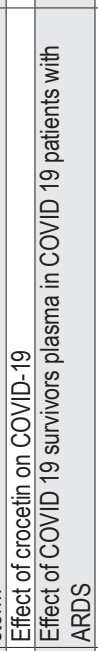 & 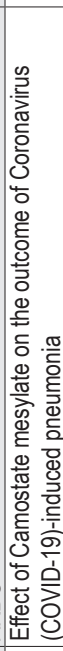 & 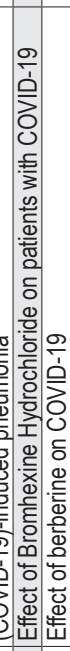 & 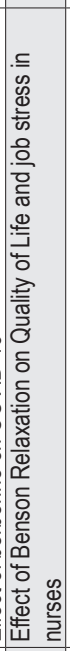 & 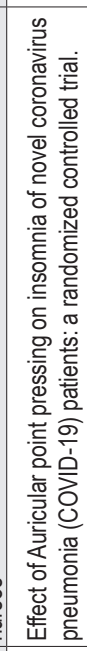 & & 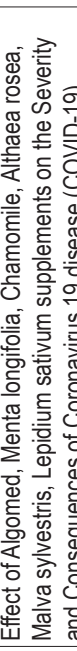 & 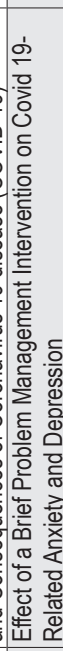 & 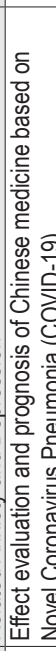 & 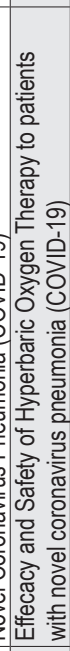 & 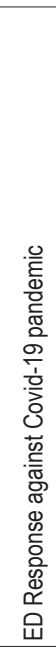 & 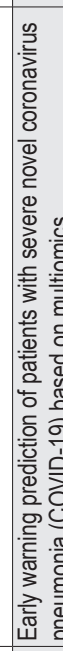 & 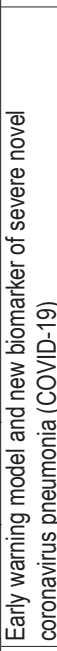 & 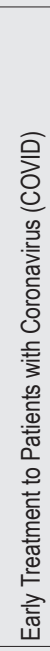 & 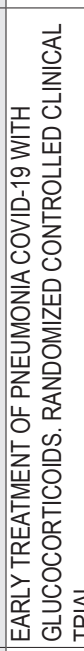 & 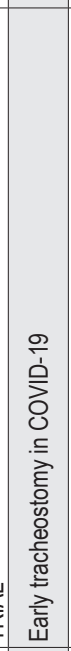 & 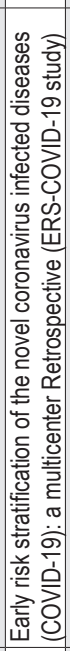 & 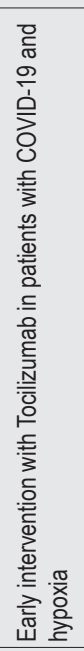 & 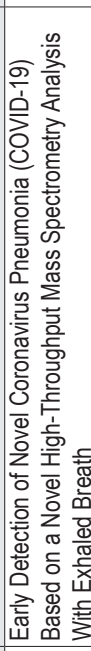 & 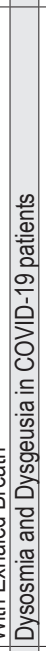 & 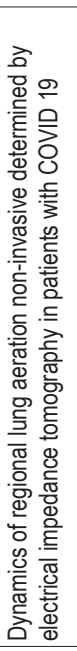 & \\
\hline 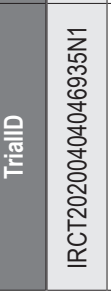 & 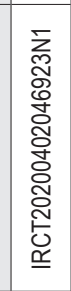 & 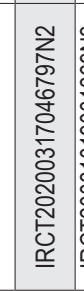 & 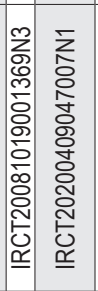 & 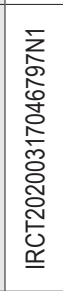 & 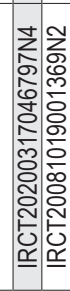 & 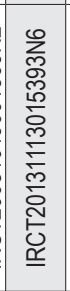 & 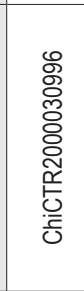 & 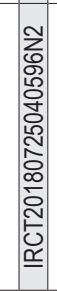 & 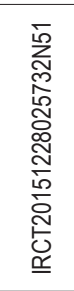 & 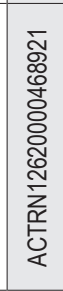 & 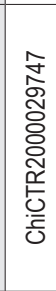 & 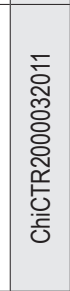 & 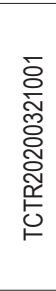 & 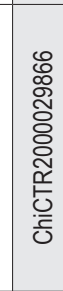 & 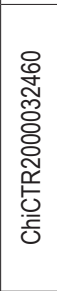 & & 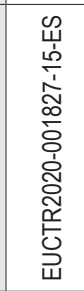 & 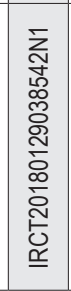 & 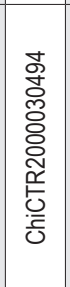 & 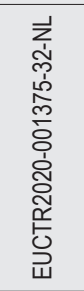 & 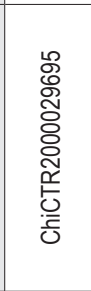 & & 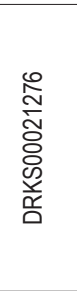 & \\
\hline
\end{tabular}




\begin{tabular}{|c|c|c|c|c|c|c|c|c|c|c|c|c|c|c|c|c|c|c|}
\hline 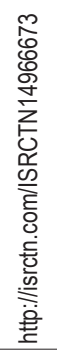 & 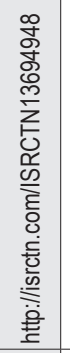 & 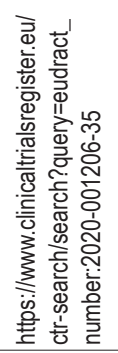 & 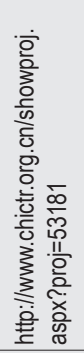 & 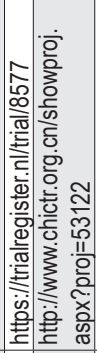 & 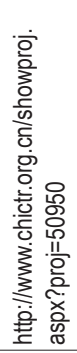 & 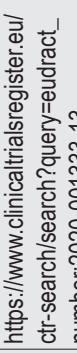 & 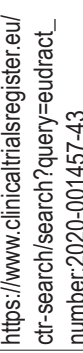 & 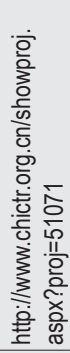 & 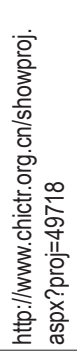 & 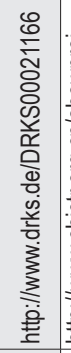 & 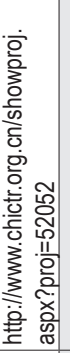 & 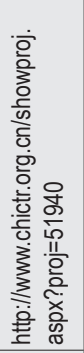 & 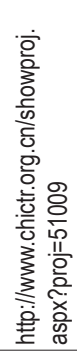 & 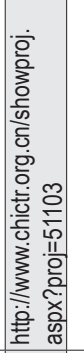 & 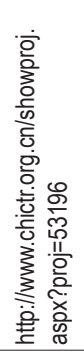 & 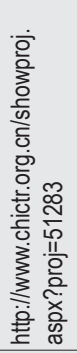 & 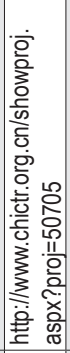 & 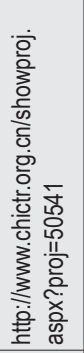 \\
\hline 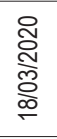 & 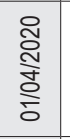 & 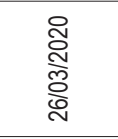 & 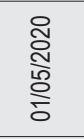 & 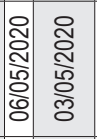 & 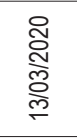 & 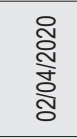 & 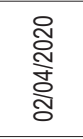 & 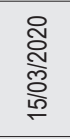 & 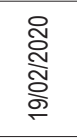 & 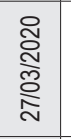 & 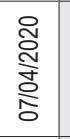 & 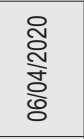 & 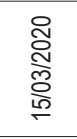 & 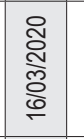 & 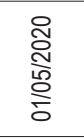 & 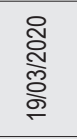 & 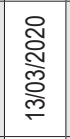 & $\begin{array}{l}\text { ल) } \\
\text { षे }\end{array}$ \\
\hline 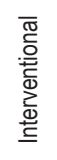 & 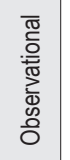 & 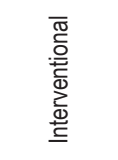 & 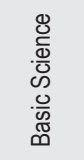 & 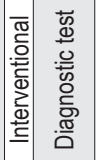 & 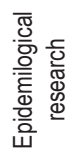 & 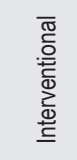 & 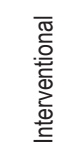 & 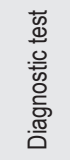 & 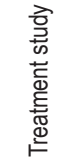 & 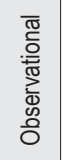 & 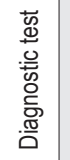 & 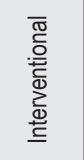 & 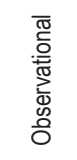 & 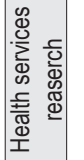 & 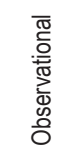 & 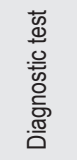 & 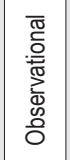 & 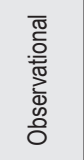 \\
\hline
\end{tabular}

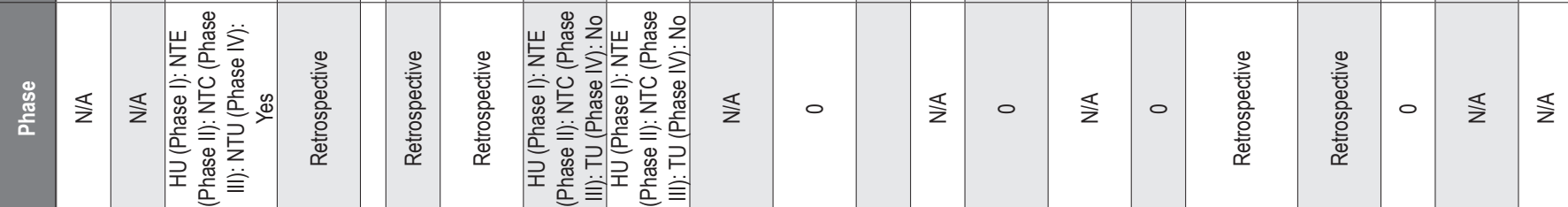

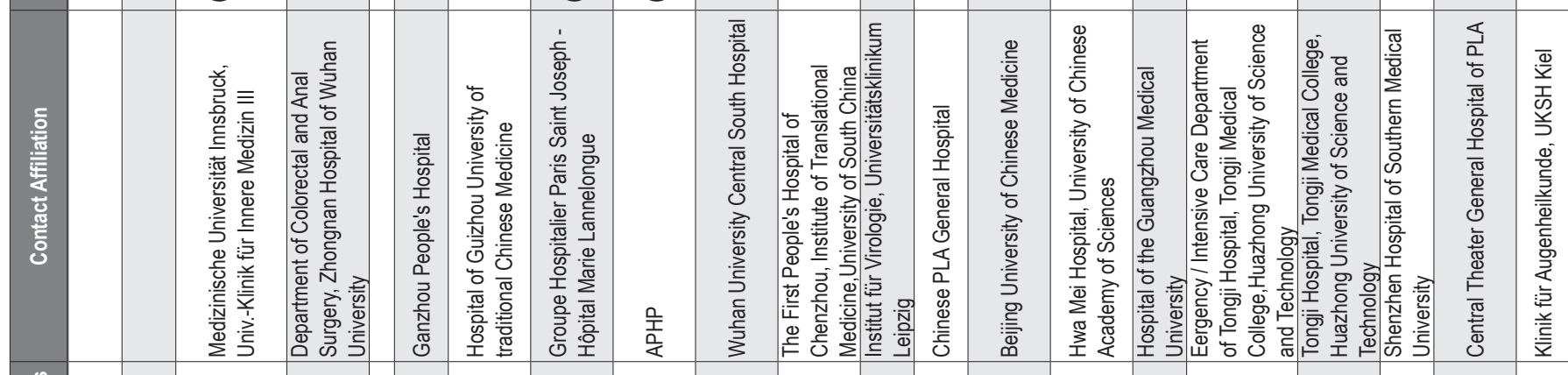

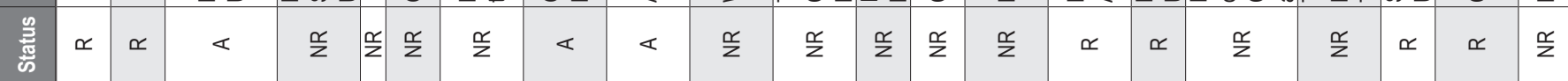

\begin{tabular}{|c|c|c|c|c|c|c|c|c|c|c|c|c|c|c|c|c|c|c|c|c|}
\hline 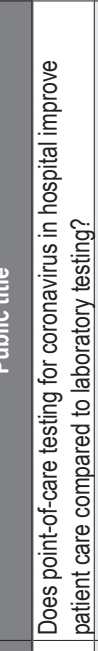 & 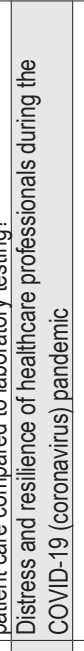 & 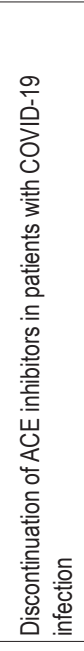 & 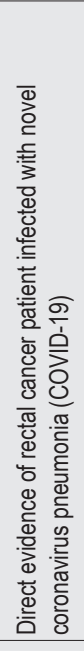 & 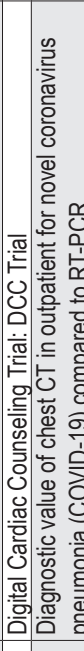 & 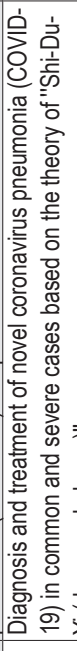 & 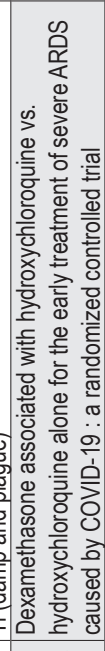 & 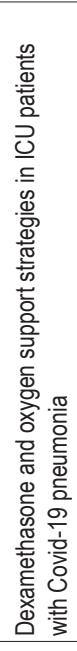 & 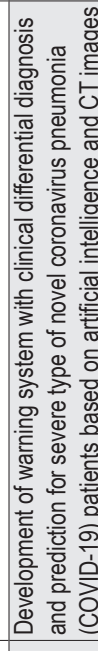 & 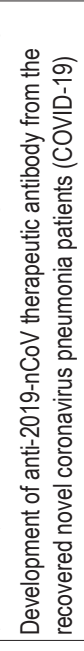 & 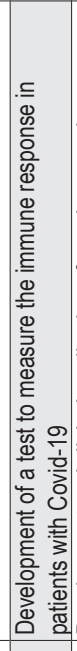 & 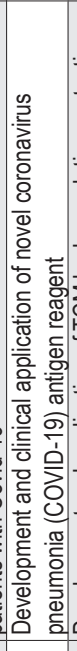 & 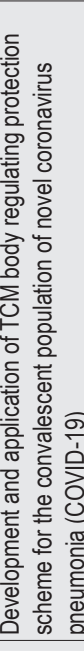 & 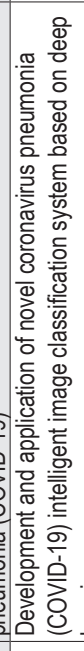 & 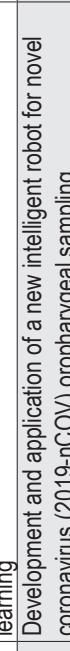 & 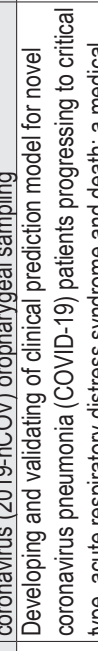 & & 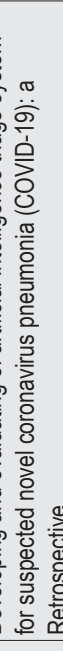 & 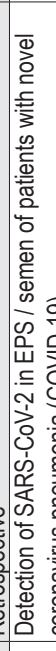 & 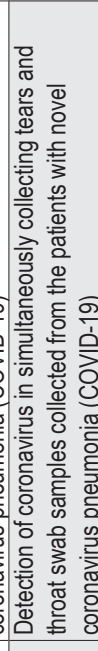 & 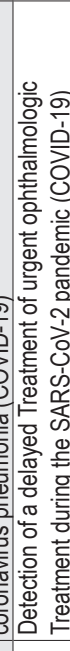 \\
\hline 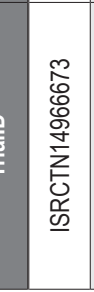 & 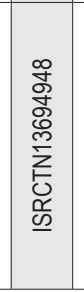 & 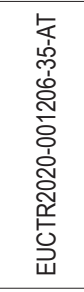 & 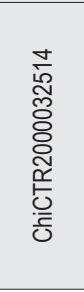 & 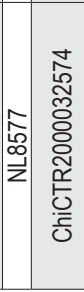 & 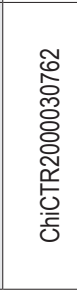 & 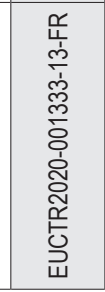 & 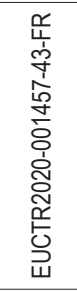 & 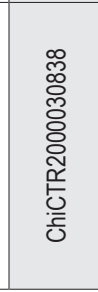 & 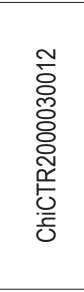 & 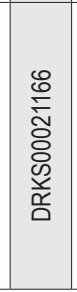 & 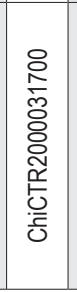 & 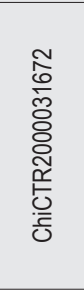 & 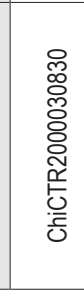 & 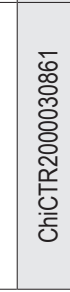 & চ্ণ & & 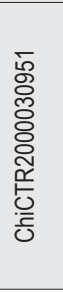 & & 0 & \\
\hline
\end{tabular}




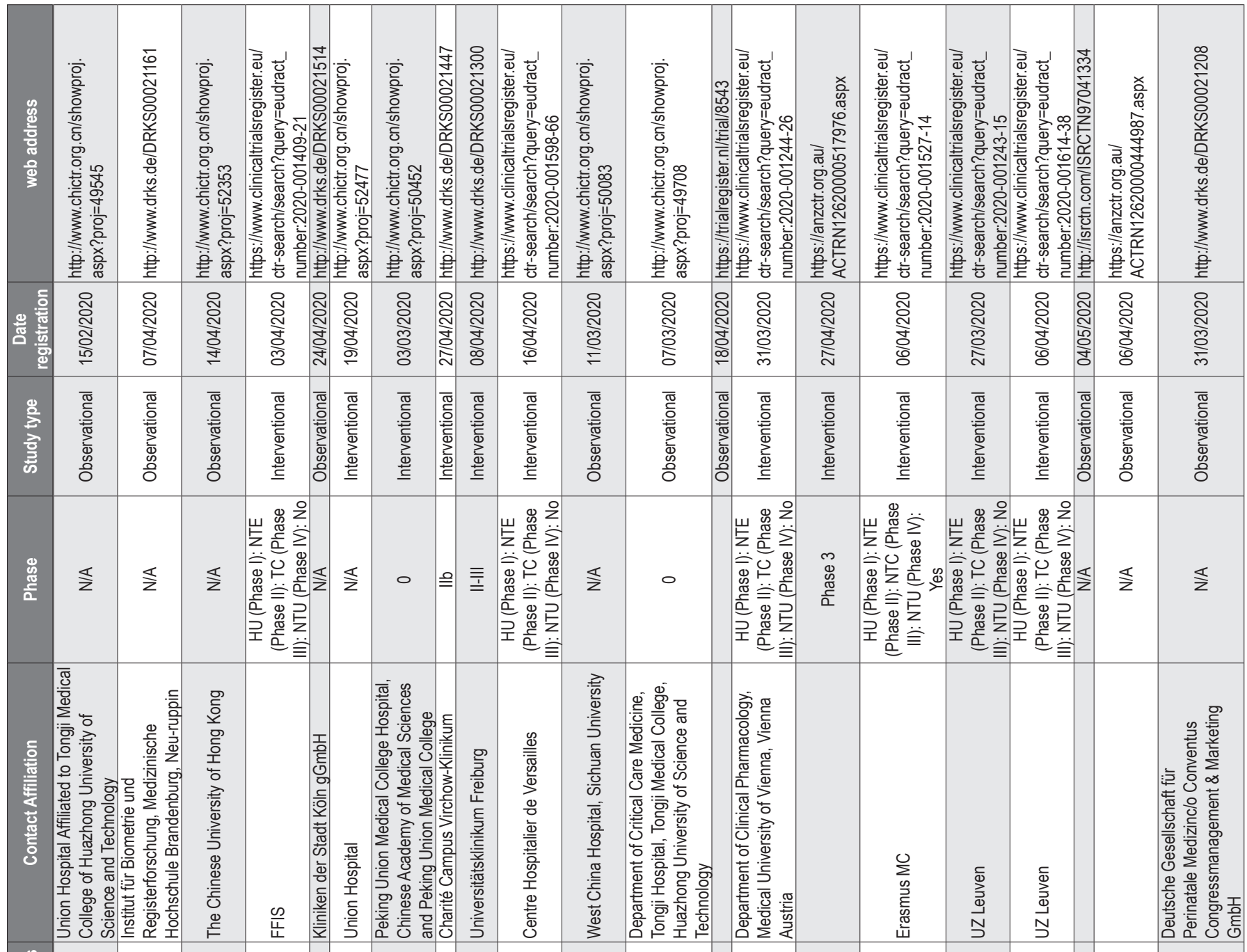

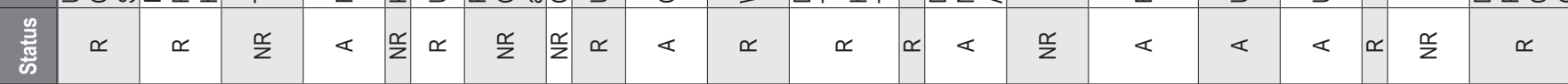

\begin{tabular}{|c|c|c|c|c|c|c|c|c|c|c|c|c|c|c|c|c|c|c|}
\hline 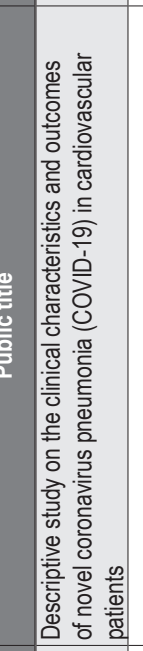 & 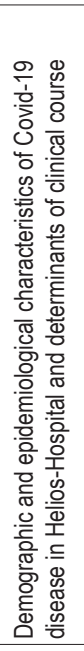 & 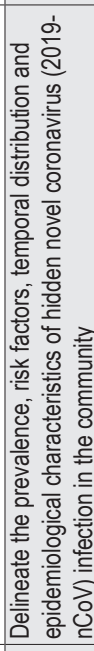 & 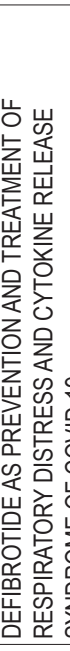 & 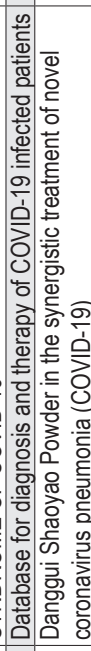 & 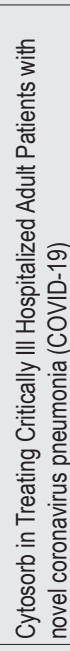 & 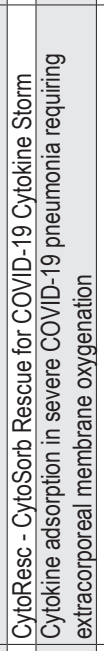 & 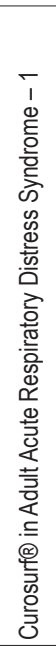 & 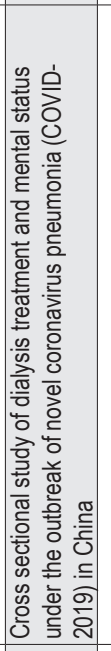 & 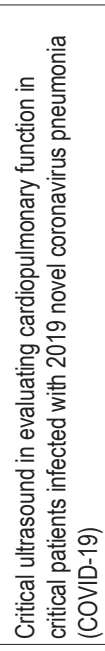 & 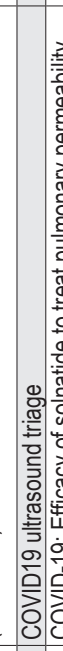 & 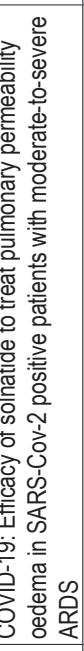 & 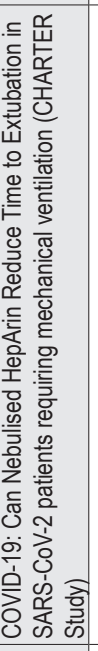 & 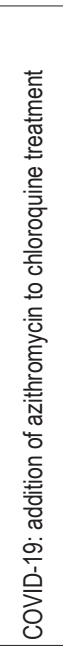 & 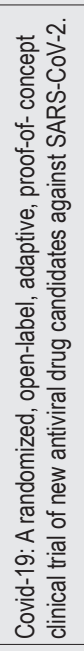 & 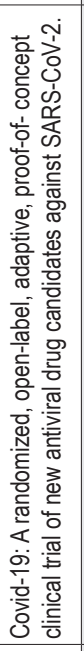 & & 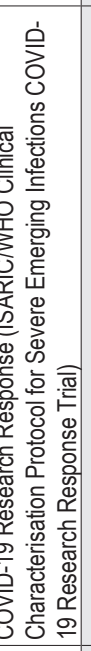 & 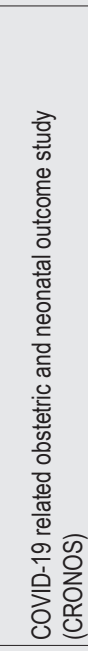 \\
\hline 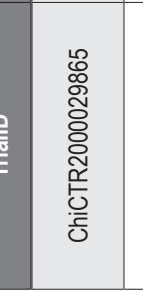 & 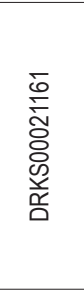 & 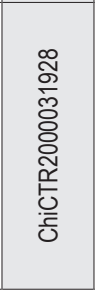 & 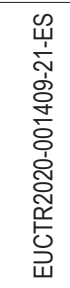 & 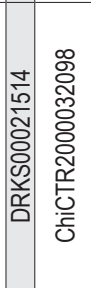 & 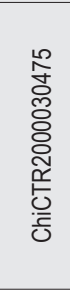 & 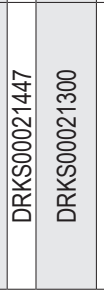 & 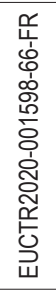 & 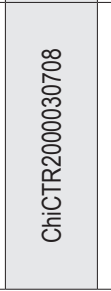 & 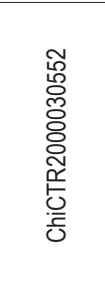 & 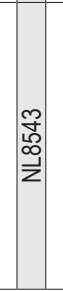 & 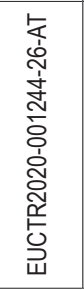 & 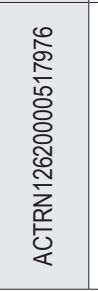 & 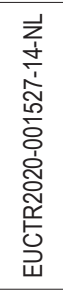 & 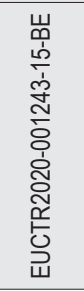 & 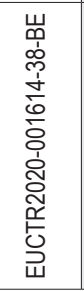 & & 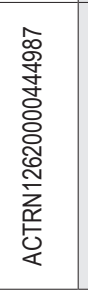 & 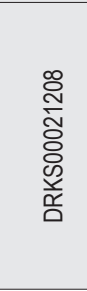 \\
\hline
\end{tabular}




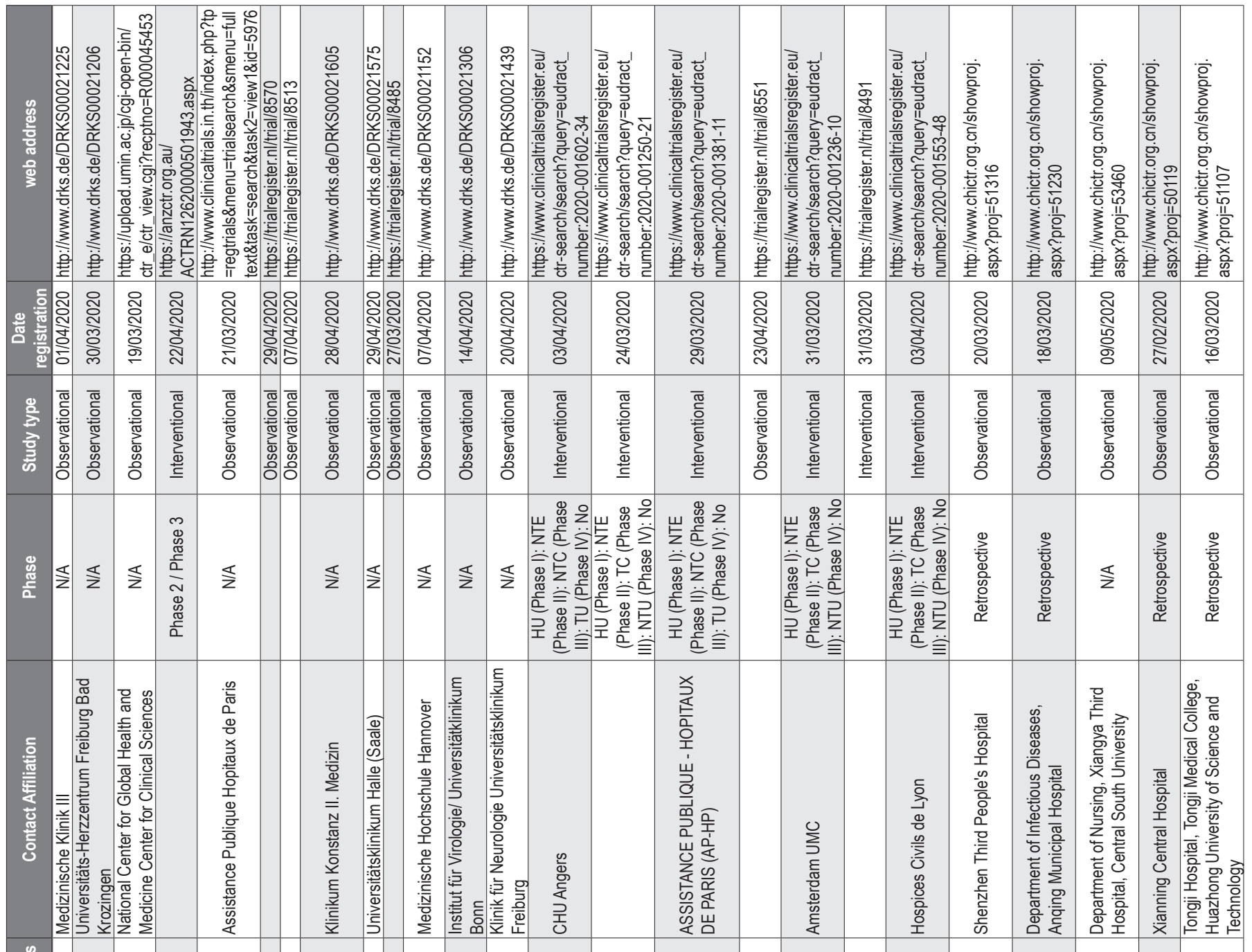

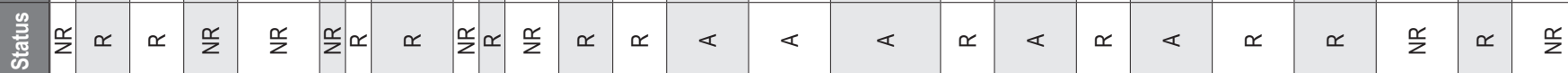

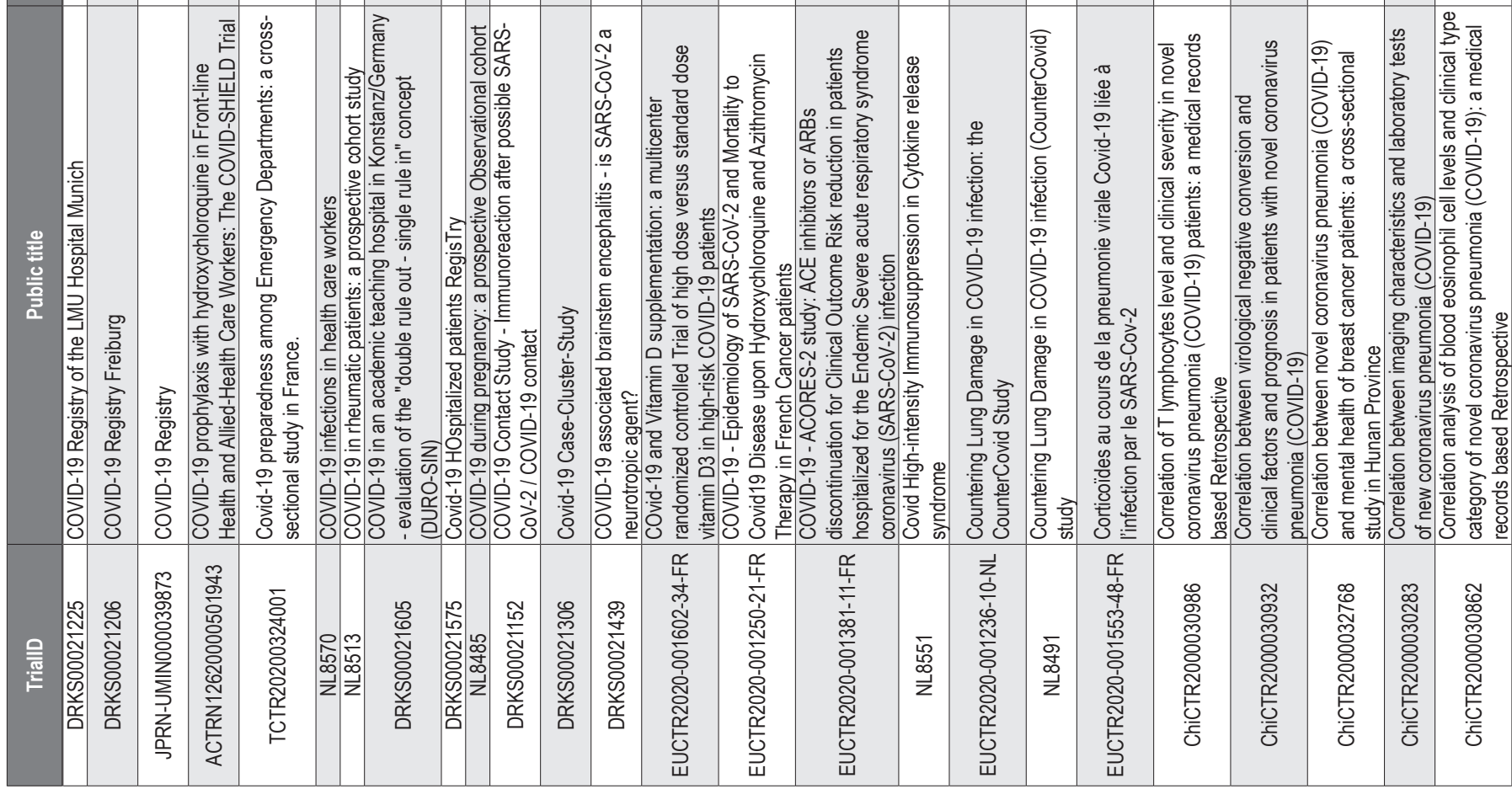




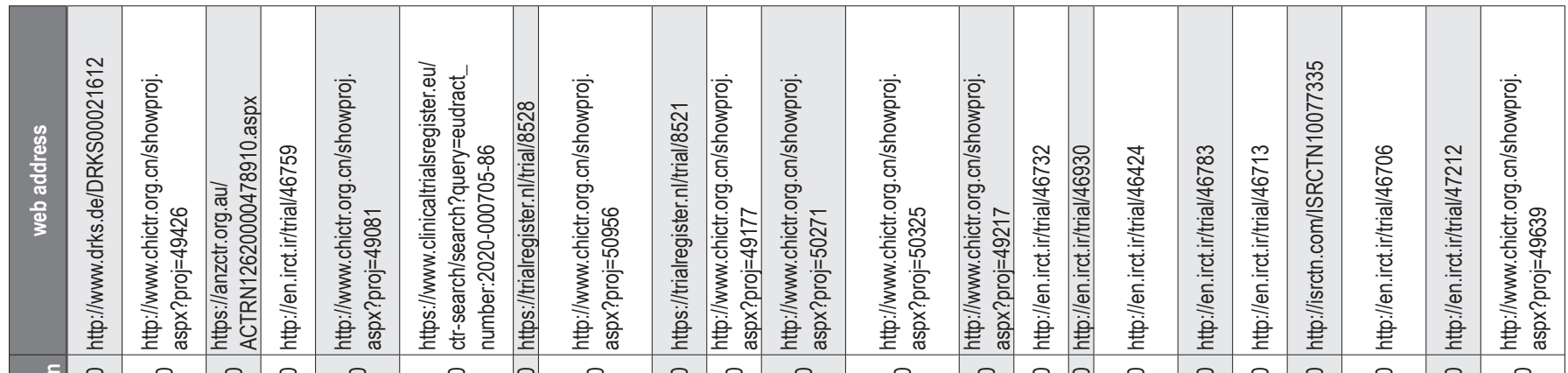

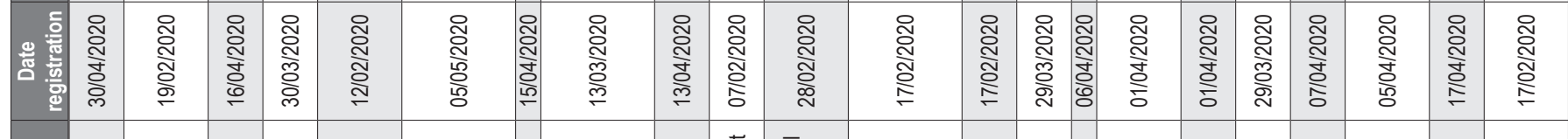

\begin{tabular}{|c|c|c|c|c|c|c|c|c|c|c|c|c|c|c|c|c|}
\hline & 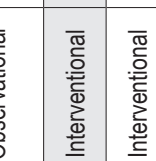 & & 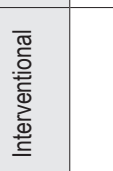 & & \multicolumn{2}{|c|}{ 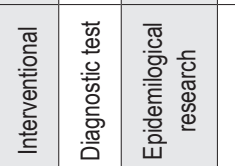 } & \multicolumn{2}{|c|}{ 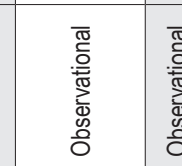 } & \multicolumn{2}{|c|}{ 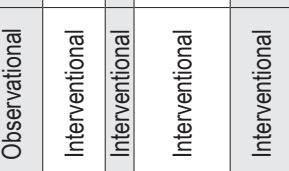 } & \multicolumn{3}{|c|}{ 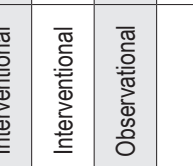 } & 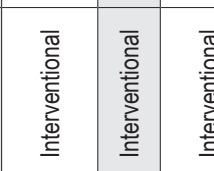 \\
\hline
\end{tabular}

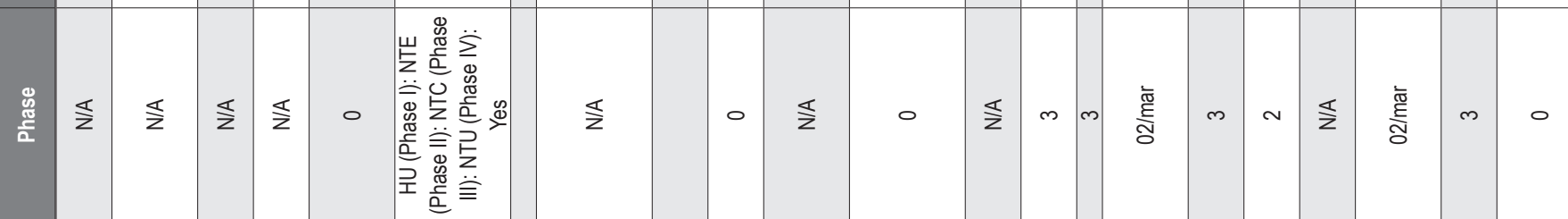

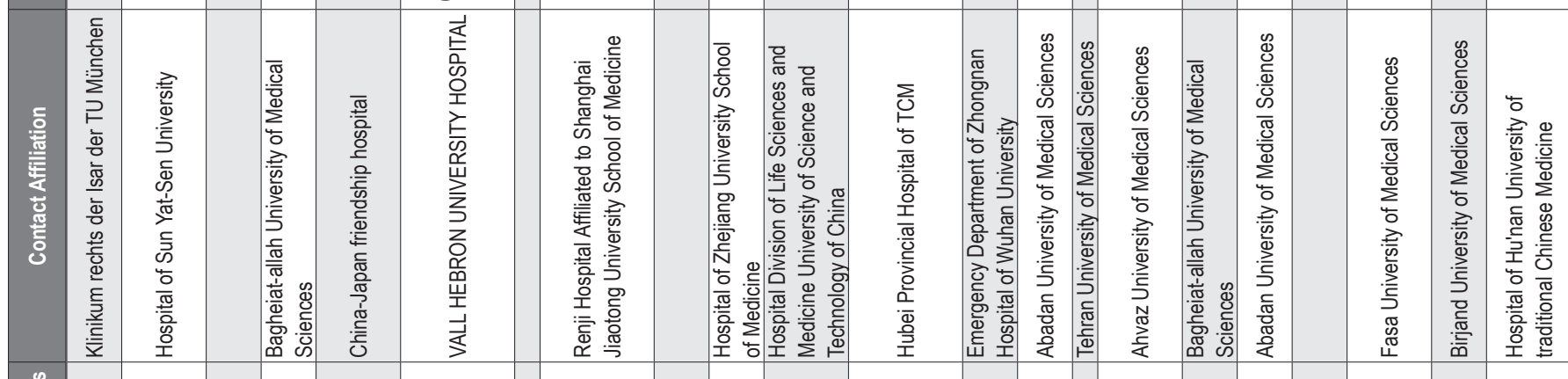

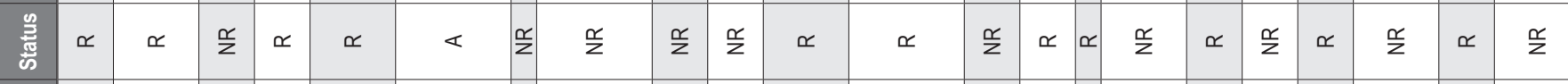

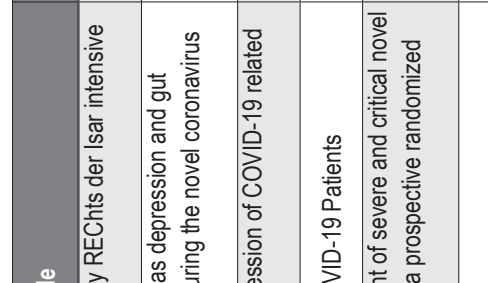

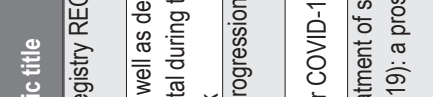

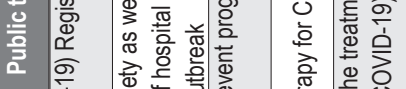

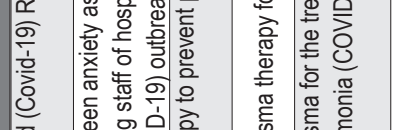

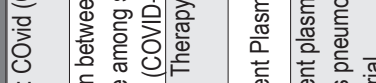

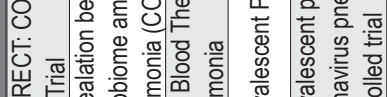

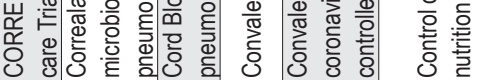

Um

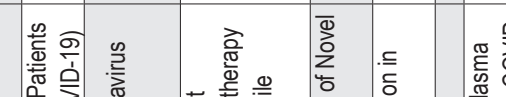

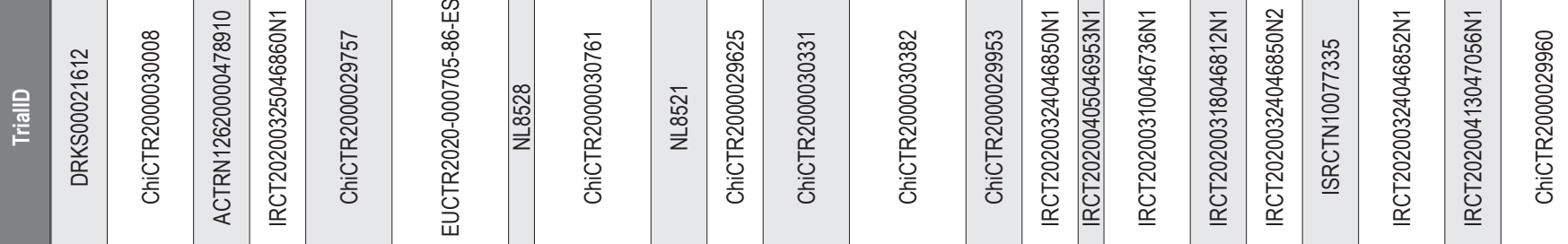




\begin{tabular}{|c|c|c|c|c|c|c|c|c|c|c|c|c|c|c|c|c|c|}
\hline 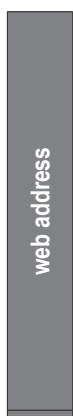 & 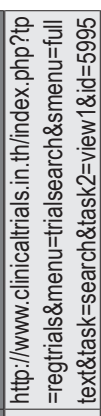 & 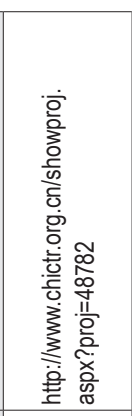 & 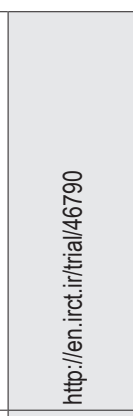 & 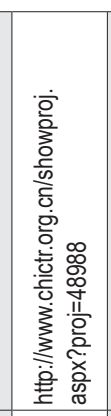 & 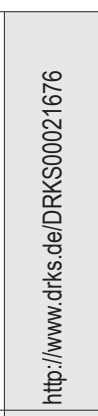 & 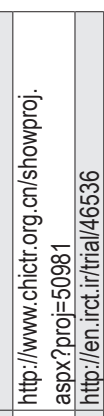 & 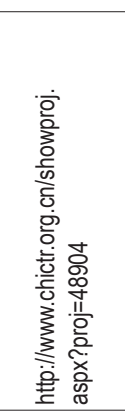 & 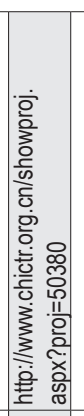 & 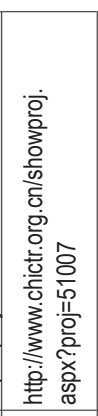 & 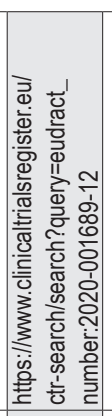 & 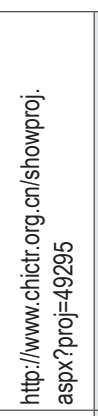 & 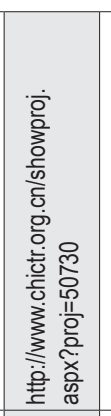 & 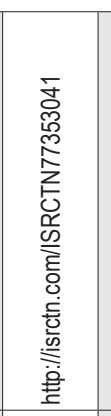 & 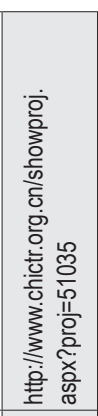 & 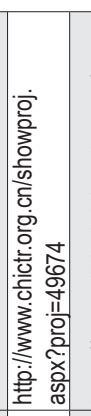 & 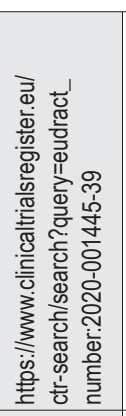 & 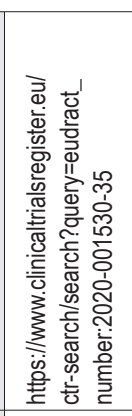 \\
\hline 흘 & 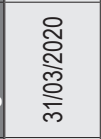 & $\begin{array}{l}\text { 令 } \\
\text { 호․ }\end{array}$ & 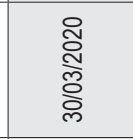 & 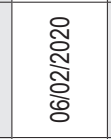 & 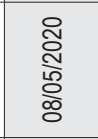 & 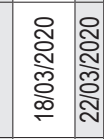 & 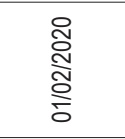 & 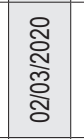 & 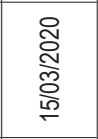 & 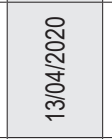 & 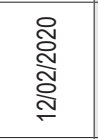 & 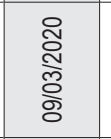 & 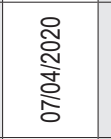 & 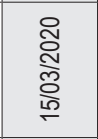 & 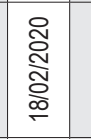 & 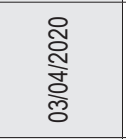 & 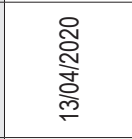 \\
\hline 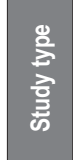 & 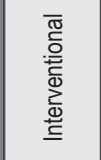 & 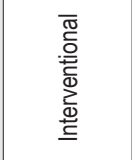 & 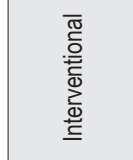 & 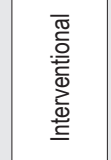 & 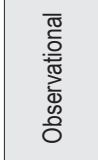 & 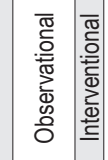 & 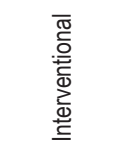 & 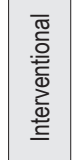 & 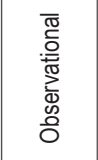 & 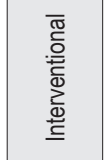 & 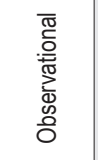 & 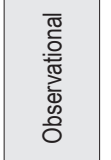 & 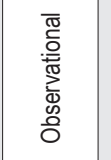 & 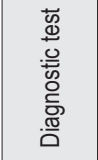 & 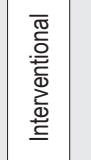 & 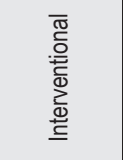 & 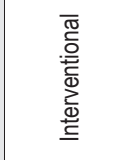 \\
\hline$\frac{g}{0}$ & $\begin{array}{l}\mathscr{\Xi} \\
\mathbb{\pi} \\
\frac{\pi}{\alpha}\end{array}$ & $\frac{\pi}{z}$ & $m$ & 0 & $\bar{s}$ & 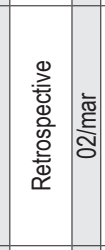 & 0 & 0 & 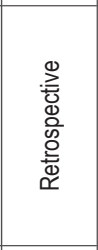 & 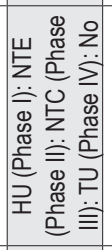 & 0 & $\bar{s}$ & $\stackrel{\Sigma}{z}$ & 0 & $\frac{\overrightarrow{\mathrm{d}}}{\bar{c}}$ & 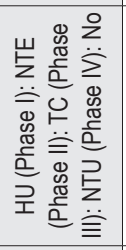 & 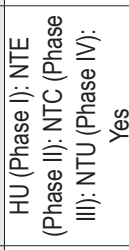 \\
\hline 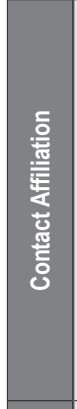 & 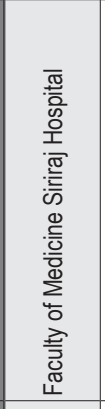 & 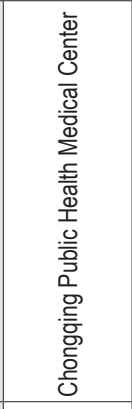 & 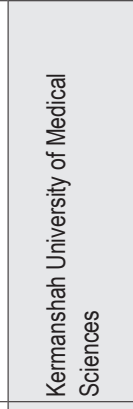 & 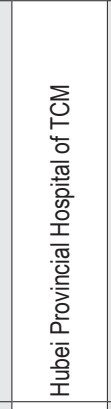 & 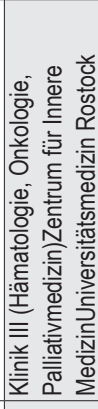 & 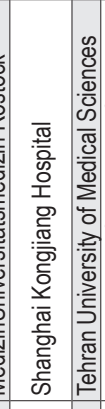 & 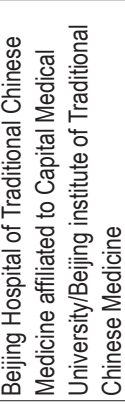 & 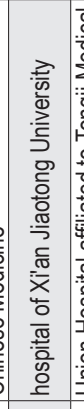 & 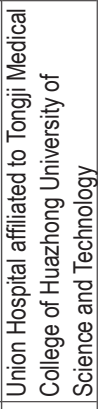 & 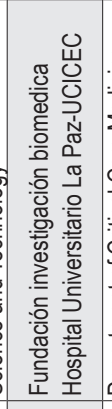 & 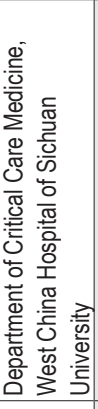 & 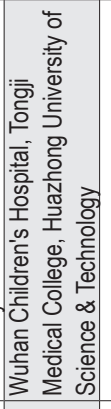 & & 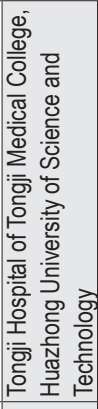 & 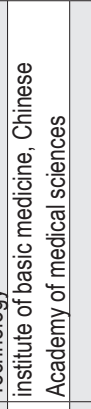 & 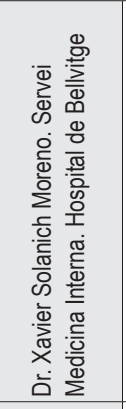 & 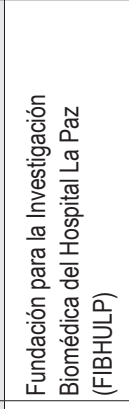 \\
\hline 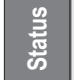 & $\frac{\alpha}{2}$ & $\propto$ & $\alpha$ & $\propto$ & $\propto$ & $\frac{\alpha}{z} \simeq$ & $z$ & $\frac{\alpha}{z}$ & $\propto$ & $\ll$ & $\simeq$ & $\propto$ & $\propto$ & $\frac{n}{2}$ & $\propto$ & $\ll$ & $\ll$ \\
\hline 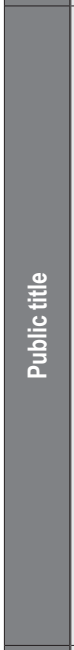 & 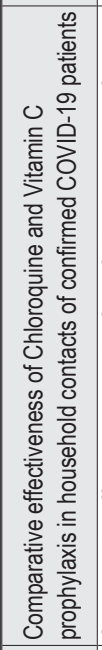 & 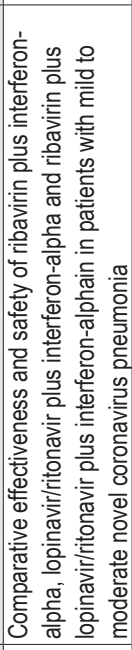 & 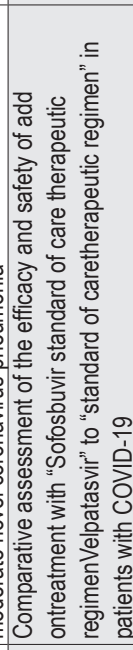 & 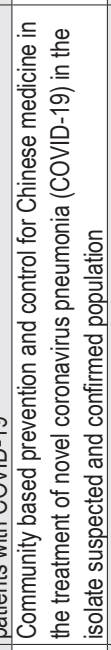 & 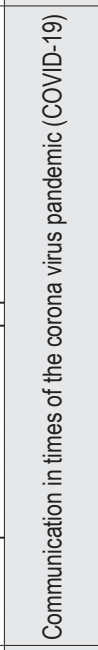 & 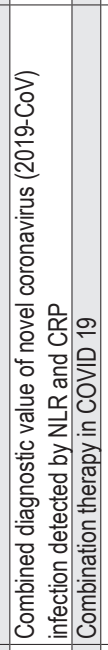 & 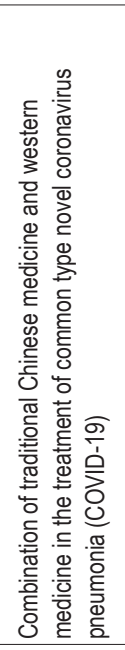 & 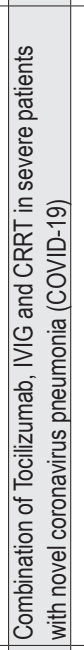 & 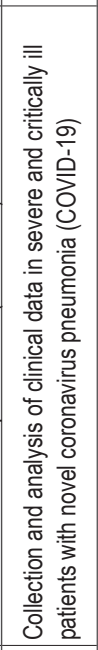 & 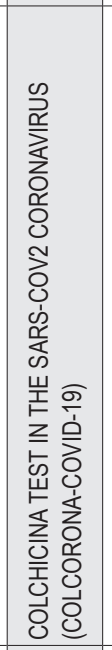 & 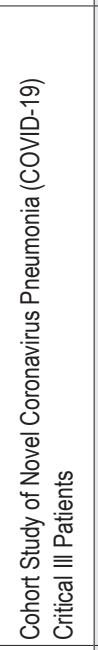 & 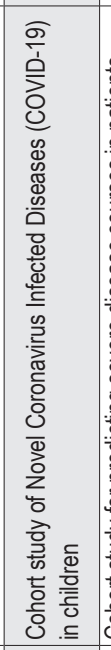 & 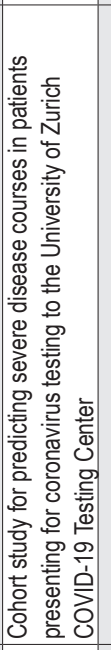 & 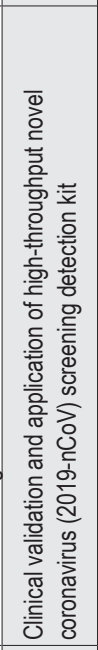 & 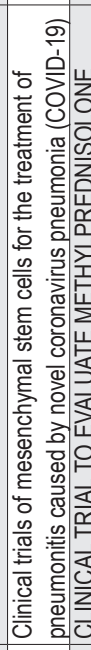 & 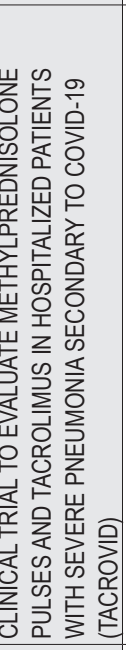 & 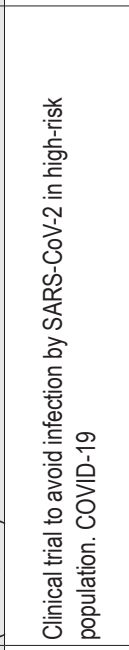 \\
\hline 害 & 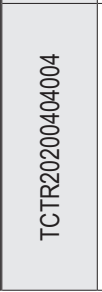 & 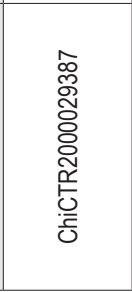 & 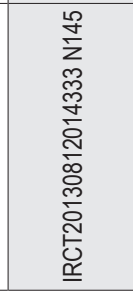 & 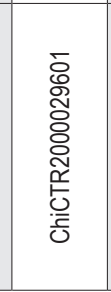 & 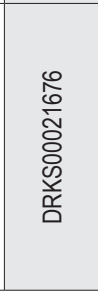 & 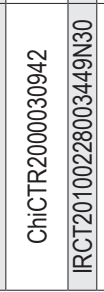 & 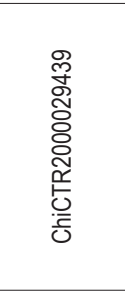 & 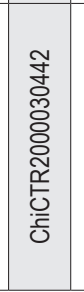 & 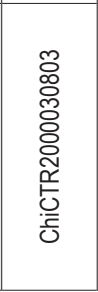 & 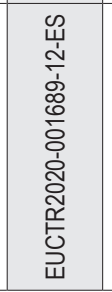 & 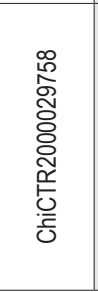 & 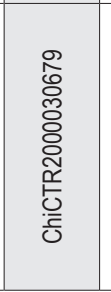 & 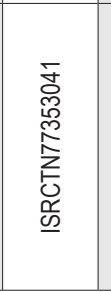 & 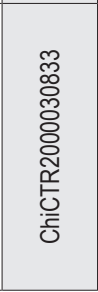 & 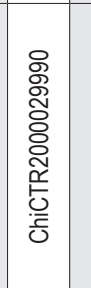 & 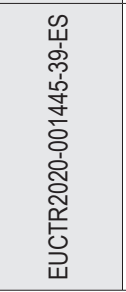 & 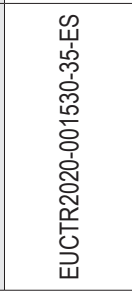 \\
\hline
\end{tabular}




\begin{tabular}{|c|c|c|c|c|c|c|c|c|c|c|c|c|c|c|c|c|}
\hline 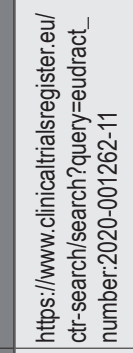 & 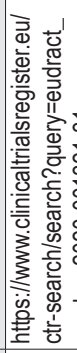 & 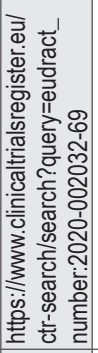 & 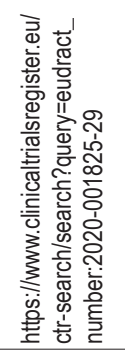 & 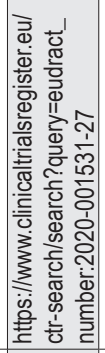 & 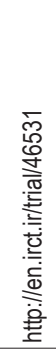 & 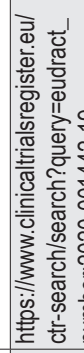 & 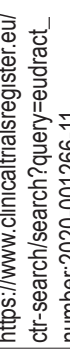 & 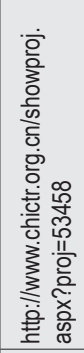 & 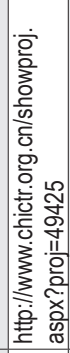 & 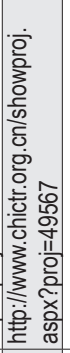 & 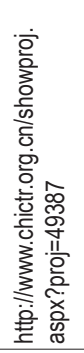 & 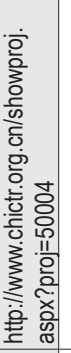 & 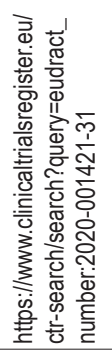 & 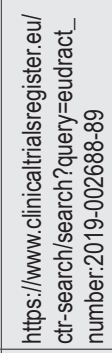 & 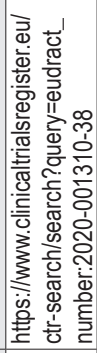 & 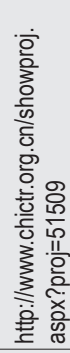 \\
\hline 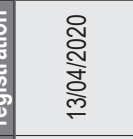 & 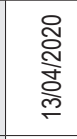 & 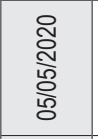 & 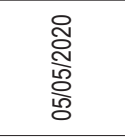 & 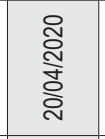 & 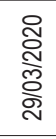 & 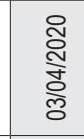 & 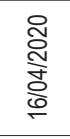 & 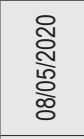 & 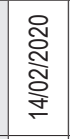 & 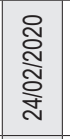 & 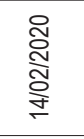 & 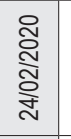 & $\begin{array}{l}\text { तิ } \\
\text { তิ } \\
\frac{0}{0}\end{array}$ & 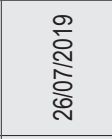 & 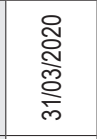 & 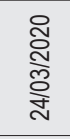 \\
\hline 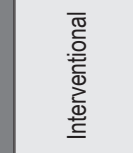 & 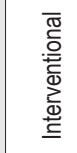 & 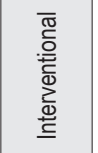 & 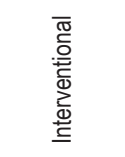 & 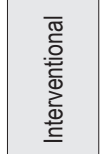 & 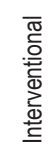 & 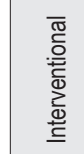 & 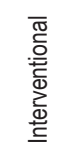 & 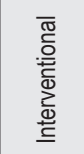 & 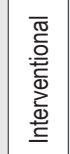 & 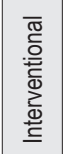 & 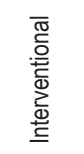 & 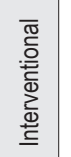 & 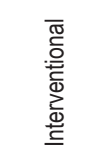 & 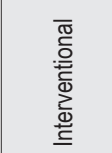 & 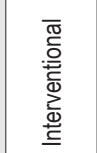 & 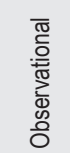 \\
\hline
\end{tabular}

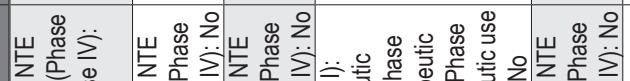

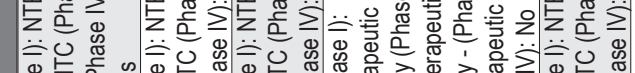

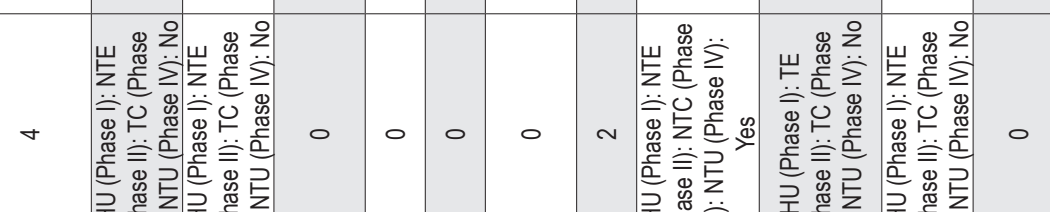
호을

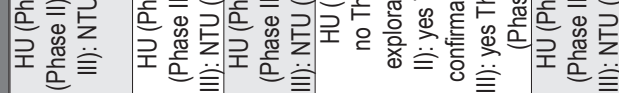

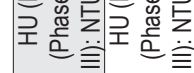

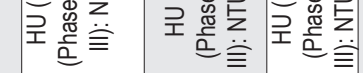

\begin{tabular}{|c|c|c|c|c|c|c|c|c|c|c|c|c|c|c|c|c|}
\hline 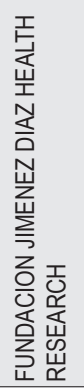 & 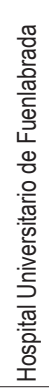 & 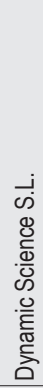 & 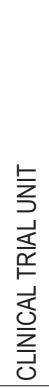 & 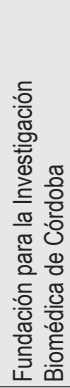 & 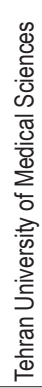 & 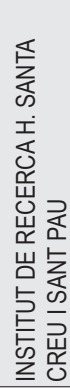 & 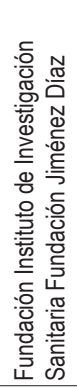 & 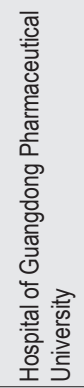 & 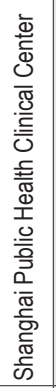 & 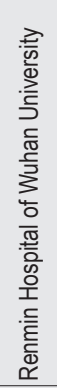 & 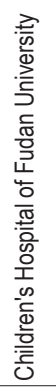 & 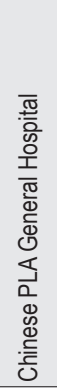 & 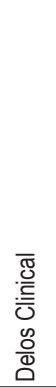 & 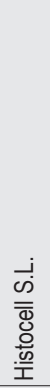 & $\begin{array}{l}\underline{\underline{\underline{\underline{s}}}} \\
\underline{\underline{\underline{y}}}\end{array}$ & 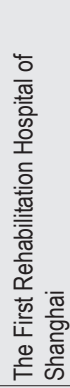 \\
\hline$\varangle$ & $\ll$ & $\varangle$ & $\varangle$ & $\varangle$ & $\propto$ & $<$ & $\varangle$ & $\frac{\alpha}{z}$ & $\propto$ & $\frac{\underline{c}}{z}$ & $\simeq$ & $\frac{\underline{\alpha}}{z}$ & $\ll$ & $\ll$ & $\ll$ & $\frac{n}{z}$ \\
\hline
\end{tabular}

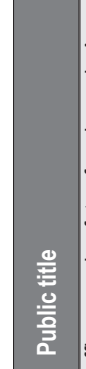

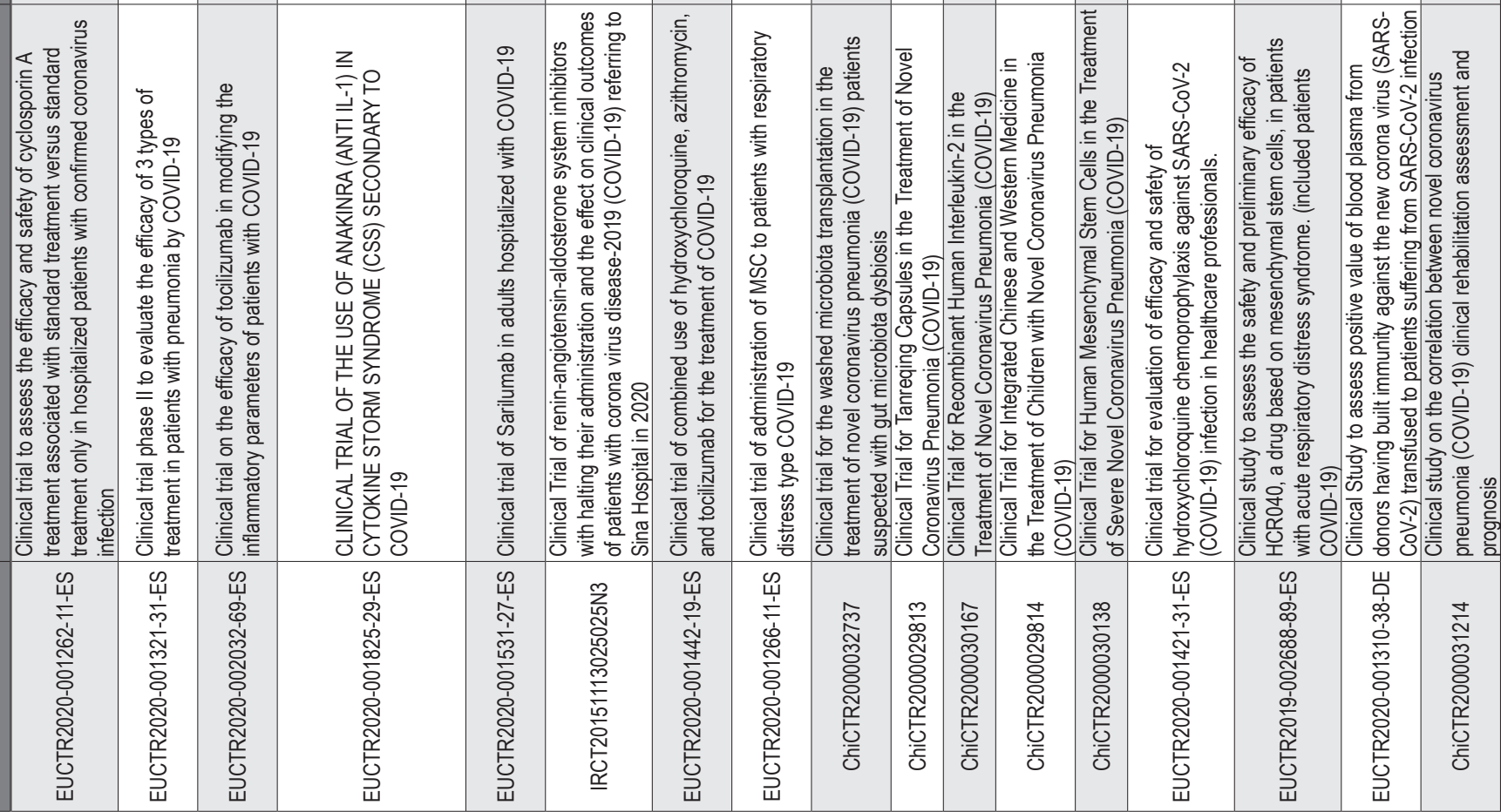




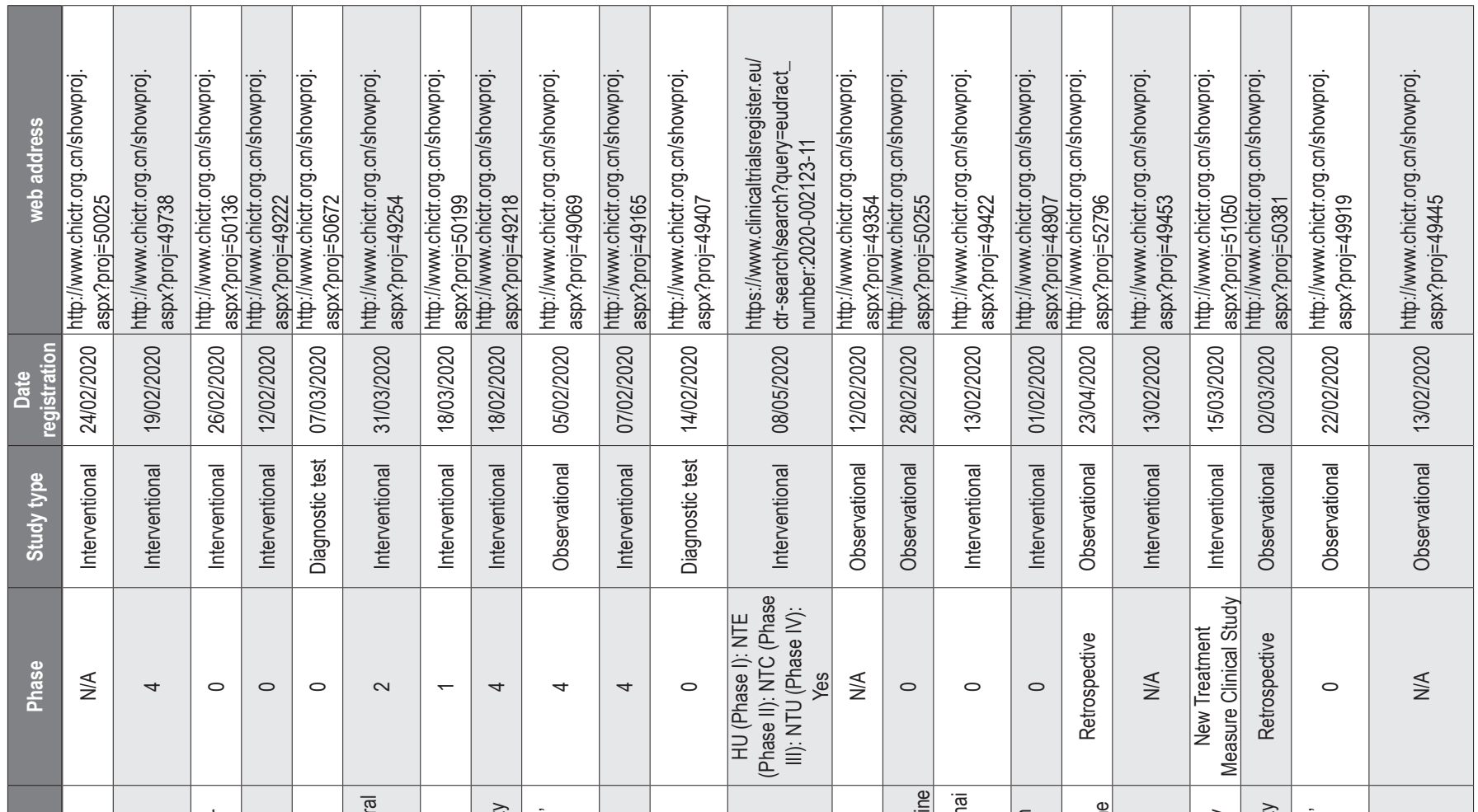

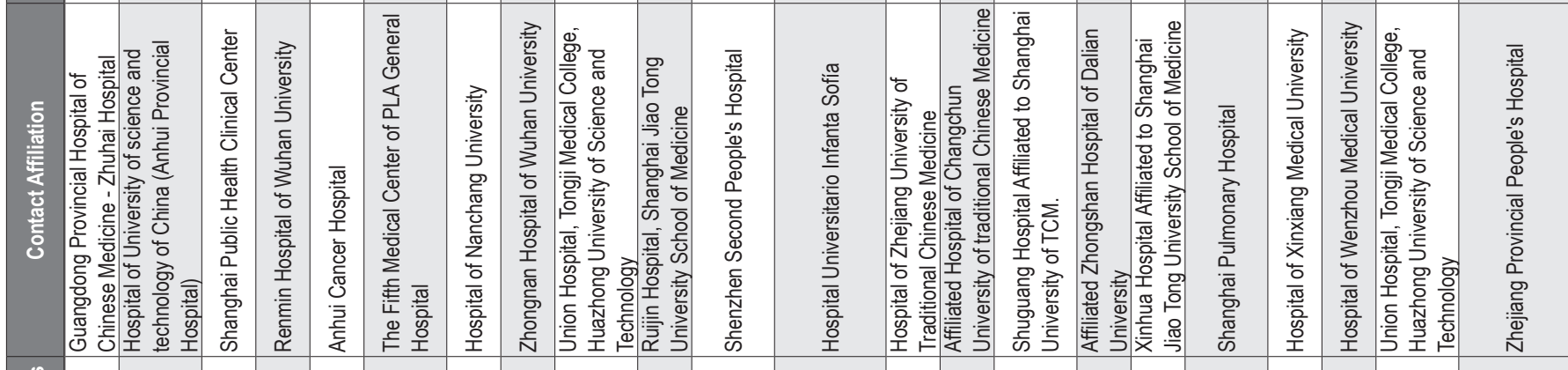

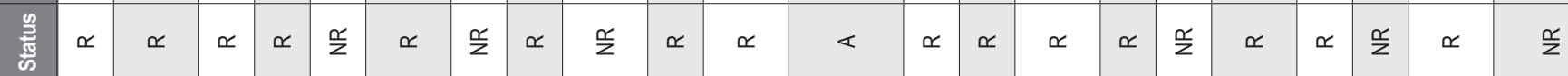

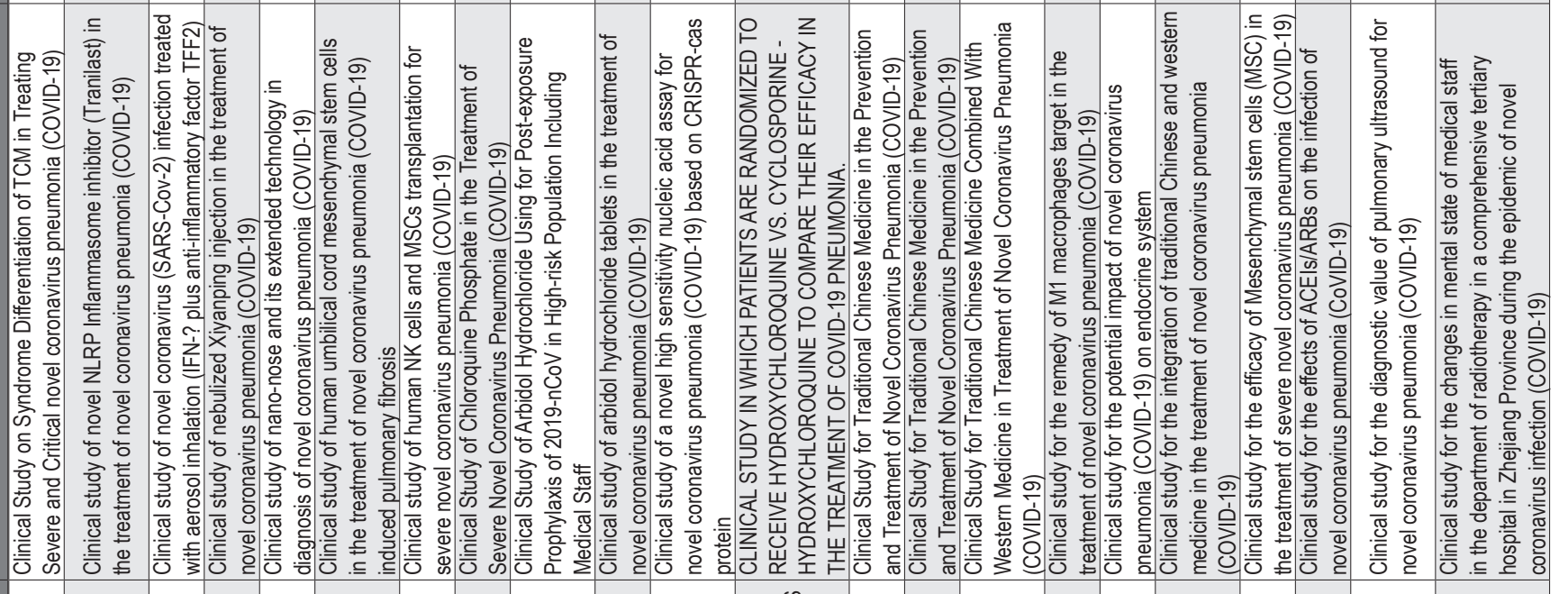

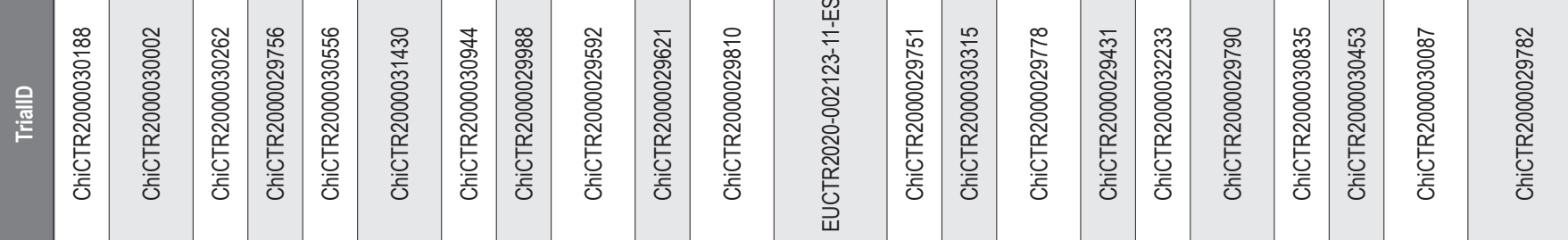




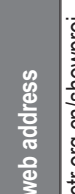

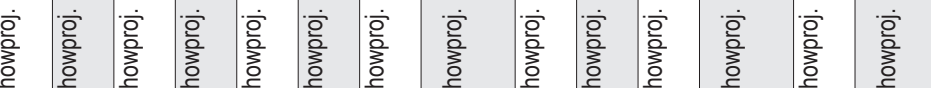
产

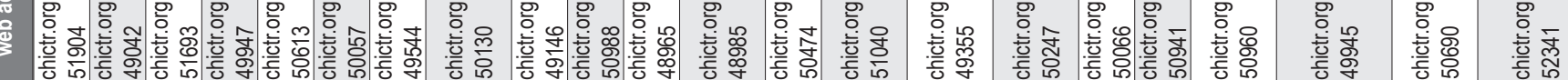

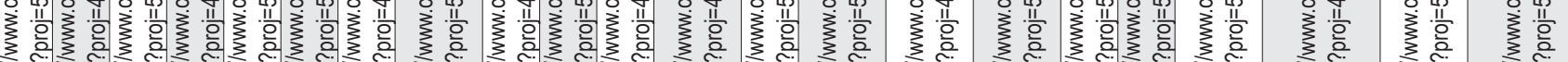

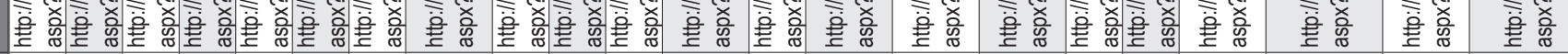

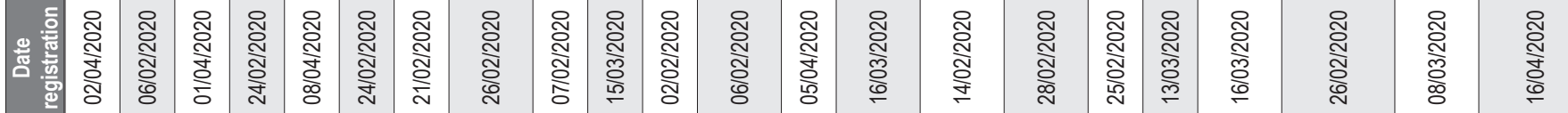

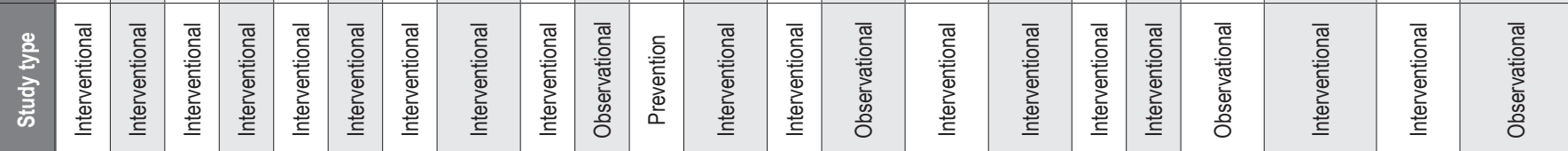

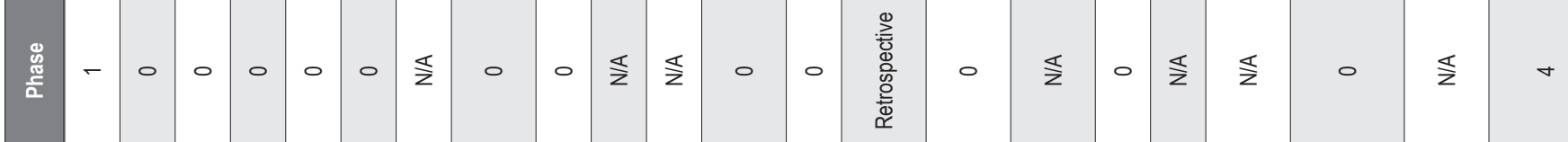

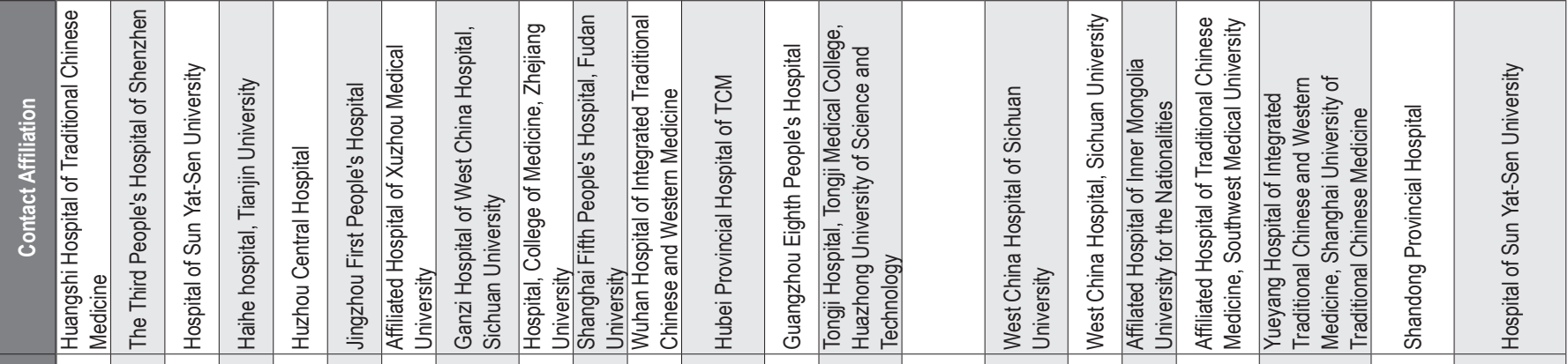

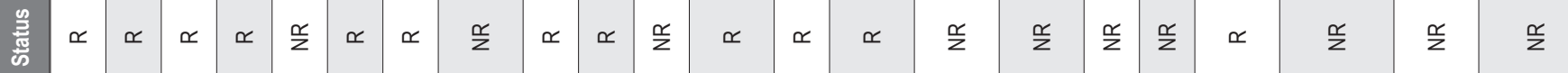

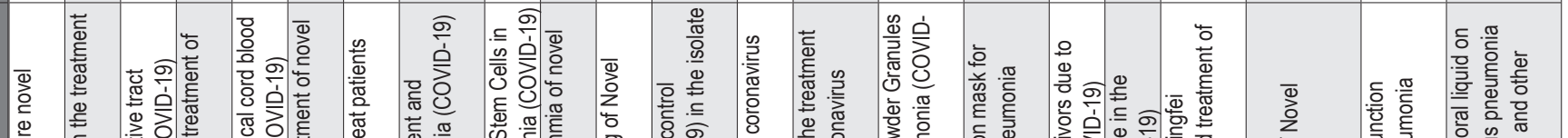

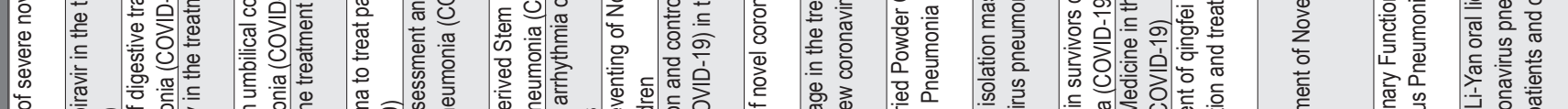
总

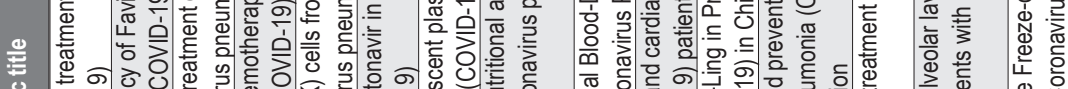

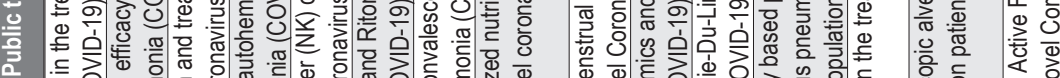
空

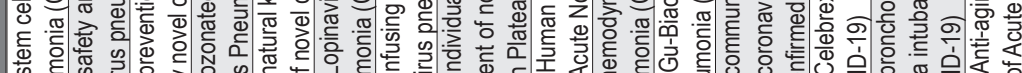

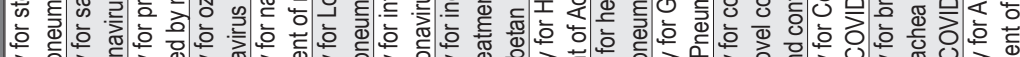

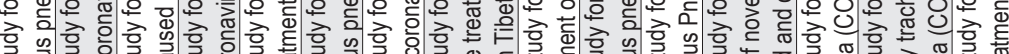

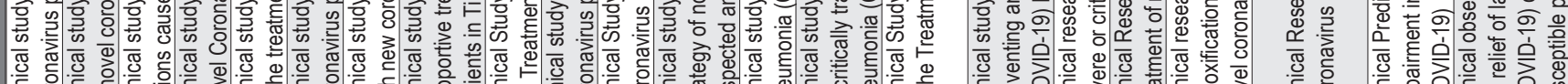

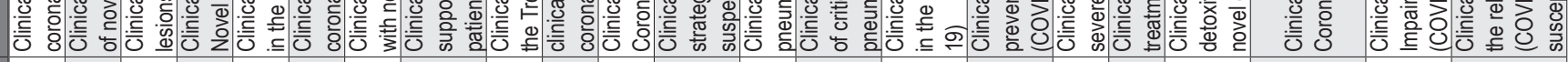

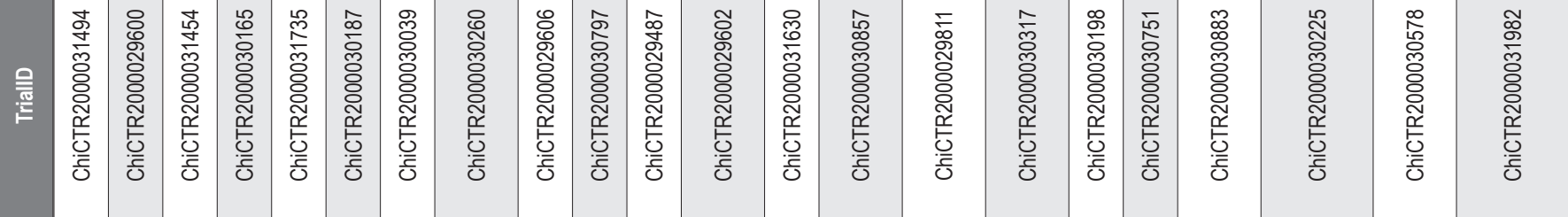




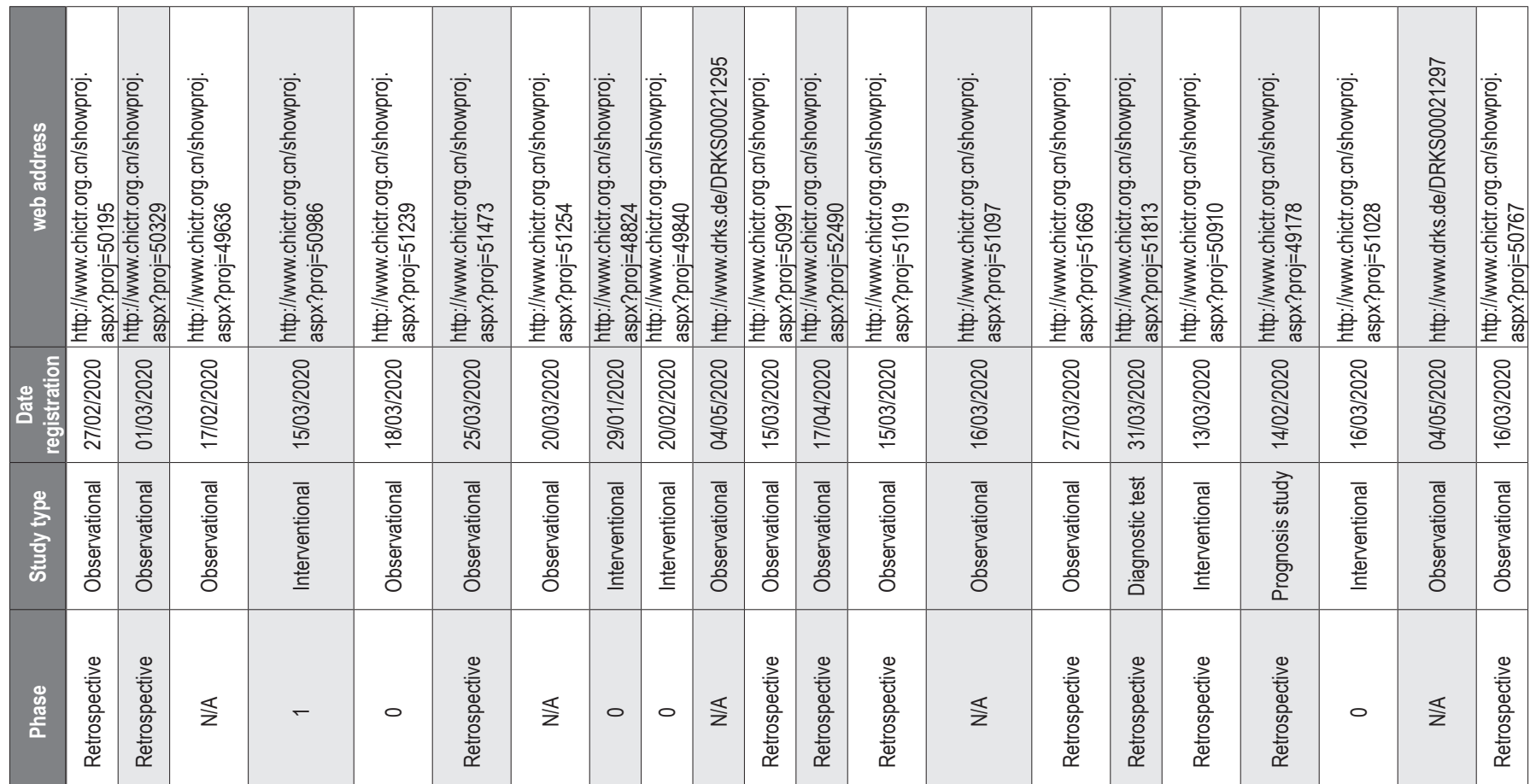

\begin{tabular}{|c|c|c|c|c|c|c|c|c|c|c|c|c|c|c|c|c|c|}
\hline 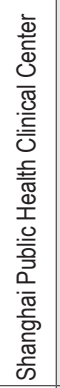 & 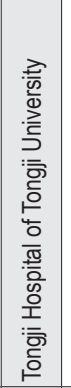 & 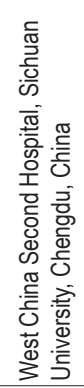 & 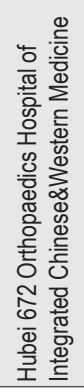 & 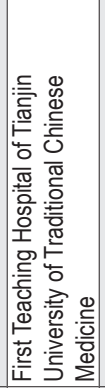 & 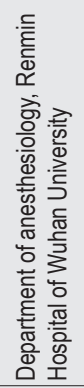 & 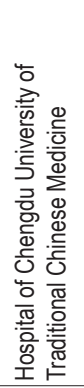 & 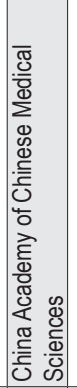 & 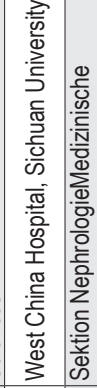 & 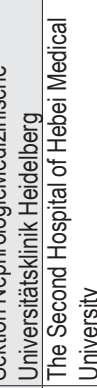 & 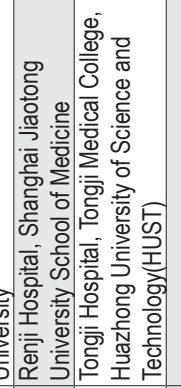 & 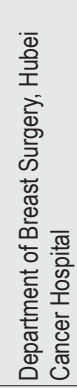 & & $\begin{array}{l}0 \\
0 \\
0 \\
0\end{array}$ & 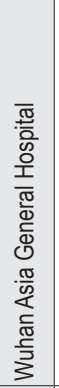 & 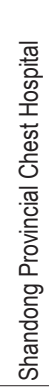 & 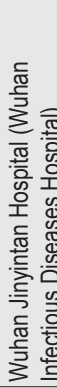 & \\
\hline
\end{tabular}

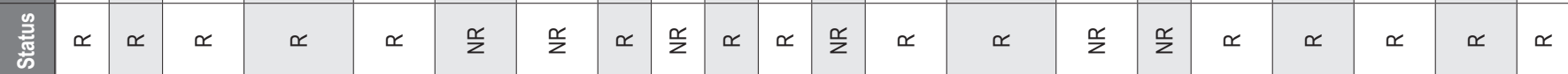

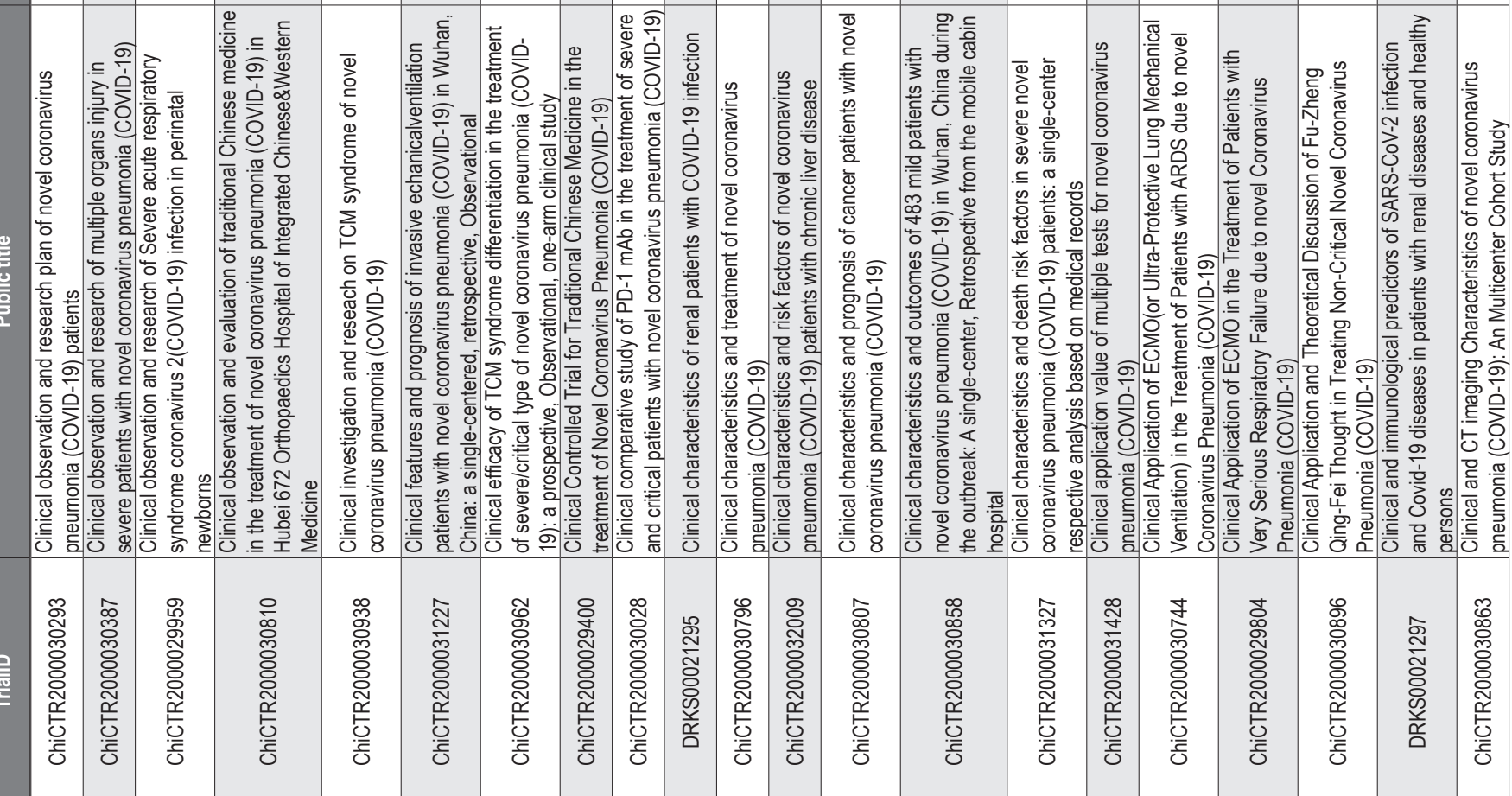




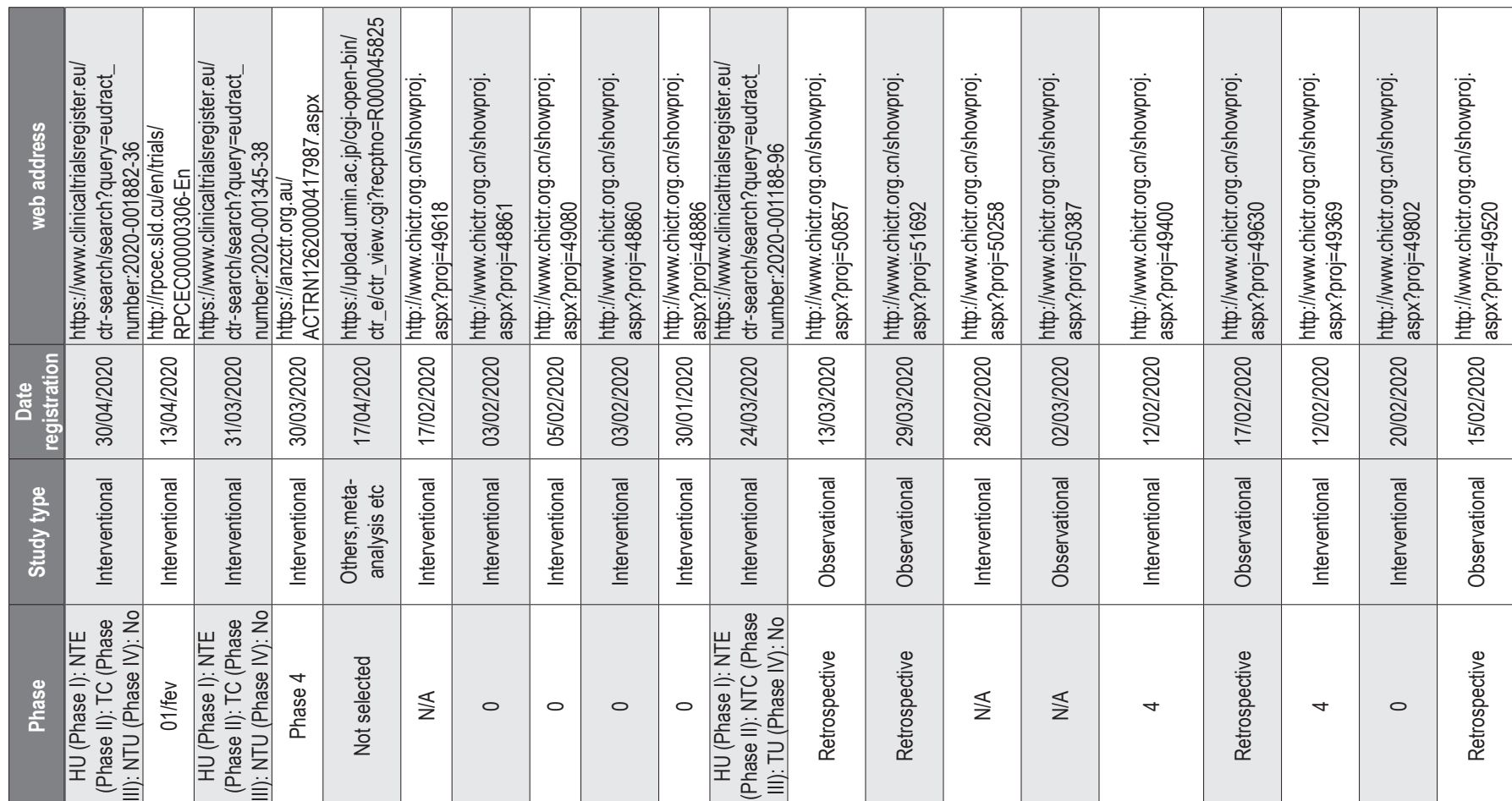

\begin{tabular}{|c|c|c|c|c|c|c|c|c|c|c|c|c|c|c|c|c|c|c|c|}
\hline 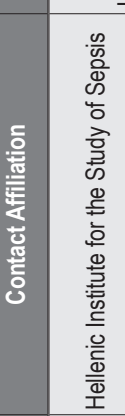 & 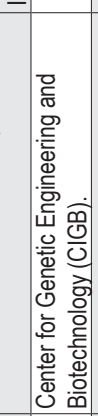 & 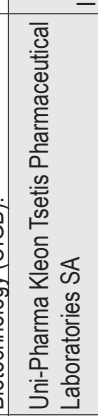 & & 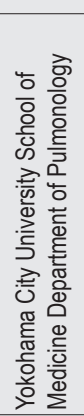 & 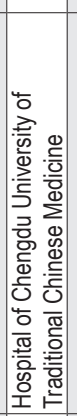 & 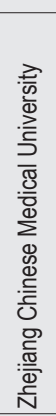 & 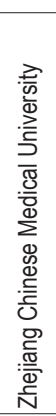 & 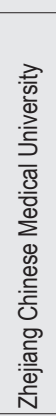 & 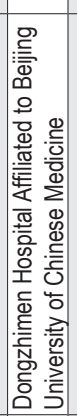 & 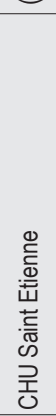 & 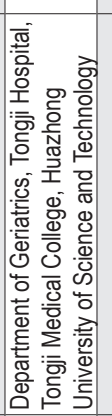 & & 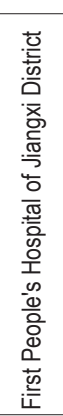 & 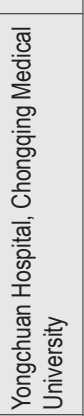 & 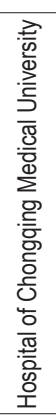 & 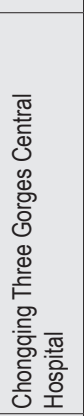 & 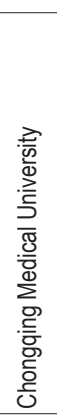 & 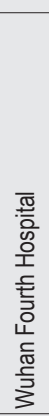 & 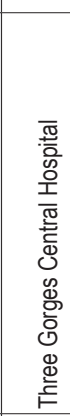 \\
\hline$\ll$ & $\propto$ & $\ll$ & $\simeq$ & $\frac{\alpha}{z}$ & $\frac{\alpha}{z}$ & $\simeq$ & $\simeq$ & $\simeq$ & $\propto$ & $\ll$ & $\frac{\alpha}{z}$ & $\frac{\underline{x}}{z}$ & $\frac{⿱ 亠 x}{z}$ & & $\propto$ & $\frac{\alpha}{z}$ & $\frac{\alpha}{z}$ & $\frac{\alpha}{z}$ & $\frac{\alpha}{z}$ \\
\hline
\end{tabular}

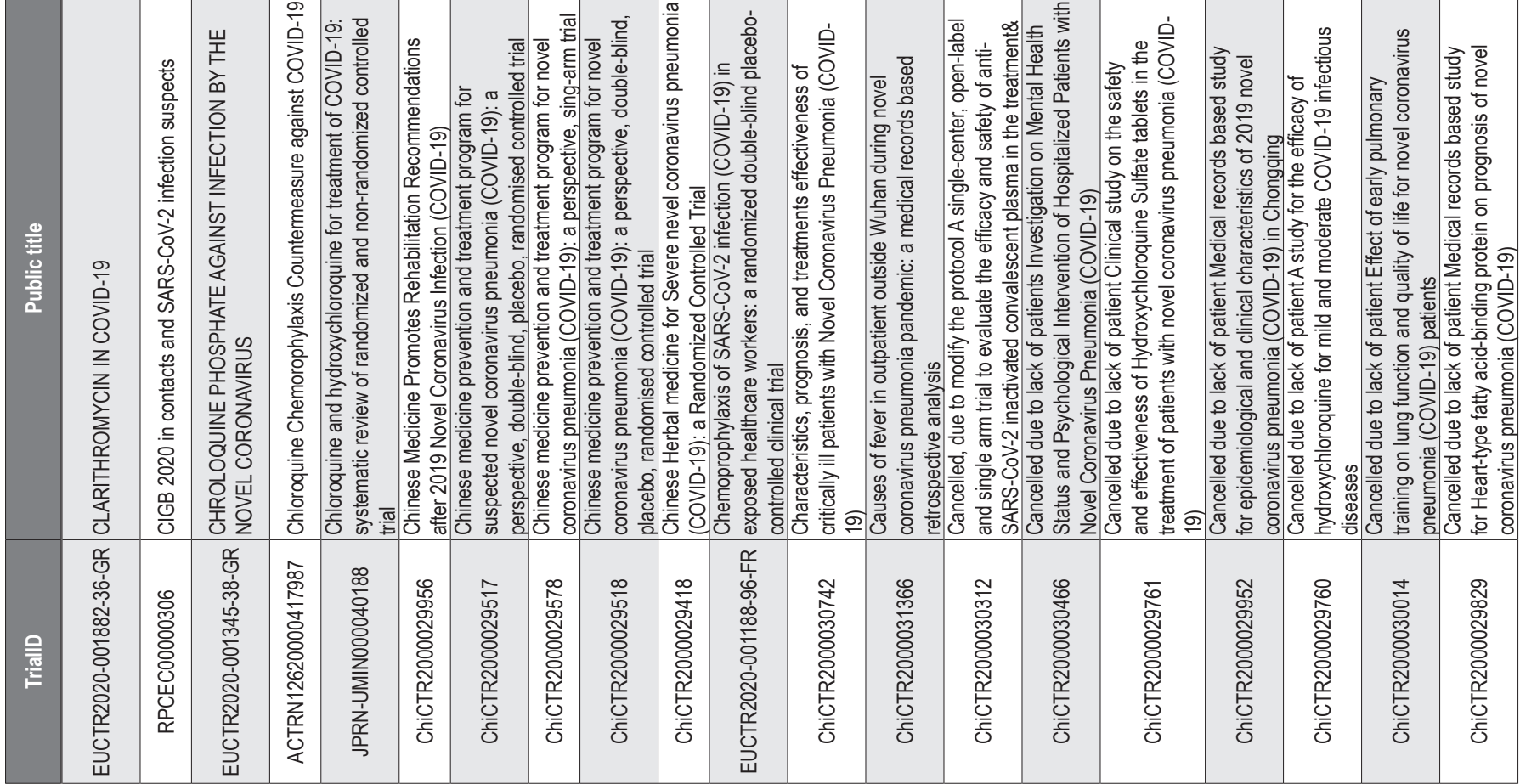




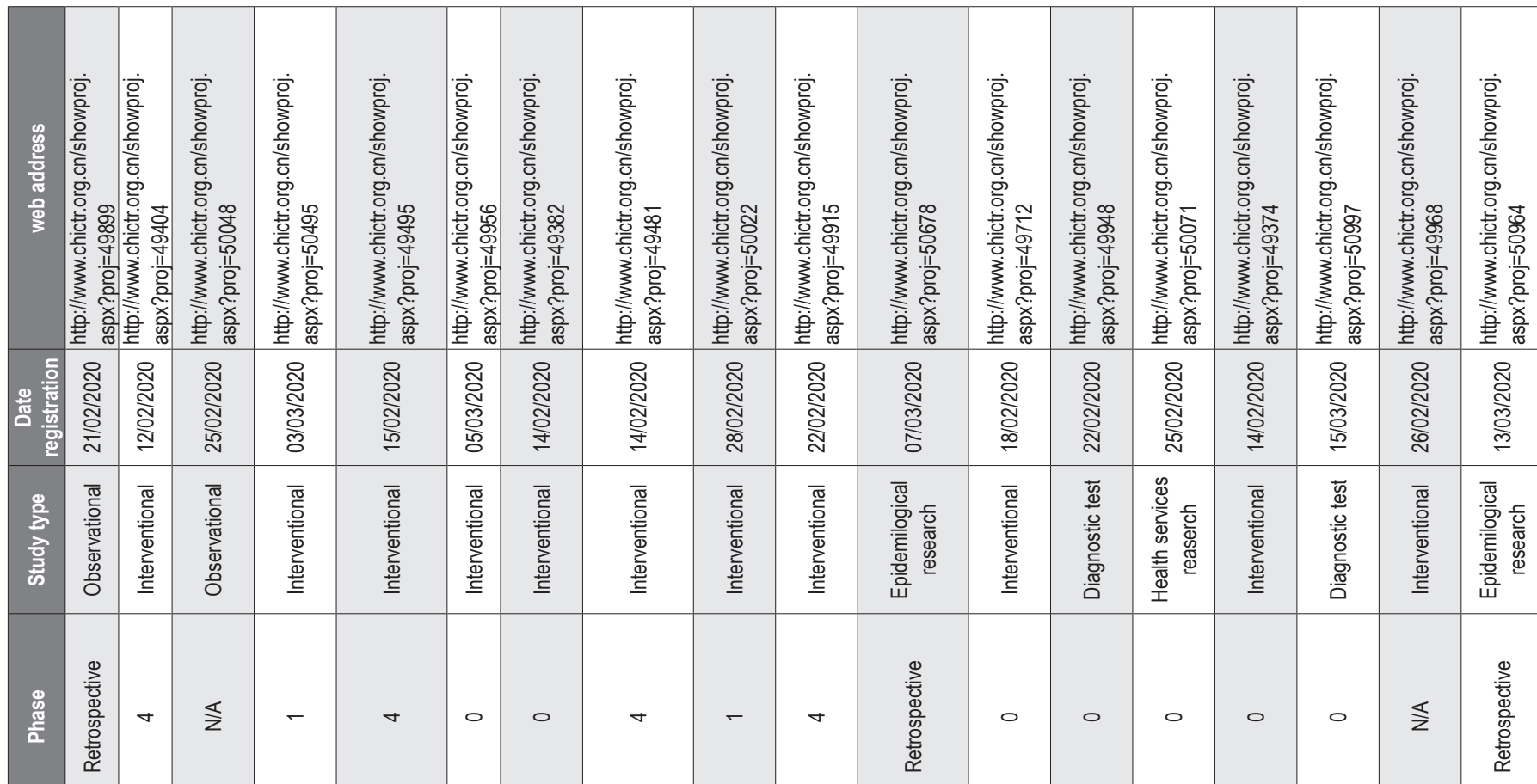

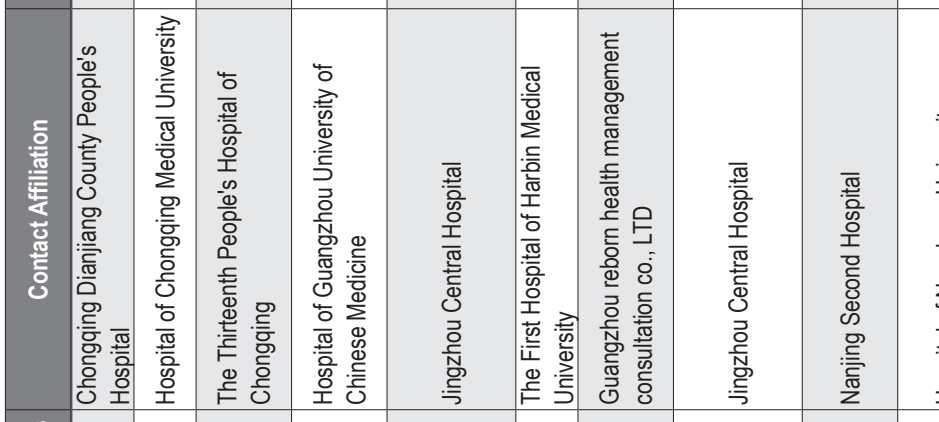

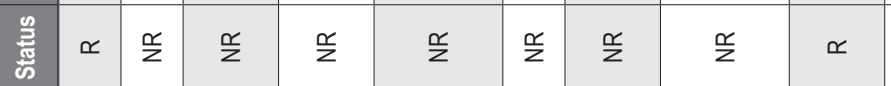

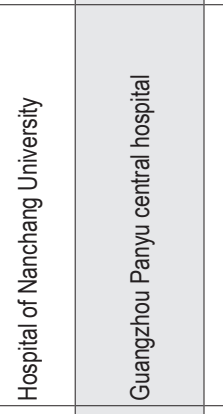

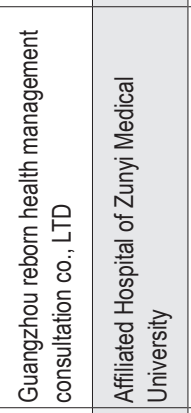

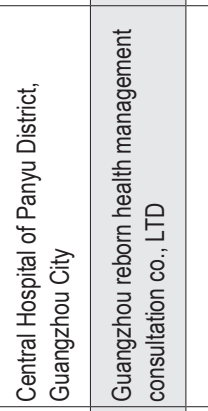

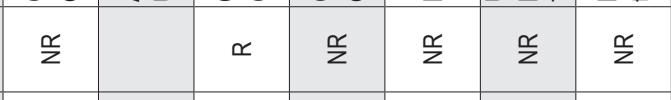

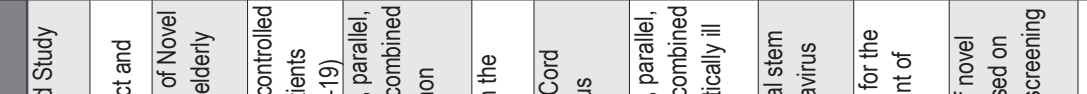

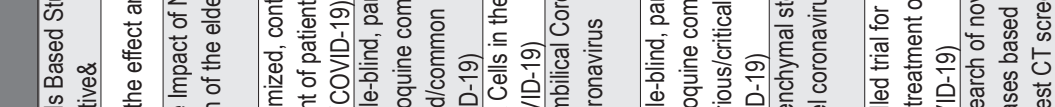

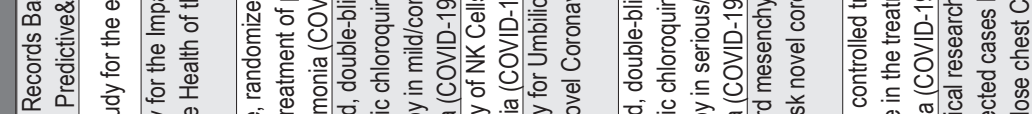

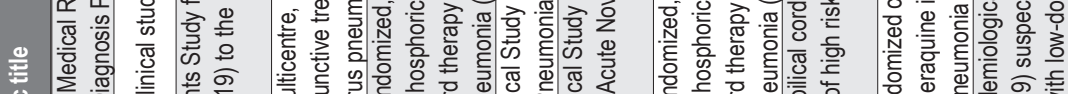

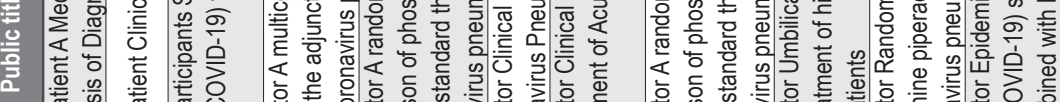

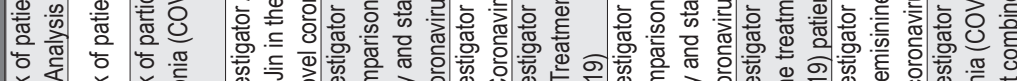

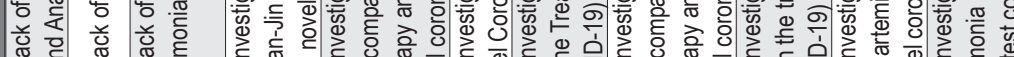

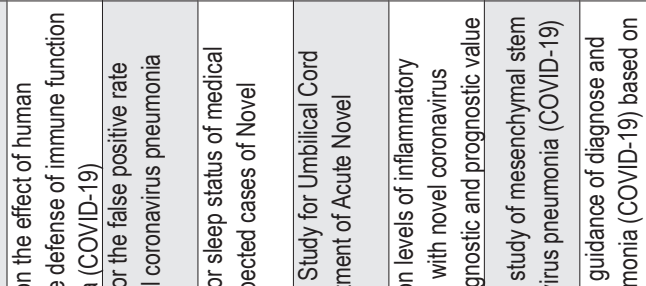

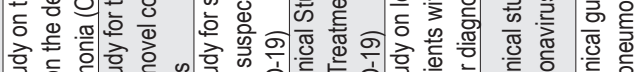

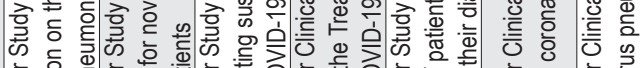

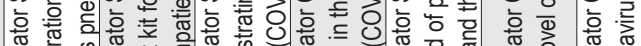

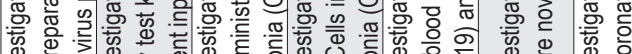

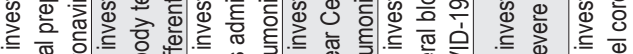

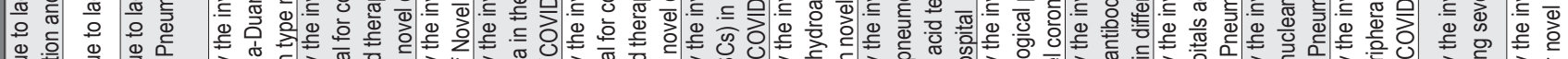

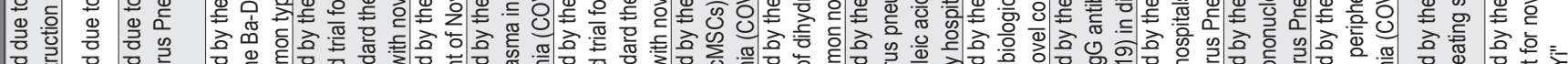

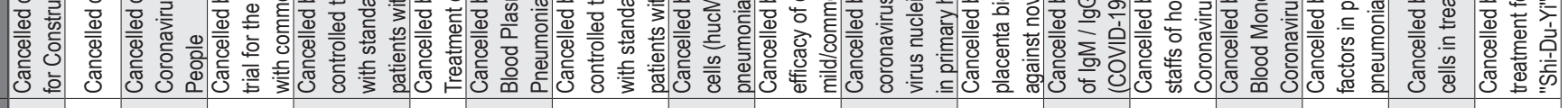

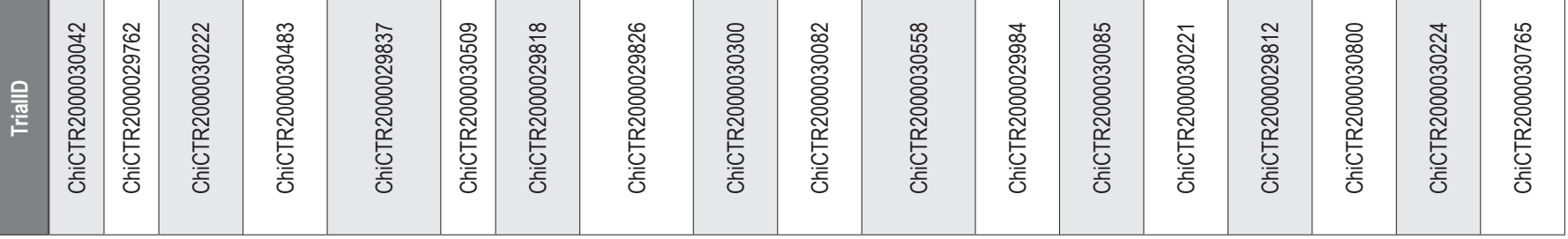




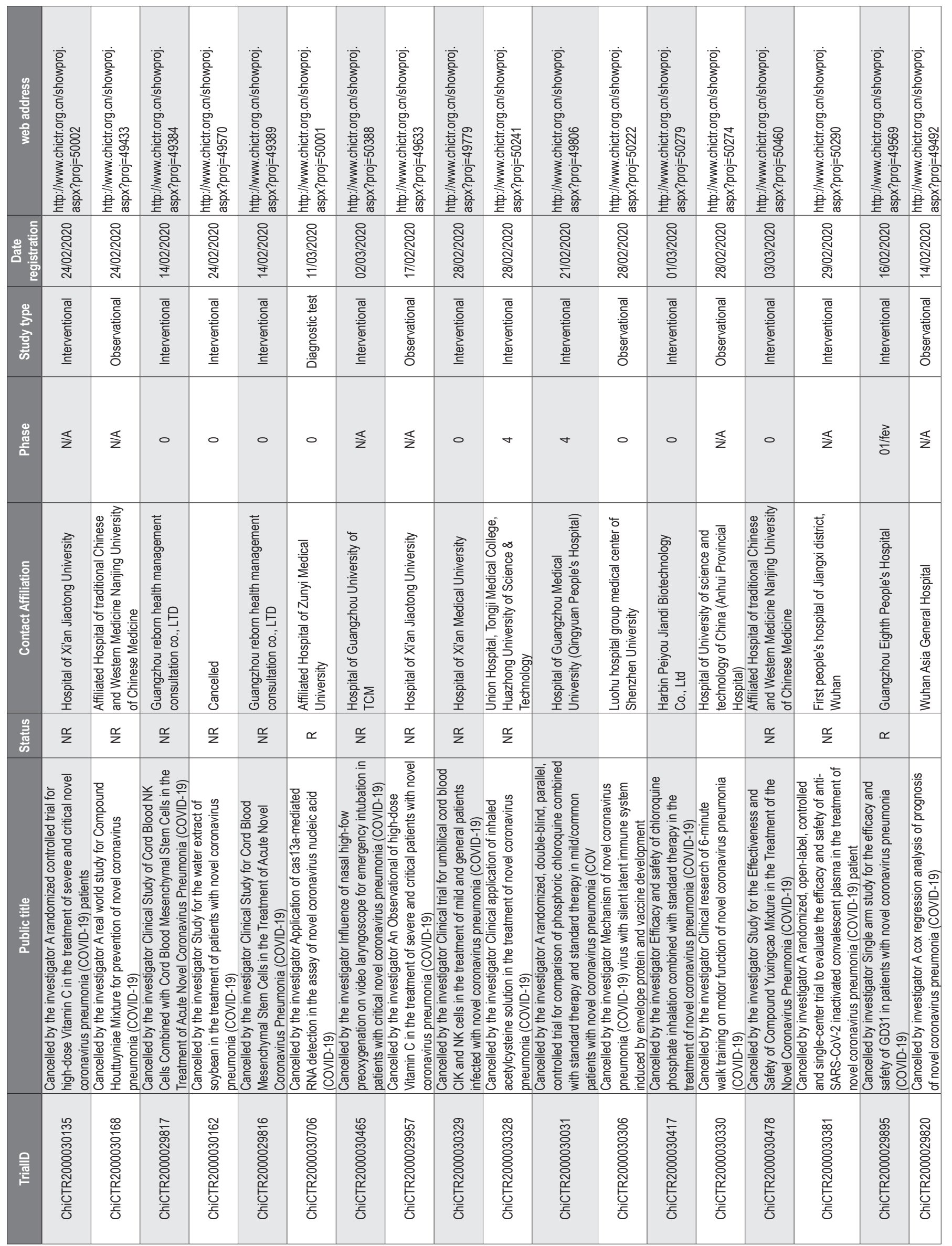




\begin{tabular}{|c|c|c|c|c|c|c|c|c|c|c|c|c|c|c|c|c|c|c|c|c|c|c|}
\hline 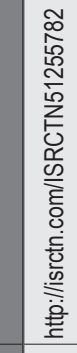 & 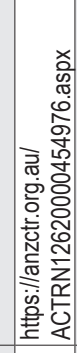 & 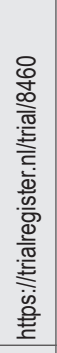 & 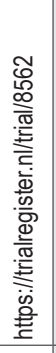 & 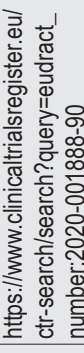 & 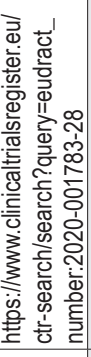 & 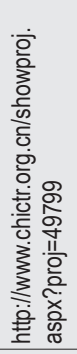 & 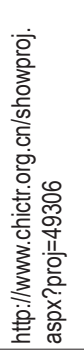 & 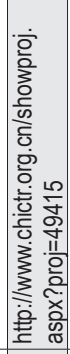 & & 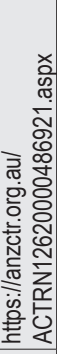 & 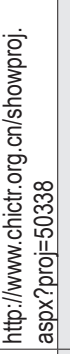 & 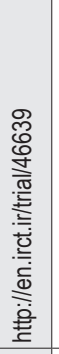 & 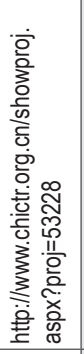 & 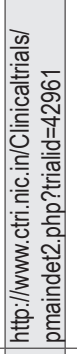 & 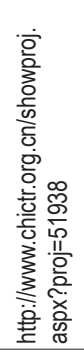 & 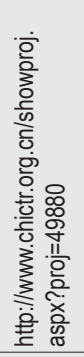 & 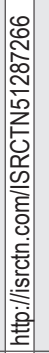 & 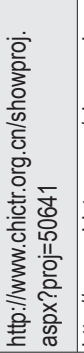 & 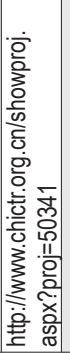 & 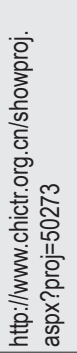 & 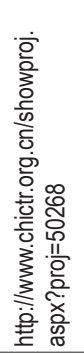 & 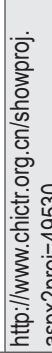 \\
\hline 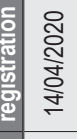 & 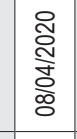 & 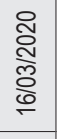 & 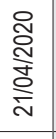 & $\begin{array}{l}\text { Dิ } \\
\text { ज्ञ } \\
\stackrel{0}{0} \\
\end{array}$ & 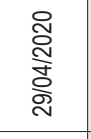 & ญิ๊ & 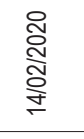 & 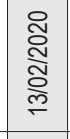 & 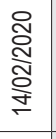 & 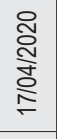 & 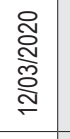 & 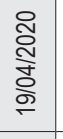 & $\begin{array}{l}\text { ్ㅗ } \\
\text { స్ } \\
\text { 응 }\end{array}$ & 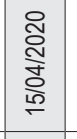 & 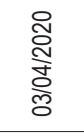 & 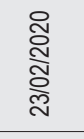 & 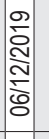 & 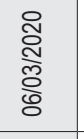 & 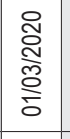 & 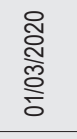 & 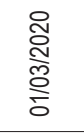 & \\
\hline 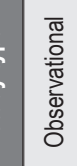 & 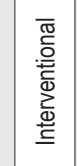 & 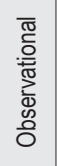 & 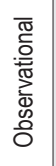 & 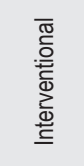 & 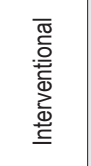 & 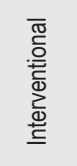 & 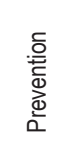 & 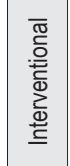 & 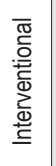 & 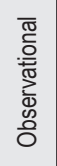 & 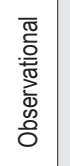 & 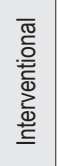 & 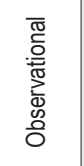 & 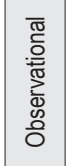 & 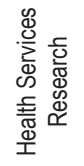 & 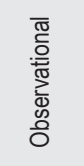 & 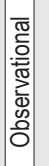 & 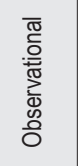 & 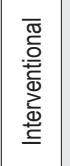 & 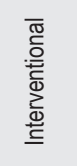 & 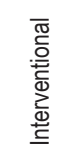 & \\
\hline 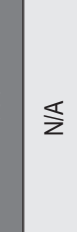 & 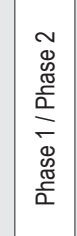 & & & 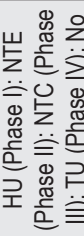 & 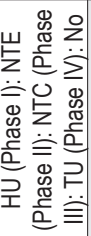 & $\nabla$ & $\stackrel{\$}{z}$ & 0 & $\sigma$ & $\stackrel{\Sigma}{z}$ & $\stackrel{\frac{1}{z}}{ }$ & 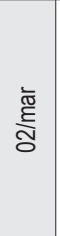 & 0 & $\stackrel{\$}{z}$ & $\stackrel{\mathbb{3}}{z}$ & 0 & $\frac{5}{z}$ & 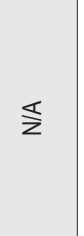 & $\stackrel{\mathbb{1}}{z}$ & - & 0 & \\
\hline
\end{tabular}

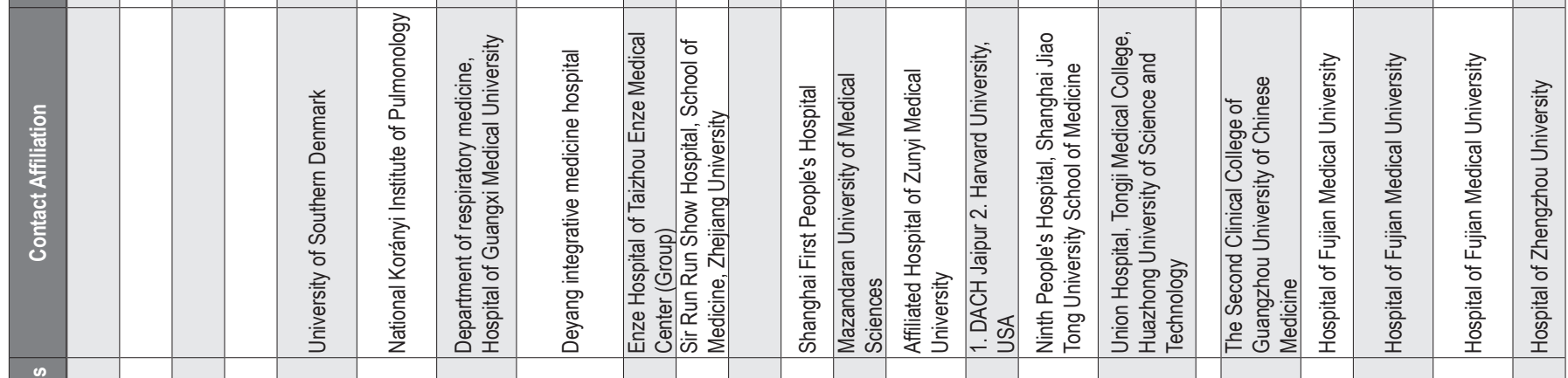

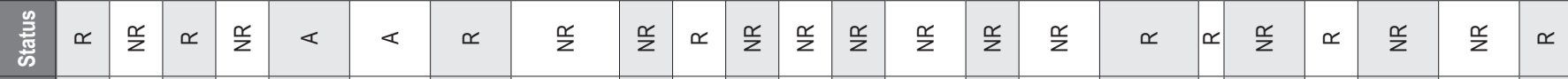

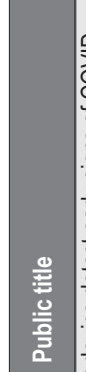

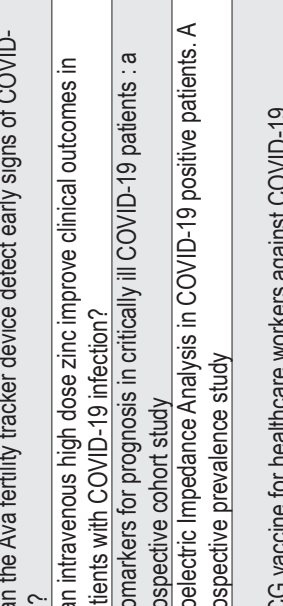

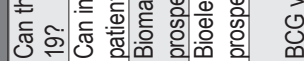

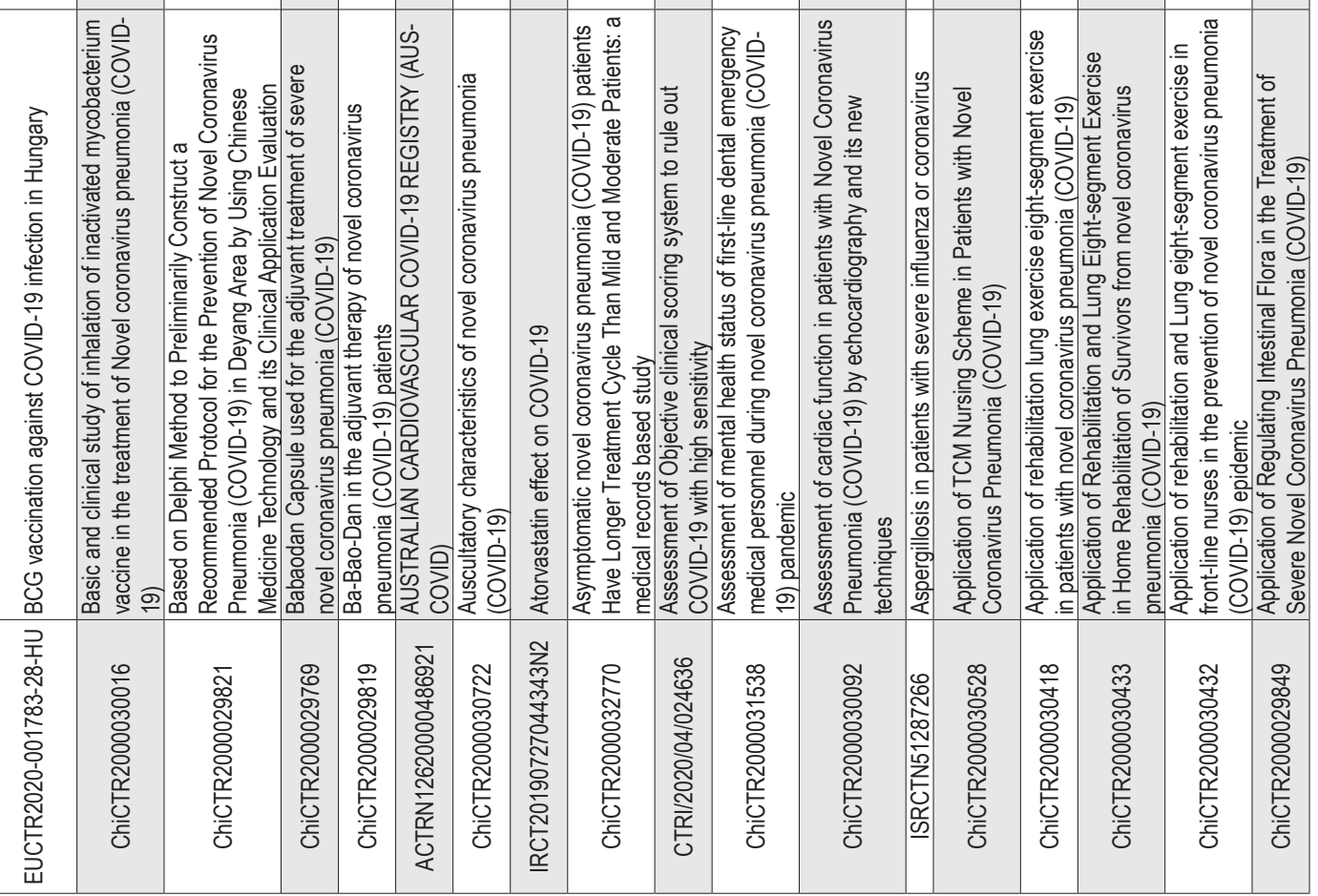




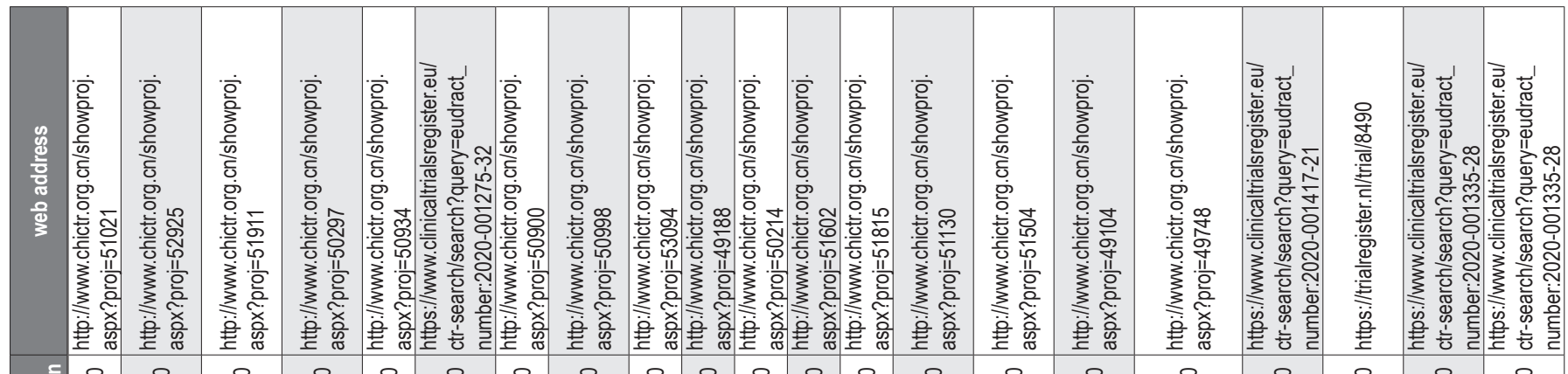

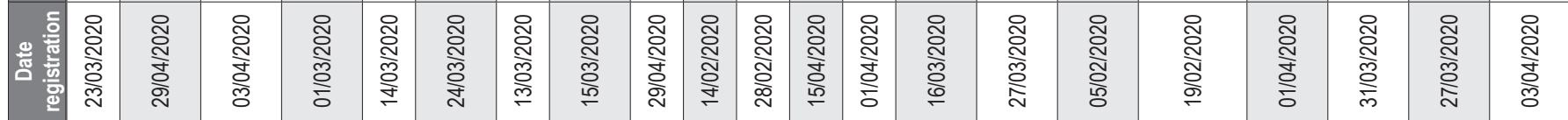

\begin{tabular}{|c|c|c|c|c|c|c|c|c|c|c|c|c|c|c|c|c|c|c|c|c|}
\hline 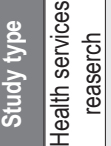 & 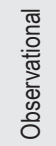 & 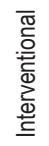 & 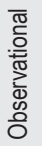 & 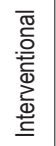 & 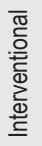 & 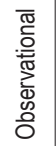 & 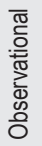 & 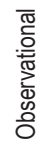 & 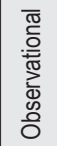 & 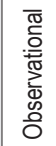 & 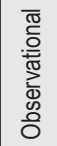 & 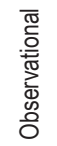 & 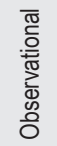 & 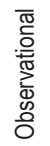 & 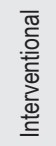 & 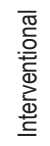 & 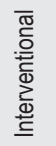 & 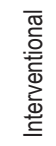 & 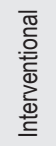 & $\overline{\widetilde{\varpi}}$ \\
\hline
\end{tabular}

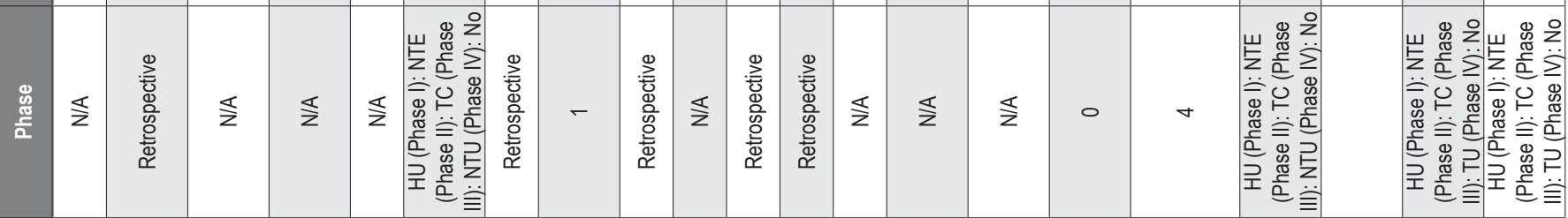

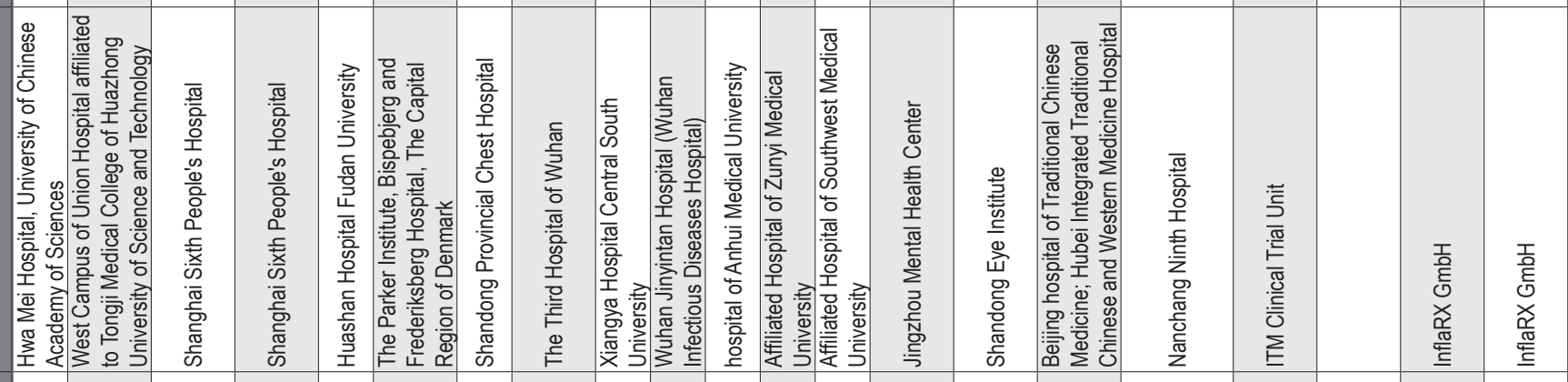

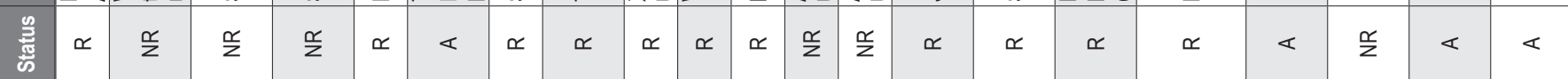

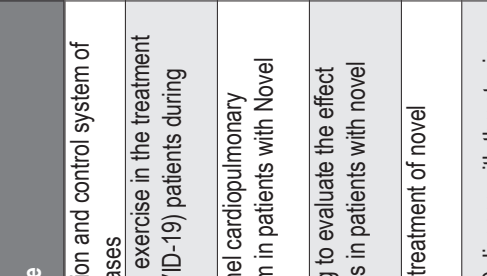

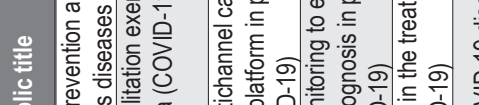

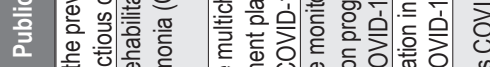

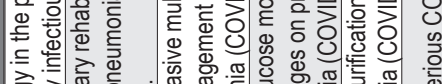

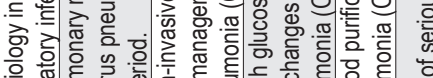
表

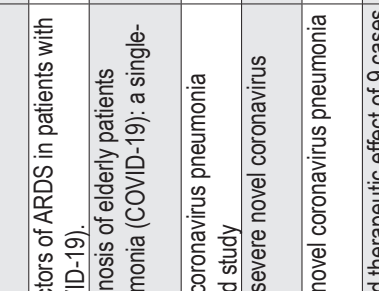

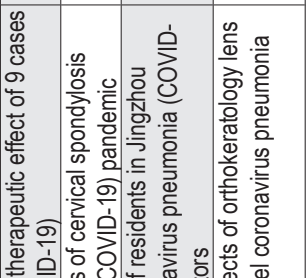

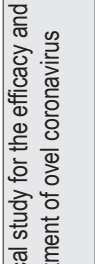

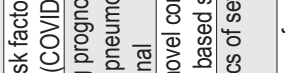

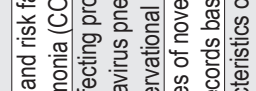

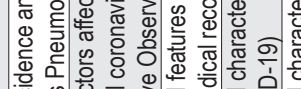

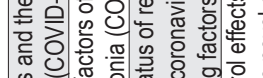

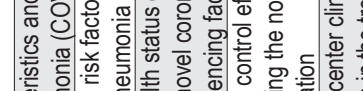

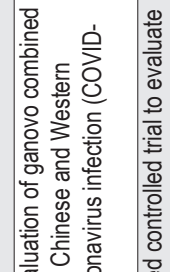

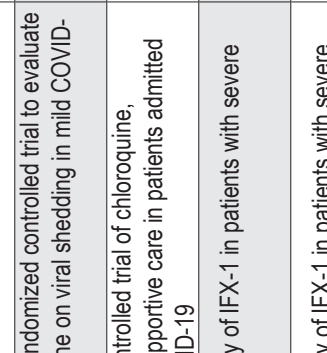

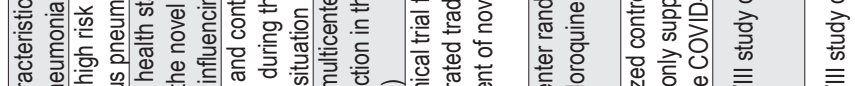

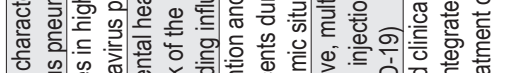

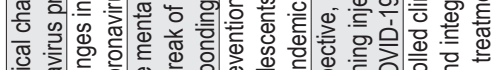

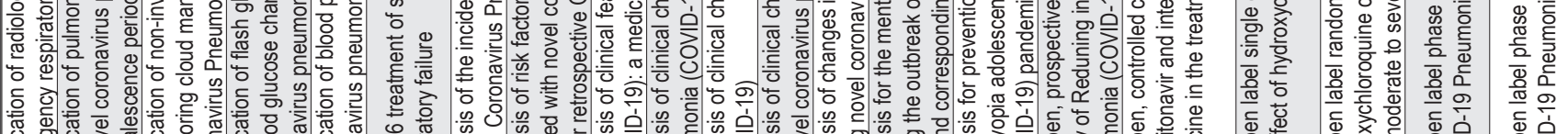

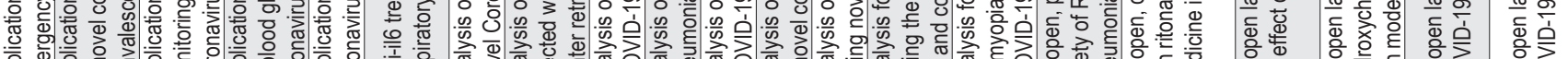

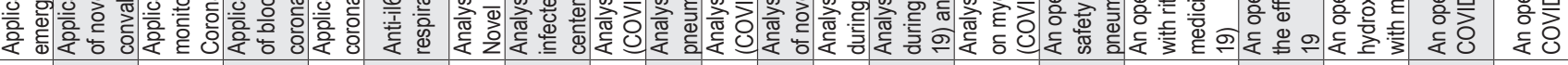

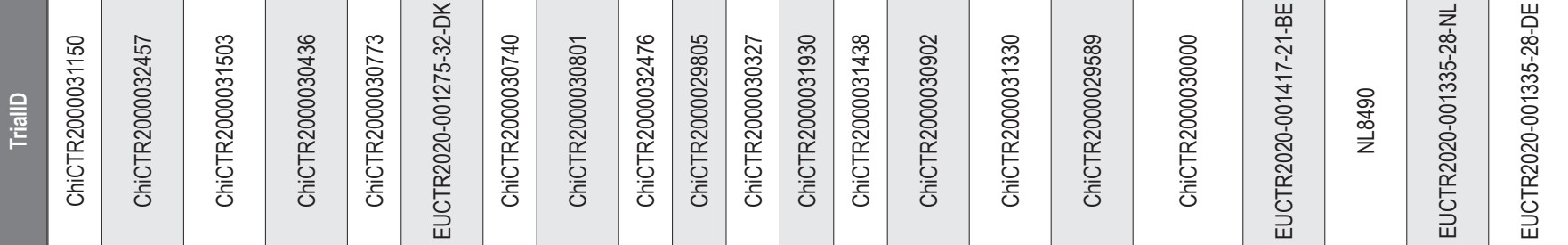




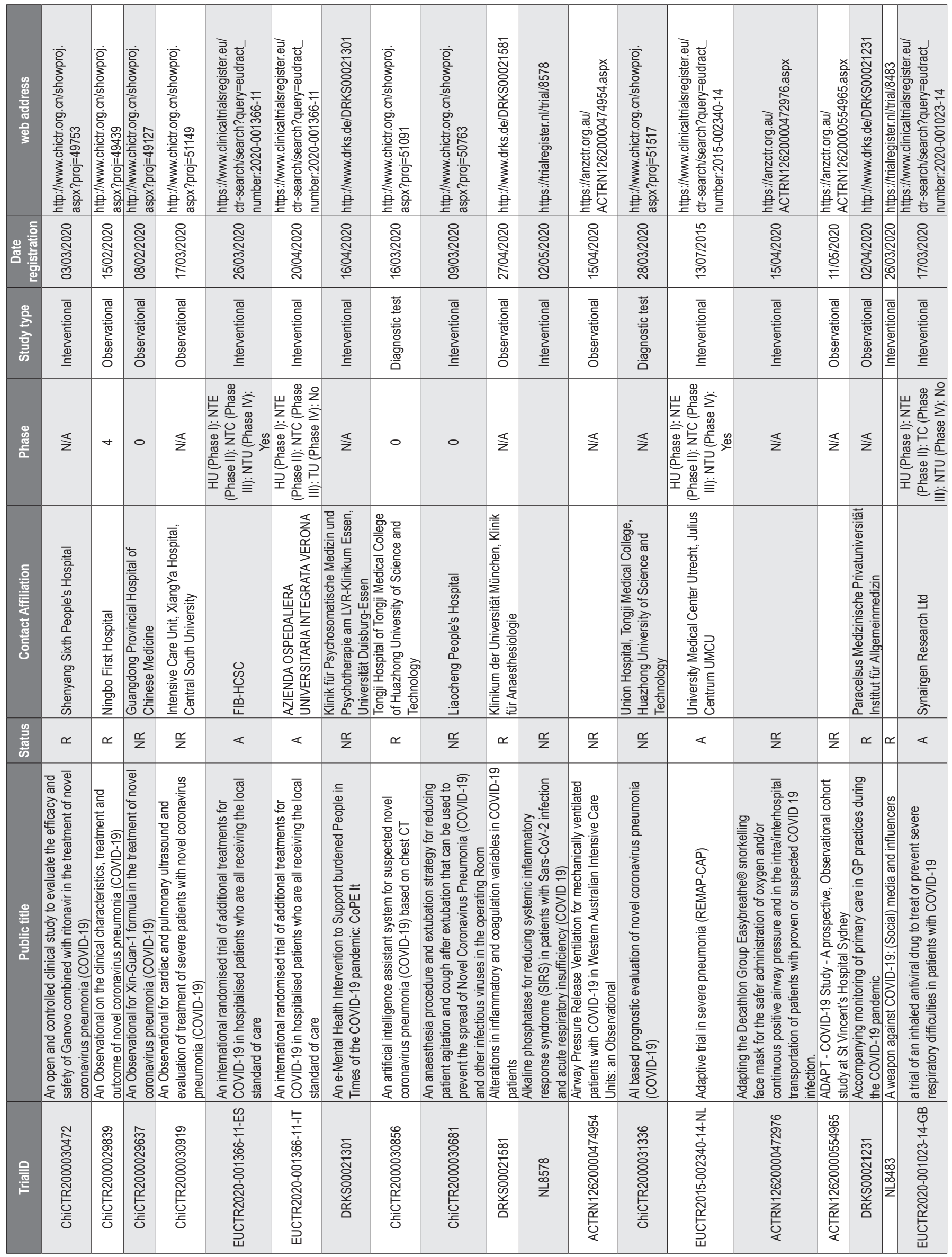




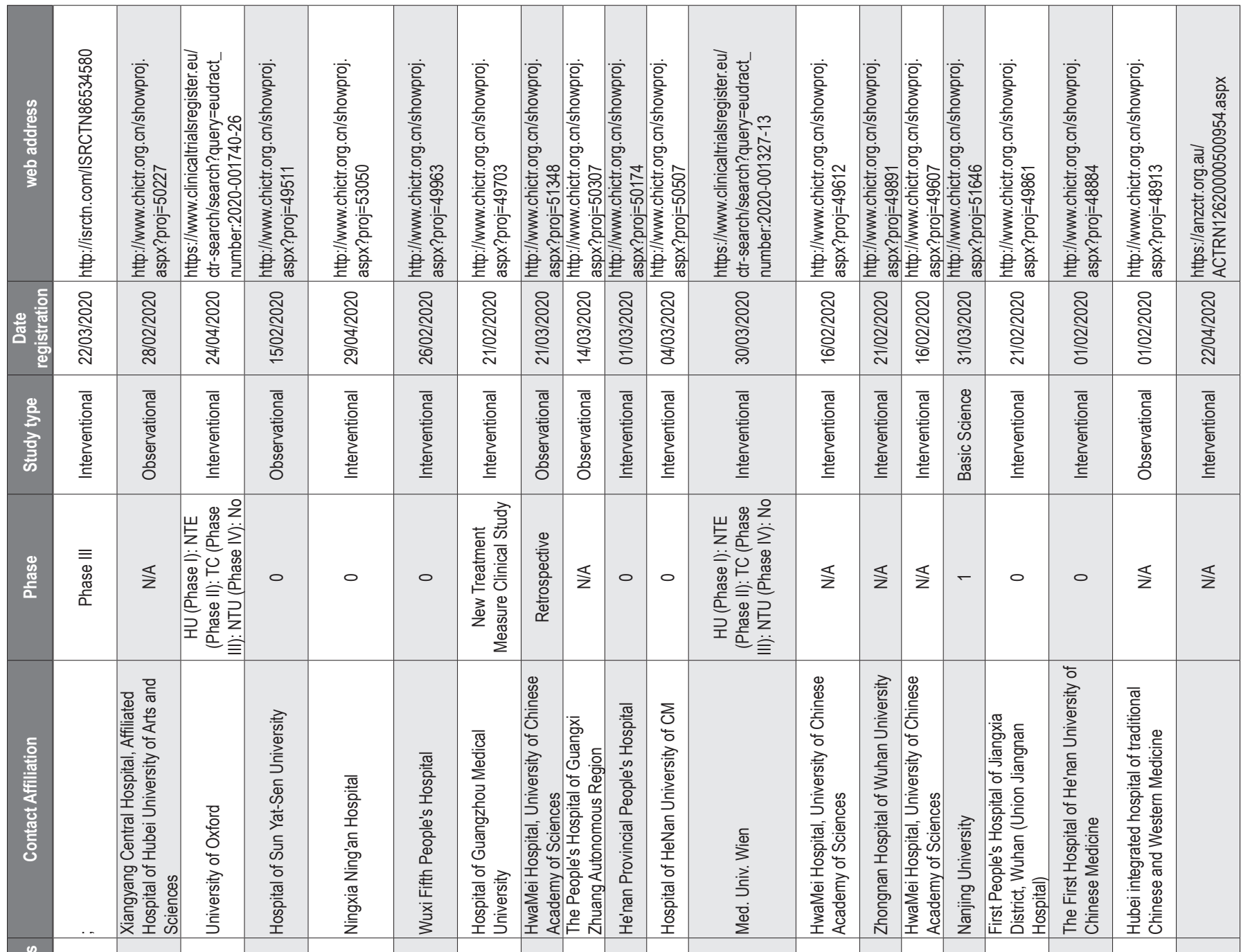

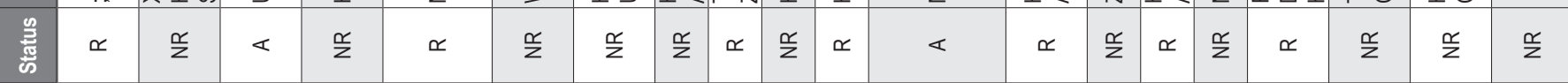

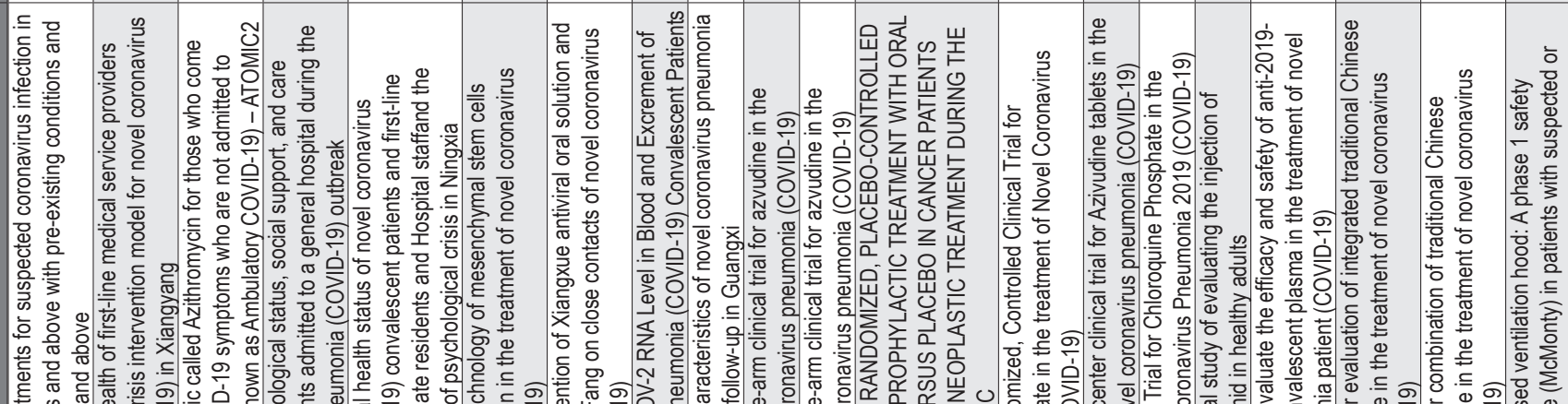

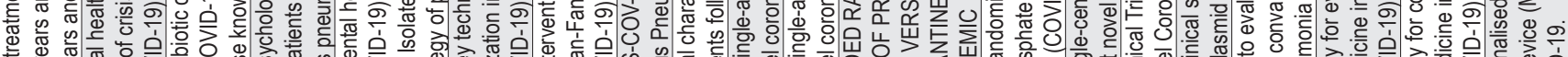

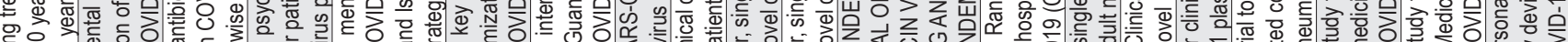
을

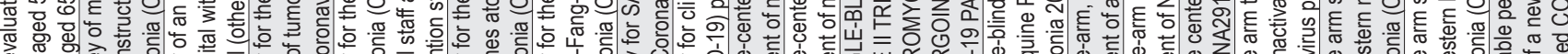

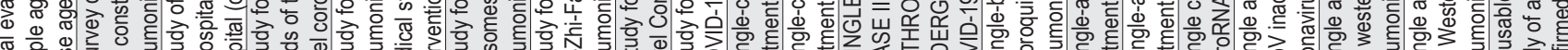

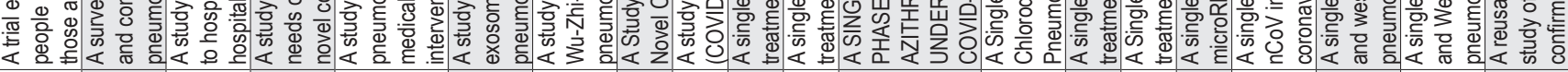

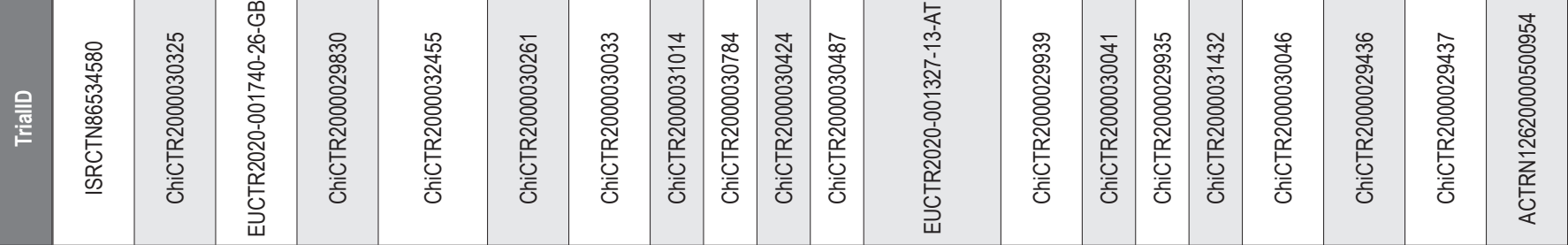




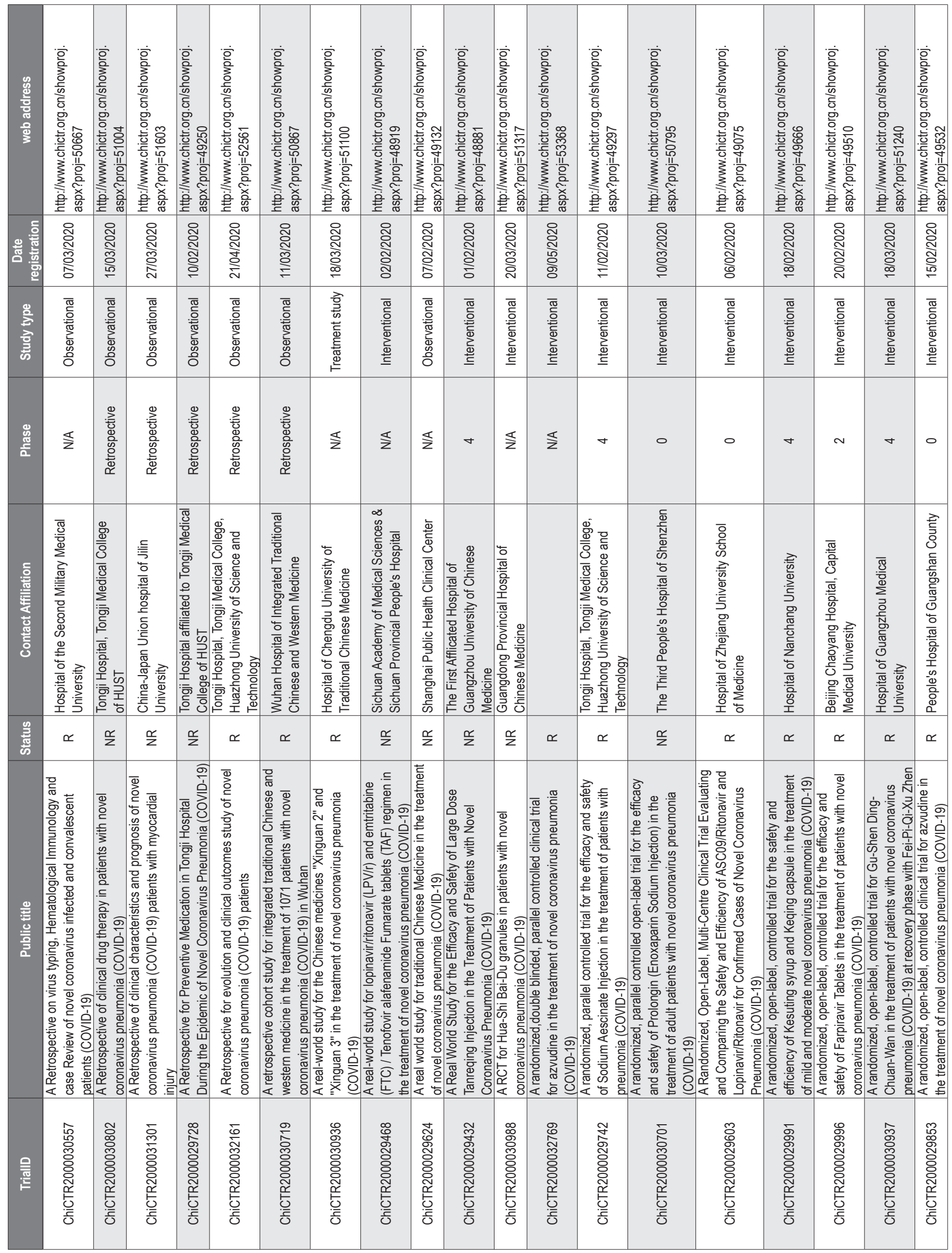




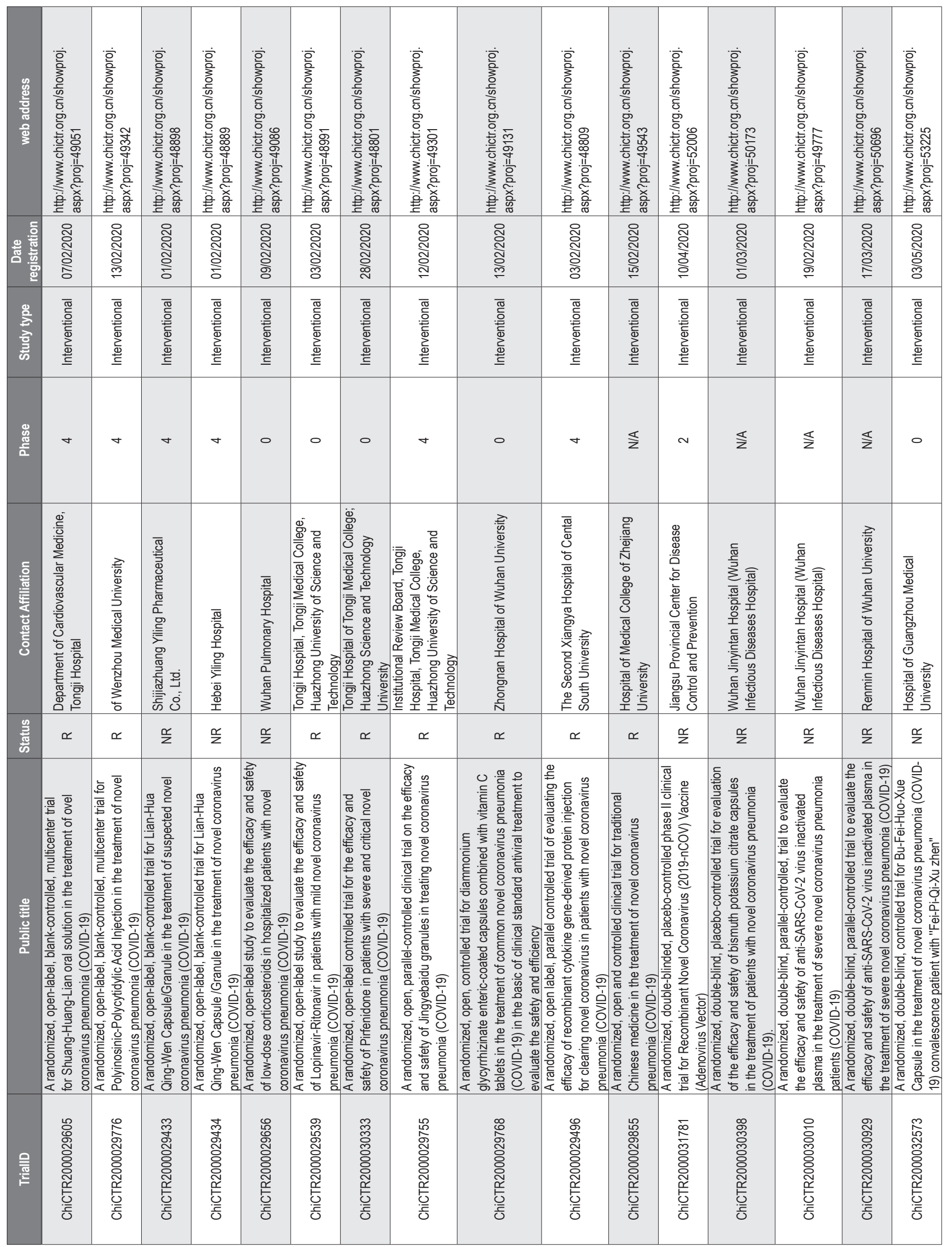




\begin{tabular}{|c|c|c|c|c|c|c|c|c|c|c|c|c|c|c|c|c|c|}
\hline 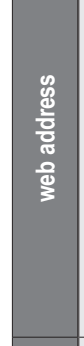 & 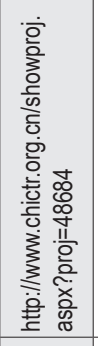 & 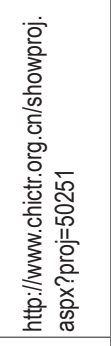 & 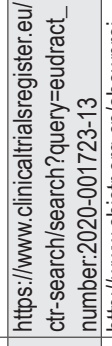 & 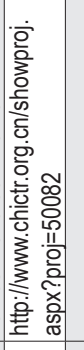 & 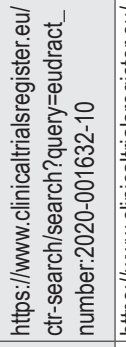 & 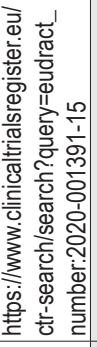 & 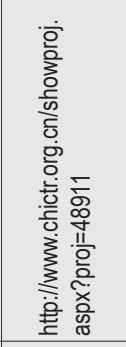 & 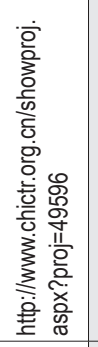 & 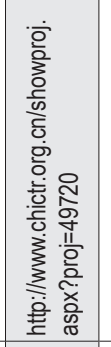 & 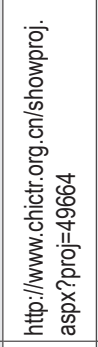 & 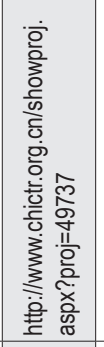 & 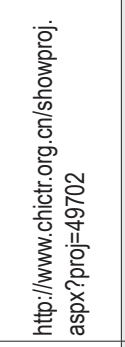 & 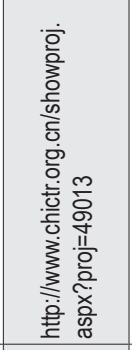 & 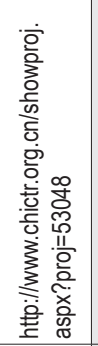 & 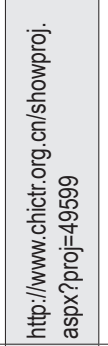 & 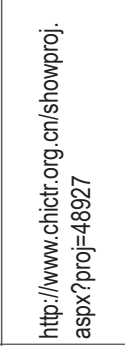 & 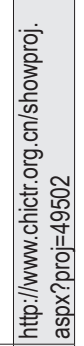 \\
\hline 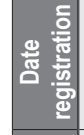 & 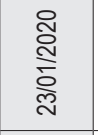 & 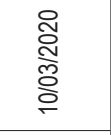 & 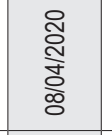 & 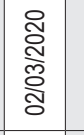 & 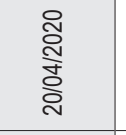 & 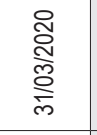 & 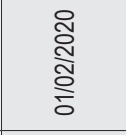 & $\begin{array}{l}\text { ָิ } \\
\text { ญิ } \\
\stackrel{0}{\circ}\end{array}$ & 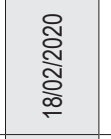 & 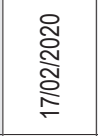 & 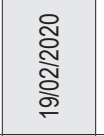 & $\begin{array}{l}\text { ్ㅗ } \\
\text { ָ̃ } \\
\stackrel{\infty}{\circ}\end{array}$ & 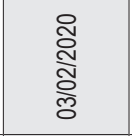 & 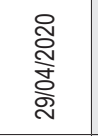 & 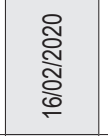 & 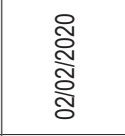 & 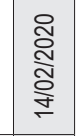 \\
\hline 竞 & 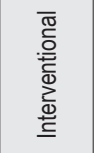 & 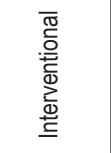 & 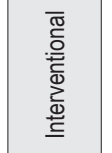 & 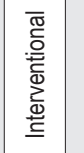 & 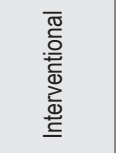 & 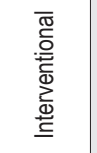 & 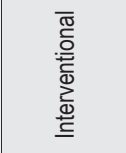 & 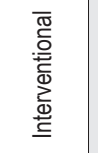 & 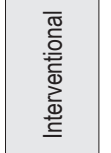 & 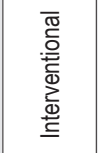 & $\sum_{\substack{\bar{N}\\
}}^{\bar{N}}$ & 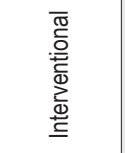 & 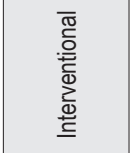 & 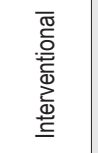 & 离 & 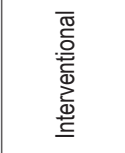 & 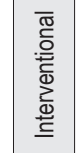 \\
\hline E & $\underline{\mathbf{s}}$ & 0 & 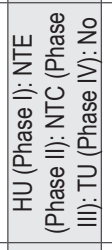 & 0 & 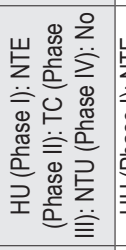 & 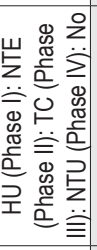 & $\sigma$ & $\underline{\Sigma}$ & $\nabla$ & $\stackrel{3}{z}$ & $\frac{s}{z}$ & $\circ$ & 0 & $\underline{\mathbf{z}}$ & $\stackrel{\mathbb{z}}{z}$ & & \\
\hline 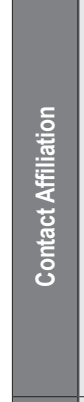 & 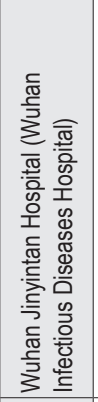 & 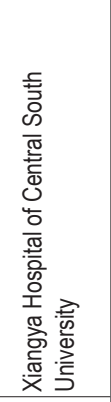 & 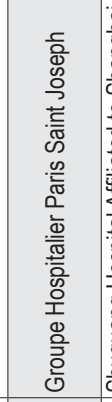 & 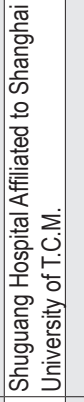 & 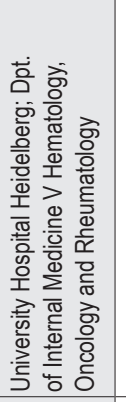 & 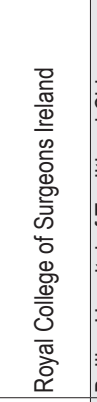 & 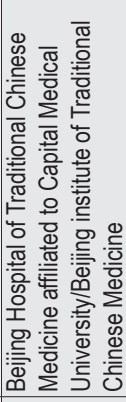 & 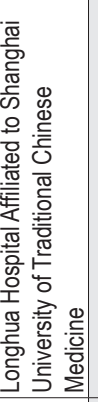 & 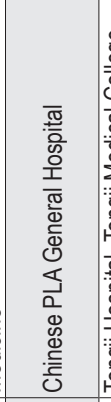 & 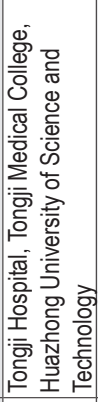 & 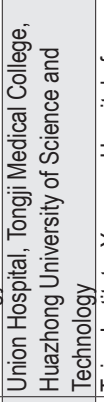 & 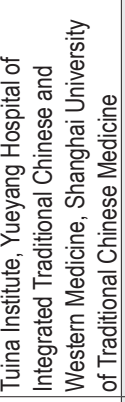 & 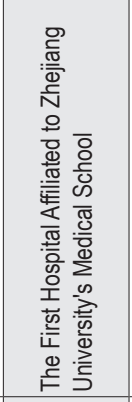 & 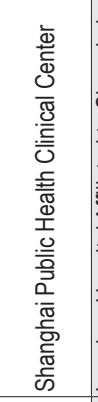 & 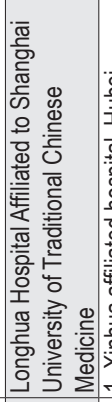 & 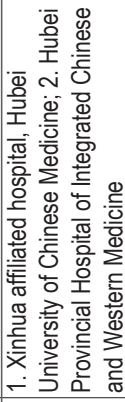 & 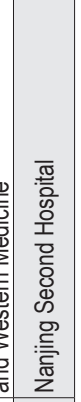 \\
\hline 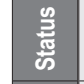 & $\propto$ & $\propto$ & $\ll$ & $\propto$ & $\ll$ & $\ll$ & $\frac{\alpha}{z}$ & $\frac{\mathscr{c}}{z}$ & $\frac{\alpha}{z}$ & $\propto$ & $\propto$ & $\frac{\alpha}{z}$ & i & $\frac{\underline{c}}{z}$ & $\frac{\alpha}{z}$ & & $\propto$ \\
\hline 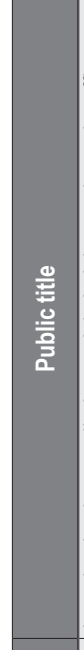 & 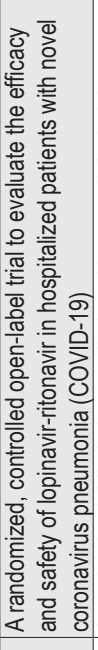 & 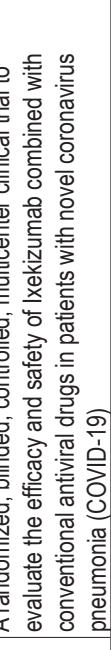 & 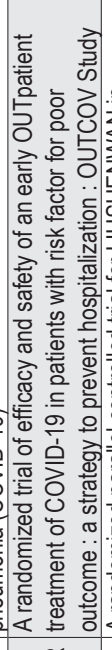 & 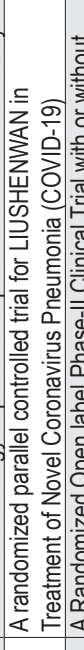 & 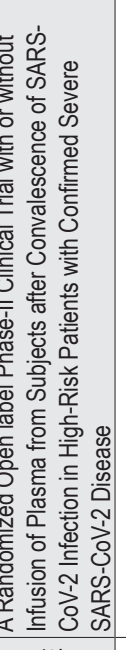 & 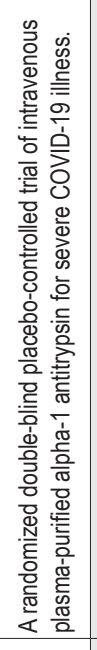 & 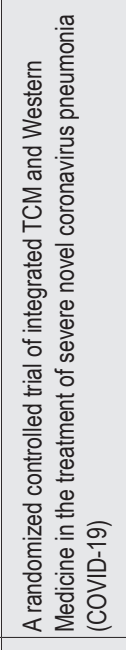 & 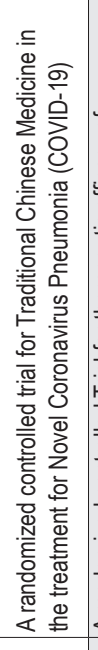 & 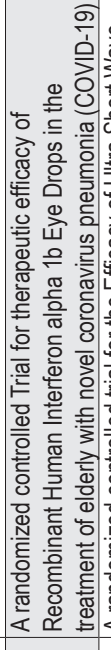 & 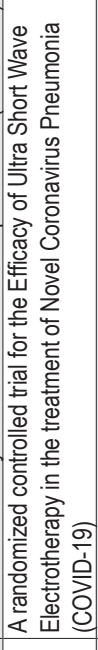 & 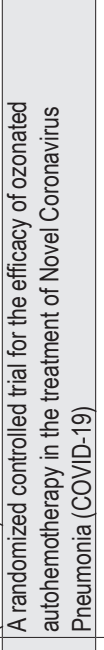 & 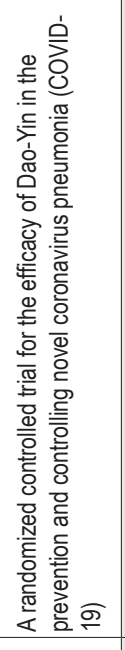 & 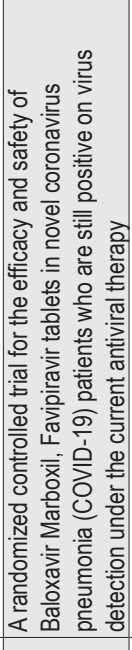 & 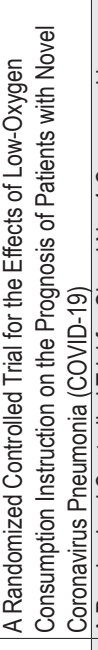 & 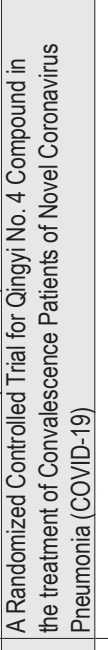 & 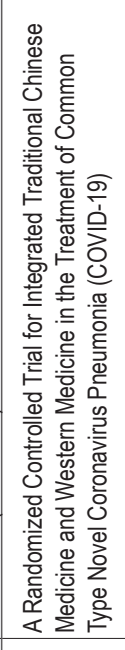 & 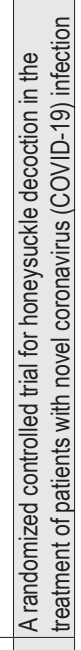 \\
\hline 言 & 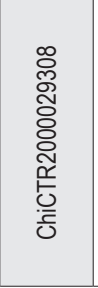 & 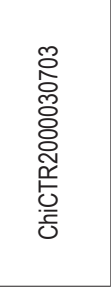 & 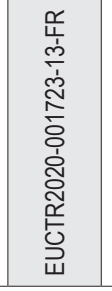 & 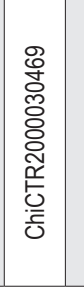 & 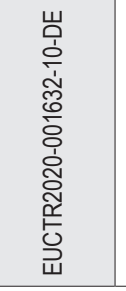 & 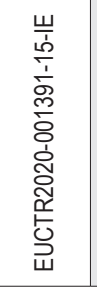 & 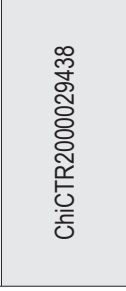 & 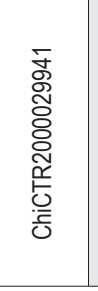 & 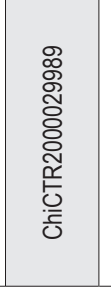 & 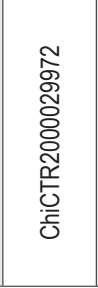 & 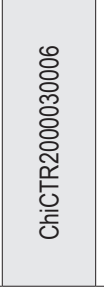 & 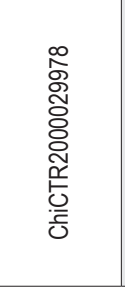 & 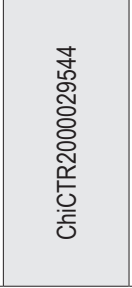 & 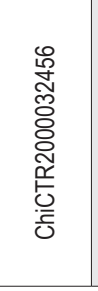 & 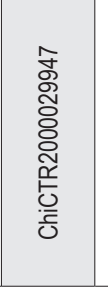 & 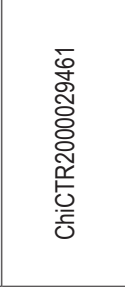 & 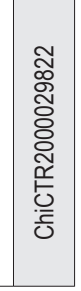 \\
\hline
\end{tabular}




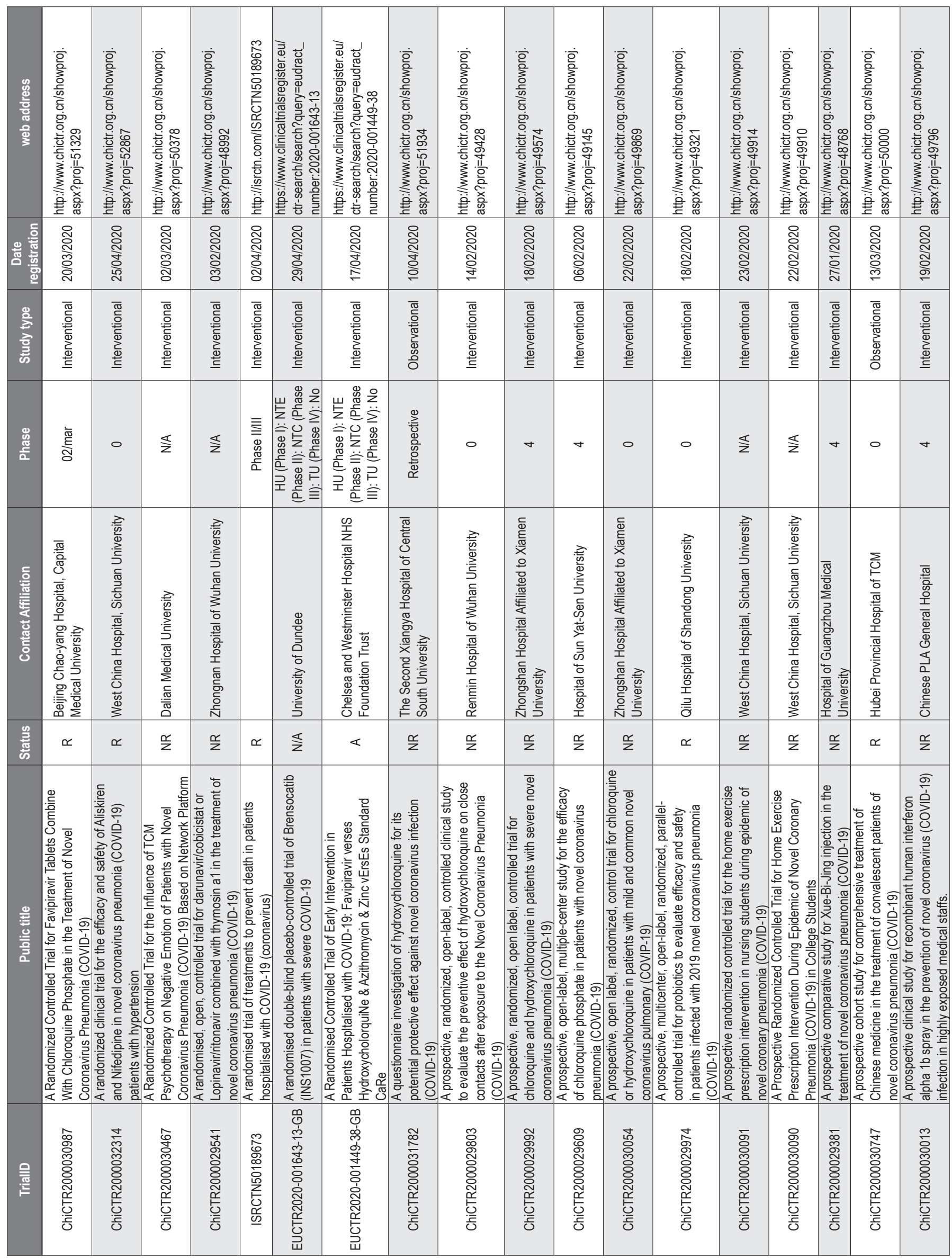




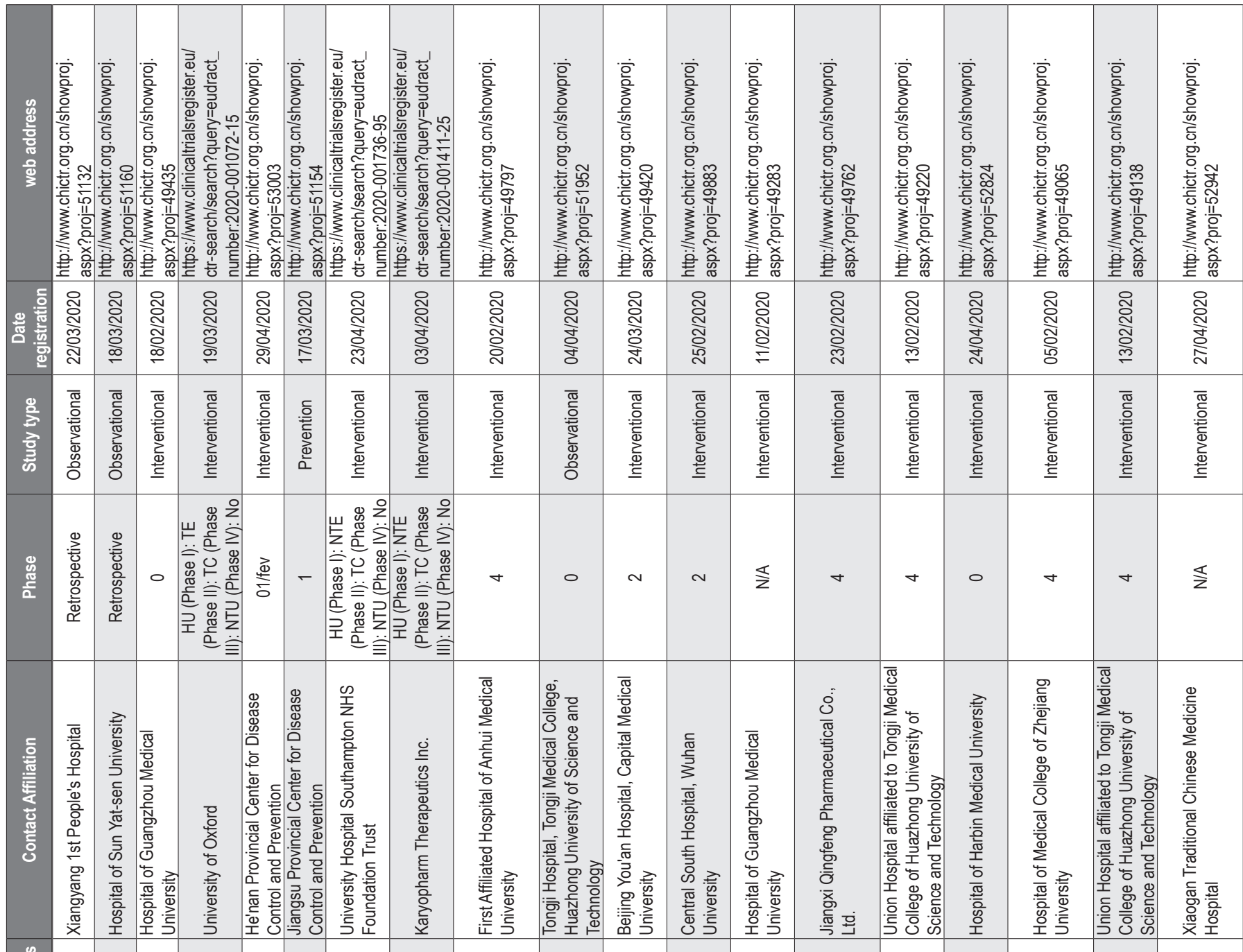

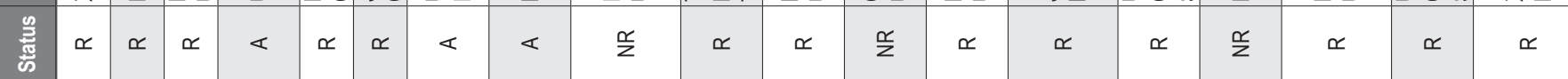

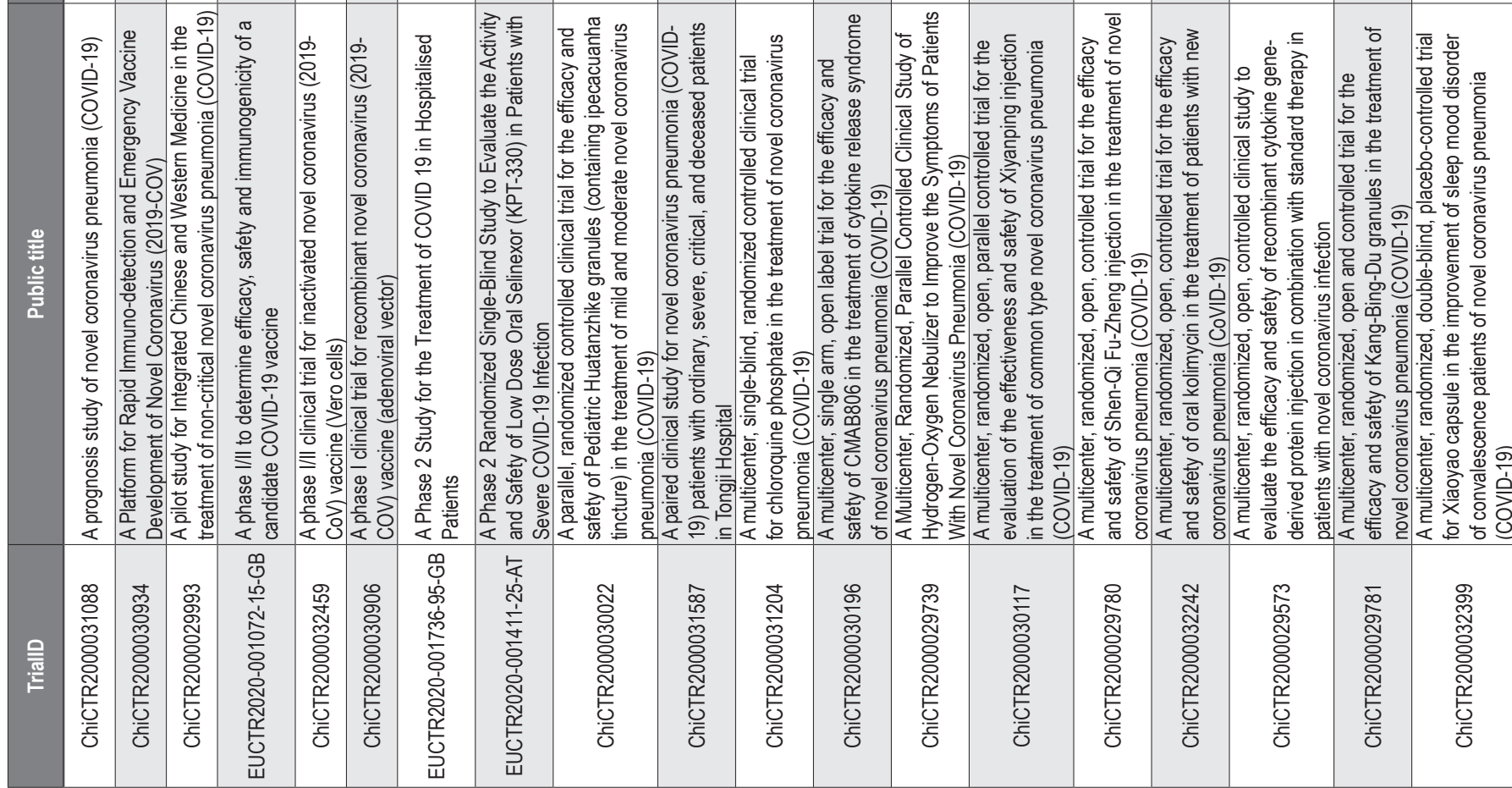




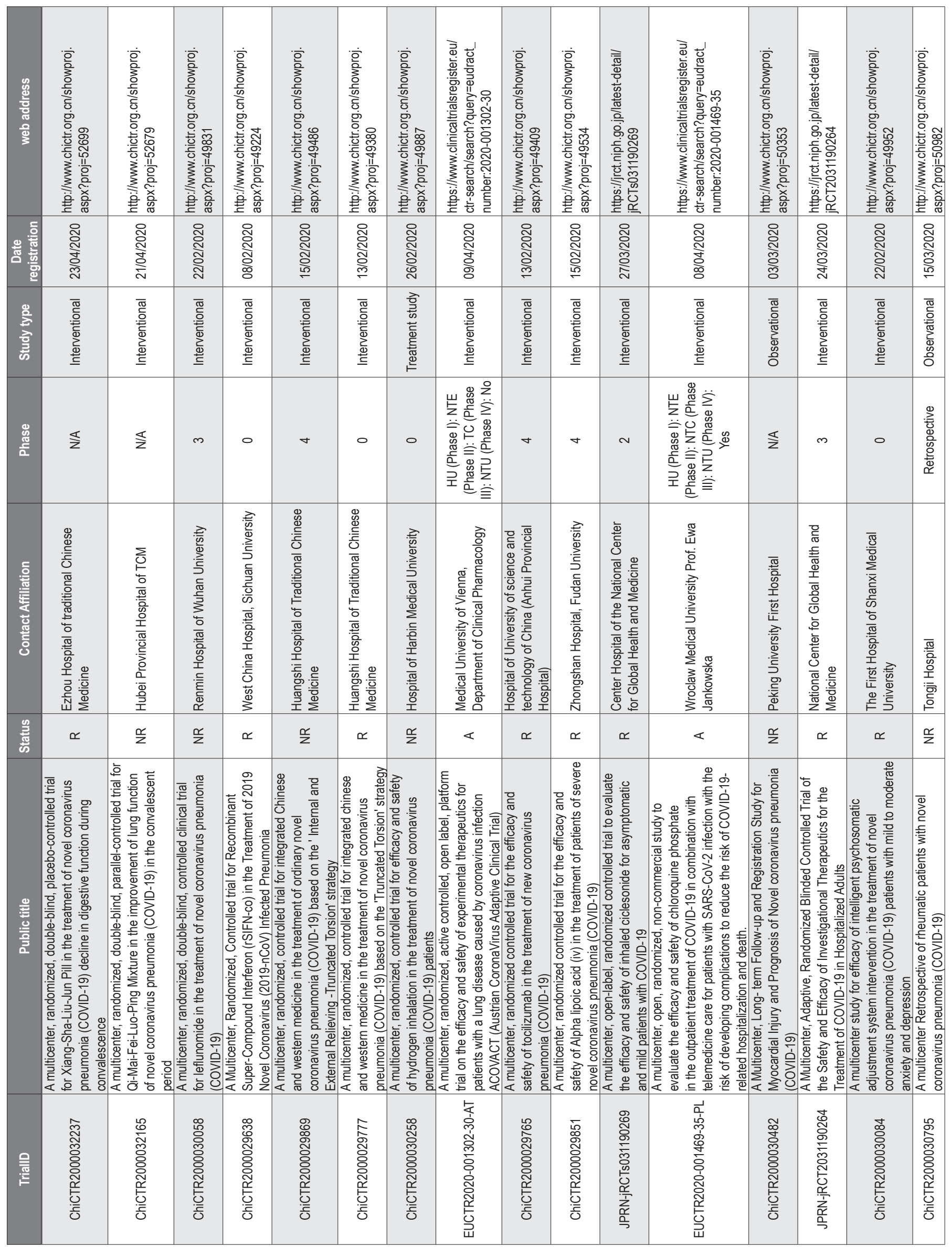




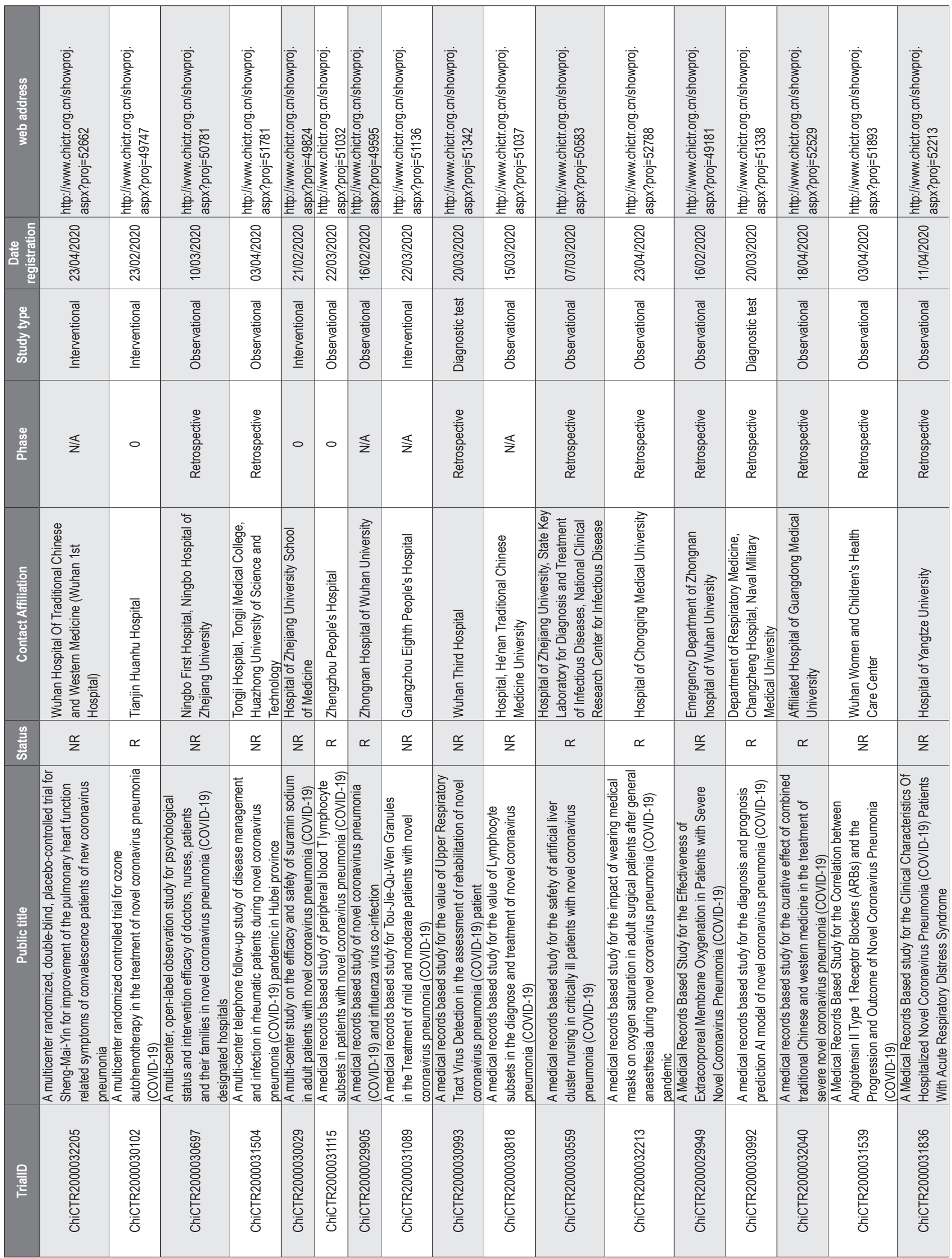




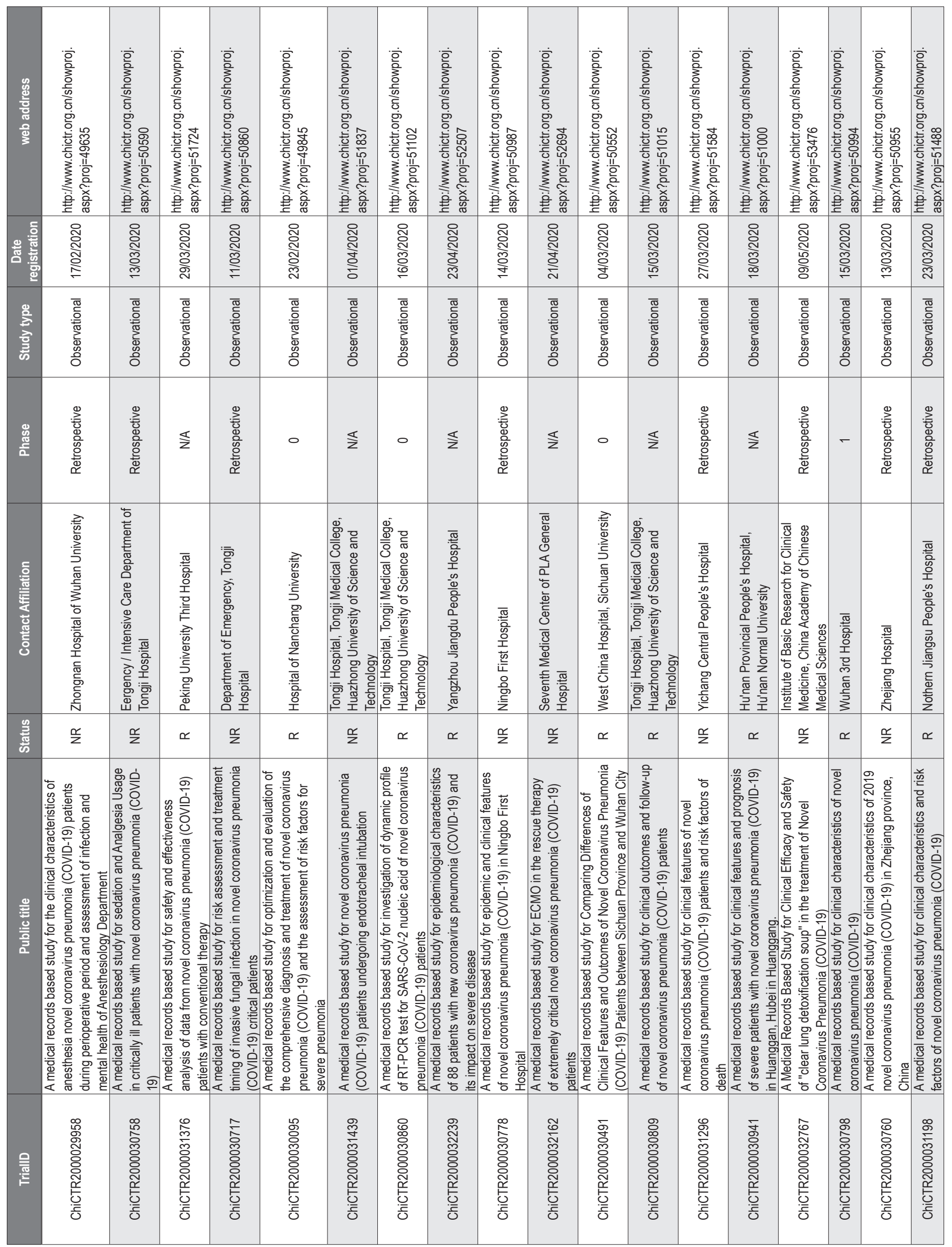




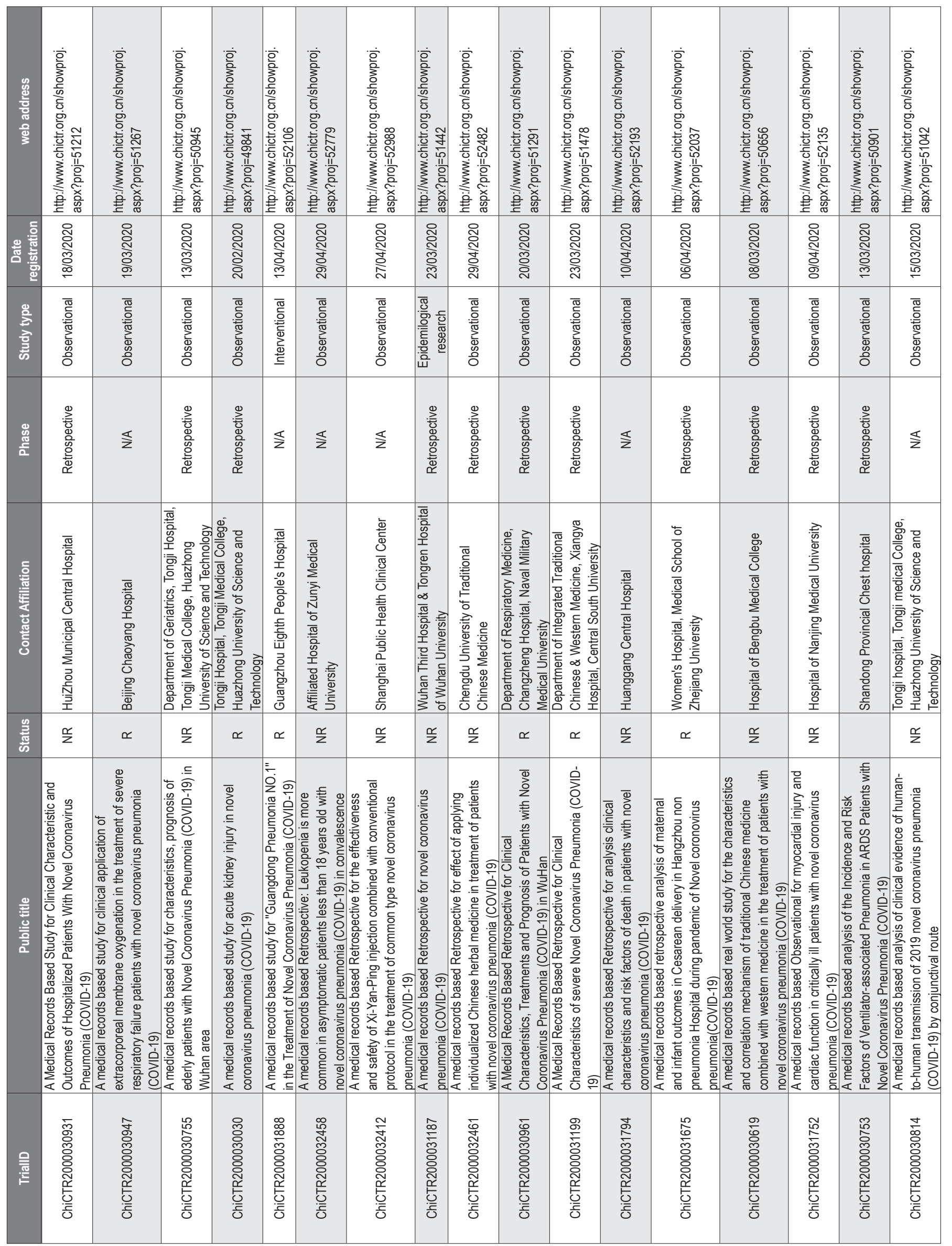




\begin{tabular}{|c|c|c|c|c|c|c|c|c|c|c|c|c|c|c|c|c|c|c|}
\hline 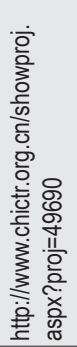 & 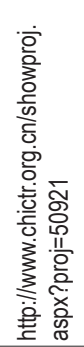 & 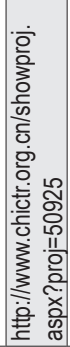 & 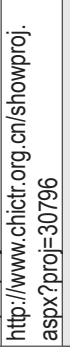 & 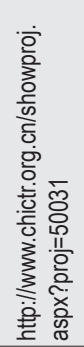 & 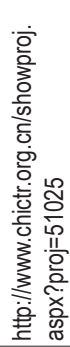 & 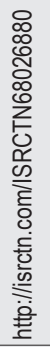 & 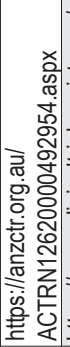 & 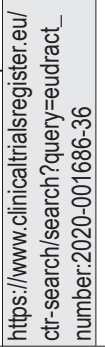 & 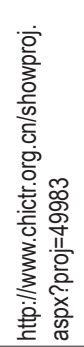 & 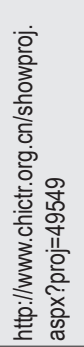 & 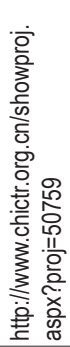 & 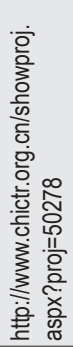 & 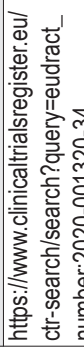 & 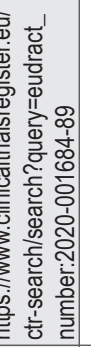 & 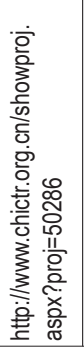 & 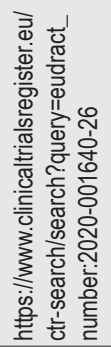 & 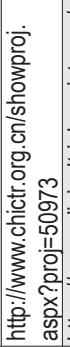 & 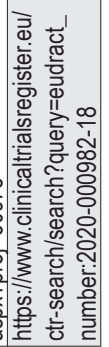 \\
\hline 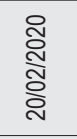 & 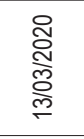 & 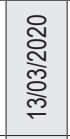 & 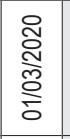 & 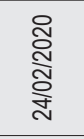 & 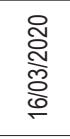 & 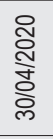 & 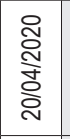 & 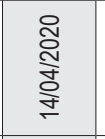 & 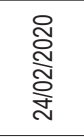 & 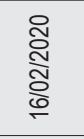 & $\begin{array}{l}\text { 芯 } \\
\stackrel{N}{\tilde{N}} \\
\text { N }\end{array}$ & 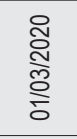 & 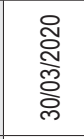 & 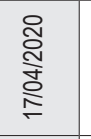 & 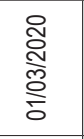 & $\begin{array}{l}\text { ్․ } \\
\text { ฐิ } \\
\text { 옹 }\end{array}$ & 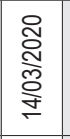 & 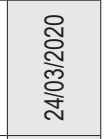 \\
\hline 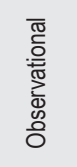 & 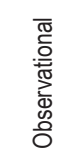 & 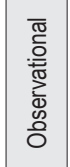 & 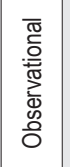 & 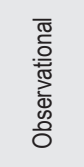 & 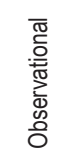 & 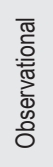 & 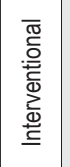 & 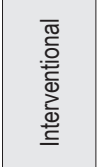 & 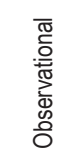 & 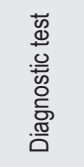 & 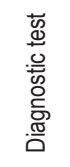 & 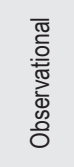 & 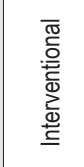 & 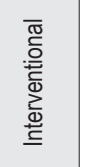 & 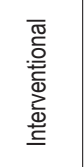 & 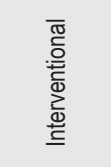 & 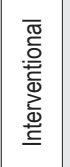 & 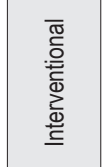 \\
\hline 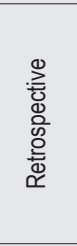 & 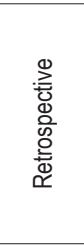 & 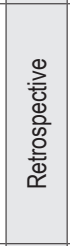 & 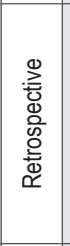 & 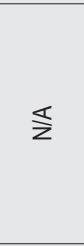 & $\stackrel{s}{z}$ & $\underline{\mathbf{z}}$ & 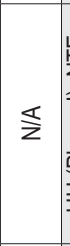 & 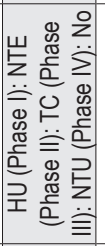 & $\stackrel{5}{z}$ & 0 & 0 & 0 & 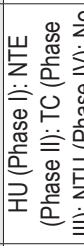 & 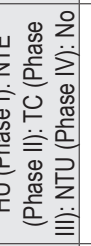 & $\stackrel{\$}{z}$ & 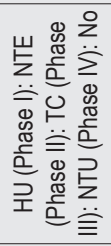 & + & 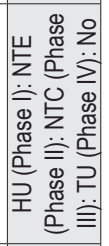 \\
\hline
\end{tabular}

\begin{tabular}{|c|c|c|c|c|c|c|c|c|c|c|c|c|}
\hline 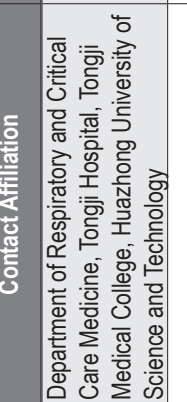 & 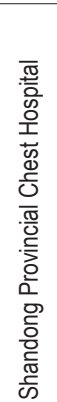 & 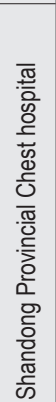 & 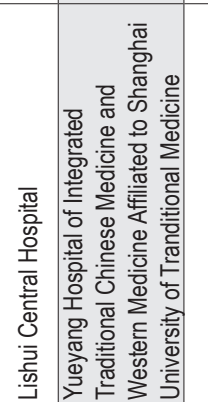 & 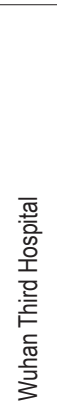 & $\bar{\tau}$ & 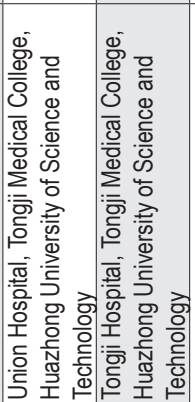 & 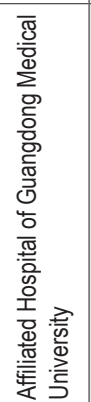 & 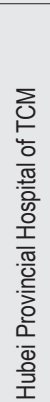 & 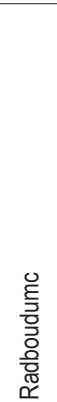 & 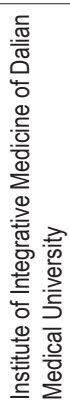 & 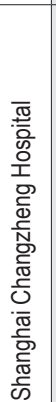 & 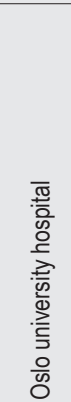 \\
\hline
\end{tabular}

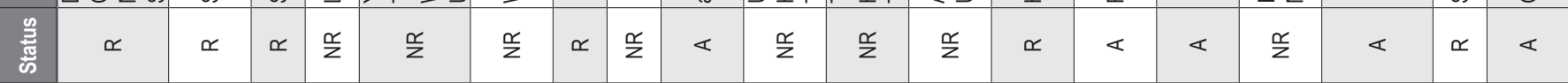

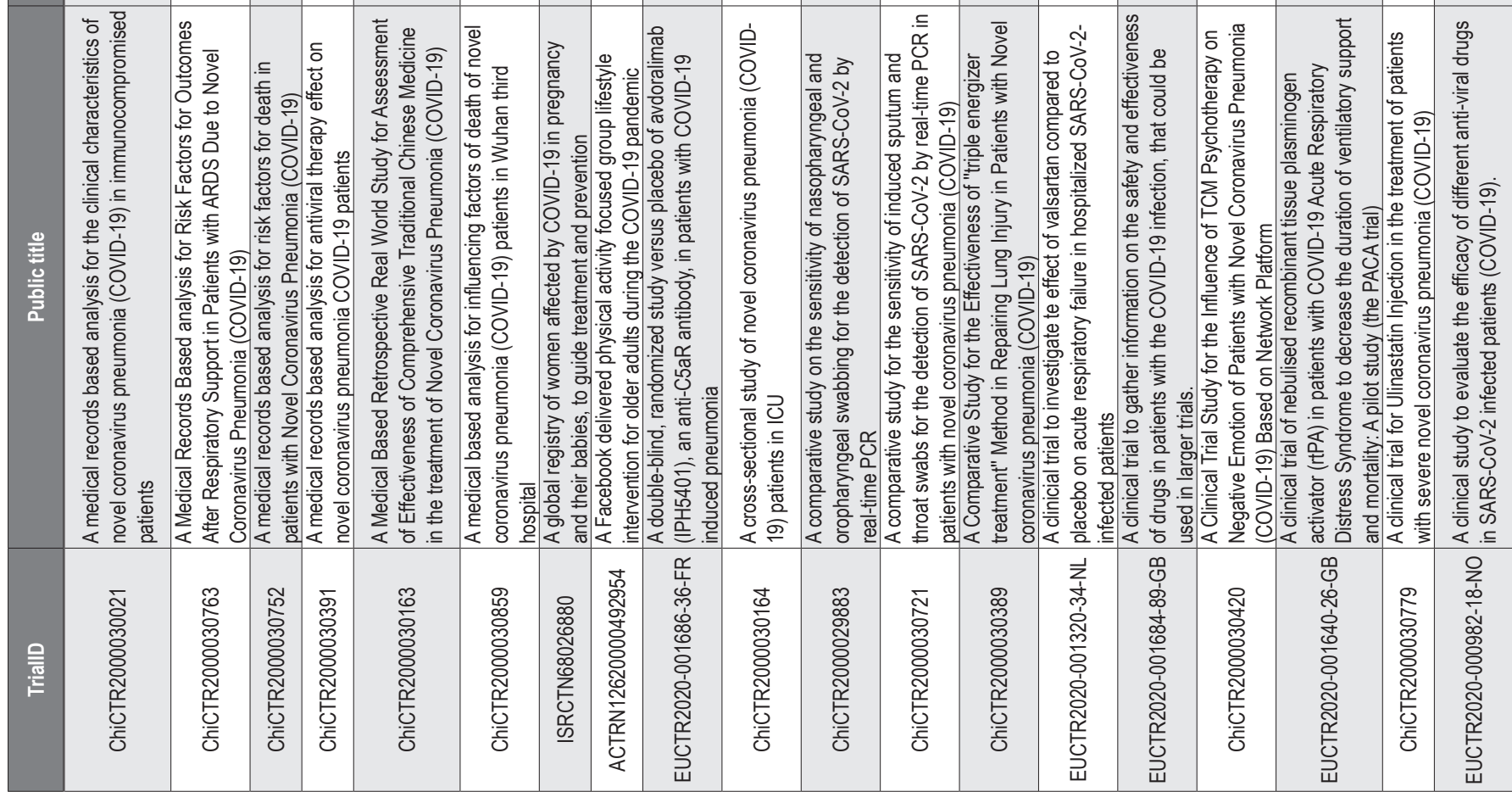

